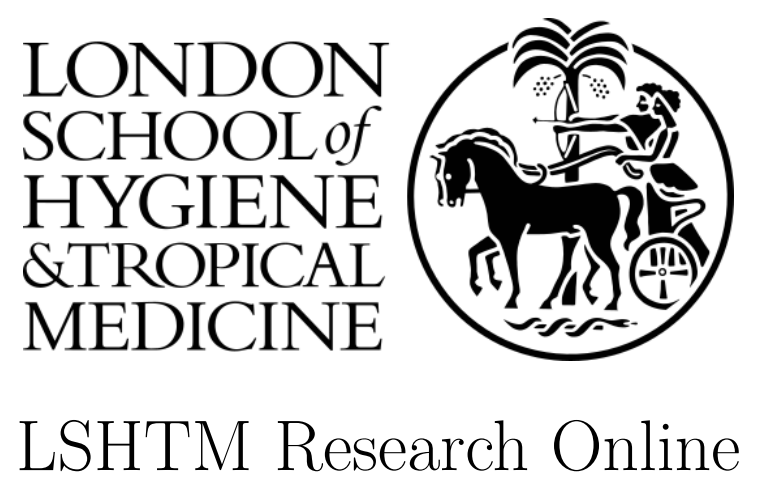

Cresswell, F; (2021) Improving the Diagnosis and Treatment of HIV-associated Tuberculous Meningitis. PhD (research paper style) thesis, London School of Hygiene \& Tropical Medicine. DOI: https://doi.org/10.17037/PUBS.04660748

Downloaded from: https://researchonline.lshtm.ac.uk/id/eprint/4660748/

DOI: https://doi.org/10.17037/PUBS.04660748

Usage Guidelines:

Please refer to usage guidelines at https://researchonline.lshtm.ac.uk/policies.html or alternatively contact researchonline@lshtm.ac.uk.

Available under license. To note, 3rd party material is not necessarily covered under this license: http://creativecommons.org/licenses/by-nc-nd/3.0/ 


\title{
Improving the Diagnosis and Treatment of HIV-associated Tuberculous Meningitis
}

\author{
Dr Fiona Cresswell
}

Date: 17th November, 2020

Clinical Research Department

Faculty of Infectious Diseases and Tropical Medicine

London School of Hygiene and Tropical Medicine

Thesis submitted in accordance with the requirements for the degree of

Doctor of Philosophy, University of London

Work contained in this thesis was supported by a Wellcome Clinical PhD Training Fellowship (210772A/Z/18/Z) 
Declaration:

I declare that the work presented in this thesis is my own. Where information has been derived from other sources I confirm that this has been indicated within the thesis.

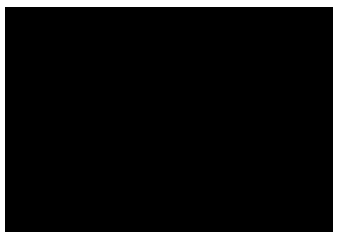

Fiona Cresswell

Date: $17^{\text {th }}$ November 2020

\section{Supervisors:}

Professor Alison Elliott

Professor of Tropical Diseases

Clinical Research Department, LSHTM, London

Programme Head LSHTM-MRC Uganda research Unit, Entebbe, Uganda

Professor David R Boulware

Division of Infectious Diseases

University of Minnesota,

Minneapolis

USA

Dr Daniel Grint

Tropical Epidemiology Group

LSHTM

London 


\section{Abstract}

HIV-1 has caused a marked shift in the epidemiology of meningitis in sub-Saharan Africa and Mycobacterium tuberculosis is now a leading cause of meningitis in adults. Diagnosis of tuberculous meningitis (TBM) is notoriously difficult, compounded by an atypical cerebrospinal (CSF) picture in HIV co-infection. The WHO-recommended standard quadruple TB therapy may not be the optimal regimen in TBM as rifampicin does not readily penetrate into the CSF at the current dose. Better diagnostics and treatments are needed to tackle the disconcertingly high TBM mortality of at least $50 \%$ in people living with HIV (PLHIV). The goals of this PhD thesis were to first identify improved approaches to the diagnosis of TBM; and second, to investigate the safety and pharmacokinetics of a novel approach to treatment with high-dose rifampicin through a phase II open-label randomised controlled trial in predominantly HIV-positive adults.

To begin, using retrospective data from two Ugandan referral hospitals, I showed that the introduction of the Cepheid Xpert MTB/Rif assay had markedly increased the microbiological confirmation of TBM. However, around half of TBM cases were treated on the basis of clinical suspicion alone, despite negative CSF TB tests. So, better diagnostics were still needed. I went on to examine the novel Xpert Ultra assay in a prospective diagnostic accuracy study, and found it to be the best CSF test for TBM, with a sensitivity of $77 \%$ and a negative predictive value of $93 \%$ for probable/ definite TBM, significantly better than Xpert MTB/Rif. A high CSF lactate and low CSF glucose were good discriminators of definite TBM. Given that CSF collection can be delayed or contraindicated, a non-CSF dependent test would be valuable, so i explored the potential of urine TB testing. Urine TBlipoarabinomannan (Alere LAM) and/or urine Xpert Ultra were positive in 50\% of probable/definite TBM cases, suggesting extra-meningeal disseminated TB disease is highly prevalent in adults with HIV/TBM. In this exploratory study urine Xpert Ultra had prognostic value in definite TBM.

The phase II randomised clinical trial demonstrated that with WHO-recommended TB treatment (containing rifampicin $10 \mathrm{mg} / \mathrm{kg} /$ day), CSF rifampicin was undetectable $(<0.25 \mathrm{mg} / \mathrm{L}$ ) in around half of participants, and only $11 \%$ achieved a CSF rifampicin concentration above the minimal inhibitory concentration (MIC) of the predominant M. tuberculosis strain in Uganda. Intravenous rifampicin 20 $\mathrm{mg} / \mathrm{kg} /$ day yielded a $\sim 6$-fold increase in serum and CSF exposures; and oral rifampicin $35 \mathrm{mg} / \mathrm{kg} /$ day yielded an $\sim 8$-fold increase in serum and CSF exposures compared to standard of care. All participants in the investigational arms had detectable CSF rifampicin, and almost all had CSF levels above the rifampicin MIC. Importantly, there was no excess toxicity in the high-dose rifampicin arms. Overall, the most common adverse event was elevated alanine transaminase, attributed to 
drug-induced liver injury, which occurred in 7 (12\%) of participants overall, 4 of which were in the control arm.

Together, this research has shown that, in Uganda, TBM is a usually a manifestation of disseminated TB disease resultant upon advanced HIV-related immune suppression, and carries a high case-fatality. Xpert Ultra is a step forward but cannot be used as a 'rule-out' test. A diagnostic test that combines pathogen-detection with a host biomarker may be the most sensitive approach for TBM. The WHOrecommended TB therapy for TBM is sub-optimal and high-dose rifampicin is a promising and safe intervention that needs to be further investigated in a phase III trial. 


\section{Preface}

I present this thesis as a research paper style thesis. The ten chapters comprise a total of seven research papers that have been submitted or published in peer-reviewed journals and are indicated in the table of contents. Cover sheets are provided with each paper that detail publication details and co-author contributions. Forewords to each chapter aim to connect the story between the different chapters. The thesis also comprises an introduction, a discussion, a conclusion and an appendix containing relevant supplementary materials. 


\section{Acknowledgements}

I am deeply grateful and indebted to my PhD supervisor Professor Alison Elliott for providing unwavering wisdom and support throughout my PhD. Thank you Alison. Likewise, Professor David Boulware who taught me that anything is possible. Equally, Dr David Meya, thank you for welcoming me into the brilliant team that you lead so gracefully. I'm hugely grateful to Dr Daniel Grint for your patience and good nature in the face of my statistical learner-plate.

I would like to express my utmost respect and heartfelt gratitude to the doctors, nurses, pharmacists and research assistants (too many to name individually) who work tirelessly on hospital wards and in the outpatient clinics. The loving and professional care given to critically ill patients and their all-toofrequently bereaved families is awe-inspiring. Thank you, Mable Kabahubya, Darlisha Williams, Ananta Bangdiwala, and Kathy HH for your phenomenal organisational skills and for keeping me on track.

The PhD would not have been possible without institutional support from the Infectious Diseases Institute. Thank you Dr Andrew Kambugu for leading such an energising and productive organisation, and the research department within which I was based (Dr Barbara Castelnuovo, Stephen Okoboi, Henry Onen, Sylvia Nabukenya, Allan Buzibye, Allan Katamba). At LSHTM, I am eternally grateful to Professor David Mabey for always finding time for words of encouragement and fun amidst your busy schedule, and also to Christina Albertsen for your guidance whilst coordinating the PhD programme. I've been fortunate to have incredibly talented collaborators at Radboud University (Dr Lindsey te Brake, Dr Elin Svensson, Professor Rob Aarnoutse) without whom the clinical trial would never have been possible. Eternal thanks to Dr Debbie Williams and Dr Duncan Churchill for being the most supportive (and patient!) educational supervisors at Brighton and Sussex University Hospital and for enabling this academic period. Thank you Professor Keith McAdam for sharing your love of Uganda with us, for introducing us to your colleagues at IDI, and for the wise words along the way.

Ultimate respect and gratitude to the incredibly stoic and resilient study participants and their devoted families and carers, who not only participated in the research but also the public engagement events that followed. I hope that being part of the research and being cared for by the research team has had a positive impact on their lives.

The PhD journey has been made all the richer by the camaraderie along the way - from Ugandan colleagues (you know who you are!), David Lawrence, Jayne Ellis, Anna, Emily, Carson, Suzaan Marais and Catriona Waitt. Finally, I'd like to thank my wonderful parents, Irene Kyomukama, my three children, and my wonderful husband Liam for sharing the load and being a constant source of laughter. 


\section{Table of Contents}

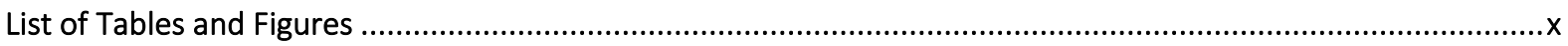

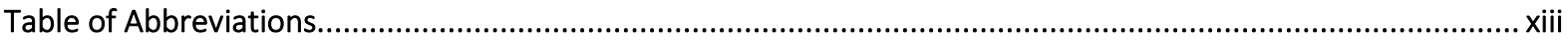

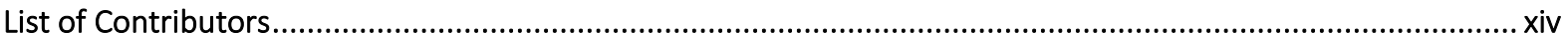

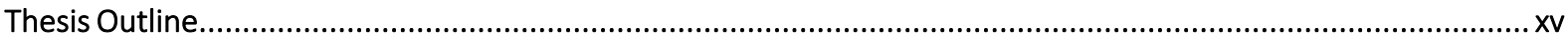

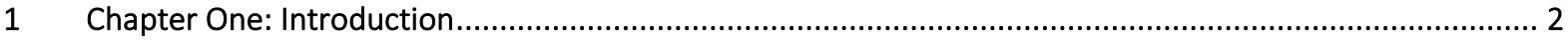

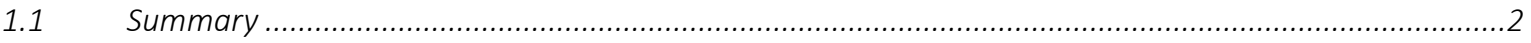

1.2 General introduction to HIV-associated Tuberculosis \& TB meningitis...........................................2

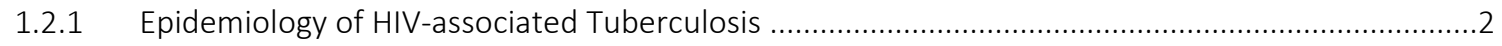

1.2.2 Immunological and clinical features of HIV-associated TB ......................................................

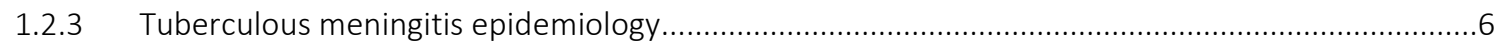

1.2.4 Pathogenesis of HIV-associated TB meningitis .................................................................

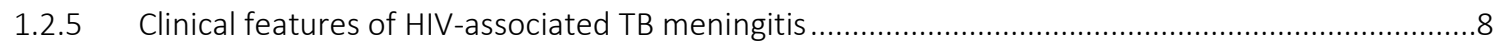

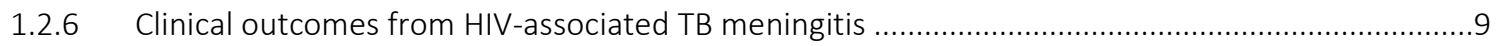

1.3 Part I introduction: Diagnostics for TB meningitis...................................................................... 11

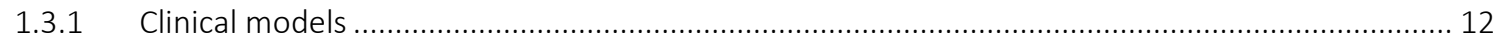

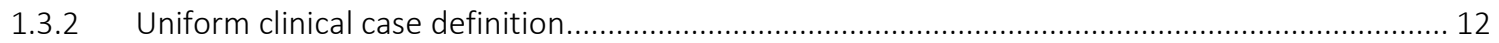

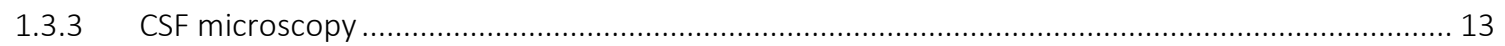

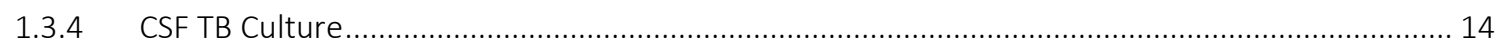

1.3.5 Molecular tests / nucleic acid amplification tests ................................................................ 14

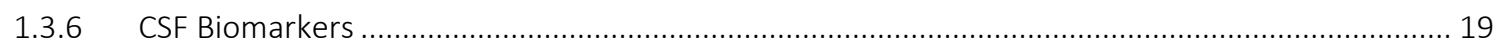

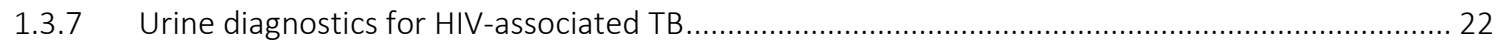

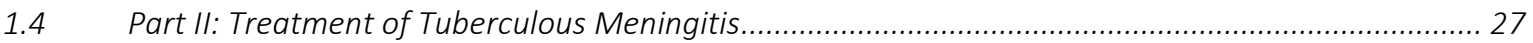

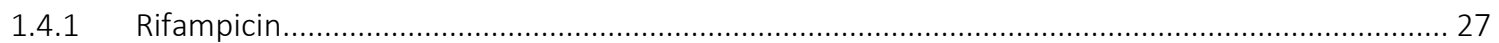

1.4.2 Current TB therapy - did we miss a trick with rifampicin dosing? ......................................... 27

1.4.3 Challenges with the current first-line TB regimen and finding better options ........................... 28

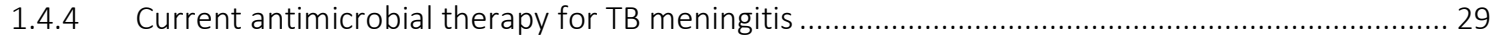

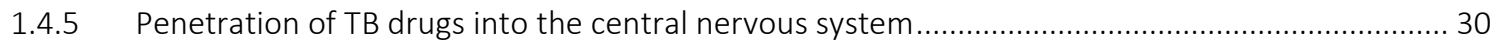

1.4.6 Can we optimise the antimicrobial treatment of TBM? ....................................................... 31

1.4.7 Defining the optimal rifampicin dose - pharmacokinetics-pharmacodynamics......................... 33

1.5 Impact of HIV-infection on pharmacokinetics and safety of TB drugs .........................................40

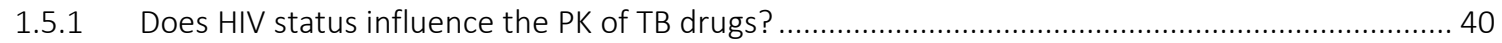


1.5.2 Does HIV status have an impact on the safety of TB therapy?

1.5.3 Does high dose rifampicin interact with antiretroviral therapy? .......................................... 43

1.5.4 Are host-directed therapies important for HIV-associated TBM? ............................................. 44

1.6 What are the pressing questions about improving the treatment of TB meningitis in Uganda? ....... 45

Bibliography

2 Chapter 2: Overview of PhD Aim, Hypotheses, Objectives, Setting and Methodology ...........................59

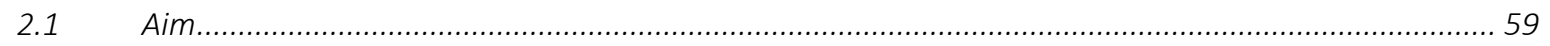

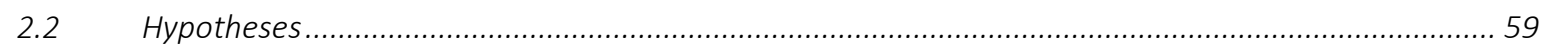

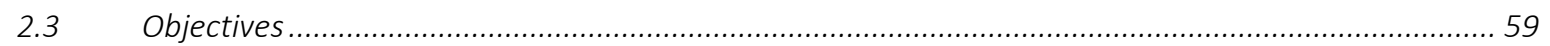

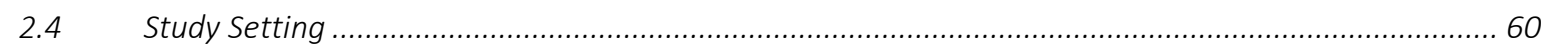

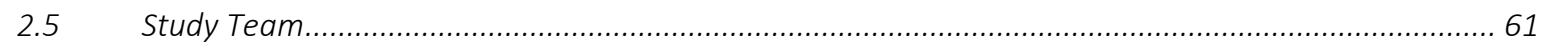

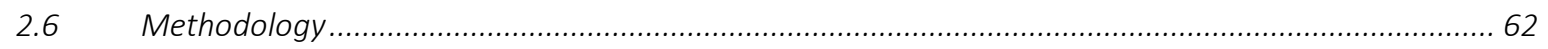

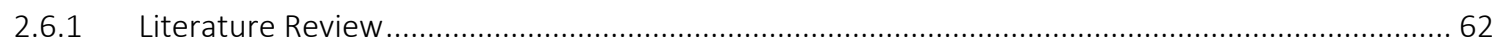

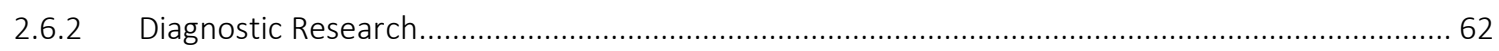

2.6.3 Therapeutic Research: A Phase II Randomised Controlled Trial of High Dose Oral and Intravenous

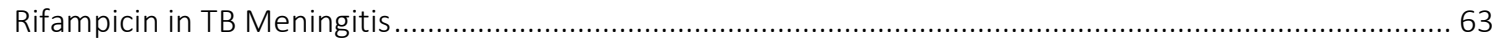

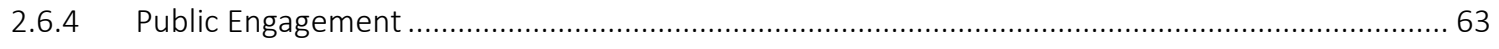

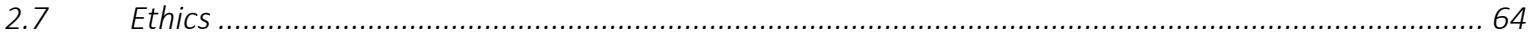

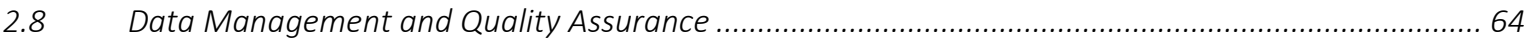

3 Chapter Three: Recent advances in Pathogenesis and Diagnostics of Tuberculous Meningitis (review paper).

4 Chapter Four: Tuberculous Meningitis Diagnosis and Outcomes During the Xpert MTB/Rif Era: Findings from a 6.5-year Cohort in Uganda (research paper) 84

5 Chapter Five: Diagnostic Accuracy of Xpert MTB/RIF Ultra for Tuberculous Meningitis in HIV-infected Adults: A Prospective Validation (research paper). 100

6 Chapter Six: Standardized Urine-based Tuberculosis Screening with TB-LAM and Xpert MTB/Rif Ultra in Ugandan Adults with Advanced HIV disease and Meningitis (research paper) 116

7 Chapter Seven: Intensified Treatment of Tuberculous Meningitis (review paper) 128

8 Chapter Eight: High Dose Intravenous and Oral Rifampicin in the Treatment of Tuberculous Meningitis: A Phase II Open Label Randomised Clinical Trial (research paper)

9 Chapter Nine: A Journey of Hope: giving research participants a voice to share their experiences and improve community engagement around advanced HIV disease in Uganda (research paper)

10 Chapter Ten: Discussion and Conclusion 203

10.1 Summary of findings 203 
10.1.1 Objective 1: To assimilate available retrospective data on adults with suspected TBM in Uganda and to describe the TBM population in terms of baseline characteristics, method of diagnosis and clinical outcomes

10.1.2 Hypothesis 1: Xpert MTB/Rif 'Ultra' is significantly more sensitive than Xpert MTB/Rif for the detection of M. tuberculosis in CSF. However, Xpert Ultra's sensitivity will not be perfect and it may fail to diagnose a small number of cases of CNS TB that are evident by metagenomic next generation sequencing, CSF culture or post-mortem examination.....

10.1.3 Hypothesis 2: Urine diagnostics such as urine TB-LAM and urine Xpert MTB/Rif Ultra will be useful adjunctive tools in diagnosing TB meningitis and will identify patients at the greatest risk of death. 206

10.1.4 Hypothesis 3: Intensified treatment of TB meningitis with intravenous rifampicin (20 mg/ $/ \mathrm{kg}$ ) or high dose oral rifampicin (35 mg/kg) will result in significantly increased blood and CSF exposure during the critical early days of TB treatment as compared to common control (10 mg/kg oral rifampicin) ....... 207

10.1.5 Hypothesis 4: High dose rifampicin will be safe in people with advanced HIV disease and may improve functional outcomes and survival at 8- and 24-weeks.

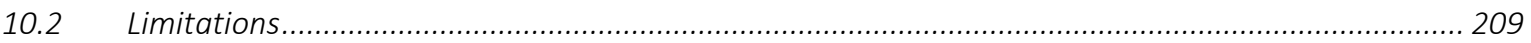

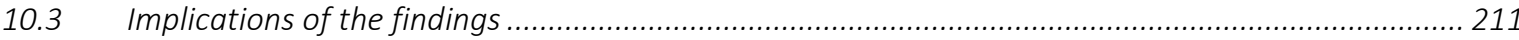

10.3.1 TBM can be prevented by upstream HIV-related interventions including earlier HIV testing, treatment and TB screening within HIV services. ........................................................................ 211

10.3.2 Xpert Ultra is the best first test for TBM but should not be the last test .............................. 212

10.3.3 HIV-associated TBM is a manifestation of disseminated TB disease so supportive care and interventions to optimise the host response are important alongside optimal antimicrobial therapy ..... 215

10.3.4 High dose rifampicin should be studied at a larger scale for TBM (and disseminated TB

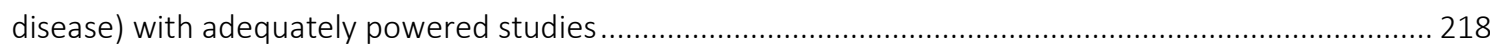

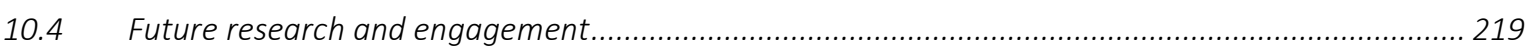

10.4.1 Model burden of disease and cost-effectiveness of upstream interventions......................... 219

10.4.2 Continue to improve low and high technology diagnostics for TBM................................... 220

10.4.3 Better characterization of the TBM population ................................................................. 223

10.4.4 Optimising antimicrobial therapy for TBM ...................................................................... 224

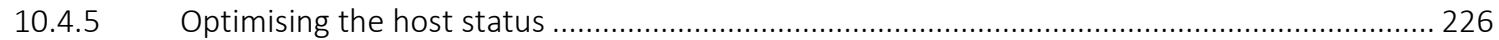

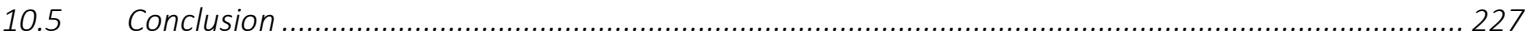

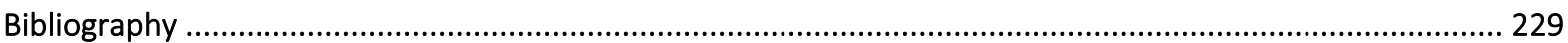




\section{List of Tables and Figures}

Chapter 1 figures $\quad P g$

1.1 TB incidence rates in WHO regions between 1990 and 2008

1.2 Global trends in estimated number of deaths caused by HIV and TB, 2000-2018 4

1.3 Spectrum of outcomes following exposure to M. tuberculosis 5

1.4 Pathological features of TB meningitis 7

1.5 Chronological illustration of paradoxical immune reconstitution inflammatory 8 syndrome (IRIS), antiretroviral therapy (ART)-associated tuberculosis (TB) and unmasking TB-IRIS

1.6 Forest plot depicting TB meningitis mortality by HIV status 9

$\begin{array}{lll}1.7 & \text { TB meningitis pooled mortality by country } & 10\end{array}$

1.8 Consensus uniform case definition for tuberculous meningitis research 13

1.9 Illustration of Xpert MTB/Rif assay and procedure $\quad 15$

1.10 Xpert and Xpert Ultra limit of detection for M. tuberculosis H37Rv 16

1.11 Venn diagram illustrating overlap of positive CSF tests for M. tuberculosis in 2018

Xpert Ultra study using cryopreserved CSF

1.12 Metagenomic next-generation sequencing in the diagnosis of meningoencephalitis 19

1.13 a) Alere determine TB-LAM assay package insert demonstrating the steps involved to 21 generate a result and ease of use

b) Photo of three Alere LAM results illustrating the real-life appearance of the negative and positive $(+3$ and +5$)$ results

1.14 Sensitivity of urine Alere TB-LAM and urine Xpert in urine samples from HIV-positive 22 patients with culture-confirmed pulmonary TB by CD4+ T cell strata

1.15 TB drug development timeline 29

1.16 Schematic overview of the relation between the blood-brain barrier (BBB), made up 31 by brain endothelial cells, and the blood-CSF barrier (BCSFB), made up by choroid plexus epithelial cells

1.17 a) Example of a PK curve and the key PK parameters for a drug that is dosed orally 34

b) Example of a concentration-response curve

1.18 Kaplan-Meier survival estimate according to rifampicin treatment arm in Ruslami et al 37 Indonesian intensification trial

1.19 Model-predicted proportion survival over time, representative for typical patients 39 (age 30 yrs, baseline $\mathrm{GCS}=13$ ) per plasma $\mathrm{AUC}_{0-24}$ at day 2

1.20 Ratio between the AUC of HIV-positive and HIV-negative TB patients 40

1.21 Kaplan-Meier survival estimate amongst PLHIV according to dexamethasone 44 treatment group

\section{Chapter 1 tables}

1.1 Reported TB meningitis data in relation to TB and extra-pulmonary TB 6

1.2 Summary of Cox regression models for 9-month mortality in Vietnamese TBM cohorts 9

1.3 Summary of studies and meta-analyses on host CSF biomarkers 20

1.4 Priority research question on epidemiology and diagnostics of TBM in Uganda 26

1.5 Priority research question on the treatment of TBM in Uganda 46

\section{Chapter 2 figures}

2.1 Map of the Republic of Uganda 54

2.2 TB incidence rate in Uganda 2000 - 2018

2.3 Meningitis Clinical Research Team 55 
3.1 Illustrative summary of the pathogenesis of tuberculous meningitis (TBM) 73

3.2 Kaplan-Meier survival curves stratified by LTA4H genotype 74

3.3 Outcomes of the host $-M$. tuberculosis interaction depicted by the basic parabola of 76 the damage-response framework

3.4 Novel Host and Pathogen biomarkers for diagnosis of tuberculous meningitis

\section{Chapter 4 figures}

4.1 Timeline illustrating evolution of diagnostic testing available in Ugandan research 91 setting

4.2 Illustration of flow of patients from screening population into TBM cohort 92

4.3 Venn diagram Illustrating the overlap of positive MGIT culture and Xpert test results in 95 the $\mathrm{n}=118$ samples tested with both assays

4.4 Illustration of odds of dying in cohort one and two compared to cohort three in a 96 multivariate model

\section{Chapter 4 tables}

4.0 TBM case series and cohorts from sub-Saharan Africa 85

4.1 Demographics, HIV details and outcomes of cohort 93

4.2 Methods of diagnosis 94

4.3 Summary of concordance between Xpert MTB/Rif and MGIT culture results 94

4.3 Hospital outcomes 95

\section{Chapter 5 figures}

5.1 Flow diagram showing the diagnostic outcomes of the study population 109

5.2 Venn diagram of positive diagnostic tests in the composite reference standard 111

\section{Chapter 5 tables}

5.1 Baseline characteristics of subjects who underwent TBM testing

5.2 Diagnostic performance of Xpert, Ultra and MGIT for the diagnosis of TBM 111

5.3 Univariate and multivariate analyses of factors associated with microbiological 112 confirmation of TBM

\section{Chapter 6 figures}

6.1 Venn Diagram of overlap between TB positive urinary assays 124

6.2 Venn diagram illustrating overlap of TB-LAM and urine Ultra amongst 26 with definite 124 tuberculous meningitis

\section{Chapter 6 tables}

6.1 Participant baseline demographics, clinical and CSF findings by disease category 123

6.2 Urine TB diagnostic test results by disease category 123

6.3 In-hospital mortality by urine diagnostic result and meningitis aetiology by univariate 125 logistic regression

\section{Chapter 7 figures}

7.1 Schematic overview of the relation between the blood-brain barrier (BBB, brain endothelial cells), and the blood-CSF barrier (BCSFB, choroid plexus epithelial cells.)

7.2 Concentration-response curves displaying the effect of rifampicin plasma $\mathrm{AUC}_{0-6 \mathrm{~h}}$ (left) and $\mathrm{C}_{\max }$ (right) exposure on survival, and the corresponding exposure threshold values associated with maximal survival. 
7.1 Clinical outcomes from TBM studies in adults and children 134

7.2 Anti-tuberculosis drugs used in TBM treatment 138

7.3 Summary of clinical studies investigating intensified antimicrobial treatments for TBM 145

7.4 Suggested intensified TBM regimen and administration for high resource settings 147

\section{Chapter 8 figures}

$\begin{array}{lll}8.1 & \text { Rift study CONSORT diagram } & 179\end{array}$

8.2 Pharmacokinetic parameters on day $2 \quad 180$

8.3 Kaplan-Meier survival by trial arm 181

\section{Chapter 8 tables}

$\begin{array}{lll}8.1 & \text { Baseline characteristics } & 179\end{array}$

8.2 Rifampicin pharmacokinetic data by treatment arm 180

8.3 Adverse events by treatment arm during the 8-week interventional period 181

\section{Chapter 8 supplementary figures}

S8.1 HIV antiretroviral therapy (ART) status 185

S8.2 Liver function test trajectories in participants who experienced drug-induced liver 190 injury

S8.3 Adverse event competing risks regressions

S8.4 Random effects linear mixed model of Glasgow coma scale score by study day 191

Chapter 8 supplementary tables

S8.1 Dosing of rifampicin by trial arm 186

S8.2 Individualised AE details 188

\section{Chapter 9 figures}

9.1 Purposes and pillars of public engagement 197

9.2 Knowledge exchange session in Western Uganda 198

9.3 Infographic leaflets distributed to share information about TB meningitis and the 199 clinical trial

9.4 Infectious Diseases Institute Community Advisory Board Meeting 199

9.5 Public audience drawn to the circus event 199

9.6 Study physician Dr Ssembambulidde records material for CBS radio broadcasts 200

9.7 Dr Kasibante, Dr Cresswell and Mr Tugume appear on National Television (NTV) station 200

9.8 Dramatisation of meningitis illness. 201

9.9 Cake cutting with the research team and Executive Director 201

\section{Chapter 10 figures}

10.1 Alanine transaminase (IU/L) by study day in the Rift trial 208

10.2 Test and test-treatment threshold in relation to pre-test probability of TBM disease 213

10.3 Schematic illustrating the natural progression of disease in adults with and without 215 HIV co-infection and the continuum of disseminated HIV disease which has TB meningitis towards the end

10.4 Non-sputum-based TB diagnostic pipeline 220

10.5 Diagnostic framework for TBM 221

10.6 Kaplan Meier survival estimate by CSF Ultra cycle threshold (Ct) from 101 patients 224 with suspected or confirmed TBM 


\section{Table of Abbreviations}

\begin{tabular}{|c|c|}
\hline$A E$ & Adverse Event/Adverse Experience \\
\hline AFB & Acid Fast Bacilli \\
\hline AIDS & Acquired Immunodeficiency Syndrome \\
\hline ALT & Alanine Transaminase \\
\hline AUC & Area Under the Curve \\
\hline BRCP & Breast Cancer Resistance Protein \\
\hline CNS & Central Nervous System \\
\hline $\mathrm{C}_{\max }$ & Maximum Concentration \\
\hline CRF & Case Report Form \\
\hline CSF & Cerebrospinal Fluid \\
\hline DAIDS & Division of AIDS, NIAID, NIH \\
\hline DILI & Drug Induced Liver Injury \\
\hline DSC & Data Safety Committee \\
\hline DTG & Dolutegravir \\
\hline E & Ethambutol \\
\hline EBA & Early Bactericidal Activity \\
\hline EFV & Efavirenz \\
\hline GCP & Good Clinical Practice \\
\hline GCS & Glasgow Coma Scale \\
\hline $\mathrm{H}$ & Isoniazid \\
\hline HDT & Host-directed therapy \\
\hline HIV & Human Immunodeficiency Virus \\
\hline $\mathrm{ICH}$ & International Conference on Harmonisation \\
\hline ICP & Intracranial Pressure \\
\hline IDI & Infectious Diseases Institute \\
\hline IFN & Interferon \\
\hline $\operatorname{lnSTI}$ & Integrase Strand Transfer inhibitor \\
\hline IMP & Investigational Medicinal Product \\
\hline IRIS & Immune Reconstitution Inflammatory Syndrome \\
\hline IRB & Institutional Review Board \\
\hline LOD & Limit of Detection \\
\hline LP & Lumbar Puncture \\
\hline MGIT & Mycobacterial Growth Indicator Tube \\
\hline MIC & Minimal Inhibitory Concentration \\
\hline N & Number (typically refers to subjects) \\
\hline NDA & New Drug Application \\
\hline NRTI & Nucleos(t)ide reverse transcriptase inhibitor \\
\hline OATP & Organic Anion Transporting Polypeptide \\
\hline P-gp & P-glycoprotein \\
\hline $\mathrm{PI}$ & Principal Investigator \\
\hline $\mathrm{PI}$ & Protease Inhibitor \\
\hline PIS & Patient Information Sheet \\
\hline $\mathrm{PK} / \mathrm{PD}$ & Pharmacokinetics / Pharmacodynamics \\
\hline $\mathrm{R}$ & Rifampicin \\
\hline RCT & Randomised Controlled Trial \\
\hline RIF & Rifampicin \\
\hline RPM & Reads Per Million \\
\hline SAE & Serious Adverse Event/Serious Adverse Experience \\
\hline SAR & Serious Adverse Reaction \\
\hline SUSAR & Serious Unexpected Adverse Reaction \\
\hline SOP & Standard Operating Procedure \\
\hline SPC & Summary of Product Characteristics \\
\hline TB & Tuberculosis \\
\hline ULN & Upper Limit of Normal \\
\hline WCC & White cell count \\
\hline WHO & World Health Organization \\
\hline Z & Pyrazinamide \\
\hline
\end{tabular}




\section{List of Contributors}

\begin{tabular}{|c|c|c|}
\hline Name & $\begin{array}{l}\text { Position } \\
\text { Institution }\end{array}$ & Contribution \\
\hline Alison Elliott & $\begin{array}{l}\text { Professor } \\
\text { Clinical Research Department } \\
\text { LSHTM, Keppel Street, London } \\
\text { MRC-UVRI Uganda Research Unit } \\
\text { Entebbe, Uganda }\end{array}$ & PhD supervisor \\
\hline David Boulware & $\begin{array}{l}\text { Professor } \\
\text { Division of Infectious Diseases and } \\
\text { International Medicine } \\
\text { University of Minnesota, } \\
\text { Minneapolis } \\
\text { USA }\end{array}$ & PhD supervisor \\
\hline Daniel Grint & $\begin{array}{l}\text { Assistant Professor } \\
\text { Tropical Epidemiology Group } \\
\text { LSHTM, Keppel Street, London }\end{array}$ & PhD supervisor \\
\hline David Meya & $\begin{array}{l}\text { Associate Professor } \\
\text { Makerere University College of Health } \\
\text { Sciences } \\
\text { Kampala, Uganda }\end{array}$ & $\begin{array}{l}\text { Group Lead } \\
\text { Co-principal investigator RifT } \\
\text { trial }\end{array}$ \\
\hline Ananta Bangdiwala & $\begin{array}{l}\text { Data Manager } \\
\text { Division of Infectious Diseases and } \\
\text { International Medicine } \\
\text { University of Minnesota, } \\
\text { Minneapolis } \\
\text { USA }\end{array}$ & $\begin{array}{l}\text { Data management } \\
\text { Case Report Form production }\end{array}$ \\
\hline Lindsey te Brake & $\begin{array}{l}\text { Post-doctoral fellow } \\
\text { Department of Pharmacy } \\
\text { Radboud University } \\
\text { Nijmegen, Netherlands }\end{array}$ & $\begin{array}{l}\text { Advisor on pharmacokinetic } \\
\text { methods }\end{array}$ \\
\hline Rob Aarnoutse & $\begin{array}{l}\text { Professor } \\
\text { Department of Pharmacy } \\
\text { Radboud University } \\
\text { Nijmegen, Netherlands }\end{array}$ & $\begin{array}{l}\text { Advisor on pharmacokinetic } \\
\text { methods }\end{array}$ \\
\hline Elin Svensson & $\begin{array}{l}\text { Post-doctoral fellow } \\
\text { Department of Pharmacy } \\
\text { Radboud University } \\
\text { Nijmegen, Netherlands }\end{array}$ & $\begin{array}{l}\text { Advisor on pharmacokinetic } \\
\text { analysis. PhD advisory group. }\end{array}$ \\
\hline Allan Buzibye & $\begin{array}{l}\text { Laboratory Scientist } \\
\text { Infectious Diseases Institute } \\
\text { Kampala, Uganda }\end{array}$ & $\begin{array}{l}\text { Pharmacokinetic assay } \\
\text { validation and sample } \\
\text { processing }\end{array}$ \\
\hline Michael Wilson & $\begin{array}{l}\text { Associate Professor \& Neurologist } \\
\text { University of California San Francisco, } \\
\text { USA }\end{array}$ & $\begin{array}{l}\text { Collaborator, next generation } \\
\text { sequencing }\end{array}$ \\
\hline
\end{tabular}




\title{
Thesis Outline
}

\section{Improving diagnosis and treatment of tuberculous meningitis}

\author{
BACKGROUND
}

Chapter 1 is a general introduction to the epidemiology, clinical features, and outcomes of Tuberculosis (TB) and tuberculous meningitis (TBM) with respect to HIV co-infection. Thereafter there is a more detailed introduction to 1) diagnosis of TBM; 2) treatment of TBM. The scientific rationale for the research conducted during the PhD is explained.

Chapter 2 is an overview of PhD aim, objectives, setting and methods. A number of methodologies are used to test the pre-specified hypotheses including a retrospective data analysis, two prospective observational cohort studies, and a randomized clinical trial. The protocol paper for the clinical trial is featured in the Appendix 5.

\section{PART I - DIAGNOSTIC RESEARCH}

Chapter 3 is a review paper that describes the recent landscape of TBM pathogenesis and diagnostic research. Research Paper: Cresswell FV, Davis A, Sharma K et al. Recent updates in pathogenesis and diagnostics of tuberculous meningitis. Wellcome Open Research; 2019.

Chapter 4 is a retrospective cohort study that describes the landscape regarding TBM diagnosis and hospital outomes in the PhD study setting. Research paper: Cresswell FV, Bangdiwala AS, Bahr NC et al. "Diagnosis and outcomes from tuberculous meningitis in the Xpert MTB/Rif era: findings from a 6.5-year HIV-positive cohort in Uganda". Wellcome Open Res.2018;3:64.

Chapter 5 Is a prospective diagnostic accuracy study of Xpert MTB/Rif Ultra on cerebrospinal fluid carried out in patients presenting to two Ugandan referral hospitals with suspected TBM. Research paper: Cresswell FV, Tugume L, Bahr NC et al. Xpert MTB/Rif Ultra for the diagnosis of HIV-associated Tuberculous meningitis: a prospective validation. Lancet Infectious Diseases. 2020;20(3):308-317.

Chapter 6 I investigate the potential of urine diagnostics including Xpert MTB/Rif Ultra and Alere TB-LAM lateral flow assay in TBM diagnosis and prognostication. Research paper: Cresswell FV, Ellis J, Kagimu E et al. Utility of urine diagnostics with TB-lipoarabinomannan and Xpert MTB/Rif Ultra in Ugandan adults with HIVassociated meningitis. Open Forum Infectious Diseases. 2020;7(4):ofaa100. 


\section{PART II - TREATMENT RESEARCH}

Chapter 7 is a review article covering the of rationale and evidence relating to intensifying the treatment of TBM. This explains why I decided that high-dose rifampicin was the right option to study in Uganda. Research paper: Cresswell FV, te Brake L, Ruslami R, Dooley K, Aarnoutse R, van Crevel R. Intensified Treatment of tuberculous meningitis. Expert Review in Clin Pharm.2019;12(3):267-288.

Chapter 8 is the largest piece of $\mathrm{PhD}$ research conducted, a phase II randomised clinical trial of high-dose intravenous and oral rifampicin with the aim of characterising the safety and pharmacokinetics of rifampicin in blood and cerebrospinal fluid of adults with TBM. The clinical trial protocol has been published as a paper and is featured in the appendix. The trial results paper presents the safety and pharmacokinetic data by trial arm. Research paper: submitted to Lancet Infectious Diseases.

\section{PUBLIC ENGAGEMENT}

Chapter 9 describes the series of public engagement activities relating to the PhD, including two community events, radio and TV pieces, and several events at HIV clinics or hospitals near the trial sites. The aims and outcomes of the public engagement activities are described in an open letter. Research paper: Cresswell FV, Ssebambulidde K, Martyn E et al. Public engagement activities in Uganda on advanced HIV disease, lumbar punctures and meningitis. Open letter published on African Academy of Sciences Open Research 2020, 3:33.

\section{DISCUSSION AND CONCLUSION}

Chapter 10: The discussion provides a summary of the scientific findings of the research conducted, limitations of the research, the implications of the findings and plans for future research.

\section{APPENDIX}

The appendix features the following items:

1. Systematic review and meta-analysis of TB meningitis outcomes.

2. The uniform TB meningitis clinical case definition.

3. The pilot study of TB-lipoarabinomannan on cerebrospinal fluid conducted during the PhD.

4. The literature search strategy for the review papers.

5. The phase II randomised controlled clinical trial (Rift study) protocol manuscript.

6. The American Academy of Neurologists abstracts published by collaborators on metagenomic next generation sequencing. 
BACKGROUND 



\section{Chapter One: Introduction}

\subsection{Summary}

This section begins by briefly reviewing the epidemiology, immunological and clinical features of Tuberculosis (TB) in relation to HIV infection and touches on why M. tuberculosis has a greater predilection for extrapulmonary sites, including the central nervous system, in people with advanced HIV-related immunosuppression. An overview on TB meningitis (TBM) epidemiology, pathogenesis, clinical features and outcomes in relation to HIV co-infection follows. Thereafter, a more focussed introduction into the diagnostics and treatment of TBM is provided.

Part 1: Diagnostics for TBM: Traditional microbiology techniques such as smear microscopy and culture have a number of shortfalls. Nucleic acid amplification tests including Xpert MTB/Rif Ultra and next generation sequencing technologies are introduced and remaining research questions around Xpert Ultra are presented. Existing urine and CSF biomarkers have poor to moderate diagnostic performance but future candidates are more promising. Evidence and research gaps relating to urine biomarkers in TBM are described.

Part 2: Treatment of TBM: Current TB treatment regimens are long, toxic and not universally effective. Repurposed or redeveloped (dose-optimised) drugs such as high-dose rifampicin may help to improve the efficacy and shorten the duration of TB therapy. Rifampicin is rapidly bactericidal and is the key sterilizing drug but inter-individual variability in exposure is large and low rifampicin exposure can lead to treatment failure and drug resistance. This is particularly relevant to TBM as only $~ 5 \%$ of plasma rifampicin penetrates across the blood-cerebrospinal fluid (CSF) barrier and rifampicin is undetectable in the CSF of the majority of TBM patients at the current dose $(10 \mathrm{mg} / \mathrm{kg})$. High-dose rifampicin is a promising approach for TBM treatment, and generation of safety and pharmacokineticpharmacodynamic data from HIV-positive adults in Africa is a priority.

\subsection{General introduction to HIV-associated Tuberculosis \& TB meningitis}

\subsubsection{Epidemiology of HIV-associated Tuberculosis}

Tuberculosis (TB), an infection caused by Mycobacterium tuberculosis complex, has been a foe since ancient times, with Egyptian mummies providing archeological evidence of spinal TB disease. In the $19^{\text {th }}$ century, TB incidence peaked at 1000 per 100,000 (1\%) population per year in the USA, and the only available treatments were open-air treatment in sanatoria or thoracic surgery. ${ }^{1}$ Thankfully, with the decline of poverty in the $20^{\text {th }}$ 
century and the evolution of antituberculous chemotherapy between 1944 and 1980s, outcomes improved and transmission declined markedly, such that in 1989 the US Department of Health and Human Services planned to eradicate TB from the USA by $20100^{2,3}$

Alas the progress was short-lived, in part due to a false sense of security that TB was a disease of the past and a resultant decline in funding for TB research and treatment, ${ }^{4}$ but largely due to the unfolding HIV epidemic in sub-Saharan Africa in the 1980s. In 2019, M. tuberculosis remained the single leading infectious cause of death and one of the top ten overall causes of death worldwide, resulting in 1.4 million deaths amongst the estimated 10 million people (range 8.9 to 11.0 million) who fell ill with the disease. ${ }^{5}$ Illustrated in Figure 1.1 is the sharp increase in TB incidence during the 1990s in HIV-endemic parts of Africa. HIV emerged as the primary risk factor for the development of TB disease, with the dual infections able to potentiate one another's pathology, a twin epidemic was born. The rapid resurgence of TB as a public health threat led to the World Health Organisation (WHO) declaring TB a global emergency in $1993 .{ }^{6}$ It is estimated that a third of the world's population in latently infected with M. tuberculosis. ${ }^{7}$

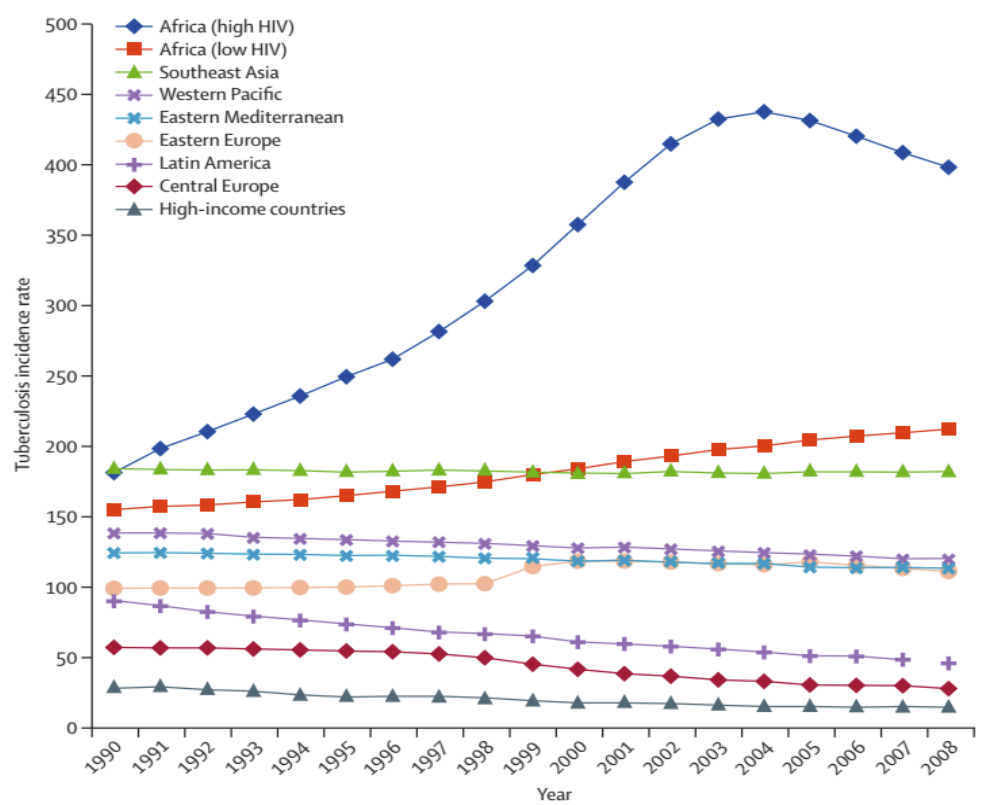

Figure 1.1 TB incidence rates in WHO regions between 1990 and $2008 .^{8}$

TB disease risk is increased at all levels of HIV-related immunosuppression, but the risk increases as CD4 T-cell count declines, and is up to 30 -fold greater in those with CD4 T-cell count $<100$ cells/ $\mu$, when compared to their HIV-negative counterparts. ${ }^{9,10,11}$ The annual risk of active TB disease in people living with HIV (PLHIV) is $\sim 10 \%$ per year, and can be as high as 30\% per year with advanced HIV disease. ${ }^{10,11}$ Unfortunately, even when HIV antiretroviral therapy (ART) has been successfully initiated and CD4 T-cell count has normalized, the TBrisk remains elevated (up to 5 -fold) compared to HIV-negative adults, ${ }^{12}$ suggesting there is a persistent deficit in the host immune response to $M$. tuberculosis. The proportion of incident TB which relates to reactivation of latent disease or de novo infection in PLHIV is likely to vary depending on regional TB prevalence; evidence 
from South African gold miners suggests that reactivation of latent disease is the main cause of TB in early HIV infection, but with more advanced HIV immune suppression active TB more commonly reflects rapid progression of disease following recent (re)infection. ${ }^{13,14}$

Despite the rapid scale up of ART and a decline in peak annual HIV incidence, 38 million [95\% confidence interval (CI) 31.6-44.5 million] people were living with HIV in 2019, two-thirds of which are in the WHO Africa region, equating to 1 in every 25 adults aged $15-49$ years (3.7\%). ${ }^{15}$ Worldwide in 2019, 815,000 [95\% Cl

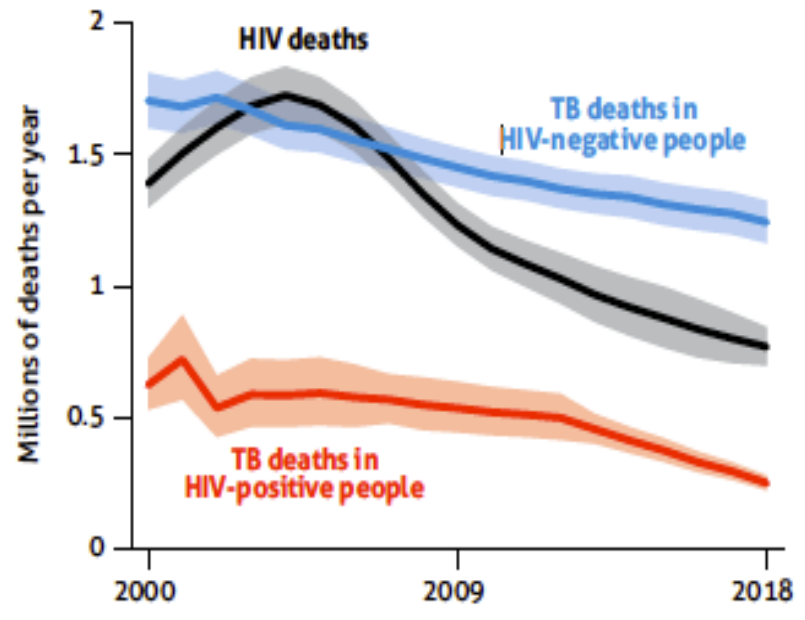
729,000-906,000] new TB infections occurred in PLHIV, $73 \%$ of which were in the WHO Africa region, resulting in 208,000 deaths from TB in PLHIV. ${ }^{5}$ TB remains the leading cause of mortality in PLHIV, but thankfully there is a gradual decline in TB mortality in both HIV-positive and negative persons (Figure 1.2), though perhaps not marked enough to meet the WHO End TB strategy goals of reducing deaths from TB by $90 \%$ by 2030 , compared with $2015 .{ }^{16}$

Figure 1.2. Global trends in estimated number of deaths caused by HIV and TB, 2000-2018. The shaded area $=$ uncertainty intervals. ${ }^{17}$

\subsubsection{Immunological and clinical features of HIV-associated TB}

Protective immune responses to TB are incredibly complex and involve both the innate and adaptive immune system and granuloma formation at the site of primary infection in the lungs. As well as depleting CD4+ T-cell numbers, HIV infection impairs CD4+ T-cell function, with potential mechanisms including impaired activation of macrophages and recruitment of monocytes, and reduced capacity to secrete chemokines and cytokines. ${ }^{18}$ HIV also impairs neutrophil function, the importance of which has more recently been discovered, through impaired phagocytosis and killing capacity. ${ }^{19,20}$

Granuloma formation, comprising a multicelluar ecosystem of activated macrophages, T-cells, B-cells, neutrophils, fibroblasts, and dentritic cells, works to contain M. tuberculosis and prevent dissemination to extra-pulmonary sites. ${ }^{11}$ However, as a result of impaired cell-mediated immunity in HIV co-infection granuloma formation fails, and whilst the host is spared the cavitating lung disease which relates to granuloma formation, the bacilli more commonly enter the lymphatic system or are haematogenously disseminated to multiple organs including the brain. In the context of HIV-associated CD4+ T-cell depletion and dysfunction M. tuberculosis bacteraemia can be detected in $10-40 \%$ of febrile TB patients, which results in the seeding of meninges (and other bodily organs) with bacilli. ${ }^{21,22}$ 
The classical spectrum of clinical outcomes of exposure to aerosolized M. tuberculosis is illustrated in Figure 1.3a, the red lines signify the more common outcomes in people with advanced HIV-related immune dysfunction and resultant failure to control bacillary replication. More recently it has been recognized that the two-state paradigm - latent TB infection (LTBI) and active TB disease - is too simplistic, in fact it there is an undulating disease course from the latent state to the active disease state which passes through the 'minimal' disease and 'sub-clinical' disease states, illustrated in Figure 1.3b. During the minimal disease state the individual remains asymptomatic but there is evidence of metabolic activity at the site of infection and incipient disease through evolving radiological changes (on X-ray, computerized tomography (CT), or positron emission tomography (PET) scan). ${ }^{23,24}$ During sub-clinical disease M. tuberculosis can be cultured from the sputum despite the absence of any symptoms of TB disease, potentially contributing to community transmission. ${ }^{25}$ The optimal way to diagnose and treat these intermediary disease states remains to be determined. It is also known that people can self-clear LTBI infection (i.e. without antimicrobial therapy) but this relies on a robust host response and is unlikely to be achievable with advanced HIV-related immune suppression, indeed it is more likely to be the case that individuals with immune suppression progress more rapidly towards the active disease state.

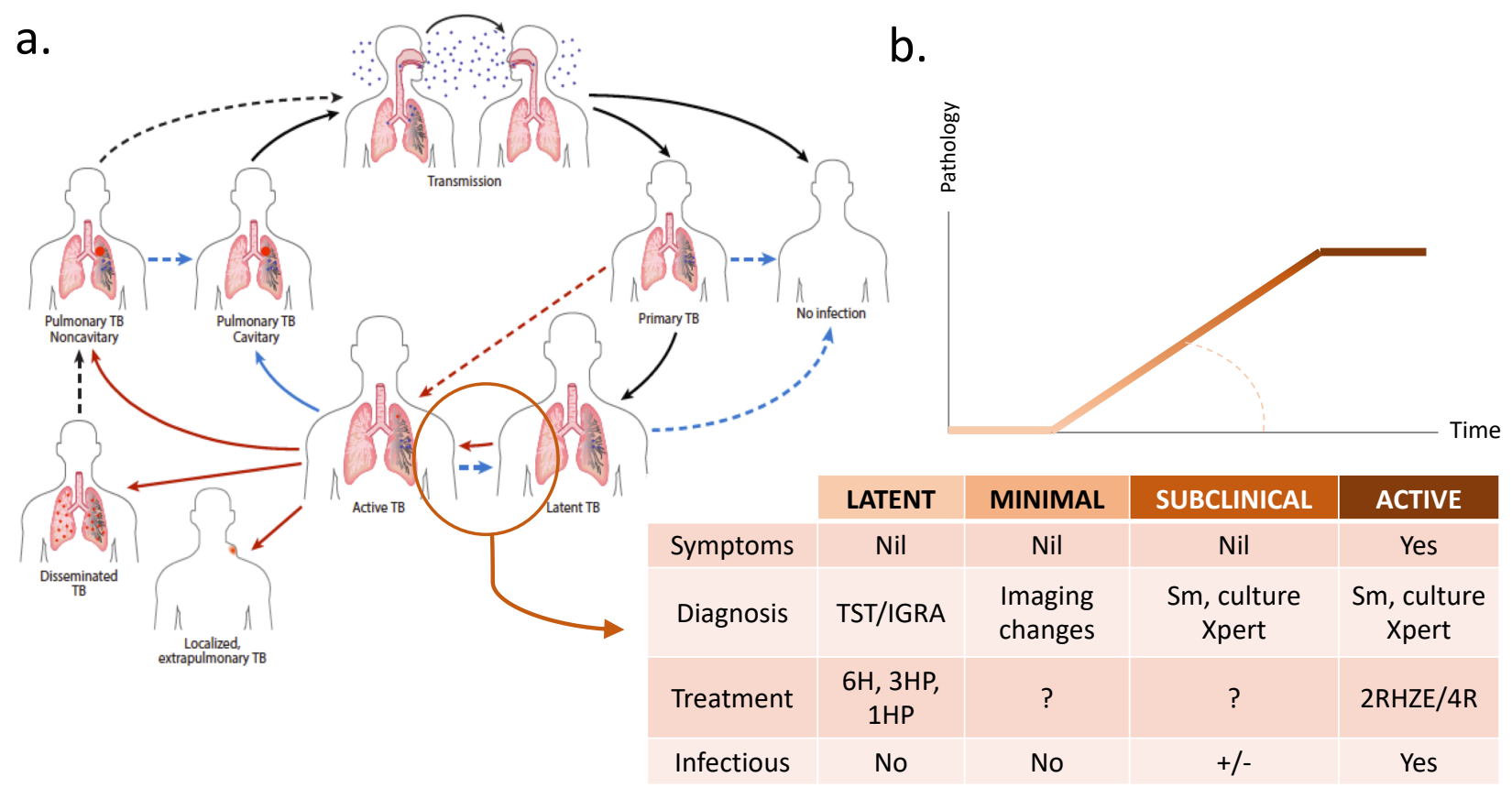

Figure 1.3a and b. Spectrum of outcomes following exposure to M. tuberculosis. Adapted from $\mathrm{H}$. Esmail11 1.3a. Solid lines show common outcomes/routes of progression. Dashed lines show infrequent routes of progression. Red lines signify that the outcome or route of progression is more common in those with impaired cell-mediated immunity (e.g. advanced HIV). Blue lines signify that the outcome/route of progression is more common in immunocompetent hosts.

1.3b. Latent TB infection progresses to the active TB disease state over a period of months or even years through states called minimal and sub-clinical TB. TST = tuberculin skin test, IGRA = interferon gamma release assay, $\mathrm{H}=$ isoniazid, $\mathrm{P}=$ rifapentine, $\mathrm{Sm}=$ smear microscopy

The clinical presentation of HIV-associated TB differs depending on the extent of host immune suppression. A study in Uganda found that at higher CD4 T-cell counts (>300-400 cells/ $\mu$ l) apical pulmonary cavitatory disease 
and sputum smear positivity is similar to HIV-negative counterparts. However, as CD4 T-cell count declines lymphadenopathy, pleural effusions, miliary disease pattern, and lower lung field disease become more common. ${ }^{26,27}$

Post-mortem studies have established that TB disease at the time of death is a commonplace in hospitalized PLHIV in sub-Saharan Africa. In a 2015 meta-analysis, TB was found in 43\% (95\% Cl 38-48\%) of HIV-positive cadavers, it was disseminated in $88 \%$ of cases, and due to the atypical presentation of TB in advanced HIV disease it was often unsuspected at the time of death. ${ }^{28}$ The lungs commonly show a miliary pattern as well as consolidation, and spleen, liver, bone marrow were also frequently affected organs. ${ }^{29}$ Given that haematogenous dissemination (miliary TB) deposits bacilli throughout the body, it is unsurprising that a significant proportion of cadavers with disseminated TB have central nervous system (CNS) involvement; a Kenyan autopsy study found that $26 \%$ of cadavers with disseminated TB had evidence of meningeal involvement. ${ }^{30}$

\subsubsection{Tuberculous meningitis epidemiology}

Dr James Hakim, a Zimbabwean Physician, described the marked change in the pattern of meningitis seen in Harare Central Hospital in the 1990s; total patient admissions increased from 53,000 to 73,000 per annum and rates of meningitis rose from 78 to 523 cases per 100,000 admissions per year between 1985 and 1995 . He goes on to describe how in 1985 bacterial meningitis was the most common cause of meningitis (92.5\%) but by 1995 this had fallen to $25.5 \%$ with TBM accounting for $28.3 \%$ of cases (up from $2.5 \%$ in 1985 ). ${ }^{31}$ This shift in meningitis aetiology has occurred in many HIV endemic high TB prevalence countries, and $M$. tuberculosis is now the second leading cause of meningitis in adults in South Africa and Uganda (after cryptococcal meningitis). ${ }^{32,33,34}$

Accurate national epidemiological data about tuberculous meningitis (TBM) incidence in many TB endemic countries is lacking, due to both challenges in making a diagnosis and weak reporting systems. Robust national level TBM data is available from only a few countries and is summarised in Table 1.1.

\begin{tabular}{|c|c|c|c|c|c|c|}
\hline COUNTRY & YEARS & $\begin{array}{l}\text { TOTAL TB } \\
\text { CASES }\end{array}$ & $\begin{array}{l}\text { EXTRA- } \\
\text { PULMONARY } \\
\text { TB }\end{array}$ & $\begin{array}{l}\text { TB } \\
\text { MENINGITIS }\end{array}$ & $\begin{array}{l}\text { TB MENINGITIS } \\
\text { AS A \% OF TB } \\
\text { CASES }\end{array}$ & $\begin{array}{l}\text { TYPE OF } \\
\text { DATA }\end{array}$ \\
\hline USA $^{35}$ & 2016 & 9272 & 1882 & 84 & 0.9 & National \\
\hline CHINA ${ }^{36}$ & 2008-2017 & 19279 & 6433 & 440 & 2.3 & Hospital \\
\hline CANADA ${ }^{37}$ & 1970-2001 & 82764 & - & 824 & 1.0 & National \\
\hline UK 38 & 2019 & 4651 & 2759 & 98 & 2.1 & National \\
\hline GERMANY 39 & 2002-2009 & 46,349 & - & 422 & 0.9 & National \\
\hline
\end{tabular}

Table 1.1 Reported TB meningitis data in relation to TB and extra-pulmonary TB.

Based on the above data, and a global TB incidence of 10 million cases in 2019, ${ }^{5}$ a conservative estimate of $\sim 1 \%$ of all TB cases being TBM would give a global burden of $\sim 100,000$ cases/year. ${ }^{40}$ However, the true burden 
is likely to be somewhat greater than 100,000 cases/year as disseminated extra-pulmonary manifestations of TB are more common in settings of high HIV and TB prevalence, from which data are not available. Some experts estimate that in sub-Saharan African countries TBM could represent up to $5 \%$ of all TB cases. ${ }^{41}$ Modelling studies are required to obtain a more accurate estimate of true global burden of TBM.

\subsubsection{Pathogenesis of HIV-associated TB meningitis}

As early as 1992, it was reported that extra-pulmonary TB, in particular TBM, occurred with greater frequency in people with TB who were HIV co-infected. ${ }^{42}$ The contemporaneous nature of disseminated TB, miliary TB and TBM observed in the post-HIV era differs from the pathological model of Arnold and Rich, whose autopsy work in the USA in 1946 lead to the proposition that TBM begins from the rupture of a single long-standing granuloma (a Rich focus) on the meninges or cortex..$^{43}$ Whilst the Arnold and Rich model may still hold true for immune competent hosts, more recent post-mortem studies in Africa suggest that meningeal involvement is part of a more broadly generalised picture in HIV/TB. ${ }^{29} \mathrm{~A}$ similar phenomenon is also observed in childhood TBM, in whom miliary TB and TBM develop most often within 3 months of primary infection, when fresh anatomic changes are still found in the primary lung focus..$^{44,45}$
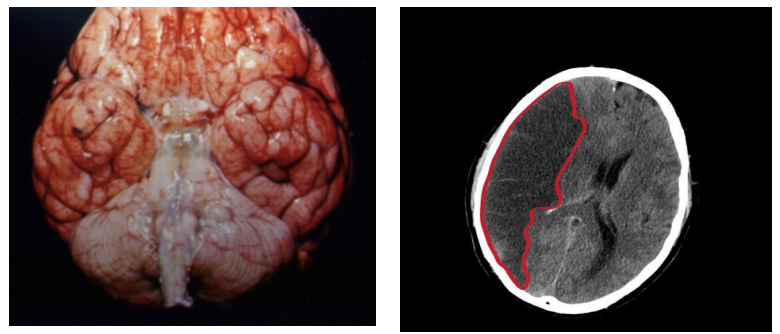

Following bacillary invasion of the subarachnoid space, host immune responses give rise to a granulomatous inflammation predominantly affecting the basal meninges. The inflammatory exudate can obstruct the normal flow of CSF, resulting in hydrocephalus, raised intracranial
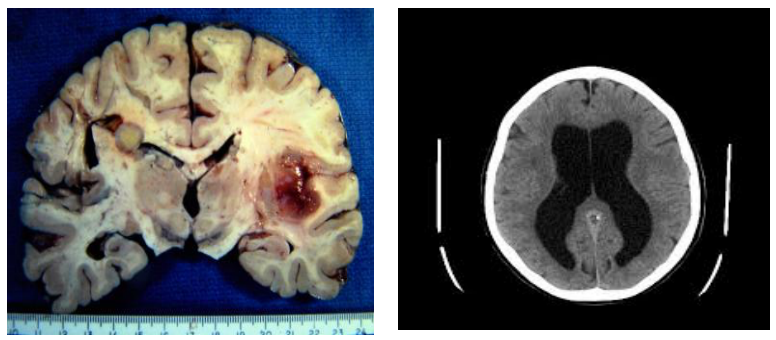
pressure, cerebral hypoxia and infarction. Even in the absence of hydrocephalus, vasculitis of small and medium size blood vessels is common resulting in stroke in up to $60 \%$ of TBM patients. ${ }^{46}$ The spectrum of brain pathology is illustrated in Figure 1.4.

Figure 1.4. Pathological features of TB meningitis. Clockwise from top left: basal meningeal exudate; cerebral middle cerebral artery territory infarct; enlarged ventricles as a result of hydrocephalus; tuberculous brain abscess.

Research is underway to fully characterize the immune response in different populations with the hope of identifying a host-directed therapy capable of regulating the aberrant response and improving outcomes. CSF cytokine profiling from Vietnamese HIV-positive adults with TBM suggest that the immune response is dysregulated and ineffective (rather than attenuated) in advanced HIV-associated TBM and highlights a role for neutrophils in the pathogenesis. ${ }^{47}$ Further detail relating to the pathogenesis of TBM are given in the chapter 3 paper Recent insights in the pathogenesis and diagnostics of Tuberculous meningitis. 


\subsubsection{Immune reconstitution inflammatory syndrome}

Antiretroviral therapy is essential in combatting TB and HIV co-infection, but can be complicated by immune reconstitution inflammatory syndrome (TB-IRIS). ART results in a rapid restoration of TB-specific immune response which can be associated with a systemic inflammatory picture or worsening of CNS symptoms in TBM. Depending on the TB treatment status at ART initiation, this immune-mediated worsening of TB pathology can take the form of paradoxical TB-IRIS, ART-associated TB or unmasking TB-IRIS, see Figure 1.5. IRIS has been reported in $8 \%-40 \%$ of people with HIV/TB following ART initiation. ${ }^{48}$ Predictors of IRIS include a baseline CD4+ T-cell count $<50$ cells/ $\mu \mathrm{L}$, rapid restoration of CD4+ T-cell counts, rapid decline in on-ART HIV viral load, and severity of TB disease. ${ }^{48,11}$ A consensus IRIS case definition has been created to support diagnosis and standardise classification, though it requires failure of TB therapy (e.g. resistance or poor adherence), drug reactions and opportunistic infections to be excluded, all challenging in LMIC settings. ${ }^{49}$

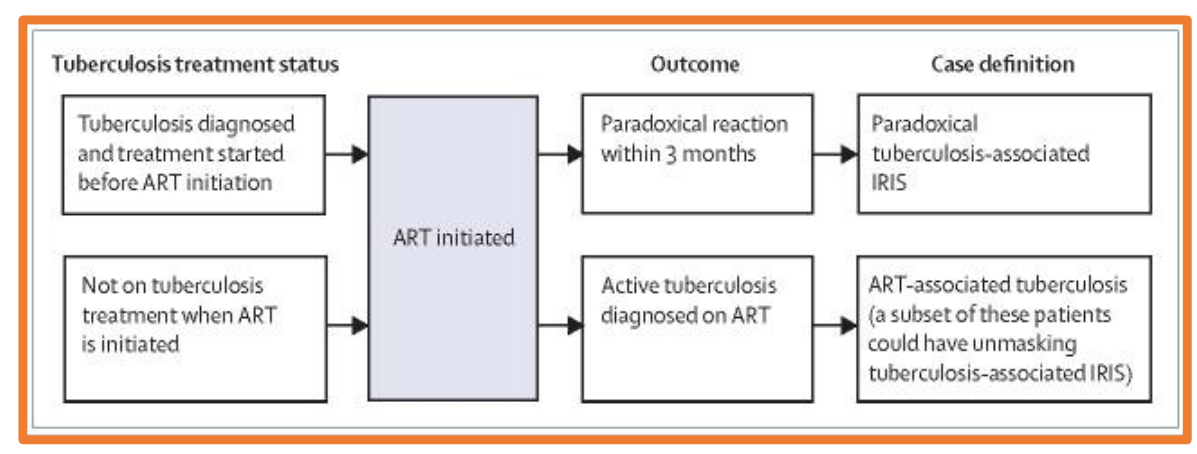

Figure 1.5. Chronological illustration of paradoxical immune reconstitution inflammatory syndrome (IRIS), antiretroviral therapy (ART)-associated tuberculosis (TB) and unmasking TBIRIS. 49

\subsubsection{Clinical features of HIV-associated TB meningitis}

After an initial non-specific prodrome, the meningitic symptoms of headache, neck stiffness and vomiting develop. As the intracranial pathology progresses altered mental status and focal neurological deficits including cranial nerve palsies and hemiplegia ensue. This progresses to certain death without treatment. Most of the evidence suggests that HIV co-infection does not significantly influence the age of clinical presentation of TBM in adults, the interval from symptoms to clinical presentation, or the neurological findings at clinical presentation. ${ }^{50}$ However, abnormal clinical findings outside the CNS occur more frequently in HIV-positive patients, including low body mass index, other features of HIV disease and TB involvement of other organs, including pulmonary, nodal, spinal, pleural and abdominal disease. ${ }^{42,51,52}$ In Vietnam, Thwaites et al. found an independent association between multidrug resistant (MDR) TBM and HIV co-infection, which occurred in $13 \%$ of HIV-positive and $4 \%$ of HIV-negative patients. ${ }^{53}$ 


\subsubsection{Clinical outcomes from HIV-associated TB meningitis}

Survival from TBM varies drastically in the literature by geographical region and HIV status, the reasons for which are unclear. Meta-analysis of data from 748 HIV-positive and 951 HIV-negative Vietnamese adults with TBM found HIV co-infection to be a strong independent predictor of 9-month mortality [hazard ratio $(\mathrm{HR})=3.94$; 95\% Cl 2.79-5.56].54 Amongst PLHIV, other baseline characteristics which were associated with 9-month mortality were lower weight, higher Medical Research Council (MRC) severity grade, lower plasma sodium, lower CD4 T-cell count and lower CSF lymphocyte count, as shown in Table 1.2.55

\begin{tabular}{|c|c|c|c|}
\hline \multicolumn{2}{|c|}{ HIV-negative } & \multicolumn{2}{|l|}{ HIV-positive } \\
\hline Variable & HR ( $p$-value) & Variable & HR (p-value) \\
\hline Age $[$ per +10 years $]$ & $1.24(<0.001)$ & Low weight $[$ per $+10 \mathrm{kgs}]$ & $0.73(<0.001)$ \\
\hline Previous TB treatment & $1.57(0.015)$ & MRC grade $I / / I I$ & $4.08(<0.001)$ \\
\hline MRC grade $I / I I I$ & $3.05(<0.001)$ & Plasma sodium [115vs125 mmol/l] & $1.63(<0.001)$ \\
\hline Focal neurological signs & $1.65(0.007)$ & CD4 count* & $0.9(0.002)$ \\
\hline No dexamethasone & $1.97(<0.001)$ & CSF lymphocytes* & $0.93(0.004)$ \\
\hline CSF lymphocyte count & $0.86(<0.001)$ & & \\
\hline \multicolumn{4}{|c|}{$\begin{array}{l}\text { HR=hazard ratio, } *=\text { HR per } 2 \text {-fold increase } \\
\text { MRC grade I = Glasgow coma scale (GCS) } 15 \text { and no focal neurology } \\
\text { MRC grade II = GCS } 15 \text { with focal neurology or GCS } 11 \text { to } 14 \text { without focal neurology } \\
\text { MRC grade III = GCS }<10 \text { with or without focal neurology }\end{array}$} \\
\hline
\end{tabular}

Table 1.2. Final Cox regression models for 9-month mortality in Vietnamese TBM cohort ${ }^{54}$

However, it is not clear whether these findings are generalizable to other geographical regions. The extent of the heterogeneity in clinical outcomes in other populations remained to be described, which prompted myself and colleagues to perform a systematic review and meta-analysis on global TBM outcomes. This has recently been published in Open Forum Infectious Disease and is included in Appendix 1. We reviewed 2,197 articles, selected 39 eligible studies including data for 5752 adults with TBM from 18 countries.

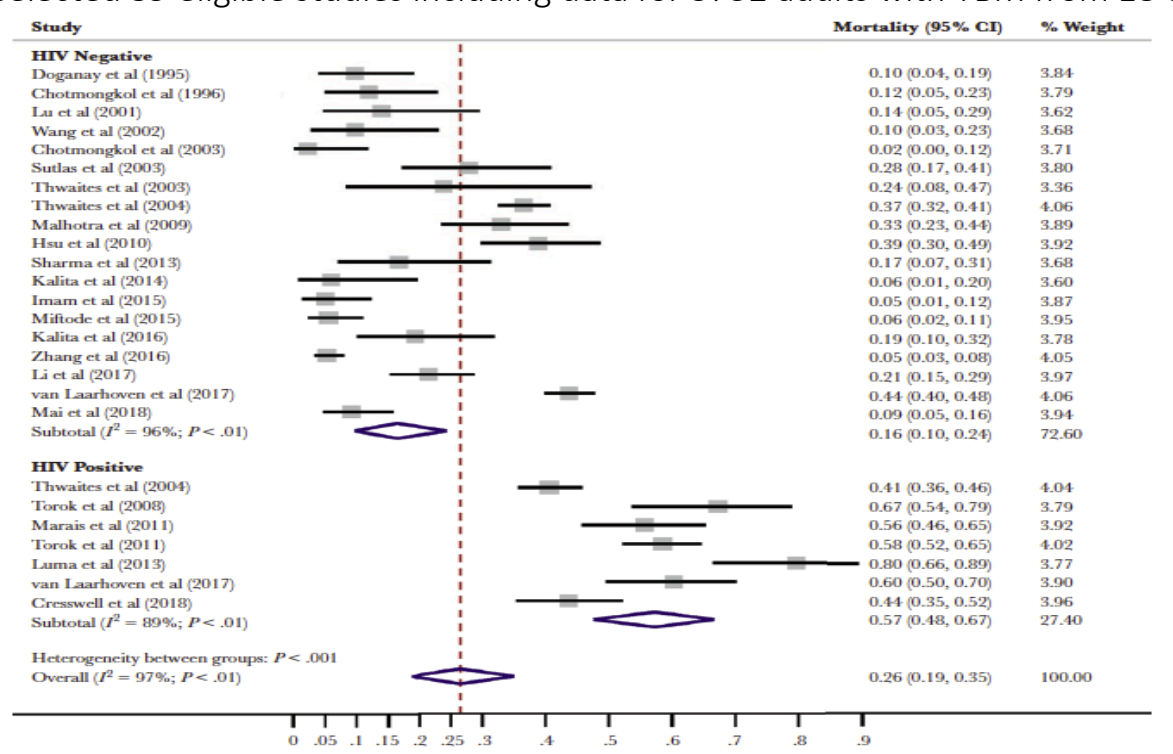

Our data demonstrated a 3fold higher pooled-mortality in PLHIV, overall mortality was $57 \%(95 \% \mathrm{Cl} 48-67 \%)$, versus $16 \%(10-24 \%)$ in HIV-negative adults. HIV status explained a significant amount of the heterogeneity in mortality between studies $(\mathrm{P}<0.01)$, Figure 1.6.56

Figure 1.6. Forest plot depicting TB meningitis mortality by HIV status. Reproduced with permission from Stadelman, OFID, 2020.56 
We also described global disparities in TBM outcomes by geographic region; mortality varied widely within and between regions with studies from sub-Saharan Africa having a range of $23-80 \%$ and Asian studies ranging from $2-67 \%$. Pooled mortality by country, and the relative paucity of studies from Africa, South and Central America is illustrated in Figure 1.7.

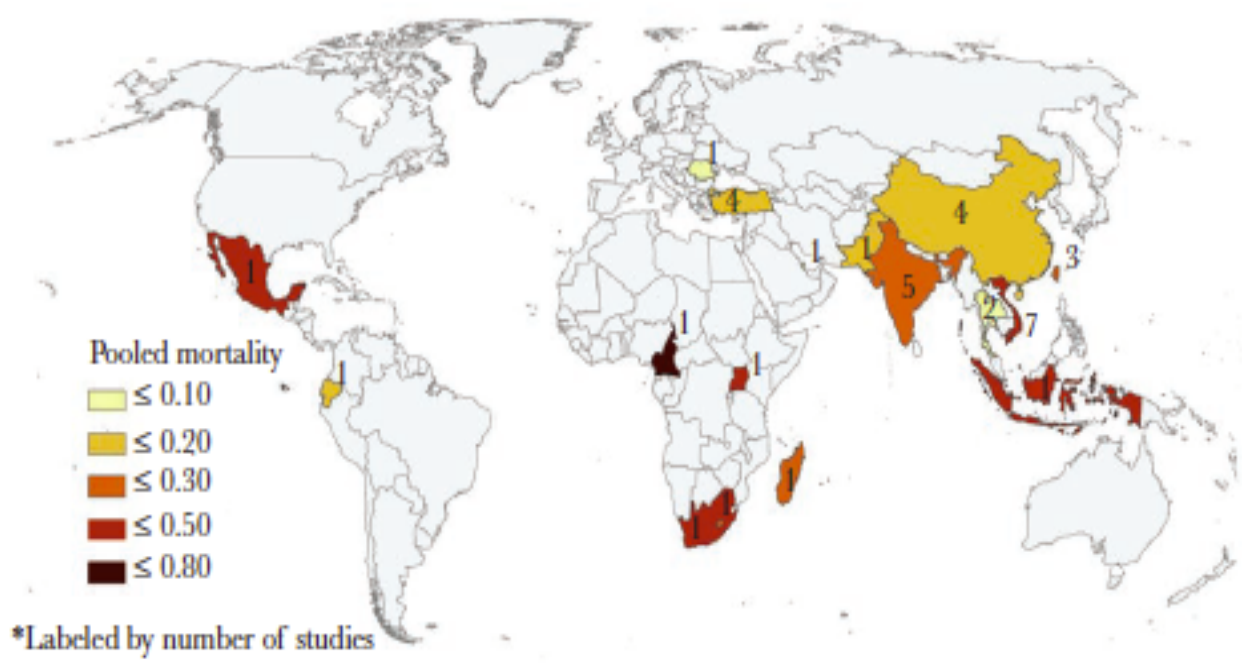

Figure 1.7. TB meningitis pooled mortality by country. For countries with only a single study the mortality shown relates to the single study. Reproduced from Stadelman, OFID, 2020.56

Additionally, temporal change in pooled mortality was reviewed in 5-year intervals between 1995 and 2018 . Reported TBM outcomes have not improved over two decades, the possible reasons for which are manifold, but the observation highlights that we still have a long way to go in optimising the diagnosis, treatment and outcomes from this illness.

Further studies in PLHIV are required to identify the unique determinants of the 3-fold increased mortality. Possible explanations include:

1. Diagnostic delay may be greater in HIV - the CSF picture can be atypical. ${ }^{57}$

2. Altered pharmacokinetics of TB drugs - adults with advanced HIV frequently suffer with diarrhoea, HIV-associated enteropathy and wasting, which have been associated with malabsorption and reduced bioavailability of antituberculous therapy. ${ }^{58}$

3. Concomitant HIV-related illnesses may contribute to the poor outcomes observed. One report suggests that AIDS-related illnesses accounted for up to $50 \%$ of the mortality in children with TBM who are not on ART. ${ }^{59}$ There is no good data co-infections/morbidities from adults.

4. Different immune response in HIV - host response could be attenuated, aberrant or excessive within the CNS or more systemically. ${ }^{60}$

5. TB immune reconstitution inflammatory syndrome may also play a role in TBM morbidity and mortality in HIV. ${ }^{61,62}$

6. Misclassification of non-TB brain infection as TBM and resultant lack of response to TB therapy and poor outcome 
As well as death, long-term disability is a major challenge in TBM, exacerbated by the lack of rehabilitation services in low- and middle-income countries (LMIC). In our systematic review, long-term disability was reported in around a third of TBM survivors (32\% [95\% Cl 22-43\%] by Barthel Index and 26\% [18-35\%] by modified Rankin scale). ${ }^{56}$

\subsection{Part I introduction: Diagnostics for TB meningitis}

Here, I focus more specifically on the diagnostics of TBM. Given my work seeks to improve diagnostics of HIVrelated TBM I give more weight to modalities that are likely to be relevant and scalable to high TB and HIV prevalence settings. The research paper on Recent Developments in Tuberculous Meningitis Pathogenesis and Diagnostics in chapter 3 covers general diagnostic insights from the last 5 years in more detail so this introduction provides further background information and probes more deeply into diagnostic considerations in HIV-associated meningitis in particular.

Making a TBM diagnosis is a challenge. There are a number of reasons for this; first, a diagnosis of TBM can only be confirmed by collecting a CSF sample via a lumbar puncture, an invasive procedure that carries risks of infection, bleeding and nerve damage if not done correctly. The procedure requires a spinal needle and an appropriately trained healthcare worker, neither of which are universally available in hospital settings in LMIC settings. In addition, the patient or their family may refuse to have the procedure due to misconceptions about lumbar punctures killing people, when actually it because they are generally only performed when a patient is very sick and therefore at high risk of dying from the underlying disease. The myths and resistance around lumbar punctures prompted me to undertake some public engagement activities which are detailed in chapter 9. Further, lumbar puncture is contraindicated in people with space occupying lesions in the brain which can be common in CNS TB infection due to tuberculomas or tuberculous abscesses. The second challenge is that the CSF sample should be processed quickly before cells decay outside the body and it requires a degree of laboratory expertise to get an accurate CSF microscopy result. Even with the best laboratory expertise and equipment the currently available diagnostics are only moderately sensitive and thus can yield a negative result when the patient actually has the disease. Thirdly, deciding whether or not to initiate TBM treatment is often a highly emotive decision; not initiating treatment in someone who has the disease will result in certain death, yet initiating treatment in someone who does not have TBM, condemns the individual to 12 months of antituberculous therapy from which pill burden, toxicity and drug-drug interactions are a real problem. Even in research settings such as clinical trials a microbiologically-confirmed diagnosis is only made in around half the participants. ${ }^{63}$

An additional challenge is encountered in HIV endemic settings - distinguishing TBM from other a host of other subacute meningoencephalitides seen in advanced HIV disease. ${ }^{64}$ Mimics of TBM include cryptococcal meningitis, cytomegalovirus encephalitis, cerebral toxoplasmosis, primary CNS lymphoma, and bacterial 
meningitis. Thankfully point of care cryptococcal antigen testing (CrAg LFA, IMMY, Norman, OK, USA) can now assist the diagnosis of cryptococcal meningitis at the beside (provided testing kits are available), or CSF can be sent to the laboratory for India Ink staining. ${ }^{64}$ Distinguishing viral meningitides from TBM is possible if viral polymerase chain reaction (PCR) assays are available in the hospital laboratory, something currently not available in government facilities in Uganda.

However, due to the lack of a widely available, rapid, accurate diagnostic test for TBM and the challenges in excluding other conditions in the differential diagnosis in LMIC settings a sensitive and specific clinical algorithm would be a useful tool in supporting clinicians in making the difficult decision about treatment initiation.

\subsubsection{Clinical models}

Nine clinical models have been created in an attempt to distinguish adult TBM from other causes of meningitis including cryptococcal meningitis and bacterial meningitis. ${ }^{65,66,67,68,69}$ The Thwaites et al. algorithm (which includes age, duration of symptoms, blood white cell count, CSF white count and neutrophilia), demonstrated high sensitivity and moderate specificity (91.7\% and $79.7 \%$ respectively) when evaluated prospectively in Vietnamese HIV-negative patients. ${ }^{70}$ However, sensitivity and specificity declined to $78 \%$ and $43 \%$ respectively when it was used in a Malawian population with high HIV prevalence. ${ }^{71}$ No published models can effectively differentiate TBM from a host of other neurological pathologies found in HIV-positive adults. ${ }^{66}$

There is therefore interest is using modern data science techniques to derive a multivariable clinical prediction model from large individual patient datasets from different geographical settings. ${ }^{72}$ This model could be accessible via a mobile phone application, giving clinicians a percentage likelihood of their patient actually having TBM based on a variety of available clinical or laboratory parameters.

\subsubsection{Uniform clinical case definition}

In 2010 a group of experts in the field defined the uniform TBM clinical case definition, which defines cases as 'definite', 'probable', 'possible' or 'not TBM' based on the number of points derived from criteria including clinical, CSF, brain imaging and evidence of TB elsewhere in the body (see Figure 1.8 and proforma in Appendix 2 for further details). ${ }^{73}$ It is important to note that this case definition was not designed as a diagnostic aid, it is a standardised tool intended for use when classifying enrolees in research studies on novel diagnostic assays or therapeutics in the absence of a perfect gold standard. This case definition was derived by expert consensus rather than being data-driven and may therefore perform better in some contexts than others. 

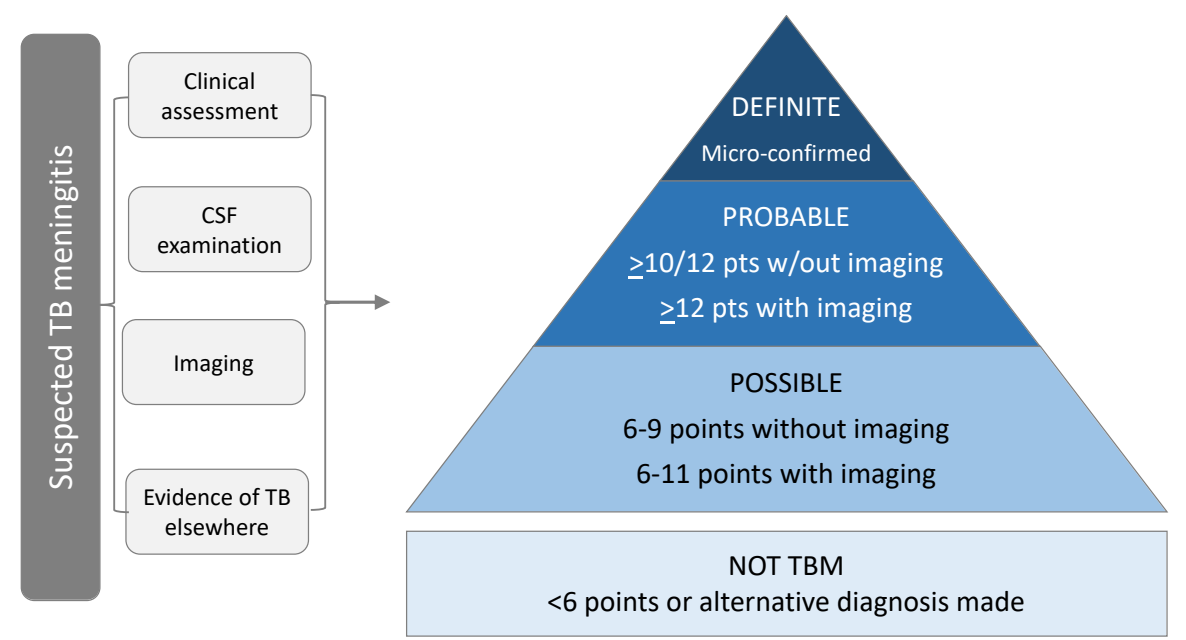

Figure 1.8. Consensus uniform case definition for tuberculous meningitis research

The potential to score points in this tool is affected by the ability to comprehensively investigate patients with brain, chest and abdominal imaging as well as microbiological sampling from outside the CNS; in LMIC settings the ability to score a high number of points may therefore be compromised.

\subsubsection{CSF microscopy}

TBM classically causes a marked CSF lymphocyte pleocytosis, high protein and low glucose (white cells $>5$ cells $/ \mu$ l, protein $>45 \mathrm{~g} / \mathrm{l}$, glucose $<2.2 \mathrm{mmol}$ ), however this "typical" pattern occurs in under two-thirds of HIVpositive TBM patients making the diagnosis more challenging. ${ }^{74}$ In our retrospective cohort of TBM patients in Kampala (chapter 4), a third (25/76) of patients with TBM microbiologically-confirmed (by Xpert MTB/Rif or culture) had an acellular CSF ( $<5$ cells $/ \mu$ l) and $4 \%(3 / 76)$ also had normal CSF protein and glucose..$^{57}$ This finding is consistent with other HIV-positive cohorts (at least outside of Asia) and highlights the additional challenge faced in diagnosing HIV-associated TBM. . $^{51,52,65,75}$

CSF Ziehl-Neelsen (ZN) staining for the presence of acid-fast bacilli (AFB) is probably the most widely available diagnostic test worldwide. However, the low bacillary burden renders CSF smear microscopy a poor test in most settings, even with the presence of an expert microscopist who can dedicate the requisite length of time to reading each slide. Centrifugation of large volume CSF followed by examination for $>30$ minutes by an expert microscopist in a Vietnamese research facility yielded a positive result in $58 \%$ of TBM cases. ${ }^{76}$ However, this is not achievable in most busy public hospital laboratories. In Uganda, sensitivity of ZN stain is at best 22\% in the Makerere University Microbiology Laboratory. ${ }^{77,78}$ A modified ZN stain with cytospin, which includes centrifugation and an additional Triton-X processing step, is intended to enhance intracellular staining of mycobacteria in CSF and make detection by the microscopist easier. Modified ZN stain was recently tested in a multicentre study (Vietnam, South Africa and Indonesia), alongside conventional ZN smear microscopy, 
Xpert MTB/Rif and culture in a 618-participant study (194, 31.4\% of whom were HIV-positive) with the uniform case definition of definite/probable/possible TBM as the reference standard. Sensitivity of conventional $Z N$ and modified $Z N$ were $33.9 \%$ and $34.5 \%$ respectively ( $P=1.0$ for the difference between tests), sensitivity of culture and Xpert were $31.8 \%$ and $25.1 \%$ respectively. When using culture as a reference, sensitivities of conventional ZN, modified ZN, and Xpert were $66.4 \%, 67.5 \%$, and $72.3 \%$, respectively. In summary, the more complex procedure did not improve performance. ${ }^{79}$ Use of an ESAT-6 intracellular immunocytochemical stain has also been studied in a small number of patients in China with performance similar to the modified ZN with cytospin. ${ }^{80,79}$ Auramine stain with fluorescence microscopy or light emitting diode-based fluorescent microscopes can improve bacillary detection further, though there is a lack of good data on this in TBM.

\subsubsection{CSF TB Culture}

The major downfall of culture is that turn-around time (around 7 to 30 days) is too slow to guide clinical care. ${ }^{77}$ Although liquid culture with a mycobacterial growth indicator tube (MGIT) theoretically has a limit of detection of $\sim 10$ colony forming units (CFU)/ml it can only detect viable bacilli and thus is of limited use in those on antituberculous therapy, following delays in sample processing or where bacilli have been damaged by host inflammatory response. The sensitivity of liquid culture ranges between 43\% (10/23) to71\% (94/132) when measured against definite/probable TBM alongside AFB smear microscopy +/- Xpert +/- Ultra. ${ }^{77,76,81,82}$ Lastly, culture techniques are not widely available in many of the highest burden settings or are limited to retreatment cases where there is a concern for drug resistance. Nonetheless, in the absence of a better test for TBM, culture techniques continue to have a place in the diagnostic repertoire and have the added advantage of being able to provide information on the number of days to positivity (which gives an indication about bacillary load) and phenotypic drug susceptibility can be performed on the isolate.

\subsubsection{Molecular tests / nucleic acid amplification tests}

and tests for TB have been available since the 1990s. Commercial assays (e.g. Roche, Amplicor M.he 1980s tuberculosis) are costly (£25-50 per test) and require experienced laboratory technologists, as well as costly equipment and reagents, precluding their use in many settings. ${ }^{83}$ Line-probe assays such as Genotype MTBDRplus (Hain LifescienceGbmH, Nehren, Germany) are widely used to genotype culture isolates and rapidly determine whether rifampicin or isoniazid-resistance is present, but they can also be done directly on the CSF specimen. ${ }^{84}$ Currently, many laboratories in higher income settings with the requisite expertise use their own 'in-house' TB PCR assay, which uses more cost-effective equipment and reagents available from diverse suppliers ( $\_15$ per test) and can involve primers for the IS6110, 16S rDNA or any other region of the 
genome which uniquely identifies $M$. tuberculosis complex..$^{85}$ A systematic review and meta-analysis including 14 studies on commercial NAATs reported a pooled sensitivity of $56 \%$ (95\% Cl 46-66\%) and specificity of $98 \%$ (95\% Cl 97-99\%). ${ }^{85}$ The summary accuracy could not be determined for the 35 'in-house' tests as the results were too heterogeneous. ${ }^{85}$ The loop-mediated isothermal amplification (LAMP) assay is a low cost, low tech option but does not have any advantages over the Xpert MTB/Rif system. ${ }^{86,87}$

\subsubsection{Xpert MTB/Rif}

The Xpert MTB/Rif (Xpert) assay, Figure 1.9, a cartridge-based system, was developed by researchers from Rutgers New Jersey Medical School and the biotech company Cepheid to overcome the need for laboratory expertise, sample processing which can generate aerosols, and reagents which are prone to stocking out. Xpert uses a hemi-nested PCR to amplify the rifampicin resistance-determining region of the $r p o B$ gene and has a run-time of about 2 hours. ${ }^{88}$ Five rpoB molecular beacons detect both the presence of $M$. tuberculosis and mutations responsible for approximately $95 \%$ of rifampicin resistance. Owing to its ease of operation and large-scale applicability outside conventional laboratories, the WHO recommended the use and rapid scale up of Xpert in 2010. An unprecedented uptake followed, with over 2300 GeneXpert instruments and 6 million cartridges procured in the public sector in 104 countries by March 2014. In 2013, the WHO expert group made a policy update which widened the recommended use of Xpert to cover paediatric TB, extra-pulmonary TB, TBM, HIV-associated TB and as an initial test (before smear microscopy) for pulmonary TB. 89,90

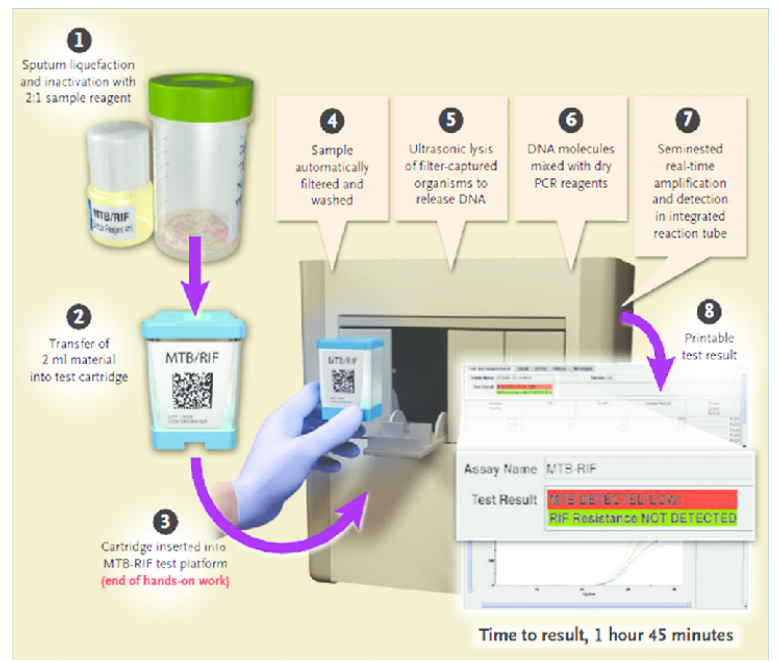
In analytical studies, Xpert has a limit of detection (LOD) of $113 \mathrm{CFU} / \mathrm{ml}\left(95 \% \mathrm{Cl}\right.$ 91.9-158.2). ${ }^{88,91}$ Using pooled data from 13 studies Xpert in CSF showed a sensitivity of $80.5 \%$ against culture and $62.8 \%$ against a clinical reference standard. Xpert had a consistently high specificity of $\sim 99 \% .{ }^{92}$ In HIV-positive adults in Uganda, a sensitivity of $72 \%$ against a culture-based reference standard has been reported when a good volume of CSF ( $>6 \mathrm{mls})$ is centrifuged prior to analysis. ${ }^{77}$

Figure 1.9. Illustration of Xpert MTB/Rif assay and procedure. ${ }^{93}$

Despite Xpert representing a huge step forward in TB diagnostics in LMIC settings, the developers of Xpert recognised its limitations in terms of sensitivity on smear-negative and extra-pulmonary samples and in the diagnosis of rifampicin resistance (which frequently gave 'indeterminate' results) and continued to refine the assay. The next generation assay, Xpert MTB/Rif Ultra (Xpert Ultra), intended to improve on these issues was endorsed by the WHO in 2017. 


\subsubsection{Xpert MTB/Rif Ultra}

The Xpert MTB/Rif Ultra assay has a number of technical optimisations: i) two additional probes targeting two multicopy genes (IS6110, IS1081) ii) a re-engineered cartridge that doubles the amount of sample that can be tested iii) optimised thermal cycling, iv) a semiquantitative 'trace' category corresponding to the lowest bacillary burden. ${ }^{91,94}$ In analytical studies Xpert Ultra has an LOD of $16 \mathrm{CFU} / \mathrm{ml}$, an 7-fold improvement on Xpert (Figure 1.10). ${ }^{91}$ The sensitivity of Xpert Ultra persists at sub-LOD concentrations, with $48 \%$ of the samples tested being positive by Ultra at $2.5 \mathrm{CFU} / \mathrm{ml} .^{91}$

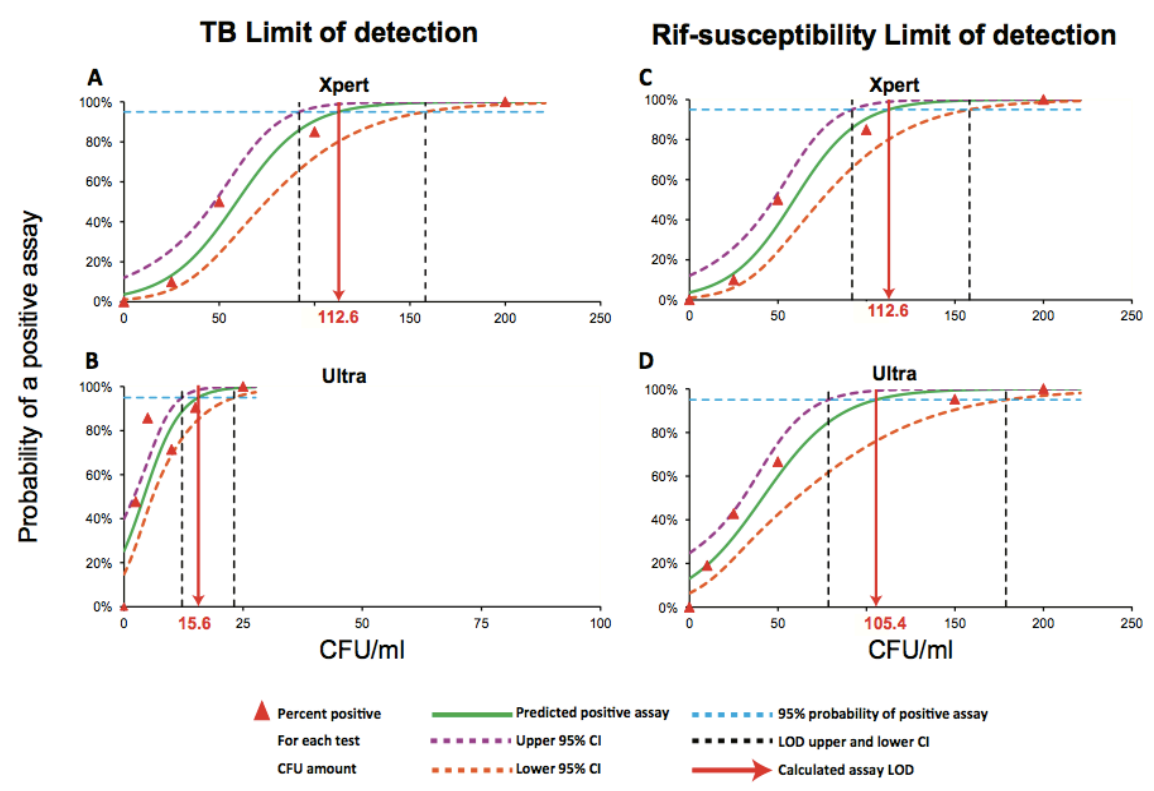

Figure 1.10. Xpert and Xpert Ultra limit of detection for M. tuberculosis H37Rv. The limit of detection of tuberculosis detection is shown for Xpert (A) versus UItra (B). The limit of detection for generating a rifampicin susceptibility rather than an indeterminate result is shown for Xpert (C) versus Ultra (D). ${ }^{91}$

The first diagnostic accuracy study comparing Xpert Ultra to Xpert MTB/Rif in pulmonary TB used culture as a reference standard and found a $5 \%$ higher sensitivity overall, with highest sensitivity increases of $+17 \%$ among smear-negative and $+12 \%$ among HIV-positive patients. However, these gains came at the expense of specificity, which was $2.7 \%$ lower, with specificity-decreases highest in patients with a history of TB who had completed treatment in the last 2 years $(-5.4 \%, 95 \% \mathrm{Cl}-9.1$ to -3.1$) .94,95$ Of the 43 participants who were positive by Xpert Ultra whilst negative by culture, next generation sequencing of amplicons from 12/14 cartridges confirmed the presence $M$. tuberculosis DNA suggesting that these are not false-positive DNA results per se, though whether the presence of DNA signified presence of active TB disease was uncertain. ${ }^{95}$ The authors believe Ultra-positive culture-negative results represent the presence of $M$. tuberculosis DNA fragments or intact bacilli (living or dead) which are not culture-viable or below the LOD on culture. ${ }^{95}$ It has since become clear that people who have been successfully treated for pulmonary TB can remain positive on Xpert Ultra (usually trace category) for years after completion of therapy. This complicates the interpretation of results in people with a history of TB and has led to difficult decisions by clinicians and stakeholders about 
the trade-off between increased sensitivity and earlier treatment of paucibacillary individuals but over treatment of people with a prior history of TB. A microsimulation modelling approach has estimated that 7 unnecessary treatments are generated per TB death averted when switching from Xpert MTB/Rif to Xpert Ultra in a South African setting, excluding the benefits of reduced transmission. ${ }^{96}$

Despite these challenges, in 2017 the WHO endorsed transitioning to Xpert Ultra in all settings. The Xpert Ultra assay can be run on the same machine, simply requiring a software update, and has a slightly shorter run time of 84 minutes. It has proven highly specific when tested on 30 different isolates of nontuberculous mycobacteria (NTM) and 18 bacteria to assess potential cross-reactivity. ${ }^{91}$

At the time of planning my PhD research, there had only been a single study of Xpert Ultra on CSF, conducted by our research team in Uganda, in which I contributed by supporting the processing of stored samples with the Xpert Ultra cartridges and manuscript write up. We evaluated Ultra on cryopreserved CSF from 129 predominantly HIV-positive individuals with suspected meningitis at Mbarara Regional Referral Hospital from February 2015 to November 2016. ${ }^{81}$ This study used MGIT culture, Xpert MTB/Rif and Xpert Ultra in the diagnostic panel and TB was microbiologically-confirmed in 22/129 (17.1\%) of CSF samples, of which 21 were positive by Xpert UItra, 10 were positive by Xpert MTB/Rif and 10 by MGIT culture (Figure 1.11).

Figure 1.11. Venn diagram illustrating overlap of positive CSF tests for M. tuberculosis in the 2018 Xpert Ultra study using cryopreserved $\mathrm{CSF}^{81}$

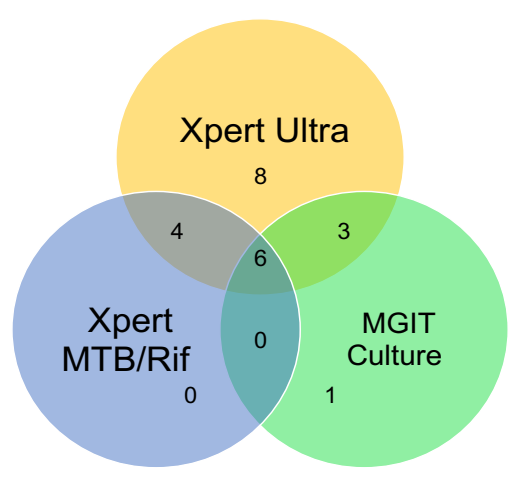

TB Negative $n=107$
In the absence of a suitable gold standard we used a composite reference standard (any positive CSF test for $M$. tuberculosis, i.e. definite TBM with inclusion of the Ultra result) for evaluating the performance of Ultra. Compared to a composite reference standard, sensitivity of Xpert Ultra was $95 \%(21 / 22,95 \% \mathrm{Cl}, 77-99 \%)$, significantly greater than either Xpert MTB/Rif or MGIT culture (both $45 \%$ sensitive, $10 / 22, \mathrm{P}<0.0034) .{ }^{97}$ Against a clinical reference standard of 'probable/definite' TBM $^{73}$ we found that Xpert Ultra had a lower sensitivity of $70 \%(95 \% \mathrm{Cl} 47$ to $87 ; 16$

of 23 cases) suggesting that up to 1 in 3 of TBM cases remain negative by all tests. Alternatively, it may be the case that the category of 'probable' TBM is non-specific and people with non-TB related brain pathology (for example tumours or viral encephalitides) are being incorrectly included in the probable category.

Following this first study on Xpert Ultra in TBM using cryopreserved samples some questions remained in my mind which warranted further investigation in a prospective study, namely:

1. What is the performance of Xpert Ultra on fresh CSF samples?

2. What is the case-fatality of TBM when Xpert Ultra is used in real-time to inform clinical decision making? 
3. If the sensitivity of Xpert Ultra is $70 \%$ for definite/probable TBM, is it possible to identify missed TBM cases by CSF culture, post-mortem examination in deceased patients without an antemortem diagnosis, and metagenomic next generation sequencing.

4. Are patients with alternative non-TB infection or pathologies misclassified as possible/probable TBM?

This prompted the prospective Xpert Ultra diagnostic accuracy study which features in chapter 5.

\subsubsection{Next-generation sequencing technologies}

The existing paradigm for diagnosing a neurological infection relies on a clinician formulating a differential diagnosis and sending CSF for a battery of tests. However, given that CSF volume is usually limited it can be impossible to perform all the tests of interest, and further the clinician may not have considered the actual pathogen responsible, especially true for rare pathogens or atypical presentations. As such, even with the diagnostics available in a higher-income setting hospital laboratory only about $50 \%$ of meningoencephalitis cases have a cause identified. Failure to obtain a timely diagnosis contributes to poor outcomes and high healthcare burden. ${ }^{98}$

Unbiased metagenomic next generation sequencing (mNGS) approaches do not require a priori selection of a pathogen and allow for the identification of a multitude of potential pathogens (spanning bacterial, viral, fungal, parasitic) to be identified by a single assay (Figure 1.12). The technique has proven useful in identifying pathogens missed by conventional microbiology techniques in recent study of 204 meningoencephalitis cases in the USA. ${ }^{98}$ Sequencing platforms are increasingly available in LMIC settings, a bioinformatic pipeline is available through a cloud-based system mitigating the need for large servers in-country, and the cost is coming down rapidly.

There is a single published small Chinese study on CSF mNGS in comparison with ZN stain, PCR and culture in 23 patients who were treated for TBM of which 12 were definite cases. Amongst definite cases mNGS had a sensitivity of $66.7 \%$ (8/12). Further, mNGS detected M. tuberculosis complex DNA in 10 of the 11 samples who were clinically treated for TBM without a positive ZN stain, Xpert or culture. ${ }^{99}$ Unfortunately the uniform case definition was not used to classify cases and no detail is given about the abundance of reads to $M$. tuberculosis.

For pathogens of interest which are usually present at very low abundance (e.g. M. tuberculosis), enrichment techniques can be used to increase the abundance of DNA prior sequencing to increase the likelihood of detection, a so called 'biased' approach. Given that TBM is a paucibacillary condition, enrichment techniques may be particularly useful. A Chinese paper has investigated a clustered regularly interspaced short palindromic repeat (CRISPR)-associated proteins (Cas) enrichment technique prior to conventional PCR, alongside Xpert and culture on 26 CSF samples from individuals treated for TBM. CRISPR-MTB had a sensitivity of $73 \%$, higher than Xpert (54\%) and culture (23\%), against a reference of clinically treated TB (uniform case definition not applied). ${ }^{100}$ 


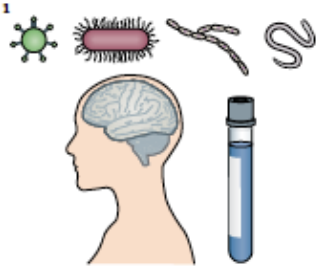

For patients with meningoencephalitis, metagenomic next generation sequencing (mNGS) of cerebrospinal fluid (CSF) or brain tissue is an unbiased tool to identify viral, bacterial, fungal or parasitic infections. DNA and RNA in a sample can degrade if the sample is not quickly frozen. CSF and brain tissue samples need to be handled in a sterile manner to avoid microbial contamination. Organisms such as Treponema pallidum, whose DNA is hard to detect by PCR in CSF, will be similarly difficult to detect by mNGS.

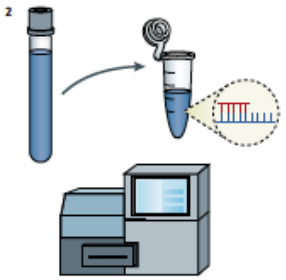

RNA and DNA are extracted and randomly amplified (after reverse transcription of the RNA) to generate cDNA molecules for sequencing. A Clinical Laboratory Improvement Amendments (CLIA)-certified technician prepares samples with care to prevent environmental and cross-sample contamination. Enough sequences are generated to ensure adequate sensitivity for detecting nonhuman sequences against the human background.

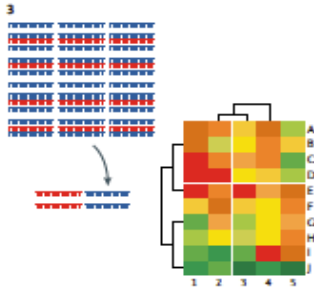

The millions of sequences that are generated for each patient sample are analysed with a standardized and clinically validated bioinformatics pipeline. Sequences mapping to the human genome, typically comprising 97-98\% of CSF mNGS data, are removed. After additional quality filtering, nonhuman sequences are searched against large genetic databases to determine the best organism matches.

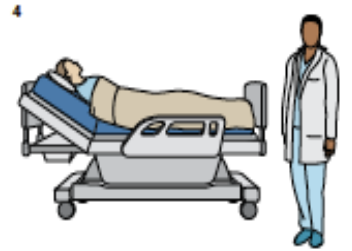

A report is provided to the clinician listing RNA or DNA viruses, parasites, fungi or

bacteria that were detected above the reporting threshold. Organisms known to be

frequent environmental contaminants

are not

reported.

Figure 1.12. Metagenomic next-generation sequencing in the diagnosis of meningoencephalitis. Adapted from Ramachandran Nature Reviews Neurology 2020. ${ }^{101}$

Next generation sequencing (Figure 1.12) for TBM is a relatively data free zone, for now at least. During the course of my PhD, we formed a collaboration with Dr Wilson and Dr Ramachandran, Neurologists at the University of California San Francisco (UCSF), supported by a US National Institute of Health R01 award (Principal Investigator Dr M Wilson, UCSF). Samples of CSF from adults with a suspected brain infection in Uganda were shipped to the UCSF Chan Zuckerberg Biohub for DNA and RNA sequencing. Firstly, Dr Wilson was keen to explore whether mNGS could be coupled with a CRISPR-Cas9 enrichment technology tool that enriches pathogen sequences of interest to improve detection of TBM. Secondly, given that $90-98 \%$ of mNGS sequencing data is derived from the host (rather than pathogen), host transcriptomic profiling (HTP) may identify pathogen-specific signatures which could be used for diagnostic purposes. Dr Wilson's team combined CSF mNGS and HTP to identify cases of TBM missed by Xpert Ultra and to identify other potential pathogens misclassified as TBM. This work, if successful, holds promise of better meningoencephalitis diagnosis in future, but thought needs to be given to how this technology can be used in real-time to guide patient care and how it can be applied to LMIC settings.

\subsubsection{CSF Biomarkers}

In light of the fact that TBM is a paucibacillary disease with marked inflammation, one could hypothesise that using an immunology-based diagnostic test would be a better approach than hunting for scarce bacilli. A biomarker is defined as 'an objective characteristic that indicates a normal or pathogenic process' and can originate from the host or the pathogen of interest. ${ }^{102}$ There is considerable research interest in developing biomarker based tests for TB but progress has been relatively limited thus far. A recent systematic review of potential TB biomarkers or biosignatures by Foundation for Innovative New Diagnostics (FIND) identified 44 
'front-runners' of which 37 (84\%) were of host origin and included antibodies, cytokines, chemokines and RNA signatures. ${ }^{102}$ The ideal TBM biomarker would ideally be instrument free so it could be performed at the point of care (or require minimal instrumentation) and would utilise readily accessible samples such as blood, urine, saliva or breath, so that lumbar puncture could be avoided. Though, even finding a biomarker that could be performed at the point-of-care (POC) on CSF would be a big step forward.

\subsubsection{CSF host biomarkers}

So far, a number of host biomarkers have been studied on CSF including adenosine deaminase (ADA, an enzyme produced by lymphocytes), IFN- $\gamma$ levels, interferon gamma release assays (IGRAs) and antibodies (anti-M37Ra, anti-M37Rv, anti-antigen-5). ${ }^{103}$ Whilst they can boast a moderate sensitivity, all are limited by high laboratory infrastructure requirements and cost. Further, heterogeneous study design and lack of reference to the uniform case definition makes cross-study comparisons challenging. Where HIV co-infection has been studied, more false-positives arise (giving reduced specificity). A number of meta-analyses have been published which summarise some of the biomarkers studied to date (Table 1.3).

\begin{tabular}{|c|c|c|c|c|c|}
\hline Diagnostic assay & $\begin{array}{l}\text { Number of studies (or } \\
\text { patients) included }\end{array}$ & Sensitivity & Specificity & $\begin{array}{l}\text { HIV co-infection } \\
\text { data reported }\end{array}$ & Comments \\
\hline $\mathrm{ADA}^{104,105}$ & $\begin{array}{l}10 \text { patients } \\
417 \text { patients }\end{array}$ & $\begin{array}{l}79 \% \\
57 \%\end{array}$ & $\begin{array}{l}91 \% \\
87 \%\end{array}$ & $\begin{array}{c}\text { Nil } \\
\text { more false positives }\end{array}$ & $\begin{array}{l}\text { High cost \& lab } \\
\text { infrastructure }\end{array}$ \\
\hline IGRA $^{106}$ & 6 studies & $77 \%$ & $88 \%$ & Nil & $\begin{array}{l}\text { Moderate } \\
\text { performance, cost }+\end{array}$ \\
\hline $\begin{array}{l}\text { Antibodies }{ }^{107} \\
\text {-Anti-M37Ra } \\
\text {-Anti-M37Rv } \\
\text {-Anti-antigen-5 }\end{array}$ & $\begin{array}{l}5 \text { studies } \\
12 \text { studies } \\
8 \text { studies }\end{array}$ & $\begin{array}{l}91 \% \\
84 \% \\
84 \%\end{array}$ & $\begin{array}{l}99 \% \\
98 \% \\
95 \%\end{array}$ & Nil & $\begin{array}{l}\text { - study heterogeneity } \\
\text { - lack of commercial } \\
\text { assays } \\
\text { - lab infrastructure }\end{array}$ \\
\hline IFN- $\gamma^{108-110}$ & $\begin{array}{l}69 \text { patients } \\
140 \text { patients } \\
128 \text { patients }\end{array}$ & $\begin{array}{l}70 \% \\
58 \% \\
70 \%\end{array}$ & $\begin{array}{l}87 \% \\
94 \% \\
94 \%\end{array}$ & $\begin{array}{c}\text { Nil } \\
\text { Ok as rule-in test } \\
\text { False positives }\end{array}$ & $\begin{array}{l}\text { - lab infrastructure, } \\
\text { cost, moderate } \\
\text { sensitivity }\end{array}$ \\
\hline
\end{tabular}

Table 1.3. Summary of studies and meta-analyses on host CSF biomarkers

No single host biomarker appears to be the perfect candidate in isolation so a combination of biomarkers, or a biomarker 'signature' may perform better. This may be particularly true if the signature combines both host and pathogen biomarkers.

\subsubsection{CSF pathogen biomarkers-TB-lipoarabinomannan}

Pathogen-based biomarkers tend to be more specific than host-biomarkers which can be elevated in the setting of other infectious or non-infectious conditions. The composition of volatile organic compounds (VOCs) in breath can be affected by the presence of $M$. tuberculosis in the lungs and are being studied for the diagnosis of pulmonary TB, but VOCs may have limited mileage in TBM as the infection can be compartmentalised to the CNS. Of all the TB antigens, lipoarabinomannan (LAM) is the marker with the largest evidence base and potential. LAM, a 17.3 kDa lipopolysaccharide mycobacterial cell wall component, has been studied on a number of bodily fluids - sputum, pleural fluid, CSF, and urine. 
In earlier studies, LAM was detected using an enzyme-linked immunosorbent assay (TB ELISA, Clearview, Inverness Medical Innovations, USA). A pilot study in South Africa using cryopreserved CSF samples with culture as a reference standard found sensitivity of CSF LAM ELISA to be quite promising (64\%) though specificity was only $69 \%$ (using a cut-point of 0.22 ). ${ }^{111}$ Since then a true POC LAM lateral flow assay (LFA) has been developed (Alere Determine TB-LAM Ag assay, Abbot, Chicago, IL, USA), which is easy to use, well priced ( $£ 3$ per test) and provides semi-quantitative results (negative, positive in the range $1+$ to $4+$ ). Minimal training is required and the results are read by comparing the visible band on the test strip with the manufacturer's reference card after exactly 25 minutes has elapsed, see Figure 1.13.

a)

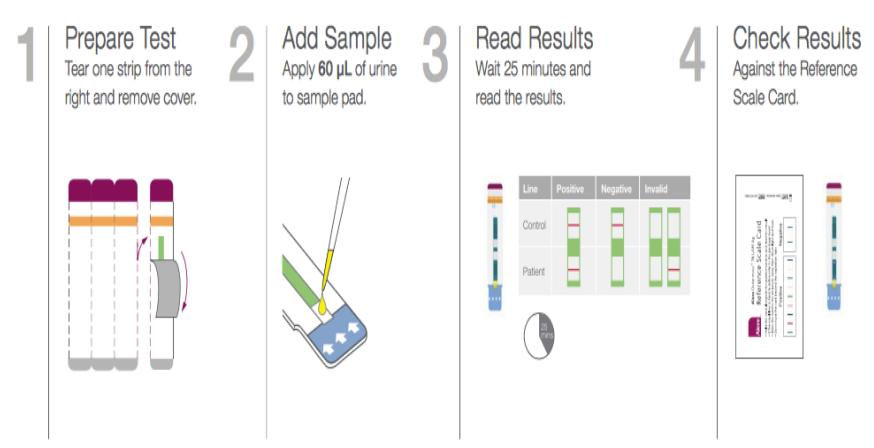

b)

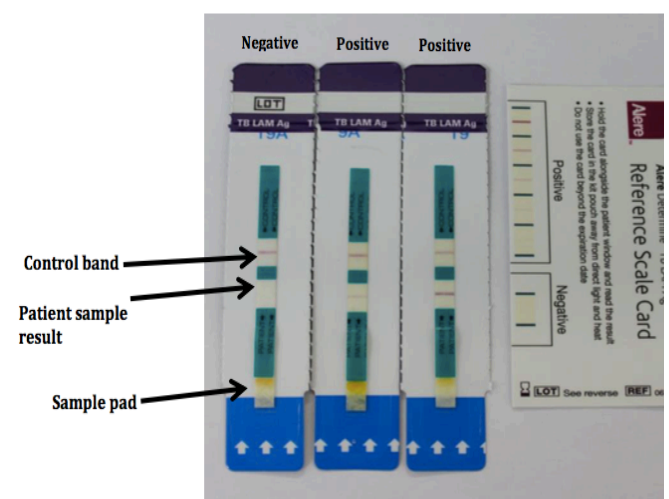

Figure 1.13. a) Alere Determine TB-LAM assay package insert demonstrating the steps involved to generate a result and ease of use, b) a photo of three Alere LAM results illustrating the real-life appearance of the negative and positive $(+3$ and +5$)$ results. Photo kindly provided by Dr A Gupta-Wright.

There was initial excitement around the performance of Alere Determine TB-LAM LFA (Alere LAM) on CSF after an autopsy study in Uganda which tested 91 cryopreserved CSF samples collected from the fourth ventricle with Alere LAM and reported a sensitivity of $75 \%$ and specificity of $87 \%$ against definite (microbiologically or histopathologically-confirmed) TBM. ${ }^{112}$ Whether such an impressive sensitivity was possible because the study design (autopsy) pre-selected patients with the highest bacillary burden, or whether ventricular CSF has a particularly high antigen load, or whether detectable TB antigen load increases after the host is deceased is unclear, but unfortunately studies of Alere LAM on lumbar CSF from living patients have been less encouraging. In 2016, the meningitis clinical research team in Uganda tested lumbar CSF samples collected from 67 patients with suspected meningitis at the bedside with Alere LAM, of which 12 had definite TBM (Xpert or culture confirmed) and 2 had probable TBM. None of the 67 tests performed yielded a positive result and the study was abandoned. ${ }^{113}$ However, the majority of patients in this small study fell into the possible or not TBM group (many actually had cryptococcal meningitis) so there was a low pretest probability of disease and further larger studies were warranted. In a diagnostic accuracy study conducted between 2014-2017, which included 550 Zambian patients with suspected meningitis and used culture-confirmed TBM as a reference standard, CSF LAM had a sensitivity of 21.9\% (23/105) and a specificity 
of $94.2 \%$ respectively. ${ }^{114}$ The culture-based reference standard is not ideal for TBM studies and the investigators did not use the uniform case definition so understanding the performance of Alere LAM in probable and possible TBM cases was not possible.

With the transition from Xpert MTB/Rif to Xpert Ultra as the WHO-recommended initial test for TBM, we revisited CSF Alere LAM in Uganda in a nested study in hospitalised patients with suspected TBM undergoing lumbar puncture (Appendix 3). The poor sensitivity (33\% for definite TBM and 24\% for definite/probable TBM) may relate to the high LAM antigen load required to yield a positive Alere LAM result.

The novel Fujifilm SILVAMP TB LAM (FujiLAM; Fujifilm, Tokyo, Japan) POC assay, which similar to Alere LAM detects the presence of LAM, but combines a pair of high affinity monoclonal antibodies directed towards largely M. tuberculosis-specific LAM epitopes and a silver-amplification step that increases the visibility of test and control lines. ${ }^{115}$ FujiLAM offers on average 30\% improved sensitivity for detecting TB (independent of whether it is pulmonary TB or extrapulmonary TB) compared to Alere LAM across subgroups stratified by CD4 strata, while maintaining high specificity. ${ }^{115}$ A single recent study has evaluated FujiLAM on 19 CSF samples and found it to have a sensitivity of $47 \%$ (compared to $16 \%$ for Alere TB-LAM) against microbiologically confirmed TBM. ${ }^{116}$

However, obtaining CSF via a lumbar puncture is not always possible in LMIC government hospital settings for the reasons outlined in section 1.3. Even in the most well-resourced hospitals lumbar punctures can often be delayed whilst brain imaging is awaited. So, a non-CSF biomarker that can support diagnosis of TBM or aid with prognostication would be very useful. In the next section I describe urine TB diagnostics of TB with a particular focus on TBM.

\subsubsection{Urine diagnostics for HIV-associated TB}

Early morning urines for TB culture are still used to diagnose TB of the genitourinary tract, albeit with a long diagnostic lead-time. Urine is easy to collect, even in the sickest patients, has low risk of generating infectious aerosols, and has relatively few contaminants. This has driven research into urine TB antigens which may be of diagnostic or prognostic value, of which the urinary LAM and Xpert MTB/Rif are the most promising to date.

\subsubsection{Urine TB-Lipoarabinomannan in HIV-associated TB}

I touched on the background of CSF LAM with regards to CSF biomarkers (section 1.3.6.2) but the volume of research on urinary LAM is far greater. Despite LAM being studied as early as 1990s, it only gained traction more recently when it became clear that its poor sensitivity of around 30\% for the detection of TB (culture- or Xpert-confirmed) in immunocompetent hosts increases to a moderate sensitivity of $56 \%(95 \% \mathrm{Cl} 41-70 \%)$ in the most immunocompromised hosts (CD4 $<100$ cells $/ \mu$ )), see Figure 1.14..$^{117,118,119}$ 
Figure 1.14. Sensitivity of urine Alere TB-LAM and urine Xpert in urine samples from HIV-positive patients with culture-confirmed pulmonary TB by CD4+ T cell strata. Reproduced from Lawn 2012. ${ }^{120}$

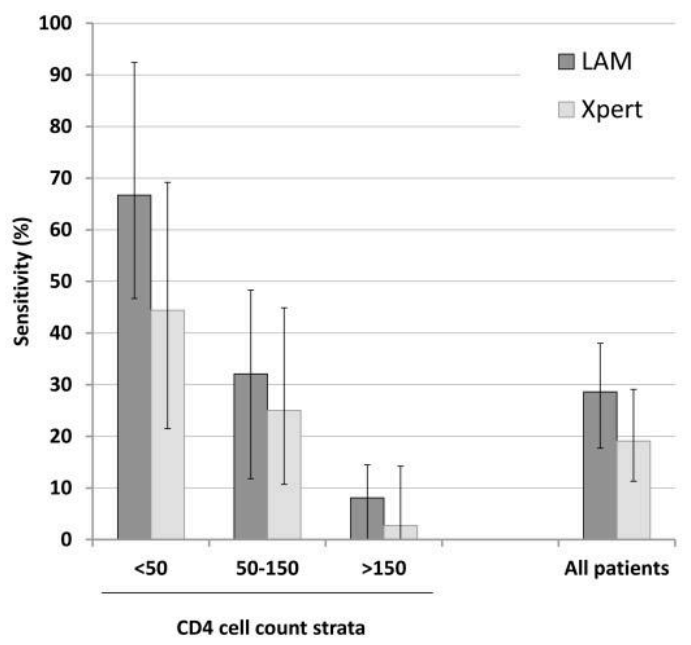

This was a welcome observation, as those with the most advanced HIV have a high early mortality ${ }^{121}$ and traditional microbiological techniques (e.g. smear microscopy) perform most poorly in this population. As CD4+ T-cell count declines M. tuberculosis becomes haematogenously disseminated and frequently affects distant organs including the genitourinary tract with renal micro-abscesses noted in $50-69 \%$ of HIVpositive cadavers. ${ }^{122}$ LAM antigenaemia may give rise to free circulating LAM being filtered into the urine (especially plausible if there is HIV-related nephropathy / proteinuria and the glycolipid molecule has not been excessively immune complexed) or $M$. tuberculosis bacilli can directly infect the renal tract and be secreted into the urine. ${ }^{118}$ The WHO recommend Alere LAM to screen for active TB in PLHIV who are hospitalised with signs or symptoms of TB and have a CD4+ T-cell count $<100$ cells/ $\mu$ l, or who are seriously ill regardless of CD4+ count. ${ }^{123}$ Persons with TBM fall into this 'seriously ill' category but unfortunately the implementation of the WHO recommendations has been slow and Alere LAM is not universally available.

The potential of the Alere LAM test as a screening tool to reduce deaths from TB was recently evaluated in an RCT in hospitalised PLHIV in Malawi and South Africa (the STAMP trial). In the intervention arm urine was tested with Alere LAM assay and Xpert MTB/Rif. Other TB diagnostics and treatment were as per normal standard of care. There was a trend towards reduced 56-day mortality overall (adjusted risk difference [aRD], $-2.8 \%, 95 \% \mathrm{Cl}-5.8$ to $0.3 \% ; 0=0.074)$ and a reduced 56 -day mortality in pre-specified sub-groups of interest: CD4+ count $<100$ cells/ $\mu \mathrm{L}$ ( $\mathrm{RRD}-7.1 \%, 95 \% \mathrm{Cl}-13.7$ to $-0.4 ; p=0.036)$, severe anaemia $(-9.0 \%,-16.6$ to -1.3 ; $p=0.021)$, and patients with clinically suspected TB $(-5.7 \%,-10.9$ to $-0.5 ; p=0.033) .{ }^{124}$ Further, Alere LAM was shown to have value as a prognostic tool for 2 month mortality as part of a 6-point risk score in two cohorts of hospitalised PLHIV in sub-Saharan Africa. ${ }^{122}$ Meta-analysis of 10 studies has confirmed that urine LAM positivity is associated with a 2.5-fold increased risk of death, even after adjustment for degree of immune suppression. ${ }^{125,126}$

With this new evidence in mind, in 2019 the WHO made a policy update and expanded its recommendations for the use of Alere LAM to include outpatient settings where an HIV-positive adult or child has signs or symptoms of TB or has a CD4+ T-cell count $<100$ cells $/ \mu L^{127}$

The novel FujiLAM assay has been compared to the Alere LAM on urine samples in HIV-positive South African hospital inpatients. Using a microbiological reference standard, the estimated sensitivity of FujiLAM was 70.4\% compared with $42 \cdot 3 \%$ for Alere LAM (difference $+28 \cdot 1 \%$ ) and the estimated specificity of FujiLAM was 
90.8\% and $95 \cdot 0 \%$ for Alere LAM (difference $-4 \cdot 2 \%$ ). ${ }^{115}$ FujiLAM appears promising as a urine diagnostic and is undergoing wider evaluation before it can be considered by WHO and other stakeholders as a routine diagnostic option (and will require scale up in manufacturing).

\subsubsection{Urine TB-Lipoarabinomannan in TB meningitis}

Given that HIV-associated TBM may occur as part of a more broadly disseminated TB picture one could hypothesize that urine TB-LAM would be a good diagnostic biomarker with reasonable sensitivity. Urine FujiLAM has not yet been studied in TBM patients and there is only a single study on Alere LAM. A Zambian study, including 550 suspected TBM patients, of which 86.2\% (474) were HIV-positive compared urine Alere LAM to culture-confirmed TBM. Urine LAM had a rather disappointing sensitivity of $24.1 \%$ (numerator, denominator and $95 \% \mathrm{Cl}$ were not reported). Specificity of urine LAM was quoted as $76.1 \%, 51$ patients had positive urine LAM whilst CSF culture was negative. Given that CSF culture is only moderately sensitivity it is an imperfect reference standard to use and it is plausible that the 51 positive urine LAM results were misclassified as false positive giving rise to the low sensitivity and specificity. The low specificity is particularly dubious given a robust South African diagnostic accuracy study of urine LAM, in which the reference standard included multiple cultures and NAATs from several extra-pulmonary sites, which found the specificity of urine LAM to be extremely high ( 99\%). ${ }^{128}$ Further, meta-analysis of 12 studies (using culture or NAAT from any bodily site as a reference standard) reported that pooled specificity was $92 \%$ (95\% Cl 80-97\%). ${ }^{117}$ To summarize, the Zambian study had sub-optimal methodology and results were not clearly reported in the paper, so the true value of urine Alere LAM assay as a diagnostic or prognostic aid in HIV/TBM remains to be established and was therefore explored within my PhD research.

\subsubsection{Urine Xpert or Ultra in the diagnosis of HIV-associated TB}

Although the WHO has endorsed Xpert for non-respiratory samples, its use on urine has not been recommended due to insufficient data. The most thorough study to date by Lawn et al, screened 427 hospital admissions in South Africa, and multiple samples were collected for culture and Xpert (including urine, sputum, blood, other samples as clinically indicated). The investigators found that sensitivity of urine Xpert was $42.4 \%$ using unconcentrated urine, but this increased to $59.0 \%$ when urine was centrifuged and the cell pellet tested with Xpert. As with urine TB-LAM, the yield from urine Xpert was greater among those with CD4+ T-cell count $<100$ cells $/ \mu$ l (77\% versus $50 \%$ in those with CD4+ T-cell count $>100$ cells $/ \mu l, p=0.001) .{ }^{129}$

A handful of studies have compared urine LAM and urine Ultra head-to-head. Urine LAM has had a higher diagnostic yield in the majority of studies including the STAMP trial. ${ }^{124}$ It may be that the limit of detection of the Xpert assay $(131 \mathrm{CFU} / \mathrm{ml})$ is greater than the antigen load required to generate a 1+ positive LAM result. Or that they are detecting discrete entities (an antigen versus DNA) which are present in different amounts depending on the disease stage/phenotype (Xpert is designed to detect the presence of bacilli, not cell free 
DNA, and TB-LAM is a circulating antigen). No studies yet have directly compared LAM and Xpert Ultra, but given that Ultra has a 7-fold lower limit of detection (16 CFU/ml) than Xpert it may prove to be superior to LAM and warrants investigation.

\subsubsection{Urine Xpert or Ultra in the diagnosis of HIV-associated TB meningitis}

To our knowledge there is only a single case report of urine Xpert Ultra being used in the diagnosis of TBM. A 56-year old Ugandan HIV+ diabetic lady presented with a 3-week history of confusion, neck pain and general body weakness. CSF examination revealed a lymphocyte pleocytosis (220 cells/ $\mu$ l) but no organisms were seen on Gram's stain, CSF protein was $48 \mathrm{mg} / \mathrm{dL}$, cryptococcal antigen was negative, CSF Xpert Ultra and bacterial culture were negative. Urine TB diagnostics were performed - Alere TB LAM was negative but urine Ultra was positive (semi-quantitative category: very low). In the absence of an alternative explanation, TBM treatment was commenced with good resolution of symptoms at 6 weeks of follow up. CSF mycobacterial growth indicator tube culture was reported as negative 6 weeks later. ${ }^{130}$ Whilst this was simply a case report, it supported the idea of Xpert Ultra being further explored in the diagnosis of TBM, and I therefore included an exploratory study of urine TB diagnostics in meningitis patients in my $\mathrm{PhD}$ research.

In summary, we know relatively little about TBM in Uganda and we currently lack a highly sensitive POC diagnostic test and prognostic marker. A multitude of research questions exist relating to diagnostics. Table 1.5 outlines some of the most pressing questions for consideration. 
Table 1.5. Priority research questions on TBM in Uganda, existing evidence and potential approaches to addressing the outlined questions.

\begin{tabular}{|c|c|c|c|}
\hline Area & Questions applicable to Africa & Evidence available & Potential approaches to address the question \\
\hline \multirow{3}{*}{$\begin{array}{l}\text { Understanding } \\
\text { epidemiology and } \\
\text { burden }\end{array}$} & $\begin{array}{l}\text { How many children / adults } \\
\text { develop TBM each year in } \\
\text { Uganda? }\end{array}$ & $\begin{array}{l}\text { Nil from Uganda. } \\
\text { EPTB cases reported } \\
\text { to WHO }\end{array}$ & $\begin{array}{l}\text { 1. Improve data collection through national } \\
\text { TB registers so that site of disease is } \\
\text { captured } \\
\text { 2. Create a national TB meningitis registry } \\
\text { 3. Modelling studies }\end{array}$ \\
\hline & $\begin{array}{l}\text { What is the annual mortality due } \\
\text { to TBM in Uganda? }\end{array}$ & Nil from Uganda & 1. Modelling studies \\
\hline & $\begin{array}{l}\text { What are the in-hospital and } \\
\text { longer-term outcomes from TBM } \\
\text { in Uganda? }\end{array}$ & Nil from Uganda & $\begin{array}{l}\text { 1. Retrospective review of available data } \\
\text { through the meningitis research team } \\
\text { 2. Prospective study to evaluate functional } \\
\text { outcomes and survival }\end{array}$ \\
\hline $\begin{array}{l}\text { Understanding risk } \\
\text { factors for developing } \\
\text { TB meningitis }\end{array}$ & $\begin{array}{l}\text { Who is at risk of developing TBM } \\
\text { disease in Uganda? }\end{array}$ & Nil from Uganda & $\begin{array}{l}\text { 1. Cross sectional or case-control study to } \\
\text { ascertain risk factors for disease }\end{array}$ \\
\hline \multirow{3}{*}{$\begin{array}{l}\text { Understanding risk } \\
\text { factors for poor } \\
\text { outcome }\end{array}$} & $\begin{array}{l}\text { Which baseline characteristics are } \\
\text { associated with risk of dying? }\end{array}$ & & $\begin{array}{l}\text { 1. Cox model to identify baseline and } \\
\text { dynamic risk factors for death }\end{array}$ \\
\hline & $\begin{array}{l}\text { Can a urine biomarker be used as } \\
\text { prognostic tools in TBM? }\end{array}$ & $\begin{array}{l}\text { Alere LAM 2.5-fold }{ }^{126} \\
\text { Xpert 2.0-fold } \\
\text { increased risk of } \\
\text { death from } \\
\text { disseminated TB } \\
\end{array}$ & $\begin{array}{l}\text { 1. Cohort study with baseline urine Alere } \\
\text { LAM and urine Ultra testing with in- } \\
\text { hospital or longer-term mortality data } \\
\text { collection }\end{array}$ \\
\hline & $\begin{array}{l}\text { Can a point of care CSF biomarker } \\
\text { be used as a prognostic tool? }\end{array}$ & $\begin{array}{l}\text { Lactate may have a } \\
\text { role in prognosis } \\
\text { (India) }^{131}(\text { Vietnam })^{132}\end{array}$ & $\begin{array}{l}\text { 1. Explore relationship between Xpert cycle } \\
\text { threshold and mortality in a cohort study } \\
\text { or with available data } \\
\text { 2. Model baseline CSF lactate \& glucose } \\
\text { with outcomes }\end{array}$ \\
\hline \multirow{6}{*}{$\begin{array}{l}\text { Improving diagnosis } \\
\text { of TB meningitis }\end{array}$} & $\begin{array}{l}\text { Can TBM be differentiated from } \\
\text { other causes of meningitis with } \\
\text { urine diagnostics? }\end{array}$ & Nil & $\begin{array}{l}\text { 1. Cross sectional study in all meningitis } \\
\text { presentations including urine Alere LAM } \\
\text { and urine Ultra and CSF diagnostic panel }\end{array}$ \\
\hline & $\begin{array}{l}\text { Will CSF Xpert Ultra improve } \\
\text { diagnosis of TBM in real-time } \\
\text { compared to Xpert MTB/Rif? }\end{array}$ & $\begin{array}{l}\text { Uganda, frozen CSF } \\
\text { sensitivity Ultra > } \\
\text { Xpert } 81\end{array}$ & $\begin{array}{l}\text { 1. Prospective cohort study using Ultra } \\
\text { alongside existing CSF diagnostic panel }\end{array}$ \\
\hline & $\begin{array}{l}\text { Can a CSF biomarker support a } \\
\text { diagnosis of TBM? }\end{array}$ & 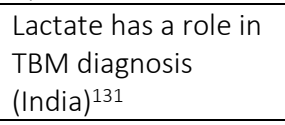 & $\begin{array}{l}\text { 1. Cross-sectional study with CSF TB-LAM in } \\
\text { combination with other diagnostics }\end{array}$ \\
\hline & $\begin{array}{l}\text { Do suspected probable and } \\
\text { possible TBM patients (negative } \\
\text { by CSF Ultra \& culture) actually } \\
\text { have TBM? If not, what do they } \\
\text { have? }\end{array}$ & & $\begin{array}{l}\text { 1. Unbiased mNGS to look for presence of } \\
\text { other pathogens } \\
\text { 2. Biased mNGS with enrichment for M.tb } \\
\text { 3. Host transcriptomic profiling } \\
\text { 4. Comprehensive meningoencephalitis } \\
\text { diagnostic evaluation for infectious and } \\
\text { non-infectious causes }\end{array}$ \\
\hline & $\begin{array}{l}\text { Can pathogen and host DNA/RNA } \\
\text { sequencing data be combined } \\
\text { into a diagnostic strategy with } \\
\text { machine learning? }\end{array}$ & & $\begin{array}{l}\text { 1. Generate large dataset with detailed } \\
\text { clinical, pathogen and host information } \\
\text { and optimise a disease classifier with } \\
\text { machine learning techniques }\end{array}$ \\
\hline & $\begin{array}{l}\text { Is CSF metagenomic next } \\
\text { generation sequencing diagnosis } \\
\text { feasible in Uganda? }\end{array}$ & Nil from Uganda & $\begin{array}{l}\text { 1. Pilot study using local sequencing and } \\
\text { bio-informatic capabilities }\end{array}$ \\
\hline
\end{tabular}

Text shown in bold denotes those that I addressed during this PhD period. $\mathrm{mNGS}=$ metagenomic next generation sequencing, LAM = lipoarabinomannan, M.tb = mycobacterium tuberculosis, EPTB = extrapulmonary TB 


\subsection{Part II: Treatment of Tuberculous Meningitis}

\subsubsection{Rifampicin}

In 1957, in a soil sample from the French Riviera, a new bacterium was discovered (Streptomyces mediteranei) which produced a novel class of molecule with antibiotic activity which was named rifamycin (after the scientist's favourite French crime story 'Rififi'). A stable semi-synthetic compound with high efficacy and good tolerability was produced in 1965, called 'rifampicin' and was USA Federal Drug Authority approved in 1971. Rifampicin binds to the $r p o B$ portion of the bacterial polymerase, thereby preventing formation of new proteins. ${ }^{133}$ Rifampicin exhibits bactericidal activity (killing actively growing organisms) and sterilizing activity (killing the persisting bacilli that are responsible for relapse). ${ }^{133,134}$ Only around $15 \%$ of the circulating drug that is unbound to plasma proteins ('free' rifampicin) is therapeutically active and able to readily cross barriers such as the intact blood-brain barrier.

Rifampicin is a clinical pharmacologist's nemesis - a promiscuous inducer of the expression of a broad array of enzymes and drug-transporting molecules through its activation of a master transcriptional regulator, the pregnane $X$ receptor. ${ }^{135}$ Rifampicin is the most powerful known inducer of the hepatic cytochrome P450 system, and through upregulation of the CYP3A4 isoenzyme it increases the metabolism of many drugs and thereby lowers their levels and clinical effect, requiring careful dose adjustments to be made. It also induces its own metabolism through clearance autoinduction, so levels reduce by about 30-50\% over the first month of therapy. ${ }^{136}$ Common side effects include gastrointestinal upset, hypersensitivity reactions (including fever and rash) and hepatotoxicity, and it turns bodily fluids alarmingly orange. As well as treating a number of other infections such as Legionnaire's disease and leprosy, rifampicin is the cornerstone of TB therapy.

\subsubsection{Current TB therapy - did we miss a trick with rifampicin dosing?}

The WHO recommended treatment for TB involves a two-month intensive phase of rifampicin $(\mathrm{R}, 8-12 \mathrm{mg} / \mathrm{kg})$, isoniazid $(\mathrm{H}, 4-6 \mathrm{mg} / \mathrm{kg})$, pyrazinamide $(\mathrm{Z}, 20-30 \mathrm{mg} / \mathrm{kg})$ and ethambutol $(\mathrm{E}, 15-20 \mathrm{mg} / \mathrm{kg})$ followed by a minimum of a 4-month continuation phase of rifampicin and isoniazid, depending on national guidelines and modifications for drug-susceptibility results where available. ${ }^{137}$ This 'short-course' TB therapy was defined in clinical trials in pulmonary TB in East Africa and India in the 1980s; rifampicin 600 mg when added to streptomycin, isoniazid and ethambutol, allowed shortening of therapy to 9-12 months. Shortly after, substitution of streptomycin with pyrazinamide allowed 6-8 months of therapy for pulmonary TB. ${ }^{138,139} \mathrm{~A}$ number of small trials in the 1970 and 80s examined rifampicin doses of up to $1200 \mathrm{mg}(20 \mathrm{mg} / \mathrm{kg}$ ) with different dosing intervals (weekly, twice/thrice weekly, and daily). Intermittent dosing of rifampicin $20 \mathrm{mg} / \mathrm{kg}$ was met with a high incidence of flu-like syndrome but despite daily dosing being well-tolerated it didn't 
become part of mainstream therapy. ${ }^{140}$ This was also in part because rifampicin was a prohibitively expensive drug in the 1970s, so with large-scale TB programming in mind, the $600 \mathrm{mg}$ dose did the job of shortening pulmonary TB therapy and became entrenched in TB treatment regimens in the decades to follow. The optimal rifampicin dose was not defined through the modern paradigm of pre-clinical in vitro studies, animal or in-human pharmacokinetic-pharmacodynamic dose-optimisation studies, or in silico modelling.

With the current rifampicin $10 \mathrm{mg} / \mathrm{kg} /$ day dose interindividual variability in exposure is large and the margin for error is minimal. ${ }^{133}$ Low rifampicin exposure has been associated with drug resistance, treatment failure and delayed bacillary clearance from sputum. ${ }^{141}$ Owing to the lack of forgiveness with the $10 \mathrm{mg} / \mathrm{kg}$ rifampicin dose evolution of rifampicin drug resistant tuberculosis has arisen and poses a significant public health threat. In South Africa around 15\% of hospitalised adults with HIV/TB have multidrug resistant tuberculosis (MDR-TB). ${ }^{142}$

\subsubsection{Challenges with the current first-line TB regimen and finding better options}

The current WHO recommended TB regimen comes with multiple challenges; these have been contributory factors to the difficultly in curbing the TB epidemic:

1. Duration of therapy leads to poor adherence $\mathrm{e}^{143,144}$

2. Pill burden leads to poor adherence ${ }^{143,144}$

3. Inter-individual variability in PK exposure to TB drugs ${ }^{145}$

4. Sub-optimal response (failure and relapse in $\sim 10 \%$ ) in those with low exposures or poor adherence ${ }^{146,138}$

5. Emergence of resistance in those with low exposures or poor adherence ${ }^{143,147}$

6. Toxicity including hepatotoxicity, peripheral neuropathy, optic neuritis ${ }^{145}$

7. Drug-drug interactions relating to rifampicin ${ }^{135}$

Identification of a novel regimen which addresses these challenges could potentially improve TB outcomes, reduce transmission and the emergence of resistance. Unfortunately, M. tuberculosis drug discovery and development is an extremely lengthy and expensive process $-M$. tuberculosis needs to be handled in a category 3 biosafety laboratory, is slow growing, and has a dormant (persistent) state where it becomes undetectable by conventional tests, clinical trials are long and not necessarily economically attractive investments to pharmaceutical companies. As such, progress has been slow with no new first-line TB drugs coming to the market since rifampicin in the 1970s, see Figure 1.15. Newer agents such as bedaquiline and delamanid are reserved for the treatment of MDR-TB, and have recently allowed MDR-TB regimens to become shorter, less toxic and free of the painful daily injections required by aminoglycosides antibiotics. ${ }^{148}$ 


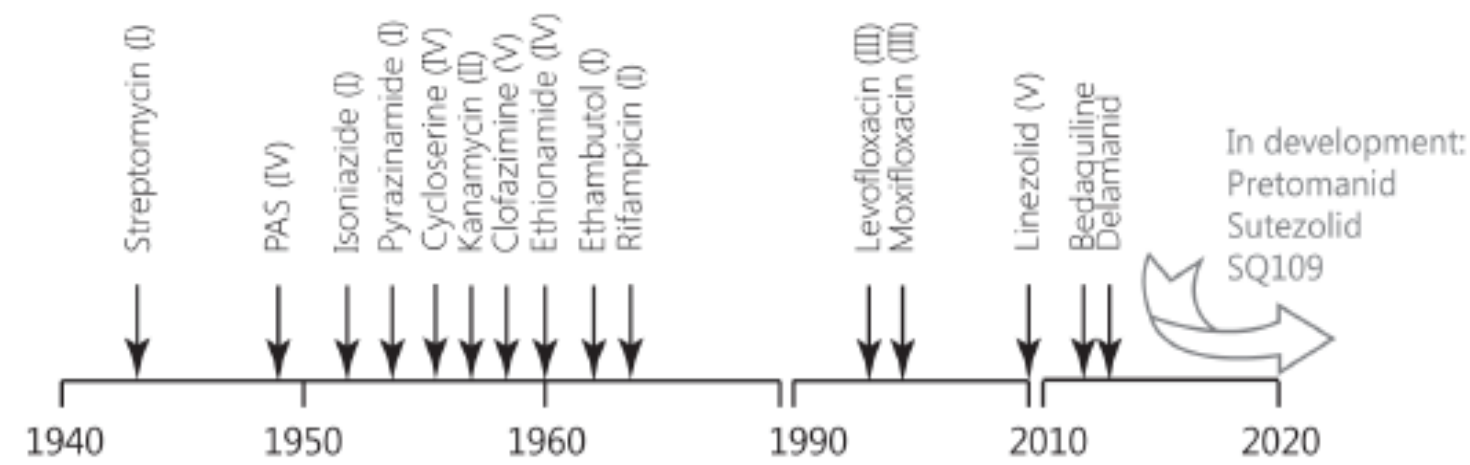

Figure 1.15. TB drug development timeline. TB drug group classification according to WHO guidelines is displayed in roman numerals. Group I drugs are first-line agents and considered the most potent and best tolerated. Group II drugs contain injectable agents previously used against multidrug-resistant TB (MDR-TB). Group III includes fluoroquinolones, such as higher generation members levofloxacin and moxifloxacin. Group IV drugs are mostly oral bacteriostatic second-line agents. Group V drugs are agents not recommended by the WHO for routine use in MDR-TB treatment because of limited data available. Not all TB drugs are depicted. Reproduced with permission from Dr L te Brake. ${ }^{149}$

Therefore, to bridge the gap until new first-line TB drugs are available, there is an urgent need to repurpose drugs that are already licensed or redevelop drugs that are already used by optimising the dose used in the standard treatment regimen to maximize bactericidal and sterilizing activities. Rifampicin is one of the drugs in need of redevelopment. With hindsight, the historical pharmacokinetic, toxicity, and cost arguments that formed the basis of the currently used dose $(10 \mathrm{mg} / \mathrm{kg}$ ) were short-sighted, leaving a lot of room for drugdosing improvements.

\subsubsection{Current antimicrobial therapy for TB meningitis}

The short course adult pulmonary TB treatment model defined in the pre-HIV era was extrapolated to all forms of extrapulmonary TB disease, including TBM, without disease-specific evidence. The WHOrecommended treatment for TBM largely follows the same model as treatment of pulmonary tuberculosis (described in section 1.4.2) simply with a prolonged continuation phase, depending on national guidelines and modifications for drug-susceptibility results where available. ${ }^{137}$ The WHO 2010 guideline does suggest switching ethambutol for streptomycin, due to concerns about ethambutol not penetrating the CNS. ${ }^{137}$ However, CNS penetration of streptomycin is only moderate and injections are poorly tolerated and difficult to implement, especially in the absence of any strong evidence to support this suggestion. So many countries simply treat TB meningitis with $2 \mathrm{RHZE} / 7 \mathrm{RH}$. The extended continuation phase of 7-10 months used in TBM is also not evidenced based, ${ }^{150}$ rather recommended by expert opinion due to concerns relating to drug penetration into the CNS. The concerns about drug penetration into the CNS are indeed valid, achieving a drug concentration at the site of disease (the meninges and CSF) for an adequate length of time is essential to sterilize the site. As I describe below, the penetration of rifampicin and ethambutol into the CNS are poor at the currently used doses making the 2RHZE/7RH therapy for TBM questionable. Whilst adoption of the short 
course pulmonary TB 'one-size-fits-all' model for the treatment of CNS TB makes a lot of sense in terms of simplifying national TB programming, it fails to consider PK-PD principals and there is a significant amount of research now going towards trying to find a more effective TBM regimen.

\subsubsection{Penetration of TB drugs into the central nervous system}

Importantly, in contrast to antibiotics used to treat pulmonary TB, antimicrobials for CNS TB must cross the blood-brain barrier (BBB) and blood-cerebrospinal fluid barrier (BCSFB), and remain in the brain or CSF at the site of infection at sufficient concentrations for a sufficient period of time to kill the TB bacilli. CSF is a unique compartment, often referred to as a 'sanctuary site' as pathogens can exist and replicate in CSF protected from antimicrobial drugs that cannot access the compartment. The composition of CSF is carefully regulated by the BBB and BCSFB, see Figure 1.16. The relationship between plasma and CSF concentrations is continuously dynamic and dependent on a number of factors:

1. Lipid solubility - lipophilic drugs cross the BBB and BCSFB by transmembrane diffusion more readily.

2. Molecular weight - low molecular weight drugs cross BBB and BCSFB by transmembrane diffusion more readily

3. Protein binding - highly protein bound drugs are unable to cross the tight junctions between the epithelial cells of the choroid plexus to enter the CSF. This is particularly relevant in the case of rifampicin, which is $\sim 85 \%$ protein bound; only the unbound or 'free' rifampicin is biologically active. In the absence of meningeal inflammation only the 'free' rifampicin crosses the BCSFB. In theory, when meninges are inflamed in meningitis the tight junctions between cells of the choroid plexus are disrupted allowing plasma proteins and protein-bound drugs (like rifampicin) to penetrate the CSF more readily, however it is not clear to what extent or for what duration this applies in TBM, and particularly HIV-associated TBM where the CSF can be acellular. ${ }^{151}$

4. Drug transporters - Organic anion-transporting polypeptide (OATP) can inwardly direct certain drugs. Conversely, transporters, like BCRP and P-glycoprotein (P-gp) in endothelial cells, actively pump potentially neurotoxic substances out of the brain and into the blood or CSF, thereby protecting the CNS. This also applies to rifampicin which is actively pumped out by P-gp. ${ }^{149}$

Rifampicin is arguably the most critical drug in the treatment of TBM, as evidenced by the universally poor outcomes of people with rifampicin-resistant disease. ${ }^{53}$ However, rifampicin has poor CSF penetration, due to the reasons outlined above, with reported CSF concentrations often reaching $<20 \%$ of those in plasma. ${ }^{152}$ CSF rifampicin concentrations were undetectable (below the limit of quantification of $0.26 \mathrm{mg} / \mathrm{L}$ ) in two-thirds of TBM patients in Indonesia and seldom exceeded the minimum inhibitory concentration (MIC) for $M$. tuberculosis. ${ }^{153,154}$ Thankfully isoniazid and pyrazinamide have excellent CSF penetration, ${ }^{154}$ though the high prevalence of isoniazid resistance in some regions (27\% in Vietnam, ${ }^{155} 5 \%$ in Uganda, ${ }^{156} 9 \%$ in South Africa ${ }^{157}$ ) further compromises TBM treatment and pyrazinamide is discontinued the end of the intensive phase of 
therapy (week 8). The CSF penetration of all the first-line, second-line, and novel antituberculous drugs is outlined in the Chapter 7 review paper - Intensified Antibiotic Treatment for Tuberculous Meningitis (Table 2).

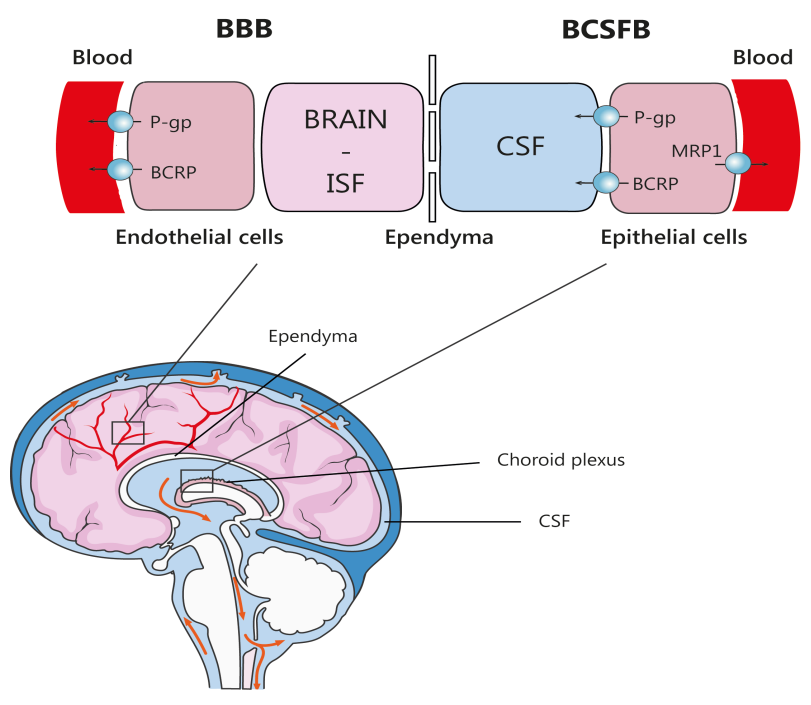

Figure 1.16. Schematic overview of the relation between the blood-brain barrier (BBB), made up by brain endothelial cells, and the blood-CSF barrier (BCSFB), made up by choroid plexus epithelial cells. The ependymal lining of the cerebral ventricles forms the barrier between brain (and its interstitial fluid, ISF) and CSF. According to this simplified paradigm, there is free exchange of drug between the brain ISF and CSF. CSF drug concentrations approximate the free drug concentration in plasma that is able to penetrate the BBB, BCSFB, or both. Displayed efflux transporters may actively disturb this equilibrium, by actively transporting drugs into the blood or CSF. Reproduced with permission by $\operatorname{Dr} L$ te Brake. ${ }^{140}$

It would seemingly make sense to treat TBM with a cocktail of drugs which is able to access the site of disease at bactericidal concentration for an adequate duration. The options for achieving this are explored in detail in the chapter 7 review paper and summarised below.

\subsubsection{Can we optimise the antimicrobial treatment of TBM?}

There are different strategies that have been considered, are under investigation, or may be relevant in future to optimise TBM treatment as listed below:

1. Prolonging TB treatment - This is unlikely to help; in a meta-analysis, the risk of relapse was extremely low $(\sim 0.8 \%)$, and virtually identical in patients who received $<6$ months versus $>6$ months of therapy. ${ }^{150}$

2. Modification of drug delivery - For example by liposome or nanoparticle-mediated permeation, however, this is not at the stage of clinical studies yet.

3. Inhibition of efflux pumps in the BBB/BCSFB - P-glycoprotein inhibitors might also increase antituberculous drug exposure in the CNS, though such approaches are not at the stage of clinical studies yet.

4. Additional drugs - Adding drugs which are effective against $M$. tuberculosis and penetrate readily into the brain and CSF to the standard quadruple TB therapy is an approach receiving considerable interest, the in-human clinical trials of which are summarized below and in the chapter 7 review paper. Adjunctive oral levofloxacin is the only drug to have been tested in a phase III trial with no 
reduction in mortality among Vietnamese adults with suspected TBM (except those with isoniazid monoresistance). ${ }^{158,159}$

5. Increase drug exposures in the CNS by increasing drug doses - a promising strategy is to increase the doses of poorly CNS penetrating drugs, where toxicity profiles allow, such that levels in the brain and CSF exceed the minimum inhibitory concentration of $M$. tuberculosis for an effective time period daily. This is particularly pertinent to rifampicin, which has been shown to be safe at considerably higher doses (up to 4 -fold increase above the standard $10 \mathrm{mg} / \mathrm{kg}$ ) in studies in pulmonary TB, albeit in a HIV-negative population. ${ }^{160}$ Paediatricians in Cape Town have for many years been using an intensified regimen of $20 \mathrm{mg} / \mathrm{kg}$ rifampicin and $40 \mathrm{mg} / \mathrm{kg}$ pyrazinamide, with isoniazid and ethionamide (in place of ethambutol) and report good outcomes with few adverse events. ${ }^{161}$ Likewise, in the Netherlands, an intensified regimen of oral rifampicin $30 \mathrm{mg} / \mathrm{kg}$ (or $15 \mathrm{mg} / \mathrm{kg}$ intravenously) along with standard dose isoniazid, pyrazinamide and moxifloxacin (in place of ethambutol) is used, albeit on the basis of expert opinion rather than high quality clinical trial data. ${ }^{140}$

6. Changing the route of drug administration - using intravenous instead of oral administration increases the bioavailability of drugs that are poorly (or variably) absorbed from the gut. This may be particularly relevant in critical illnesses, such as TBM, where patients are often receiving drugs though a nasogastric tube, have paralytic ileus, enteropathy, vomiting or diarrhoea. Such factors could explain the lower plasma rifampicin concentrations observed in patients with TBM compared to those with pulmonary TB taking the same dose from the same setting in Indonesia. ${ }^{153,162}$ Currently, few TB drugs are available parenterally, namely rifampicin, isoniazid, quinolones, linezolid and aminoglycosides. Intrathecal streptomycin was trialed in a small number of patients the $1940 \mathrm{~s},{ }^{163}$ but adverse reactions (including death) were frequent so the study was not continued and this has not been explored further since as it is a highly risky strategy, especially in LMIC settings.

7. Personalised therapy based on host pharmacogenomics - isoniazid is metabolised by the genetically polymorphic $\mathrm{N}$-acetyltransferase 2 (NAT2) and rapid acetylators have lower isoniazid exposures in both plasma and CSF. Isoniazid exposure was strongly related to survival in the recent PK-PD analysis of the landmark Vietnam intensified treatment trial. ${ }^{164,158}$ Rapid acetylators may therefore benefit from increased isoniazid doses, something which has been piloted in pulmonary TB but not yet in TBM, though a study is planned in China (NCT03787940). ${ }^{165}$ Rifampicin metabolism is also believed to be under a degree of genomic regulation, though pharmacogenomic data from Africa is relatively sparse. The SLC01B1, AADAC and CES-1 gene loci (amongst others) have been studied in African adults, with conflicting results in different countries, possibly relating to the magnitude of genetic diversity across the African continent or the different study methodologies deployed. ${ }^{166,167}$ The 
SLC01B1 genotype has been investigated in Uganda in patients with pulmonary TB but was not found to influence rifampicin PK. ${ }^{168}$

8. Personalising therapy based on specific disease categories - designing specific regimens for patients with tuberculomas, spinal TB, HIV coinfection or drug mono-resistance may result in better outcomes than the current 'one-size-fits-all' approach.

Of all of these options, and based on accumulating evidence that high-dose rifampicin is safe and more efficacious in pulmonary TB, it makes sense to optimise the dose of rifampicin in the treatment of TBM. Rifampicin is now a cheap drug with numerous generic options, it is already available in many TB endemic countries so could be implemented quickly. But is high-dose rifampicin safe in HIV-associated TB?

\subsubsection{Defining the optimal rifampicin dose - pharmacokinetics-pharmacodynamics}

Pharmacokinetics (PK) is the study of how the human body processes the drug of interest, in relation to the absorption, distribution, metabolism and excretion of the drug. Pharmacokinetic studies allow for the generation of a PK curve (see Figure 1.17) from which pharmacokinetic parameters can be calculated including key parameters such as the following:

1. $C_{\max }$ - the peak concentration of the drug after administration, also known as peak exposure. Rifampicin is subject to huge (6 to 10 -fold) interindividual variability in peak concentration, the reasons for which are multifactorial and not fully elucidated.

2. $T_{\max }$ - the time to reach $C_{\max }$. This depends on the route of administration and volume and rate of distribution of the drug into different compartments (e.g. plasma, tissue, brain etc)

3. $T_{1 / 2}-$ the elimination half-life. This is the time it takes for the concentration to reduce by $50 \%$

4. $\mathrm{AUC}_{0-24 \mathrm{hr}}$ - the area under the time concentration curve from 0 to 24-hours after drug administration. This is often referred to as 'total exposure' and depends on the peak concentration and the rate of clearance

5. F - bioavailability - the fraction of the administered drug that reaches the systemic circulation

6. $\mathrm{Cl}$ - clearance - the volume of plasma cleared of the drug per unit time

7. $V d-$ volume of distribution - the apparent volume in which the drug is distributed

8. AUC/MIC - the area of the time concentration curve which is spent above the minimal inhibitory concentration (MIC) for the M. tuberculosis isolate. The MIC is the drug concentration which arrests visible growth of bacteria in vitro after overnight incubation under unrestricted growth conditions with static drug exposure. ${ }^{169}$

Pharmacodynamics is the study of how the drug affects the organism or the body, be it a therapeutic or toxic effect, and the relationship between the dose, the drug concentration/exposure, and the response (Figure 1.17). 


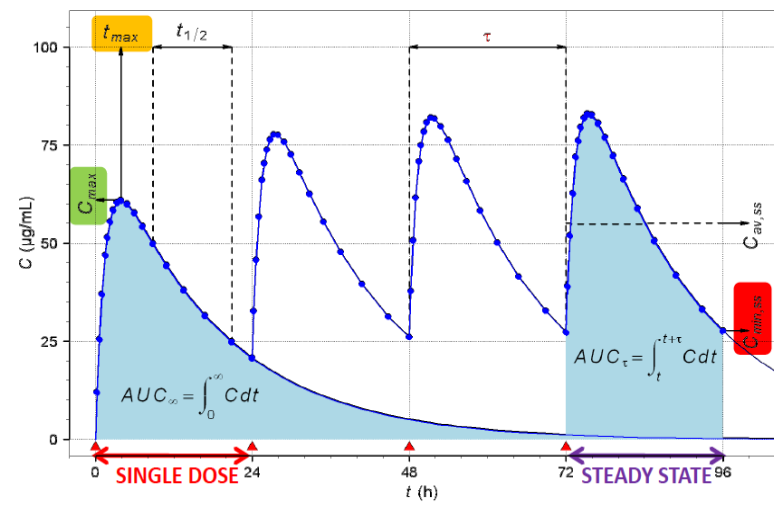

Fig 1.17a. Example of a PK curve and the key PK parameters for a drug that is dosed orally. The blue shaded area on the left illustrates the total exposure (AUC) from a single dose from administration til elimination. The right-hand blue shaded area illustrates the $\mathrm{AUC}_{0-24 \mathrm{hr}}$ if the drug is administered once daily.

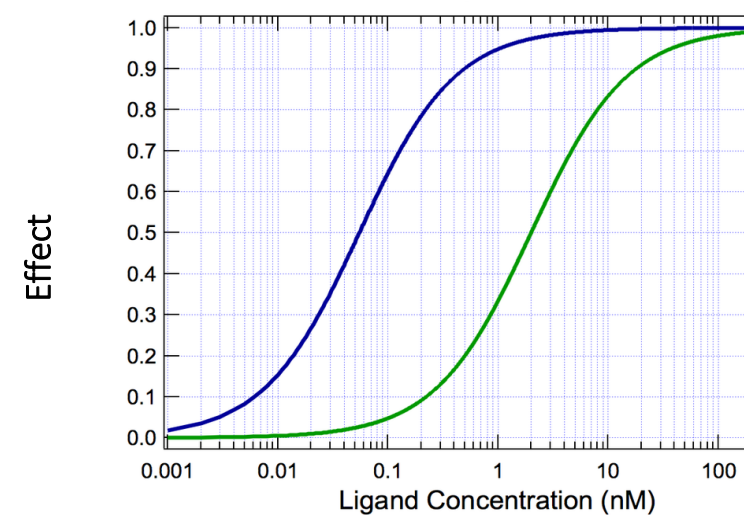

Fig 1.17b. Example of a concentration-response curve. The response variable used in studies of pulmonary TB is 'time to sputum culture conversion' and in TBM studies has been 'survival'.

The simplest bacteriological pharmacodynamic measure of activity is the minimal inhibitory concentration (MIC), the drug concentration which arrests visible growth of bacteria in vitro after overnight incubation under unrestricted growth conditions with static drug exposure. ${ }^{169} \mathrm{~A}$ clinical dose can be derived by comparing drug exposures in preclinical pharmacokinetic models or in-human PK studies with the MICs of clinical mycobacterial isolates. However, this simplistic approach fails to understand the relationship between dose, exposure, and clinical response. In vitro pharmacodynamic models (e.g. hollow-fibre models) and animal models of infection have also been used to create a more dynamic environment in which to examine the activity of an antibiotic. These models provide a better understanding of how changes in antibiotic exposure over time may influence activity, yet they remain limited in their ability to accurately reflect the human infection and host environment. ${ }^{170}$ While in-human pharmacodynamic studies are challenging to conduct, their influence on our understanding of antimicrobial activity in vivo is invaluable for evaluating the doseexposure-response relationship and understanding how co-variates (e.g. sex, body weight, comorbid conditions) affect that relationship. ${ }^{170}$ Modern in silico modelling approaches are also extremely useful in defining the dose-exposure-response relationship. In silico modelling can be used either at a pre-clinical stage or in combining in-human data from multiple studies to be able to analyse the dose-exposure-response relationship with greater power or in different populations.

In the 1970/80s, at the time of defining short-course TB therapy, far less attention was paid to establishing the relationship between dose, exposure and pharmacodynamic responses. Furthermore, there was little interest for differences among disease-type subsets, such as TBM. No PD targets had been defined. For therapeutic drug monitoring purposes in the 1990s a target rifampicin concentration of $8-22 \mathrm{mcg} / \mathrm{ml}$ at the site of disease was suggested, though this was largely based on expert opinion of observed pharmacokinetic data from the pre-HIV era and not on pharmacodynamic effect on bacilli. ${ }^{171}$ Since then, mouse models 
suggest that the index which best predicts microbial kill is the total exposure $\left(\mathrm{AUC}_{0-24}\right)$ over the $M$. tuberculosis MIC (AUC $0-24 / \mathrm{MIC}$ ). Measuring individual level M. tuberculosis MICs is a laborious, complex and costly process so a regional or national MIC can be used where available.

\subsubsection{What is the optimal rifampicin dose from animal models?}

A number of murine studies have shown that mice can tolerate rifampicin up to a daily dose of $160 \mathrm{mg} / \mathrm{kg}$ (current in-human dose is $10 \mathrm{mg} / \mathrm{kg} /$ day). A series of experiments from St Georges Hospital, London which used escalating doses of rifampicin in a Cornell mouse model found that high-dose rifampicin at 30, 40 and $50 \mathrm{mg} / \mathrm{kg}$ accelerated the rate of bacterial clearance and killed 'persistent' organisms in the lungs and spleen so that treatment duration could be shortened without relapse. ${ }^{134,172}$ With the 30 to $50 \mathrm{mg} / \mathrm{kg}$ dose there was true sterility - negative for CFU count and no resuscitatable bacteria - after 8 weeks of treatment. The 10 $\mathrm{mg} / \mathrm{kg}$ dose (and even the $20 \mathrm{mg} / \mathrm{kg}$ dose) was unable to eliminate the dormant bacilli leading to disease relapse. ${ }^{134}$

Mouse models must be extrapolated to humans with caution; mice do not form necrotic caseating granulomas in the same way that humans do, the extent of protein binding (and therefore free rifampicin) can be different, treatment is usually initiated about two weeks after infection in mouse models whereas it can be many months in humans, comorbid conditions such as diabetes and HIV coinfection cannot be studied to name but a few of the differences between mice and men. Despite these considerations, compelling animal evidence points towards the fact that rifampicin doses of above $30 \mathrm{mg} / \mathrm{kg}$ are likely to be the most beneficial in humans. ${ }^{134,172}$

\subsubsection{What is the optimal rifampicin dose from clinical studies in pulmonary TB?}

In a small ( $n=14$ ) proof of concept study in 2007, South African patients with smear positive pulmonary TB received rifampicin $20 \mathrm{mg} / \mathrm{kg}$ (in isolation before other TB drugs were commenced) and early bactericidal activity (EBA) was assessed at day 2 and 5. Compared to historical controls, the 2-day EBA with $1200 \mathrm{mg}$ was double that with standard dose rifampicin $(600 \mathrm{mg}) .{ }^{173}$ Larger studies with pharmacokinetic evaluation, and robust microbiological and clinical endpoints for PK-PD analysis were called for and have been led by the PanACEA (Pan African Consortium for Evaluation of Antituberculous Antibiotics) consortium, and the InterTB group at St Georges, London.

The use of serial sputum colony counting as a surrogate efficacy endpoint, and the use of innovative clinical trial designs including factorial and multi-arm multistage studies (MAMS), allows the efficacy of drugs to be explored efficiently in the PanACEA series of studies. In 2015, a rifampicin dose escalation (20, 25, 30,35 $\mathrm{mg} / \mathrm{kg}$ ) study by Boeree et al. (HIGHRIF1) aimed to find the highest tolerated dose in patients with cultureconfirmed pulmonary TB. In all patients, rifampicin was given alone for the first week, with isoniazid, pyrazinamide, and ethambutol added in standard doses for the second 7 days of treatment. Dose-limiting 
toxicity was not found even at the higher dose $(35 \mathrm{mg} / \mathrm{kg}$ ) and there was a more rapid fall in bacterial load (CFU count) over 14 days with the higher doses of rifampicin. ${ }^{160}$ The results of the $40 \mathrm{mg} / \mathrm{kg}$ and $50 \mathrm{mg} / \mathrm{kg}$ arms have just been published in pre-print though only one participant was HIV-positive so it is not clear whether the results can be generalized to PLHIV. Whilst $50 \mathrm{mg} / \mathrm{kg} /$ day had very impressive early EBA all participants experienced tolerability-related AEs (grade $\leq 3$, largely gastrointestinal, jaundice, pruritis) during monotherapy and 11/17 withdrew or stopped the study medication. The $40 \mathrm{mg} / \mathrm{kg} / \mathrm{day}$ dose was better tolerated and only one participant discontinued treatment. The authors concluded that rifampicin $40 \mathrm{mg} / \mathrm{kg}$ is the maximum tolerated dose which should be explored in phase IIB/C treatment shortening trials. ${ }^{174} \mathrm{~A}$ multisite treatment shortening study of rifampicin $35 \mathrm{mg} / \mathrm{kg}$ in pulmonary TB is almost fully recruited (RIFASHORT) and results are eagerly awaited.

In 2016, the RIFATOX study conducted in Uganda, Bolivia and Nepal found that rifampicin $20 \mathrm{mg} / \mathrm{kg} /$ day was safe but PLHIV were excluded due to concerns about drug interactions with ART. ${ }^{175}$ Safety of rifampicin $1200 \mathrm{mg}\left(20 \mathrm{mg} / \mathrm{kg}\right.$ ) administered over a longer period of 2 months was also established in a Peruvian study ${ }^{176}$ including $n=5$ PLHIV and a second PanACEA study (HIGHRIF2) which included $n=15$ PLHIV. The HIGHRIF2 study did not detect an improvement in bacteriological response, and concluded that yet higher rifampicin doses may be required to maximize efficacy. ${ }^{177}$ This was tested in the larger MAMs study which combined rifampicin 10,20 or $35 \mathrm{mg} / \mathrm{kg}$ with other drugs (including isoniazid, pyrazinamide, ethambutol, moxifloxacin, and a new compound SQ109) for 12 weeks. The primary endpoint (time to culture conversion in liquid media within 12 weeks) was faster in the $35 \mathrm{mg} / \mathrm{kg}$ rifampicin group than in the control group (median 48 days vs 62 days, adjusted HR 1.78; 95\% Cl, 1.22-2.58, p=0.003), but not in other experimental arms. Clinical outcome (recurrence of disease) was similar between groups though numbers were small (11 in total, with one in the $R$ $35 \mathrm{mg} / \mathrm{kg}$ arm). ${ }^{178}$

Taken together evidence from pulmonary TB suggests that doses of $35-40 \mathrm{mg} / \mathrm{kg}$ are safe (at least in HIVnegative adults) and have greater early bactericidal activity, but long-term clinical benefits remain to be confirmed.

\subsubsection{What is the optimal rifampicin dose from TB meningitis studies?}

\subsection{The Indonesian trio of TBM trials}

The first study on high-dose rifampicin in TBM was a phase II open-label randomized controlled trial in Indonesia (2013), in which Ruslami et al. investigated the safety and PK of intravenous rifampicin (600 mg) as well as oral moxifloxacin (400 mg or $800 \mathrm{mg}$ daily), in adults with suspected TBM. ${ }^{153}$ Although not powered to detect a mortality benefit, improved survival was observed in patients receiving intravenous rifampicin ( $\sim 13$ $\mathrm{mg} / \mathrm{kg} / \mathrm{day}, \mathrm{n}=29$ ) compared to the oral standard dose ( $10 \mathrm{mg} / \mathrm{kg} /$ day, $\mathrm{n}=31$ ) during the initial 2-weeks of TB treatment. Participants who received intravenous rifampicin had a more rapid resolution of coma (4 vs 5 days) 
and markedly reduced mortality at 6 -months ( $34 \%$ versus $65 \%$; adjusted $\mathrm{HR}=0.42 ; 95 \% \mathrm{Cl}, 0.20-0.87$, $\mathrm{P}=0.0193)$, see Figure 1.18 .

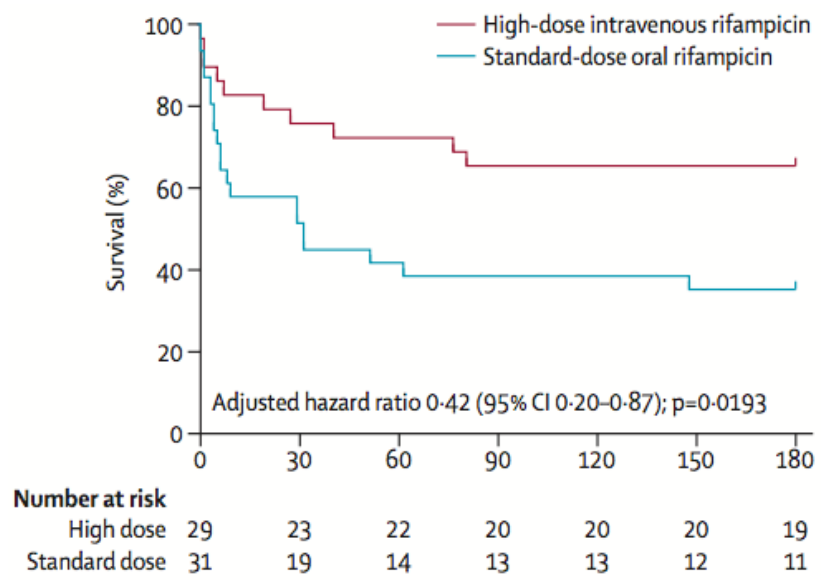

Intravenous administration was associated with a 3fold increase in plasma $\mathrm{AUC}_{0-6}$ (total exposure up to 6 hours post dose), $\mathrm{C}_{\max }$ and a 3 -fold increase in CSF $C_{\text {highest }}$ from 0.21 to $0.60 \mathrm{mg} / \mathrm{L}$. Due to the small sample size in this trial it could be argued that the observed effect was due to chance (type 1 error). However, further pharmacodynamic analysis of this trial demonstrated that there was evidence of a rifampicin concentration-effect relationship. ${ }^{179}$

Figure 1.18. Kaplan-Meier survival estimate according to rifampicin treatment arm in Ruslami et al 2013 Indonesian intensification trial

This promising result prompted the investigators to seek a safe and effective oral rifampicin dose which might have a similar impact on survival. In the Indonesian group's second open-label phase II randomised trial (Yunivita et al. 2016), they compared the safety and PK of intravenous rifampicin $600 \mathrm{mg} / \mathrm{day}$ with oral rifampicin $750 \mathrm{mg}$ ( $17 \mathrm{mg} / \mathrm{kg} /$ day) and $900 \mathrm{mg}$ ( $20 \mathrm{mg} / \mathrm{kg} /$ day) in 30 patients with suspected TBM (in combination with standard doses of $\mathrm{H}, \mathrm{Z}, \mathrm{E})$. In the first days of treatment, the geometric mean plasma $\mathrm{AUC}_{0-}$ 24 values following rifampicin $750 \mathrm{mg}$ orally, $900 \mathrm{mg}$ orally and $600 \mathrm{mg}$ intravenously were 131, 165, and 146 $\mathrm{h} \cdot \mathrm{mg} / \mathrm{L}$ respectively. Exposures to rifampicin varied substantially between individuals and decreased by $30-$ $50 \%$ at the end of week 2 due to autoinduction. A microbiological confirmation of TBM was only made in 13\% (4/30) patients and survival was not described as this was primarily a PK study. Transient grade 3 alanine transaminase (ALT) increases occurred in a quarter of patients (8/30 patients) and one grade 4 ALT increase occurred, though not related to rifampicin exposure. ${ }^{180}$

Having established the safety of $20 \mathrm{mg} / \mathrm{kg} /$ day orally the Indonesian investigators (Dian et al., 2018) conducted their third phase II trial, this time double-blinded and placebo-controlled, examining $900 \mathrm{mg}$ ( 20 $\mathrm{mg} / \mathrm{kg} /$ day) and $1350 \mathrm{mg}$ ( $30 \mathrm{mg} / \mathrm{kg} /$ day) of oral rifampicin (in combination with standard doses of $\mathrm{H}, \mathrm{Z}, \mathrm{E}$ ) versus standard of care TB treatment amongst 60 patients with suspected TBM. The total exposure (AUC $\mathrm{C}_{-24 h r}$ ) in the standard of care arm was $54 \mathrm{~h} \cdot \mathrm{mg} / \mathrm{L}$, this increased by 3-fold to 171 and 5-fold to $294 \mathrm{~h} \cdot \mathrm{mg}$. /L in the 20 $\mathrm{mg} / \mathrm{kg}$ and $30 \mathrm{mg} / \mathrm{kg}$ respectively with no increase in adverse events. In CSF, concentrations were 0.28, 0.59 and $0.74 \mathrm{mg} / \mathrm{L}$ in the standard of care, $20 \mathrm{mg} / \mathrm{kg}$ and $30 \mathrm{mg} / \mathrm{kg}$ arms respectively ( $p<0.001$ ). In this study an impressive $71 \%$ of participants had microbiologically-confirmed TBM. There was a trend toward lower 6month mortality in the $30 \mathrm{mg} / \mathrm{kg} /$ day arm compared to standard of care in participants with 'definite' TBM (7\% vs $36 \%, p=0.07) .^{181}$ 


\subsection{The Vietnam trial}

Conversely, in the landmark Vietnamese double-blinded, randomised placebo-controlled trial, ${ }^{155}$ Heemskerk et al. randomized 817 adults with suspected TB meningitis to receive either A) an intensified regimen containing i) increased oral dose of rifampicin at $15 \mathrm{mg} / \mathrm{kg} /$ day and ii) additional oral levofloxacin 20 $\mathrm{mg} / \mathrm{kg} /$ day (plus standard of care doses of $\mathrm{H}, \mathrm{Z}$ and $\mathrm{E}$ ); or B) standard of care TB treatment during the first 2 months of therapy. During 9-months of follow-up, 113 (27.7\%) patients in the intensified-treatment group and 114 (27.9\%) patients in the standard-treatment group died (HR $0.94 ; 95 \% \mathrm{Cl}, 0.73$ to $1.22 ; \mathrm{P}=0.66$ ). There was no evidence of a differential effect of intensified treatment in the overall population, although there was a marked survival benefit for patients with isoniazid-resistant infections (HR 0.34; 95\% $\mathrm{Cl} 0.15$ to 0.76; $\mathrm{P}=0.01) .{ }^{159,63}$ The results of the PK-PD sub-study within this trial have recently been published. There was an approximate doubling of plasma and CSF rifampicin exposures: day 14 plasma $\mathrm{AUC}_{0-24}$ increased from 48.2 $\mathrm{h} \cdot \mathrm{mg} / \mathrm{L}$ (range 18.2-93.8) to $82.5 \mathrm{~h} \cdot \mathrm{mg} / \mathrm{L}$ (range 8.7-161.0) and CSF AUC $\mathrm{U}_{-24}$ from $3.5 \mathrm{~h} \cdot \mathrm{mg} / \mathrm{L}$ (range 1.2-9.6) to $6.0 \mathrm{~h} \cdot \mathrm{mg} / \mathrm{L}$ (range 0.7-15.1) in the intensified arm compared to the control arm. However, there was no observed association between rifampicin exposure and survival within the range of exposures observed. ${ }^{164}$ There was also no relationship between levofloxacin exposure and survival. ${ }^{164}$ The increase in rifampicin dose to $15 \mathrm{mg} / \mathrm{kg}$ may have been too modest to elicit a microbiological and clinical impact, bearing in mind that the PanACEA MAMs study only found higher early bactericidal activity at a rifampicin dose of $35 \mathrm{mg} / \mathrm{kg}{ }^{178}$ The rifampicin total exposure achieved in the Vietnam study was substantially lower (geometric mean $\mathrm{AUC}_{0-24}$ of $82.5 \mathrm{~h} \cdot \mathrm{mg} / \mathrm{L}$ ) than the most recent Indonesian trial (Dian et al.) where there was a trend towards improved outcomes with $30 \mathrm{mg} / \mathrm{kg} / \mathrm{day}\left(\mathrm{AUC}_{0-24} 294 \mathrm{~h} \cdot \mathrm{mg}\right.$. /L). ${ }^{181}$ Total exposure cannot be compared with the 2013 Ruslami et al. study of IV rifampicin as, due to the timing of sample collection, the investigators were only able to calculate the area under the time concentration curve up to 6-hours post dose rather than $\mathrm{AUC}_{0-24 \text {. The }}$ hypothesis that the rifampicin exposure in the Vietnam trial was too low to elicit a pharmacodynamic effect is supported by the results of a recent meta-analysis of the Indonesian data.

\subsection{Model-based meta-analysis}

Given the modest sample size in each of the three Indonesian trials the individual datasets provided limited scope for understanding dose-exposure-response relationships. However, pooling data from the three trials and performing a combined analysis with advanced modelling methodologies provided greater statistical power to be able to characterize the relationship between individual exposures and mortality, and exposure and toxicity. The analysis included data from 1150 PK measurements (including 170 from CSF) and survival data included 148 individuals of whom 58 died and 15 dropped out. Nonlinear mixed effects modelling was utilised to describe the PK data. The final model featured two disposition compartments, saturable clearance and autoinduction. Oral bioavailability was estimated at 78\%, clearance decreased with increasing concentration, intrinsic clearance was $48 \%$. Only a tiny proportion of rifampicin penetrated into CSF (5.5\%; 
95\% $\mathrm{Cl}, 4.5$ to $6.4 \%$ ), and CSF concentration was directly related to plasma concentration and distribution to CSF did not saturate within the studied range of rifampicin exposures. Higher levels of protein in the CSF were linked to higher CSF rifampicin concentrations (with each 10-fold change in protein concentration there was a 63\% increase in CSF penetration). The model was used to generate individual exposure metrics ( $\mathrm{AUC}_{0-24}$ and $\mathrm{C}_{\max }$ ) to evaluate in the survival model. In the parametric time to event survival model the three main factors affecting survival to 6 months were age, baseline Glasgow coma score, and plasma rifampicin $\mathrm{AUC}_{0-24}$. Out of the individual PK metrics, plasma $\mathrm{AUC}_{0-24}$ was a better predictor of survival than plasma $\mathrm{C}_{\max }$ or $\mathrm{CSF} \mathrm{AUC}_{0-24}$. The predicted survival over time per rifampicin dose group included in the three trials is shown in Figure 1.19, illustrating that an increase from $450 \mathrm{mg}$ to $1350 \mathrm{mg}$ could be expected to increase survival from $50 \%$ to $70 \%$ in the same population. ${ }^{152}$

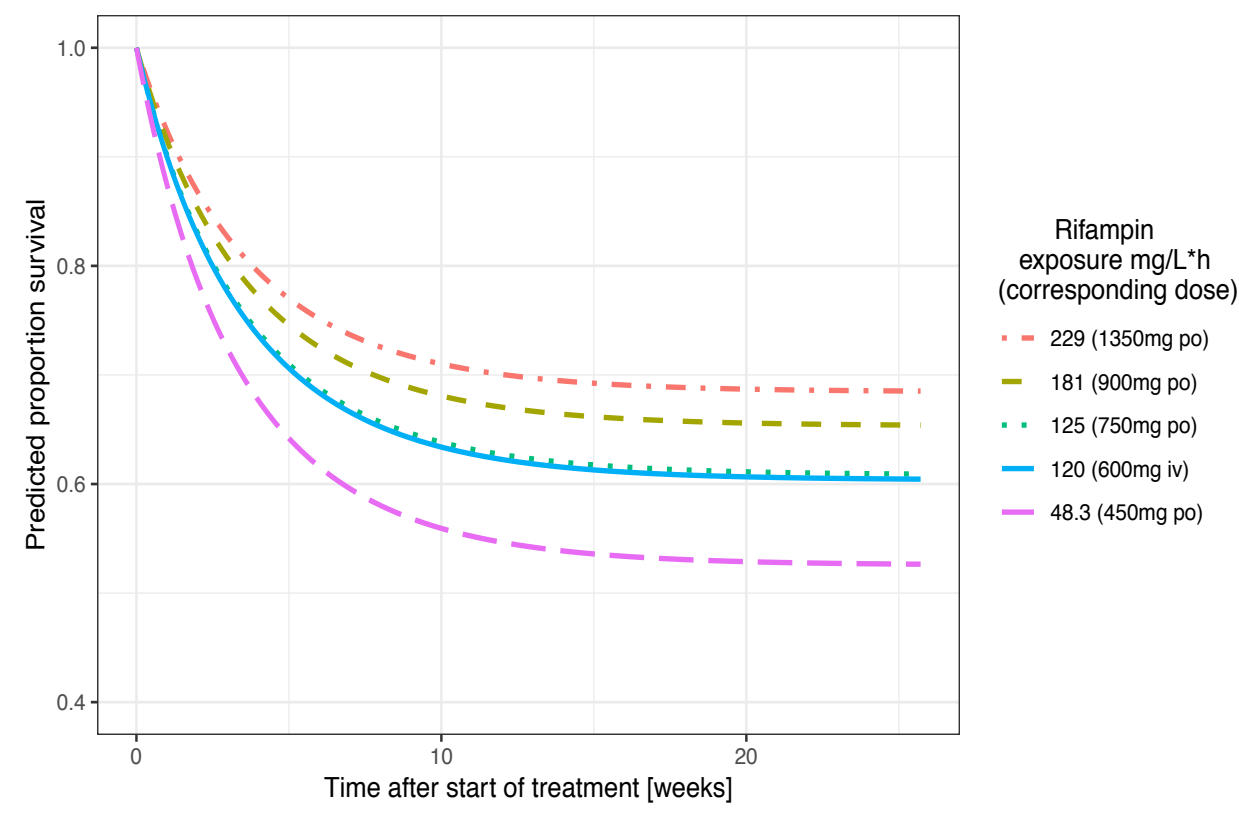

Figure 1.19: Model-predicted proportion survival over time representative for typical patients (age 30 yrs, baseline GCS $=13$ ) per plasma $\mathrm{AUC}_{0-24}$ at day 2.152

The plasma $\mathrm{AUC}_{0-24}$ at day 2 corresponding to a $50 \%$ and a $65 \%$ decrease in the hazard was estimated to be $171 \mathrm{~h} \cdot \mathrm{mg} / \mathrm{L}$. and $300 \mathrm{~h} \cdot \mathrm{mg} / \mathrm{L}$ respectively. A rifampicin dose of $1800 \mathrm{mg}(30 \mathrm{mg} / \mathrm{kg}$ in a $60 \mathrm{~kg}$ individual, or 35 $\mathrm{mg} / \mathrm{kg}$ in a $50 \mathrm{~kg}$ individual) is likely to attain the desired exposure $(300 \mathrm{~h} \cdot \mathrm{mg} / \mathrm{L})$ in the vast majority of TBM patients. The investigators did not find a relationship between rifampicin exposure and adverse events, suggesting drug toxicity may be idiosyncratic rather than exposure-related. In summary, a model-based metaanalysis of data from three Indonesian phase II trials supports the notion that higher dose rifampicin improves survival from TBM.

HIV status did not affect the hazard though there were only a total of 18 HIV-positive people in the whole dataset and all participants were Indonesian, so it is unclear whether these findings can be generalised to other ethnicities and PLHIV. It is therefore important to generate data from other populations and using the 
$30-40 \mathrm{mg} / \mathrm{kg}$ dose suggested by the model. There are a number of trials planned to do exactly this, summarised in the chapter 7 review paper 'Intensified Antibiotic Treatment for Tuberculous Meningitis' (Table 3), including the Rift study conducted during my PhD, the findings of which are presented in chapter 8.

\subsection{Impact of HIV-infection on pharmacokinetics and safety of TB drugs}

Several factors are known to cause interindividual variability in the PK of TB drugs including body weight, sex, pharmacogenomics, and comorbid conditions such as diabetes mellitus and possibly HIV infection. ${ }^{182}$ For this reason it would be remiss to extrapolate safety and PK-PD data from HIV-negative Indonesians to an HIVpositive African population. In addition, adverse drug reactions during TB treatment are 2 - to 4 -fold ${ }^{183,135}$ in PLHIV, including drug induced liver injury. ${ }^{184}$ This highlights the importance of examining the safety and PK-PD of a drug within the population where it will be used.

\subsubsection{Does HIV status influence the PK of TB drugs?}

HIV status may have an impact on the PK of TB drugs, though this has been a point of deliberation. Several studies have conflicting results and disentangling other confounding factors has not been straightforward. Early studies from the 1980s and 90s reported that PLHIV achieve lower plasma concentrations of the orally administered first-line antituberculous drugs than their HIV-negative counterparts, though this was largely in people with advanced AIDS in the pre-ART era. ${ }^{185,186,187}$ Though even in a stable outpatient population, subtherapeutic range peak rifampicin concentrations $\left(C_{\max }<8 \mathrm{mg} / \mathrm{L}\right.$ ) were reported in the majority of patients in the Ugandan Infectious Diseases Institute HIV/TB clinic cohort, with a median plasma $C_{\max }$ of $7.1 \mathrm{mg} / \mathrm{L} .{ }^{145}$

To explore the impact of HIV status on PK, a systematic review was conducted by the University of Cape Town Pharmacometrics group, over half (11/20) of studies included showed a statistically significant difference in

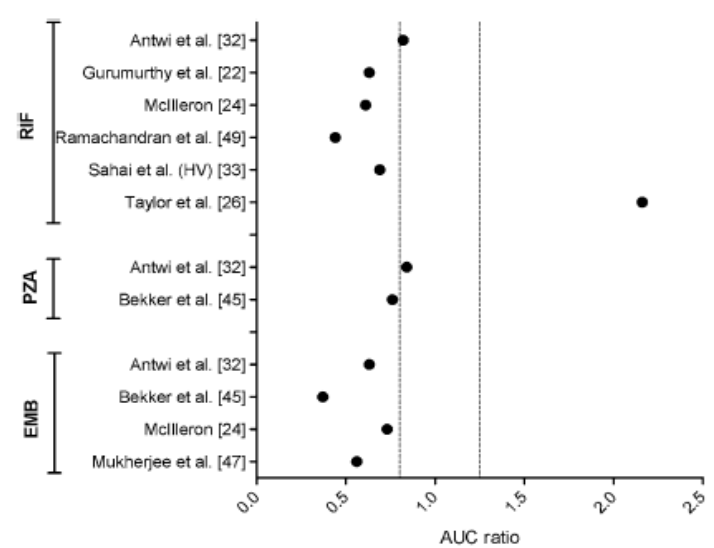

plasma AUC for a first-line drug by HIV status. The ratio of AUC between HIV-positive and HIV-negative participants was generally $<1.0$, as shown in Figure 1.20. In the studies that did not find lower drug exposures among PLHIV, the majority had higher $\mathrm{CD} 4+\mathrm{T}$ cell count and were receiving ART, suggesting that stage of HIV infection may also influence PK. It is possible that successful ART mitigates the effect of HIV infection on TB drug PK parameters.

Figure 1.20. Ratio between the AUC of HIV-positive and HIV-negative TB patients.

The figure illustrates the 11 studies that identified a difference in AUC between HIV-positive and HIV negative participants. In all but one study (Taylor) where a difference was identified, the AUCs were lower in the HIV-positive than HIV-negative (ratio <1) participants. Dashed lines represent the $0.8-1.25$ range, studies with a ratio outside this range were considered to have a clinically relevant reduction in AUC. RIF = rifampicin, $\mathrm{PZA}=$ pyrazinamide, $\mathrm{EMB}=$ ethambutol. ${ }^{182}$ 
Comparison between individual small studies is limited by sample size, differing sampling strategies and analytical methodologies. Therefore, a meta-analysis has been undertaken by the University of Liverpool group to definitively answer this question in relation to rifampicin. The meta-analysis included rifampicin PK data for 931 individuals, and concluded that HIV status affects the total exposure to rifampicin in the early days of treatment (AUC $37.2 \mathrm{~h} \cdot \mathrm{mg} / \mathrm{L}$ in HIV-positive versus $56.7 \mathrm{~h} \cdot \mathrm{mg} / \mathrm{L}$ in HIV-negative, $p=0.003$ ) but not once steady state has been achieved. ${ }^{171}$ The lower plasma rifampicin exposure in HIV-positive individuals in the initial days of therapy may be particularly relevant in TBM, which carries a high early mortality. Possible explanations are HIV enteropathy, diarrhoea, and low bodyweight due to HIV wasting (the latter leading to under-dosing using current weight-based algorithms). ${ }^{58,185,186,188}$

Additionally, with regards to CSF PK, PLHIV commonly have less marked meningeal inflammation in TBM, as measured by lower CSF white blood cell count or protein. ${ }^{52,57,189,190}$ When the meninges are inflamed the tight junctions between cells of the choroid plexus are disrupted allowing plasma proteins and protein-bound drugs (like rifampicin) to penetrate the CSF more readily. ${ }^{151}$ The Indonesian meta-analysis found a correlation between CSF protein and CSF rifampicin concentration. ${ }^{152}$ One could therefore hypothesise that reduced meningeal inflammation in HIV-associated TB meningitis may result in lower CSF levels of rifampicin. If this hypothesis is valid, then intensified therapies that penetrate effectively into the CNS, regardless of degree of meningeal inflammation, may be particularly important in HIV/TBM.

\subsubsection{Does HIV status have an impact on the safety of TB therapy?}

Possible reasons for the increased risk of adverse drug reactions in PLHIV include 1) overlapping toxicities of antitubercular and antiretroviral drugs (including skin rashes, gastrointestinal intolerance, hepatotoxicity, central nervous system symptoms and peripheral neuropathy); 2) polypharmacy exacerbated by concomitant prophylactic antibiotic medications such as fluconazole and trimethoprim-sulfamethoxazole; 3 ) high rates of concomitant viral hepatitis giving rise to liver toxicity; 4) pre-existing HIV-related liver disease giving rise to liver toxicity; 5) immune dysregulation in advanced HIV disease; 6) immune reconstitution inflammatory syndrome which can be difficult to disentangle from drug toxicity.

With regards to hepatotoxicity in particular, there is good evidence that being HIV-positive increases the risk of developing drug-induced liver injury (DILI) (OR 4.40, 95\% Cl 1.06 to 18.3) even after adjustment for age, sex and baseline liver function tests. ${ }^{184}$ Though most studies examining drug reactions are retrospective and do not allow accurate attribution of the risks conferred by HIV infection itself, by ART, by other concomitant medications, or by IRIS. ${ }^{135}$ 


\subsubsection{Immune reconstitution inflammatory syndrome and the timing of ART}

As discussed in section 1.2.4.1 TB IRIS is caused by ART-induced restoration of TB-specific immune responses, resulting in either the deterioration of a treated infection (paradoxical IRIS) or a new presentation of a previously subclinical infection (ART-associated TB or unmasking IRIS). Neurological TB-IRIS, the most severe form of IRIS, presents with worsening of headache, onset/exacerbation of focal neurology or seizures. Neurological TB-IRIS can be life-threatening and carries a mortality of up to $30 \%{ }^{61}$

The decision on when to start ART is a balance of the competing risks of HIV disease progression versus severe IRIS, overlapping drug toxicities and pill burden. This quandary has been investigated in a number of large randomized controlled trials in suspected TB. ${ }^{191,192,193}$ There has also been one trial specifically In TBM patients. ${ }^{194}$ The balance of evidence from these studies has given rise to the current recommendations which differ between TBM and other forms of TB:

- CD4 T-cell count $<50$ - begin ART within the first 2 weeks of TB treatment. ${ }^{195}$ Early initiation of ART increases AIDS-free survival (e.g. reduces risk of death from other opportunistic infections / illnesses). ${ }^{192}$

- CD4 T-cell count $>50-$ TB treatment should begin first, followed by ART within the first 8 weeks of treatment. ${ }^{195}$ ART initiation within 8 weeks of TB treatment is associated with a reduction in overall mortality, compared with ART initiated after 8 weeks of TB treatment or after TB treatment is completed. ${ }^{195}$ In SAPIT study, deferral of ART to the first 4 weeks of the continuation phase of TB therapy in those with higher CD4+ T-cell counts reduced the risk of IRIS and other adverse events without increasing the risk of AIDS or death. ${ }^{192}$

- TBM - Caution is needed in TBM as immediate ART is associated with more severe adverse events. ${ }^{195}$ TBM is unlike other forms of TB in that the infection surrounds a crucial structure (the brain) with a very limited ability to expand within the skull should excess inflammation occur. Therefore, ART is usually deferred until the intensive phase of TB treatment has been completed regardless of CD4 count. This is based on the results of a double-blind placebo-controlled RCT of immediate (within 7 days of TB treatment initiation) or delayed (after week 8 of TB treatment) ART in 253 HIV-positive Vietnamese adults with suspected TBM. Immediate ART did not did not improve survival from HIVassociated TBM and was associated with more grade 4 adverse events (102 in the immediate ART group vs 87 in the deferred ART group; $P=0.04$ ). Interestingly there was no reported increase in neurological events in the immediate ART group which one would have anticipated due to CNS IRIS. It is possible that the adjunctive corticosteroids in all participants may have suppressed CNS IRIS. The difference between arms was partly driven by the most common laboratory AE - hepatitis (transaminases $>5 x$ ULN) which occurred in $25 \%$ of the immediate ART group and $17 \%$ of the deferred ART group, but difference did not reach statistical significance. ${ }^{194}$ The study did not formally adjudicate suspected IRIS events. In cryptococcal meningitis early ART was proven to increase early mortality due to IRIS, ${ }^{196}$ so clinicians who managed TBM are cautious about early ART unless there is a strong clinical indication. 


\subsubsection{Does high dose rifampicin interact with antiretroviral therapy?}

As mentioned in section 1.4.1, rifampicin induces a plethora of metabolic processes inducing expression of enzymes and drug transporters (e.g. CYP3A4, CYP2B6, UGT1A1, and P-gp) involved in the metabolism and transport of many HIV drugs. Expert opinion suggests that maximal enzyme induction occurs with standard dose rifampicin but a dose-related effect has not been excluded. ${ }^{141}$ Considering that high-dose rifampicin may be increasingly considered as a way of improving TB outcomes, establishing the safety and extent of drugdrug interactions with ART, and their impact on HIV virological control, is key. There is no clinically significant interaction between rifampicin and tenofovir or lamivudine, which are the most widely used nucleotidereverse transcriptase inhibitor (NRTI) backbone used. Though interactions with the third ART agent cannot be avoided.

The recent RIFAVIRENZ trial, conducted in Uganda, is the only study to have examined the impact of high dose rifampicin (20 mg/kg/day) with efavirenz (EFV), dosed at either $600 \mathrm{mg}$ or $800 \mathrm{mg}$, versus standard dose rifampicin (10 mg/kg/day) with EFV 600mg. Despite a slight decrease in EFV AUC ${ }_{0-24}$ with rifampicin 20mg/kg, the geometric mean ratios remained within a preset allowable interval (0.70 to 1.43). The proportion of patients with subtherapeutic EFV concentrations in the high-dose rifampicin arms was no greater then with the standard dose rifampicin. Strangely, despite everyone switching to standard dose rifampicin at week 8 and the HIV virological response being equal at week 12, there was a lower proportion with virological suppression after 24-weeks among the high-dose rifampicin group with acquisition of the K103N resistance mutation in four participants. The possibility of adherence-related failure has been suggested. Adverse events were evenly distributed across treatment arms and the proportion of patients with grade $\geq 3$ increase of transaminase $(<10 \%)$ was comparable to standard dose rifampicin in other studies. ${ }^{197}$ The upcoming SAEFRIF study in Uganda plans to examine the safety and PK of rifampicin $35 \mathrm{mg} / \mathrm{kg}$ with EFV and dolutegravir (DTG).

Dolutegravir, an integrase strand transfer inhibitor (InSTI), is now recommended first-line alongside a backbone of two NRTIs in many African countries. DTG is arguably better tolerated than EFV, there is little or no population level resistance and it has a higher barrier to evolution of resistance. DTG is a substrate of drug efflux pumps, such as breast cancer resistance protein (BCRP) and P-gp, and is predominantly metabolized by uridine 5'-diphospho-glucuronosyltransferase 1A1 (UGT1A1), with CYP3A4 as a minor route. ${ }^{198}$ Coadministration of standard dose rifampicin with DTG results in a clinically significant reduction in DTG exposure but this can be overcome by doubling the DTG dose to 50mg twice daily or 100mg once daily (though the former is preferable). ${ }^{198,199}$ There is no data on high dose rifampicin with DTG but this will also be studied in SAEFRIF. ${ }^{141}$ Standard dose rifampicin and HIV protease inhibitor (PI) administration is not recommended due to significant interaction, which cannot be easily overcome (other than super-boosted 
lopinavir/ritonavir) and there are no plans to test the administration of high-dose rifampicin with protease inhibitors (PIs).

In summary, rifampicin and ART drug-drug interactions are an important consideration in the treatment of HIV/TB and data is needed on the safety and PK of high-dose rifampicin with ART (specifically DTG and EFV which are the most widely used agents in Africa) and on long-term HIV virological outcomes.

\subsubsection{Are host-directed therapies important for HIV-associated TBM?}

We know that inflammatory response contributes to poor outcomes in TBM. ${ }^{60}$ Limiting damaging inflammation may be just as important as controlling the infection and recent research has yielded fascinating results thanks to the use of 'omics' technologies, as further described in the chapter 3 review paper 'Recent developments in pathogenesis and diagnostics of tuberculous meningitis'.

Adjunctive corticosteroids are currently recommended for TBM based on the results of a meta-analysis: at follow-up from 3- to 18-months, steroids reduced deaths by almost one quarter ( $\mathrm{RR} 0.75,95 \% \mathrm{Cl}, 0.65$ to 0.87; nine trials, 1337 participants). ${ }^{200}$ Disabling neurological deficit is common in survivors, and unfortunately steroids may have little or no effect on this outcome (RR 0.92, 95\% Cl 0.71 to 1.20; eight trials, 1314 participants). The Thwaites et al 2004 trial followed participants for five years, and the effect on death was no longer apparent at this time-point (RR 0.93, 95\% Cl 0.78 to 1.12; one trial, 545 participants). ${ }^{201}$

The Thwaites et al. 2004 trial is the only study has reported results by HIV status. The study included 98 PLHIV and the sub-group analysis by HIV status was underpowered and did not quite achieve statistical significance

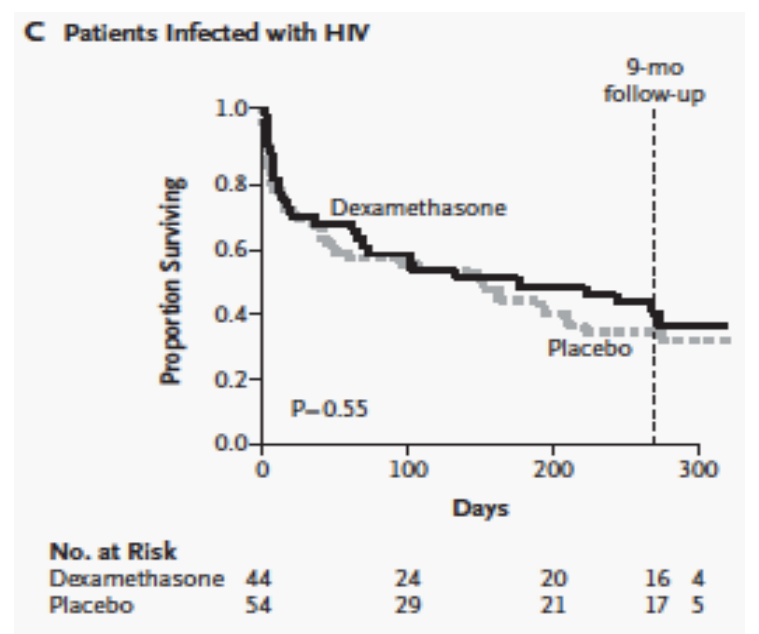
(stratified RR, 0.78; 95\% Cl, 0.59 to 1.04; $\mathrm{P}=0.08$ ), Figure 1.21. ${ }^{202}$ In light of this inconclusive result in PLHIV, a randomized placebo-controlled trial of the same dexamethasone regimen is underway in Vietnam and Indonesia (NCT03092817) with a longer follow-up duration of 24-months to observe for any late adverse effects of steroids in people with an already compromised immune system (e.g. Kaposi's sarcoma, other cancers, fungal or invasive bacterial infections).

Figure 1.21. Kaplan-Meier survival estimate amongst PLHIV according to dexamethasone treatment group. ${ }^{202}$

TBM is associated with a hypercoagulable inflammatory state with platelet dysfunction and cerebral infarction occurs in at least a quarter of cases. Aspirin is increasingly being studied in an effort to avert stroke and the ensuing death or long-term disability. ${ }^{203,204,205} \mathrm{~A}$ recent promising pilot study in Vietnam demonstrated a reduction in new infarcts and death with $1000 \mathrm{mg}$ aspirin in addition to the standard corticosteroids. The 
effect of $1000 \mathrm{mg}$ aspirin was most marked in people with definite TBM, new infarct or death occurred in $10.7 \%(3 / 28)$ versus $34.4 \%(11 / 32)$ with corticosteroids alone $(p=0.06)$. A dose-dependent inhibition of thromboxane A2 and upregulation of pro-resolving protectins in the CSF was identified as a possible mechanism of action. ${ }^{206}$ Larger studies are planned in both adults and children. Thalidomide and other host directed therapies are touched upon in the review paper in chapter 3. I have not gone on to discuss pathogenesis and host-directed therapy in detail, as whilst it is an extremely important and relevant topic it is beyond the scope of this thesis.

\subsection{What are the pressing questions about improving the treatment of TB meningitis in Uganda?}

Specific considerations relevant to HIV/TBM outlined in the chapter 7 research paper Intensified Antibiotic Treatment for Tuberculous Meningitis (Box 1) and a multitude of research questions relevant to TBM in Africa in general outlined in Table 1.6 below. Currently, high-dose rifampicin appears to be the most promising option for intensification of TBM treatment. There is however scant data on the safety and PK of high-dose rifampicin in PLHIV in Africa and no CSF PK data. Therefore, the most pressing question that I addressed in my PhD research is whether high-dose rifampicin can be safely used in advanced HIV disease and whether highdose intravenous or oral rifampicin achieve therapeutic CSF levels. I plan to address through a phase II openlabel RCT, the study design and results are outlined in chapter 8. 
Table 1.6. Research questions relating to the treatment of TBM in Africa

$\mathrm{RCT}=$ randomised controlled trial $\mathrm{IV}=$ intravenous $\mathrm{mAb}=$ monoclonal antibody $\mathrm{IL}=$ interleukin $\mathrm{LZD}=$ linezolid $\mathrm{H}=$ isoniazid Items shown in bold are those which I hope to address through my PhD research.

\begin{tabular}{|c|c|c|c|}
\hline Area & Questions applicable to Africa & $\begin{array}{l}\text { Evidence available from } \\
\text { Uganda or elsewhere }\end{array}$ & $\begin{array}{l}\text { Potential research to address the } \\
\text { question }\end{array}$ \\
\hline \multirow{10}{*}{$\begin{array}{l}\text { Improving } \\
\text { antimicrobial } \\
\text { treatment of } \\
\text { HIV-associated } \\
\text { TB meningitis }\end{array}$} & $\begin{array}{l}\text { Is high-dose rifampicin safe and well- } \\
\text { tolerated in Ugandans? }\end{array}$ & $\begin{array}{l}\text { Rifatox, }{ }^{175} \text { Rifavirenz }{ }^{197} \\
\text { dose } 20 \mathrm{mg} / \mathrm{kg} / \text { day is safe }\end{array}$ & $\begin{array}{l}\text { 1. Rifashort study in PTB is } \\
\text { investigating R } 35 \mathrm{mg} / \mathrm{kg} \\
\text { 2. Phase II RCT of } 30-40 \mathrm{mg} / \mathrm{kg}\end{array}$ \\
\hline & $\begin{array}{l}\text { Can high-dose rifampicin be safely used } \\
\text { in people with advanced HIV disease? }\end{array}$ & $\begin{array}{l}\text { Rifavirenz }{ }^{197} \mathrm{R} 20 \mathrm{mg} / \mathrm{kg} \\
\text { outpatient study, median } \\
\text { CD4 } 120 \text { to } 144 \text {. } \\
\text { PanACEA MAMs study } \\
\mathrm{N}=24 \text { HIV+ CD4 }>200 \text {, only } \\
\mathrm{n}=4 \text { received } \mathrm{R} 35 \mathrm{mg} / \mathrm{kg}^{178}\end{array}$ & $\begin{array}{l}\text { 1. RCT of high dose rifampicin versus } \\
\text { standard therapy in hospitalised } \\
\text { PLHIV }\end{array}$ \\
\hline & $\begin{array}{l}\text { Can people with AIDS and critical illness } \\
\text { adequately absorb oral rifampicin or is } \\
\text { an IV regimen preferable? }\end{array}$ & $\begin{array}{l}\text { Yunivita }{ }^{180} \text { compared IV to } \\
\text { PO rifampicin included } n=6 \\
\text { PLHIV (Indonesia) }\end{array}$ & $\begin{array}{l}\text { 1. PK study with IV and oral rifampicin } \\
\text { in hospitalised PLHIV }\end{array}$ \\
\hline & $\begin{array}{l}\text { What are the blood and CSF PK } \\
\text { characteristics of high-dose rifampicin } \\
\text { in Ugandans with TB and HIV? }\end{array}$ & $\begin{array}{l}\operatorname{Dian}^{181} \mathrm{~N}=4 \text { PLHIV } \\
\text { R } 30 \mathrm{mg} / \mathrm{kg} \text { (Indonesia) }\end{array}$ & $\begin{array}{ll}\text { 1. } & \text { PK study of R } 35 \mathrm{mg} / \mathrm{kg} \text { in } \\
\text { hospitalised PLHIV with CSF } \\
\text { sampling } \\
\text { 2. PK results from Rifashort (blood) }\end{array}$ \\
\hline & $\begin{array}{l}\text { Can high-dose rifampicin improve } \\
\text { clinical outcomes from TBM? }\end{array}$ & $\begin{array}{l}\text { Svennson et al. }{ }^{152} \text { PK-PD } \\
\text { meta-analysis (Indonesia) }\end{array}$ & 1. Phase III RCT R $35 \mathrm{mg} / \mathrm{kg}$ in TBM \\
\hline & $\begin{array}{l}\text { Can higher dose isoniazid (in rapid } \\
\text { acetylators) improve outcomes? }\end{array}$ & $\begin{array}{l}\text { Ding164 PK-PD study, found } \\
\text { H exposure predicted } \\
\text { survival }\end{array}$ & 1. Phase III trial planned in China \\
\hline & Can linezolid improve outcomes? & $\begin{array}{l}\text { Sun } 207 \text { non-randomized } \\
\text { study: faster GCS \& temp } \\
\text { resolution with LZD }\end{array}$ & $\begin{array}{l}\text { 1. Phase IIB RCT (LASER), phase II RCT } \\
\text { (ALTER), phase III RCT (INTENSE- } \\
\text { TBM) in Africa }\end{array}$ \\
\hline & $\begin{array}{l}\text { What is the optimal regimen for MDR- } \\
\text { TBM, tuberculomas, spinal TB? }\end{array}$ & $\begin{array}{l}\text { Expert opinion, case } \\
\text { reports only. }{ }^{208}\end{array}$ & $\begin{array}{l}\text { 1. Rare subgroups of disease would } \\
\text { require multicentre approach }\end{array}$ \\
\hline & $\begin{array}{l}\text { How do other co-infections or co- } \\
\text { morbidities contribute to poor } \\
\text { outcomes in TBM? }\end{array}$ & $\begin{array}{l}\text { Post-mortem studies from } \\
\text { South Africa describe co- } \\
\text { infections in PLHIV209 }\end{array}$ & $\begin{array}{l}\text { 1. Prospective cohorts with detailed } \\
\text { clinical, microbiological, } \\
\text { radiological work up, and post } \\
\text { mortem examination }\end{array}$ \\
\hline & $\begin{array}{l}\text { Does high-dose rifampicin affect } \\
\text { dolutegravir levels more than standard } \\
\text { dose rifampicin? }\end{array}$ & nil & $\begin{array}{l}\text { 1. SAEFRIF study planned R } 35 \mathrm{mg} / \mathrm{kg} \\
\text { 2. Nested PK studies in RCTs }\end{array}$ \\
\hline \multirow{3}{*}{$\begin{array}{l}\text { Improving host- } \\
\text { directed } \\
\text { therapy in HIV- } \\
\text { associated TB } \\
\text { meningitis }\end{array}$} & $\begin{array}{l}\text { Are corticosteroids safe in PLHIV? What } \\
\text { is the risk of cryptococcal disease or } \\
\text { Kaposi's sarcoma? }\end{array}$ & $\begin{array}{l}\text { 9-month data on n-98 } \\
\text { Thwaites } 2004 \text { trial202 } \\
\text { African studies suggest } \\
\text { elevated risk of Kaposi's } \\
\text { sarcoma with steroids } 210,211\end{array}$ & $\begin{array}{l}\text { 1. Vietnamese ACT HIV trial } \\
\text { 2. Observational studies describing } \\
\text { incidence of cancers and fungal } \\
\text { infections in HIV/TBM }\end{array}$ \\
\hline & $\begin{array}{l}\text { Is there an alternative effective host- } \\
\text { directed therapy for TBM? }\end{array}$ & $\begin{array}{l}\text { Thalidomide, }{ }^{212} \\
\text { statins, TNF- } \alpha \text { mAb, IL-1Ra } \\
\text { used at case report level }\end{array}$ & $\begin{array}{l}\text { 1. Animal models } \\
\text { 2. Phase II trial of other novel options }\end{array}$ \\
\hline & $\begin{array}{l}\text { Is aspirin effective in averting stroke in } \\
\text { PLHIV/ TBM? }\end{array}$ & $\begin{array}{l}\text { Aspirin } 81 \mathrm{mg} \text { and } 1000 \mathrm{mg}, \\
\text { phase II (Vietnam)206 }\end{array}$ & $\begin{array}{ll}\text { 1. } & \text { Phase III trial (INTENSE, Uganda) } \\
\text { 2. } & \text { Phase II trial (LASER-TBM, S. Africa) } \\
\end{array}$ \\
\hline \multirow{4}{*}{ Supportive care } & $\begin{array}{l}\text { How should we manage } \\
\text { hyponatraemia? }\end{array}$ & $\begin{array}{l}\text { Observational reports } \\
\text { (India) }\end{array}$ & $\begin{array}{l}\text { 1. Cross sectional study to understand } \\
\text { cause of hyponatraemia }\end{array}$ \\
\hline & $\begin{array}{l}\text { What is the best management for } \\
\text { hydrocephalus in Uganda? }\end{array}$ & $\mathrm{Nil}$ & $\begin{array}{l}\text { 1. Begin with observational study } \\
\text { describing prevalence and } \\
\text { outcomes }\end{array}$ \\
\hline & $\begin{array}{l}\text { Is deep vein thrombosis prophylaxis } \\
\text { indicated? }\end{array}$ & $\mathrm{Nil}$ & $\begin{array}{l}\text { 1. Begin with observational study } \\
\text { describing prevalence and } \\
\text { outcomes }\end{array}$ \\
\hline & $\begin{array}{l}\text { Is prophylactic fluconazole warranted in } \\
\text { PLHIV + TBM receiving corticosteroids? }\end{array}$ & $\mathrm{Nil}$ & $\begin{array}{l}\text { 1. Observational study on incident } \\
\text { fungal infections }\end{array}$ \\
\hline
\end{tabular}




\section{Bibliography}

1. Daniel TM. The history of tuberculosis. Respir Med 2006; 100(11): 1862-70.

2. United States Center for Disease Control. A Strategic Plan for the Elimination of Tuberculosis in the United States. Morbidity and Mortality Weekly Report 1989; 38: 1-25.

3. Murray JF, Schraufnagel DE, Hopewell PC. Treatment of Tuberculosis. A Historical Perspective. Ann Am Thorac Soc 2015; 12(12): 1749-59.

4. World Health Organisation. Bugs, Drugs \& Smoke: stories from public health. Geneva, Switzerland: World Health Organisation; 2011.

5. World Health Organisation. Global tuberculosis report. Geneva, 2020.

6. World Health Organisation. Tuberculosis: a global emergency. Geneva 1994.

7. Rangaka MX, Cavalcante SC, Marais BJ, et al. Controlling the seedbeds of tuberculosis: diagnosis and treatment of tuberculosis infection. Lancet 2015; 386(10010): 2344-53.

8. World Health Organisation. Global Tuberculosis Report, 2009.

9. Lawn SD, Zumla Al. Tuberculosis. Lancet 2011; 378(9785): 57-72.

10. Aaron L, Saadoun D, Calatroni I, et al. Tuberculosis in HIV-infected patients: a comprehensive review. Clin Microbiol Infect 2004; 10(5): 388-98.

11. Esmail H, Riou C, Bruyn ED, et al. The Immune Response to Mycobacterium tuberculosis in HIV-1Coinfected Persons. Annu Rev Immunol 2018; 36: 603-38.

12. Gupta A, Wood R, Kaplan R, Bekker LG, Lawn SD. Tuberculosis incidence rates during 8 years of follow-up of an antiretroviral treatment cohort in South Africa: comparison with rates in the community. PLoS One 2012; 7(3): e34156.

13. Middelkoop K, Bekker LG, Shashkina E, Kreiswirth B, Wood R. Retreatment tuberculosis in a South African community: the role of re-infection, HIV and antiretroviral treatment. Int J Tuberc Lung Dis 2012; 16(11): 1510-6.

14. Sonnenberg P, Murray J, Glynn JR, Shearer S, Kambashi B, Godfrey-Faussett P. HIV-1 and recurrence, relapse, and reinfection of tuberculosis after cure: a cohort study in South African mineworkers. Lancet 2001; 358(9294): 1687-93.

15. World Health Organisation. Global Health Observatory data. 2019. https://www.who.int/gho/hiv/en/ (accessed 31st August 2020, .

16. World Health Organisation. The End TB Strategy. Geneva, Switzerland: World Health Organisations, 2015.

17. World Health Organisation. Global Tuberculosis Report 2019. Geneva: World Health Organisation, 2019.

18. Orme IM, Robinson RT, Cooper AM. The balance between protective and pathogenic immune responses in the TB-infected lung. Nat Immunol 2015; 16(1): 57-63.

19. Lowe DM, Redford PS, Wilkinson RJ, O'Garra A, Martineau AR. Neutrophils in tuberculosis: friend or foe? Trends Immunol 2012; 33(1): 14-25.

20. Bangani N, Nakiwala J, Martineau AR, Wilkinson RJ, Wilkinson KA, Lowe DM. Brief Report: HIV-1 Infection Impairs CD16 and CD35 Mediated Opsonophagocytosis of Mycobacterium tuberculosis by Human Neutrophils. J Acquir Immune Defic Syndr 2016; 73(3): 263-7. 
21. Nakiyingi L, Ssengooba W, Nakanjako D, et al. Predictors and outcomes of mycobacteremia among HIV-infected smear- negative presumptive tuberculosis patients in Uganda. BMC Infect Dis 2015; 15: 62.

22. Shafer RW, Goldberg R, Sierra M, Glatt AE. Frequency of Mycobacterium tuberculosis bacteremia in patients with tuberculosis in an area endemic for AIDS. Am Rev Respir Dis 1989; 140(6): 1611-3.

23. Barry CE, 3rd, Boshoff HI, Dartois $\mathrm{V}$, et al. The spectrum of latent tuberculosis: rethinking the biology and intervention strategies. Nat Rev Microbiol 2009; 7(12): 845-55.

24. Esmail $\mathrm{H}$, Lai RP, Lesosky $\mathrm{M}$, et al. Characterization of progressive HIV-associated tuberculosis using 2-deoxy-2-[(18)F]fluoro-D-glucose positron emission and computed tomography. Nat Med 2016; 22(10): 1090-3.

25. Dowdy DW, Basu S, Andrews JR. Is passive diagnosis enough? The impact of subclinical disease on diagnostic strategies for tuberculosis. Am J Respir Crit Care Med 2013; 187(5): 543-51.

26. Chamie G, Luetkemeyer A, Walusimbi-Nanteza M, et al. Significant variation in presentation of pulmonary tuberculosis across a high resolution of CD4 strata. Int J Tuberc Lung Dis 2010; 14(10): 1295-302.

27. Chamie G, Luetkemeyer A, Charlebois E, Havlir DV. Tuberculosis as part of the natural history of HIV infection in developing countries. Clin Infect Dis 2010; 50 Suppl 3: S245-54.

28. Gupta RK, Lucas SB, Fielding KL, Lawn SD. Prevalence of tuberculosis in post-mortem studies of HIVinfected adults and children in resource-limited settings: a systematic review and meta-analysis. AIDS 2015; 29(15): 1987-2002.

29. Lucas SB, De Cock KM, Hounnou A, et al. Contribution of tuberculosis to slim disease in Africa. BMJ 1994; 308(6943): 1531-3.

30. Rana FS, Hawken MP, Mwachari C, et al. Autopsy study of HIV-1-positive and HIV-1-negative adult medical patients in Nairobi, Kenya. J Acquir Immune Defic Syndr 2000; 24(1): 23-9.

31. Hakim JG, Gangaidzo IT, Heyderman RS, et al. Impact of HIV infection on meningitis in Harare, Zimbabwe: a prospective study of 406 predominantly adult patients. AIDS 2000; 14(10): 1401-7.

32. Jarvis JN, Meintjes G, Williams A, Brown Y, Crede T, Harrison TS. Adult meningitis in a setting of high HIV and TB prevalence: findings from 4961 suspected cases. BMC Infect Dis 2010; 10: 67.

33. Ellis J, Bangdiwala AS, Cresswell FV, et al. The Changing Epidemiology of HIV-Associated Adult Meningitis, Uganda 2015-2017. Open Forum Infect Dis 2019; 6(10): ofz419.

34. Bergemann A, Karstaedt AS. The spectrum of meningitis in a population with high prevalence of HIV disease. Q J M: an international journal of medicine 1996; 89(7): 499-504.

35. Centre for Disease Control. Reported Tuberculosis in the United States, 2016, 2017.

36. Pang Y, An J, Shu W, et al. Epidemiology of Extrapulmonary Tuberculosis among Inpatients, China, 2008-2017. Emerg Infect Dis 2019; 25(3): 457-64.

37. Phypers M, Harris T, Power C. CNS tuberculosis: a longitudinal analysis of epidemiological and clinical features. Int J Tuberc Lung Dis 2006; 10(1): 99-103.

38. Public Health England. Tuberculosis in England. London, UK. , 2019.

39. Ducomble T, Tolksdorf $\mathrm{K}$, Karagiannis I, et al. The burden of extrapulmonary and meningitis tuberculosis: an investigation of national surveillance data, Germany, 2002 to 2009. Euro Surveill 2013; 18(12).

40. Wilkinson RJ, Rohlwink U, Misra U.K., van Crevel R, Mai N.T.T., Dooley K.E. et al. . Tuberculous Meningitis. Nature Reviews: Neurology 2017. 
41. Donovan J, Thwaites GE, Huynh J. Tuberculous meningitis: where to from here? Curr Opin Infect Dis 2020; 33(3): 259-66.

42. Berenguer J, Moreno S, Laguna F, et al. Tuberculous meningitis in patients infected with the human immunodeficiency virus. N Engl J Med 1992; 326(10): 668-72.

43. Rich AR. The Pathogenesis of Tuberculosis: C. C. Thomas; 1946.

44. Donald PR, Schaaf HS, Schoeman JF. Tuberculous meningitis and miliary tuberculosis: the Rich focus revisited. J Infect 2005; 50(3): 193-5.

45. Wallgreen A. Some aspects of tuberculous meningtiis and the possibility of its prevention J Pediatric 1934; (5): 291-8.

46. Brancusi F, Farrar J, Heemskerk D. Tuberculous meningitis in adults: a review of a decade of developments focusing on prognostic factors for outcome. Future Microbio/ 2012; 7(9): 1101-16.

47. Marais S, Wilkinson KA, Lesosky M, et al. Neutrophil-associated central nervous system inflammation in tuberculous meningitis immune reconstitution inflammatory syndrome. Clin Infect Dis 2014; 59(11): 1638-47.

48. Letang E, Ellis J, Naidoo K, et al. Tuberculosis-HIV Co-Infection: Progress and Challenges After Two Decades of Global Antiretroviral Treatment Roll-Out. Arch Bronconeumol 2020; 56(7): 446-54.

49. Meintjes G, Lawn SD, Scano F, et al. Tuberculosis-associated immune reconstitution inflammatory syndrome: case definitions for use in resource-limited settings. Lancet Infect Dis 2008; 8(8): 516-23.

50. Marais S, Pepper DJ, Marais BJ, Torok ME. HIV-associated tuberculous meningitis--diagnostic and therapeutic challenges. Tuberculosis (Edinb) 2010; 90(6): 367-74.

51. Thwaites GE, Duc Bang N, Huy Dung N, et al. The influence of HIV infection on clinical presentation, response to treatment, and outcome in adults with Tuberculous meningitis. J Infect Dis 2005; 192(12): 2134-41.

52. Katrak SM, Shembalkar PK, Bijwe SR, Bhandarkar LD. The clinical, radiological and pathological profile of tuberculous meningitis in patients with and without human immunodeficiency virus infection. J Neurol Sci 2000; 181(1-2): 118-26.

53. Thwaites GE, Lan NT, Dung NH, et al. Effect of antituberculosis drug resistance on response to treatment and outcome in adults with tuberculous meningitis. J Infect Dis 2005; 192(1): 79-88.

54. Thao LTP, Heemskerk AD, Geskus RB, et al. Prognostic Models for 9-Month Mortality in Tuberculous Meningitis. Clin Infect Dis 2018; 66(4): 523-32.

55. Thao LTP, Heemskerk AD, Geskus RB, et al. Prognostic models for 9 month mortality in tuberculous meningitis. Clin Infect Dis 2017.

56. Stadelman AM, Ellis J, Samuels THA, et al. Treatment Outcomes in Adult Tuberculous Meningitis: A Systematic Review and Meta-analysis. Open Forum Infect Dis 2020; 7(8): ofaa257.

57. Cresswell FV, Bangdiwala AS, Meya DB, et al. Absence of cerebrospinal fluid pleocytosis in tuberculous meningitis is a common occurrence in HIV co-infection and a predictor of poor outcomes. Int J Infect Dis 2018; 68: 77-8.

58. Mcllleron $\mathrm{H}$, Rustomjee $\mathrm{R}$, Vahedi $\mathrm{M}$, et al. Reduced antituberculosis drug concentrations in HIVinfected patients who are men or have low weight: implications for international dosing guidelines. Antimicrob Agents Chemother 2012; 56(6): 3232-8.

59. van der Weert EM, Hartgers NM, Schaaf HS, et al. Comparison of diagnostic criteria of tuberculous meningitis in human immunodeficiency virus-infected and uninfected children. Pediatr Infect Dis J 2006; 25(1): 65-9. 
60. Thuong NTT, Heemskerk D, Tram TTB, et al. Leukotriene A4 Hydrolase Genotype and HIV Infection Influence Intracerebral Inflammation and Survival From Tuberculous Meningitis. J Infect Dis 2017; 215(7): 1020-8.

61. Marais S, Meintjes G, Pepper DJ, et al. Frequency, severity, and prediction of tuberculous meningitis immune reconstitution inflammatory syndrome. Clin Infect Dis 2013; 56(3): 450-60.

62. Pepper DJ, Marais S, Maartens G, et al. Neurologic manifestations of paradoxical tuberculosisassociated immune reconstitution inflammatory syndrome: a case series. Clin Infect Dis 2009; 48(11): e96-107.

63. Heemskerk AD, Bang ND, Mai NT, et al. Intensified Antituberculosis Therapy in Adults with Tuberculous Meningitis. N Engl J Med 2016; 374(2): 124-34.

64. Bahr NC, Boulware DR. Methods of rapid diagnosis for the etiology of meningitis in adults. Biomark Med 2014; 8(9): 1085-103.

65. Thwaites GE, Chau TT, Stepniewska K, et al. Diagnosis of adult tuberculous meningitis by use of clinical and laboratory features. Lancet 2002; 360(9342): 1287-92.

66. Cohen DB, Zijlstra EE, Mukaka M, et al. Diagnosis of cryptococcal and tuberculous meningitis in a resource-limited African setting. Trop Med Int Health 2010; 15(8): 910-7.

67. Torok ME, Nghia HD, Chau TT, et al. Validation of a diagnostic algorithm for adult tuberculous meningitis. Am J Trop Med Hyg 2007; 77(3): 555-9.

68. Dendane T, Madani N, Zekraoui A, et al. A simple diagnostic aid for tuberculous meningitis in adults in Morocco by use of clinical and laboratory features. Int J Infect Dis 2013; 17(6): e461-5.

69. Boyles TH, Lynen L, Seddon JA, Tuberculous Meningitis International Research C. Decision-making in the diagnosis of tuberculous meningitis. Wellcome Open Res 2020; 5: 11.

70. Sunbul M, Atilla A, Esen S, Eroglu C, Leblebicioglu H. Thwaites' diagnostic scoring and the prediction of tuberculous meningitis. Med Princ Pract 2005; 14(3): 151-4.

71. Checkley AM, Njalale Y, Scarborough M, Zjilstra EE. Sensitivity and specificity of an index for the diagnosis of TB meningitis in patients in an urban teaching hospital in Malawi. Trop Med Int Health 2008; 13(8): 1042-6.

72. Boyles T, Stadelman S, Ellis J, et al. The diagnosis of tuberculous meningitis in adults and adolescents: protocol for a systematic review and individual patient data meta-analysis to inform a multivariable prediction model. Wellcome Open Res 2020; 4.

73. Marais S, Thwaites G, Schoeman JF, et al. Tuberculous meningitis: a uniform case definition for use in clinical research. Lancet Infect Dis 2010; 10(11): 803-12.

74. Croda MG, Vidal JE, Hernandez AV, Dal Molin T, Gualberto FA, de Oliveira AC. Tuberculous meningitis in HIV-infected patients in Brazil: clinical and laboratory characteristics and factors associated with mortality. Int J Infect Dis 2010; 14(7): e586-91.

75. Karstaedt AS, Valtchanova S, Barriere R, Crewe-Brown HH. Tuberculous meningitis in South African urban adults. QJM 1998; 91(11): 743-7.

76. Thwaites GE, Chau TT, Farrar JJ. Improving the bacteriological diagnosis of tuberculous meningitis. J Clin Microbiol 2004; 42(1): 378-9.

77. Bahr NC, Tugume L, Rajasingham R, et al. Improved diagnostic sensitivity for tuberculous meningitis with Xpert((R)) MTB/RIF of centrifuged CSF. Int J Tuberc Lung Dis 2015; 19(10): 1209-15.

78. Cresswell FV, Bangdiwala AS, Bahr NC, et al. Can improved diagnostics reduce mortality from Tuberculous meningitis? Findings from a 6.5-year cohort in Uganda. Wellcome Open Res 2018; 3: 64. 
79. Heemskerk AD, Donovan J, Thu DDA, et al. Improving the microbiological diagnosis of tuberculous meningitis: A prospective, international, multicentre comparison of conventional and modified ZiehlNeelsen stain, GeneXpert, and culture of cerebrospinal fluid. J Infect 2018.

80. Feng GD, Shi M, Ma L, et al. Diagnostic accuracy of intracellular mycobacterium tuberculosis detection for tuberculous meningitis. Am J Respir Crit Care Med 2014; 189(4): 475-81.

81. Bahr NC, Nuwagira E, Evans EE, et al. Diagnostic accuracy of Xpert MTB/RIF Ultra for tuberculous meningitis in HIV-infected adults: a prospective cohort study. Lancet Infect Dis 2018; 18(1): 68-75.

82. Cresswell FV, Tugume L, Bahr NC, et al. Xpert MTB/RIF Ultra for the diagnosis of HIV-associated tuberculous meningitis: a prospective validation study. Lancet Infect Dis 2020.

83. Peralta G, Barry P, Pascopella L. Use of Nucleic Acid Amplification Tests in Tuberculosis Patients in California, 2010-2013. Open Forum Infect Dis 2016; 3(4): ofw230.

84. Duo L, Ying B, Song $X$, et al. Molecular profile of drug resistance in tuberculous meningitis from southwest china. Clin Infect Dis 2011; 53(11): 1067-73.

85. Pai M, Flores LL, Pai N, Hubbard A, Riley LW, Colford JM, Jr. Diagnostic accuracy of nucleic acid amplification tests for tuberculous meningitis: a systematic review and meta-analysis. Lancet Infect Dis 2003; 3(10): 633-43.

86. Sun WW, Sun $Q$, Yan LP, Zhang Q. The application of IS6110-baced loop-mediated isothermal amplification (LAMP) in the early diagnosis of tuberculous meningitis. Oncotarget 2017; 8(34): 57537-42.

87. Nagdev KJ, Kashyap RS, Parida MM, et al. Loop-mediated isothermal amplification for rapid and reliable diagnosis of tuberculous meningitis. J Clin Microbiol 2011; 49(5): 1861-5.

88. Helb D, Jones $M$, Story $E$, et al. Rapid detection of Mycobacterium tuberculosis and rifampin resistance by use of on-demand, near-patient technology. J Clin Microbiol 2010; 48(1): 229-37.

89. World Health Organisation. Xpert MTB/Rif: WHO policy update and implementation manual. https://www.who.int/tb/laboratory/xpert launchupdate/en/ (accessed 2nd September 2020.

90. World Health Organisation. Automated real-time nucleic acid amplification technology for rapid and simultaneous detection of tuberculosis and rifampicin resistance: Xpert MTB/RIF assay for the diagnosis of pulmonary and extrapulmonary TB in adults and children: policy update. Geneva, Switzerland: World Health Organisation, 2014.

91. Chakravorty S, Simmons AM, Rowneki M, et al. The New Xpert MTB/RIF Ultra: Improving Detection of Mycobacterium tuberculosis and Resistance to Rifampin in an Assay Suitable for Point-of-Care Testing. MBio 2017; 8(4).

92. Cox. JA, Lukande. RL, Kalungi. S, Van Marck. E, Lammens. M, al. VdVKe. Accuracy of Lipoarabinomannan and Xpert MTB/RIF Testing in Cerebrospinal Fluid To Diagnose Tuberculous Meningitis in an Autopsy Cohort of HIV-Infected Adults. J Clin Microbiol 2015; 53(8): 2667-73.

93. Boehme CC, Nabeta P, Hillemann D, et al. Rapid molecular detection of tuberculosis and rifampin resistance. N Engl J Med 2010; 363(11): 1005-15.

94. World Health Organisation Technical Expert Group. Non-inferiority analysis of Xpert MTB/RIF Ultra compared to Xpert MTB/RIF. Geneva: World Health Organisation 2017.

95. Dorman SE, Schumacher SG, Alland D, et al. Xpert MTB/RIF Ultra for detection of Mycobacterium tuberculosis and rifampicin resistance: a prospective multicentre diagnostic accuracy study. Lancet Infect Dis 2018; 18(1): 76-84. 
96. Kendall EA, Schumacher SG, Denkinger CM, Dowdy DW. Estimated clinical impact of the Xpert MTB/RIF Ultra cartridge for diagnosis of pulmonary tuberculosis: A modeling study. PLoS Med 2017; 14(12): e1002472.

97. Bahr NC, Nuwagira E, Evans EE, et al. Diagnostic accuracy of Xpert MTB/RIF Ultra for tuberculous meningitis in HIV-infected adults: a prospective cohort study. Lancet Infect Dis 2017.

98. Wilson MR, Sample HA, Zorn KC, et al. Clinical Metagenomic Sequencing for Diagnosis of Meningitis and Encephalitis. N Engl J Med 2019; 380(24): 2327-40.

99. Wang $S$, Chen $Y$, Wang D, et al. The Feasibility of Metagenomic Next-Generation Sequencing to Identify Pathogens Causing Tuberculous Meningitis in Cerebrospinal Fluid. Front Microbiol 2019; 10: 1993.

100. Ai JW, Zhou X, Xu T, et al. CRISPR-based rapid and ultra-sensitive diagnostic test for Mycobacterium tuberculosis. Emerg Microbes Infect 2019; 8(1): 1361-9.

101. Ramachandran PS, Wilson MR. Metagenomics for neurological infections - expanding our imagination. Nat Rev Neurol 2020.

102. MacLean E, Broger T, Yerlikaya S, Fernandez-Carballo BL, Pai M, Denkinger CM. A systematic review of biomarkers to detect active tuberculosis. Nat Microbio/ 2019; 4(5): 748-58.

103. Bahr NC, Meintjes G, Boulware DR. Inadequate diagnostics: the case to move beyond the bacilli for detection of meningitis due to Mycobacterium tuberculosis. J Med Microbiol 2019; 68(5): 755-60.

104. Xu HB, Jiang RH, Li L, Sha W, Xiao HP. Diagnostic value of adenosine deaminase in cerebrospinal fluid for tuberculous meningitis: a meta-analysis. Int J Tuberc Lung Dis 2010; 14(11): 1382-7.

105. Corral I, Quereda C, Navas E, et al. Adenosine deaminase activity in cerebrospinal fluid of HIVinfected patients: limited value for diagnosis of tuberculous meningitis. Eur J Clin Microbiol Infect Dis 2004; 23(6): 471-6.

106. Yu J, Wang ZJ, Chen LH, Li HH. Diagnostic accuracy of interferon-gamma release assays for tuberculous meningitis: a meta-analysis. Int J Tuberc Lung Dis 2016; 20(4): 494-9.

107. Huang TY, Zhang XX, Wu QL, et al. Antibody detection tests for early diagnosis in tuberculous meningitis. International Journal of Infectious Diseases 2016; 48: 64-9.

108. Lu D, Chen C, Yu S, Chen S. Diagnosis of Tuberculous Meningitis Using a Combination of Peripheral Blood T-SPOT.TB and Cerebrospinal Fluid Interferon-gamma Detection Methods. Lab Med 2016; 47(1): 6-12.

109. JuanRafael S, S nchez-Su rez C, Rebollo MJ, et al. Interferon gamma quantification in cerebrospinal fluid compared with PCR for the diagnosis of tuberculous meningitis. Journal of Neurology 2006; 253(10): 1323-30.

110. Luca MC, Petrovici CM, Vata A, et al. [Gamma interferon testing in blood and cerebrospinal fluid-rapid method for the diagnosis of tuberculous meningitis]. Rev Med Chir Soc Med Nat lasi 2008; 112(1): 108-10.

111. Patel VB, Bhigjee Al, Paruk HF, et al. Utility of a novel lipoarabinomannan assay for the diagnosis of tuberculous meningitis in a resource-poor high-HIV prevalence setting. Cerebrospinal Fluid Res 2009; 6: 13.

112. Cox JA, Lukande RL, Kalungi S, et al. Accuracy of Lipoarabinomannan and Xpert MTB/RIF Testing in Cerebrospinal Fluid To Diagnose Tuberculous Meningitis in an Autopsy Cohort of HIV-Infected Adults. J Clin Microbiol 2015; 53(8): 2667-73. 
113. Bahr NC, Tugume L, Boulware DR. A Word of Caution in Considering the Use of the Lipoarabinomannan Lateral Flow Assay on Cerebrospinal Fluid for Detection of Tuberculous Meningitis. J Clin Microbiol 2016; 54(1): 241-2.

114. Siddiqi OK, Birbeck GL, Ghebremichael M, et al. Prospective Cohort Study on Performance of Cerebrospinal Fluid (CSF) Xpert MTB/RIF, CSF Lipoarabinomannan (LAM) Lateral Flow Assay (LFA), and Urine LAM LFA for Diagnosis of Tuberculous Meningitis in Zambia. J Clin Microbiol 2019; 57(8).

115. Broger $\mathrm{T}$, Sossen B, du Toit E, et al. Novel lipoarabinomannan point-of-care tuberculosis test for people with HIV: a diagnostic accuracy study. Lancet Infect Dis 2019; 19(8): 852-61.

116. Kerkhoff AD, Sossen B, Schutz C, et al. Diagnostic sensitivity of SILVAMP TB-LAM (FujiLAM) point-ofcare urine assay for extra-pulmonary tuberculosis in people living with HIV. Eur Respir J 2020; 55(2).

117. Shah $M$, Hanrahan $C$, Wang $Z Y$, et al. Lateral flow urine lipoarabinomannan assay for detecting active tuberculosis in HIV-positive adults. Cochrane Database Syst Rev 2016; (5): CD011420.

118. Lawn SD. Point-of-care detection of lipoarabinomannan (LAM) in urine for diagnosis of HIVassociated tuberculosis: a state of the art review. BMC Infect Dis 2012; 12: 103.

119. Ankur Gupta-Wright, Elizabeth L. Corbett, Joep J. van Oosterhout, et al. URINE-BASED SCREENING FOR TUBERCULOSIS: A RANDOMIZED TRIAL IN HIV-POSITIVE INPATIENTS. Conferecne for Retroviruses and Opportunisitic Infections. Boston, USA; 2018.

120. Lawn SD, Kerkhoff AD, Vogt M, Wood R. High diagnostic yield of tuberculosis from screening urine samples from HIV-infected patients with advanced immunodeficiency using the Xpert MTB/RIF assay. J Acquir Immune Defic Syndr 2012; 60(3): 289-94.

121. Gupta-Wright A, Fielding K, Wilson D, et al. Tuberculosis in hospitalised patients with HIV: clinical characteristics, mortality, and implications from the STAMP trial. Clin Infect Dis 2019.

122. Lawn SD, Gupta-Wright A. Detection of lipoarabinomannan (LAM) in urine is indicative of disseminated TB with renal involvement in patients living with HIV and advanced immunodeficiency: evidence and implications. Trans R Soc Trop Med Hyg 2016; 110(3): 180-5.

123. World Health Organisation. The Use of Lateral FlowUrine Lipoarabinomannan Assay (LF-LAM) for the Diagnosis and Screening of Active Tuberculosis in People Living with HIV. Geneva, 2015.

124. Gupta-Wright A, Corbett EL, van Oosterhout JJ, et al. Rapid urine-based screening for tuberculosis in HIV-positive patients admitted to hospital in Africa (STAMP): a pragmatic, multicentre, parallelgroup, double-blind, randomised controlled trial. Lancet 2018; 392(10144): 292-301.

125. Gupta-Wright A, Corbett EL, Wilson D, et al. Risk score for predicting mortality including urine lipoarabinomannan detection in hospital inpatients with HIV-associated tuberculosis in sub-Saharan Africa: Derivation and external validation cohort study. PLoS Med 2019; 16(4): e1002776.

126. Gupta-Wright A, Peters JA, Flach C, Lawn SD. Detection of lipoarabinomannan (LAM) in urine is an independent predictor of mortality risk in patients receiving treatment for HIV-associated tuberculosis in sub-Saharan Africa: a systematic review and meta-analysis. BMC Med 2016; 14: 53.

127. World Health Organisation. Lateral flow urine lipoarabinomannan assay (LF-LAM) for the diagnosis of active tuberculosis in people living with HIV. Policy update 2019. Geneva, 2019.

128. Lawn SD, Kerkhoff AD, Burton R, et al. Diagnostic accuracy, incremental yield and prognostic value of Determine TB-LAM for routine diagnostic testing for tuberculosis in HIV-infected patients requiring acute hospital admission in South Africa: a prospective cohort. BMC Med 2017; 15(1): 67.

129. Lawn SD, Kerkhoff AD, Burton R, et al. Rapid microbiological screening for tuberculosis in HIVpositive patients on the first day of acute hospital admission by systematic testing of urine samples using Xpert MTB/RIF: a prospective cohort in South Africa. BMC Med 2015; 13: 192. 
130. Atherton RR, Cresswell FV, Ellis J, et al. Detection of Mycobacterium tuberculosis in urine by Xpert MTB/RIF Ultra: A useful adjunctive diagnostic tool in HIV-associated tuberculosis. Int J Infect Dis 2018; 75: 92-4.

131. Siddiqi Z, Siddiqi MS, Fatma J, Karoli R, Singhal V, Gupta M. Cerebrospinal Fluid Lactate in Tubercular Meningitis: Diagnostic or Prognostic Marker? J Assoc Physicians India 2018; 66(5): 18-21.

132. Thwaites GE, Simmons CP, Than Ha Quyen N, et al. Pathophysiology and prognosis in vietnamese adults with tuberculous meningitis. J Infect Dis 2003; 188(8): 1105-15.

133. Mitchison D, Davies G. The chemotherapy of tuberculosis: past, present and future. Int J Tuberc Lung Dis 2012; 16(6): 724-32.

134. Liu Y, Pertinez H, Ortega-Muro F, et al. Optimal doses of rifampicin in the standard drug regimen to shorten tuberculosis treatment duration and reduce relapse by eradicating persistent bacteria. $J$ Antimicrob Chemother 2018; 73(3): 724-31.

135. Mcllleron H, Meintjes G, Burman WJ, Maartens G. Complications of antiretroviral therapy in patients with tuberculosis: drug interactions, toxicity, and immune reconstitution inflammatory syndrome. $J$ Infect Dis 2007; 196 Suppl 1: S63-75.

136. Loos U, Musch E, Jensen JC, Mikus G, Schwabe HK, Eichelbaum M. Pharmacokinetics of oral and intravenous rifampicin during chronic administration. Klin Wochenschr 1985; 63(23): 1205-11.

137. World Health Organisation. Treatment of Tuberculosis Guidelines, Fourth Edition. Geneva: World Health Organisation, 2010.

138. Mitchison DA. Shortening the treatment of tuberculosis. Nat Biotechnol 2005; 23(2): 187-8.

139. Mitchison DA. The diagnosis and therapy of tuberculosis during the past 100 years. Am J Respir Crit Care Med 2005; 171(7): 699-706.

140. Cresswell FV, Te Brake L, Atherton R, et al. Intensified antibiotic treatment of tuberculosis meningitis. Expert Rev Clin Pharmacol 2019; 12(3): 267-88.

141. Nabisere R, Musaazi J, Denti P, et al. Pharmacokinetics, SAfety/tolerability, and EFficacy of high-dose RIFampicin in tuberculosis-HIV co-infected patients on efavirenz- or dolutegravir-based antiretroviral therapy: study protocol for an open-label, phase II clinical trial (SAEFRIF). Trials 2020; 21(1): 181.

142. Schutz C, Barr D, Andrade BB, et al. Clinical, microbiologic, and immunologic determinants of mortality in hospitalized patients with HIV-associated tuberculosis: A prospective cohort study. PLoS Med 2019; 16(7): e1002840.

143. Vernon A, Fielding K, Savic R, Dodd L, Nahid P. The importance of adherence in tuberculosis treatment clinical trials and its relevance in explanatory and pragmatic trials. PLoS Med 2019; 16(12): e1002884.

144. Menzies R, Rocher I, Vissandjee B. Factors associated with compliance in treatment of tuberculosis. Tuber Lung Dis 1993; 74(1): 32-7.

145. Sekaggya-Wiltshire $C$, von Braun A, Scherrer AU, et al. Anti-TB drug concentrations and drugassociated toxicities among TB/HIV-coinfected patients. J Antimicrob Chemother 2017; 72(4): 11727.

146. Imperial MZ, Nahid P, Phillips PPJ, et al. A patient-level pooled analysis of treatment-shortening regimens for drug-susceptible pulmonary tuberculosis. Nat Med 2018; 24(11): 1708-15.

147. Mitchison DA. How drug resistance emerges as a result of poor compliance during short course chemotherapy for tuberculosis. Int J Tuberc Lung Dis 1998; 2(1): 10-5. 
148. Seung KJ, Hewison C. Now is the time for shorter all-oral regimens for multidrug-resistant tuberculosis. Lancet Glob Health 2019; 7(6): e706.

149. Te Brake L. Translational research in tuberculosis: crossing barriers between molecular and clinical pharmacology; 2016.

150. Jullien S, Ryan H, Modi M, Bhatia R. Six months therapy for tuberculous meningitis. Cochrane Database Syst Rev 2016; 9: CD012091.

151. Nau R, Sorgel F, Eiffert H. Penetration of drugs through the blood-cerebrospinal fluid/blood-brain barrier for treatment of central nervous system infections. Clin Microbiol Rev 2010; 23(4): 858-83.

152. Svensson EM, Dian S, Te Brake L, et al. Model-based meta-analysis of rifampicin exposure and mortality in Indonesian tuberculosis meningitis trials. Clin Infect Dis 2019.

153. Ruslami R, Ganiem AR, Dian S, et al. Intensified regimen containing rifampicin and moxifloxacin for tuberculous meningitis: an open-label, randomised controlled phase 2 trial. Lancet Infect Dis 2013; 13(1): 27-35.

154. Donald PR. Cerebrospinal fluid concentrations of antituberculosis agents in adults and children. Tuberculosis (Edinb) 2010; 90(5): 279-92.

155. Heemskerk D, Day J, Chau TT, et al. Intensified treatment with high dose rifampicin and levofloxacin compared to standard treatment for adult patients with tuberculous meningitis (TBM-IT): protocol for a randomized controlled trial. Trials 2011; 12: 25.

156. Lukoye D, Adatu F, Musisi K, et al. Anti-tuberculosis drug resistance among new and previously treated sputum smear-positive tuberculosis patients in Uganda: results of the first national survey. PLoS One 2013; 8(8): e70763.

157. Diseases NIfC. South African Tuberculosis Drug Resistance Survey 2012-2014. 2015.

158. Heemskerk AD, Bang ND, Thwaites GE. Therapy for Tuberculous Meningitis. N Eng/ J Med 2016; 374(22): 2188-9.

159. Heemskerk AD, Nguyen MTH, Dang HTM, et al. Clinical Outcomes of Patients With Drug-Resistant Tuberculous Meningitis Treated With an Intensified Antituberculosis Regimen. Clin Infect Dis 2017; 65(1): 20-8.

160. Boeree MJ, Diacon AH, Dawson R, et al. A dose-ranging trial to optimize the dose of rifampin in the treatment of tuberculosis. Am J Respir Crit Care Med 2015; 191(9): 1058-65.

161. van Toorn R, Schaaf HS, Laubscher JA, van Elsland SL, Donald PR, Schoeman JF. Short intensified treatment in children with drug-susceptible tuberculous meningitis. Pediatr Infect Dis J 2014; 33(3): 248-52.

162. Ruslami R, Nijland HM, Alisjahbana B, Parwati I, van Crevel R, Aarnoutse RE. Pharmacokinetics and tolerability of a higher rifampin dose versus the standard dose in pulmonary tuberculosis patients. Antimicrob Agents Chemother 2007; 51(7): 2546-51.

163. Cairns H, Duthie ES. Intrathecal streptomycin in meningitis; clinical trial in tuberculous, coliform, and other infections. Lancet 1946; 2(6414): 153-5.

164. Ding J, Thuy Thuong Thuong N, Pham TV, et al. Pharmacokinetics and Pharmacodynamics of Intensive Antituberculosis Treatment of Tuberculous Meningitis. Clin Pharmacol Ther 2020.

165. Azuma J, Ohno M, Kubota R, et al. NAT2 genotype guided regimen reduces isoniazid-induced liver injury and early treatment failure in the 6-month four-drug standard treatment of tuberculosis: a randomized controlled trial for pharmacogenetics-based therapy. Eur J Clin Pharmacol 2013; 69(5): 1091-101. 
166. Naidoo A, Chirehwa M, Ramsuran V, et al. Effects of genetic variability on rifampicin and isoniazid pharmacokinetics in South African patients with recurrent tuberculosis. Pharmacogenomics 2019; 20(4): 225-40.

167. Sloan DJ, McCallum AD, Schipani A, et al. Genetic Determinants of the Pharmacokinetic Variability of Rifampin in Malawian Adults with Pulmonary Tuberculosis. Antimicrobial Agents and Chemotherapy 2017; 61(7): e00210-17.

168. Mukonzo JK, Kengo A, Kutesa B, et al. Role of pharmacogenetics in rifampicin pharmacokinetics and the potential effect on TB-rifampicin sensitivity among Ugandan patients. Trans R Soc Trop Med Hyg 2019.

169. Andrews JM. Determination of minimum inhibitory concentrations. J Antimicrob Chemother 2001; 48 Suppl 1: 5-16.

170. McKinnon PS, Davis SL. Pharmacokinetic and pharmacodynamic issues in the treatment of bacterial infectious diseases. Eur J Clin Microbiol Infect Dis 2004; 23(4): 271-88.

171. Stott KE, Pertinez $\mathrm{H}$, Sturkenboom MGG, et al. Pharmacokinetics of rifampicin in adult TB patients and healthy volunteers: a systematic review and meta-analysis. J Antimicrob Chemother 2018.

172. Hu Y, Liu A, Ortega-Muro F, Alameda-Martin L, Mitchison D, Coates A. High-dose rifampicin kills persisters, shortens treatment duration, and reduces relapse rate in vitro and in vivo. Front Microbiol 2015; 6: 641.

173. Diacon AH, Patientia RF, Venter A, et al. Early bactericidal activity of high-dose rifampin in patients with pulmonary tuberculosis evidenced by positive sputum smears. Antimicrob Agents Chemother 2007; 51(8): 2994-6.

174. te Brake L, de Jager V, Narunsky K, et al. Increased Bactericidal Activity But Dose-Limiting Tolerability at $50 \mathrm{mg} / \mathrm{kg}$ Rifampicin SSRN 2020.

175. Jindani A, Borgulya G, de Patino IW, et al. A randomised Phase II trial to evaluate the toxicity of highdose rifampicin to treat pulmonary tuberculosis. Int J Tuberc Lung Dis 2016; 20(6): 832-8.

176. Velasquez GE, Brooks MB, Coit JM, et al. Reply to te Brake et al.: Conflicting Findings on an Intermediate Dose of Rifampicin for Pulmonary Tuberculosis. Am J Respir Crit Care Med 2019; 199(9): 1167-8.

177. Aarnoutse RE, Kibiki GS, Reither K, et al. Pharmacokinetics, Tolerability, and Bacteriological Response of Rifampin Administered at 600, 900, and 1,200 Milligrams Daily in Patients with Pulmonary Tuberculosis. Antimicrob Agents Chemother 2017; 61(11).

178. Boeree MJ, Heinrich N, Aarnoutse R, et al. High-dose rifampicin, moxifloxacin, and SQ109 for treating tuberculosis: a multi-arm, multi-stage randomised controlled trial. Lancet Infect Dis 2017; 17(1): 39-49.

179. Te Brake L, Dian S, Ganiem AR, et al. Pharmacokinetic/pharmacodynamic analysis of an intensified regimen containing rifampicin and moxifloxacin for tuberculous meningitis. Int J Antimicrob Agents 2015; 45(5): 496-503.

180. Yunivita V, Dian S, Ganiem AR, et al. Pharmacokinetics and safety/tolerability of higher oral and intravenous doses of rifampicin in adult tuberculous meningitis patients. Int J Antimicrob Agents 2016; 48(4): 415-21.

181. Dian S, Yunivita V, Ganiem AR, et al. Double-Blind, Randomized, Placebo-Controlled Phase II DoseFinding Study To Evaluate High-Dose Rifampin for Tuberculous Meningitis. Antimicrob Agents Chemother 2018; 62(12). 
182. Daskapan A, Idrus LR, Postma MJ, et al. A Systematic Review on the Effect of HIV Infection on the Pharmacokinetics of First-Line Tuberculosis Drugs. Clin Pharmacokinet 2019; 58(6): 747-66.

183. Dean GL, Edwards SG, Ives NJ, et al. Treatment of tuberculosis in HIV-infected persons in the era of highly active antiretroviral therapy. AIDS 2002; 16(1): 75-83.

184. Abbara A, Chitty S, Roe JK, et al. Drug-induced liver injury from antituberculous treatment: a retrospective study from a large TB centre in the UK. BMC Infect Dis 2017; 17(1): 231.

185. Choudhri SH, Hawken M, Gathua S, et al. Pharmacokinetics of antimycobacterial drugs in patients with tuberculosis, AIDS, and diarrhea. Clin Infect Dis 1997; 25(1): 104-11.

186. Gurumurthy P, Ramachandran G, Hemanth Kumar AK, et al. Malabsorption of rifampin and isoniazid in HIV-infected patients with and without tuberculosis. Clin Infect Dis 2004; 38(2): 280-3.

187. Daskapan A, Idrus LR, Postma MJ, et al. A Systematic Review on the Effect of HIV Infection on the Pharmacokinetics of First-Line Tuberculosis Drugs. Clin Pharmacokinet 2018.

188. Court R, Chirehwa MT, Wiesner L, et al. Quality assurance of rifampicin-containing fixed-drug combinations in South Africa: dosing implications. Int J Tuberc Lung Dis 2018; 22(5): 537-43.

189. Cecchini D, Ambrosioni J, Brezzo C, et al. Tuberculous meningitis in HIV-infected patients: drug susceptibility and clinical outcome. AIDS 2007; 21(3): 373-4.

190. Thwaites GE, Chau TT, Caws M, et al. Isoniazid resistance, mycobacterial genotype and outcome in Vietnamese adults with tuberculous meningitis. Int J Tuberc Lung Dis 2002; 6(10): 865-71.

191. Blanc FX, Sok T, Laureillard D, et al. Earlier versus later start of antiretroviral therapy in HIV-infected adults with tuberculosis. N Engl J Med 2011; 365(16): 1471-81.

192. Abdool Karim SS, Naidoo K, Grobler A, et al. Timing of initiation of antiretroviral drugs during tuberculosis therapy. N Engl J Med 2010; 362(8): 697-706.

193. Havlir DV, Kendall MA, Ive P, et al. Timing of antiretroviral therapy for HIV-1 infection and tuberculosis. N Engl J Med 2011; 365(16): 1482-91.

194. Torok ME, Yen NT, Chau TT, et al. Timing of initiation of antiretroviral therapy in human immunodeficiency virus (HIV)--associated tuberculous meningitis. Clin Infect Dis 2011; 52(11): 137483.

195. World Health Organisation. Consolidated guidelines on the use of antiretroviral drugs for treating and preventing HIV infection Geneva Switzerland, 2016.

196. Boulware DR, Meya DB, Muzoora C, et al. Timing of antiretroviral therapy after diagnosis of cryptococcal meningitis. N Engl J Med 2014; 370(26): 2487-98.

197. Atwine D, Baudin E, Gele T, et al. Effect of high-dose rifampicin on efavirenz pharmacokinetics: drugdrug interaction randomized trial. J Antimicrob Chemother 2020; 75(5): 1250-8.

198. Wang $X$, Cerrone M, Ferretti F, et al. Pharmacokinetics of dolutegravir $100 \mathrm{mg}$ once daily with rifampicin. Int J Antimicrob Agents 2019; 54(2): 202-6.

199. Dooley KE, Kaplan R, Mwelase N, et al. Dolutegravir-based Antiretroviral Therapy for Patients Coinfected With Tuberculosis and Human Immunodeficiency Virus: A Multicenter, Noncomparative, Open-label, Randomized Trial. Clin Infect Dis 2020; 70(4): 549-56.

200. Prasad K SM, Ryan H. Corticosteroids for managing tuberculous meningitis., 2016.

201. Torok ME, Nguyen DB, Tran TH, et al. Dexamethasone and long-term outcome of tuberculous meningitis in Vietnamese adults and adolescents. PLoS One 2011; 6(12): e27821.

202. Thwaites GE, Nguyen DB, Nguyen HD, et al. Dexamethasone for the treatment of tuberculous meningitis in adolescents and adults. N Engl J Med 2004; 351(17): 1741-51. 
203. Misra UK, Kalita J, Nair PP. Role of aspirin in tuberculous meningitis: a randomized open label placebo controlled trial. J Neurol Sci 2010; 293(1-2): 12-7.

204. Schoeman JF, Janse van Rensburg A, Laubscher JA, Springer P. The role of aspirin in childhood tuberculous meningitis. J Child Neurol 2011; 26(8): 956-62.

205. Misra UK, Kalita J, Sagar B, Bhoi SK. Does adjunctive corticosteroid and aspirin therapy improve the outcome of tuberculous meningitis? Neurol India 2018; 66(6): 1672-7.

206. Mai NT, Dobbs N, Phu NH, et al. A randomised double blind placebo controlled phase 2 trial of adjunctive aspirin for tuberculous meningitis in HIV-uninfected adults. Elife 2018; 7.

207. Sun F, Ruan Q, Wang J, et al. Linezolid manifests a rapid and dramatic therapeutic effect for patients with life-threatening tuberculous meningitis. Antimicrob Agents Chemother 2014; 58(10): 6297-301.

208. Marais S VTR, Chow FC et al., . Management of intracranial tuberculous mass lesions: how long should we treat for? Wellcome Open Res, 2020; 4: 158.

209. Karat AS, Omar T, von Gottberg A, et al. Autopsy Prevalence of Tuberculosis and Other Potentially Treatable Infections among Adults with Advanced HIV Enrolled in Out-Patient Care in South Africa. PLoS One 2016; 11(11): e0166158.

210. Mayosi BM, Ntsekhe M, Bosch J, et al. Prednisolone and Mycobacterium indicus pranii in tuberculous pericarditis. N Engl J Med 2014; 371(12): 1121-30.

211. Elliott AM, Luzze H, Quigley MA, et al. A randomized, double-blind, placebo-controlled trial of the use of prednisolone as an adjunct to treatment in HIV-1-associated pleural tuberculosis. J Infect Dis 2004; 190(5): 869-78.

212. Schoeman JF, Springer P, Ravenscroft A, et al. Adjunctive thalidomide therapy of childhood tuberculous meningitis: possible anti-inflammatory role. Journal of Child Neurology 2000; 15(8): 497503.

213. Misra UK, Kalita J, Bhoi SK, Singh RK. A study of hyponatremia in tuberculous meningitis. J Neurol Sci 2016; 367: 152-7. 



\section{Chapter 2: Overview of PhD Aim, Hypotheses, Objectives, Setting and Methodology}

\subsection{Aim}

The overarching aim of this research was to identify better ways of diagnosing and treating TBM in subSaharan Africa.

\subsection{Hypotheses}

1. The next generation assay Xpert MTB/Rif 'Ultra' is significantly more sensitive than Xpert MTB/Rif for the detection of M. tuberculosis in CSF. However, Xpert Ultra's sensitivity will be imperfect and it will fail to diagnose a small number of cases of TBM that are evident by CSF culture, post-mortem examination, or metagenomic next generation sequencing.

2. Urine diagnostics such as urine TB-LAM and urine Xpert Ultra will be useful adjunctive tools in diagnosing TBM and will identify patients at the greatest risk of death.

3. Intensified treatment of TBM with intravenous rifampicin $(20 \mathrm{mg} / \mathrm{kg}$ ) or high-dose oral rifampicin (35 $\mathrm{mg} / \mathrm{kg}$ ) will result in significantly increased blood and CSF exposure during the critical early days of TB treatment as compared to common control (10mg/kg oral rifampicin).

4. High-dose rifampicin will be safe in people with advanced HIV disease and may improve functional outcomes and survival at 8 - and 24-weeks.

\subsection{Objectives}

1. To assimilate available retrospective data on adults with suspected TBM in Uganda and to describe the TBM population in terms of baseline characteristics, method of diagnosis and clinical outcomes.

2. To review the literature on novel diagnostics for TBM and summarise key findings in a review paper

3. To prospectively determine the diagnostic accuracy of Xpert Ultra for the diagnosis of TBM in a Ugandan population. To explore whether metagenomic next generation sequencing can help differentiate suspected TBM cases who are negative by Xpert MTB/Rif Ultra.

4. To investigate the utility of urine diagnostics, specifically urine TB-LAM and urine Xpert Ultra, in the diagnosis and prognostication of TBM.

5. To review the literature relating to the rationale and best options for intensified antibiotic treatment for TBM and summarise findings in a review article. 
6. To determine, through a phase II open-label randomised controlled trial, whether higher-dose rifampicin, delivered orally at $35 \mathrm{mg} / \mathrm{kg} /$ day or delivered intravenously at $20 \mathrm{mg} / \mathrm{kg} / \mathrm{day}$ provide blood and CSF exposure profiles that are favourable compared to the $10 \mathrm{mg} / \mathrm{kg}$ standard dose oral rifampicin.

7. To describe the safety of high-dose rifampicin, and clinical outcomes including functional status and survival, overall and by treatment arm.

8. To deliver a series of public engagement events to increase the community and district healthcare worker's awareness of TBM, advanced HIV disease, and the research available.

\subsection{Study Setting}

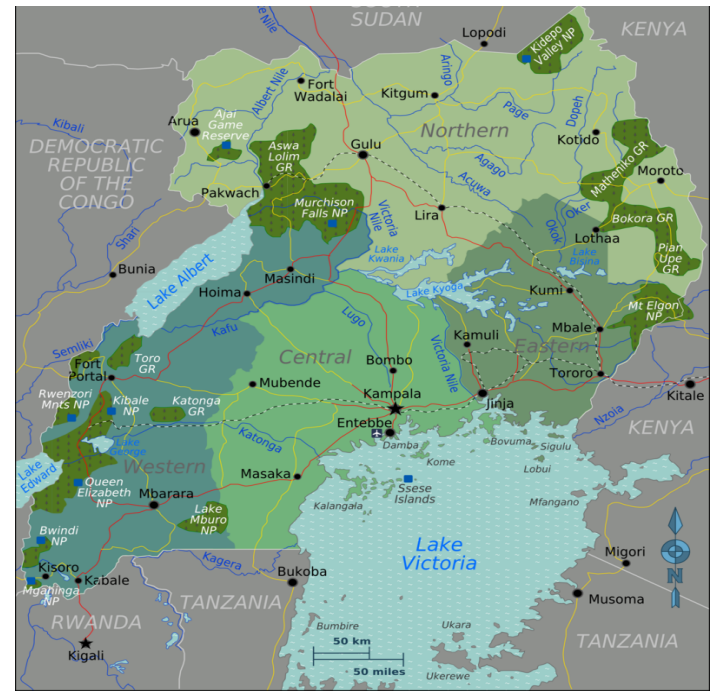

The Republic of Uganda is a country in East-Central Africa bordered by Kenya, South Sudan, Democratic Republic of Congo and Rwanda, Figure 2.1. Uganda is listed as a WHO 'high' TB prevalence country and TB is a major driver of morbidity and mortality, particularly in PLWHIV. An autopsy study at Mulago Hospital discovered disseminated TB in 32\% (17/53) of all autopsies, and 46\% (16/35) HIV-positive cadavers. ${ }^{1}$ In an outpatient setting at the Infectious Diseases Institute clinic in Kampala, TB remains the most frequent cause of death in PLWHIV $(24 \%, 17 / 70)$ despite $>15$-years of ART roll-out. ${ }^{2}$

Figure 2.1. Map of the Republic of Uganda

The TB and HIV epidemic in Uganda are inextricably intertwined with a HIV prevalence of 40\% amongst TB cases with a known HIV status. ${ }^{3}$ Of HIV-positive TB cases, $97 \%$ report being on ART, though the timing of ART initiation, adherence to ART and virological suppression is not reported. ${ }^{3}$

In 2019, there were 88,000 (range 52-134,000) cases of TB, giving an incidence of 200/100,000 population. This included $\sim 35,000$ cases amongst PLWHIV, resulting in 8,500 (range 5100 to 13000) deaths in PLWHIV. ${ }^{3}$ The incidence of TB is declining slowly, Figure 2.2. The Ugandan national TB reporting system captures data on TB cases as 'pulmonary' or 'extrapulmonary' and reports that $94 \%$ of notified cases were pulmonary in 2019. So, whilst we can estimate that there was $\sim 5280$ cases of extrapulmonary TB in 2019, there is no national level data on TBM incidence or outcomes.

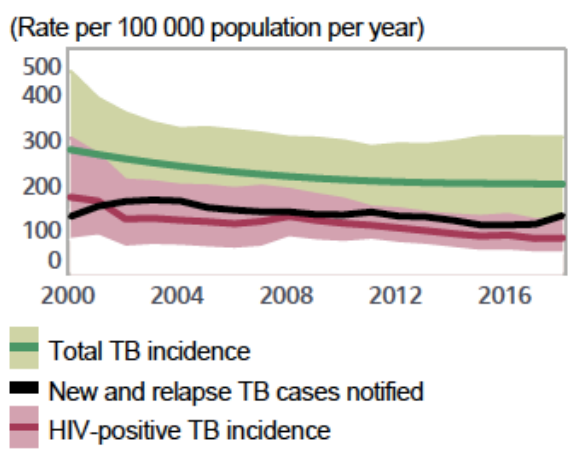

Figure 2.2. TB incidence rate in Uganda 2000 - 2018 


\subsection{Study Team}

Professor Alison Elliott, based at the Medical Research Council - Uganda Virus Research Institute and London School of Hygiene and Tropical Medicine Uganda Research Unit, is my primary supervisor and has overseen the PhD process from initial fellowship application, through PhD upgrading and write up of the thesis. I regularly attended Professor Elliott's lab meetings to discuss ideas, share progress and receive guidance and inspiration.

This research was based within the activities of the Infectious Diseases Institute (IDI) Meningitis Clinical Research Team, Figure 2.3, led by Dr David Meya (Makerere University) and Professor David Boulware (University of Minnesota, UoM) who was also my PhD supervisor. The team is made up of Physicians, Medical Officers, Nurses, Research Assistants, Laboratory Technicians, and an HIV Counsellor. The team's outputs are the result of a 10-year collaboration between IDI and UoM, and include the conduct of several clinical trials (COAT - Cryptococcal Optimal Antiretroviral Timing, and ASTRO - Adjunctive Sertraline for the Treatment of Cryptococcal Meningitis) and the generation of an extensive amount of research around meningitis diagnostics and clinical management of cryptococcal meningitis. More recently the team has expanded its scope to cover TB meningitis.

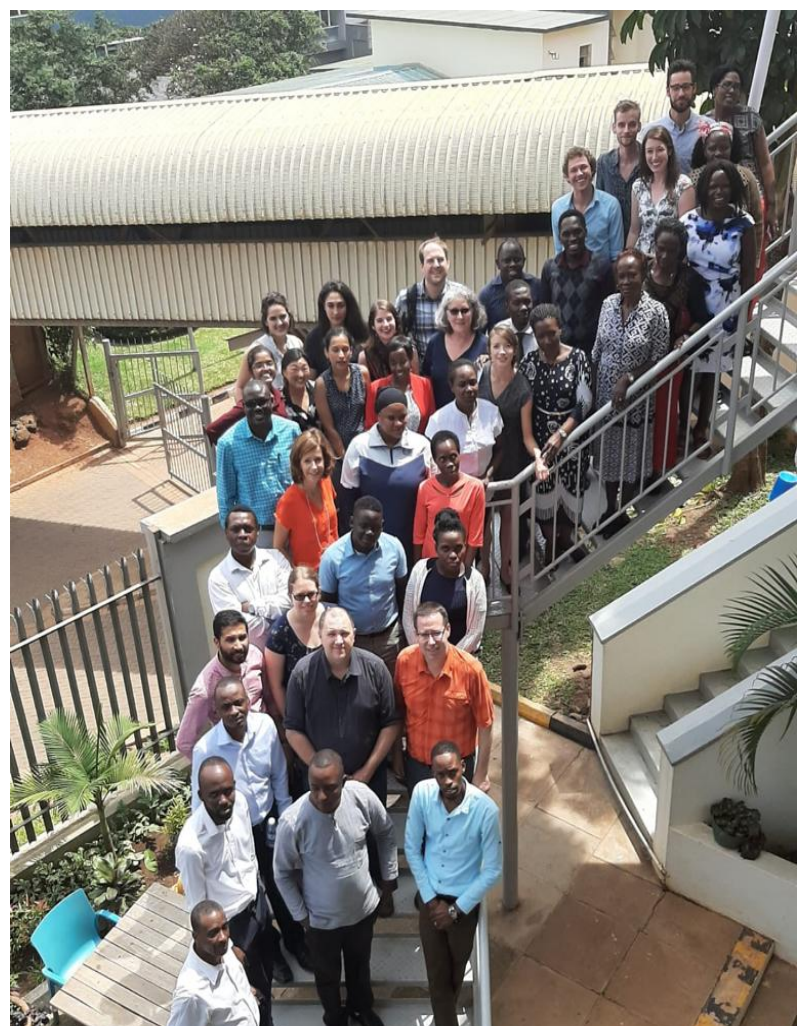

Figure 2.3. IDI Meningitis Clinical Research Team

The team conduct their activities on the Infectious Diseases and Neurology wards of Kiruddu National Referral Hospital (formerly part of Mulago National Referral Hospital and now an independent entity) in Kampala and the General Medicine wards at Mbarara Regional Referral Hospital. After hospital discharge patients are followed-up at the Infectious Diseases Institute Clinic in Kampala and in the Outpatient Department in Mbarara Hospital. The hospital laboratories have access to a GeneXpert machine but other laboratory capabilities were limited so samples were transported to the Makerere University Microbiology Laboratory (CSF analysis) or Makerere University TB Laboratory (CSF TB culture) or to the IDI Translational Laboratory (Xpert MTB/Rif Ultra) and IDI Core Laboratory for blood work (complete blood count, renal and liver function tests) and storage. In Mbarara the Joint Clinical Research Centre Laboratory was used. 


\subsection{Methodology}

I utilised a broad range of research methodologies during my PhD to appropriately address the 8 objectives described in above. The methodologies and statistical techniques used are described in detail in the respective research papers. Broadly, the research methodologies can be broken down into five components as detailed below.

\subsubsection{Literature Review}

I conducted literature reviews to familiarize myself with the background evidence in the field of TBM outcomes, diagnostics and intensified TBM treatment. This culminated in a number of papers:

- Systematic review and meta-analysis on outcome of TBM (Appendix 1)

- Review article on recent updates in pathogenesis and diagnostics of TBM (chapter 3)

- Review article on Intensified antimicrobial therapy for TBM (chapter 7).

\subsubsection{Diagnostic Research}

\subsubsection{Retrospective cohort study on TB Meningitis Diagnosis and Outcomes in Uganda}

To more fully understand the nature of TBM in Uganda I utilized existing data collected by the IDI Meningitis Research Team during prior studies between 2010 - 2016. I identified patients who may have had TBM by looking at those patients in whom tests for cryptococcal meningitis were negative, as well as searching for variables that related to TB diagnostic testing, TB treatment or 'suspected TBM' being listed by the study doctor. Where there was uncertainty I revisited archived research files to clarify the patient history and data. After extensive reviewing of research notes and data cleaning I generated a retrospective TBM dataset, containing 195 patients treated for TBM (suspected or confirmed diagnosis), which I used to analyse and write up the research paper in chapter 4 'Tuberculous meningitis diagnosis and during the Xpert MTB/Rif era: a 6.5year cohort study in Uganda'.

\subsubsection{Prospective Diagnostic Research on CSF and Urine}

Throughout my PhD research period I undertook ward rounds with the study team on the Infectious Diseases Ward at Kiruddu National Referral Hospital in Kampala. On a daily basis we assessed adults presenting with suspected meningitis (e.g. headache or altered mental status), sought informed consent (from the patient or their caregiver) and thereafter made a clinical assessment and collect baseline data and a CSF sample, as well as a urine sample. We performed imaging as clinically indicated and where possible. We returned diagnostic results to the local ward team and followed the patient through to the time of hospital discharge or death. CSF samples from patients with suspected TB were used for the prospective diagnostic accuracy study of CSF 
Xpert MTB/Rif Ultra (chapter 5). Urine samples were used to explore the utility of urine diagnostics for TBM diagnosis and management (chapter 6).

\subsubsection{Therapeutic Research: A Phase II Randomised Controlled Trial of High Dose Oral and Intravenous Rifampicin in TB Meningitis}

During the first 6 months of the PhD I invested time in developing the protocol, standard operating procedures (SOPs) and case report forms (CRFs) for the Rift trial, a phase II open-label randomized controlled trial investigating high-dose rifampicin for adult TBM. The trial primary endpoints were: 1) pharmacokinetic parameters (serum total and peak exposure and CSF concentration), 2) composite safety endpoint (grade 3-5 AEs, serious AEs or discontinuation of rifampicin for $>5$ days) during the 8-week intervention period. The study was powered for primary endpoints with a sample size of 60 across the three arms. Secondary endpoints were primarily exploratory and included clinical outcomes: 1) mortality at 8 and 24 weeks, 2) time to normalization of conscious level, 3) functional status by modified Rankin score). I published the clinical trial protocol as a paper on Wellcome Open Research, featured in the Appendix 5.

Thereafter, I navigated the regulatory approvals from the UK and Ugandan authorities, including three rounds of Institutional Review Board submissions at Mulago Hospital and LSHTM, Uganda National Council of Science and Technology approval and Ugandan National Drug Authority approval. Donation of study drug was sought from Sanofi. The trial began enrolment on 14 $4^{\text {th }}$ January 2019 and recruited through til $17^{\text {th }}$ December 2019. The individual follow-up period was 6 months, so the final participant completed follow-up on the $26^{\text {th }}$ May 2020. The Rift main trial paper is included in chapter 8.

\subsubsection{Public Engagement}

I realized that there was a major need for public engagement work around meningitis to address a number of challenges:

1. Firstly, I observed how people present to hospital with very advanced disease, often after weeks of illness, by which time the TBM is advanced (Medical Research Council, MRC stage II/III), irreversible brain damage already exists and the chance of any therapeutic intervention having an impact is limited.

2. Secondly, even after presentation to hospital some patients languish at regional hospitals or private hospitals for days or weeks without having a lumbar puncture, appropriate treatment being initiated or onwards referral to the research team for diagnostic or therapeutic research being made.

3. Thirdly, there is a misperception in the community that lumbar punctures kill people (often because they are only performed at the terminally stage of the illness for the reasons mentioned above) and this misperception can lead to a delay in consent for the LP on arrival at hospital.

4. Lastly, misconceptions are held by some community members about the aims and nature of clinical research relating to fears about being experimented upon and exploited. 
These are challenging problems to address and no work had been done on public engagement around meningitis research previously in Uganda. I was successful in winning three public engagement grants, the first a small public engagement grant from LSHTM, the second an Endeavour award from Makerere-UVRI Infection and Immunity (MUII) Centre of Excellence, and the third a continued development public engagement award from LSHTM. The overall aim of the public engagement work was to increase dialogue between the community and research team to increase awareness of HIV-associated meningitis the ongoing clinical research, and to dispel myths and anxiety around lumbar punctures.

Along with the research team, and with guidance from the Infectious Diseases Community Advisory Board, I put together a series of different events over 15 months. These events included a circus in an informal settlement in central Kampala to engage with hard-to-reach individuals, continued medical education sessions with regional healthcare workers, testimonials from trial participants broadcast on CBS national radio, a television show on NTV national TV including a trial participant and an 'Event of Hope' research results dissemination with trial participants and other stakeholders. The activities are detailed in an open letter in chapter 9 published on the African Academy of Sciences Open Research.

\subsection{Ethics}

All elements of the research were conducted in-line with Good Clinical Practice in Research and the Declaration of Helsinki. Ethical approvals were obtained from Mulago Hospital Institutional Review Board (MREC 1260) and the LSHTM Ethics Committee (14388).

Informed written consent (signature or thumbprint where illiterate) was obtained from participants or their surrogate (if participant lacked mental capacity due to altered mental state, which is common in brain infections). Clinical examination and study procedures were undertaken by experienced and appropriately trained study staff. Study drugs were administered by research nurses and drug management was overseen by the IDI research pharmacist and the Ugandan National Drug Authority.

\subsection{Data Management and Quality Assurance}

Data was collected using the DataFax system which relies on paper case report forms (CRFs) that were scanned, emailed to a server, and data entered by intelligent character recognition. After an initial automated error-checking, secondary review for accuracy was performed by the DataFax team at the Infectious Diseases Institute, Uganda. A detailed data management plan was written and reviewed by the data management team at LSHTM. To ensure data collected was of the highest quality a monitoring plan was implemented which involved both internal monitoring by IDI and external monitoring by Mrs Mirriam Akello and Mrs Sarah Coutinho from Makerere-UVRI Infection and Immunity Centre of Excellence. 


\section{Bibliography}

1. Cox JA, Lukande RL, Nelson AM, et al. An autopsy study describing causes of death and comparing clinico-pathological findings among hospitalized patients in Kampala, Uganda. PLoS One 2012; 7(3): e33685.

2. Kiragga AN, Mubiru F, Kambugu AD, Kamya MR, Castelnuovo B. A decade of antiretroviral therapy in Uganda: what are the emerging causes of death? BMC Infect Dis 2019; 19(1): 77.

3. World Health Organisation. Tuberculosis Country Data. .

https://worldhealthorg.shinyapps.io/tb profiles/? inputs \&lan=\%22EN\%22\&iso2=\%22UG\%22\&mai n tabs=\%22est tab\%22 (accessed 11th September 2020. 


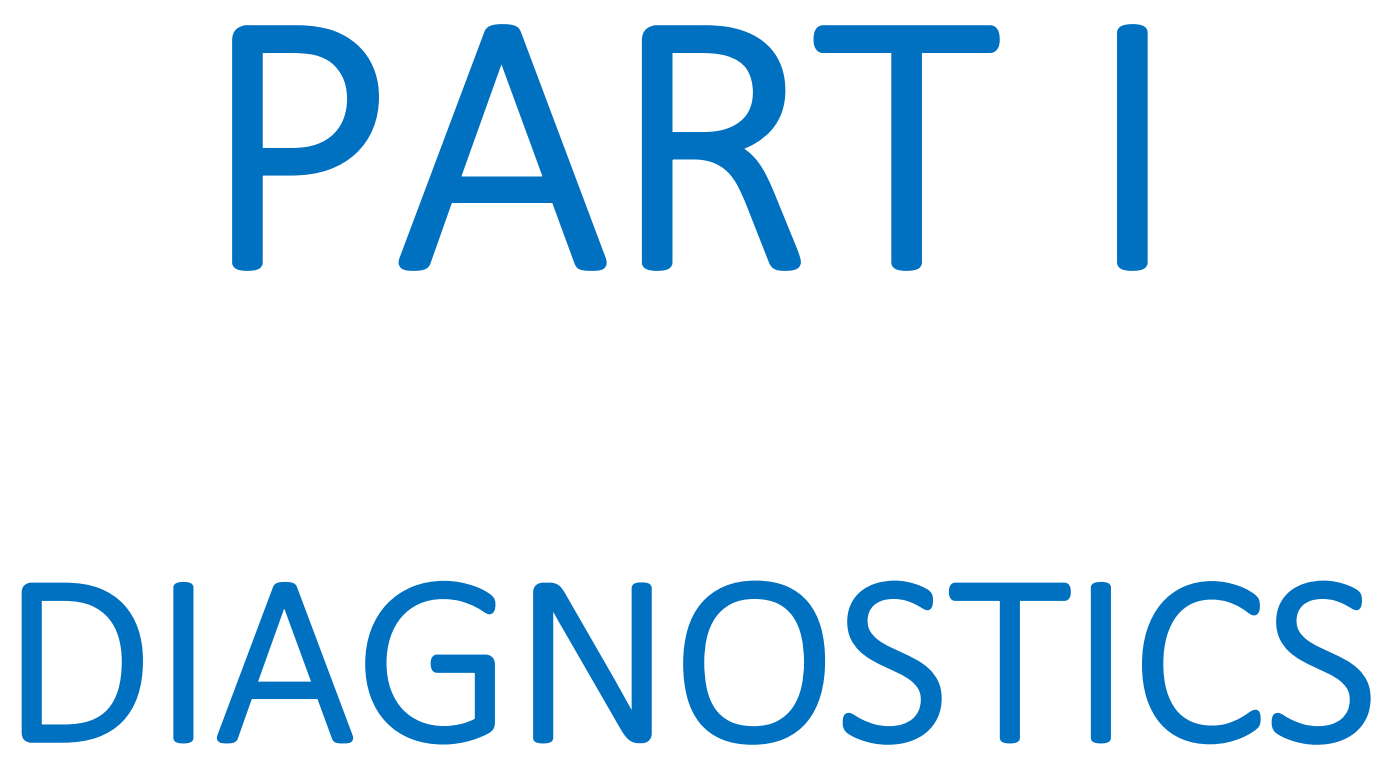



\section{Chapter Three: Recent advances in Pathogenesis and Diagnostics of Tuberculous Meningitis (review paper)}

\section{Foreword}

The number of publications relating to

TBM has steadily increased since the

turn of the century, equating to

almost 2000 in total. The increasing

use of 'omics' techniques in the last 5-

10 years is increasingly illuminating

the complex pathogenesis of this

disease and will hopefully lead to

better diagnostics and treatments.

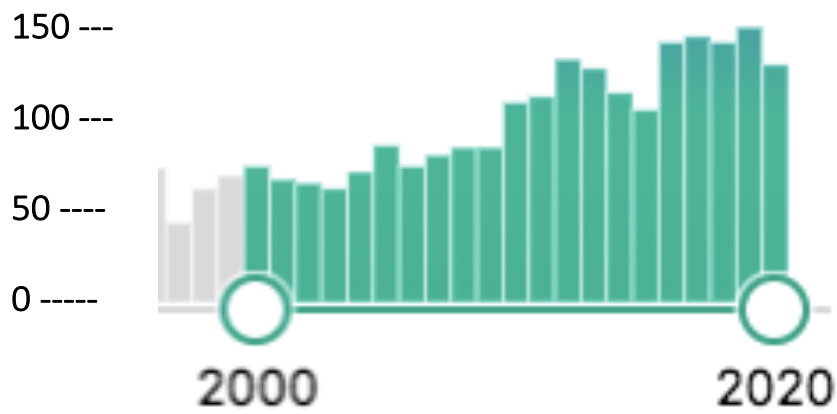

Year of publication

Annual publications, PubMed. Search terms tuberculous meningitis OR TB meningitis in title/abstract

The Tuberculous Meningitis International Research Consortium is comprised of basic scientists, translational and clinical researchers interested in TBM and meets biennially. Along with fellow multi-disciplinary members of the consortium I led the writing of a review paper on 'Recent advances in pathogenesis and diagnostics of tuberculous meningitis'. This paper involved pulling together a group of ten authors from seven different countries. The article has become part of a 'collection' of 11 articles published on the Wellcome Open Research platform by the consortium (https://wellcomeopenresearch.org/collections/tbmeningitis). I laid out the framework for the article, wrote the section on molecular diagnostics and harmonised and edited the other sections. I handled the submission process and the response to reviewer comments. Literature search was conducted on PubMed using the terms tubercul* meningitis OR TB meningitis within the dates 10/10/2014 and 10/10/2019, yielding 742 titles/abstracts which were the sifted for relevance.

The article specifically focusses on insights from the last 5 years and does not aim to be a comprehensive or systematic review of all literature relating to TBM. The article serves as a broad overview and introduction to immunology and diagnostics and introduces topics relevant to the rest of the thesis. 



\section{RESEARCH PAPER COVER SHEET}

\section{PLEASE NOTE THAT A COVER SHEET MUST BE COMPLETED FOR EACH RESEARCH PAPER INCLUDED IN A THESIS.}

\section{SECTION A - Student Details}

\begin{tabular}{|l|l|}
\hline Student & Fiona Cresswell \\
\hline Principal Supervisor & Alison Elliott \\
\hline Thesis Title & $\begin{array}{l}\text { Improving diagnosis and treatment of HIV-associated } \\
\text { Tuberculous meningitis }\end{array}$ \\
\hline
\end{tabular}

If the Research Paper has previously been published please complete Section B, if not please move to Section C

\section{SECTION B - Paper already published}

\begin{tabular}{|l|l|l|l|}
\hline Where was the work published? & Wellcome Open Research \\
\hline When was the work published? & 31 st Oct 2019 \\
\hline $\begin{array}{l}\text { If the work was published prior to } \\
\text { registration for your research degree, } \\
\text { give a brief rationale for its inclusion }\end{array}$ & \multicolumn{3}{|l|}{} \\
\hline $\begin{array}{l}\text { Have you retained the copyright for the } \\
\text { work?* }\end{array}$ & Yes & $\begin{array}{l}\text { Was the work subject to } \\
\text { academic peer review? }\end{array}$ & Yes \\
\hline
\end{tabular}

*If yes, please attach evidence of retention. If no, or if the work is being included in its published format, please attach evidence of permission from the copyright holder (publisher or other author) to include this work.

\section{SECTION C - Prepared for publication, but not yet published}

\begin{tabular}{|l|l|}
\hline $\begin{array}{l}\text { Where is the work intended to be } \\
\text { published? }\end{array}$ & \\
\hline $\begin{array}{l}\text { Please list the paper's authors in the } \\
\text { intended authorship order: }\end{array}$ & \\
\hline Stage of publication & Choose an item. \\
\hline
\end{tabular}

\section{SECTION D - Multi-authored work}

For multi-authored work, give full details of your role in the research included in the paper and in the preparation of the paper. (Attach a further sheet if necessary)
I laid out the format for the manuscript and coordinated a group of 9 co-authors from 7 countries through the writing process. I wrote the section on molecular diagnostics and helped to write other sections. I handled all the referencing, submission, review and response and re-submission process. 
Student Signature:

Supervisor Signature:
Date: _ 24th Sept 2020

Date: 8th October 2020 


\title{
REVISED Recent Developments in Tuberculous Meningitis
}

\section{Pathogenesis and Diagnostics [version 2; peer review: 1}

\section{approved]}

\author{
Fiona V Cresswell (iD1-3, Angharad G. Davis (D) 4-6, Kusum Sharma7, \\ Robindra Basu Roy1, Ahmad Rizal Ganiem', Enock Kagimu (iD), \\ Regan Solomons (ii) 9 , Robert J. Wilkinson (ii)5,6,10, Nathan C Bahr (iD11, \\ Nguyen Thuy Thuong Thuong12, \\ Tuberculous Meningitis International Research Consortium
}

\footnotetext{
${ }^{1}$ Clinical Research Department, London School of Hygiene and Tropical Medicine, London, WC1E 7HT, UK

${ }^{2}$ Research Department, Infectious Diseases Institute, Kampala, PO Box 22418, Uganda

${ }^{3}$ MRC-UVRI-London School of Hygiene and Tropical Medicine Uganda Research Unit, Entebbe, Uganda

${ }^{4}$ University College London, London, WC1E6BT, UK

${ }^{5}$ Francis Crick Institute, London, NW1 1AT, UK

${ }^{6}$ Department of Medicine, Institute of Infectious Diseases and Molecular Medicine, University of Cape Town, 7925, South Africa

7 Department of Medical Microbiology, Post-graduate Department of Medical Education and Research, Chandigahr, India

${ }^{8}$ Department of Neurology, Hasan Sadikin Hospital, Faculty of Medicine. Universitas Padjadjaran, Bandung, Indonesia

${ }^{9}$ Department of Paediatrics and Child Health, Faculty of Medicine and Health Sciences, Stellenbosch University, South Africa

${ }^{10}$ Department of Infectious Diseases, Imperial College, London, W2 1PG, UK

${ }^{11}$ Division of Infectious Diseases. Department of Medicine., University of Kansas, Kansas City, USA

${ }^{12}$ Oxford University Clinical Research Unit, Ho Chi Minh City, Vietnam
}

V2 First published: 31 Oct 2019, 4:164

https://doi.org/10.12688/wellcomeopenres.15506.1

Latest published: $06 \mathrm{Jul}$ 2020, 4:164

https://doi.org/10.12688/wellcomeopenres.15506.2

\section{Abstract}

The pathogenesis of Tuberculous meningitis (TBM) is poorly understood, but contemporary molecular biology technologies have allowed for recent improvements in our understanding of TBM. For instance, neutrophils appear to play a significant role in the immunopathogenesis of TBM, and either a paucity or an excess of inflammation can be detrimental in TBM. Further, severity of HIVassociated immunosuppression is an important determinant of inflammatory response; patients with the advanced immunosuppression (CD4+ T-cell count of $<150$ cells/ $\mu \mathrm{L}$ ) having higher CSF neutrophils, greater CSF cytokine concentrations and higher mortality than those with CD4+ T-cell counts $>150$ cells $/ \mu \mathrm{L}$. Host genetics may also influence outcomes with LT4AH genotype predicting inflammatory phenotype, steroid responsiveness and survival in Vietnamese adults with TBM. Whist in Indonesia, CSF tryptophan level was a predictor of survival, suggesting tryptophan metabolism may be important in TBM pathogenesis. These varying responses mean that we must consider whether a "one-size-fits-all"
Open Peer Review

Reviewer Status

Invited Reviewers

1

version 2

(revision)

06 Jul 2020

version 1

31 Oct 2019

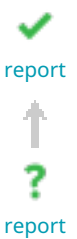

1. James E. Scriven ID, University of

Birmingham, Birmingham, UK

Any reports and responses or comments on the 
approach to anti-bacillary or immunomodulatory treatment in TBM is truly the best way forward. Of course, to allow for proper treatment, early and rapid diagnosis of TBM must occur. Diagnosis has always been a challenge but the field of TB diagnosis is evolving, with sensitivities of at least $70 \%$ now possible in less than two hours with GeneXpert MTB/Rif Ultra. In addition, advanced molecular techniques such as CRISPR-MTB and metagenomic next generation sequencing may hold promise for TBM diagnosis. Host-based biomarkers and signatures are being further evaluated in childhood and adult TBM as adjunctive biomarkers as even with improved molecular assays, cases are still missed. A better grasp of host and pathogen behaviour may lead to improved diagnostics, targeted immunotherapy, and possibly biomarker-based, patient-specific treatment regimens.

\section{Keywords}

Tuberculous meningitis, TBM, TB, HIV, pathogenesis, diagnostics, article can be found at the end of the article.

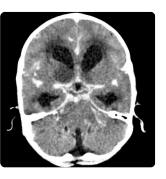

This article is included in the Tuberculous

Meningitis International Research Consortium

collection.

Corresponding author: Fiona V Cresswell (fiona.cresswell@lshtm.ac.uk)

Author roles: Cresswell FV: Conceptualization, Methodology, Project Administration, Writing - Original Draft Preparation, Writing Review \& Editing; Davis AG: Conceptualization, Methodology, Writing - Original Draft Preparation, Writing - Review \& Editing; Sharma K: Writing - Original Draft Preparation; Basu Roy R: Writing - Original Draft Preparation, Writing - Review \& Editing; Ganiem AR: Writing Original Draft Preparation; Kagimu E: Writing - Original Draft Preparation; Solomons R: Methodology, Writing - Original Draft

Preparation, Writing - Review \& Editing; Wilkinson RJ: Methodology, Writing - Original Draft Preparation, Writing - Review \& Editing; Bahr NC: Conceptualization, Methodology, Writing - Original Draft Preparation, Writing - Review \& Editing; Thuong NTT: Conceptualization, Methodology, Writing - Original Draft Preparation, Writing - Review \& Editing;

Competing interests: No competing interests were disclosed.

Grant information: This work is supported by the Wellcome Trust through a Wellcome Clinical PhD Fellowship to FVC [210772], a UCL Wellcome Trust PhD Programme for Clinicians Fellowship award to AGD [175479], a Wellcome Trust intermediate Fellowship to NTTT [206724], support to the Francis Crick Institute [FC0010218] and grants to RJW [104803, 203135]. NCB is supported by the National Institute of Neurological Disorders and Stroke (K23 NS110470) within the National Institutes of Health. RJW is also supported by the Francis Crick Institute which receives support from Wellcome [FC0010218], CRUK [FC0010208]; Meningitis Now; and the European and Developing Countries Clinical Trials Partnership (EDCTP). RS is supported by the National Research Foundation of South Africa [109437]. This work was supported by the Wellcome Trust through funding to the Tuberculous Meningitis International Research Consortium. The funders had no role in study design, data collection and analysis, decision to publish, or preparation of the manuscript.

Copyright: (c) 2020 Cresswell FV et al. This is an open access article distributed under the terms of the Creative Commons Attribution License, which permits unrestricted use, distribution, and reproduction in any medium, provided the original work is properly cited. The author(s) is/are employees of the US Government and therefore domestic copyright protection in USA does not apply to this work. The work may be protected under the copyright laws of other jurisdictions when used in those jurisdictions.

How to cite this article: Cresswell FV, Davis AG, Sharma K et al. Recent Developments in Tuberculous Meningitis Pathogenesis and Diagnostics [version 2; peer review: 1 approved] Wellcome Open Research 2020, 4:164

https://doi.org/10.12688/wellcomeopenres.15506.2

First published: 31 Oct 2019, 4:164 https://doi.org/10.12688/wellcomeopenres.15506.1 


\section{REVISED Amendments from Version 1}

In this version we have taken on board the suggestions of reviewer 1 . We have further developed the evidence on TBM pathogenesis including host genotype and brain injury biomarkers, and made other minor changes.

Any further responses from the reviewers can be found at the end of the article

\section{Introduction}

The pathogenesis of Tuberculous meningitis (TBM) is poorly understood. Mechanisms by which Mycobacteria disseminate from lung to the brain, key factors driving a dysregulated host response, and the pathogen specific factors influencing presentation and severity, compared to other forms of TB, are not well described. In recent years application of contemporary molecular biology 'omics' techniques to clinical samples, greater availability of advanced neuroradiology, emphasis on immunemediated contributions to pathology, and use of refined experimental models of TBM have better illuminated its pathogenesis. A better grasp of these processes may also lead to improved diagnostics, targeted immunotherapy as well as a biomarkerbased, patient-specific approach to personalized treatment. Diagnosis has been traditionally insensitive (AFB smear) and slow (culture). This has improved with the addition of GeneXpert MTB/Rif (Xpert) which gave sensitivities similar to culture in 2 hours (versus 2-4 weeks with culture). Subsequently, GeneXpert MTB/Rif Ultra (Ultra), a re-engineered version, has shown better sensitivities than culture in some settings. Yet, none of these technologies has adequate negative predictive value to 'rule-out' TBM. In this article we review important recently published studies that have informed our current understanding of TBM pathogenesis and diagnostics. We do not seek to present a comprehensive review of the history of TBM pathogenesis and diagnostics as a number of detailed papers that have addressed this recently ${ }^{1-3}$. Rather we provide a commentary of key studies published within the last 5 years and summarise knowledge gaps and future considerations to enable progress in the field.

\section{TBM pathogenesis}

\section{Dissemination to the central nervous system}

Understanding of the microbial and immune processes that allow $M$. tuberculosis to disseminate from the respiratory epithelium to reach the meninges remains incomplete ${ }^{2,4}$. The foundations of what is known were laid through natural history and autopsy studies in the pre-chemotherapy era. The necessary steps to develop TBM include the pathogen surviving its initial encounter with the innate immune system at the respiratory epithelium and establishment of primary infection in the lung parenchyma with characteristic granulomatous inflammation ${ }^{5-7}$. Spread beyond the lungs likely occurs through the blood and may be preceded by local invasion to the lymphatic system. Donald and Schoeman have highlighted the possibility of coincident miliary TB in cases of TBM, particularly in young children, where tubercles of different sizes and ages have been described on the meninges and confirmed by magnetic resonance imaging $(\mathrm{MRI})^{8,9}$. In children, miliary TB and TBM develop most often within 3 months of primary infection, when fresh anatomical changes are still found in the primary lung focus $^{10}$. In addition to children, people living with HIV (PLWHIV) are another vulnerable group who may be unable to control the infection in the lungs and therefore at risk of coincident miliary TB and TBM secondary to haematogenous dissemination of $M . t b^{8,11}$. The contemporaneous nature of TBM and miliary TB potentially challenges the "Rich focus" model (of a single meningeal/sub-cortical granuloma rupturing years after initial haematogenous dissemination discharging acid-fast bacilli into the sub-arachnoid space) $)^{12}$.

\section{Host immune response to TB infection in the CNS}

The host immune response to TB bacilli in the sub-arachnoid space gives rise to a granulomatous inflammation predominantly affecting the basal meninges. Inflammatory exudates may obstruct the passage of cerebrospinal fluid (CSF), leading to hydrocephalus. Small and medium-sized intracerebral arteries can become inflamed and occluded, leading to cerebral infarcts. The majority of TBM pathology is believed to result from the host inflammatory response, which has been reviewed in depth elsewhere; ${ }^{2}$ several pro- and anti-inflammatory cytokines such as tumour necrosis factor- $\alpha$ (TNF- $\alpha$ ), interferon- $\gamma$ (IFN- $\gamma$ ), interleukin (IL) $1 \beta$, IL-6, IL-8, and IL-10 are shown to be induced in $\mathrm{TBM}^{13,14}$. Disequilibrium of pro- and anti-inflammatory cytokines influence the severity and course of TBM. Current understanding of key established mechanisms known to play a role in host immune response in TBM are summarised in Figure $1^{2,15}$.

In the recent literature, the long-standing belief that excessive inflammation is the cause of death in TBM was brought into question by a recent immunopathogenesis study in Vietnam. In HIV-negative adults, associations between death and both lower CSF cytokine concentrations and lower CSF leucocyte counts (median $59 \times 10^{3}$ cells $/ \mathrm{mL}\left(\mathrm{IQR} 13-240 \times 10^{3}\right.$ cells $/ \mathrm{mL}$ ) in those who died versus $135 \times 10^{3}$ cells $/ \mathrm{mL}$ (IQR, 48-298 $\times 10^{3}$ cells $/ \mathrm{mL}$ ) in survivors) were noted ${ }^{16}$. These data support the notion that poor outcome from TBM, in the context of immunosuppressive treatment (adjunctive corticosteroids), is associated with an inadequate pretreatment inflammatory response in HIV-negative individuals. In a study of 120 Vietnamese adults with TBM included in a trial of adjunctive aspirin treatment, it was shown that there was an aspirin dose-dependent inhibition of thromboxane $\mathrm{A}_{2}$ and upregulation of pro-resolving CSF protectins, resulting in potential reduction in new infarcts and deaths by day 60 of treatment in microbiologically confirmed TBM patients ${ }^{17}$. A further study investigated concentrations of host protective lipid mediators (specialized proresolving mediators, SPMs) in CSF. Prostaglandins and cysteinyl leukotrienes were found to be reduced in more severe cases, while the lipoxygenase 5-derived 13-series resolvin (RvT)2, RvT4, and 15-epi-lipoxin B4, were significantly increased in survivors. These data suggest SPMs may play an important role in TBM pathogenesis ${ }^{18}$.

Among 608 Indonesian adults with suspected TBM, higher CSF and blood neutrophil counts (HR 1.10 (95\%CI 1.04-1.16) per $10 \%$ increase and HR 1.06 per $10^{9}$ neutrophils/L increase; 


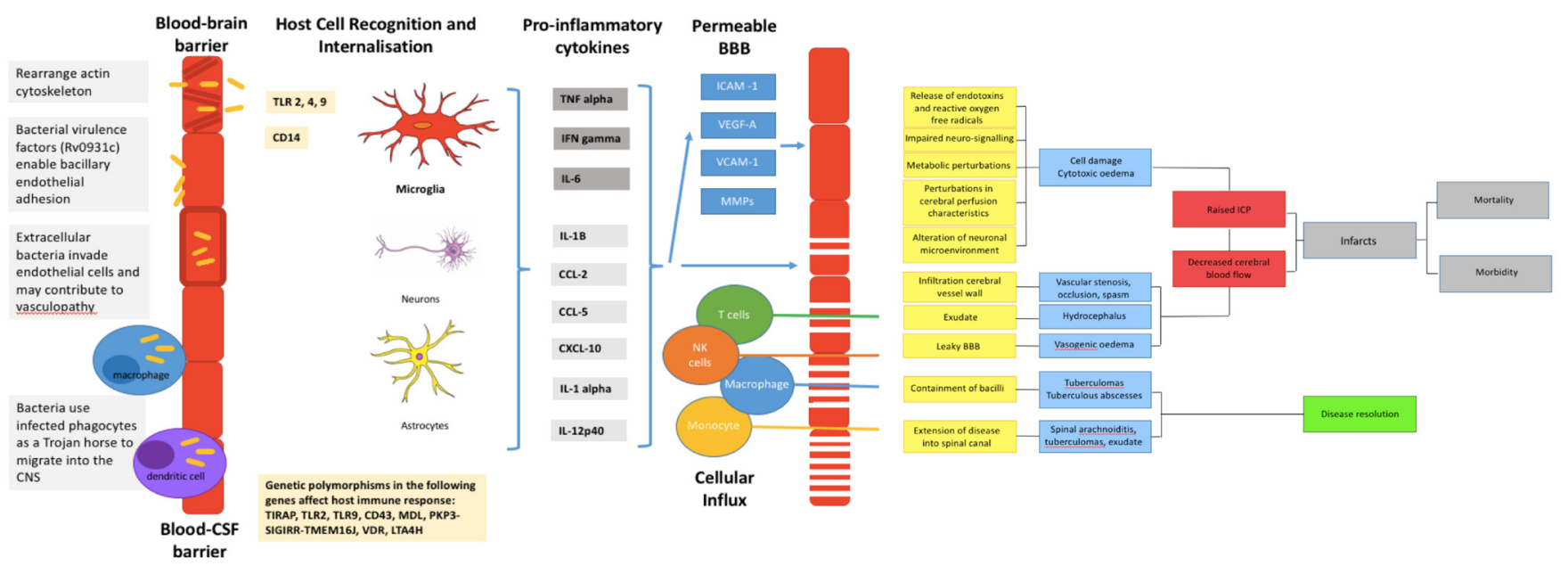

Figure 1. Illustrative summary of the pathogenesis of tuberculous meningitis (TBM). Reproduced with permission from author and Journal of Leukocyte Biology². A: Mycobacterium tuberculosis bacilli (M.tb) disseminate from the primary site of infection in the lung to seed the brain. The bacilli traverse the blood brain barrier (BBB) and blood cerebrospinal fluid barrier (BCSFB) through various virulence factors that enable the invasion of and migration through cerebral vascular endothelial cells, or are carried into the CNS by infected peripheral innate immune cells. B: In the CNS antigen recognition and internalization by microglia, neurons and astrocytes occurs, mediated by numerous host genetic factors. C: The resulting immune response stimulates the release of proinflammatory cytokines and chemokines and other immune mediators that contribute to the breakdown of the BBB and the influx of innate and adaptive immune cells from the periphery. D: A prolific inflammatory response ensues. The inflammatory exudate in the basal cisterns contributes to cerebral vascular pathology and the development of hydrocephalus and raised intracranial pressure. Vasogenic edema due to an influx of proteins through the leaky BBB, and cytotoxic edema as a result of cellular damage contribute to the raised pressure. The overall decrease in cerebral blood flow puts the brain at risk of ischemia, infarction and poor patient outcomes. In some cases the infection is controlled in discrete tuberculomas or abscesses, which may resolve with treatment and time.

(95\% CI 1.03-1.10), respectively) were associated with mortality ${ }^{19}$. Flow-cytometry on blood in a subset of 160 HIV-negative adults with TBM showed lower $\alpha \beta \mathrm{T}$ and $\gamma \delta \mathrm{T}$ cells, NK cells and MAIT cells in TBM subjects compared to 26 pulmonary TB adults $(2.4$ to 4-fold, all $\mathrm{p}<0.05)$ and 27 healthy controls (2.7-7.6-fold, $\mathrm{p}<0.001)$, but higher neutrophils and classical monocytes $(2.3$ - 3.0-fold, $\mathrm{p}<0.001)$. CSF flow cytometry of TBM patients showed a predominance of $\alpha \beta T$ and NK cells, associated with better survival, as well as the presence of MAIT cells, previously undescribed in $\mathrm{CSF}^{20}$. Indonesian HIV-negative TBM patients showed a strong myeloid blood response and a remarkably broad lymphoid CSF response including innate lymphocytes, however there was little correlation between blood and CSF compartments ${ }^{20}$.

These recent studies in Vietnamese and Indonesian adults with TBM, aimed at gaining insights into mechanisms of the inflammatory response in disease pathogenesis, used novel and highresolution methods to look at lipid mediator profiles and immune cell populations. Data indicated specific lipid mediator signatures and cell populations that are associated with disease severity before treatment and mortality; these should be considered for host-directed therapy of TBM.

\section{Host genetic and metabolic factors.}

More efficient and cost-effective genomics platforms have enabled of late better understanding of variable host responses in TBM through the study of host genetics. Polymorphisms in CD43 encoding a surface glycoprotein involved in M.tb adhesion and proinflammatory cytokine induction and PKP3-SIGIRRTMEM16J gene region encoding a negative regulator of TLR/IL-1R signalling have both been linked to survival in $\mathrm{TBM}^{21,22}$. However, the greatest interest has been around the role of leukotriene A4 hydrolase (LTA4H). LTA4H catalyzes the final step in the synthesis of leukotriene B4 (LTB4), a potent chemoattractant and pro-inflammatory eicosanoid. A common functional promoter variant rs17525495 in the LTA4H gene can predict survival and dexamethasone responsiveness in HIV-uninfected adults with $\mathrm{TBM}^{16,23}$. This human candidate gene association study was guided by findings in a zebra fish model where LTA4H was found to determine the balance of pro-inflammatory and anti-inflammatory eicosanoids in response to mycobacterial infection ${ }^{16,24}$. In a retrospective study in Vietnamese HIV-uninfected adults with TBM, while LTA4H rs 17525495 TT and CC genotypes were both associated with susceptibility to mycobacterial infection, the associations involved opposing inflammatory states: high inflammation for the TT genotype and low inflammation for the CC genotype. CT genotype had an intermediate inflammatory response and were more likely to survive TBM. Dexamethasone treatment improved survival in TT genotype patients with hyperinflammatory response but was possibly harmful to CC patients with hypo-inflammatory response ${ }^{23}$. A later prospective study in Vietnam reported that in TBM HIV-uninfected adults, LTA4H genotype influences cytokine inflammatory response and correlates with TBM severity, Figure $2^{16}$. More importantly, this study confirmed that the LTA $4 H$ genotype determined corticosteroid responsiveness and survival. 

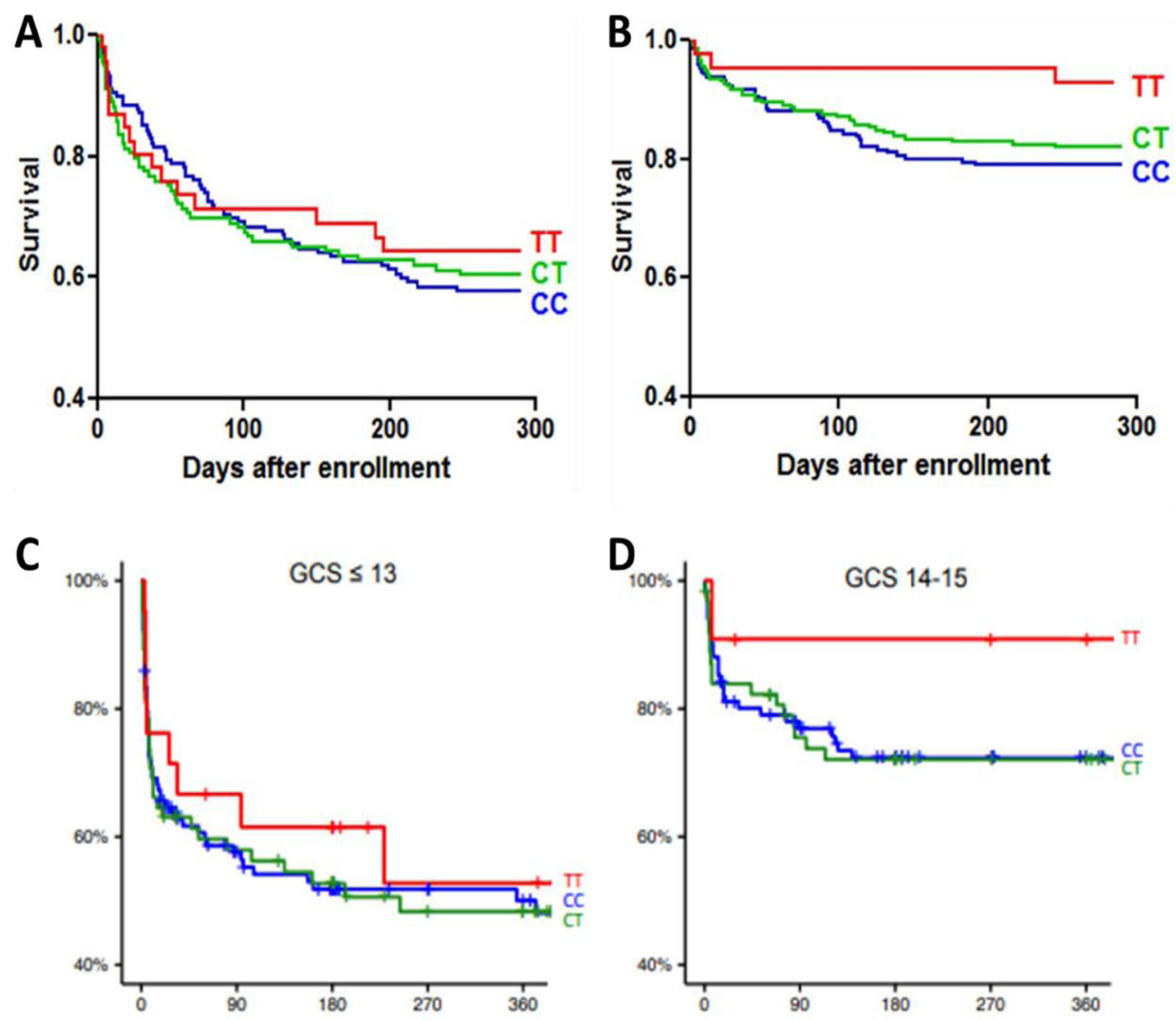

Figure 2. Kaplan-Meier survival curves stratified by LTA4H genotype. Figures $\mathbf{A}$ and $\mathbf{B}$ show survival in 763 patients with tuberculous meningitis in Vietnam ${ }^{16}$, with human immunodeficiency virus (HIV) infection (A), and without HIV infection (B). In HIV-infected patients, case-fatality rates were $34.8 \%$ (16 of 46 ) in those with TT genotype, $42.1 \%$ (61 of 145) in CT genotype, and $38.8 \%$ (52 of 134 ) in CC genotype. In HIV-uninfected patients, case-fatality rates were $7.1 \%$ (3 of 42) in TT, 21.4\% (40 of 187) in CT, and 18.7\% (39 of 209) in CC. Figures C and D show survival in 375 patients with tuberculous meningitis in Indonesia ${ }^{19}$. These patients are HIV-uninfected with severe (GCS $\left.\leq 13\right)(\mathbf{C})$ or milder (GCS 14-15) disease (D). In a recessive model, TT genotype versus CT/TT combined had HR $0.81(95 \% \mathrm{Cl} 0.41-1.62, \mathrm{p}=.550)$ in severe and $0.31(95 \% \mathrm{Cl} 0.04-2.25, \mathrm{p}=.156)$ in milder disease.

Interestingly, LTA4H genotype did not predict outcomes in Indonesian adults with TBM, but there was a trend towards improved survival with TT genotype compared to CC or CT genotype, Figure $2^{19}$. A clinical trial is currently underway in Vietnam (NCT03100786) to evaluate LTA4H genotype-directed corticosteroid therapy, an exciting example of personalised medicine in $\mathrm{TBM}^{25}$.

Although prior studies have considered sodium, glucose and lactate as related to TBM pathogenesis, recent developments in the application of targeted metabolomics have provided greater insight in the role of tryptophan, a potential key metabolite in TBM. This amino acid required for protein biosynthesis is a precursor to serotonin and melatonin (serotonin pathway) and kynurenine and quinolinic acid (kynurenine pathway). The latter is stimulated at the expense of the former by pro-inflammatory cytokine such as IL-6, TNF-alpha and IFN-gamma via indoleamine 2, 3-dioxygenase. In a recent study of serum and CSF metabolites, low levels of tryptophan were associated with survival ${ }^{26}$. One theory regarding this association could be the neuroprotective effects of the associated kynurenine pathway downstream metabolites. Either this pathway, or the 11 genetic foci related to CSF tryptophan metabolism could have novel clinical implications for $\mathrm{TBM}^{26}$.

\section{HIV co-infection and immune reconstitution inflammatory syndrome}

HIV infection is a strong independent predictor of death from TBM (hazard ratio, 3.94; 95\% confidence interval (CI), 2.79-5.56 $)^{27}$. The role of adjunctive corticosteroids in HIVassociated TBM is inconclusive (relative risk of death with adjunctive steroids, $0.78 ; 95 \% \mathrm{CI}, 0.59$ to $1.04 ; \mathrm{P}=0.08)^{28}$ and a randomized placebo-controlled trial is underway (NCT03092817) to address the use of steroids in HIV-associated TBM. Pathogenesis studies in PLWHIV are required to identify the unique pathogenic determinants of poor prognosis. Thuong et al. compared the pretreatment CSF cells and cytokine profiles of 764 HIV-positive and HIV-negative participants in Vietnamese TBM clinical trials. HIV-positive individuals had higher mean CSF neutrophil percentage $(17 \%$ vs $5 \%$; $<.0001)$ and global 
cytokine expression (aside from IL-10 which inhibits response to M. tuberculosis) than their HIV-negative counterparts. PLWHIV with CD4+ T-cell counts $<150$ cells $/ \mu \mathrm{L}$ showed higher median CSF neutrophil percentage $(25 \%)$, than those with a count $\geq 150$ cells $/ \mu \mathrm{L}$ (neutrophils $10 \% ; \mathrm{P}=0.021$ ) and patients without HIV infection (neutrophils 5\%; $\mathrm{P}<0.0001$ ). Of patients with a CD4+ T-cell count of $<150$ cells $/ \mu \mathrm{L}, 44 \%$ (105 of 238) died, compared with $13 \%$ (5 of 39 ) with a count of $\geq 150$ cells $/ \mu \mathrm{L}$ and $19 \%$ ( 83 of 439 ) without HIV infection ${ }^{16}$. These findings, amongst others, suggest a role for neutrophils in the immunopathogenesis of HIV-associated TBM.These findings, amongst others, suggest a role for neutrophils in the immunopathogenesis of HIV-associated $\mathrm{TBM}^{29}$.

Marais et al. conducted longitudinal analyses of paired blood and CSF samples in South Africans with HIV-associated TBM, describing the relationships between the development of immune reconstitution inflammatory syndrome (IRIS) and CSF leucocytes, the concentrations of $>30$ blood and CSF inflammatory mediators, and blood transcriptional profiles. They found TBM-associated CNS IRIS to have an inflammatory signature characterized by neutrophil and inflammasome-mediated proinflammatory responses ${ }^{30,31}$. The neutrophil-dependent inflammatory activation could be detected in peripheral blood before the start of TB treatment and therefore has potential to predict who will develop IRIS.

\section{Brain Injury Markers.}

The study of neurodegenerative-associated proteins to describe extent and type of brain injury post TBM has recently been explored through omics analysis, approaches which strive to understand genetic or molecular profiles of humans, particularly in paediatric TBM. In lumbar CSF of children with TBM, S100B and NSE (structural proteins of the CNS, and biomarkers of CNS tissue damage) at disease onset were associated with poor outcome, as was highest concentration overall and an increasing profile over time in S100B, NSE, and GFAP neuromarker concentrations increased over time in those who died (whilst inflammatory markers decreased), and were overall highest in those with cerebral infarction ${ }^{32}$. It is of interest that despite markers of inflammation reducing, proteins traditionally associated with neurodegenerative processes continued to rise.

In ventricular CSF of children with TBM, transcriptome analysis has revealed significant enrichment of transcripts associated with neuro-excititoxicity predominantly driven by glutamate release and NMDA binding and receptor uptake ${ }^{33}$. Upregulation of genes associated with nitric oxide, cytochrome $\mathrm{c}$, brain injury proteins like myelin basic protein, and proteins including tau, amyloidbeta and apo- lipoprotein were also seen ${ }^{33}$; many of which have also been described in in neurodegenerative conditions such as Alzhiemer's disease and traumatic brain injury ${ }^{34}$.

These findings raise the possibility of ongoing brain injury which in TBM seem to occur following ischaemic injury, despite resolving acute inflammation ${ }^{32}$. Further studies, including those which investigate the longer-term pathogenic processes in $\mathrm{TBM}$ are required to validate these results and understand further neurological sequelae including those which may indicate a post-infectious process in TBM.

\section{Neuroimaging in pathogenesis studies}

Technical advances and increasing availability of imaging modalities has recently enabled research in which imaging is used to assess pathogenic mechanisms in TBM in vivo in animal and human subjects. In a blood and CSF biomarker study of childhood TBM tuberculomas, magnetic resonance imaging has been used to note an association between tuberculomas and elevated interleukin (IL) 12p40, interferon-inducible protein 10 , and monocyte chemoattractant protein 1 concentrations, whereas infarcts were associated with elevated TNF- $\alpha$, macrophage inflammatory protein $1 \alpha$, IL-6, and IL- $8^{32}$. Specific sequences can also be used to describe morphology of structural damage and correlate this to meaningful clinical measures. For instance poorer Diffusion Tensor Imaging (DTI) parameters of white matter integrity in the anterior cingulate gyrus parahippocampal gyrus and globus pallidus are associated with worse neuropsychological performance ${ }^{35}$. A further study by the same group used Diffeomorphic Anatomical Registration Through Exponentiated Lie Algebra (DARTEL) voxel-based morphometry (VBM) to assess the integrity of grey matter in these same TBM patients ${ }^{36}$. Patients with TBM performed significantly poorer on the digit symbol, similarities, block design, matrix reasoning, and letternumber sequencing subtests of the Wechsler Adult Intelligence Scale compared to healthy adults. These changes correlated with smaller grey matter volumes in the right thalamus, right superior temporal gyrus, right precuneus, right middle temporal gyrus, left putamen, right caudate nucleus, and right middle temporal gyrus ${ }^{36}$. These studies suggest that structural damage can be cortical as well as subcortical which may in turn be related to degree of long-term impairment. This has implications for understanding long term outcomes particularly neurocognitive impairment in TBM, which in light of these findings may share features with other forms of dementia (including vascular and HIV associated neurocognitive impairment) where a subcortical pattern of neurocognitive impairment (including frontal and executive functions) can be observed.

A rabbit model study of childhood TBM, utilized ionized calcium binding adapter molecule (Iba-1) to approximate microglial activation with flurodeoxyglucose-positron emission tomography (FDG-PET) and demonstrated the presence of activated microglia and macrophages localized to $\mathrm{TB}$ lesions ${ }^{37}$. In humans, case reports and a prospective study have advocated the use of FDG-PET as a diagnostic tool, as it has been effective in detecting extra-cranial evidence supportive of a TBM diagnosis ${ }^{38-40}$. The role of FDG-PET in unravelling time course of inflammation in TBM remains to be seen, although it has played a role in understanding Alzheimer's, a disease in which, similar to TBM, inflammation plays a key pathogenic role ${ }^{41,42}$.

\section{Pathogen factors: bacillary load, pathogen strain and virulence factors}

TBM patients generally have low bacterial loads in CSF which causes difficulties in both diagnosis and ability to study bacterial load evolution-related pathophysiology. The time-to-positivity 
of a culture and cycle threshold $(\mathrm{Ct})$ of nucleic acid amplification tests such as GeneXpert MTB/Rif (Xpert) can provide an indication of likely bacterial burden ${ }^{43}$. Over $50 \%$ of diagnosed cases are microbiologically undetectable and defined as 'probable' or 'possible' TBM which obviously limits this approach ${ }^{44}$. Marais et al. showed that in patients where M.tb was cultured from CSF taken before and after two weeks of anti-tuberculosis treatment, there was a 9.3-fold increased risk of subsequently developing TBM-IRIS, although the sample size is small with 15 TBM-IRIS patients compared with 6 non-TBM-IRIS patients $^{45}$. Thuong et al. found that among 692 Vietnamese adults with TBM, pre-treatment CSF M.tb load (by Xpert Ct) was correlated with increased CSF neutrophil counts, increased cytokine production, and new neurological events after treatment initiation, but not death ${ }^{43}$.

In addition, epidemiological trends of M.tb lineage from TBM $(n=73)$ and pulmonary TB $(n=220)$ patients in Thailand showed that the Indo-Oceanic lineage is more frequently found in TBM patients $(41 \% \text { versus } 13 \% \text { in PTB })^{46}$. This association did not hold true in Indonesia, though specific genetic variations were identified which were associated with TB phenotype, including one (Rv0218) whose encoded protein may play a role in hostpathogen interaction ${ }^{47}$.

\section{Host-pathogen interactions}

It is estimated that the global burden of latent TB infection (LTBI) is approximately $23.0 \%$ (95\% CI $20.4 \%-26.4 \%$ ), amounting to approximately 1.7 billion people ${ }^{48}$. Innate immune responses are critical to control TB infection yet also contribute to tissue damage. This delicate balance is illustrated in the damage response framework which provides a theory of microbial pathogenesis that incorporates the contributions of both host and microbe to host damage that stems from host-microbe interaction ${ }^{49,50}$. This framework likely applies to TBM based on evidence of both failed immunity and excessive inflammation being linked to increased TBM pathology, see Figure $3^{23,51,52}$. Both the microbe and the host contribute to host damage and where an individual patient's immune response lies on the continuum of the damage response framework parabola determines the nature of the disease process ${ }^{16,43,53}$. Evidence from recent studies shows LTA4H genotype, CSF cytokines and CSF immune cells such as neutrophils are determinants of inflammatory state, which impacts both bacterial growth and host damage and thus leads to different outcomes. The current one-size-fits-all approach to TBM treatment fails to recognize divergent pathologies and may explain the poor outcomes in certain populations. Being able to identify where on the parabola an individual lies and tailoring therapy to achieve the optimal milieu is an approach that
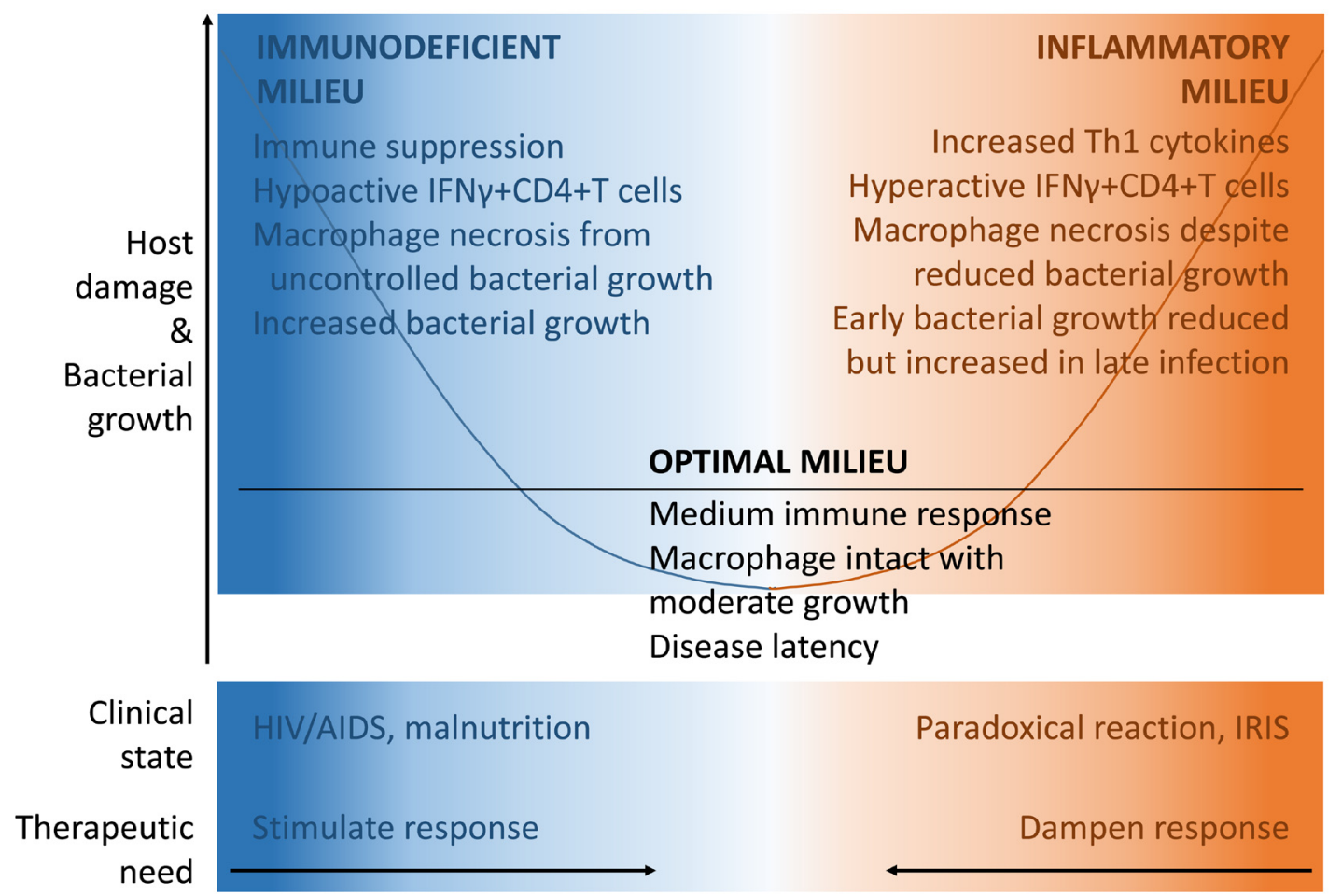

Figure 3. Outcomes of the host $-M$. tuberculosis interaction depicted by the basic parabola of the damage-response framework. On the left side of the parabola, shaded in blue, the immune system fails to limit mycobacterial growth and invasion which results in host damage. On the right side, shaded in red, the immune response is excessive and the resultant inflammation and host-damage. The proportion of the parabola lying below the black line represents disease latency, which is not associated with clinically evident host damage. On the blue side therapeutic interventions could be targeted at stimulating an immune response, whilst on the red side therapeutic interventions could aim to dampen immune response. 
warrants further investigation. LTA4H genotype is an example of using host genotype to predict inflammatory response and to tailor treatment by host directed therapy. Omics technology are now being used to identify additional host genetic markers and treatment targets in TBM.

\section{TBM diagnostics}

\section{Host-based diagnostic biomarkers}

Traditional diagnostic techniques for TBM include CSF smear microscopy for acid fast bacilli (rapid and cheap but insensitive in most settings, 10-15\%) and CSF culture (improved sensitivity of $50-60 \%$ but results in 2-6 weeks with a biosafety lab level three requirement) ${ }^{3}$. Given the limitations of traditional, diagnostic tests for TBM that focus on bacillary detection, there is interest in the utilization of host-based diagnostic biomarkers for diagnosis of TBM, Figure 4. Adenosine deaminase (ADA), produced by lymphocytes, is an important regulator of follicular helper T-cells. ADA is commonly used for diagnosis of TB from other, typically extra-pulmonary locations and numerous studies have considered ADA for diagnosis of $\mathrm{TBM}^{54}$. One 2017 meta-analysis found ADA to have a pooled sensitivity of $89 \%$ (95\% CI $84-92 \%$ ) with pooled specificity $91 \%$ $(95 \% \text { CI, } 87-93 \%)^{54}$. Yet ADA use for TBM diagnosis has been limited by the high cost of the test, required sophisticated lab infrastructure, study heterogeneity, inadequate negative predictive values, and variable test performance.
A number of studies have considered unstimulated CSF interferon gamma (IFN- $\gamma$ ) levels as a diagnostic test, in general, a high number of false positive results has limited the utility of CSF IFN- $\gamma^{55,56}$. For instance, in one study of 39 controls ( $\mathrm{n}=12$ viral, $\mathrm{n}=16$ purulent, $\mathrm{n}=11$ cryptococcal meningitis) and 30 subjects with TBM while median IFN- $\gamma$ levels where higher amongst subjects with TBM, diagnostic accuracy was inadequate $^{56}$. At the strongest cut-point $(81 \mathrm{pg} / \mathrm{mL})$ determined by receiver operator curve analysis, positive predictive value was only $81 \%$ with positive results occurring in $2 / 12$ (17\%) with viral meningitis, 3/16 (19\%) with purulent meningitis, and $1 / 11(9 \%)$ with cryptococcal meningitis ${ }^{56}$.

Interferon gamma release assays (IGRAs) are commonly used to infer LTBI. A 2016 meta-analysis of six studies performing CSF IGRA's found a pooled sensitivity and specificity of $77 \%$ (95\% CI 69\%-84\%) and 88\% (95\% CI 74\%-95\%), respectively, for TB meningitis, though reference standards varied by study $^{57}$. Limitations of IGRA include high cost, the need for advanced lab infrastructure, frequent "indeterminate" results, and false positives associated with other causes of meningitis. Additional host biomarkers including delta-like ligand 1, vitamin D binding protein, and fetuin have been evaluated in CSF though none were found to have satisfactory performance ${ }^{58}$. Numerous CSF antibodies to M.tb in CSF has also been evaluated. Huang and colleagues found pooled sensitivities of $91 \%$

\section{Concerns limiting use in TBM diagnosis}

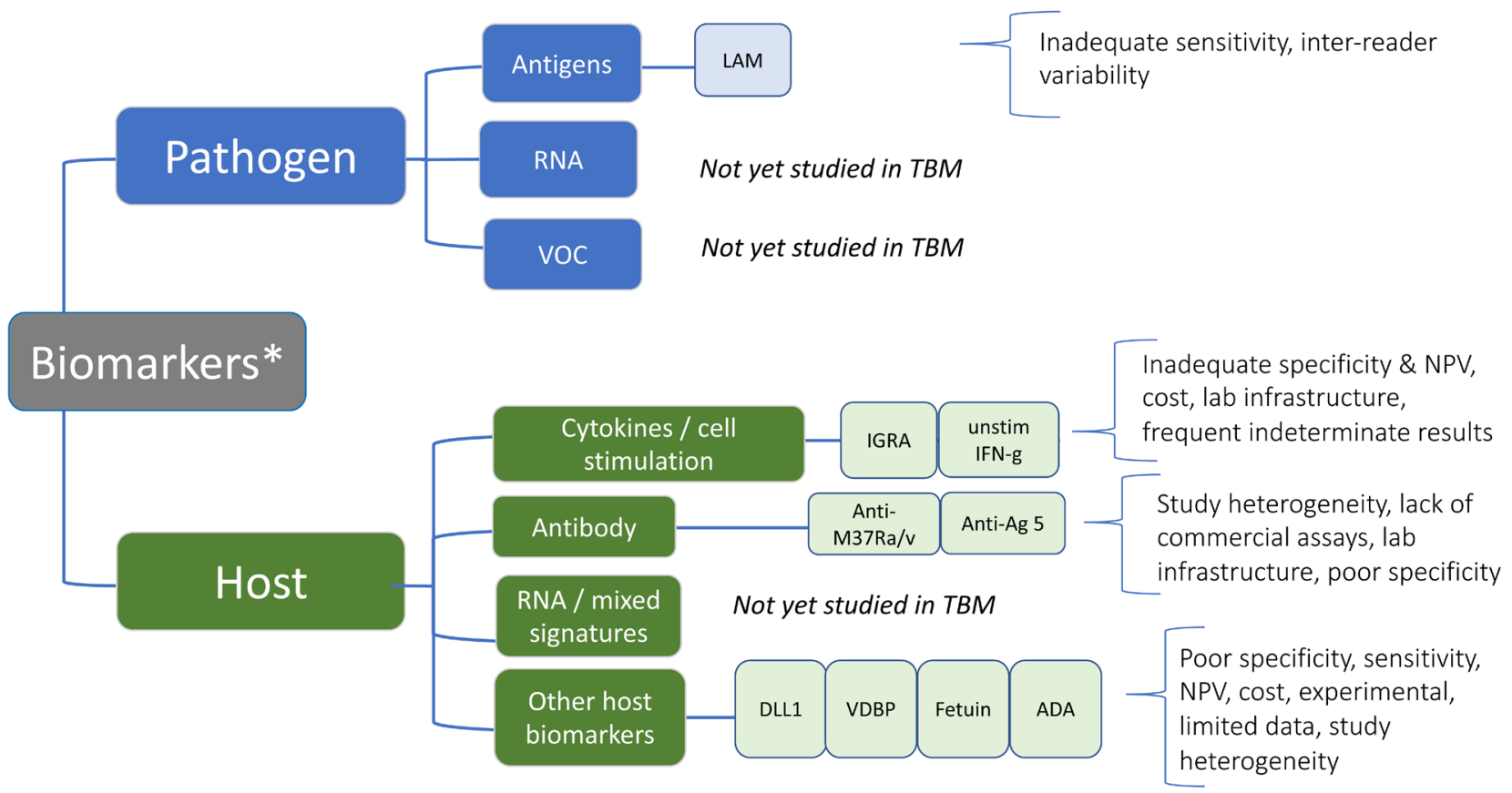

Figure 4. Novel Host and Pathogen biomarkers for diagnosis of tuberculous meningitis ${ }^{54-60}$. LAM $=$ lipoarabinomannan, RNA $=$ riboneucleic acid, VOC = volatile organic chemicals, IGRA = inferferon gamma resease assay, DLL1 = delta-like ligand 1 , VDBP $=$ vitamin d binding protein, ADA = adenosine deaminase. ${ }^{*}$ This image focuses only on novel host or TB biomarkers for diagnosis of TBM and does not incorporate traditional tools such as culture or AFB smear, or newer nucleic acid amplification tests in use commonly such as GeneXpert or GeneXpert Ultra, or experimental techniques such as metagenomic next generation sequencing. 
(95\% CI 71-98\%) for anti-M37Ra across five studies, $84 \%$ (95\% CI 71-92\%) for anti-antigen-5 across eight studies, and $84 \%$ (95\% CI 71-92\%) across 12 studies for anti-M37Rv, again using a variety of reference standards (making the pooled estimates somewhat flawed $)^{59}$. Use of blood antibody assays are discouraged for the diagnosis of TB, and their utility in CSF is limited by heterogeneity and the lack of a uniform reference standard across research studies as well as a lack of commercial assays.

Though on the surface, many of these markers look to have promise, uptake has been limited. Heterogeneity in study design and widely variable study performance has limited the consensus regarding the utilities for most host-based tests. Further, many of these tests require sophisticated laboratory infrastructure, are costly, and in some cases are not commercially available. None of these tests are routinely used and as far as the authors are aware, none are actively being studied further.

\section{Biomarkers in children}

The often-dismal outcome of TBM is contributed to by delayed diagnosis and/or initiation of treatment, especially in high burden settings ${ }^{4}$. Currently available diagnostic tests performance is especially poor in young children with TBM. Thus, diagnosis of childhood TBM is mostly based on a combination of clinical findings, CSF analysis and radiological findings ${ }^{61 .}$ Even so, there are often multiple missed opportunities prior to a diagnosis of childhood $\mathrm{TBM}^{62}$. Since it can be challenging to identify bacilli in paediatric extrapulmonary $\mathrm{TB}$, the use of host or pathogen biomarkers to aid diagnosis is being explored. Host biomarker-based tests have shown promise in extrapulmonary TB outside of the CNS and therefore have potential applications in $\mathrm{TBM}^{63}$. Recent technological advances have made it possible to screen for many biomarkers in as little as $3 \mu \mathrm{l}$ of sample using the Luminex multiplex cytokine beaded arrays, albeit in research context currently, rather than routine clinical practice.

A three-marker CSF biosignature comprising IL-13, VEGF and cathelicidin LL-37, diagnosed childhood TBM with a sensitivity of $52 \%$, specificity of $95 \%$, with positive and negative predictive values of $91 \%$ and $66 \%$ respectively. Cut-off values for VEGF, IL-13 and cathelicidin LL-37 were $42.92 \mathrm{pg} / \mathrm{mL}, 37.26 \mathrm{pg} / \mathrm{mL}$ and $3221.01 \mathrm{pg} / \mathrm{mL}$ respectively ${ }^{15}$. Further evaluation of this three-marker CSF biosignature in a different cohort revealed positive and negative predictive values of $90 \%$ and $59.5 \%$ respectively, however with different cut-off values for VEGF, IL-13 and cathelicidin LL-37 of $9.4 \mathrm{pg} / \mathrm{ml}$, $524.9 \mathrm{pg} / \mathrm{ml}$ and optical density of 0.045 respectively ${ }^{64}$. In a study investigating potentially useful host biomarkers in CSF for childhood TBM (23 children with TBM and 24 controls), 28 proteins including IFN- $\gamma$, TNF- $\alpha$, MPO, MMP-8, MMP-9, MIP-4 and CXCL9 amongst others, when analysed individually, showed areas under the receiver-operating curve (AUC) $\geq 0.80$. When combined, biomarkers IFN- $\gamma$, MPO and VEGF showed good accuracy (AUC $=0.97$, up to $91.3 \%$ sensitivity and up to $100 \%$ specificity), as well as ICAM-1, MPO, CXCL8, and IFN- $\gamma$ (AUC of 0.97 , up to $87.0 \%$ sensitivity and up to $95.8 \%$ specificity). Cut-off values for VEGF, IFN- $\gamma$,
MPO, ICAM-1 and CXCL8 were $>9.4 \mathrm{pg} / \mathrm{ml},>99.5 \mathrm{pg} /$ $\mathrm{ml},>25823.0 \mathrm{pg} / \mathrm{ml},>1372.0 \mathrm{pg} / \mathrm{ml}$ and $>394.8 \mathrm{pg} / \mathrm{ml}$, respectively ${ }^{64}$.

Despite the potential of CSF-based biosignatures, collection of CSF is invasive, and blood or urine-based inflammatory biosignatures require exploration. In a study evaluating serum biomarkers, the combination of CRP, IFN- $\gamma$, IP-10, CFH, Apo-A1 and SAA showed moderate diagnostic accuracy for clinically-defined TBM, including both 'definite' and 'probable' TBM (AUC of 0.75 , sensitivity of $69.6 \%$ and specificity of $62.5 \%$ ). A three-biomarker combination of adipsin, $\mathrm{A} \beta 42$ and IL-10 showed improved accuracy (AUC of 0.84 , sensitivity of $82.6 \%$ and specificity of $75.0 \%$ ). Cut-off values for CRP, IFN- $\gamma$, IP-10, CFH, Apo-A1, SAA, adipsin, A 342 and IL-10 were $>80721.0 \mathrm{ng} / \mathrm{ml},<61.5 \mathrm{pg} / \mathrm{ml},<57.2 \mathrm{pg} / \mathrm{ml},>350185.0 \mathrm{ng} / \mathrm{ml}$, $>287512.0 \mathrm{ng} / \mathrm{ml},>59894.0 \mathrm{ng} / \mathrm{ml},<2393.0 \mathrm{ng} / \mathrm{ml},<278.4 \mathrm{pg} / \mathrm{ml}$ and $<7.0 \mathrm{pg} / \mathrm{ml}$, respectively. Although sample size was small, these biomarkers warrant further exploration ${ }^{65}$.

\section{Pathogen-based diagnostics}

The absence of a perfect gold standard for use in TBM diagnostic studies means that the results must be interpreted with an awareness of the pros and cons of the reference standard used. The 2010 uniform TBM case definition which defines cases as 'definite', 'probable', 'possible' or 'not TBM' is the most standardised tool to use when defining a case definition ${ }^{61}$. This case definition was derived by expert consensus rather than being data-driven and, although designed to be applicable to any age, HIV infection status or geographical setting, may perform better in some contexts than others. In HIV-negative populations a reference standard of 'definite, probable or possible' is often used, however in PLWHIV including 'possible' in the reference standard can be imprecise due to the wide variety of infectious and non-infectious aetiologies that can fall into this category. It must also be noted that the potential to score points is affected by the ability to comprehensively investigate patients with brain, chest and abdominal imaging as well as microbiological sampling from outside the CNS; in resource constrained settings the ability to score a high number of points may therefore be compromised. We do strongly advocate the use of the case definition to standardise results, allow for greater comparison between studies and meta-analysis of data; use of other standards must be interpreted with a degree of caution.

\section{Nucleic-acid amplification tests.}

To address the limitations of conventional microscopy and culture techniques, NAATs have emerged as important tools for rapid and accurate diagnosis of $\mathrm{TBM}^{44}$. A recent meta-analysis evaluating NAATs in TBM reported heterogeneity in results with a pooled sensitivity of $82 \%$ against culture and $68 \%$ against a clinical reference standard ${ }^{66}$. This variability, especially in in-house NAATs, is subject to difference in volume of sample, method of extraction, choice of targets used, presence of inhibitors in the sample and lack of optimal reference standard. Traditional NAATs require expensive equipment, stringent operational conditions and technical expertise limiting their use in routine clinical practice in lower-resource, high endemic settings. 
To circumvent these challenges, loop mediated isothermal amplification (LAMP) assays were developed and can be conveniently carried out under isothermal conditions in an ordinary laboratory water bath or heating block within one hour. Though LAMP has outperformed PCR in an Indian study on $\mathrm{TBM}^{67}$, the assay is still in its infancy and needs further validation. Another method to potentially reduce the overall cost of NAAT would be to utilize magnetic bead assay technology, thus obviating the need of gel electrophoresis system or expensive dyes.

Xpert is a rapid (90 min run-time) fully-automated cartridgebased real-time PCR assay that detects the presence of M.tb complex DNA, as well as $r p o B$ gene mutations responsible for rifampicin resistance. The pooled sensitivity and specificity of Xpert against culture in 33 studies on TBM, was $71.1 \%$ and $98 \%$, respectively ${ }^{68}$. Xpert has been shown to significantly increase microbiological confirmation of TBM in Uganda over a 6.5-year period but its impact on clinical outcomes in unknown ${ }^{69}$. Individual studies have also found inferior performance for Xpert compared to multiplex $\mathrm{PCR}^{70}$ or Amplicor assay ${ }^{71}$ in diagnosing TBM although these results have not been confirmed. The next generation, GeneXpert MTB/Rif Ultra (Ultra) has an 8-fold lower limit of detection than Xpert $(16 \mathrm{CFU} / \mathrm{ml}$ versus $113 \mathrm{CFU} / \mathrm{ml}$ ) attributable to a larger chamber allowing double the volume of sample to reach the PCR reaction and two additional DNA probes (IS1081 and IS6110) ${ }^{72}$. In a Ugandan study of Ultra using cryopreserved CSF, Ultra demonstrated a sensitivity of $95 \%$ against a composite microbiological reference and $70 \%$ against probable/definite $\mathrm{TBM}$ in comparison to $45 \%$ and $43 \%$, respectively for each Xpert and culture ${ }^{73,74}$. In 2017, Ultra was endorsed by the WHO as the best initial test for TBM and is being rolled out currently worldwide, superseding Xpert ${ }^{75}$.

In January 2020, two larger prospective studies evaluating Ultra were published. In the Ugandan study, 204 (96\% HIVpositive) adults with suspected meningitis had CSF Xpert Ultra performed. Compared with a reference of definite/probable TBM, test sensitivities were $77 \%$ (95\% CI $63-87 \%$ ) for Ultra, $56 \%(95 \%$ CI $44-70 \%$ ) for Xpert, and $61 \%$ (95\% CI $45-76 \%$ ) for mycobacterial culture ${ }^{76}$. In this study 'possible TBM' cases were not included in the reference standard as this category is non-specific in HIV co-infection due to concomitant infectious and non-infectious brain pathologies associated with advanced immunosuppression. In the second study, Donovan et al employed a different study design and randomised 205 Vietnamese adults (15\% HIV co-infected) with meningitis to either Ultra or Xpert testing. Against a reference standard of definite, probable, or possible TBM, test sensitivities were $47 \%$ (95\%CI, $34-60 \%$ ) for Ultra, 40\% (95\%CI, $28-53 \%$ ) for Xpert, and $48 \%(95 \%$ CI, 38 - 58\%) for mycobacterial culture $^{77}$. Specificity of Ultra for TBM diagnosis was high in both studies. The sensitivity of Ultra statistically superior to that of Xpert in Uganda but not in a Vietnamese predominantly HIV-negative population. How can we rationalise and interpret these differing results? Firstly, diagnostic tests cannot be expected to perform identically in all settings. Differences in tested CSF volume, CSF processing, HIV co-infection, genetics influencing host response to M.tb, and M.tb lineages (the number of copies of IS1081 and IS6110 genes varies by lineage) could all contribute to these different results, as could the differences in study design (e.g. head-to-head comparison versus randomizing samples) and smear microscopy sensitivity and reference standards used. Secondly, and most importantly, regardless of the differences in the exact performance of Ultra, the key point is that while Ultra demonstrates some improvement on the performance of Xpert, its negative predictive value is not sufficiently high to exclude TBM when the result is negative.

Another commercial NAAT, the MTBDRplus assay, has been evaluated only in few cases of TBM and needs further validation $^{78}$. Accurate and rapid detection of drug resistance is another challenge, rifampicin resistance detection by Xpert has imperfect sensitivity (93\%) and where detected and ideally requires confirmation by sequencing or culture $\mathrm{e}^{70,79}$. Ultra uses melt curve analysis to improve detection of rifampicin resistance but both are about $95 \%$ sensitive ${ }^{80,81}$. Ultra will not be able to adequately define rifampin resistance in samples with a low quantity of bacilli (trace category positive) ${ }^{82}$. In summary NAATs, are a major diagnostic advance but they cannot yet fully replace culture methods. Ultra is too insensitive to rule out TBM, and like Xpert, should be considered as the first test and not the last in TBM diagnosis ${ }^{83}$. Ultra is an important step in the right direction but the result should be considered in the context of the clinical probability of $\mathrm{TBM}^{84}$.

CRISPR-MTB and metagenomic next generation sequencing. Clustered regularly interspaced palindromic repeat (CRISPR) associated proteins (Cas) have the ability to cleave DNA at specific sites and are being used widely in gene-editing and more recently in infectious disease diagnostics. When combined with DNA amplification, the CRISPR system can detect nucleic acid molecules at extremely low abundance. There is one recent report of utilizing the CRISPR system for detection of M.tb (CRISPR-MTB). The study included $26 \mathrm{CSF}$ specimens and found CRISPR-MTB to have a sensitivity of $73 \%$ compared to $54 \%$ for Xpert and $23 \%$ for culture against a reference standard of 'clinical TBM'. The specificity of the test was $98 \%$ when tested against 63 non-TB cases. CRISPR-MTB is isothermal and can be performed in under 2 hours using only $500 \mu \mathrm{l}$ of CSF. CRISPR-MTB remains to be tested against Ultra and requires a higher level of laboratory expertise, resources, and time than the Xpert platform but may be an advance in TB diagnostics if these findings can be confirmed in other settings with more standardized reference stanrdards ${ }^{85}$.

Metagenomic next generation sequencing (mNGS) is a rapidly developing technology that has proved useful in determining aetiologies for CNS infections that have evaded detection by conventional techniques. Further, mNGS, as opposed to organismspecific molecular tests has the ability to detect any low abundance infection with a single test ${ }^{86}$. A recent small study applied mNGS to stored CSF samples from 23 TBM cases and found a sensitivity of $67 \%(8 / 12)$ against a reference standard of definite TBM, higher than AFB stain (33\%, 4/12), PCR (25\%, 
$3 / 12)$ and culture $(8 \%, 1 / 12)^{87}$. Paucibacillary conditions such as TBM where the bacillary load may fall below the LOD of commercial NAATs, or where mutations exist around specific PCR primer binding sites may find particular use for mNGS. Targeted enrichment of low abundance genes with Finding Low Abundance Sequences by Hybridization (FLASH), a novel CRISPR-Cas9 technology can increase DNA read abundance by up to $10^{5}$-fold before sequencing occurs ${ }^{88}$. Combining FLASH and mNGS technologies could improve detection of TB DNA and associated antimicrobial resistance mutations mutations ${ }^{89}$. A first pilot of FLASH technology in TB demonstrated up to a 100-fold increase in TB read abundance, detection of $6 / 6$ cases of TBM positive with Ultra and detection of an additional case of TBM that had been missed by Xpert, Ultra and MGIT culture $^{90}$. Here again, large studies need to be performed to better understand this technology's performance and the cost, laboratory infrastructure, and degree of expertise will need to be improved upon to permit widespread usage.

Pathogen-based biomarkers. A urine lateral flow assay (LFA) that detects M.tb lipoarabinomannan (TB-LAM), a $17 \mathrm{kDa}$ glycolipid found in the outer cell wall of MTB, has recently been recommended by the World Health Organization for the diagnosis of $\mathrm{HIV}$-associated $\mathrm{TB}$ in HIV-positive inpatients (Alere Determine TB-LAM, Abbott, Chicago, USA). The unique characteristic of the test is that its sensitivity increases as CD4 T-cell count falls, with a sensitivity of $56 \%$ in those with CD4 $<100$ cells $/ \mathrm{ml}^{60}$. Yet, in CSF, despite some initial optimism related to an autopsy-based study in Uganda, the Alere TB-LAM has shown poor sensitivity on lumbar CSF in Uganda ${ }^{91,92}$, along with a larger Zambian study which examined culture positive TBM in Zambia (TB-LAM sensitivity $22 \%(23 / 105))^{91,93}$. The Alere TB-LAM is also limited by is susceptibility to individual reader interpretation of the darkness of the test line compared to the reference card (Figure 4). A novel LAM assay (Fujifilm SILVAMP TB-LAM, Fujifilm, Japan) is able to detect concentration of LAM at approximately 30-fold lower than Alere TB-LAM due to design differences, including a silver amplification step and gives a result in one hour ${ }^{94}$. The Fujifilm LAM was recently tested on 968 urine samples (600 with definite pulmonary TB) in South Africa and showed a sensitivity of $70 \%$ (95\% CI 53 - 83\%) compared to $42 \%$ (95\% CI $32-52 \%$ ) for the Alere TB-LAM against a microbiological reference standard ${ }^{94}$. There is no data on this assay for diagnosis of TBM published to date.

\section{Clinical prediction rules.}

Work is underway to develop a more accurate multivariable clinical prediction rule derived from large international cohorts using individual patient data ${ }^{95}$. The hope is that a data-driven scoring system will be developed for use in a range of clinical settings by using common, readily available clinical or laboratory parameters to aide in clinical decision making.

\section{Discussion}

In the last five years, the pathogenesis of TBM has been better elucidated, in part thanks to detailed immunological studies on clinical samples preemptively stored during clinical trials. These advances highlight the importance of collecting and storing samples appropriately for future research to maximize scientific outputs, as highlighted in the paper on sampling strategies in this collection. HIV infection is a major predictor of mortality in TBM and advanced HIV infection (CD4 T cell count $<150$ cell/ $\mu \mathrm{l})$ appears to drive a dysregulated, hyperinflammatory phenotype with very poor outcomes. In HIV-endemic sub-Saharan African settings around $90 \%$ of all adult TBM occurs amongst HIV-positive individuals ${ }^{69,96}$, often with either untreated advanced HIV or having recently initiated ART - both driving a hyperinflammatory response. In Vietnamese adults with TBM, LTA4H genotype is a strong predictor of mortality though this finding was not duplicated in Indonesia.

Recent insights have shown that neutrophils play a significant role in the immunopathogenesis of TBM, and that both a paucity and an excess of inflammation can be equally damaging in TBM. It has become increasingly clear that a 'one-size-fits-all' approach is too simplistic in TBM treatment, as in other infections such as pulmonary TB and sepsis ${ }^{97,98}$. The damage-response framework may provide a useful structure for understanding hostpathogen interactions in TBM, illustrating how immune response could be exploited for therapeutic purposes. Additional antiinflammatory therapy with aspirin ${ }^{17,99}$ or more targeted immunotherapy could have a role in persons with an excessive inflammatory response; whilst individuals with an inadequate response might do better without corticosteroid treatment or might even benefit from immunomodulating therapy to boost their immune response ${ }^{100}$. Future trials of novel specific hostdirected therapies are needed and must include immune markers to allow for post-hoc identification of subgroups benefitting from the initiated therapy. Because of the lack of correlation between blood and CSF compartments we advocate inclusion of both blood and CSF markers when studying adjuvant therapies.

The field of TBM diagnosis is rapidly evolving with GeneXpert MTB/Rif Ultra being the most promising test to date for diagnosis of TBM. Ultra is rapid and has potential to confirm more cases of TBM at lower bacillary loads, though whether this will improve outcomes remains to be determined. Most importantly, Ultra does not appear to have adequate predictive value to 'rule-out' TBM and so it cannot meet the potential of an ideal TBM diagnostic test to avoid long, toxic TBM therapy in persons without TBM. Novel sequencing technologies hold potential to provide increased understanding of pathogen genomics and behavior and further illuminate host response, which may in turn lead to novel diagnostic and therapeutic targets. Sequencing technologies are increasingly available in TB endemic settings but will need further improvements in affordability and speed in addition to more data on accuracy to unlock their potential as diagnostic tools for TBM. It is now a realistic hope that a test (or set of tests) will one day be available that will be able to confirm or rule out TBM, provide M.tb resistance information, and direct clinicians to targeted, adjunctive hostdirected therapy within hours.

\section{Acknowledgements}

Tuberculosis Meningitis International Research Consortium

Rob E. Aarnoutse; Suzanne T. B. Anderson; Nathan C. Bahr; Nguyen D. Bang; David R. Boulware; Tom Boyles; Lindsey H. M. te Brake; Satish Chandra; Felicia C. Chow; Fiona V. Cresswell; 
Reinout van Crevel; Angharad G. Davis; Sofiati Dian; Joseph Donovan; Kelly E. Dooley; Anthony Figaji; A. Rizal Ganiem; Ravindra Kumar Garg; Diana M. Gibb; Raph L. Hamers; Nguyen T. T. Hiep; Darma Imran; Akhmad Imron; Sanjay K. Jain; Sunil K. Jain; Byramee Jeejeebhoy; Jayantee Kalita; Rashmi Kumar; Vinod Kumar; Arjan van Laarhoven; Rachel P-J. Lai; Abi Manesh; Suzaan Marais; Vidya Mave; Graeme Meintjes; David B. Meya;
Usha K. Misra; Manish Modi; Alvaro A. Ordonez; Nguyen H. Phu; Sunil Pradhan; Kameshwar Prasad; Alize M. Proust; Lalita Ramakrishnan; Ursula Rohlwink; Rovina Ruslami; Johannes F. Schoeman; James A. Seddon; Kusum Sharma; Omar Siddiqi; Regan S. Solomons; Nguyen T. T. Thuong; Guy E. Thwaites; Ronald van Toorn; Elizabeth W. Tucker; Sean A. Wasserman; Robert J. Wilkinson.
1. Wilkinson RJ, Rohlwink U, Misra UK, et al:: Tuberculous meningitis. Nat Rev Neurol. 2017; 13(10): 581-598. PubMed Abstract | Publisher Full Text

2. Davis AG, Rohlwink UK, Proust A, et al:: The pathogenesis of tuberculous meningitis. J Leukoc Biol. 2019; 105(2): 267-80.

PubMed Abstract | Publisher Full Text | Free Full Text

3. Bahr NC, Meintjes G, Boulware DR: Inadequate diagnostics: the case to move beyond the bacilli for detection of meningitis due to Mycobacterium tuberculosis. J Med Microbiol. 2019; 68(5): 755-60.

PubMed Abstract | Publisher Full Text

4. Chiang SS, Khan FA, Milstein MB, et al:: Treatment outcomes of childhood tuberculous meningitis: a systematic review and meta-analysis. Lancet Infect Dis. 2014; 14(10): 947-57.

PubMed Abstract | Publisher Full Text

5. Gupta N, Kumar R, Agrawal B: New Players in Immunity to Tuberculosis: The Host Microbiome, Lung Epithelium, and Innate Immune Cells. Front Immunol. 2018; 9: 709 .

PubMed Abstract | Publisher Full Text | Free Full Text

6. Turner RD, Chiu C, Churchyard GJ, et al.: Tuberculosis Infectiousness and Host Susceptibility. J Infect Dis. 2017; 216(suppl_6): S636-S43. PubMed Abstract | Publisher Full Text | Free Full Text

7. Torrelles JB, Schlesinger LS: Integrating Lung Physiology, Immunology, and Tuberculosis. Trends Microbiol. 2017; 25(8): 688-97. PubMed Abstract | Publisher Full Text | Free Full Text

8. Donald PR, Schaaf HS, Schoeman JF: Tuberculous meningitis and miliary tuberculosis: the Rich focus revisited. J Infect. 2005; 50(3): 193-5. PubMed Abstract | Publisher Full Text

9. Dekker G, Andronikou S, van Toorn R, et al:: MRI findings in children with tuberculous meningitis: a comparison of HIV-infected and non-infected patients. Childs Nerv Syst. 2011; 27(11): 1943-9. PubMed Abstract | Publisher Full Text

10. Wallgren A: Some aspects of tuberculous meningitis and the possibility of its prevention. J Pediatric. 1934; 5(3): 291-8.

Publisher Full Text

11. Marais S, Pepper DJ, Marais BJ, et al:: HIV-associated tuberculous meningitisdiagnostic and therapeutic challenges. Tuberculosis. 2010; 90(6): 367-74. PubMed Abstract | Publisher Full Text

12. Rich AR, HA M: The pathogenesis of tuberculous meningitis. Bull Johns Hopkins Hosp. 1933; 52: 413-29.

13. Simmons CP, Thwaites GE, Quyen NT, et al.: Pretreatment intracerebral and peripheral blood immune responses in Vietnamese adults with tuberculous meningitis: diagnostic value and relationship to disease severity and outcome. J Immunol. 2006; 176(3): 2007-14. PubMed Abstract | Publisher Full Text

14. Donald PR, Schoeman JF, Beyers N, et al:: Concentrations of interferon gamma, tumor necrosis factor alpha, and interleukin-1 beta in the cerebrospinal fluid of children treated for tuberculous meningitis. Clin Infect Dis. 1995; 21(4): 924-9.

PubMed Abstract | Publisher Full Text

15. Visser $\mathrm{DH}$, Solomons RS, Ronacher $\mathrm{K}$, et al:: Host immune response to tuberculous meningitis. Clin Infect Dis. 2015; 60(2): 177-87. PubMed Abstract | Publisher Full Text

16. Thuong NTT, Heemskerk D, Tram TTB, et al:: Leukotriene A4 Hydrolase Genotype and HIV Infection Influence Intracerebral Inflammation and Survival From Tuberculous Meningitis. J Infect Dis. 2017; 215(7): 1020-8. PubMed Abstract | Publisher Full Text | Free Full Text

17. Mai NT, Dobbs N, Phu NH, et al:: A randomised double blind placebo controlled phase 2 trial of adjunctive aspirin for tuberculous meningitis in HIV-uninfected adults. eLife. 2018; 7: pii: e33478. PubMed Abstract | Publisher Full Text | Free Full Text

18. Colas RA, Nhat LTH, Thuong NTT, et al.: Proresolving mediator profiles in cerebrospinal fluid are linked with disease severity and outcome in adults with tuberculous meningitis. FASEB J. 2019; 5: fj201901590R.

PubMed Abstract | Publisher Full Text

19. van Laarhoven A, Dian S, Ruesen C, et al.: Clinical Parameters, Routine Inflammatory Markers, and LTA4H Genotype as Predictors of Mortality Among 608 Patients With Tuberculous Meningitis in Indonesia. J Infect Dis. 2017; 215(7): 1029-39.

PubMed Abstract | Publisher Full Text

20. van Laarhoven A, Dian S, van Dorp S, et al.: Immune cell characteristics and cytokine responses in adult HIV-negative tuberculous meningitis: an observational cohort study. Sci Rep. 2019; 9(1): 884. PubMed Abstract | Publisher Full Text | Free Full Text

21. Campo M, Randhawa AK, Dunstan S, et al:: Common polymorphisms in the CD43 gene region are associated with tuberculosis disease and mortality. Am J Respir Cell Mol Biol. 2015; 52(3): 342-8. PubMed Abstract | Publisher Full Text | Free Full Text

22. Horne DJ, Randhawa AK, Chau TT, et al:: Common polymorphisms in the PKP3-SIGIRR-TMEM16J gene region are associated with susceptibility to tuberculosis. J Infect Dis. 2012; 205(4): 586-94. PubMed Abstract | Publisher Full Text | Free Full Text

23. Tobin DM, Roca FJ, Oh SF, et al.: Host genotype-specific therapies can optimize the inflammatory response to mycobacterial infections. Cell. 2012; 148(3): 434-46.

PubMed Abstract | Publisher Full Text | Free Full Text

24. Tobin DM, Vary JC Jr, Ray JP, et al.: The Ita4h locus modulates susceptibility to mycobacterial infection in zebrafish and humans. Cell. 2010; 140(5): 717-30. PubMed Abstract | Publisher Full Text | Free Full Text

25. Donovan J, Phu NH, Thao LTP, et al.: Adjunctive dexamethasone for the treatment of HIV-uninfected adults with tuberculous meningitis stratified by Leukotriene A4 hydrolase genotype (LAST ACT): Study protocol for a randomised double blind placebo controlled non-inferiority trial [version 1 ; peer review: 2 approved]. Wellcome Open Res. 2018; 3: 32 PubMed Abstract | Publisher Full Text | Free Full Text

26. van Laarhoven A, Dian S, Aguirre-Gamboa R, et al:: Cerebral tryptophan metabolism and outcome of tuberculous meningitis: an observational cohort study. Lancet Infect Dis. 2018; 18(5): 526-35. PubMed Abstract | Publisher Full Text

27. Thao LTP, Heemskerk AD, Geskus RB, et al:: Prognostic Models for 9-Month Mortality in Tuberculous Meningitis. Clin Infect Dis. 2018; 66(4): 523-32. PubMed Abstract | Publisher Full Text | Free Full Text

28. Thwaites GE, Nguyen DB, Nguyen HD, et al.: Dexamethasone for the treatment of tuberculous meningitis in adolescents and adults. N Engl J Med. 2004; 351(17): 1741-51 PubMed Abstract | Publisher Full Text

29. Marais S, Wilkinson KA, Lesosky M, et al.: Neutrophil-associated central nervous system inflammation in tuberculous meningitis immune reconstitution inflammatory syndrome. Clin Infect Dis. 2014; 59(11): 1638-47. PubMed Abstract | Publisher Full Text | Free Full Text

30. Marais S, Lai RPJ, Wilkinson KA, et al.: Inflammasome Activation Underlying Central Nervous System Deterioration in HIV-Associated Tuberculosis. J Infect Dis. 2017; 215(5): 677-86. PubMed Abstract | Publisher Full Text | Free Full Text

31. Thuong NTT, Thwaites GE: Treatment-Associated Inflammatory Deterioration in Tuberculous Meningitis: Unpicking the Paradox. J Infect Dis. 2017; 215(5): 665-7. PubMed Abstract | Publisher Full Text | Free Full Text

32. Rohlwink UK, Mauff K, Wilkinson KA, et al.: Biomarkers of Cerebral Injury and Inflammation in Pediatric Tuberculous Meningitis. Clin Infect Dis. 2017; 65(8): 1298-307.

PubMed Abstract | Publisher Full Text | Free Full Text

33. Rohlwink UK, Figaji A, Wilkinson KA, et al.: Tuberculous meningitis in children 
is characterized by compartmentalized immune responses and neural excitotoxicity. Nat Commun. 2019; 10(1): 3767 PubMed Abstract | Publisher Full Text | Free Full Text

34. Pinon JM, Puygauthier-Toubas D, Lepan $\mathrm{H}$, et al.: Rapid detection of proteins by enzyme-linked immunofiltration assay after transfer onto nitrocellulose membranes. Electrophoresis. 1990; 11(1): 41-5. PubMed Abstract | Publisher Full Text

35. Lin WC, Chen PC, Wang HC, et al:: Diffusion tensor imaging study of white matter damage in chronic meningitis. PLoS One. 2014; 9(6): e98210. PubMed Abstract | Publisher Full Text | Free Full Text

36. Chen $\mathrm{HL}, \mathrm{Lu} \mathrm{CH}$, Chang $\mathrm{CD}$, et al:: Structural deficits and cognitive impairment in tuberculous meningitis. BMC Infect Dis. 2015; 15: 279. PubMed Abstract | Publisher Full Text | Free Full Text

37. Tucker EW, Pokkali S, Zhang Z, et al:: Microglia activation in a pediatric rabbit model of tuberculous meningitis. Dis Model Mech. 2016; 9(12): 1497-506. PubMed Abstract | Publisher Full Text | Free Full Text

38. Kim DW, Kim CG, Park SA, et al:: Experience of Dual Time Point Brain F-18 FDG $\mathrm{PET} / \mathrm{CT}$ Imaging in Patients with Infectious Disease. Nucl Med Mol Imaging. 2010; 44(2): 137-42.

PubMed Abstract | Publisher Full Text | Free Full Text

39. Sharma P, Marangmei C: Tubercular Meningitis on 18F-FDG PET/CT: Incidentally Detected and Masquerading as Relapse in a Patient With Ovarian Burkitt Lymphoma. Clin Nucl Med. 2015; 40(7): 606-7. PubMed Abstract | Publisher Full Text

40. Rangan K, Ravina M, Yadav N, et al:: 18F-FDG PET/CT of Tuberculosis Meningitis and Carotid Pseudoaneurysm. Clin Nucl Med. 2017; 42(6): e304-e5. PubMed Abstract | Publisher Full Text

41. Bateman RJ, Xiong C, Benzinger TL, et al.: Clinical and biomarker changes in dominantly inherited Alzheimer's disease. N Engl J Med. 2012; 367(9): 795-804 PubMed Abstract | Publisher Full Text | Free Full Text

42. Vlassenko AG, Benzinger TL, Morris JC: PET amyloid-beta imaging in preclinica Alzheimer's disease. Biochim Biophys Acta. 2012; 1822(3): 370-9. PubMed Abstract | Publisher Full Text | Free Full Text

43. Thuong NTT, Vinh DN, Hai HT, et al:: Pretreatment Cerebrospinal Fluid Bacterial Load Correlates With Inflammatory Response and Predicts Neurological Events During Tuberculous Meningitis Treatment. J Infect Dis. 2019; 219(6): 986-95.

PubMed Abstract | Publisher Full Text | Free Full Text

44. Heemskerk AD, Donovan J, Thu DDA, et al:: Improving the microbiological diagnosis of tuberculous meningitis: A prospective, international, multicentre comparison of conventional and modified Ziehl-Neelsen stain, GeneXpert, and culture of cerebrospinal fluid. J Infect. 2018; 77(6): 509-15.

PubMed Abstract | Publisher Full Text | Free Full Text

45. Marais S, Meintjes G, Pepper DJ, et al.: Frequency, severity, and prediction of tuberculous meningitis immune reconstitution inflammatory syndrome. Clin Infect Dis. 2013; 56(3): 450-60.

PubMed Abstract | Publisher Full Text | Free Full Text

46. Faksri K, Xia E, Ong RT, et al:: Comparative whole-genome sequence analysis of Mycobacterium tuberculosis isolated from tuberculous meningitis and pulmonary tuberculosis patients. Sci Rep. 2018; 8(1): 4910. PubMed Abstract | Publisher Full Text | Free Full Text

47. Ruesen C, Chaidir L, van Laarhoven A, et al.: Large-scale genomic analysis shows association between homoplastic genetic variation in Mycobacterium tuberculosis genes and meningeal or pulmonary tuberculosis. BMC Genomics. 2018; 19(1): 122

PubMed Abstract | Publisher Full Text | Free Full Text

48. Houben RM, Dodd PJ: The Global Burden of Latent Tuberculosis Infection: A Re-estimation Using Mathematical Modelling. PLoS Med. 2016; 13(10): e1002152.

PubMed Abstract | Publisher Full Text | Free Full Text

49. Casadevall A, Pirofski LA: Host-pathogen interactions: redefining the basic concepts of virulence and pathogenicity. Infect Immun. 1999; 67(8): 3703-13. PubMed Abstract | Free Full Text

50. Pirofski LA, Casadevall A: Immune-Mediated Damage Completes the Parabola: Cryptococcus neoformans Pathogenesis Can Reflect the Outcome of a Weak or Strong Immune Response. mBio. 2017; 8(6). PubMed Abstract | Publisher Full Text | Free Full Text

51. Agarwal N, Lamichhane G, Gupta R, et al.: Cyclic AMP intoxication of macrophages by a Mycobacterium tuberculosis adenylate cyclase. Nature. 2009; 460(7251): 98-102.

PubMed Abstract | Publisher Full Text

52. Berg RD, Ramakrishnan L: Insights into tuberculosis from the zebrafish model. Trends Mol Med. 2012; 18(12): 689-90. PubMed Abstract | Publisher Full Text

53. Kumar P: IFN $\gamma$-producing $\mathrm{CD}^{+} \mathrm{T}$ lymphocytes: the double-edged swords in tuberculosis. Clin Transl Med. 2017; 6(1): 21.

PubMed Abstract | Publisher Full Text | Free Full Text

54. $\mathrm{Xu} \mathrm{HB}$, Jiang RH, Li L, et al:: Diagnostic value of adenosine deaminase in cerebrospinal fluid for tuberculous meningitis: a meta-analysis. Int $J$ Tuberc Lung Dis. 2010; 14(11): 1382-7. PubMed Abstract

55. Juan RS, Sánchez-Suárezz C, Rebollo MJ, et al:: Interferon gamma quantification in cerebrospinal fluid compared with PCR for the diagnosis of tuberculous meningitis. J Neurol. 2006; 253(10): 1323-30.

PubMed Abstract | Publisher Full Text

56. Lu D, Chen C, Yu S, et al: Diagnosis of Tuberculous Meningitis Using a Combination of Peripheral Blood T-SPOT.TB and Cerebrospinal Fluid Interferon- $\gamma$ Detection Methods. Lab Med. 2016; 47(1): 6-12. PubMed Abstract | Publisher Full Text

57. Yu J, Wang ZJ, Chen LH, et al:: Diagnostic accuracy of interferon-gamma release assays for tuberculous meningitis: a meta-analysis. Int J Tuberc Lung Dis. 2016; 20(4): 494-9. PubMed Abstract | Publisher Full Text

58. Bahr NC, Halupnick R, Linder G, et al.: Delta-like 1 protein, vitamin D binding protein and fetuin for detection of Mycobacterium tuberculosis meningitis. Biomark Med. 2018; 12(7): 707-16.

PubMed Abstract | Publisher Full Text | Free Full Text

59. Huang TY, Zhang XX, Wu QL, et al:: Antibody detection tests for early diagnosis in tuberculous meningitis. Int $J$ Infect Dis. 2016; 48: 64-9. PubMed Abstract | Publisher Full Text

60. Shah M, Hanrahan C, Wang ZY, et al.: Lateral flow urine lipoarabinomannan assay for detecting active tuberculosis in HIV-positive adults. Cochrane Database Syst Rev. 2016; (5): CD011420.

PubMed Abstract | Publisher Full Text | Free Full Text

61. Marais S, Thwaites G, Schoeman JF, et al:: Tuberculous meningitis: a uniform case definition for use in clinical research. Lancet Infectious Dis. 2010; 10(11): 803-12.

PubMed Abstract | Publisher Full Text

62. Solomons R, Grantham M, Marais BJ, et al:: IMCI indicators of childhood TBM at primary health care level in the Western Cape Province of South Africa. Int $J$ TB and Lung Dis. 2016; 20(10): 1309-13.

PubMed Abstract | Publisher Full Text

63. Chegou NN, Walzl G, Bolliger CT, et al.: Evaluation of adapted whole-blood interferon-gamma release assays for the diagnosis of pleural tuberculosis. Respiration. 2008; 76(2): 131-8.

PubMed Abstract | Publisher Full Text

64. Manyelo $\mathrm{CM}$, Solomons RS, Snyders $\mathrm{Cl}$, et al:: Application of Cerebrospinal Fluid Host Protein Biosignatures in the Diagnosis of Tuberculous Meningitis in Children from a High Burden Setting. Mediators Inflamm. 2019; 2019: 7582948. PubMed Abstract | Publisher Full Text | Free Full Text

65. Manyelo CM, Solomons RS, Snyders Cl, et al.: Potential of Host Serum Protein Biomarkers in the Diagnosis of Tuberculous Meningitis in Children. Front Pediatr. 2019; 7: 376 .

PubMed Abstract | Publisher Full Text | Free Full Text

66. Pormohammad A, Nasiri MJ, McHugh TD, et al.: A Systematic Review and Meta-analysis of the Diagnostic Accuracy of Nucleic Acid Amplification Tests for Tuberculous Meningitis. J Clin Microbiol. 2019; 57(6) PubMed Abstract | Publisher Full Text | Free Full Text

67. Modi M, Sharma K, Sharma M, et al.: Multitargeted loop-mediated isothermal amplification for rapid diagnosis of tuberculous meningitis. Int $J$ Tuberc Lung Dis. 2016; 20(5): 625-30 PubMed Abstract | Publisher Full Tex

68. Kohli M, Schiller I, Dendukuri N, et al:: Xpert $^{\oplus}$ MTB/RIF assay for extrapulmonary tuberculosis and rifampicin resistance. Cochrane Database Syst Rev. 2018; 8(8): tuberculosis Cd012768.
PubMed Abstract | Publisher Full Text | Free Full Text

69. Cresswell FV, Bangdiwala AS, Bahr NC, et al:: Can improved diagnostics reduce mortality from Tuberculous meningitis? Findings from a 6.5-year cohort in Uganda [version 2; peer review: 3 approved]. Wellcome Open Res. 2018; 3: 64 PubMed Abstract | Publisher Full Text | Free Full Text

70. Sharma K, Sharma M, Chaudhary L, et al: Comparative evaluation of Xpert MTB/RIF assay with multiplex polymerase chain reaction for the diagnosis of tuberculous meningitis. Tuberculosis (Edinb). 2018; 113: 38-42. PubMed Abstract | Publisher Full Text

71. Patel VB, Connolly C, Singh R, et al:: Comparison of amplicor and GeneXpert MTB/RIF tests for diagnosis of tuberculous meningitis. J Clin Microbiol. 2014; 52(10): 3777-80.

PubMed Abstract | Publisher Full Text | Free Full Text

72. Chakravorty S, Simmons AM, Rowneki M, et al.: The New Xpert MTB/RIF UItra: Improving Detection of Mycobacterium tuberculosis and Resistance to Rifampin in an Assay Suitable for Point-of-Care Testing. mBio. 2017; 8(4) PubMed Abstract | Publisher Full Text | Free Full Text

73. Bahr NC, Nuwagira E, Evans EE, et al:: Diagnostic accuracy of Xpert MTB/RIF Ultra for tuberculous meningitis in HIV-infected adults: a prospective cohort study. Lancet Infect Dis. 2018; 18(1): 68-75. PubMed Abstract | Publisher Full Text | Free Full Text

74. Cresswell F, Tugume L, Bahr NC, et al.: Xpert MTB/Rif Ultra for the Diagnosis of HIV-Associated Tuberculous Meningitis: A Prospective Validation. 2019. Reference Source

75. Group WHOTE: Non-inferiority analysis of Xpert MTB/RIF Ultra compared to Xpert MTB/RIF. Geneva: World Health Organisation. 2017.

76. Cresswell FV, Tugume L, Bahr NC, et al:: Xpert MTB/RIF Ultra for the diagnosis of HIV-associated tuberculous meningitis: a prospective validation study. Lancet Infect Dis. 2020; 20(3): 308-317.

PubMed Abstract | Publisher Full Text | Free Full Text

77. Donovan J, Thu DDA, Phu NH, et al:: Xpert MTB/RIF Ultra versus Xpert 
MTB/RIF for the diagnosis of tuberculous meningitis: a prospective, randomised, diagnostic accuracy study. Lancet Infect Dis. 2020; 20(3): 299-307. PubMed Abstract | Publisher Full Text | Free Full Text

78. Gupta R, Thakur R, Gupta P, et al:: Evaluation of Geno Type MTBDRplus Line Probe Assay for Early Detection of Drug Resistance in Tuberculous Meningitis Patients in India. J Glob Infect Dis. 2015; 7(1): 5-10. PubMed Abstract | Publisher Full Text | Free Full Text

79. Zong $\mathrm{K}$, Luo $\mathrm{C}$, Zhou $\mathrm{H}$, et al:: Xpert MTB/RIF assay for the diagnosis of rifampicin resistance in different regions: a meta-analysis. BMC Microbiol. 2019; 19(1): 177

PubMed Abstract | Publisher Full Text | Free Full Text

80. Nhu NT, Heemskerk D, Thu do DA, et al: Evaluation of GeneXpert MTB/RIF for diagnosis of tuberculous meningitis. J Clin Microbiol. 2014; 52(1): 226-33. PubMed Abstract | Publisher Full Text | Free Full Text

81. Patel VB, Theron G, Lenders L, et al.: Diagnostic accuracy of quantitative PCR (Xpert MTB/RIF) for tuberculous meningitis in a high burden setting: a prospective study. PLoS Med. 2013; 10(10): e1001536. PubMed Abstract | Publisher Full Text | Free Full Text

82. Bahr NC, Nuwagira E, Evans EE, et al.: Diagnostic accuracy of Xpert MTB/RIF Ultra for tuberculous meningitis in HIV-infected adults: a prospective cohort study. Lancet Infect Dis. 2018; 18(1): 68-75. PubMed Abstract | Publisher Full Text | Free Full Text

83. Bahr NC, Marais S, Caws M, et al.: GeneXpert MTB/Rif to Diagnose Tuberculous Meningitis: Perhaps the First Test but not the Last. Clin Infect Dis. 2016; 62(9): 1133-5.

PubMed Abstract | Publisher Full Text | Free Full Text

84. Boyles T: Xpert Ultra's place in the diagnosis of tuberculous meningitis. Lancet Infect Dis. 2018; 18(3): 248-9.

PubMed Abstract | Publisher Full Text

85. Ai JW, Zhou X, Xu T, et al.: CRISPR-based rapid and ultra-sensitive diagnostic test for Mycobacterium tuberculosis. Emerg Microbes Infect. 2019; 8(1): 1361-9. PubMed Abstract | Publisher Full Text

86. Wilson MR, Sample HA, Zorn KC, et al.: Clinical Metagenomic Sequencing for Diagnosis of Meningitis and Encephalitis. N Engl J Med. 2019; 380(24): 2327-40.

PubMed Abstract | Publisher Full Text | Free Full Text

87. Wang S, Chen Y, Wang D, et al.: The Feasibility of Metagenomic Next-Generation Sequencing to Identify Pathogens Causing Tuberculous Meningitis in Cerebrospinal Fluid. Front Microbiol. 2019; 10: 1993. PubMed Abstract | Publisher Full Text | Free Full Text

88. Quan J, Langelier C, Kuchta A, et al.: FLASH: a next-generation CRISPR diagnostic for multiplexed detection of antimicrobial resistance sequences. Nucleic Acids Res. 2019; 47(14): e83. PubMed Abstract | Publisher Full Text | Free Full Text

89. Coll F, McNerney R, Preston MD, et al:: Rapid determination of anti-tuberculosis drug resistance from whole-genome sequences. Genome Med. 2015; 7(1): 51. PubMed Abstract | Publisher Full Text | Free Full Text

90. Ramachandran $\mathrm{P}$, Cresswell $\mathrm{F}$, Langelier $\mathrm{C}$, et al:: Illuminating Tuberculosis Meningitis with Metagenomics and FLASH Enrichment. Neurology. 2019; S45:
005

Reference Source

91. Bahr NC, Tugume L, Boulware DR: A Word of Caution in Considering the Use of the Lipoarabinomannan Lateral Flow Assay on Cerebrospinal Fluid for Detection of Tuberculous Meningitis. J Clin Microbiol. 2016; 54(1): 241-2. PubMed Abstract | Publisher Full Text | Free Full Text

92. Kwizera R, Cresswell FV, Mugumya G, et al.: Performance of Lipoarabinomannan Assay using Cerebrospinal fluid for the diagnosis of Tuberculous meningitis among HIV patients [version 2; peer review: 2 approved]. Wellcome Open Res. 2019; 4: 123.

PubMed Abstract | Publisher Full Text | Free Full Text

93. Cox JA, Lukande RL, Kalungi S, et al: Accuracy of Lipoarabinomannan and Xpert MTB/RIF Testing in Cerebrospinal Fluid To Diagnose Tuberculous Meningitis in an Autopsy Cohort of HIV-Infected Adults. J Clin Microbiol. 2015; 53(8): 2667-73.

PubMed Abstract | Publisher Full Text | Free Full Text

94. Broger $\mathrm{T}$, Sossen $\mathrm{B}$, du Toit $\mathrm{E}$, et al:: Novel lipoarabinomannan point-of-care tuberculosis test for people with HIV: a diagnostic accuracy study. Lancet Infect Dis. 2019; 19(8): 852-61.

PubMed Abstract | Publisher Full Text | Free Full Text

95. Boyles T, Stadelman A, Ellis JP, et al:: The diagnosis of tuberculous meningitis in adults and adolescents: protocol for a systematic review and individual patient data meta-analysis to inform a multivariable prediction model [version 2; peer review: 1 approved, 1 approved with reservations]. Wellcome Open Res. 2019; 4: 19.

Publisher Full Text

96. Siddiqi OK, Birbeck GL, Ghebremichael M, et al.: Prospective Cohort Study on Performance of Cerebrospinal Fluid (CSF) Xpert MTB/RIF, CSF Lipoarabinomannan (LAM) Lateral Flow Assay (LFA), and Urine LAM LFA for Diagnosis of Tuberculous Meningitis in Zambia. J Clin Microbiol. 2019; 57(8): pii: e00652-19.

PubMed Abstract | Publisher Full Text | Free Full Text

97. Cohen J, Vincent JL, Adhikari NK, et al.: Sepsis: a roadmap for future research. Lancet Infect Dis. 2015; 15(5): 581-614.

PubMed Abstract | Publisher Full Text

98. Imperial MZ, Nahid P, Phillips PPJ, et al.: A patient-level pooled analysis of treatment-shortening regimens for drug-susceptible pulmonary tuberculosis. Nat Med. 2018; 24(11): 1708-15

PubMed Abstract | Publisher Full Text | Free Full Text

99. Misra UK, Kalita J, Nair PP: Role of aspirin in tuberculous meningitis: a randomized open label placebo controlled trial. J Neurol Sci. 2010; 293(1-2): $12-7$

PubMed Abstract | Publisher Full Text

100. Coulter JB, Baretto RL, Mallucci CL, et al.: Tuberculous meningitis: protracted course and clinical response to interferon-gamma. Lancet Infect Dis. 2007; 7(3): 225-32.

PubMed Abstract | Publisher Full Text

101. Thwaites GE, van Toorn R, Schoeman J: Tuberculous meningitis: more questions, still too few answers. Lancet Neurol. 2013; 12(10): 999-1010. PubMed Abstract | Publisher Full Text 


\title{
4 Chapter Four: Tuberculous Meningitis Diagnosis and Outcomes During
}

\author{
the Xpert MTB/Rif Era: Findings from a 6.5-year Cohort in Uganda \\ (research paper)
}

\section{Foreword}

At the beginning my PhD in 2017 there was no literature on TBM in Uganda, and a collection of case series from other African countries, see Table 4.0. My understanding of the condition locally was based on clinical experience and anecdotes from colleagues. So, my first aim was to use available data to describe the demographics of the local TBM population, method of diagnosis and clinical outcomes. No national level data on TBM is collected by the Ministry of Health and the government hospitals generally do not have a medical records system which readily allows for retrospective review of notes. I therefore used data collected by the IDI meningitis research team during diagnostic studies and in the screening process for meningitis clinical trials at Mulago and Mbarara Hospitals in the period $16^{\text {th }}$ November 2010 to $28^{\text {th }}$ May 2017. Data was stored in a number of databases at the University of Minnesota or in research files at IDI archive in Kampala. I identified TBM cases by searching for variables relating to TB diagnostics and TB treatment to generate a TBM dataset of $n=195$ treated cases, which I analysed and wrote up as a retrospective cohort.

This paper describes the demographics of TBM cases, the nature of their diagnosis, and hospital survival over three time periods: 1 ) the period before Xpert MTB/Rif was available; 2) the period around the introduction of Xpert MTB/Rif when the assay was only run on stored samples and results were not available to guide clinical management; 3) the period when Xpert MTB/Rif was used as a real-time diagnostic tool to guide clinical management. It was not possible to comment on annual number of TBM cases or incidence of TBM as screening intensity varied over the 6.5-year time period depending on whether a trial was active. This research paper was in fulfilment of PhD objective $\# 1$ - to assimilate available retrospective data on adults with suspected TBM in Uganda and to describe the TBM population in terms of baseline characteristics, method of diagnosis and clinical outcomes. The findings of this paper were presented as a poster at the Royal Society of Tropical Medicine and Hygiene Research in Progress conference in Moshi, Tanzania in 2018.

I found that 12\% (195/1672) of patients who received a lumbar puncture at Mbarara and Mulago Referral hospitals were treated for TBM in the 2010-2017 period and 96\% were PLHIV. Despite ART coverage in Uganda improving rapidly during the study period, from $0 \%$ of patients presenting to hospital on ART in 2010 to $61 \%$ being on ART in the most recent period, the degree of immune suppression at presentation did not improve significantly and median CD4+ T cell count was 78 cells/ $\mu$ l overall. The introduction of Xpert MTB/Rif had a marked impact on the proportion of TBM cases which are microbiologically confirmed: from 3\% (1/33, with AFB smear only) to 41\% (60/147, with Xpert MTB/Rif and culture). TBM In-hospital mortality declined 
from $57 \%$ in the earliest period to $41 \%(51 / 124)$ in the most recent period where Xpert MTB/Rif has been used in real-time to guide decision-making. There are multiple confounding factors that may have contributed to this observed difference in hospital outcomes, alongside missing outcome data, so it is not possible to draw any conclusions about the impact of Xpert MTB/Rif on TBM survival. However, it is clear that even with the routine use of Xpert MTB/Rif and culture, the majority of TBM patients $(59 \%, 87 / 147)$ are still treated on clinical suspicion alone despite negative TB tests and case-fatality in hospital is concerningly high. There was therefore a need for more sensitive, affordable, rapid diagnostic test to support clinical decision-making and prompt treatment initiation, with the hope of reducing death and disability. In light of this, I went on to prospectively investigate whether Xpert Ultra would be more sensitive than Xpert MTB/Rif in TBM diagnosis and whether other point of care biomarkers such as lactate has a role in HIV/TBM diagnosis (chapter 5), and thereafter whether urine TB diagnostics (Alere LAM, Xpert Ultra) have a role in TBM management (chapter 6).

\section{Table 4.0. TBM case series and cohorts from sub-Saharan Africa}

\begin{tabular}{|c|c|c|c|c|c|c|c|c|c|}
\hline $\begin{array}{l}\text { Table } 4.0 \\
\text { Author }\end{array}$ & Year & Country & $\begin{array}{l}\text { Number } \\
\text { TBM } \\
\text { included }\end{array}$ & Confirmed TBM & $\begin{array}{l}\text { CSF TB } \\
\text { diagnostics }\end{array}$ & $\begin{array}{l}\text { HIV } \\
\text { prevalence }\end{array}$ & Mortality & $\begin{array}{l}\text { Study } \\
\text { type }\end{array}$ & Comment \\
\hline Patel ${ }^{1}$ & $\begin{array}{l}2008 \\
\text { to } \\
2011\end{array}$ & $\begin{array}{l}\text { South } \\
\text { Africa }\end{array}$ & $\begin{array}{l}59 \text { def } \\
64 \text { prob }\end{array}$ & & $\begin{array}{l}\text { Xpert, PCR, } \\
\text { Culture, ZN } \\
\text { stain }\end{array}$ & $87 \%$ & $\begin{array}{l}\text { Not } \\
\text { reported }\end{array}$ & $\begin{array}{l}141 \\
\text { meningitis } \\
\text { patients }\end{array}$ & $\begin{array}{l}\text { Sensitivity of Xpert } 62 \% \\
(48-75 \%)\end{array}$ \\
\hline Siddiqui ${ }^{2}$ & $\begin{array}{l}2014 \\
\text { to } \\
2017\end{array}$ & Zambia & 105 & $100 \%$ & $\begin{array}{l}\text { Culture } \\
\text { Xpert }\end{array}$ & $86.2 \%$ & $\begin{array}{l}43 \% \text { (in } \\
\text { hospital) } \\
57 \% \text { (1 } \\
\text { year) }\end{array}$ & $\begin{array}{l}19 \% \text { of } n=550 \\
\text { meningitis } \\
\text { admissions }\end{array}$ & \\
\hline Karstaedt ${ }^{3}$ & $\begin{array}{l}1994 \\
\text { to } \\
1997\end{array}$ & $\begin{array}{l}\text { South } \\
\text { Africa }\end{array}$ & 56 & $100 \%$ & $\begin{array}{l}\text { ZN stain } \\
\text { Culture }\end{array}$ & $69.6 \%$ & $69.1 \%$ & $\begin{array}{l}\text { Cohort of } \\
\text { culture- } \\
\text { confirmed } \\
\text { TBM }\end{array}$ & $\begin{array}{l}\text { HIV+ had more } \\
\text { extrameningeal TB }(76.9 \% \\
\text { vs } 9.1 \%) .21 .4 \% \text { acellular } \\
\text { CSF. CSF neuts in } 39.3 \% \text {. } \\
5.4 \% \text { AFB + }\end{array}$ \\
\hline Cohen ${ }^{4}$ & 2007 & Malawi & 46 & $\begin{array}{l}100 \% \\
\text { Culture-positve }\end{array}$ & Culture & $89.1 \%$ & $\begin{array}{l}\text { Not } \\
\text { reported }\end{array}$ & $\begin{array}{l}18 \% \text { of } n=263 \\
\text { meningitis } \\
\text { inpatients }\end{array}$ & \\
\hline Hakim 5 & 1994 & Zimbabwe & 24 & $\begin{array}{l}100 \% \\
\text { Culture-confirmed }\end{array}$ & $\begin{array}{l}\text { ZN stain } \\
\text { Culture (U) }\end{array}$ & $88 \%$ & $66.7 \%$ & $\begin{array}{l}12 \% \text { of } n=200 \\
\text { meningitis } \\
\text { inpatients }\end{array}$ & $26.3 \%$ acellular CSF \\
\hline Bergmann ${ }^{6}$ & $\begin{array}{l}1994 \\
\text { to } \\
1995\end{array}$ & $\begin{array}{l}\text { South } \\
\text { Africa }\end{array}$ & 72 & $\begin{array}{l}\text { a) Culture, b) TB at } \\
\text { another site, c) clinical } \\
\text { Dx + ATT response }\end{array}$ & $\begin{array}{l}\text { ZN stain } \\
\text { culture }\end{array}$ & $\begin{array}{l}54 \% \text { of } \\
\text { those } \\
\text { tested }\end{array}$ & $>40 \%$ & $\begin{array}{l}25 \% \text { of } n=284 \\
\text { meningitis } \\
\text { inpatients }\end{array}$ & $\begin{array}{l}\text { TBM the most common } \\
\text { cause of meningitis }\end{array}$ \\
\hline Patel 7 & $\begin{array}{l}1999 \\
\text { to } \\
2002\end{array}$ & $\begin{array}{l}\text { South } \\
\text { Africa }\end{array}$ & $\begin{array}{l}30 \\
\text { MDR- } \\
\text { TBM }\end{array}$ & $\begin{array}{l}100 \% \\
\text { Culture-confirmed }\end{array}$ & $\begin{array}{l}\text { ZN stain } \\
\text { Culture }\end{array}$ & $60 \%$ & $56.6 \%$ & $\begin{array}{l}\text { MDR-TBM } \\
\text { cohort }\end{array}$ & $\begin{array}{l}73 \% \text { prior TB ATT } \\
3 \% \text { no prior ATT } \\
17 \% \text { unknown ATT }\end{array}$ \\
\hline Jarvis $^{8}$ & $\begin{array}{l}2006 \\
\text { to } \\
2008\end{array}$ & $\begin{array}{l}\text { South } \\
\text { Africa }\end{array}$ & 227 & $100 \%$ & $\begin{array}{l}\text { Auramine } \\
\text { MGIT } \\
\text { Culture, PCR }\end{array}$ & $94 \%$ & $\begin{array}{l}\text { Not } \\
\text { reported }\end{array}$ & $\begin{array}{l}4549 \text { patient } \\
\text { CSF samples } \\
\text { analysed }\end{array}$ & \\
\hline Berhe 9 & $\begin{array}{l}2002 \\
\text { to } \\
2009\end{array}$ & Ethiopia & 78 & Not specified & ZN stain & $100 \%$ & $53.8 \%$ & $\begin{array}{l}22 \% \text { of } n=347 \\
\text { meningitis } \\
\text { inpatients }\end{array}$ & \\
\hline $\begin{array}{l}\text { Osuntokun } \\
10\end{array}$ & $\begin{array}{l}1965 \\
\text { to } \\
1969\end{array}$ & Nigeria & 52 & $15 \%$ & ZN stain & N/A & $44 \%$ & $\begin{array}{l}52 \text { TBM case } \\
\text { series }\end{array}$ & \\
\hline Marais 11 & 2009 & $\begin{array}{l}\text { South } \\
\text { Africa }\end{array}$ & 120 & $39 \%$ & $\begin{array}{l}\text { ZN stain } \\
\text { Culture } \\
\text { PCR }\end{array}$ & $88 \%$ & $49 \%$ & $\begin{array}{l}57 \% \text { of } 211 \\
\text { meningitis } \\
\text { inpatients }\end{array}$ & $\begin{array}{l}\text { ART initiation during TB } \\
\text { therapy improved survival }\end{array}$ \\
\hline Luma 12 & $\begin{array}{l}2004 \\
\text { to } \\
2009\end{array}$ & Cameroon & 54 & $2 \%$ & ZN stain & $100 \%$ & $80 \%$ & $\begin{array}{l}\text { notes review } \\
672 \text { HIV+ } \\
\text { inpatients }\end{array}$ & \\
\hline $\begin{array}{l}\text { Raberahona } \\
13\end{array}$ & $\begin{array}{l}2007 \\
\text { to } \\
2014\end{array}$ & Madagascar & 75 & $11 \%$ & $\begin{array}{l}\text { ZN stain } \\
\text { Culture } \\
\text { PCR }\end{array}$ & $3 \%$ & $28 \%$ & $\begin{array}{l}\text { hospital } \\
\text { notes review }\end{array}$ & \\
\hline \multicolumn{10}{|c|}{$\begin{array}{l}\text { ATT = antituberculous therapy ZN = Ziehl Neelsen } \\
\text { Bibliography: 1.Patel VB, Theron G, Lenders L, et al. PLoS Med 2013; 10(10): e1001536. 2.Siddiqi OK, Birbeck GL, Ghebremichael M, et al. Journal of clinical microbiology 2019; 57(8). 3. Karstaedt } \\
\text { AS, Valtchanova S, Barriere R, Crewe-Brown HH. QJM 1998; 91(11): 743-7. 4.Cohen DB, Zijstra EE, Mukaka M, et al. Tropical medicine \& international health : TM \& IH 2010; 15(8): } 910-7 \text {. 5. Hakim } \\
\text { JG, Gangaidzo IT, Heyderman RS, et al. AIDS 2000; 14(10): 1401-7. 6. Bergemann A, Karstaedt AS. Q J M: an international journal of medicine 1996; 89(7): 499-504. 7.Patel VB, Padayatchi N, Bhigjee } \\
\text { Al, et al. Clinical infectious diseases. 2004; 38(6): 851-6. 8.Jarvis JN, Meintjes G, Williams A, Brown Y, Crede T, Harrison TS. BMC Infectious Diseases 2010; 10: 67. 9. Berhe T, Melkamu Y, Amare A. } \\
\text { AIDS research and therapy 2012; 9: 11. 10.Osuntokun BO, Adeuja AO, Familusi JB. Trop Geogr Med 1971; 23(3): 225-31. 11. Marais S, Pepper DJ, Schutz C, Wilkinson RJ, Meintjes G. PloS one 2011; } \\
\text { 6(5): e20077. 12.Luma HN, Tchaleu BC, Ngahane BH, et al. AIDS research and therapy 2013; 10(1): 16. 13. Raberahona M, Rakotoarivelo RA, Razafinambinintsoa T, Andrianasolo RL, Randria MJ. } \\
\text { BioMed research international 2017; 2017: 9316589. }\end{array}$} \\
\hline
\end{tabular}




\section{RESEARCH PAPER COVER SHEET}

\section{PLEASE NOTE THAT A COVER SHEET MUST BE COMPLETED FOR EACH RESEARCH PAPER INCLUDED IN A THESIS.}

\section{SECTION A - Student Details}

\begin{tabular}{|l|l|}
\hline Student & Fiona Cresswell \\
\hline Principal Supervisor & Alison Elliott \\
\hline Thesis Title & $\begin{array}{l}\text { Improving diagnosis and treatment of HIV-associated } \\
\text { Tuberculous meningitis }\end{array}$ \\
\hline
\end{tabular}

If the Research Paper has previously been published please complete Section B, if not please move to Section C

\section{SECTION B - Paper already published}

\begin{tabular}{|l|l|l|l|}
\hline Where was the work published? & Wellcome Open Research \\
\hline When was the work published? & 29th May 2018 \\
\hline $\begin{array}{l}\text { If the work was published prior to } \\
\text { registration for your research degree, } \\
\text { give a brief rationale for its inclusion }\end{array}$ & \multicolumn{3}{|l|}{} \\
\hline $\begin{array}{l}\text { Have you retained the copyright for the } \\
\text { work?* }\end{array}$ & Yes & $\begin{array}{l}\text { Was the work subject to } \\
\text { academic peer review? }\end{array}$ & Yes \\
\hline
\end{tabular}

*If yes, please attach evidence of retention. If no, or if the work is being included in its published format, please attach evidence of permission from the copyright holder (publisher or other author) to include this work.

\section{SECTION C - Prepared for publication, but not yet published}

\begin{tabular}{|l|l|}
\hline $\begin{array}{l}\text { Where is the work intended to be } \\
\text { published? }\end{array}$ & \\
\hline $\begin{array}{l}\text { Please list the paper's authors in the } \\
\text { intended authorship order: }\end{array}$ & \\
\hline Stage of publication & Choose an item. \\
\hline
\end{tabular}

\section{SECTION D - Multi-authored work}

For multi-authored work, give full details of your role in the research included in the paper and in the preparation of the paper. (Attach a further sheet if necessary)
I came up with the concept for the study and explored available data collected by the group over the last 7 years of meningitis research in Uganda. I cleaned the data, merged datasets, undertook the analysis, interpreted the results and wrote the first draft of the manuscript. I handled all the referencing, submission, reviewer responses and re-submission process. 
Student Signature:

Supervisor Signature:
Date: _ 24th Sept 2020

Date: 8th October 2020 

Xpert MTB/Rif era: a 6.5-year cohort study in Uganda [version 2;

\section{referees: 3 approved]}

Previously titled: Can improved diagnostics reduce mortality from Tuberculous meningitis? Findings from a

6.5-year cohort in Uganda

Fiona V. Cresswell (iD) 1,2, Ananta S. Bangdiwala3 ${ }^{3}$, Nathan C. Bahr (iD) 4, Emily Trautner (10) 5, Edwin Nuwagira ${ }^{6}$, Jayne Ellis ${ }^{\text {(i) } 7 \text {, Radha Rajasingham }}{ }^{8}$, Joshua Rhein (iD) 2,8, Darlisha A. Williams², Conrad Muzoora ${ }^{6}$, Alison M. Elliott (i) 1,9, David B. Meya ${ }^{2,10}$, David R. Boulware (iD 8

\footnotetext{
${ }^{1}$ Department of Clinical Research, London School of Hygiene and Tropical Medicine, London, WC1E 7HT, UK

2Infectious Diseases Institute, Kampala, Uganda

${ }^{3}$ Division Biostatistics, School of Public Health, University of Minnesota, Minneapolis, MN, 55455, USA

${ }^{4}$ Division of Infectious Diseases, Department of Medicine, University of Kansas, Kansas City, KS, 66160, USA

5 University of Utah, Salt Lake City, UT, 84112, USA

6 Mbarara University of Science and Technology, Mbarara, Uganda

${ }^{7}$ Doctors.net.uk, Abingdon, OX14 4SH, UK

${ }^{8}$ Division of Infectious Disease and International Medicine, Department of Medicine, University of Minnesota, Minneapolis, MN, 55455, USA

${ }_{9}$ Medical Research Council/Uganda Virus Research Institute and London School of Hygiene \& Tropical Medicine Uganda Research Unit on AIDS, Entebbe, Uganda

${ }^{10}$ College of Health Sciences, Makerere University, Kampala, Uganda
}

V2

First published: 29 May 2018, 3:64 (doi: 10.12688/wellcomeopenres.14610.1)
Latest published: 03 Jul 2018, 3:64 (doi: 10.12688/wellcomeopenres.14610.2)

\section{Abstract}

Background: Tuberculous meningitis (TBM), a leading cause of meningitis in sub-Saharan Africa, is notoriously difficult to diagnose. In our Ugandan setting TB diagnostics have evolved rapidly in recent years, with introduction of Xpert MTB/Rif (Xpert) in 2011 and culture in 2013. We aim to describe the impact of improved TBM diagnostics at two Ugandan hospitals between 2010 and 2017.

Methods: Adults presenting with meningitis (headache and objective meningism) were assessed for eligibility for enrolment in two consecutive trials investigating cryptococcal meningitis. Cohort one received cerebrospinal fluid (CSF) smear microscopy only (2010-2013). Cohort two received smear microscopy and Xpert on $1 \mathrm{ml}$ unprocessed CSF at physician discretion (2011-2013). Cohort three received smear microscopy, routine liquid-media culture and Xpert on large volume CSF (2013-2017) for all meningitis suspects with a negative CSF cryptococcal antigen (crAg). In a post-hoc analysis of three prospective cohorts, we compare rates of microbiologically confirmed TBM and hospital outcomes over time.

Results: 1672 predominantly HIV-infected adults underwent lumbar puncture, of which $33 \%$ (558/1672) had negative CSF crAg and 12\% (195/1672) were treated for TBM. Over the study period, microbiological confirmation of TBM

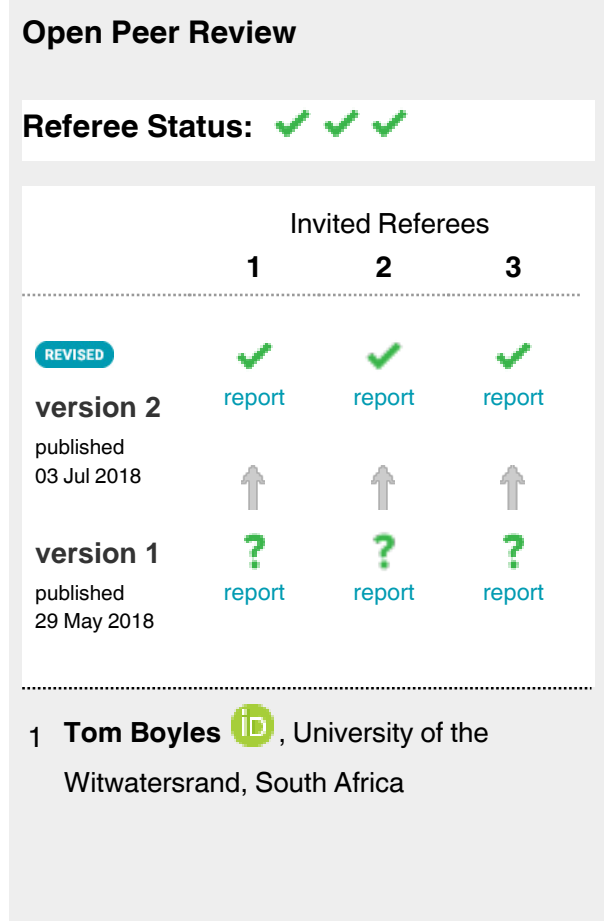


increased from $3 \%$ to $41 \%(\mathrm{P}<0.01)$ and there was a decline in in-hospital mortality from $57 \%$ to $41 \%(\mathrm{P}=0.27)$. Adjusting for definite TBM and antiretroviral therapy, and using imputed data, the odds of dying were nearly twice as high in cohort one (adjusted odds ratio $1.7,95 \% \mathrm{Cl} 0.7$ to 4.4 ) compared to cohort three. Sensitivity of Xpert was 63\% (38/60) and culture was $65 \%$ (39/60) against a composite reference standard.

Conclusions: Since 2010, as TBM diagnostics have evolved, microbiologically-confirmed TBM diagnoses have increased significantly. There has been a non-significant decline in TBM in-hospital mortality but due to multiple possible confounding factors it is not possible to conclude what has driven this decline in mortality.

Keywords

Tuberculous meningitis, TBM, HIV, diagnosis, outcomes
2 Raph L. Hamers (D), Eijkman-Oxford Clinical Research Unit, Indonesia

3 Sean Wasserman, University of Cape Town, South Africa

Angharad Davis, University College London Hospitals, UK

\section{Discuss this article}

Comments (0)

\section{Corresponding author: Fiona V. Cresswell (fiona.cresswell@Ishtm.ac.uk)}

Author roles: Cresswell FV: Conceptualization, Data Curation, Formal Analysis, Investigation, Writing - Original Draft Preparation, Writing Review \& Editing; Bangdiwala AS: Conceptualization, Data Curation, Formal Analysis, Investigation, Methodology, Project Administration, Writing - Original Draft Preparation, Writing - Review \& Editing; Bahr NC: Conceptualization, Methodology, Writing - Original Draft Preparation, Writing Review \& Editing; Trautner E: Data Curation, Writing - Review \& Editing; Nuwagira E: Data Curation; Ellis J: Data Curation, Writing - Review \& Editing; Rajasingham R: Data Curation, Investigation; Rhein J: Conceptualization, Data Curation, Methodology, Writing - Review \& Editing; Williams DA: Conceptualization, Data Curation, Investigation, Methodology, Resources, Writing - Review \& Editing; Muzoora C: Investigation, Methodology; Elliott AM: Supervision, Writing - Original Draft Preparation, Writing - Review \& Editing; Meya DB: Conceptualization, Funding Acquisition, Investigation, Project Administration, Resources, Supervision, Writing - Review \& Editing; Boulware DR: Conceptualization, Data Curation, Funding Acquisition, Investigation, Methodology, Project Administration, Resources, Supervision, Writing - Original Draft Preparation, Writing - Review \& Editing

Competing interests: No competing interests were disclosed.

Grant information: FVC is supported by the Wellcome Trust [210772/Z/18/Z]. ASB, DBM, and DRB are supported by Fogarty International Center and National Institute of Neurologic Diseases and Stroke [R01NS086312].

The funders had no role in study design, data collection and analysis, decision to publish, or preparation of the manuscript.

Copyright: @ 2018 Cresswell FV et al. This is an open access article distributed under the terms of the Creative Commons Attribution Licence, which permits unrestricted use, distribution, and reproduction in any medium, provided the original work is properly cited. The author(s) is/are employees of the US Government and therefore domestic copyright protection in USA does not apply to this work. The work may be protected under the copyright laws of other jurisdictions when used in those jurisdictions.

How to cite this article: Cresswell FV, Bangdiwala AS, Bahr NC et al. Tuberculous meningitis diagnosis and outcomes during the Xpert MTB/Rif era: a 6.5-year cohort study in Uganda [version 2; referees: 3 approved] Wellcome Open Research 2018, 3:64 (doi:

10.12688/wellcomeopenres.14610.2)

First published: 29 May 2018, 3:64 (doi: 10.12688/wellcomeopenres.14610.1) 


\section{REVISED Amendments from Version 1}

Title

The title has been changed to: Tuberculous meningitis diagnosis and outcomes during the Xpert MTB/Rif era: a 6.5-year retrospective cohort study in Uganda

\section{Methods:}

As per reviewer suggestions we have expanded on the nature of the parent trials. To clarify who was eligible to be included in the diagnostic analysis and TBM cohort $(n=195)$ we have added to the methods that "any patient from the 1672 patients screened who received testing for TBM (AFB smear, Xpert or culture) was eligible to be included in the diagnostic analysis and any patient who was treated for TBM was eligible to be included in the TBM cohort".

We have added a $2 \times 2$ table and a study schematic to make the flow of patients clearer as per the suggestion of Dr Wasserman and Dr Davis. It is not a classic Consort diagram as this population were not enrolled into a clinical trial.

We have added a detail to the methods section to define empiric TBM treatment "TBM treatment given in the absence of a positive microbiologic result, based on a high index of clinical suspicion, was defined as 'empiric TBM treatment'".

\section{Results}

The reviewers kindly pointed out a typographical error in the manuscript. The number of microbiologically confirmed cases is 74 (not 76) and the text has been amended accordingly.

Regarding the sensitivity of Xpert and Ultra against a composite reference standard a $2 \times 2$ table has been added to accompany Figure 2.

\section{Discussion}

In light of all three reviewer's comments we have expanded the discussion to further acknowledge the other potential confounding factors and concluding that it is not possible to draw a conclusion about whether Xpert has reduced TBM mortality in this setting.

\section{See referee reports}

\section{Introduction}

Tuberculous meningitis (TBM) is the second most common cause of adult meningitis in sub-Saharan Africa, ${ }^{1,2}$, accounting for one to five percent of the 10.4 million tuberculosis (TB) cases reported worldwide in $2016^{3}$. Despite treatment, TBM outcomes are poor with 19-28\% mortality in HIV-uninfected persons and 40-67\% mortality in HIV-infected patients in addition to long-term disability is frequent among survivors ${ }^{4-6}$.

Insidious symptom onset in persons with TBM leads to delay in seeking care and increasing disease severity at presentation correlates with higher mortality ${ }^{7}$. Further, the paucibacillary nature of TBM increases the difficulty in confirming diagnosis once care is sought, also contributing to high mortality ${ }^{8}$. Cerebrospinal fluid (CSF) smear microscopy for acid-fast bacilli (AFB smear) has poor sensitivity $(\sim 10-20 \%)$ in routine practice? Culture has improved sensitivity $(\sim 50-60 \%)$ but is not widely available in many resource constrained settings and commonly takes at least 2-3 weeks for liquid culture growth, which is too slow to guide decision-making at the time of presentation $^{8}$.

In 2013, the World Health Organization endorsed the Xpert MTB/RIF (Xpert) assay (Cepheid, Sunnyvale, California, USA), a cartridge-based, polymerase chain reaction assay with a run time of 113 minutes, as the preferred initial test to investigate TB meningitis on the basis of a meta-analysis of 13 studies ${ }^{9}$. Of the two major studies included in the meta-analysis, Patel and colleagues reported $67 \%$ sensitivity against microbiologically proven TBM and $36 \%$ against consensus clinical case definitions, while Nhu and colleagues showed 59\% sensitiv-ity against the same case definitions ${ }^{10-12}$. Additionally, use of a larger volume of centrifuged CSF improves sensitivity of $\operatorname{Xpert}^{10,13}$. Yet, inadequate negative predictive value means that a negative Xpert result has limited influence on clinical decision making ${ }^{14}$.

There is evidence that use of Xpert for diagnosis of pulmonary TB reduces diagnostic delay, increases the rate of same day treatment, and decreases usage of empiric treatment ${ }^{15,16}$. However, for pulmonary TB, Xpert has not been shown to decrease mortality ${ }^{16-18}$. Yet, lessening diagnostic delay in persons with TBM may be more likely to lead to improved outcomes as compared to pulmonary TB given the high early mortality of $\mathrm{TBM}^{19}$. Whether routine use of Xpert for investigation of suspected TBM has made an impact on diagnosis or mortality has not yet been investigated.

Herein we describe TBM diagnosis and outcomes over a 6.5-year period in prospective cohorts at two Ugandan referral hospitals.

\section{Methods}

Study population

Uganda is a high burden HIV setting, with a prevalence of $6.2 \%$ among adults aged 15 to 64 years with an estimated $60 \%$ viral load suppression in 2017 among all HIV-infected adults ${ }^{20}$. Adults presenting with suspected meningitis (headache and neck stiffness +/- vomiting, fever, seizures, focal neurological deficits, or altered consciousness), to Mulago National Referral Hospital, Kampala, and Mbarara Regional Referral Hospital, were assessed for eligibility for enrolment in two consecutive randomised clinical trials investigating cryptococcal meningitis. The first trial Cryptococcal Optimal Antiretroviral Timing (COAT) investigated early versus delayed antiretroviral therapy in HIV-related cryptococcal meningitis (www.clinicaltrials.gov: NCT01075152) and the second, Adjunctive Sertraline for the Treatment of Cryptococcal Meningitis (ASTRO-CM) evaluated whether sertraline when added to standard amphotericin-based therapy for cryptococcal meningi-tis, lead to improved survival (NCT01802385). Screening began on $22^{\text {nd }}$ November 2010 and continued until $28^{\text {th }}$ May 2017. After an informed consent process for trial screening a diagnostic lumbar puncture was performed and baseline demographics and clinical information were recorded on all. Participants with non-cryptococcal meningitis were not enrolled into the clinical trials but followed until hospital discharge.

Any patient who received testing for TBM (CSF AFB smear, Xpert or mycobacterial culture) during this period was eligible to be included in the diagnostic accuracy analysis. Any patient who was ultimately treated for TBM was eligible to be included in one of the three TBM cohorts, from which data as used to compare rates of microbiological confirmation and outcomes. Cohort was determined by what type of TB testing they individual had undergone. 
Microbiologically proven (definite) TB meningitis was defined as any positive AFB smear, culture or Xpert result from CSF testing. TBM treatment given in the absence of a positive microbiologic result, due to high index of clinical suspicion, was defined as 'empiric TBM treatment'. Consensus uniform case definitions were used to categorise patients as definite, probable, possible or not $\mathrm{TBM}^{11}$. TBM treatment included 12 months of antituberculous therapy with 6-8 weeks of adjunctive corticosteroids as per Ugandan guidelines ${ }^{21}$.

\section{Cohort definitions and diagnostic tests used}

Cohort one (16th November 2010 until 28 $8^{\text {th }}$ May 2013) received only CSF AFB smear testing (Figure 1). If available, $1 \mathrm{~mL}$ cryopreserved CSF was later tested with Xpert MTB/Rif when Xpert became available. Cohort two (1 ${ }^{\text {st }}$ April 2011 until $10^{\text {th }}$ November 2013) underwent CSF AFB smear and Xpert $\mathrm{MTB} /$ Rif on a $1 \mathrm{ml}$ sample of uncentrifuged CSF. Testing was performed at physician discretion when there was lymphocytic pleocytosis and/or high degree of clinical suspicion. In the period of overlap of cohort one and two (April 2011-May 2013), Xpert testing was not being done on a routine basis; subjects were included in cohort two when Xpert was done in real-time and in cohort one if Xpert was not done, or only done at a later date on cryopreserved specimens.

In cohort three $\left(11^{\text {th }}\right.$ November 2013 until $28^{\text {th }}$ May 2017) all cryptococcal antigen negative (IMMY, Norman, Oklahoma, USA) patients were systematically investigated for the presence of TB meningitis, irrespective of physician discretion. Subjects had comprehensive testing for TBM with CSF AFB smear (Mulago Hospital only), Xpert MTB/Rif on large volume centrifuged $\mathrm{CSF}^{13}$ and CSF Mycobacteria Growth Inhibitor Tube culture (MGIT, Becton Dickinson, Franklin Lakes, USA). AFB smear was discontinued in Mbarara in 2013 as the sensitivity was deemed too low to justify further use. In patients with a confirmed diagnosis of cryptococcal meningitis (CM), if TBM co-infection was suspected, patients would be investigated for TBM at the physician's discretion.

\section{Assessment of outcome}

In-hospital outcome was determined from case report forms, hospital medical records or follow-up telephone calls with the patient or their surrogate where hospital outcome was unknown. The outcome was categorised as discharged alive, deceased prior to hospital discharge or unknown (i.e. self-discharged against medical advice in an imminently terminal patient, hospital outcome undetermined, transferred to another facility).

\section{Statistical methods}

Comparisons of categorical and continuous demographic and clinical characteristics by cohort were performed using Fisher's exact tests and Kruskal-Wallis tests, respectively. Sensitivity of Xpert MTB/Rif was evaluated against a composite reference standard (any positive CSF test - AFB smear, Xpert or culture i.e. definite TBM according to the uniform case definition $)^{11}$. A separate analysis was conducted against the uniform case definition of probable or definite $\mathrm{TBM}^{11}$. Concordance between Xpert MTB/Rif and culture was evaluated with a kappa statistic and McNemar's test. Invalid tests (e.g. culture contamination, Xpert error) were counted as negative results. Mortality was first compared by cohort for participants with a known outcome using Fisher's exact test. Data for patients with unknown outcome was imputed to assume first that 50\% within each cohort died, or that $75 \%$ died (both within the expected mortality range for this population). Odds ratios and $95 \%$ confidence intervals were computed from multivariable logistic regression models with these imputed data, adjusted for 1) ART status, and 2) ART status and definite TBM diagnosis. Imputations were repeated with new random assignments to confirm results. Analyses were conducted using SAS version 9.4 (The SAS Institute, Cary, NC) and p-values <0.05 were considered statistically significant.

\section{Ethics}

Institutional review board approvals for the studies and the associated screening process were obtained locally in Uganda [ASTRO: Mulago Hospital Research Ethics Committee (approval

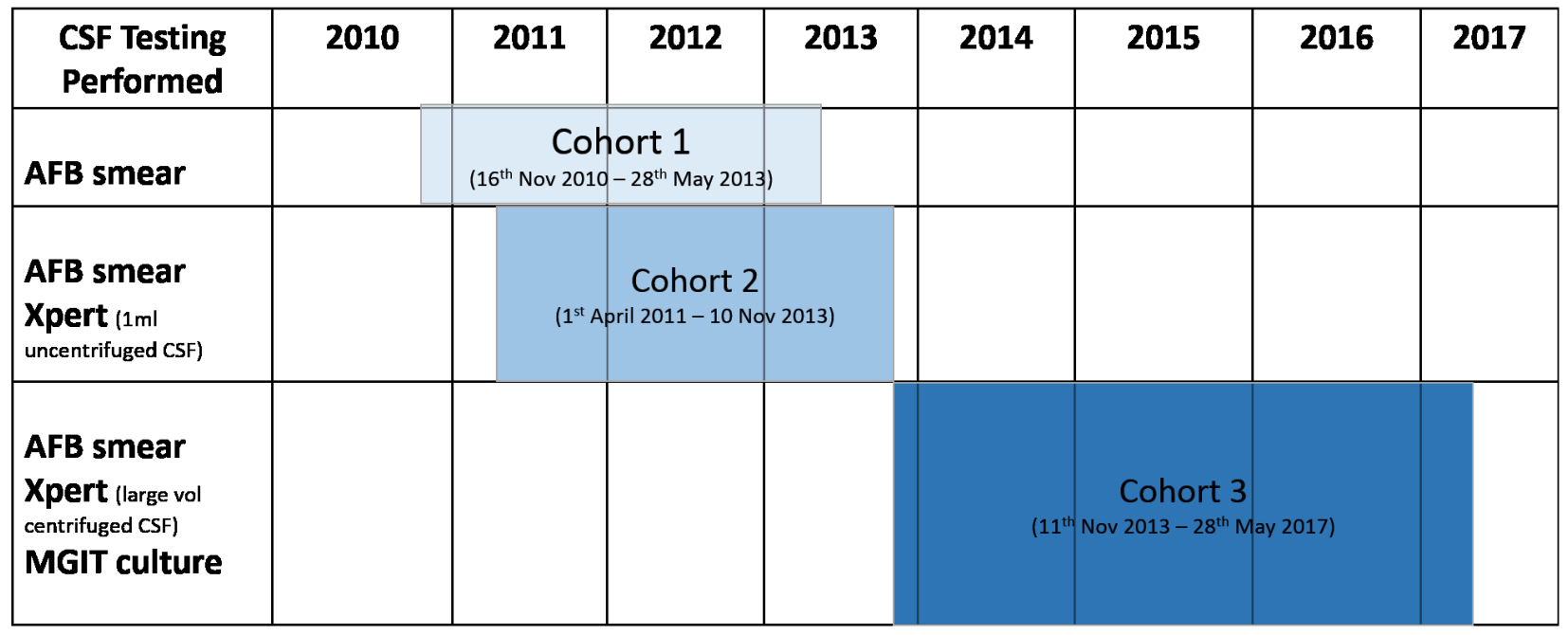

Figure 1. Timeline illustrating evolution of diagnostic testing. 
number, MREC 429); COAT: Makerere University School of Medicine Research and Ethics Committee (approval number, REC Ref No. 2009-022)], from the University of Minnesota (USA), and by the Uganda National Council of Science and Technology. Written informed consent for screening or participation in the studies was obtained from all participants or from their surrogates (e.g. family member or guardian) where the patient had altered mental status and did not have the capacity to provide consent.

\section{Results}

Participant characteristics

Over the study period, 1672 patients with meningitis symptoms were assessed and underwent lumbar puncture: 1058 (63\%) had a positive CSF cryptococcal antigen test, 558 (33\%) had negative CSF cryptococcal antigen test (data missing, $n=56$ ). A total of 195 subjects were treated for TBM, see Figure 2. Overall $61 \%$ were male, median age was 35 years (IQR 30-42), 96\% were HIV-positive, median CD4 count was 78 cells/ $\mu \mathrm{L}$ (IQR 26-191) and the majority (69\%) presented with British Medical Research Council severity grade II disease, see Table 1. Baseline characteristics were similar between cohorts with the exception of antiretroviral (ART) experience; $0 \%$ of participants were on ART in cohort one compared to $61 \%$ in cohort three $(\mathrm{P}<0.01)$.

Among the 74 cases of microbiologically proven TBM in this population with advanced HIV infection, 34\% (25/74) had an acellular CSF (white cells $<5$ cells $\mu \mathrm{L}$ ) at presentation, and $4 \%(3 / 74)$ had a normal CSF profile $(\mathrm{CSF}$ cells $<5$ cells $/ \mu \mathrm{L}$, protein $<45 \mathrm{mg} / \mathrm{dL}$, and glucose $>2.2 \mathrm{mmol} / \mathrm{l})$.

\section{Method of diagnosis}

Microbiological confirmation of TBM was made in $38 \%$ (74/195) of cases. The proportion of cases with microbiologically confirmed TBM (definite TBM) increased significantly, from $3 \%(1 / 33)$ in cohort one to $87 \%(13 / 15)$ in cohort 2 and $41 \%(60 / 147)$ in cohort $3(\mathrm{P}<0.01)$. Categorisation by uniform case definition is summarised in Table 2.

There was a marked difference in physician threshold for empiric TBM therapy between the two clinical sites. In cohort three, Mulago Hospital recorded 44 cases of which $77 \%$ (34/44) were microbiologically confirmed and $23 \%$ (10/44) were empirically treated, whilst Mbarara Hospital recorded 103 cases of which $25 \%$ (26/103) were microbiologically confirmed and $75 \%(77 / 103)$ were empirically treated.

\section{Diagnostic accuracy of Xpert MTB/Rif}

Xpert MTB/Rif was positive in 51 of 455 tested $(11 \%)$, MGIT culture positive in 39 of $321(12 \%)$ tested, AFB stain positive on 5 of 818 tested $(1 \%)$, as summarised in Table 2.

Diagnostic accuracy of Xpert and MGIT were analysed in cohort three, when both assays were done routinely, and 60 participants had a microbiologically confirmed diagnosis (composite reference standard). Sensitivity of Xpert was 63\% (38/60) against the composite reference standard and 54\% (38/71) against the uniform case definition (probable or definite TBM). Sensitivity of MGIT culture was $65 \%$ (39/60) against the composite reference standard of definite microbiologic-confirmed TBM

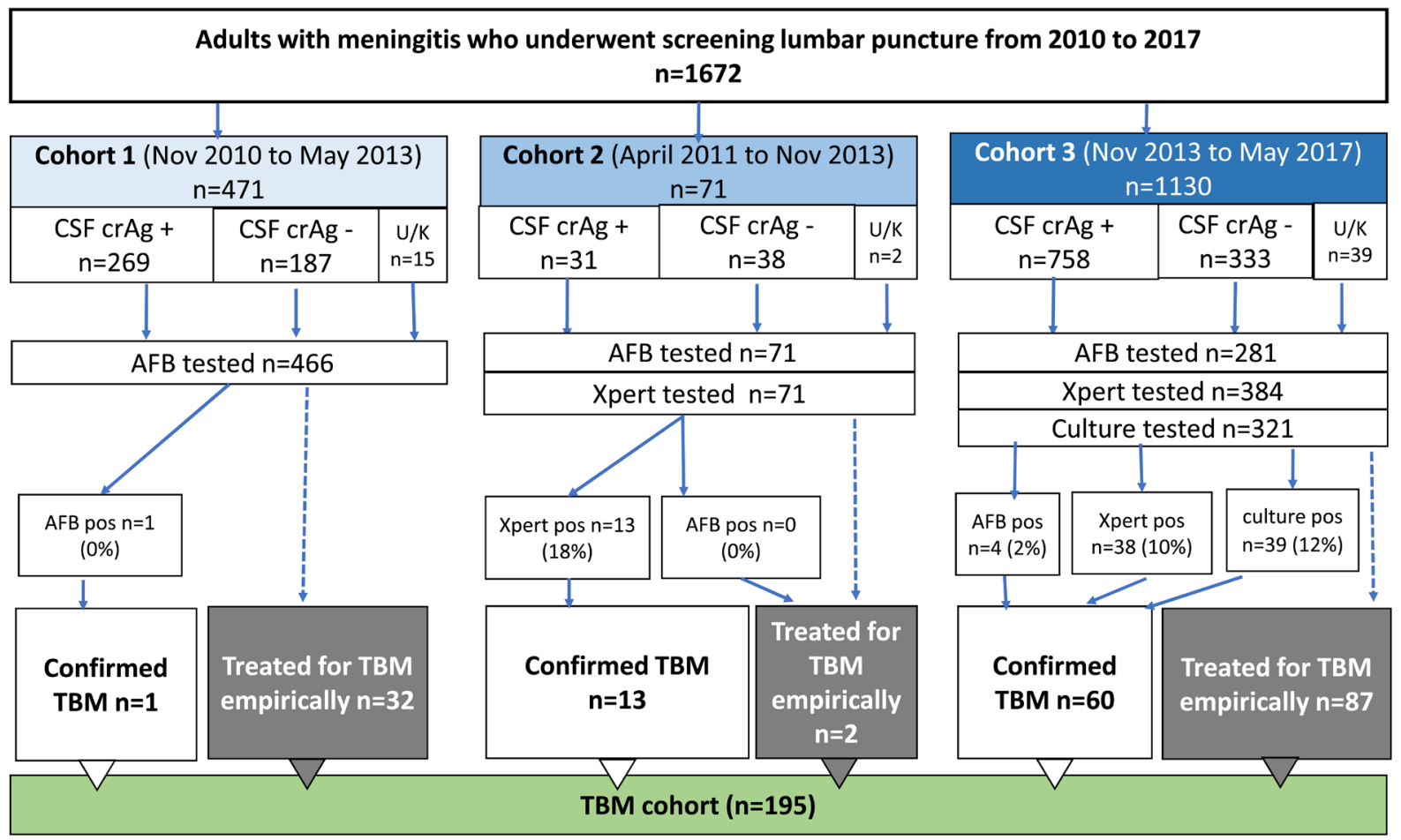

Figure 2. Illustration of flow of patients from the screening population into the TBM cohort. 


\begin{tabular}{|c|c|c|c|c|c|c|}
\hline & $\begin{array}{c}N \text { with } \\
\text { data }\end{array}$ & $\begin{array}{l}\text { Cohort } 1 \\
\text { Nov } 2010 \text { to } \\
\text { May } 2013\end{array}$ & $\begin{array}{l}\text { Cohort } 2 \\
\text { Apr } 2011 \text { to } \\
\text { Nov } 2013\end{array}$ & $\begin{array}{l}\text { Cohort } 3 \\
\text { Nov } 2013 \text { to } \\
\text { May } 2017\end{array}$ & & \\
\hline Diagnostics used & & AFB smear & $\begin{array}{l}\text { AFB smear } \\
\text { Xpert }\end{array}$ & $\begin{array}{c}\text { AFB smear } \\
\text { Xpert } \\
\text { Culture }\end{array}$ & Total & P-value* \\
\hline $\mathrm{N}$ in TBM case cohort & & 33 & 15 & 147 & 195 & \\
\hline \multicolumn{7}{|l|}{ Demographics } \\
\hline Sex & 195 & & & & & 0.58 \\
\hline Male & & $18(55 \%)$ & $8(53 \%)$ & $92(63 \%)$ & $118(61 \%)$ & \\
\hline Age & 195 & & & & & 0.33 \\
\hline Median (IQR) & & $33(29,38)$ & $35(29,40)$ & $35(30,43)$ & $35(30,43)$ & \\
\hline \multicolumn{7}{|l|}{ HIV details } \\
\hline HIV status, n (\%) & 195 & & & & & 1.00 \\
\hline HIV-positive & & $32(97 \%)$ & $15(100 \%)$ & $141(96 \%)$ & $188(96 \%)$ & \\
\hline ART status, n (\%) & 179 & & & & & $<0.01$ \\
\hline On ART & & $0(0 \%)$ & $4(27 \%)$ & $80(61 \%)$ & $84(47 \%)$ & \\
\hline ART naive & & $32(100 \%)$ & $11(73 \%)$ & $52(39 \%)$ & $95(53 \%)$ & \\
\hline CD4 & 131 & & & & & 0.30 \\
\hline Median (IQR) & & $12(7,121)$ & $148(54,169)$ & $78(26,206)$ & $78(26,191)$ & \\
\hline \multicolumn{7}{|l|}{ TBM details } \\
\hline $\begin{array}{l}\text { MRC severity grade, } \\
\text { n (\%) }\end{array}$ & 191 & & & & & 0.13 \\
\hline I & & $9(27 \%)$ & $4(31 \%)$ & $20(14 \%)$ & $33(17 \%)$ & \\
\hline ॥ & & $22(67 \%)$ & $7(54 \%)$ & $102(70 \%)$ & $131(69 \%)$ & \\
\hline III & & $2(6 \%)$ & $2(15 \%)$ & $23(16 \%)$ & $27(14 \%)$ & \\
\hline
\end{tabular}

${ }^{*}$ P-values from Fisher's exact tests for categorical variables and Kruskal-Wallis tests for continuous variables.

and 55\% (39/71) against uniform case definition for probable or definite TBM.

Concordance between Xpert MTB/Rif and MGIT culture was analysed in the 118 with both Xpert and MGIT culture results available (Table 3). Either Xpert or MGIT culture was positive in 56 patients, of which only $30 \%(17 / 56)$ were positive by both modalities (kappa 0.23 95\% CI [0.04, 0.41], p=0.01) (Figure 3). Neither method diagnosed significantly more cases than the other $(\mathrm{p}=0.42)$.

\section{Outcomes}

Hospital outcome was known for 142 participants, 53 had unknown outcomes or self-discharged against medical advice. Median time to death was 3 days (IQR 1-9 days) among those known to have died, and median length of hospitalization was 7 days (IQR 4-10 days) for participants known to have survived to hospital discharge. Among those with known outcomes, there was a non-significant decline in mortality from $57 \%$ in cohort one to $41 \%$ in cohort three $(\mathrm{p}=0.27)$ (Table 4$)$. Assuming that $50 \%$ of those with unknown outcome died, and adjusting for
ART status and definite TBM diagnosis at hospitalization, the odds of dying were approximately twice as high for cohort one (aOR 1.7 95\% CI $[0.7,4.4])$ and cohort two $(1.8[0.6,5.6])$ as compared to cohort three. Assuming that $75 \%$ of those with unknown outcome died, adjusted odds of death increase further, cohort one $(4.0[1.5,10.9])$ and cohort two $(2.0[0.6,6.7])$ compared to cohort three (Table 4, Figure 4).

\section{Discussion}

Rapid molecular diagnostics have been predicted to reduce TBrelated mortality ${ }^{22}$ but little is reported about the impact of Xpert on TBM-related mortality. Here we report diagnosis and clinical outcomes among hospitalized Ugandans treated for TB meningitis over a 6.5 -year period. In-hospital mortality was high in the cohort overall (44\% 95\% CI [36,52\%]), similar to other research settings with high HIV prevalence ${ }^{7,19,23,24}$. The adjusted model found that odds of in-hospital mortality were almost two-fold higher in the earliest cohort, tested by CSF smear microscopy only, compared to that of the most recent cohort in which Xpert (and culture) were routinely performed. Though severity of TBM at presentation was similar over the study period 
Table 2. Methods of Diagnosis.

\begin{tabular}{|c|c|c|c|c|c|}
\hline & Cohort 1 & Cohort 2 & Cohort 3 & & \\
\hline & AFB smear & $\begin{array}{c}\text { AFB smear } \\
\text { Xpert }\end{array}$ & $\begin{array}{c}\text { AFB smear } \\
\text { Xpert } \\
\text { Culture }\end{array}$ & Total & P-value ${ }^{s}$ \\
\hline \multicolumn{6}{|l|}{ All meningitis patients screened } \\
\hline Total number & 471 & 71 & 1130 & 1672 & \\
\hline Cryptococcal Antigen positive & 269 & 31 & 758 & 1058 & \\
\hline Cryptococcal Antigen negative & 187 & 38 & 333 & 558 & \\
\hline \multicolumn{6}{|l|}{ TBM diagnostic tests performed } \\
\hline \multicolumn{6}{|l|}{ CSF AFB smear microscopy* } \\
\hline N AFB performed & 466 & 71 & 281 & 818 & \\
\hline N AFB positive & $1(0 \%)$ & $0(0 \%)$ & $4(2 \%)$ & $5(1 \%)$ & \\
\hline \multicolumn{6}{|l|}{ CSF TB culture } \\
\hline N TB culture performed & 0 & 0 & 321 & 321 & \\
\hline N TB culture positive & 0 & 0 & $39(12 \%)$ & $39(12 \%)$ & \\
\hline \multicolumn{6}{|l|}{ CSF Xpert MTB/Rif } \\
\hline N Xpert performed (realtime) & 0 & 71 & 384 & 455 & \\
\hline N Xpert positive & 0 & $13(18 \%)$ & $38(10 \%)$ & $51(11 \%)$ & \\
\hline \multicolumn{6}{|l|}{ Uniform case definition } \\
\hline Definite & $1(3 \%)$ & $13(87 \%)$ & $60(41 \%)$ & $74(38 \%)$ & $<.01$ \\
\hline Probable & $5(15 \%)$ & $2(13 \%)$ & $11(7 \%)$ & 18 (9\%) & \\
\hline Possible & $22(67 \%)$ & $0(0 \%)$ & $53(36 \%)$ & 75 (38\%) & \\
\hline Not & $5(15 \%)$ & $0(0 \%)$ & $23(16 \%)$ & $28(14 \%)$ & \\
\hline
\end{tabular}

Prior to November, 2013 any patient not prospectively tested with Xpert was considered in Cohort $1{ }^{*}$ AFB smear was initially performed on all meningitis patients regardless of CSF Cryptococcal antigen result. From October 2013, it was only performed on those with a negative Cryptococcal antigen, and was later stopped altogether in Mbarara. ${ }^{\$}$ P-value from Fisher's exact test

Table 3. Summary of concordance between Xpert MTB/Rif and MGIT culture results.

\begin{tabular}{|l|c|c|c|c|c|}
\hline \multicolumn{2}{|c|}{ Diagnostic Test } & \multicolumn{2}{c|}{ Xpert MTB/Rif } & Total & P-value \\
\hline & positive & negative & & 0.423 \\
\hline \multirow{2}{*}{ MGIT culture } & positive & 17 & 22 & 39 & \\
\cline { 2 - 6 } & negative & 17 & 62 & 79 & \\
\hline Total & & 34 & 84 & 118 & \\
\hline
\end{tabular}

P-value from McNemar's test

$\mathrm{N}=118$ (with both Xpert and culture results) 


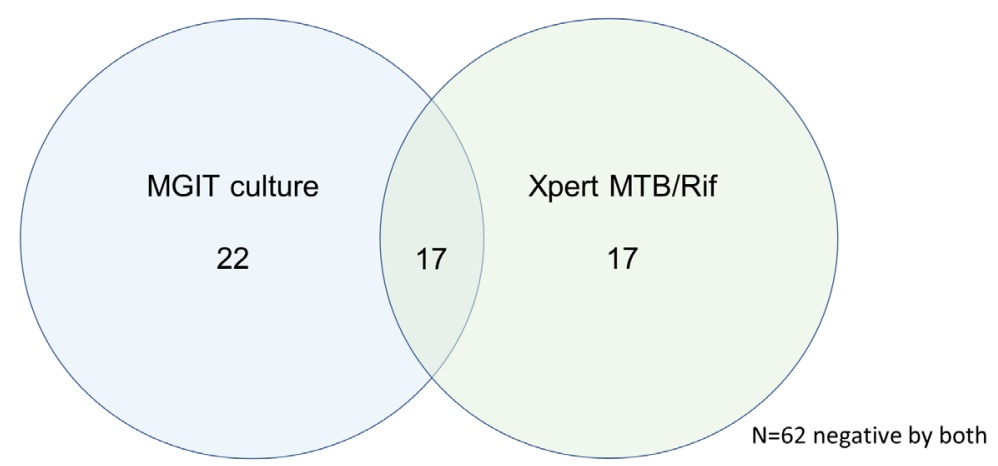

Figure 3. Venn diagram Illustrating the overlap of positive MGIT culture and Xpert test results in the $\mathrm{n}=118$ samples tested with both assays. A total of 118 adults were tested with both MGIT culture and Xpert, of which 22 were positive by MGIT culture, 17 by Xpert and 17 by both tests. Neither test performed better than the other, $p=0.423$ by McNemar's. A kappa statistics value of $0.2395 \% \mathrm{Cl}[0.04,0.41], p=0.01$, suggests only slight agreement of the two assays.

Table 4. Hospital outcomes.

\begin{tabular}{|c|c|c|c|c|c|}
\hline & $\begin{array}{l}\text { Cohort } 1 \\
\text { Nov } 2010 \text { to } \\
\text { May } 2013\end{array}$ & $\begin{array}{l}\text { Cohort } 2 \\
\text { Apr } 2011 \text { to } \\
\text { Nov } 2013\end{array}$ & $\begin{array}{l}\text { Cohort } 3 \\
\text { Nov } 2013 \text { to } \\
\text { May } 2017\end{array}$ & & \\
\hline Diagnostics used & AFB smear & $\begin{array}{c}\text { AFB smear } \\
\text { Xpert }\end{array}$ & $\begin{array}{c}\text { AFB smear } \\
\text { Xpert } \\
\text { Culture }\end{array}$ & Total & P-value* \\
\hline $\mathrm{N}$ in TBM case cohort & 33 & 15 & 147 & 195 & \\
\hline \multicolumn{6}{|c|}{ Outcome of hospitalization } \\
\hline Unknown & $26(79 \%)$ & $4(27 \%)$ & $23(16 \%)$ & $53(27 \%)$ & \\
\hline Known & $7(21 \%)$ & $11(73 \%)$ & $124(84 \%)$ & $142(73 \%)$ & \\
\hline Discharged Alive & $3(43 \%)$ & $4(36 \%)$ & $73(59 \%)$ & $80(56 \%)$ & 0.27 \\
\hline Died & $4(57 \%)$ & $7(64 \%)$ & $51(41 \%)$ & $62(44 \%)$ & \\
\hline
\end{tabular}

Assuming $50 \%$ of unknowns died

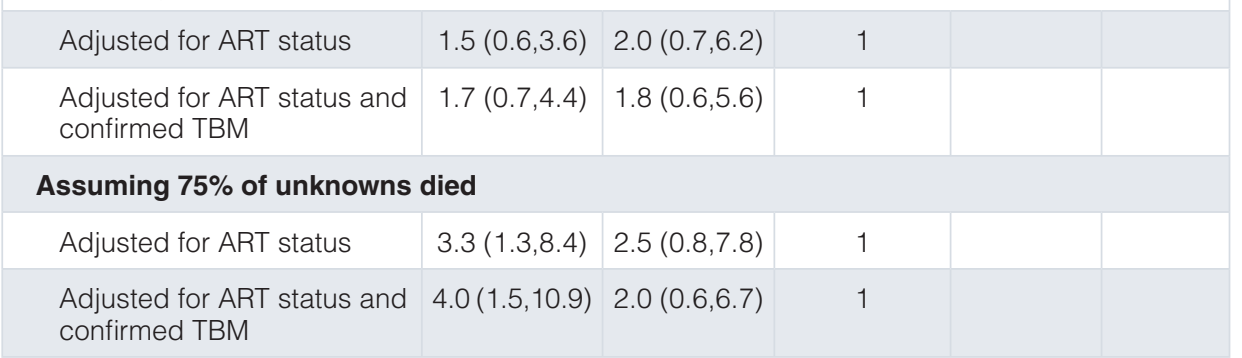

Overall median $(\mathrm{IQR})$ time in hospital was $7(4,10)$ days among those who were known to be discharged alive, and $3(1,9)$ days among those who were known to have died in hospital

*P-value from Fisher's exact test comparing KNOWN discharged alive vs KNOWN died; Odds ratios are the odds of being discharged alive, assuming $50 \%$ and $75 \%$ of those with unknown outcome died

and TBM treatment recommendations have not changed for Uganda, there are multiple other potential confounding factors due to the nature of the study. Improved access to ART, strengthening in healthcare services, increased awareness of TBM amongst communities and healthcare workers or changes in employment of empiric treatment all may have occurred over the study period and if present, may have impacted our findings. Due to these potential confounding factors, it is not possible to draw a conclusion about whether Xpert has reduced TBM mortality in this setting. 


\section{Odds Ratios for Mortality (by TBM Testing Cohort)}

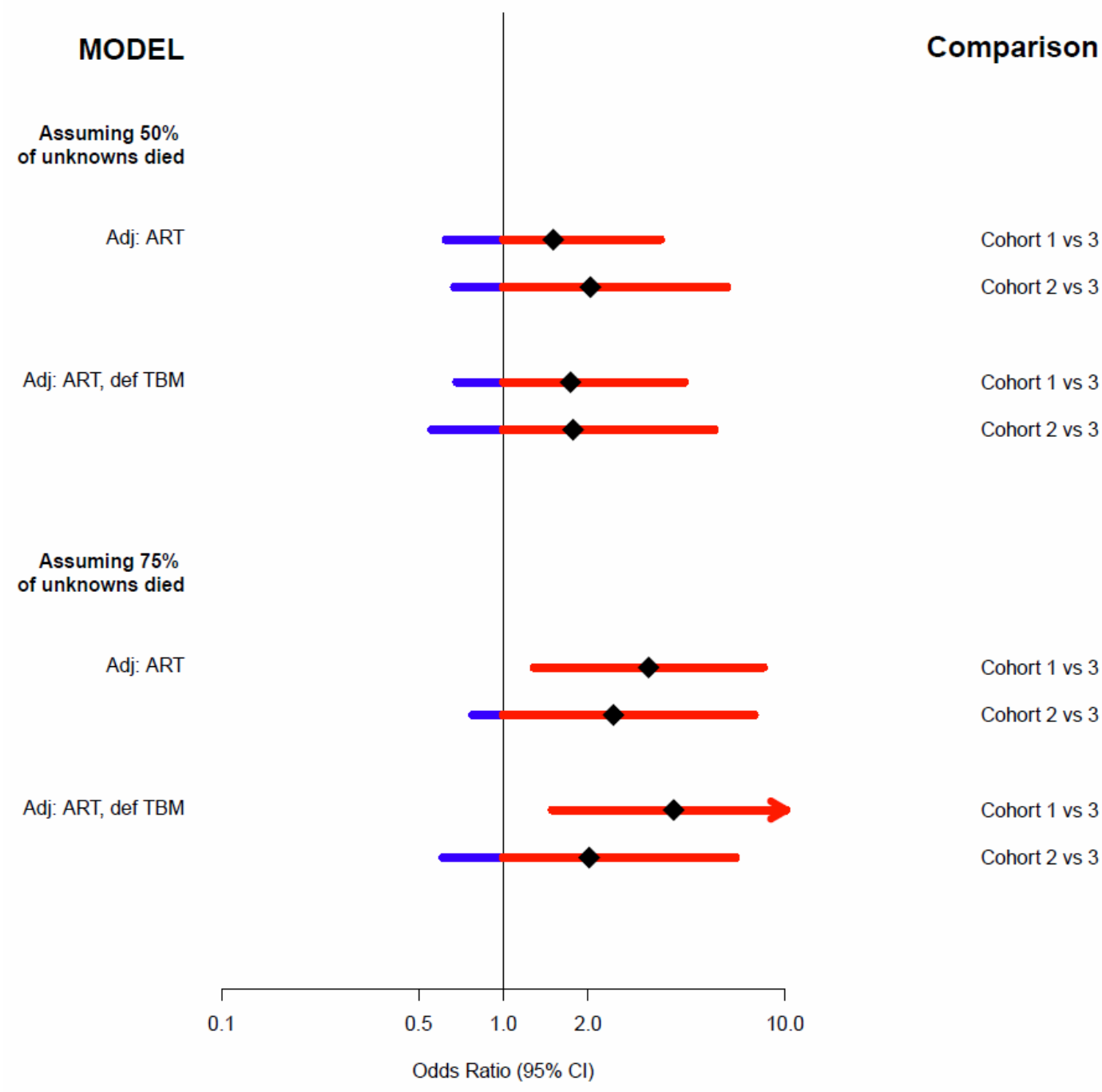

Figure 4. Illustration of odds of dying in cohort one and two compared to cohort three in a multivariate model. Odds ratios (and 95\% confidence intervals) for death by the end of hospitalization comparing cohorts 1 and 2 to cohort 3 , computed from multivariable logistic regression models with imputed data, adjusted for (1) ART status, and (2) ART status and definite TBM diagnosis. Data for patients with unknown outcome was imputed to assume that $50 \%$ within each cohort died, or that $75 \%$ died. In all models, neither ART nor definite TBM status had a significant association with in-hospital mortality, adjusted for cohort.

The proportion of ART experienced subjects increased significantly over time with improved access to ART treatment in Uganda and because the parent trial in cohort one enrolled only ART naïve subjects ${ }^{25}$. Although ART status was not associated with mortality, we adjusted for ART in multivariable models due to the large discrepancy in ART status between cohorts. Nonetheless the impact of ART use is likely to have played a role in the observed decline in mortality.

Despite a non-significant decline in mortality, a current casefatality rate of $41 \%$ remains unacceptably high and highlights the remaining work required to achieve the WHO goal of reducing TB-related deaths by $90 \%$ by $2030^{26}$. Initiating treatment in the early stage of disease is the single most important factor in improving outcomes ${ }^{7}$. Earlier presentation to the hospital is essential for prompt diagnosis and treatment initiation, yet, $83 \%$ of our cohort presented with MRC grade II or III disease.

Once the patient presents to care, an affordable, rapid, and reliable test that can effectively confirm or rule out TBM is crucial for prompt diagnosis. In this predominantly HIV-positive TBM cohort, sensitivity of Xpert was $63 \%$ against the composite reference standard. Thus, even though results were available rapidly, Xpert missed over one in three cases. The next generation assay Xpert MTB/Rif Ultra has an analytic limit of detection of 15 colony forming units $(\mathrm{CFU}) / \mathrm{ml}$, compared to $113 \mathrm{CFU} / \mathrm{ml}$ for $\mathrm{Xpert}^{27}$. Ultra appears to be significantly more sensitive than Xpert or culture for the diagnosis of TBM $(95 \%$ 
versus $45 \%$ and $45 \%$ respectively, $\mathrm{P}<0.001)^{28}$. Whether Ultra can reduce diagnostic delay and improve outcome from TBM requires further prospective evaluation.

Where both Xpert and MGIT had been done, less than a third $(23 \%, 17 / 74)$ of confirmed cases were positive by both modalities. This is consistent with prior findings and is likely due to the relatively higher sensitivity of culture versus Xpert, and the ability of Xpert to detect non-viable TB bacilli damaged by host-immune response and/or antituberculous drug therapy ${ }^{13,28}$. Neither test performed better than the other $(\mathrm{P}=0.42)$ and currently CSF culture continues to earn a place in TBM diagnostics testing algorithms.

Until a rapid test with suitable negative predictive value is widely available, there is likely to be on-going heterogeneity in clinical practice regarding frequency of empiric TBM therapy (TB treatment in the absence of a positive result). Here, Mulago Hospital participants were treated for TBM on an empiric basis in under one quarter of cases as opposed to over three quarters of cases at Mbarara Hospital, despite the same diagnostic armamentarium being available in both settings. In our study settings, it is likely that individual or departmental clinical practice and risk thresholds for TB treatment initiation dictate this variation. Though empiric TBM therapy is potentially life-saving, significant risks such as side effects, drug-interactions and adjunctive steroids in an already immunosuppressed population need to be considered. Ideally, a rapid, accurate test allows therapy for TB meningitis to be started promptly only in those who actually have TBM. Overall, the proportion with microbiologically confirmed TBM increased significantly from $3 \%$ in cohort one to $41 \%$ in cohort three $(\mathrm{P}<0.01)$. In cohort two, selection bias could have impacted on results as Xpert was only performed in cases where there was extremely high index of suspicion and empiric treatment was given only twice in those with a negative Xpert $(4 \%, 2 / 56)$. The low number of empiric diagnoses during this period were likely due to over-confidence in Xpert's ability to rule-out TBM. As understanding regarding the limitations of Xpert for the diagnosis of TBM became known, empiric TBM treatment rose ${ }^{14}$.

Limitations of this study relate to the nature of available data namely missing data on hospital and long-term outcomes, the time to starting TB treatment, unbalanced numbers in each cohort, and other un-adjusted confounding factors and potential sources of bias. When imputing data in the model we assumed that either $50 \%$ or $75 \%$ of patients with unknown outcome actually died, which is a clinically reasonable judgment for this population ${ }^{4}$.
Here we present important data on diagnostic confirmation and TBM mortality during a period of TB diagnostic evolution. There has been a significant increase in microbiological confirmation and a modest, albeit non-significant, decline in mortality since introduction of Xpert and culture in our study setting. An on-going multifaceted approach is needed to further reduce death and disability from TBM.

\section{Consent}

Written informed consent for publication of the anonymised data was obtained from the participants or their surrogates.

\section{Data availability}

The database contains individual level data and as such is not available through an open-access data repository. The database is stored on a secure server at University of Minnesota. Researchers interested in accessing the data can contact the corresponding author (FVC), the last author (DRB) or the Division of Biostatistics at the University of Minnesota. Data access will be granted to active researchers in the field with the agreement of the authors.

\section{Competing interests}

No competing interests were disclosed.

\section{Grant information}

FVC is supported by the Wellcome Trust [210772/Z/18/Z]. ASB, DBM, and DRB are supported by Fogarty International Center and National Institute of Neurologic Diseases and Stroke [R01NS086312].

The funders had no role in study design, data collection and analysis, decision to publish, or preparation of the manuscript.

\section{Acknowledgements}

FVC is an honorary fellow of the Makerere University - Uganda Virus Research Institute Centre of Excellence for Infection and Immunity Research and Training (MUII-plus). MUII-plus is supported through the DELTAS Africa Initiative (grant no. 107743). The DELTAS Africa Initiative is an independent funding scheme of the African Academy of Sciences (AAS), Alliance for Accelerating Excellence in Science in Africa (AESA), and supported by the New Partnership for Africa's Development Planning and Coordinating Agency (NEPAD Agency) with funding from the Wellcome Trust (grant no. 107743) and the UK Government.
1. Jarvis JN, Meintjes G, Williams A, et al.: Adult meningitis in a setting of high HIV and TB prevalence: findings from 4961 suspected cases. BMC Infect Dis. 2010; 10: 67 .

PubMed Abstract | Publisher Full Text | Free Full Text
2. Durski KN, Kuntz KM, Yasukawa K, et al:: Cost-effective diagnostic checklists for meningitis in resource-limited settings. J Acquir Immune Defic Syndr. 2013; 63(3): e101-8.

PubMed Abstract | Publisher Full Text | Free Full Text 
3. World Health Organization: Global Tuberculosis Report 2017. Geneva: World Health Organisation, 2017.

Reference Source

4. Woldeamanuel YW, Girma B: A 43-year systematic review and meta-analysis: case-fatality and risk of death among adults with tuberculous meningitis in Africa. J Neurol. 2014; 261(5): 851-65.

PubMed Abstract | Publisher Full Text

5. Mai NT, Thwaites GE: Recent advances in the diagnosis and management of tuberculous meningitis. Curr Opin Infect Dis. 2017; 30(1): 123-8. PubMed Abstract

6. Brancusi F, Farrar J, Heemskerk D: Tuberculous meningitis in adults: a review of a decade of developments focusing on prognostic factors for outcome. Future Microbiol. 2012; 7(9): 1101-16.

PubMed Abstract | Publisher Full Text

7. Wilkinson RJ, Rohlwink U, Misra UK, et al:: Tuberculous meningitis. Nat Rev Neurol. 2017; 13(10): 581-598.

PubMed Abstract | Publisher Full Text

8. Boulware DR: Utility of the Xpert MTB/RIF assay for diagnosis of tuberculous meningitis. PLoS Med. 2013; 10(10): e1001537.

PubMed Abstract | Publisher Full Text | Free Full Text

9. Denkinger CM, Schumacher SG, Boehme CC, et al:: Xpert MTB/RIF assay for the diagnosis of extrapulmonary tuberculosis: a systematic review and metaanalysis. Eur Respir J. 2014; 44(2): 435-46. PubMed Abstract | Publisher Full Text

10. Patel VB, Theron G, Lenders $L$, et al.: Diagnostic accuracy of quantitative PCR (Xpert MTB/RIF) for tuberculous meningitis in a high burden setting: a prospective study. PLoS Med. 2013; 10(10): e1001536. PubMed Abstract | Publisher Full Text | Free Full Text

11. Marais S, Thwaites G, Schoeman JF, et al:: Tuberculous meningitis: a uniform case definition for use in clinical research. Lancet Infect Dis. 2010; 10(11): 803-12.

PubMed Abstract | Publisher Full Text

12. Nhu NT, Heemskerk D, Thu do DA, et al.: Evaluation of GeneXpert MTB/RIF for diagnosis of tuberculous meningitis. J Clin Microbiol. 2014; 52(1): 226-33. PubMed Abstract | Publisher Full Text | Free Full Text

13. Bahr NC, Tugume L, Rajasingham R, et al:: Improved diagnostic sensitivity for tuberculous meningitis with Xpert(®) MTB/RIF of centrifuged CSF. Int J Tuberc Lung Dis. 2015; 19(10): 1209-15.

PubMed Abstract | Publisher Full Text | Free Full Text

14. Bahr NC, Marais S, Caws M, et al.: GeneXpert MTB/Rif to Diagnose Tuberculou Meningitis: Perhaps the First Test but not the Last. Clin Infect Dis. 2016; 62(9): $1133-5$.

PubMed Abstract | Publisher Full Text | Free Full Text

15. Yoon C, Cattamanchi A, Davis JL, et al.: Impact of Xpert MTB/RIF testing on tuberculosis management and outcomes in hospitalized patients in Uganda. PLoS One. 2012; 7(11): e48599.

PubMed Abstract | Publisher Full Text | Free Full Text
16. Theron G, Zijenah L, Chanda D, et al:: Feasibility, accuracy, and clinical effect of point-of-care Xpert MTB/RIF testing for tuberculosis in primary-care settings in Africa: a multicentre, randomised, controlled trial. Lancet. 2014; 383(9915): 424-35.

PubMed Abstract | Publisher Full Text

17. Boehme CC, Nabeta P, Hillemann D, et al.: Rapid molecular detection of tuberculosis and rifampin resistance. N Engl J Med. 2010; 363(11): 1005-15. PubMed Abstract | Publisher Full Text | Free Full Text

18. Boehme CC, Nicol MP, Nabeta $P$, et al.: Feasibility, diagnostic accuracy, and effectiveness of decentralised use of the Xpert MTB/RIF test for diagnosis of tuberculosis and multidrug resistance: a multicentre implementation study. Lancet. 2011; 377(9776): 1495-505.

PubMed Abstract | Publisher Full Text | Free Full Text

19. Török ME, Yen NT, Chau TT, et al.: Timing of initiation of antiretroviral therapy in human immunodeficiency virus (HIV)--associated tuberculous meningitis. Clin Infect Dis. 2011; 52(11): 1374-83.

PubMed Abstract | Publisher Full Text | Free Full Text

20. Uganda Population-Based HIV Impact Assessment. 2016. Accessed April 11, 2018 Reference Source

21. Uganda National Tuberculosis and Leprosy Control Programme: Manual for Management and Control of Tuberculosis and Leprosy. 2017. Reference Source

22. Ramsay A, Steingart KR, Pai M: Assessing the impact of new diagnostics on tuberculosis control. Int J Tuberc Lung Dis. 2010; 14(12): 1506-7. PubMed Abstract

23. Heemskerk AD, Bang ND, Mai NT, et al.: Intensified Antituberculosis Therapy in Adults with Tuberculous Meningitis. N Engl J Med. 2016; 374(2): 124-34. PubMed Abstract | Publisher Full Text

24. Ruslami R, Ganiem AR, Aarnoutse RE, et al.: Rifampicin and moxifloxacin fo tuberculous meningitis--authors' reply. Lancet Infect Dis. 2013; 13(7): 570. PubMed Abstract | Publisher Full Text

25. Boulware DR, Meya DB, Muzoora C, et al:: Timing of antiretroviral therapy after diagnosis of cryptococcal meningitis. $N$ Engl $J$ Med. 2014; 370(26): 2487-98.

PubMed Abstract | Publisher Full Text | Free Full Text

26. World Health Organization: The End TB Strategy. Geneva, Switzerland: World Health Organisations, 2015 Reference Source

27. Chakravorty S, Simmons AM, Rowneki M, et al: The New Xpert MTB/RIF Ultra: Improving Detection of Mycobacterium tuberculosis and Resistance to Rifampin in an Assay Suitable for Point-of-Care Testing. MBio. 2017; 8(4): pii: e00812-17.

PubMed Abstract | Publisher Full Text | Free Full Text

28. Bahr NC, Nuwagira E, Evans EE, et al.: Diagnostic accuracy of Xpert MTB/RIF Ultra for tuberculous meningitis in HIV-infected adults: a prospective cohort study. Lancet Infect Dis. 2018; 18(1): 68-75.

PubMed Abstract | Publisher Full Text | Free Full Text 


\section{Chapter Five: Diagnostic Accuracy of Xpert MTB/RIF Ultra for Tuberculous Meningitis in HIV-infected Adults: A Prospective Validation (research paper)}

\section{Foreword}

The findings of the retrospective cohort study in chapter 4 supported the idea that molecular diagnostics can improve microbiological confirmation of TBM, and may be a contributory factor in improving survival from the disease. It is known that TBM diagnostic delay leads to poor outcomes and therefore near-point-of-care diagnostics are particularly needed to reduce time to treatment initiation. ${ }^{1}$ However, with only a quarter of treated TBM cases in chapter 4 having a positive CSF Xpert MTB/Rif (26\%, 38/147 in cohort 3, Nov 2013 to May 2017), it suggested that a more sensitive molecular test was urgently needed. The lack of a reliable POC TBM diagnostic test contributes to missed or delayed diagnosis in some patients and over treatment in others, with associated drug toxicity, drug-drug interactions, pill burden and the risks of corticosteroids in an already immunocompromised population.

The next generation Xpert MTB/Rif Ultra (Xpert Ultra) assay, re-engineered to be more sensitive in paucibacillary forms of TBM than Xpert MTB/Rif, was recommended by the WHO in 2017. ${ }^{2}$ The diagnostic performance of Xpert Ultra needs to be evaluated in different populations and types of TB so that clinicians and policy-makers know how to interpret the results.

The evaluation of novel diagnostics in TBM is complicated by the fact that there is no single 'gold standard' test to use as a reference. Typically, in pulmonary TB, novel tests are measured against mycobacterial culture alone, but in TBM we know this is only $26-67 \%$ sensitive so its use as a gold standard leads to misclassification of the index test. ${ }^{3}$ A composite microbiological reference standard (e.g. a combination of TB-specific tests) is more accurate than culture alone or alternatively the consensus uniform clinical case definition (as described in section 1.3.2 and Appendix 2) should be used. ${ }^{4}$ Though, should we use probable/definite or possible/probable/definite as the reference standard in TBM? Which one is likely to represent the most genuine TBM cases? Each of these options has its own limitations and presenting a combination of these possibilities gives the reader the most complete picture of test performance, albeit with caveats. In the prior study of Xpert Ultra on cryopreserved CSF samples (described in section 1.3.5) we used 'probable/definite' as the reference standard as we were concerned that 'possible' TBM would be a non-TB-specific heterogeneous category which might include a variety of other infectious and non-infectious aetiologies in advanced immunosuppression. As such, including 'possible' in the reference standard would give rise to a falsely low sensitivity of the TB index test. The recent Heemskerk et al. multi-center diagnostic study of modified ZN stain, used 'possible/probable/definite' as the reference standard. Inclusion of the 'possible' category in the 
reference standard in this study, which included one-third PLHIV, gave rise to a (perhaps falsely) low sensitivity of Xpert of $25 \%$ and culture of $32 \%{ }^{5}$

The ideal is to perform a full diagnostic work-up with the aim of identifying an alternative explanation for the patient's symptoms; when an alternative diagnosis is confirmed microbiologically (stain, culture, or NAAT), serologically, or histopathologically, that individual leaves the 'possible or probable' group and enters the 'not' TBM group, thereby making the uniform case definition a more accurate reference standard. The 2010 uniform case definition states "the list of alternative diagnoses that should be considered include: pyogenic bacterial meningitis, cryptococcal meningitis, syphilitic meningitis, viral meningoencephalitis, cerebral malaria, parasitic or eosinophilic meningitis (Angiostrongylus cantonesis, Gnathostoma spinigerum, toxocariasis, cysticercosis), cerebral toxoplasmosis and bacterial brain abscess (space-occupying lesion on cerebral imaging) and malignancy (e.g. lymphoma)" ${ }^{4}$ Unbiased metagenomic next generation sequencing (mNGS) might therefore be particularly helpful in identifying alternative aetiologies of disease in the 'possible' or 'probable' group, allowing reclassification as 'not' TBM. Equally, biased sequencing with prior enrichment of M. tuberculosis DNA might identify TB that had been missed in the 'possible or probable' group allowing it to be classified as 'definite'.

In this prospective study of 204 patients with suspected TBM at two referral hospitals in Uganda I aimed to accurately assess of the diagnostic accuracy of Xpert Ultra by optimising the reference standard through inclusion of the Biofire Filmarray meningoencephalitis PCR (for 14 viral and bacterial pathogens), post-mortem examination in people who died without a confirmed diagnosis, and storage of CSF samples for mNGS. This research paper tested hypothesis \#1: Xpert Ultra is significantly more sensitive than Xpert MTB/Rif for the detection of M. tuberculosis in CSF. However, Xpert Ultra's sensitivity will be imperfect and it will fail to diagnose a small number of cases of TBM that are evident by CSF culture, post-mortem examination, or metagenomic next generation sequencing.

In this study I confirmed that Xpert Ultra is significantly more sensitive that Xpert MTB/Rif against both the reference standards used when testing fresh CSF samples of patients with suspected TBM. The sensitivity of Xpert Ultra was $77 \%(95 \% \mathrm{Cl}, 63-87 \%)$ against the reference standard of probable/definite TBM and negative predictive value was $93 \%(95 \% \mathrm{Cl}, 87-96 \%)$. The specificity of Xpert Ultra was $96 \%(95 \% \mathrm{Cl}, 91-98 \%)$ when the Ultra result was excluded in assigning the case definition. A total of 4 post-mortem examinations were performed of which one had macroscopic evidence of meningitis and one had evidence of disseminated TB. If these two cases were categorised as definite TBM then the sensitivity and negative predictive value of Xpert Ultra would be $89 \%$ and $97 \%$ respectively against the composite reference standard, and $74 \%$ and $92 \%$ respectively against probable/definite TBM. 
In the univariate analysis the following baseline factors were associated with definte TBM: low GCS, CSF white cell pleocytosis, low CSF glucose, high CSF lactate, high CSF protein. Every doubling in CSF lactate resulted in a $\sim 6$-fold increase in the odds of definite TBM. In the multivariate analysis low CSF glucose remained strongly associated with definite TBM - every doubling of CSF glucose resulted in a $70 \%$ decrease in the odds of definite TBM. It was not possible to examine CSF lactate in the multivariable model.

At the time of writing, 84 of the mNGS results for the 204 patients in the prospective Xpert Ultra cohort were available. Among the 9 participants with 'probable' TBM who were included in the 'probable/definite' TBM reference standard there were no alternative pathogens and no missed $M$. tuberculosis infections identified by mNGS (mNGS results were available for 5/9 participants). Therefore, the number of participants in the 'probable' TBM reference category remained unchanged by mNGS and Xpert Ultra's diagnostic accuracy results stand as they are in the published paper. Although reads to cytomegalovirus (CMV, 10 reads per million (rpm)), Epstein Barr virus (EBV, 4 rpm) and pegivirus (5998 rpm) were identified in the 'probable' group these were deemed to be non-clinically significant. In the 'possible' TBM group mNGS were available for 27 patients including 3 clinically significant infections (Wesselbrons virus 1414 rpm, Varicella Zoster virus 98933 rpm, CMV 68503 rpm).

Of the 39 cases in the 'definite' TBM group who were positive by Xpert Ultra we have mNGS results available for 17 patients. mNGS detected M. tuberculosis DNA in 65\% (11/17, 2 to 540 rpm) but did not identify $M$. tuberculosis DNA in 35\% (6/17) definite TBM cases. We therefore cannot hold out a great deal of hope that mNGS is going to be more sensitive than Xpert Ultra in the detection of M. tuberculosis DNA, though improvements in the collection, storage and the sequencing process may improve its performance in future.

As described in section 1.3.5.3, the mNGS research is part of a wider ongoing collaboration between Dr Meya and Professor Boulware's Ugandan team and Dr Michael Wilson at UCSF. The mNGS sample processing and bioinformatic work was not undertaken by myself, rather by Dr Prashanth Ramachandran (Dr Wilson's postdoctoral researcher) and the results of the wider sequencing study are being finalised and written up by $\mathrm{Dr}$ Ramachandran currently. To date, three relevant abstracts (a) viral pathogens in a TBM cohort, b) a combined host and pathogen classifier, c) enrichment techniques for TB] have been presented at the American Academy of Neurology conference, Appendix 6a-c and are referred to in the chapter 10 discussion. 
1. Sheu JJ, Hsu CY, Yuan RY, Yang CC. Clinical characteristics and treatment delay of cerebral infarction in tuberculous meningitis. Intern Med J 2012; 42(3): 294-300.

2. Chakravorty S, Simmons AM, Rowneki M, et al. The New Xpert MTB/RIF Ultra: Improving Detection of Mycobacterium tuberculosis and Resistance to Rifampin in an Assay Suitable for Point-of-Care Testing. MBio 2017; 8(4).

3. Donovan J, Cresswell FV, Thuong NTT, et al. Xpert MTB/RIF Ultra for the Diagnosis of Tuberculous Meningitis: A Small Step Forward. Clin Infect Dis 2020.

4. Marais S, Thwaites G, Schoeman JF, et al. Tuberculous meningitis: a uniform case definition for use in clinical research. Lancet Infect Dis 2010; 10(11): 803-12.

5. Heemskerk AD, Donovan J, Thu DDA, et al. Improving the microbiological diagnosis of tuberculous meningitis: A prospective, international, multicentre comparison of conventional and modified Ziehl-Neelsen stain, GeneXpert, and culture of cerebrospinal fluid. J Infect 2018. 


\section{RESEARCH PAPER COVER SHEET}

\section{PLEASE NOTE THAT A COVER SHEET MUST BE COMPLETED FOR EACH RESEARCH PAPER INCLUDED IN A THESIS.}

\section{SECTION A - Student Details}

\begin{tabular}{|l|l|}
\hline Student & Dr Fiona Cresswell \\
\hline Principal Supervisor & Prof Alison Elliott \\
\hline Thesis Title & $\begin{array}{l}\text { Improving diagnosis and treatment of HIV-associated } \\
\text { Tuberculous meningitis }\end{array}$ \\
\hline
\end{tabular}

If the Research Paper has previously been published please complete Section B, if not please move to Section C

\section{SECTION B - Paper already published}

\begin{tabular}{|l|l|l|l|}
\hline Where was the work published? & Lancet Infectious Diseases \\
\hline When was the work published? & 7 th Jan 2020 \\
\hline $\begin{array}{l}\text { If the work was published prior to } \\
\text { registration for your research degree, } \\
\text { give a brief rationale for its inclusion }\end{array}$ & \multicolumn{3}{|l|}{} \\
\hline $\begin{array}{l}\text { Have you retained the copyright for the } \\
\text { work?* }\end{array}$ & Yes & $\begin{array}{l}\text { Was the work subject to } \\
\text { academic peer review? }\end{array}$ & Yes \\
\hline
\end{tabular}

*If yes, please attach evidence of retention. If no, or if the work is being included in its published format, please attach evidence of permission from the copyright holder (publisher or other author) to include this work.

\section{SECTION C - Prepared for publication, but not yet published}

\begin{tabular}{|l|l|}
\hline $\begin{array}{l}\text { Where is the work intended to be } \\
\text { published? }\end{array}$ & \\
\hline $\begin{array}{l}\text { Please list the paper's authors in the } \\
\text { intended authorship order: }\end{array}$ & \\
\hline Stage of publication & Choose an item. \\
\hline
\end{tabular}

\section{SECTION D - Multi-authored work}

For multi-authored work, give full details of your role in the research included in the paper and in the preparation of the paper. (Attach a further sheet if necessary)
I spend time on the hospital wards reviewing meningitis suspects, performing lumbar punctures and collecting clinical samples for the study. I followed up results in real-time, cleaned the data, undertook the analysis, interpreted the results and wrote the first draft of the manuscript. I handled all the referencing, submission, reviewer responses and re-submission process. 
Student Signature:

Supervisor Signature:
Date: _ 24th Sept 2020

Date: 8th October 2020 


\section{Xpert MTB/RIF Ultra for the diagnosis of HIV-associated tuberculous meningitis: a prospective validation study}

Fiona V Cresswell, Lillian Tugume, Nathan C Bahr, Richard Kwizera, Ananta S Bangdiwala, Abdu K Musubire, Morris Rutakingirwa, Enock Kagimu, Edwin Nuwagira, Edward Mpoza, Joshua Rhein, Darlisha A Williams, Conrad Muzoora, Daniel Grint, Alison M Elliott, David B Meya, David R Boulware, on behalf of the ASTRO-CM team

\section{Summary}

Introduction Tuberculous meningitis accounts for 1-5\% of tuberculosis cases. Diagnostic delay contributes to poor outcomes. We evaluated the performance of the new Xpert MTB/RIF Ultra (Xpert Ultra) for tuberculous meningitis diagnosis.

Methods In this prospective validation study, we tested the cerebrospinal fluid (CSF) of adults presenting with suspected meningitis (ie, headache or altered mental status with clinical signs of meningism) to the Mulago National Referral Hospital and Mbarara Regional Referral Hospital in Uganda. We centrifuged the CSF, resuspended the cell pellet in $2 \mathrm{~mL} \mathrm{CSF}$, and tested $0.5 \mathrm{~mL}$ aliquots with Xpert Ultra, Xpert MTB/RIF (Xpert), and mycobacterial growth indicator tube (MGIT) culture. We quantified diagnostic performance against the uniform case definition of probable or definite tuberculous meningitis and a composite microbiological reference standard.

Findings From Nov 25, 2016, to Jan 24, 2019, we screened 466 adults with suspected meningitis and tested 204 for tuberculous meningitis. Uniform clinical case definition classified 51 participants as having probable or definite tuberculous meningitis. Against this uniform case definition, Xpert Ultra had 76.5\% sensitivity (95\% CI 62 ·5-87.2; 39 of 51 patients) and a negative predictive value of $92 \cdot 7 \%(87 \cdot 6-96 \cdot 2 ; 153$ of 165), compared with $55 \cdot 6 \%$ sensitivity $(44 \cdot 0-70 \cdot 4 ; 25$ of $45 ; p=0 \cdot 0010)$ and a negative predictive value of $85 \cdot 8 \%(78 \cdot 9-91 \cdot 1 ; 121$ of 141$)$ for Xpert and $61.4 \%$ sensitivity $(45 \cdot 5-75 \cdot 6 ; 27$ of $44 ; \mathrm{p}=0.020)$ and negative predictive value of $85 \cdot 2 \%(77 \cdot 4-91 \cdot 1 ; 98$ of 115$)$ for MGIT culture. Against the composite microbiological reference standard, Xpert Ultra had sensitivity of $92.9 \%(80 \cdot 5-98 \cdot 5 ; 39$ of 42$)$, higher than Xpert at $65 \cdot 8 \%(48 \cdot 6-80 \cdot 4 ; 25$ of 38; $\mathrm{p}=0.0063)$ and MGIT culture at $72 \cdot 2 \%(55 \cdot 9-86 \cdot 2 ; 27$ of $37 ; \mathrm{p}=0 \cdot 092)$. Xpert Ultra detected nine tuberculous meningitis cases missed by Xpert and MGIT culture.

Interpretation Xpert Ultra detected tuberculous meningitis with higher sensitivity than Xpert and MGIT culture in this HIV-positive population. However, with a negative predictive value of $93 \%$, Xpert Ultra cannot be used as a ruleout test. Clinical judgment and novel highly sensitive point-of-care tests are still required.

Funding Wellcome Trust, National Institute of Health, National Institute of Neurologic Diseases and Stroke, Fogarty International Center, and National Institute of Allergy and Infectious Diseases.

Copyright (C) 2020 The Author(s). Published by Elsevier Ltd. This is an Open Access article under the CC BY 4.0 license.

\section{Introduction}

Worldwide, Mycobacterium tuberculosis affected more than 10 million people in 2018 , with devastating consequences, including around 1.5 million deaths. ${ }^{1}$ Tuberculous meningitis, the most serious form of tuberculosis infection, accounts for $1-5 \%$ of new cases of tuberculosis and results in death or substantial disability in more than half of those affected. ${ }^{2}$ Outcomes are particularly poor in patients with HIV co-infection, which can triple the risk of death from tuberculous meningitis, reaching approximately $50 \% .^{3}$ Another major driver of poor outcomes is diagnostic delay. ${ }^{4}$

Cerebrospinal fluid (CSF) smear microscopy with Ziehl-Neelsen staining for acid-fast bacilli is the cheapest and most widely available test for tuberculous meningitis diagnosis, but it is insensitive in most settings without expert microscopists. ${ }^{5-7}$ Culture takes a minimum of 2 weeks to provide results (too slow for clinical utility), has only moderate sensitivity (30-60\%), and is not readily available in most settings within low-income tuberculosis-endemic countries. ${ }^{8,6}$ Xpert MTB/RIF (Xpert; Cepheid, Sunnyvale, CA, USA)—a rapid, automated, cartridge-based molecular test-was endorsed by WHO in 2015 as the best initial test for tuberculous meningitis. ${ }^{9}$ Xpert has been deployed in 130 of 145 countries eligible for concessional pricing as of 2016. ${ }^{10}$ Xpert provides $45-67 \%$ sensitivity to detect microbiologically proven tuberculous meningitis, meaning a negative result does not provide adequate confidence that tuberculous meningitis is not present. . $^{8,1}$ Thus, empirical antituberculous therapy for tuberculous meningitis, with its associated drug toxicities, drugdrug interactions, pill burden, and cost, is still commonly used, often unnecessarily.

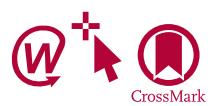

Lancet Infect Dis 2020

Published Online January 7,2020 https://doi.org/10.1016/ S1473-3099(19)30550-X

See Online/Comment https://doi.org/10.1016/ S1473-3099(19)30718-2 See Online/Articles https://doi.org/10.1016/ S1473-3099(19)30649-8

Clinical Research Department (FV Cresswell MBChB A M Elliott MD) and Tropical Epidemiology Group (D Grint PhD), London School of Hygiene \& Tropical Medicine, London, UK; Infectious Diseases Institute, College of Health Sciences, Makerere University, Kampala, Uganda (FV Cresswell, LTugume MBChB, R Kwizera MSc, A K Musubire MD, $M$ Rutakingirwa MBChB E Kagimu MBChB, E Mpoza MBChB, J Rhein MD D A Williams MPH, D B Meya PhD); Medical Research Council/Uganda Virus Research Institute and London School of Hygiene \& Tropical Medicine Uganda Research Unit, Entebbe, Uganda (FV Cresswell, A M Elliott); Division of Infectious Diseases, Department of Medicine, University of Kansas, Kansas City, KS, USA (Nathan C Bahr MD); Division of Infectious Diseases and International Medicine, Department of Medicine (D R Boulware MD, J Rhein) and Division of Biostatistics, School of Public Health (A S Bangdiwala MS), University of Minnesota, Minneapolis, MN, USA; and Mbarara University of Science and Technology, Mbarara, Uganda (E Nuwagira MMed, ( Muzoora MD)

Correspondence to: Dr Fiona V Cresswell, Research Department, Infectious Diseases Institute, Kampala, Uganda fiona.cresswell@Ishtm.ac.uk 
Research in context

\section{Evidence before this study}

We searched PubMed Central for reports of Xpert MTB/RIF Ultra (Xpert Ultra) for the diagnosis of tuberculosis using the terms ("Xpert MTB/RIF Ultra" or "Xpert Ultra") and ("tuberculosis" or "TB" or "tuberculous meningitis" or "TBM" or "extrapulmonary"). The search was done on Feb 14, 2019, with no search date or language restrictions.

In-vitro studies of Xpert MTB/RIF (Xpert) and Xpert Ultra assays have been done on sputum samples spiked with decreasing numbers of Mycobacterium tuberculosis H37Rv colony-forming units (CFUs), which found the limit of detection to be 16 CFUs per $\mathrm{mL}$ for Xpert Ultra versus 113 CFUs per $\mathrm{mL}$ for Xpert-a seven-times reduction in the limit of detection.

This improvement signified a promising step for the diagnosis of paucibacillary tuberculosis. Since then, several prospective and retrospective studies have evaluated Xpert Ultra in diagnosis of adult pulmonary tuberculosis (four), paediatric tuberculosis (two), extrapulmonary tuberculosis (two), paucibacillary tuberculosis (one), and tuberculous meningitis (one), all of which have found Xpert Ultra to be more sensitive than Xpert. The largest prospective diagnostic accuracy study to date, including 1753 participants with suspected pulmonary tuberculosis, found Xpert Ultra to have superior sensitivity to Xpert in smear-negative pulmonary tuberculosis ( $63 \%$ vs $46 \%$ ) and in HIV-associated pulmonary tuberculosis ( $90 \%$ vs $77 \%$ ), albeit with a more modest difference of 5.4 percentage points in the general pulmonary tuberculosis population. To date, only Bahr and colleagues have specifically focused on tuberculous meningitis, testing 129 cryopreserved CSF samples from HIV-positive Ugandan adults and finding sensitivities of $70 \%$ (16 of 23 patients) for Xpert Ultra and $43 \%$ (ten of 23 ) for Xpert against a uniform reference standard of probable or definite tuberculous meningitis. The sensitivity against a composite microbiological reference standard was 95\% (21 of 22) for Xpert Ultra and 45\% (ten of 22) for Xpert $(p=0.0010)$.

\section{Added value of this study}

To our knowledge, this prospective study is the largest to date to investigate the diagnostic performance of Xpert Ultra for tuberculous meningitis. It corroborates the findings from cryopreserved CSF samples and adds to the data on use of Xpert Ultra in HIV-positive populations and in paucibacillary forms of tuberculosis. We tested each sample with Xpert and Xpert Ultra to allow direct head-to-head comparisons of the performance of the two assays. To optimise the uniform case definition reference standard, we endeavoured to exclude other non-tuberculosis causes of meningitis by testing CSF with a meningoencephalitis PCR panel (testing for 14 viral, bacterial, and fungal pathogens) and offering post-mortem examinations when possible for participants who died without a microbiologically confirmed diagnosis.

\section{Implications of all the available evidence}

Our results support the WHO recommendations to use Xpert Ultra as the initial test for suspected tuberculous meningitis. With its run-time of $84 \mathrm{~min}$, Xpert Ultra, in the context of appropriate infrastructure, holds potential to provide same-day results and facilitate prompt tuberculous meningitis treatment. Nonetheless, Xpert Ultra's imperfect negative predictive value (92.7\% [95\% Cl 87.6-96.2] vs the uniform clinical case definition in this study) means that clinical judgment must override a negative Xpert Ultra result and empirical tuberculosis treatment is still warranted in cases where there is a high index of suspicion.
Subsequently, Xpert MTB/RIF Ultra (Xpert Ultra) was developed, which has a larger chamber $(50 \mu \mathrm{L})$ for DNA amplification than Xpert, allowing twice the volume of sample to reach the PCR reaction. Xpert Ultra also has two new multicopy DNA targets (IS6110 and IS1081), incorporates fully nested nucleic acid amplification, and uses melting temperature-based analysis instead of real-time PCR to improve the accuracy of rifampicinresistance detection. In vitro, these changes have made the limit of detection of $M$ tuberculosis seven times lower (16 vs 113 colony forming units [CFUs] per $\mathrm{mL}$ ). ${ }^{12,13}$ Another important benefit of Xpert Ultra is the shortened run time of $84 \mathrm{~min}$ (compared with $112 \mathrm{~min}$ for Xpert), enabling an extra five tests to be run on each module in a working day, equating to an extra 40 tests per day on an eight-module unit. To upgrade from Xpert, only new software and the Xpert Ultra cartridges are required-the same instrument can be used. Previous studies have shown that Xpert Ultra's sensitivity is markedly improved over Xpert's in smear-negative pulmonary tuberculosis (63\% vs $46 \%$ ) ${ }^{14} \mathrm{HIV}$-associated pulmonary tuberculosis (90\% vs 77\%), ${ }^{14}$ and paediatric tuberculosis $(74 \%$ vs $63 \%) .{ }^{15}$ The largest study to date of Xpert Ultra in tuberculous meningitis used cryopreserved CSF samples and found sensitivities of 95\% (95\% CI 77-99) for Xpert Ultra and the same sensitivity of $45 \%$ (24-68) for each of Xpert and mycobacterial growth indicator tube (MGIT) culture against a composite microbiological reference standard. ${ }^{8}$ Against the uniform clinical case definition of probable or definite tuberculous meningitis, ${ }^{16}$ sensitivity was $70 \%$ (47-87; 16 of 23 patients) for Xpert Ultra and $43 \%$ (23-66; ten of 23) for both culture and Xpert. ${ }^{8}$ Four additional small studies on CSF (containing a minimum of four and a maximum of 43 samples) corroborated the increased sensitivity of Xpert Ultra. ${ }^{17-20}$ Additional larger studies using real-time CSF samples and prespecified reference standards are needed to better inform the current understanding of Xpert Ultra's performance in tuberculous meningitis.

The aim of this study was to prospectively assess the diagnostic accuracy of Xpert Ultra for tuberculous 
meningitis compared with Xpert using fresh CSF specimens from an HIV-positive population.

\section{Methods}

\section{Study design and population}

In this diagnostic accuracy study, we prospectively evaluated adults (aged $\geq 18$ years) presenting consecutively to Mulago National Referral Hospital, Kampala, and Mbarara Regional Referral Hospital, Mbarara, Uganda, with suspected meningitis (headache for $>3$ days or altered mental status [Glasgow Coma Scale $<15$ ] with clinical signs of meningism at examination-ie, neck stiffness or Kernig's sign). The period of enrolment ran from Nov 26, 2016, to Jan 24, 2019, at Mulago National Referral Hospital and from Nov 25, 2016, to June 13, 2017, at Mbarara Regional Referral Hospital. Ethical approval was obtained from the Mulago Hospital, London School of Hygiene \& Tropical Medicine, and University of Minnesota institutional review boards and Uganda National Council of Science and Technology, and informed consent was obtained from participants or their surrogate (in patients with altered mental status) as part of the screening process for a meningitis clinical trial (NCT01802385) that subsequently rolled into an observational diagnostic study. The study was conducted in line with the Standards for Reporting Diagnostic Accuracy Studies. ${ }^{21}$

\section{Procedures}

Given the high prevalence of cryptococcus and the similar initial presentations between cryptococcal meningitis and tuberculous meningitis, fingerstick cryptococcal antigen lateral flow assay (LFA; Immy, Norman, OK, USA) was done at the participant's bedside. Thereafter, lumbar puncture was done for all participants, CSF opening pressure was recorded, and CSF was collected into a sterile tube with a target volume of more than $6 \mathrm{~mL}$. At the bedside, CSF glucose and lactate were measured from a drop of CSF collected into an Eppendorf tube using a handheld OneTouch glucose meter (OneTouch; Lifescan, Inverness, UK) and a point-of-care lactate meter (Nova Biomedical; Waltham, MA, USA). Xpert and Xpert Ultra results were returned to the study team within $24 \mathrm{~h}$ to guide treatment decisions.

Clinical history, physical examination, and detailed neurological assessment findings were recorded as recommended by the Tuberculous Meningitis International Research Consortium. ${ }^{22}$ Other diagnostic tests, including urine tuberculosis lipoarabinomannan (Alere Determine; Alere, Waltham, MA, USA), chest radiograph, abdominal ultrasonography, brain imaging, and sputum Xpert, were done as clinically indicated and when locally available.

\section{Microbiological testing}

Laboratory CSF testing included white blood cell count, lymphocyte percentage, differential Gram stain, aerobic bacterial culture, Ziehl-Neelsen acid-fast bacilli stain (Kampala only), total protein, CSF cryptococcal antigen, and Biofire FilmArray meningoencephalitis PCR panel (bioMérieux; Durham, NC, USA) for 14 meningoencephalitis pathogens (Streptococcus pneumoniae, Neisseria meningitidis, Listeria monocytogenes, Haemophilus influenzae, Streptococcus agalactiae, Escherichia coli, herpes simplex virus types 1 and 2, cytomegalovirus, varicella zoster virus, human herpes virus 6 , enterovirus, human parechovirus, and cryptococcus). All samples that were negative for CSF cryptococcal antigen underwent comprehensive tuberculosis diagnostic studies. Samples positive for cryptococcal antigen underwent fungal culture and, in the event of fungal growth, quantitative fungal culture count.

For tuberculosis analysis, the CSF was centrifuged at $3000 \mathrm{~g}$ for $20 \mathrm{~min}$ to concentrate bacilli into the cell pellet. Supernatant was pipetted out to leave a residual volume of $2 \mathrm{~mL}$ in which the cell pellet was re-suspended by vortexing. The resuspended cell pellet was then divided into four $0.5 \mathrm{~mL}$ aliquots for Xpert, Xpert Ultra, MGIT culture, and cryopreservation at $-80^{\circ} \mathrm{C}$ in case of invalid results from Xpert or Xpert Ultra or for future sequencing work. The aliquots for Xpert and Xpert Ultra were mixed with $1.5 \mathrm{~mL}$ of sample reagent, shaken vigorously, allowed to sit for $15 \mathrm{~min}$, and then run on the Cepheid platform. The $0.5 \mathrm{~mL}$ aliquot for MGIT culture was added to the MGIT tube and incubated in a Bactec 960 instrument (Becton Dickinson; Franklin Lakes, NJ, USA). The algorithm for CSF diagnostic testing is illustrated in the appendix (p 2). Where the volume of CSF was below $6 \mathrm{~mL}$, a stepwise approach was used to maximise the likelihood of yielding a diagnosis for the participant, as shown in the appendix ( $\mathrm{p} 4)$. At the clinician's discretion, participants with a positive test for CSF cryptococcal antigen were allowed to undergo tuberculous meningitis testing as cryptococcosis coinfection with tuberculous meningitis does occur, although rarely. ${ }^{23}$ In Mbarara, tuberculosis testing was done at the Médecins Sans Frontières Epicentre laboratory. In Kampala, testing was done at the Makerere University Microbiology Laboratory, Makerere University Mycobacteriology (BSL-3) laboratory (MGIT culture), and the Infectious Diseases Institute Translational laboratory (Xpert and Xpert Ultra). Clinical information was not available to the performers of the index or reference tests. The same laboratory technologist performed Xpert and Xpert Ultra, but MGIT results were read and reported by a different technologist.

Xpert results are reported as follows: $M$ tuberculosis not detected; invalid, if the internal quality control checks fail; or, if $M$ tuberculosis DNA is detected, a semiquantitative category of very low, low, medium, or high is given. Xpert Ultra results are reported in the same way but with the addition of the trace semi-quantitative category for the lowest bacillary loads (less than approximately 113 CFUs per $\mathrm{mL}$ ). Rifampicin resistance is reported as indeterminate for tests positive in the trace category as the DNA quantity is too low to adequately detect rifampicin resistance conferring mutations. 


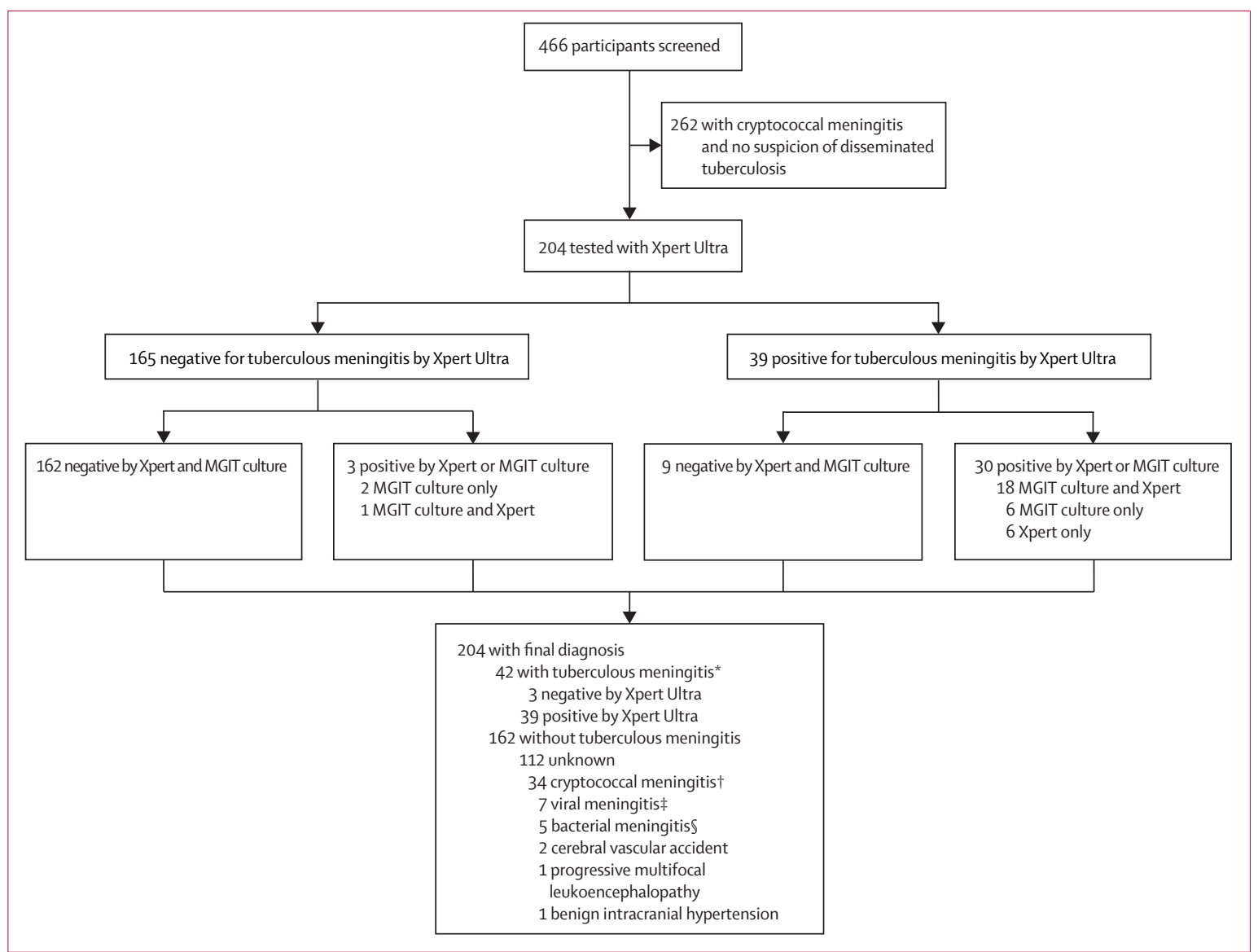

Figure 1: Flow diagram showing the diagnostic outcomes of the study population

Xpert=Xpert MTB/RIF. MGIT=mycobacterial growth indicator tube. HSV=herpes simplex virus. CSF=cerebrospinal fluid. *Five participants with confirmed tuberculous meningitis had a positive CSF cryptococcal antigen test, of whom two had culture-confirmed cryptococcal meningitis. $†$ Culture or PCR positive. $¥ T$ Two HSV type 1 , two HSV type 2, two varicella zoster virus, and one cytomegalovirus. SOne confirmed Streptococcus pneumoniae on PCR, four clinical diagnosis of bacterial meningitis based on CSF picture (ie, high CSF white blood cell count with neutrophil predominance suggestive of bacterial meningitis).

For participants who died during hospitalisation at Mulago hospital and did not have a microbiologically confirmed diagnosis, the families were invited to provide informed consent for post-mortem examination in the Makerere University mortuary by a trained pathologist. Post-mortem examinations were unavailable in Mbarara. Additionally, CSF samples collected from participants with cryptococcal meningitis and who survived without tuberculosis therapy (ie, negative controls) were run on Xpert Ultra from the same parent study (NCT01802385).

\section{Statistical analysis}

We assessed the diagnostic accuracy of Xpert Ultra against two reference standards. For the primary reference standard, we used the consensus uniform case definition of probable ( $\geq 10$ points on the diagnostic scoring system when brain imaging is not available or $\geq 12$ points when brain imaging is available) or definite (microbiologically confirmed $M$ tuberculosis) tuberculous meningitis. ${ }^{16}$ We also used a composite microbiological reference standard of any positive CSF test (Ziehl-Neelsen stain microscopy,
Xpert, Xpert Ultra, and MGIT) for $M$ tuberculosis. Here, we included the index test (Xpert Ultra) in the reference standard as the existing tests are imperfect, and we judged the likelihood of false-positive detection of $M$ tuberculosis DNA in the CSF of an HIV-positive person with aseptic meningitis in a tuberculosis-endemic area to be extremely low.

Baseline clinical characteristics were compared between those with definite tuberculous meningitis and the remaining participants, who were classified as other meningitis, via Wilcoxon rank-sum test for continuous variables and Pearson's $\chi^{2}$ squared or Fisher's exact test for categorical variables. We directly compared the sensitivity of Xpert Ultra with that of Xpert or MGIT using McNemar's test for paired categorical data. For the comparison of two diagnostic test performances against the primary reference standard, we used the Cochran-Mantel-Haenszel statistic for comparing matched categorical data across two strata of diagnostic tests versus the uniform case definition. ${ }^{16}$ Negative predictive value was calculated against the primary reference standard, both with and without the 
Xpert Ultra result being used to assign the uniform case definition category. Calculation of specificity and positive predictive value was done against the composite endpoint and the uniform case definition using $2 \times 2$ tables. We counted invalid tests (eg, culture contamination or Xpert error) as negative results. Additionally, a sensitivity analysis was done including only participants who received all three tests. We did univariate and multivariable logistic regression analysis to identify variables that correlate with microbiological confirmation of tuberculous meningitis. Variables which showed association in the univariate analysis (likelihood ratio test $\mathrm{p}<0 \cdot 1$ ) were eligible to be included in the multivariable model. Variables that were not normally distributed were log transformed.

Sample size calculation was done using the composite microbiological reference standard. For the paired analysis comparing the sensitivity of Xpert Ultra with that of Xpert or MGIT among participants positive for tuberculous meningitis, we assumed a third of pairs would give discordant results and that the sensitivity of Xpert Ultra would be $25 \%$ higher than that of Xpert and MGIT. Under these assumptions, we required 39 tuberculous meningitis cases to give $80 \%$ power with $\alpha$ set to $5 \%$. Assuming the prevalence of tuberculous meningitis would be $20 \%$, we aimed to recruit 200 participants.

We did a post-hoc analysis of the sensitivity of all assays according to the British Medical Research Council (MRC) tuberculous meningitis grade of disease severity grade against both reference standards (appendix p 12).

Stata version 13.1 was used for statistical analyses.

\section{Role of the funding source}

The funders had no role in study design, data collection, data analysis, data interpretation, or writing of the report. The corresponding author had full access to all the data in the study and had final responsibility for the decision to submit for publication.

\section{Results}

During the study period, 466 HIV-positive adults presented with suspected meningitis and consented to diagnostic lumbar puncture. Of these, 262 participants were diagnosed with cryptococcal meningitis and had no suspicion for disseminated tuberculosis warranting CSF tuberculosis diagnostics, so were excluded from this study. 204 were tested for tuberculous meningitis with Xpert Ultra (figure 1), of whom 195 (96\%) were HIV positive with a median CD4 T cell count of 46 cells $/ \mu \mathrm{L}$ (IQR 11-130). This number included 39 (19\%) patients who had a positive CSF cryptococcal antigen test but were still tested for tuberculous meningitis because of suspected tuberculosis coinfection, and an additional $31(15 \%)$ of those tested for tuberculous meningitis tested positive for cryptococcal antigen in serum but negative in CSF. Biofire meningoencephalitis PCR was done on 80 CSF samples; not all samples were tested owing to a technical issue with the machine. We collected a median

\begin{tabular}{|c|c|c|c|}
\hline & $\begin{array}{l}\text { Definite tuberculous } \\
\text { meningitis }(n=42)\end{array}$ & $\begin{array}{l}\text { Other meningitis } \\
(n=162)\end{array}$ & $p$ value \\
\hline Age, years & $32(29-38)$ & $35(28-42)$ & 0.43 \\
\hline Sex & .. & .. & 0.73 \\
\hline Female & $19(45 \%)$ & $68(42 \%)$ &.. \\
\hline Male & $23(55 \%)$ & $94(58 \%)$ &.. \\
\hline HIV status & .. &.. & .. \\
\hline Positive & $41(98 \%)$ & $154(95 \%)$ & 0.47 \\
\hline Negative & $1(2 \%)$ & $6(4 \%)$ &.. \\
\hline Unknown & 0 & $2(1 \%)$ & .. \\
\hline On antiretroviral therapy & $23(55 \%)$ & $99(62 \%)$ & 0.48 \\
\hline Headache duration, days & $14(7-21)$ & $14(5-21)$ & 0.40 \\
\hline Glasgow Coma Scale & $13(10-14)$ & $14(13-15)$ & 0.0012 \\
\hline CD4 count, cells per $\mu L^{*}$ & $57(13-108)$ & $46(10-188)$ & 0.83 \\
\hline CSF opening pressure, $\mathrm{cmH}_{2} \mathrm{O}$ & $20(10-32)$ & $18(13-26)$ & 0.91 \\
\hline Acellular CSF $<5$ cells per $\mu \mathrm{L}$ & $11(26 \%)$ & $99(61 \%)$ & $<0.0001$ \\
\hline CSF white blood cells per $\mu \mathrm{L} \dagger$ & $170(70-283)$ & $100(40-275)$ & 0.085 \\
\hline CSF lymphocytes & $100 \%(84-100)$ & $100 \%(80-100)$ & 0.61 \\
\hline CSF total protein, $\mathrm{g} / \mathrm{L}$ & $1 \cdot 2(0 \cdot 9-2 \cdot 0)$ & $0.3(0.2-0.8)$ & $<0.0001$ \\
\hline CSF glucose, $\mathrm{mmol} / \mathrm{L}$ & $1 \cdot 2(0.9-2 \cdot 0)$ & $2 \cdot 9(1 \cdot 9-4 \cdot 4)$ & $<0.0001$ \\
\hline CSF lactate, $\mathrm{mmol} / \mathrm{mL} \ddagger$ & $9 \cdot 5(4 \cdot 6-11)$ & $3 \cdot 6(2 \cdot 4-5 \cdot 1)$ & $<0.0001$ \\
\hline Alive at hospital discharge & $25(60 \%)$ & $101 / 143(71 \%) \S$ & 0.18 \\
\hline \multicolumn{4}{|c|}{$\begin{array}{l}\text { Values are } \mathrm{n}(\%), \mathrm{n} / \mathrm{N}(\%) \text {, or median (IQR), unless otherwise stated. } \mathrm{p} \text { values are from Wilcoxon rank-sum for } \\
\text { continuous data and Fisher's exact test for categorical data. Patients with definite tuberculous meningitis were positive } \\
\text { for tuberculous meningitis by the composite microbiological reference standard. Patients with other meningitis were } \\
\text { the remaining participants, including } 112 \text { with unknown causes and } 50 \text { with known causes of meningitis (see figure } 1 \text { ). } \\
\text { * } 63 \text { participants had CD } 4 \text { count data ( } 12 \text { in the definite tuberculous meningitis group and } 51 \text { in the other meningitis } \\
\text { group). † Median values in participants with more than five white blood cells per } \mu \mathrm{L} \text { of CSF. } \text { Lactate concentrations } \\
\text { were available for } 18 \text { participants in the tuberculous meningitis definite group and } 70 \text { in the other meningitis group. } \\
\$ 19 \text { participants had unknown status at discharge in the other meningitis group. }\end{array}$} \\
\hline
\end{tabular}

of $11 \mathrm{~mL}$ (IQR 7-15) of CSF per participant, which left a median of $8 \mathrm{~mL}(5-11)$ of CSF to be spun down for tuberculosis testing after routine microbiology and chemistry testing had been done. Collected CSF was clear in 186 (91\%) participants, with the remaining $18(9 \%)$ having turbid CSF. Brain imaging was rarely done (ie, in 18 participants) because of a lack of functioning CT scanners in the study sites. No adverse events were attributable to the diagnostic testing (data not shown).

At baseline, lack of white blood cell pleocytosis and concentrations of glucose, protein, and lactate differed significantly between those with and without microbiologically confirmed tuberculous meningitis (table 1). The demographic, clinical, and diagnostic details of the 42 participants with microbiologically confirmed tuberculous meningitis are shown in the appendix (pp 5-6).

Of the 204 participants who underwent tuberculous meningitis diagnostic testing, Xpert Ultra was positive in 39, of whom Xpert was also positive in 24 and MGIT culture in 24 (figure 1). Xpert Ultra was negative in 165 participants, of whom 162 were negative and three were positive by the composite microbiological reference standard (figure 1). Xpert Ultra detected nine cases of 
tuberculous meningitis that were not identified by Xpert or MGIT culture (figure 2). We diagnosed probable or definite tuberculous meningitis in 51 (25\%) of 204 participants when including the Xpert Ultra result in assigning the uniform case definition. When the Xpert Ultra result was excluded, $44(22 \%)$ participants were classified as having probable or definite tuberculous meningitis. Of the 42 participants with definite tuberculous meningitis, five (12\%) tested positive for cryptococcal antigen in CSF, including two (5\%) who had positive fungal cultures (quantitative cryptococcal culture 520000 and 20000 CFUs per mL; figure 1).

We identified alternative causes of meningitis in $49(30 \%)$ of the 162 participants without confirmed tuberculous meningitis (figure 1). In four patients without confirmed tuberculous meningitis who died in hospital, post-mortem examinations were done and causes of

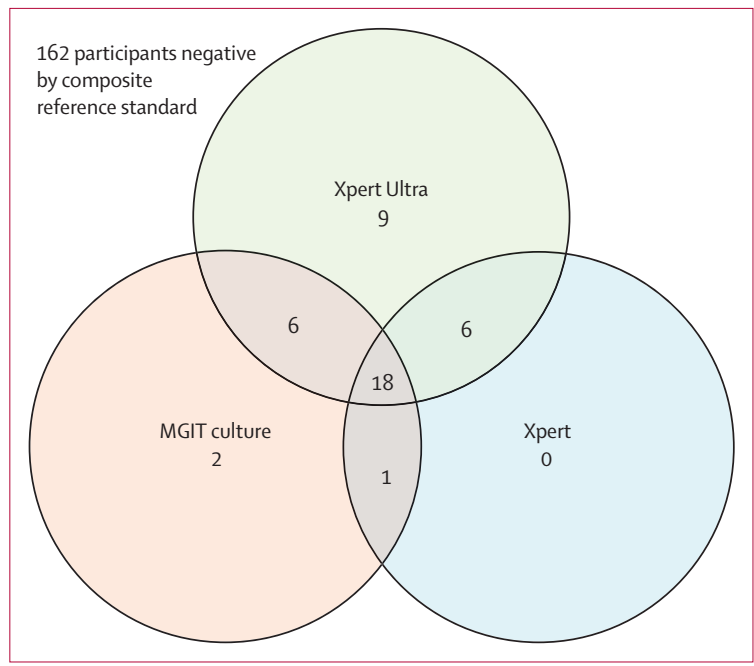

Figure 2: Venn diagram of positive diagnostic tests in the composite microbiological reference standard

The Venn diagram displays 42 participants with microbiologically confirmed tuberculous meningitis by either Xpert, Xpert Ultra, or MGIT culture. Xpert=Xpert MTB/RIF. MGIT=mycobacterial growth indicator tube. death were herpes simplex virus meningoencephalitis, progressive multifocal leukoencephalopathy, and disseminated tuberculosis without obvious CNS involvement in one patient; progressive multifocal leukoencephalopathy (PML) in one patient; pneumonia with no obvious CNS pathology in one patient; and meningoencephalitis with macroscopic appearance that was compatible with tuberculous meningitis, with histopathological confirmation awaited, in one patient.

Among the 42 participants with definite tuberculous meningitis, 17 (40\%) died in hospital, with a median time to death of 4 days (IQR 2-6). Among the 162 patients in the other meningitis group, hospital outcome was known for 143, of whom 19 (13\%) died in hospital.

When compared with the uniform case definition (probable or definite tuberculous meningitis), sensitivities were $76 \cdot 5 \%$ (95\% CI $62 \cdot 5-87 \cdot 2)$ for Xpert Ultra, $55 \cdot 6 \%(44 \cdot 0-70 \cdot 4)$ for Xpert, and 61.4\% (45.5-75.6) for MGIT (table 2). Against the composite microbiological reference standard, sensitivities were $92 \cdot 9 \%(80 \cdot 5-98 \cdot 5)$ for Xpert Ultra, $65 \cdot 8 \%(48 \cdot 6-80 \cdot 4)$ for Xpert, and $72 \cdot 2 \%$ $(55 \cdot 9-86 \cdot 2)$ for MGIT culture (table 2). Xpert Ultra was superior to Xpert in detecting tuberculous meningitis when either the composite microbiological reference standard or uniform case definition was used (table 2). Xpert Ultra was significantly more sensitive than MGIT culture when the uniform case definition was used but not when the composite microbiological reference standard was used (table 2). The negative predictive value of Xpert Ultra was $92 \cdot 7 \%(87 \cdot 6-96 \cdot 2)$ against the uniform case definition (table 2). When including only the 117 participants who had received all three tests, no major difference in performance of any of the tests was observed (appendix p 7).

Of the 39 positive Xpert Ultra results, 14 were categorised as trace, ten as very low, seven as low, five as medium, and none as high; three results were unknown. Of the 14 Xpert Ultra trace samples, only four were positive on Xpert (three very low and one low) and six were positive on culture. Median time to CSF culture positivity was 14 days

\begin{tabular}{|c|c|c|c|c|c|c|c|}
\hline & Number & $\begin{array}{l}\text { Sensitivity vs } \\
\text { composite } \\
\text { microbiological } \\
\text { reference standard* }\end{array}$ & $\mathrm{p}$ value $†$ & $\begin{array}{l}\text { Sensitivity vs } \\
\text { uniform case } \\
\text { definition }\end{array}$ & p value $\ddagger$ & $\begin{array}{l}\text { Negative predictive } \\
\text { value } v \text { s uniform case } \\
\text { definition }\end{array}$ & $\begin{array}{l}\text { Specificity vs uniform } \\
\text { case definition }\end{array}$ \\
\hline Xpert Ultra & 204 & $\begin{array}{l}92 \cdot 9 \%(80 \cdot 5-98 \cdot 5) \\
39 / 42\end{array}$ & .. & $\begin{array}{l}76 \cdot 5 \%(62 \cdot 5-87 \cdot 2) \\
39 / 51\end{array}$ & .. & $\begin{array}{l}92 \cdot 7 \%(87 \cdot 6-96 \cdot 2) \\
153 / 165\end{array}$ & $\begin{array}{l}100 \%(97 \cdot 6-100) \\
153 / 153\end{array}$ \\
\hline Xpert & 166 & $\begin{array}{l}65 \cdot 8 \%(48 \cdot 6-80 \cdot 4) \\
25 / 38\end{array}$ & 0.0063 & $\begin{array}{l}55 \cdot 6 \%(44 \cdot 0-70 \cdot 4) \\
25 / 45\end{array}$ & 0.0010 & $\begin{array}{l}85 \cdot 8 \%(78 \cdot 9-91 \cdot 1) \\
121 / 141\end{array}$ & $\begin{array}{l}100 \%(97 \cdot 0-100) ; \\
121 / 121\end{array}$ \\
\hline MGIT culture & 142 & $\begin{array}{l}72 \cdot 2 \%(55 \cdot 9-86 \cdot 2) \\
27 / 37\end{array}$ & 0.092 & $\begin{array}{l}61 \cdot 4 \%(45 \cdot 5-75 \cdot 6) \\
27 / 44\end{array}$ & 0.020 & $\begin{array}{l}85 \cdot 2 \%(77 \cdot 4-91 \cdot 1) \\
98 / 115\end{array}$ & $\begin{array}{l}100 \%(96 \cdot 3-100) \\
98 / 98\end{array}$ \\
\hline \multicolumn{8}{|c|}{$\begin{array}{l}\text { Values are percentage }(95 \% \mathrm{Cl}) \text {; numerator/denominator unless stated otherwise. Xpert=Xpert MTB/RIF. MGIT=mycobacterial growth indicator tube. }{ }^{*} \text { The composite } \\
\text { microbiological reference standard included a positive CSF test on any of Ziehl-Neelsen stain microscopy, Xpert, Xpert Ultra, and MGIT culture. Specificity (and the positive } \\
\text { predictive value) versus the composite endpoint is by definition } 100 \% \text { as the index test is included in the reference standard of definite tuberculous meningitis. If the Xpert } \\
\text { Ultra result is excluded when assigning the case definition, the specificity of Xpert Ultra is } 96 \%(95 \% \mathrm{CI} 91-98 ; 153 \text { of } 160 \text { patients) and the positive predictive value is } 82 \% \\
(66-93 ; 32 \text { of } 39) \text {. †McNemar's test comparing the sensitivity of Xpert or MGIT culture with that of Xpert Ultra. \#Cochran-Mantel-Haenszel test comparing the distribution of } \\
\text { Xpert or MGIT results with that of Xpert Ultra results against the uniform clinical standard of definite or probable tuberculous meningitis. }\end{array}$} \\
\hline
\end{tabular}




\begin{tabular}{|c|c|c|c|c|c|c|}
\hline & \multicolumn{3}{|c|}{ Univariate model } & \multicolumn{3}{|c|}{ Multivariable model } \\
\hline & $n$ & Odds ratio $(95 \% \mathrm{Cl})$ & $p$ value & $n$ & Adjusted odds ratio ( $95 \% \mathrm{Cl})$ & $\mathrm{p}$ value \\
\hline Age, peryear & 204 & $0.98(0.95-1.01)$ & 0.26 & .. & .. & .. \\
\hline Male sex & 204 & $1.14(0.58-2.26)$ & $0 \cdot 70$ &.. & .. & .. \\
\hline Duration of headache, per day & 168 & $1.00(0.98-1.01)$ & 0.95 & .. & .. & .. \\
\hline \multicolumn{7}{|l|}{ Glasgow Coma Scale score } \\
\hline $15^{*}$ & 72 & 1 (ref) & .. & 35 & 1 (ref) & .. \\
\hline $11-14$ & 97 & $3.62(1 \cdot 39-9 \cdot 39)$ & 0.0083 & 46 & $3.71(0.84-16 \cdot 48)$ & 0.084 \\
\hline$\leq 10$ & 33 & $5 \cdot 50(1.82-16 \cdot 62)$ & 0.0025 & 21 & $4.71(0.93-23.82)$ & 0.061 \\
\hline $\log _{2}$ CD4 count, cells per $\mu \mathrm{L}$ & 63 & $0.94(0.71-1 \cdot 34)$ & 0.66 & .. & .. & .. \\
\hline On antiretroviral therapy & 202 & $1.34(0.67-2.66)$ & 0.40 &.. & .. & .. \\
\hline $\log _{2}$ CSF volume, $\mathrm{mL}$ & 198 & $1.20(0.84-1.72)$ & 0.32 & .. & .. & .. \\
\hline $\log _{2}$ CSF volume spun down, $\mathrm{mL}$ & 96 & $1.40(0.84-2 \cdot 35)$ & $0 \cdot 20$ & .. & .. & .. \\
\hline CSF pleocytosis $\dagger$ & 189 & $4.94(2 \cdot 28-10 \cdot 72)$ & $<0.0001$ & 101 & $2.04(0.33-12.74)$ & 0.44 \\
\hline Log $_{2}$ CSF glucose, $\mathrm{mmol} / \mathrm{L}$ & 122 & $0.31(0.18-0.54)$ & $<0.0001$ & 101 & $0.33(0.17-0.65)$ & 0.0010 \\
\hline $\log _{2}$ CSF lactate, $\mathrm{mmol} / \mathrm{L} \neq$ & 88 & $5 \cdot 76(2 \cdot 45-13 \cdot 52)$ & $<0.0001$ & .. & .. & .. \\
\hline $\log _{2}$ CSF protein, $\mathrm{mg} / \mathrm{dL}$ & 166 & $2.10(1.52-2.89)$ & $<0.0001$ & 101 & $1.02(0.56-1.87)$ & 0.25 \\
\hline \multicolumn{7}{|c|}{$\begin{array}{l}\text { Variables that were significant }(p<0.1) \text { in the univariate model were included in the multivariable model. Odds ratios for log-transformed variables are per log }{ }_{2} \text { increase. } \\
\text { CSF=cerebrospinal fluid. }{ }^{*}=0.0001 \text { with the likelihood ratio test. } \dagger>5 \text { lymphocytes per } \mu \mathrm{L} \text { of CSF. } \neq C S F \text { lactate was excluded from the multivariable model because of the } \\
\text { amount of missing data. }\end{array}$} \\
\hline
\end{tabular}

(IQR 10-15). Median time to positivity by Xpert Ultra semiquantitative category was 15 days (13-15) for trace, 15 days (12-15) for very low, 12 days (9-21) for low, and 9 days (8-9) for medium (appendix pp 3,8).

No cases of rifampicin resistance were identified with either Xpert or Xpert Ultra. Positive samples classed in the Xpert Ultra trace category have indeterminate rifampicin resistance because more than 16 CFUs per $\mathrm{mL}$ of $M$ tuberculosis and amplification of rpob DNA is required to generate rifampicin susceptibility results; ${ }^{13}$ thus all 14 trace positive results had indeterminate rifampicin resistance in our study. Culture-based drug susceptibility testing was not done. MGIT culture reported contaminated results in $13(9 \%)$ of 142 cultures performed.

When analysing by MRC grade, the sensitivity of Xpert Ultra against the composite microbiological reference standard was $100 \%$ in grade 1 disease, $96 \%$ in grade 2 disease, and $82 \%$ in grade 3 disease. Using the uniform case definition, the sensitivity of Xpert Ultra was $100 \%$ in grade 1 disease, $74 \%$ in grade 2 disease, and $69 \%$ in grade 3 disease. The observation of decreasing sensitivity with advancing disease MRC severity grade held true for Xpert and MGIT (appendix p 9).

The CSF from 45 participants with confirmed cryptococcal meningitis and no clinical suspicion of tuberculosis coinfection were all negative on Xpert Ultra, suggesting false positivity (eg, laboratory contamination) is a rare occurrence (specificity 100\%, 95\% CI 92-100).

Univariate analysis found that Glasgow Coma Scale score of less than 15, CSF pleocytosis, and lower CSF glucose, higher CSF protein, and higher CSF lactate concentrations were all positively associated with microbiological confirmation of tuberculous meningitis (table 3). $\log _{2}$ CSF lactate concentration was not included in the multivariate analysis because of the large amount of missing data. In the multivariable logistic regression model, lower $\log _{2}$ CSF glucose concentration remained positively associated with microbiological confirmation of tuberculous meningitis, although the multivariable model analysis was restricted to 101 participants with a complete dataset for the variables included in the model (table 3). The median volume of CSF spun down for tuberculosis testing was $8 \mathrm{~mL}$ (IQR 5-11) and each doubling (ie, $\log _{2}$ increase) of CSF volume spun down increased the odds of tuberculosis confirmation by $40 \%$, although this did not achieve statistical significance (table 3).

Of the nine participants who were positive only by Xpert Ultra, six had started antiretroviral therapy within the 6 weeks preceding the study (median duration 28 days, IQR 17-35), a timing consistent with unmasking immune reconstitution inflammatory syndrome.

\section{Discussion}

In this HIV-positive population with suspected meningitis, Xpert Ultra showed higher sensitivity to detect tuberculous meningitis than either Xpert or MGIT culture against both considered reference standards, although the difference was not significant when Xpert Ultra was compared with MGIT culture against the composite microbiological reference standard. These findings from fresh CSF corroborate our earlier findings from cryopreserved CSF, supporting the robustness of these data. ${ }^{8}$

Importantly, more than a third (14 [36\%] of 39) of positive Xpert Ultra results were in the trace category 
(correlating to $<100 \mathrm{CFUs}$ per $\mathrm{mL}$ ), which is usually below the limit of detection of Xpert and potentially below the limit of detection of tuberculosis culture. Of these 14 trace positive samples, only four were positive by Xpert and six by culture. The ability to detect nonviable organisms is another reason why detection of tuberculosis might be improved with molecular tests versus culture.

As part of the uniform case definition, we did not include participants with possible tuberculous meningitis $(n=74)$ in our analysis because of the non-specific nature of this category in this study population with advanced HIV-eg, due to concurrent neurological pathology such as PML (study population median CD4 $\mathrm{T}$ cell count 46 cells/ $\mu \mathrm{L}$, IQR 11-130).

With increasing market penetration of the Xpert platform in countries with high tuberculosis burden ${ }^{24}$ and a turnaround time of $84 \mathrm{~min}$, Xpert Ultra is the best pointof-care test for tuberculous meningitis currently available and has been endorsed by WHO as the best initial test for tuberculous meningitis. ${ }^{25}$ Yet, our study shows a moderate $76 \cdot 5 \%$ sensitivity of Xpert Ultra against a uniform case definition of probable or definite tuberculous meningitis, giving a negative predictive value of $92.7 \%$. In other words, nearly a fifth of patients (nine [18\%] of 51) with clinical phenotypes and CSF profiles highly suggestive of tuberculous meningitis and negative for all other tested pathogens were negative by Xpert Ultra (and by Xpert and culture). Four of these participants died from their illness, one with macroscopic post-mortem findings compatible with tuberculous meningitis. Our belief is that a proportion of these are false-negative results, but this remains to be determined in future studies. CSF could also be tested with Xpert Ultra on multiple days if safe to do so and a false-negative result is suspected; we have employed this strategy with success in several patients (outside of this study). Markers of host response should also be considered as potential future adjunctive diagnostic tests, although to date, none have been successful. ${ }^{26}$ Currently, clinical judgment remains relevant when making treatment decisions regarding tuberculous meningitis. ${ }^{11}$

Multivariable analysis showed low CSF glucose to be associated with microbiologically confirmed tuberculous meningitis. CSF lactate could not be taken through to the multivariable model because of the amount of missing data but was found to be strongly associated with microbiologically confirmed tuberculous meningitis in the univariate model and thus warrants further investigation as a diagnostic marker. Handheld glucometers and lactate meters are true point-of-care tests, with results available at the bedside within a matter of seconds. A low CSF glucose concentration $(<2 \cdot 2 \mathrm{mmol} / \mathrm{L},<40 \mathrm{mg} / \mathrm{dL}$, or $\mathrm{CSF}$ to plasma glucose ratio $<50 \%$ ) in a patient with symptoms and signs suggestive of tuberculous meningitis, once bacterial meningitis and cryptococcus have been excluded, is a good indication to consider antituberculous therapy regardless of tuberculosis-specific test results.
The potential for using CSF glucose and lactate as components of a comprehensive diagnostic algorithm focused on probability of diagnosis should be explored in future studies.

We believe that using Xpert Ultra on CSF samples is highly specific for the diagnosis of tuberculous meningitis, although the absence of a perfect reference standard makes this difficult to prove. Furthermore, the inclusion of Xpert Ultra in the composite reference standard risked incorporation bias and is a limitation of this study. Yet, unlike sputum from the lungs, which can remain positive in the trace category on Xpert Ultra even years after treatment of prior pulmonary tuberculosis, ${ }^{27} \mathrm{CSF}$ is a sterile body fluid that is replenished four to five times a day. ${ }^{28}$ Presence of $M$ tuberculosis in the CSF progresses to death if untreated. In this study of hospitalised patients with advanced HIV who were from a tuberculosisendemic country and had symptoms of subacute meningitis, we feel that the probability of a false-positive DNA-based test is negligible.

The median total volume of CSF spun down for tuberculosis testing was $8.0 \mathrm{~mL}$; however, this volume was then divided into four, with a median volume of approximately $2.0 \mathrm{~mL}$ per diagnostic test. We used an algorithm for stepwise dropping of tests when the CSF volume collected was less than $6 \mathrm{~mL}$ so the minimal input CSF volume would always be at least $2.0 \mathrm{~mL}$ per diagnostic test to maximise the likelihood of obtaining an accurate result for the patient. Each doubling of CSF volume spun down increased the odds of tuberculosis confirmation by $40 \%$, although this association was not significant. Xpert has been noted in some studies to have improved performance when higher CSF volumes are used; this study cannot inform whether the same is true for Xpert Ultra. ${ }^{5,6}$ The stepwise use of tests on low-volume samples resulted in small samples receiving fewer tests and thus imperfect matching, with CSF from 204 patients tested with Xpert Ultra compared with only 166 with Xpert. There were also the common problems of culture, beyond diagnostic delay; only 142 culture results were received (including 13 that were contaminated) because of a number being lost when sent to an external mycobacterial laboratory.

$11(26 \%)$ of 42 patients with microbiologically confirmed tuberculous meningitis had an acellular CSF (fewer than five white blood cells per $\mu \mathrm{L}$ ). All those with acellular CSF also had CSF opening pressure and total protein within the normal range and lower median CSF lactate concentrations (CSF lactate $4.6 \mathrm{mmo} / \mathrm{mL}$ vs $9.5 \mathrm{mmol} / \mathrm{mL}$ in the definite tuberculous meningitis group). We previously reported that one in three patients with definite tuberculous meningitis have acellular CSF, a finding that has also been noted in other HIVpositive cohorts outside of Asia. ${ }^{29}$ This observed absence of an inflammatory response in patients with microbiologically confirmed tuberculous meningitis could be explained by immune paresis secondary to 
advanced HIV disease (CD4 count was available in two of 11 patients and was three cells per $\mu \mathrm{L}$ and six cells per $\mu \mathrm{L}$, respectively), or by early presentation (three of 11 patients had MRC grade 1 disease). Mortality was high in this subgroup despite the apparent absence of CSF inflammation, with six (55\%) patients dying in hospital. Low CSF white blood cell count has been associated with death in a multivariate prognostic model in Vietnamese adults with tuberculous meningitis, and it is becoming increasingly clear that both absence of inflammation, as well as excessive inflammation, are deleterious in tuberculous meningitis. ${ }^{2,30}$

The sensitivity of all assays was highest in early disease (MRC grade 1), and sensitivity decreased as disease severity progressed. Although the numbers are small and the analysis of sensitivity by MRC grade was post hoc, this finding supports the notion that an increasing immune response (ie, inflammation) aimed at controlling bacillary load is the mediator of disease. Thereby, an inverse relationship might actually exist between CSF bacillary load and disease severity in this population. This hypothesis warrants further investigation in immunology studies and highlights the importance of finding the optimal host-directed therapy to control damaging inflammation in this population.

When translating these results to the field, it is important to consider that in many hospital laboratory settings, centrifuging the CSF might not be feasible, in which case we would advise loading $2 \mathrm{~mL}$ of CSF directly into the Xpert Ultra cartridge to maximise the bacillary load, as dilution with sample reagent is not required for CSF. Additionally, although our cohort cannot inform diagnostic performance in an HIV-negative population, our paired analysis of specimens shows that Xpert Ultra performs better than Xpert in an HIV-positive population. Whether Xpert Ultra can improve outcomes of tuberculous meningitis or lessen unnecessary exposure to tuberculous meningitis medications remain to be determined. In this prospective study, with real-time Xpert Ultra results available to the clinical team within $24 \mathrm{~h}$, inhospital mortality was $40 \%$ (17 of 42 patients) compared with $50 \%$ (11 of 22) in our previous study on cryopreserved CSF in which results were not available to guide management decisions. ${ }^{7.8}$ We cannot say whether this observed reduction in mortality is attributable to the availability of real-time Xpert Ultra results because this study was not designed to study the effect of Xpert Ultra on clinical outcomes. Additionally, this study included two clinical sites (Mulago and Mbarara hospitals), whereas the previous study was done solely in Mbarara hospital. Furthermore, given the time between this study and the previous study, additional potential confounding factors might be present that contribute towards the mortality difference. In addition to access to improved point-of-care diagnostics, earlier presentation to hospital, better supportive care, and optimised antimicrobial and anti-inflammatory treatments are required to reduce the high case-fatality in this population. Larger structural factors, such as poverty and weak health systems, also continue to play a role in the high mortality of tuberculosis and HIV coinfection and need to be addressed. At present, regular use of empirical therapy continues to be required for tuberculous meningitis.

In conclusion, Xpert Ultra offers a substantial improvement in rapid and accurate diagnosis of tuberculous meningitis, and is superior to Xpert. However, Xpert Ultra does not represent a perfect rule-out test. Clinical judgment and empirical therapy remain important to improve outcomes for patients with tuberculous meningitis. In this exciting era of rapidly evolving molecular diagnostics and biomarkers, the development of a highly sensitive point-ofcare test that can facilitate rapid treatment and give clinicians confidence in rationalising the use of empirical tuberculosis treatment is a realistic goal.

\section{Contributors}

DRB, NCB, FVC, and DBM contributed to study concept and design. LT, AKM, EK, MR, EM, EN, CM, DAW, and FVC contributed to acquisition of clinical data. RK contributed to acquisition of laboratory data. ASB, DG, FVC, DRB, and NCB contributed to statistical analysis. DG, FVC, DRB, AME, and NCB contributed to data interpretation.

FVC and NCB contributed to initial manuscript drafting. AME, NCB, FVC DRB, DG, JR, AKM, LT, and RK contributed to revisions for intellectual content. All authors approved the final version to be published.

\section{Declaration of interests}

We declare no competing interests.

\section{Acknowledgments}

This research was made possible through support from the National Institute of Neurologic Disorders and Stroke (R01NS086312, K23NS110470), the Fogarty International Center (K01TW010268, K43TW010718), and National Institute of Allergy and Infectious Diseases (T32AI055433). FVC is supported through a Wellcome Trust Clinical PhD Fellowship (grant number 210772/Z/18/Z). FVC is an honorary fellow of the Makerere University-Uganda Virus Research Institute (UVRI) Centre of Excellence for Infection and Immunity Research and Training (MUII-plus). MUII-plus is supported through the Developing Excellence in Leadership, Training and Science Africa Initiative (grant number 107743), which is an independent funding scheme of the African Academy of Sciences and the Alliance for Accelerating Excellence in Science in Africa, and is supported by the New Partnership for Africa's Development Planning and Coordinating Agency, with funding from the Wellcome Trust (grant number 107743) and the UK Government. The MRC/UVRI \& LSHTM Uganda Research Unit is jointly funded by the UK MRC and the UK Department for International Development (DFID) under the MRC/DFID Concordat agreement and is also part of the European \& Developing Countries Clinical Trials Partnership 2 programme supported by the EU. We would like to acknowledge the technologists at Makerere University Microbiology Laboratory and MSF Epicentre Laboratory and the study participants and their caregivers. We thank Joshua Matovu for assisting with the running of some of the assays in the Infectious Diseases Institute Translational Laboratory.

\section{References}

1 WHO. Global tuberculosis report 2019. Geneva: World Health Organization, 2019.

2 Thao LTP, Heemskerk AD, Geskus RB, et al. Prognostic models for 9-month mortality in tuberculous meningitis. Clin Infect Dis 2018; 66: 523-32.

3 Heemskerk AD, Bang ND, Thwaites GE. Therapy for tuberculous meningitis. N Engl J Med 2016; 374: 2188-89.

4 Sheu JJ, Yuan RY, Yang CC. Predictors for outcome and treatment delay in patients with tuberculous meningitis. Am J Med Sci 2009 338: $134-39$. 
5 Bahr NC, Tugume L, Rajasingham R, et al. Improved diagnostic sensitivity for tuberculous meningitis with Xpert MTB/RIF of centrifuged CSF. Int J Tuberc Lung Dis 2015; 19: 1209-15.

6 Heemskerk AD, Donovan J, Thu DDA, et al. Improving the microbiological diagnosis of tuberculous meningitis: a prospective, international, multicentre comparison of conventional and modified Ziehl-Neelsen stain, GeneXpert, and culture of cerebrospinal fluid. J Infect 2018; 77: 509-15.

7 Cresswell FV, Bangdiwala AS, Bahr NC, et al. Tuberculous meningitis diagnosis and outcomes during the Xpert MTB/RIF era: a 6-5-year cohort in Uganda. Wellcome Open Res 2018; 3: 64.

8 Bahr NC, Nuwagira E, Evans EE, et al. Diagnostic accuracy of Xpert MTB/RIF Ultra for tuberculous meningitis in HIV-infected adults: a prospective cohort study. Lancet Infect Dis 2018; 18: 68-75.

9 WHO. Policy update: Xpert MTB/RIF assay for the diagnosis of pulmonary and extrapulmonary TB in adults and children. Geneva: World Health Organization, 2015.

10 WHO. Xpert MTB/RIF roll out 2016. http://www.who.int/tb/areasof-work/laboratory/mtb-rif-rollout/en (accessed March 1, 2019).

11 Bahr NC, Marais S, Caws M, et al. GeneXpert MTB/RIF to diagnose tuberculous meningitis: perhaps the first test but not the last. Clin Infect Dis 2016; 62: 1133-35.

12 WHO. Non-inferiority analysis of Xpert MTB/RIF Ultra compared to Xpert MTB/RIF. Geneva: World Health Organization, 2017.

13 Chakravorty S, Simmons AM, Rowneki M, et al. The new Xpert MTB/RIF Ultra: improving detection of Mycobacterium tuberculosis and resistance to rifampin in an assay suitable for point-of-care testing. mBio 2017; 8: e00812-17.

14 Dorman SE, Schumacher SG, Alland D, et al. Xpert MTB/RIF Ultra for detection of Mycobacterium tuberculosis and rifampicin resistance: a prospective multicentre diagnostic accuracy study. Lancet Infect Dis 2018; 18: 76-84.

15 Nicol MP, Workman L, Prins M, et al. Accuracy of Xpert Mtb/RIF Ultra for the diagnosis of pulmonary tuberculosis in children. Pediatr Infect Dis J 2018; 37: e261-63.

16 Marais S, Thwaites G, Schoeman JF, et al. Tuberculous meningitis: a uniform case definition for use in clinical research. Lancet Infect Dis 2010; 10: 803-12.

17 Wu X, Tan G, Gao R, et al. Assessment of the Xpert MTB/RIF Ultra assay on rapid diagnosis of extrapulmonary tuberculosis. Int J Infect Dis 2019; 81: 91-96.
18 Perez-Risco D, Rodriguez-Temporal D, Valledor-Sanchez I, Alcaide F. Evaluation of the Xpert MTB/RIF Ultra assay for direct detection of Mycobacterium tuberculosis complex in smear-negative extrapulmonary samples. J Clin Microbiol 2018; 56: e00659-18.

19 Chin JH, Musubire AK, Morgan N, et al. Xpert MTB/RIF Ultra for the detection of Mycobacterium tuberculosis in cerebrospinal fluid. J Clin Microbiol 2019; 57: e0249-19.

20 Wang G WS, Jiang G, Yang X, Huang M, Huo F. Xpert MTB/RIF Ultra improved the diagnosis of paucibacillary tuberculosis: a prospective cohort study. J Infect 2019; 78: 311-16.

21 Cohen JF, Korevaar DA, Altman DG, et al. STARD 2015 guidelines for reporting diagnostic accuracy studies: explanation and elaboration. BMJ Open 2016; 6: e012799.

22 Marais BJ, Heemskerk AD, Marais SS, et al. Standardized methods for enhanced quality and comparability of tuberculous meningitis studies. Clin Infect Dis 2017; 64: 501-09.

23 Ellis J, Cresswell FV, Joshua R, Kenneth S, Boulware DR. Cryptococcal meningitis and tuberculous meningitis co-infection in HIV-infected Ugandan adults. Open Forum Infect Dis 2018; 5: ofy193.

24 Cazabon D, Alsdurf H, Satyanarayana S, et al. Quality of tuberculosis care in high burden countries: the urgent need to address gaps in the care cascade. Int J Infect Dis 2017; 56: 111-16.

25 WHO. WHO meeting report of a technical expert consultation: non-inferiority analysis of Xpert MTB/RIF Ultra compared to Xpert MTB/RIF. 2017. http://www.who.int/tb/publications/2017/ XpertUltra/en (accessed March 24, 2017).

26 Bahr NC, Meintjes G, Boulware DR. Inadequate diagnostics: the case to move beyond the bacilli for detection of meningitis due to Mycobacterium tuberculosis. J Med Microbiol 2019; 68: 755-60.

27 Theron G, Venter R, Calligaro G, et al. Xpert MTB/RIF results in patients with previous tuberculosis: can we distinguish true from false positive results? Clin Infect Dis 2016; 62: 995-1001.

28 Sakka L, Coll G, Chazal J. Anatomy and physiology of cerebrospinal fluid. Eur Ann Otorhinolaryngol Head Neck Dis 2011; 128: 309-16.

29 Cresswell FV, Bangdiwala AS, Meya DB, et al. Absence of cerebrospinal fluid pleocytosis in tuberculous meningitis is a common occurrence in HIV co-infection and a predictor of poor outcomes. Int J Infect Dis 2018; 68: 77-78.

30 Thuong NTT, Heemskerk D, Tram TTB, et al. Leukotriene A4 hydrolase genotype and HIV infection influence intracerebra inflammation and survival from tuberculous meningitis. J Infect Dis 2017; 215: 1020-28. 


\section{Chapter Six: Standardized Urine-based Tuberculosis Screening with TB-LAM and Xpert MTB/Rif Ultra in Ugandan Adults with Advanced HIV disease and Meningitis (research paper)}

\section{Foreword}

In chapter 4 and 5 I have shown that introduction of the Xpert MTB/Rif assay has had a marked impact on microbiological confirmation of TBM and that the Xpert Ultra assay is more sensitive than Xpert MTB/Rif when testing the CSF of patients with suspected TBM. However, CSF analysis is not without operational challenges including the following: 1) lumbar punctures (LPs) are not necessarily available in smaller hospitals in Uganda and other LMIC settings due to a lack of expertise and equipment; 2) LPs can be delayed whilst brain imaging is awaited; 3) LPs can be medically contraindicated due to space occupying lesions, thrombocytopaenia or coagulopathy; 4) LPs carry risks including nerve damage, bleeding, infection, and cerebellar herniation. A nonCSF dependent diagnostic test that can support TBM treatment decisions would be incredibly valuable, especially if it also had value in prognostication.

Urine-based TB diagnostics have the potential to reduce missed TB diagnoses and mortality in patients with advanced HIV disease and disseminated TB. ${ }^{1}$ Urine is an easily obtainable sample and can be tested using either a lateral flow assay for TB-lipoarabinomannan (Alere LAM) or Xpert Ultra. To date, urine LAM and Xpert Ultra have not been explored specifically in a suspected meningitis population and there are a number of questions of interest:

1. Can urine TB diagnostics help to differentiate aetiologies of meningitis and support TBM treatment decisions?

2. What's the prevalence of disseminated TB detectable by urine testing in hospitalised patients with suspected meningitis?

3. Does urine TB test positivity serve as prognostic tool in TBM?

I sought to explore some of these questions in a pilot study of adults presenting with suspected meningitis (including cryptococcal meningitis, TBM or meningitis of unknown cause) to Kiruddu National Referral Hospital and Mbarara Regional Referral Hospital. This research paper explores hypothesis \#2: urine diagnostics such as urine TB-LAM and urine Xpert Ultra will be useful adjunctive tools in diagnosing TBM and will identify patients at the greatest risk of death.

The key finding of this exploratory study was that there was evidence of disseminated TB by positive urine TB test (Alere LAM or Xpert Ultra) in a surprisingly high proportion of all meningitis cases: $22 \%$ (44/198) of cryptococcal meningitis, 50\% (20/40) of definite TBM, 50\% (11/22) probable TBM, 17\% (15/88) of other/unknown cases. So, a positive urine Alere LAM or Ultra in isolation cannot reliably support TBM 
treatment decisions, but might be more useful when considered alongside the cryptococcal antigen result. Given that urine Alere LAM and urine Xpert MTB/Rif are only moderately sensitive the true burden of disseminated TB disease could be even higher than that detectable by urine. The findings therefore suggest that TBM is commonly part of a disseminated TB disease picture, rather than a single compartment infection, in PLHIV and this may be relevant when considering optimising management, as further discussed in chapter 10. In patients with definite TBM, urine Ultra may have prognostic value, though this needs to be further investigated through a larger study with appropriate adjustment for $\mathrm{CD} 4+\mathrm{T}$-cell count and other baseline factors.

The key results in this paper were presented in a poster at the Conference on Retroviruses and Opportunistic Infections in Boston, March 2020.

\section{Bibliography}

1. Gupta-Wright A, Corbett EL, van Oosterhout JJ, et al. Rapid urine-based screening for tuberculosis in HIV-positive patients admitted to hospital in Africa (STAMP): a pragmatic, multicentre, parallelgroup, double-blind, randomised controlled trial. Lancet 2018; 392(10144): 292-301. 


\section{RESEARCH PAPER COVER SHEET}

\section{PLEASE NOTE THAT A COVER SHEET MUST BE COMPLETED FOR EACH RESEARCH PAPER INCLUDED IN A THESIS.}

\section{SECTION A - Student Details}

\begin{tabular}{|l|l|}
\hline Student & Dr Fiona Cresswell \\
\hline Principal Supervisor & Prof Alison Elliott \\
\hline Thesis Title & $\begin{array}{l}\text { Improving diagnosis and treatment of HIV-associated } \\
\text { Tuberculous meningitis }\end{array}$ \\
\hline
\end{tabular}

If the Research Paper has previously been published please complete Section B, if not please move to Section C

\section{SECTION B - Paper already published}

\begin{tabular}{|l|l|l|l|}
\hline Where was the work published? & Open Forum Infectious Diseases \\
\hline When was the work published? & 20th March 2020 \\
\hline $\begin{array}{l}\text { If the work was published prior to } \\
\text { registration for your research degree, } \\
\text { give a brief rationale for its inclusion }\end{array}$ & \multicolumn{3}{|l|}{} \\
\hline $\begin{array}{l}\text { Have you retained the copyright for the } \\
\text { work?* }\end{array}$ & Yes & $\begin{array}{l}\text { Was the work subject to } \\
\text { academic peer review? }\end{array}$ & Yes \\
\hline
\end{tabular}

*If yes, please attach evidence of retention. If no, or if the work is being included in its published format, please attach evidence of permission from the copyright holder (publisher or other author) to include this work.

\section{SECTION C - Prepared for publication, but not yet published}

\begin{tabular}{|l|l|}
\hline $\begin{array}{l}\text { Where is the work intended to be } \\
\text { published? }\end{array}$ & \\
\hline $\begin{array}{l}\text { Please list the paper's authors in the } \\
\text { intended authorship order: }\end{array}$ & \\
\hline Stage of publication & \\
\hline
\end{tabular}

\section{SECTION D - Multi-authored work}

For multi-authored work, give full details of your role in the research included in the paper and in the preparation of the paper. (Attach a further sheet if necessary)
I spent time on the hospital wards assessing participants, collecting clinical samples and actioning results. I cleaned the data, undertook the analysis, interpreted the results, wrote the first draft of the manuscript, coordinated comments from coauthors, handled submission and review responses with input from co-authors 
Student Signature:

Supervisor Signature:
Date: _24th Sept 2020

8th October 2020

Date: 


\title{
Standardized Urine-Based Tuberculosis (TB) Screening With TB-Lipoarabinomannan and Xpert MTB/RIF Ultra in Ugandan Adults With Advanced Human Immunodeficiency Virus Disease and Suspected Meningitis
}

\begin{abstract}
Fiona V. Cresswell, ${ }^{1,2,3, a, \odot}$ Jayne Ellis, ${ }^{4, a, \odot}$ Enock Kagimu, ${ }^{1}$ Ananta S. Bangdiwala, ${ }^{5}$ Michael Okirwoth, ${ }^{1}$ Gerald Mugumya, ${ }^{6}$ Morris Rutakingirwa, John Kasibante, ${ }^{1}$ Carson M. Quinn, ${ }^{1}$ Kenneth Ssebambulidde, ${ }^{1}$ Joshua Rhein, ${ }^{1,8, \odot}$ Edwin Nuwagira, ${ }^{9}$ Lillian Tugume, ${ }^{1}$ Emily Martyn, ${ }^{1}$ Caleb P. Skipper, ${ }^{1,7}$ Conrad Muzoora, ${ }^{9}$ Daniel Grint, ${ }^{10}$ David B. Meya, ${ }^{1,7}$ Nathan C. Bahr, ${ }^{8}$ Alison M. Elliott, ${ }^{2,3}$ and David R. Boulware

${ }^{1}$ Infectious Diseases Institute, Makerere University, Kampala, Uganda, ${ }^{2}$ Clinical Research Department, London School of Hygiene and Tropical Medicine, London, United Kingdom, ${ }^{3}$ MRC-UVRILondon School of Hygiene and Tropical Medicine Uganda Research Unit, Entebbe, Uganda, ${ }^{4}$ Hospital for Tropical Diseases, University College London Hospitals NHS Foundation Trust, London, United Kingdom, ${ }^{5}$ Division of Biostatistics, School of Public Health, University of Minnesota, Minneapolis, Minnesota, USA, ${ }^{6}$ Microbiology Laboratory, Kiruddu Referral Hospital, Kampala, Uganda, ${ }^{7}$ Division of Infectious Diseases and International Medicine, Department of Medicine, University of Minnesota, Minneapolis, Minnesota, USA, ${ }^{8}$ Division of Infectious Diseases, Department of Medicine, University of Kansas, Kansas City, Kansas, USA, ${ }^{9}$ Mbarara University of Science and Technology, Mbarara, Uganda, ${ }^{10}$ Tropical Epidemiology Group, London School of Hygiene and Tropical Medicine, London, United Kingdom
\end{abstract}

Background. Diagnosis of extrapulmonary tuberculosis (TB) remains challenging. We sought to determine the prevalence of disseminated TB by testing urine with TB-lipoarabinomannan (TB-LAM) lateral flow assay and Xpert MTB/RIF Ultra (Ultra) in hospitalized adults.

Methods. We prospectively enrolled human immunodeficiency virus (HIV)-positive adults with suspected meningitis in Uganda during 2018-2020. Participants underwent standardized urine-based TB screening. Urine (60 mcL) was tested with TB-LAM (Alere), and remaining urine was centrifuged with the cell pellet resuspended in $2 \mathrm{~mL}$ of urine for Xpert Ultra testing.

Results. We enrolled 348 HIV-positive inpatients with median CD4 of 37 cells $/ \mathrm{mcL}$ (interquartile range, 13-102 cells $/ \mathrm{mcL}$ ). Overall, $26 \%$ ( 90 of 348 ; $95 \%$ confidence interval [CI], 21\%-30\%) had evidence of disseminated TB by either urine assay. Of 243 participants with both urine TB-LAM and Ultra results, 20\% (48 of 243) were TB-LAM-positive, 12\% (29 of 243) were Ultra-positive, and $6 \%$ (14 of 243) were positive by both assays. In definite and probable TB meningitis, 37\% (14 of 38) were TB-LAM-positive and $41 \%$ ( 15 of 37 ) were Ultra-positive. In cryptococcal meningitis, 22\% (40 of 183) were TB-LAM-positive and 4.4\% (6 of 135) were Ultra-positive. Mortality trended higher in those with evidence of disseminated TB by either assay (odds ratio $=1.44 ; 95 \% \mathrm{CI}$, $0.83-2.49 ; P=.19$ ) and was 6 -fold higher in those with definite $\mathrm{TB}$ meningitis who were urine Ultra-positive (odds ratio $=5.67 ; 95 \%$ CI, $1.13-28.5 ; P=.04)$.

Conclusions. In hospitalized Ugandans with advanced HIV disease and suspected meningitis, systematic screening with urine TB-LAM and Ultra found a high prevalence of urine TB test positivity (26\%). In those with TB meningitis, urine tests were positive in over one third. There was little concordance between Ultra and TB-LAM, which warrants further investigation.

Keywords. HIV; meningitis; TB-LAM; tuberculosis; Xpert MTB/RIF Ultra.

Tuberculosis (TB) is a major cause of death in people with human immunodeficiency virus (HIV). Human immunodeficiency virus-associated TB is often disseminated, defined as having 2 or more noncontiguous sites resulting from

Received 3 January 2020; editorial decision 17 March 2020; accepted 20 March 2020.

aF. V. C. and J. E. contributed equally to this work.

Correspondence: Fiona Cresswell, MBChB, BSc, Research Department, Infectious Diseases Institute, Kampala, PO Box 22418, Uganda (fiona.cresswell@Ishtm.ac.uk).

Open Forum Infectious Diseases ${ }^{\circledR}$

(C) The Author(s)2020. Published by Oxford UniversityPress onbehalf of Infectious Diseases Society of America. This is an Open Access article distributed under the terms of the Creative Commons Attribution License (http://creativecommons.org/licenses/by/4.0/), which permits unrestricted reuse, distribution, and reproduction in any medium, provided the original work is properly cited. DOl: 10.1093/ofid/ofaa100 lymphohematogenous dissemination of Mycobacterium tuberculosis $[1,2]$. Postmortem studies have shown that $>90 \%$ of cases involve multiple organ systems including spleen, liver, multiple lymph node groups, kidneys, or central nervous system [3]. Diagnosis of disseminated TB is taxing due to challenges in accessing clinical samples from the affected sites. In addition, in the context of HIV-associated meningitis, of which cryptococcal meningitis is the most common cause $[4,5]$, disseminated TB may not be considered due to the considerable overlap in the clinical presentation of the 2 diseases: ie, fevers, night sweats, cough, and wasting. There are case reports and case series of cryptococcal meningitis coinfection with $\mathrm{TB}$ $[6,7]$, including TB meningitis [8], but no published studies 
systematically screened for TB among HIV-positive adults presenting with neurological infections. It is unknown to what extent undiagnosed disseminated TB infection contributes to the high mortality in HIV-infected patients with neurological infections.

Urine-based TB diagnostics have the potential to reduce missed TB diagnoses and reduce mortality in patients with advanced HIV disease. Urine is easily obtainable and can be tested using either a lateral flow assay (LFA) for TB-lipoarabinomannan (TB-LAM) or the Xpert MTB/RIF assay (Xpert; Cepheid, Sunnyvale, CA). Tuberculosis-LAM is emerging as the key urine-based TB diagnostic. Published data, including a 2016 Cochrane review, demonstrate that the first-generation Alere Determine TB-LAM Ag assay (TB-LAM; Alere, Waltham, MA) has a sensitivity of $40 \%-70 \%$ and a specificity of $\geq 98 \%$ in TB/HIV-coinfected patients with CD4 counts $<100$ cells $/ \mu \mathrm{L}$ [9-11]. The STAMP trial evaluated the use of the Alere Determine TB-LAM Ag assay and Xpert MTB/RIF among HIV-positive patients hospitalized in Malawi and South Africa [12] and demonstrated reduced all-cause 56-day mortality with urine-based TB-LAM screening in certain high-risk subgroups of those with CD4 T-cell counts $<100$ cells $/ \mu \mathrm{L}$, severe anemia (hemoglobin $<8 \mathrm{~g} / \mathrm{dL}$ ), or clinically suspected TB. These findings corroborate those of an earlier study that found a $4 \%$ (95\% confidence interval [CI], 1\%-7\%) reduction in 8-week all-cause mortality in hospitalized HIV-positive adults with urine TB-LAM screening [13]. STAMP found that TB-LAM provided a 2-fold higher diagnostic yield than urine Xpert MTB/RIF. As a result, the World Health Organization (WHO) has recently updated their guidelines to support the expanded use of the Alere TB-LAM assay in all patients hospitalized with symptoms and signs of TB, CD 4 count $<200$ cells/ $\mu \mathrm{L}$ (irrespective of symptoms), or clinically advanced HIV disease, or who are seriously ill [14] .

Due to the limited sensitivity of urine Xpert MTB/RIF, urine screening with Xpert is not currently recommended by the WHO. In 2017, however, Cepheid introduced the re-engineered Xpert MTB/RIF Ultra (Ultra) with technical enhancements resulting in 7-fold improved analytical sensitivity and addition of a "trace" category for the lowest bacillary load [15]. Two diagnostic accuracy studies of Ultra on cerebrospinal fluid (CSF) in HIV-positive Ugandan adults with suspected TB meningitis found Ultra to be significantly more sensitive than Xpert MTB/ RIF against a composite reference standard [16, 17]. However, publications on Xpert Ultra use in urine have thus far been limited, and include a case report [18] and a small number of samples in diagnostic accuracy studies of Ultra in extrapulmonary samples [19, 20], without evaluation as a screening tool. In a Ugandan study, the performance of urine Xpert Ultra in diagnosing pulmonary $\mathrm{TB}$ was assessed, and researchers found that Xpert Ultra had significantly higher sensitivity than TB-LAM, with $50 \%$ sensitivity in patients with CD $<100$ cells/ $\mu \mathrm{L}$ [21]. This study, which also included HIV-negative patients, used sputum-based diagnostics as the reference standard. The suboptimal sensitivity of urine diagnostics in pulmonary TB diagnosis is perhaps not surprising because sputum studies are often discordant with urine diagnostics if the TB has not disseminated beyond the lungs, as may often be the case in HIVnegative patients [22]. In our study, we sought to determine the prevalence of disseminated TB infection by testing urine with the Alere TB-LAM LFA and Xpert Ultra in HIV-positive adults presenting with suspected meningitis.

\section{METHODS}

\section{Study Population}

We conducted a prospective cohort study as part of screening for an observational meningitis diagnostic study and a TB meningitis randomized controlled trial (RifT trial: ISRCTN 15668391). Consecutive HIV-infected adults presenting with suspected meningitis to Mulago National Referral Hospital, Kampala or Mbarara Regional Referral Hospital were screened for infectious meningitis etiologies using a stepwise diagnostic algorithm, as described elsewhere [23], after provision of written informed consent. Inclusion criteria for diagnostic testing were HIV-positive adults ( $\geq 18$ years) presenting with suspected meningitis (eg, headache or altered mental status, clinical signs of meningism). From March 27, 2018 to February 6, 2020, urine-based screening for TB was conducted using an Alere Determine TB-LAM LFA assay (TB-LAM; Alere) and Xpert Ultra (Cepheid). Urine TB testing was conducted for all patients within 48 hours of admission to hospital. Tuberculosis screening was performed irrespective of TB symptoms, past or current TB therapy, or CD4 T-cell count among consented participants. Cryptococcal meningitis was diagnosed by positive CSF cryptococcal antigen (CrAg) LFA at the bedside and later confirmed by culture. Participants with suspected or confirmed TB meningitis were categorized according to the 2010 uniform case definition; with "definite TB meningitis" comprising those with microbiologically confirmed $M$ tuberculosis in CSF and "probable TB meningitis" comprising those who score $\geq 10$ points (or $\geq 12$ points if brain imaging is performed) on clinical, CSF, and imaging criteria but who are negative for TB on CSF testing [24]. Due to its low specificity in a high HIV-burden setting "possible TB meningitis" was not included as a discrete diagnostic category.

\section{Study Procedures}

A suggested minimum of $30 \mathrm{~mL}$ of midstream, early morning urine was collected from participants able to provide a sample. In the laboratory, $60 \mu \mathrm{L}$ of unconcentrated urine was tested with the TB-LAM according to the manufacturers' instructions, and test samples were classified according to the reference card with grade 1 or above considered positive according to the WHO guidelines [14]. The remainder of the urine specimen 
was measured using calibrated Falcon tubes, the volume was recorded, and the urine was processed for Ultra testing. The urine was concentrated by centrifugation at $3000 \times g$ for 15 minutes at room temperature. Surplus urine was decanted off leaving $2 \mathrm{~mL}$ in which the cell pellet was resuspended and loaded into the cartridge for Xpert Ultra testing. No buffer was used. TuberculosisLAM and Xpert Ultra testing were performed simultaneously by a laboratory technologist blinded to the patient history, and the TB-LAM result was reported independently without awareness of the Ultra result. Both results were reported in real-time to the clinical team.

Clinical management, including timing of commencement of anti-TB therapy and additional TB investigation, was at the discretion of the attending physician; wherever possible, anti-TB therapy was started as soon as possible for all patients with a positive TB-LAM and/or Xpert Ultra result, and additional work-up, including collection of additional clinical samples where possible for TB microbiology (Xpert Ultra and/or Mycobacteria Growth Inhibitor Tube [MGIT] culture; Becton Dickinson, Franklin Lakes, NJ), chest radiography, and abdominal ultrasound, was performed where indicated and feasible.

Baseline demographics, clinical characteristics including antiretroviral therapy (ART) use, and laboratory data were collected as part of the screening process. Consented participants were followed up until hospital discharge or through $>10$ weeks if enrolled into a meningitis clinical trial.

\section{Ethics}

All participants (or a surrogate in cases of mental incapacity) provided written informed consent for urine testing, lumbar puncture, CSF testing, CSF storage, and data collection. Approval for the studies was obtained from the Mulago Hospital Research Ethics Committee (MHREC1260 and MHREC1246), Uganda National Council for Science and Technology, University of Minnesota, and the London School of Hygiene and Tropical Medicine.

\section{Statistical Analysis}

Statistical analysis is primarily descriptive with the primary endpoint of interest being the prevalence of disseminated TB detected by urine testing. We compared baseline characteristics across meningitis etiologies, with Kruskall-Wallis tests comparing continuous variables and $\chi^{2}$ tests comparing categorical variables. We compared the proportion of positive Urine TB-LAM results and the distribution of TB-LAM grade across meningitis etiologies using a $\chi^{2}$ test. We compared congruency in positive TB-LAM versus Xpert Ultra tests by McNemar's test, and we compared the distribution of urine Xpert Ultra semiquantitative grade across meningitis etiologies using a $\chi^{2}$ test and Fisher's exact test (as appropriate according to sample size). We tested the relationship between urine volume and a positive Xpert Ultra result using a $\chi^{2}$ test with volume dichotomized at the median $24 \mathrm{~mL}$ (range, $4.5-45 \mathrm{~mL}$ ). We reported the proportion surviving hospitalization by each urine TB diagnostics result, and we compared test results and mortality using univariable logistic regression. $P$ values were not adjusted for multiple testing. All analyses were conducted using SAS (version 9.4; SAS Institute, Cary, NC).

\section{RESULTS}

\section{Patient Demographics}

We enrolled 348 HIV-positive hospitalized participants, 58\% (201 of 348) of whom were men. The median age was 35 years (interquartile range [IQR], 29-42). The study participants had advanced HIV disease (median CD4 = 37 cells/ $\mu \mathrm{L} ; \mathrm{IQR}, 13-102$ cells/mcL), despite $48 \%$ (167 of 347 ) reporting ART use at the time of presentation (implying virologic failure, suboptimal adherence, or recent initiation). The majority of participants had cryptococcal meningitis (57\%, 198 of 348); 18\% (62 of 348) had definite or probable $\mathrm{TB}$ meningitis according to the uniform clinical case definition [24]. Cerebrospinal fluid features by meningitis etiology are shown in Table 1.

\section{Diagnostic Yield of Urine Tuberculosis-Lipoarabinomannan and Ultra}

Overall, $26 \%$ (90 of $348 ; 95 \%$ CI, 21\%-30\%) of the study participants had evidence of disseminated TB by positive TB urine assay: $23 \%$ ( 75 of $327 ; 95 \% \mathrm{CI}, 18 \%-27 \%$ ) positive by urine TB-LAM, and $11 \%$ ( 29 of $264 ; 95 \%$ CI, 7\%-15\%) positive by urine Xpert Ultra. In cryptococcal patients, $22 \%$ (40 of 183) had evidence of disseminated TB by urine TB-LAM and 4.4\% (6 of 135) by urine Xpert Ultra. In definite $T B$ meningitis patients, $37 \%$ (14 of 38) had a positive urine TB-LAM and 41\% (15 of 37) had a positive urine Xpert Ultra. In probable TB meningitis patients, $41 \%$ ( 9 of 22 ) had a positive urine TB-LAM and $21 \%$ (4 of 19) had a positive on urine Xpert Ultra. In a composite of definite and probable TB meningitis, 37\% (23 of 62) had a positive urine TB-LAM and 34\% (19 of 56) had a positive Xpert Ultra (Table 2 and Supplemental Figure 1). Among urine TB-LAMnegative subjects, the number needed to test with Xpert Ultra to diagnose an additional case of disseminated TB was 13 persons (95\% CI, 8.0-22.9).

\section{Concordance Between Tuberculosis-Lipoarabinomannan and Ultra}

Overall, 243 participants had both urine TB-LAM and Xpert Ultra results available for paired analysis. Of 63 participants with a positive urine TB assay, $76 \%$ (48 of 63) were positive by TB-LAM and $46 \%$ (29 of 63) were positive by Xpert Ultra $(P=.01$ ). Only $22 \%$ (14 of 63) of those with a positive urine TB test were positive by both TB-LAM and Ultra (Figure 1). The overlap of TB-LAM and Ultra was greater among those with definite TB meningitis. Of the 35 definite TB meningitis participants tested with both assays, 51\% (18 of 35) had positive urine test, $34 \%$ (12 of 35 ) were urine TB-LAM positive, and $43 \%$ (15 of 35 ) were urine Ultra positive with an overlap of $50 \%$ ( 9 of 
Table 1. Participant Baseline Demographics, Clinical, and CSF Findings by Disease Category

\begin{tabular}{|c|c|c|c|c|}
\hline Characteristics & Cryptococcal Meningitis & TBM (Confirmed) & TBM (Probable) & Unknown Other ${ }^{a}$ \\
\hline N & 198 & 40 & 22 & 88 \\
\hline Age, years & 35 [29-42] & 32 [28-36] & 38 [30-48] & 37 [30-44] \\
\hline Women & $79(40 \%)$ & $17(43 \%)$ & $11(50 \%)$ & $40(45 \%)$ \\
\hline \multicolumn{5}{|l|}{ HIV Factors } \\
\hline $\mathrm{CD}^{+} \mathrm{T}$ cells $/ \mu \mathrm{L}$ & $23[8-70]$ & $72[41-111]$ & $55[22-186]$ & $205[45-340]$ \\
\hline Currently on ART & $91(46 \%)$ & $17(43 \%)$ & $8(38 \%)$ & $51(58 \%)$ \\
\hline Months on $\mathrm{ART}^{\mathrm{b}}$ & $2.3[0.6-29.4]$ & $2.2[0.7-27.9]$ & $1.5[0.5-11.3]$ & $6.9[1.6-73.9]$ \\
\hline \multicolumn{5}{|l|}{ Clinical } \\
\hline Fever & $77(52 \%)$ & $34(97 \%)$ & $20(91 \%)$ & $46(68 \%)$ \\
\hline Headache & $190(98 \%)$ & $34(94 \%)$ & $15(68 \%)$ & $61(76 \%)$ \\
\hline Duration of headache & $14[7-21]$ & $14[8-21]$ & $14[7-21]$ & $14[7-30]$ \\
\hline Focal neurologic deficit & $7(4 \%)$ & $15(38 \%)$ & $8(36 \%)$ & $21(24 \%)$ \\
\hline Wasting & $81(42 \%)$ & $23(58 \%)$ & $13(59 \%)$ & $35(41 \%)$ \\
\hline Glasgow come score $<15$ & $70(35 \%)$ & $36(92 \%)$ & $19(86 \%)$ & $45(52 \%)$ \\
\hline Seizure & $41(30 \%)$ & $11(38 \%)$ & $4(21 \%)$ & $23(38 \%)$ \\
\hline Weight, kg & $52[45-60]$ & $55[45-58]$ & $53[50-60]$ & $50[50-59]$ \\
\hline Cough & $56(39 \%)$ & $25(74 \%)$ & $10(53 \%)$ & $39(61 \%)$ \\
\hline Night sweats ${ }^{c}$ & $31(25 \%)$ & $22(85 \%)$ & $13(72 \%)$ & $24(44 \%)$ \\
\hline \multicolumn{5}{|l|}{ Baseline CSF Results } \\
\hline Opening pressure, $\mathrm{cm} \mathrm{H}_{2} \mathrm{O}$ & $23[14-32]$ & $19[10-29]$ & 15 [10-24] & 18 [12-23] \\
\hline Opening pressure $<20 \mathrm{~cm} \mathrm{H}_{2} \mathrm{O}$ & $66(38 \%)$ & $16(52 \%)$ & $10(67 \%)$ & $40(62 \%)$ \\
\hline Total WBC count cells $\mu \mathrm{L}$ & $<5[<5-50]$ & $30[<5-220]$ & $<5[<5-200]$ & $<5[<5-30]$ \\
\hline Total WBC count $<5 \mu \mathrm{L}$ & $117(60 \%)$ & $17(45 \%)$ & $11(52 \%)$ & $61(71 \%)$ \\
\hline CSF protein, mg/dL & $69[36-100]$ & $128[61-200]$ & $88[31-151]$ & $68[28-111]$ \\
\hline CSF WBC $<5 \mu \mathrm{L}$ and protein $<45 \mathrm{mg} / \mathrm{dL}$ & $51(28 \%)$ & $5(15 \%)$ & $4(20 \%)$ & $26(36 \%)$ \\
\hline
\end{tabular}

Abbreviations: ART, antiretroviral therapy; CSF, cerebrospinal fluid; HIV, human immunodeficiency virus; IQR, interquartile range; TBM, tuberculous meningitis; WBC, white blood cells. NOTE: Data are $\mathrm{n}(\%)$ or median [IQR].

${ }^{a}$ Includes possible and nontuberculous meningitis diagnoses.

${ }^{\mathrm{b}}$ Among those on ART at diagnosis.

${ }^{\mathrm{c}}$ Data on night sweats were only available for 122 of cryptococcosis, 26 definite TB meningitis, 18 probable TB meningitis, and 54 other.

Table 2. Urine TB Diagnostic Test Results by Disease Category

\begin{tabular}{|c|c|c|c|c|c|}
\hline Urine TB Testing Details & Cryptococcal Meningitis & TBM (Confirmed) & TBM (Probable) & Unknown Other ${ }^{a}$ & $P$ Value ${ }^{b}$ \\
\hline N & 198 & 40 & 22 & 88 & \\
\hline \multicolumn{6}{|l|}{ Urine TB-LAM } \\
\hline Tested with TB-LAM, N & 183 & 38 & 22 & 84 & \\
\hline Urine TB-LAM positive, $n(\%)$ & $40(21.9 \%)$ & $14(36.8 \%)$ & $9(40.9 \%)$ & $12(14.3 \%)$ & $<.01$ \\
\hline LAM Grade 1 & $25(13.7 \%)$ & $5(13.2 \%)$ & $4(18.2 \%)$ & $7(8.3 \%)$ & \\
\hline LAM Grade 2 & $9(4.9 \%)$ & $0(0.0 \%)$ & $4(18.2 \%)$ & $4(4.8 \%)$ & \\
\hline LAM Grade 3 & $5(2.7 \%)$ & $7(18.4 \%)$ & $1(4.5 \%)$ & $0(0.0 \%)$ & \\
\hline LAM Grade 4 & $1(0.5 \%)$ & $2(5.3 \%)$ & $0(0.0 \%)$ & $1(1.2 \%)$ & \\
\hline \multicolumn{6}{|l|}{ Urine Xpert Ultra } \\
\hline Tested with Xpert Ultra, N & 135 & 37 & 19 & 73 & \\
\hline Urine Xpert Ultra positive, $\mathrm{n}(\%)$ & $6(4.4 \%)$ & $15(40.5 \%)$ & $4(21.1 \%)$ & $4(5.5 \%)$ & $<.001$ \\
\hline Semiquantitative trace & $1(0.7 \%)$ & $4(10.8 \%)$ & $1(5.3 \%)$ & $0(0.0 \%)$ & \\
\hline Semiquantitative very low & $1(0.7 \%)$ & $4(10.8 \%)$ & $0(0.0 \%)$ & $1(1.4 \%)$ & \\
\hline Semiquantitative low & $2(1.5 \%)$ & $4(10.8 \%)$ & $3(15.8 \%)$ & $0(0.0 \%)$ & \\
\hline Semiquantitative medium & $0(0.0 \%)$ & $2(5.4 \%)$ & $0(0.0 \%)$ & $1(1.4 \%)$ & \\
\hline Grade unknown & $2(1.5 \%)$ & $1(2.7 \%)$ & $0(0.0 \%)$ & $2(2.7 \%)$ & \\
\hline \multicolumn{6}{|l|}{ Either TB Test Positive } \\
\hline TB-LAM and/or Xpert Ultra positive & $44(22 \%)$ & $20(50 \%)$ & $11(50 \%)$ & $15(17 \%)$ & $<.001$ \\
\hline
\end{tabular}

Abbreviations: TB, tuberculosis; TB-LAM, TB-lipoarabinomannan; TBM, tuberculous meningitis.

ancludes possible and nontuberculous meningitis diagnoses.

${ }^{\mathrm{b}} P$ value assessed by $\chi^{2}$ distribution of LAM positivity and Ultra positivity across disease groups. 


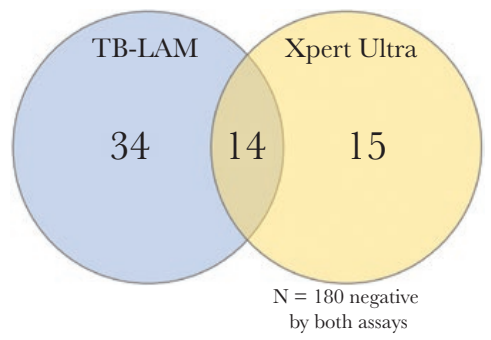

Figure 1. Venn diagram of overlap between tuberculosis (TB)-positive urinary assays. Of 243 persons with both TB-lipoarabinomannan (TB-LAM) and urine Xpert Ultra results, 63 were positive by either TB urinary assay, $76 \%$ (48 of 63 ) of whom were positive by TB-LAM and 46\% (29 of 63) by Xpert Ultra ( $P=.01$ ). Only 22\% (14 of 63) of those with a positive urine TB test were positive by both TB-LAM and Ultra. Among the 195 persons with a negative TB-LAM, 15 (7.7\%) were positive by Xpert Ultra, with a number needed to test with Ultra to diagnose an additional case of disseminated TB being 13 (95\% Cl, 8.0-22.9).

18 of positive tests). Overall, 17 persons with microbiologically confirmed TB meningitis had negative urinary tests (Figure 2).

\section{Urine Diagnostics as a Predictor of In-Hospital Mortality}

Among those who are known to have died in hospital, the median time to death was 7 days (IQR, 3-12 days). Among participants with disseminated TB with either urine assay positive, the mortality was $33 \%$ (27 of 82 ), compared with $25 \%$ (59 of 232) among those with negative urine assays (odds ratio $=1.44$; 95\% CI, $0.83-2.49 ; P=.19$ ). The mortality was also higher (albeit nonsignificantly) among participants with positive urine TB-LAM ( $32 \%$ positive LAM vs $26 \%$ negative LAM, $P=.32$ ) and among urine Xpert Ultra positive participants ( $36 \%$ vs $24 \%$, $P=.18)$. However, among those with definite TB meningitis, there was $50 \%$ mortality ( 7 of 14 ) in those with positive urine Xpert Ultra compared with 23\% (3 of 20) in those with negative urine Xpert Ultra (odds ratio $=5.67 ; 95 \%$ CI, 1.13-28.5;

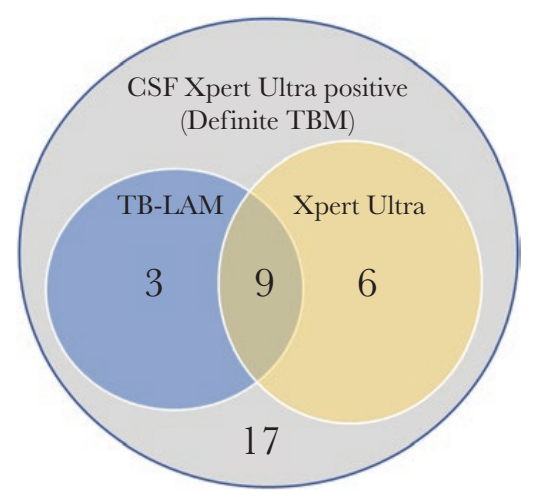

Figure 2. Venn diagram illustrating overlap of tuberculosis-lipoarabinomannan (TB-LAM) and urine Ultra among those with definite TB meningitis (TBM). Of 35 persons with definite TBM tested with both assays, a total of $18(51 \%)$ had positive urine test, 12 (34\%) were urine TB-LAM positive, and $15(43 \%)$ were urine Ultra positive with an overlap of 9 of $18(50 \%)$ positive participants being positive by both assays. Overall, 49\% (17 of 35) with definite TBM, diagnosed by positive CSF Xpert Ultra, had negative urinary tests.
$P=.04)$. With either urine TB-LAM or Ultra positive, the odds of dying were 3 -fold higher (odds ratio $=3.00$; $95 \% \mathrm{CI}, 0.71$ $12.7 ; P=.14$ ) in those with definite TB meningitis (Table 3).

\section{Relationship Between Urine Volume and Ultra Positivity}

Among patients with urine volume measured and recorded, $13 \%$ ( 8 of 64 ) of patients with $<24 \mathrm{~mL}$ tested positive by Ultra, and $9 \%$ (6 of 65 ) of patients with $\geq 24 \mathrm{~mL}$ tested positive by Ultra $(P=.55)$. The distribution of urine volume among those with definite TB meningitis was the same as the rest of the study population, thus larger centrifuged volumes did not yield a higher prevalence of detecting TB.

We tested for differences in the demographics, clinical, or laboratory parameters of patients who were positive by a single urine assay or both assays. We explored available laboratory parameters by urine test positivity, and there were no significant differences in baseline CD4 count, hemoglobin, or creatinine level in patients who had discordant results (Supplementary Table 1).

\section{DISCUSSION}

We have demonstrated that the prevalence of urine TB test positivity is $26 \%$ amongst HIV-infected patients presenting to hospital with suspected meningitis and advanced HIV disease in Uganda. The urine TB-LAM assay had more positive results (23\%) than urine Xpert Ultra (11\%), yet Xpert Ultra positivity was associated with higher risk of mortality (36\% in-hospital mortality), particularly in those with TB meningitis (50\% in-hospital mortality). Uganda has a high TB incidence rate (200 of 100000 population in 2018); our findings may be generalizable to many other countries in sub-Saharan Africa with similar TB incidence rates [25].

Cryptococcosis and TB coinfection (including TB meningitis) has been described in case reports, and in the Cryptococcal Optimal ART Timing (COAT) trial where 18\% (32 of 177) of participants with cryptococcal meningitis were treated for concurrent active TB disease during the 46-week study period [26]. However, this is the first study in which disseminated TB has been systematically screened for in an HIVassociated meningitis cohort. Among the 198 cryptococcal meningitis patients, one quarter had a positive urine TB test, TB-LAM (22\%) or Xpert Ultra (4.4\%). The true prevalence of disseminated TB could be higher than this given that urine TB testing may not detect all forms of disseminated TB. Given the considerable overlap in symptoms between cryptococcal meningitis and disseminated TB in advanced HIV disease, it is likely that without routine screening for $\mathrm{TB}$, some of these diagnoses would be missed, and therefore they may contribute to the high mortality associated with cryptococcal meningitis. Consistent with other studies, in-hospital mortality was high in our cryptococcal meningitis subgroup and trended towards there being 
Table 3. In-Hospital Mortality by Urine Diagnostic Result and Meningitis Aetiology by Univariate Logistic Regression ${ }^{\mathrm{a}}$

\begin{tabular}{|c|c|c|c|c|c|c|c|c|c|c|}
\hline \multirow[b]{2}{*}{$\begin{array}{l}\text { Study } \\
\text { Population }\end{array}$} & \multicolumn{4}{|c|}{ Alere TB-LAM } & \multicolumn{4}{|c|}{ Xpert MTB/RIF Ultra } & \multicolumn{2}{|c|}{ Either TB Assay Positive ${ }^{b}$} \\
\hline & Positive & Negative & $\begin{array}{l}\text { Odds Ratio } \\
(95 \% \mathrm{Cl})\end{array}$ & $\begin{array}{c}P \\
\text { Value }\end{array}$ & Positive & Negative & $\begin{array}{l}\text { Odds Ratio } \\
(95 \% \mathrm{Cl})\end{array}$ & $\begin{array}{c}P \\
\text { Value }\end{array}$ & $\begin{array}{l}\text { Odds Ratio } \\
(95 \% \mathrm{Cl})\end{array}$ & $\begin{array}{c}P \\
\text { Value }\end{array}$ \\
\hline Overall & $32 \%$ (22 of 68 ) & $26 \%$ (60 of 229$)$ & $1.35(0.75-2.42)$ & .32 & $36 \%(10$ of 28$)$ & $24 \%$ (51 of 214 ) & $1.78(0.77-4.09)$ & .18 & $1.44(0.83-2.49)$ & .19 \\
\hline Cryptococcal & $32 \%(12$ of 38$)$ & $27 \%$ (36 of 133 ) & $1.24(0.57-2.72)$ & .59 & $50 \%(3$ of 6$)$ & $23 \%$ (28 of 121$)$ & $3.32(0.63-17.4)$ & .16 & $1.41(0.67-2.96)$ & .37 \\
\hline TBM, definite & $38 \%(5$ of 13$)$ & $32 \%(7$ of 22$)$ & $1.34(0.32-5.61)$ & .69 & $50 \%(7$ of 14$)$ & $15 \%(3$ of 20$)$ & $5.67(1.13-28.5)$ & .04 & $3.00(0.71-12.7)$ & .14 \\
\hline TBM, probable & $50 \%$ (4 of 8 ) & $38 \%$ (5 of 13$)$ & $1.60(0.27-9.49)$ & .60 & $0 \%(0$ of 4$)$ & $50.0 \%$ (7 of 14$)$ & & & & \\
\hline Other/unknown & $11.1 \%$ (1 of 9 ) & $19.7 \%(12$ of 61$)$ & $0.51(0.06-4.48)$ & .54 & $0 \%(0 / 4)$ & $22 \%(13 / 59)$ & & & $0.34(0.04-2.84)$ & .32 \\
\hline
\end{tabular}

Abbreviations: $\mathrm{Cl}$, confidence interval; TB, tuberculosis; TB-LAM, TB-lipoarabinomannan; TBM, tuberculous meningitis.

${ }^{a}$ Data represent mortality by TB test status. Hospital outcome was unknown for 34 participants (7.5\% of cryptococcal, 7.5\% TBM definite, $5 \%$ TBM probable, $17 \%$ unknown/other meningitis). Among those who are known to have died in hospital, the median time to death was 7 days (interquartile range, 3-12 days).

${ }^{b}$ Either TB assay positive is any positive urine diagnostic (TB-LAM or Ultra or both) versus both assays being negative.

persistent increased mortality in coprevalent disseminated TB infection despite clinical teams having real-time access to TB urine diagnostic results; however, this study was not designed nor powered to determine an impact of coprevalent $\mathrm{TB}$ on mortality.

We investigated the clinical utility of TB urine diagnostics in $\mathrm{TB}$ meningitis, a condition that is notoriously challenging to diagnose even with molecular CSF diagnostics. Overall, 50\% (20 of 40; with both urine tests) of definite TB meningitis participants had either a positive TB-LAM or urine Xpert Ultra. Among probable TB meningitis cases, patients with highly suggestive clinical features but in whom rapid CSF diagnostics (acid-fast bacilli [AFB] smear and CSF Xpert Ultra) were negative, $41 \%$ were urine TB-LAM positive and $21 \%$ urine Ultra positive. A lack of sensitive and timely diagnostics has historically made $\mathrm{TB}$ meningitis diagnoses extremely difficult, and therefore treatment for probable TB meningitis rests upon the presence of symptoms or signs indicative of meningitis, CSF features plus suggestive imaging criteria or confirmed evidence of extra-neural TB. The uniform case definition agreed by consensus in 2010 does not include the TB-LAM assay as "evidence of extra-neural TB" [24], but our data suggest that this may be warranted. In this study a positive urine TB test was interpreted as evidence of extra-neural TB when assigning points in the uniform case definition. Urine TB diagnostics have the potential to improve our classification of TB meningitis cases and warrant investigation in a larger study. Although not routinely used as part of a TB meningitis diagnostic work-up, urine is an easily obtained sample, and TB-LAM and Ultra are relatively low cost; therefore, we advocate their use as adjunctive tests in the investigation of patients with suspected TB meningitis.

Urine Xpert Ultra has previously been used to diagnose disseminated TB in a case report [18] and has been evaluated as a marker of pulmonary TB in a cross-sectional study [21], but this is the first time that urine Xpert Ultra has been evaluated as a screening test for disseminated TB in a prospective cohort and correlated with survival. Urine Xpert Ultra was positive in $11 \%$ of our (tested) cohort overall, demonstrating that urine is an additional viable clinical sample for use with Ultra. It is interesting to note that in patients with definite TB meningitis, Xpert Ultra and TB-LAM were positive in a similar proportion of patients $(41 \%$ and $37 \%$ respectively), whilst there were considerably more positive TB-LAM tests than Xpert Ultra tests in patients with cryptococcal meningitis (22\% and $4 \%$ respectively). Our data raise several questions about the MTB antigen load required for respective test positivity, the performance of urine Xpert Ultra at different degrees of immunosuppression, the impact of renal TB infection versus TB bacteremia on test performance, and the specificity of TB-LAM in patients with disseminated fungal diseases or other opportunistic infections [27].

In our subgroup analysis, in-hospital mortality was 6 -fold higher $(50 \%$ vs $15 \%$; odds ratio $=5.67 ; 95 \%$ CI, $1.13-28.5 ; P$ $=.04)$ in patients with definite TB meningitis and a positive urine Xpert Ultra in comparison to patients with definite TB meningitis and a negative urine tests. These data suggest that urine Ultra testing may be a useful test to identify TB meningitis patients with at particularly higher risk of death and thus requiring more intensive $\mathrm{TB}$ therapy or alternative or adjunctive host-directed immunotherapy. Future research is needed to explore whether urine Ultra positivity and the increased mortality relates to burden of MTB infection, host factors, or both.

Overall, there was little concordance between urine Xpert Ultra and TB-LAM positivity, only $22 \%$ of those positive by either test were positive by both assays. A similar observation was made in the STAMP trial, in which only $23 \%$ of patients had both urine TB-LAM and urine Xpert MTB/RIF positivity [12]. The reasons for this are unclear. We observed no statistically significant differences in the demographics, clinical, or laboratory parameters of patients who were positive by a single urine assay or both assays, although our study was not powered to detect a clear association between these variables and urine assay positivity. We need to further investigate the lack of concordance between TB-LAM and urine Xpert Ultra and to explore whether this relates to the following: (1) 
variation in the $M$ tuberculosis burden required for test positivity; (2) interpatient differences in the renal parenchymal changes associated with renal TB or HIV-related nephropathy; or (3) differences in host immunity that may impact on the degree of immune complexing of circulating TB-LAM antigen and thereby its potential to cross the basement membrane [28]. Such a study could include immunological and renal parameters (such as proteinuria or urine-protein to creatinine ratio) to investigate whether the degree of immunodeficiency or glomerular pathology may contribute towards the likelihood of excreting $M$ tuberculosis bacilli or the TB-LAM glycolipid-the latter is only $17.5 \mathrm{kDa}$ without immune complexing but $150-1000 \mathrm{kDa}$ when complexed with anti-LAM antibodies-into the urine.

One strength of our study is that we studied a large cohort of 348 adults and this is the first time that urine TB diagnostics have been evaluated in a prospective manner among HIV-associated meningitis patients. However, we do recognize limitations to our study. Due to either suboptimal volume of urine sample or due to Ultra test availability, not all patients had TB-LAM and Xpert Ultra test results available for analysis. In instances in which urine volume was limited, TB-LAM testing was prioritized as per WHO guidelines, and overall more TB-LAM tests were conducted because bedside TB-LAM tests were consistently more available than Ultra. To ensure a fair comparison of the diagnostic performance of TB-LAM and urine Xpert Ultra, the concordance and diagnostic yield of each test was only compared in 243 participants who had both test results available. Second, due to the high rates of urinary incontinence in this critically ill population, not all participants managed to provide the suggested $>30 \mathrm{~mL}$ of urine. Low urine volume may have impacted the sensitivity of Ultra, although we did not detect a difference in TB test positivity in urine samples above and below the median $24-\mathrm{mL}$ volume. Third, our study was exploratory and not powered to detect a clear association between TB-LAM and/or Xpert Ultra positivity and death. Although there was a trend towards worse outcomes in patients with evidence of disseminated TB, our data herein provide estimates necessary for future sample size calculations. Finally, due to the study setting and population (critically ill meningitis patients), we were unable to perform an extensive array of other TB diagnostics tests such as imaging or sputum collection to corroborate the urine results. However, urine TB-LAM has a high specificity ( $\geq 98 \%$ in TB/HIV-coinfected patients), and although there is a lack of published data on the specificity of urine Ultra, Ultra has a high specificity when used on other clinical samples [11, 15]; therefore, in this study population with advanced HIV disease in a setting known to have a high prevalence of $\mathrm{TB}$, we believe the risk of false-positive results is minimal. The duration of urine Xpert Ultra positivity after prior TB treatment is unknown, but given the continuous filtration of the kidneys and that urine is normally a sterile fluid, it is unlikely that nonviable bacilli would persist months or years after prior treatment, although further study of this point is warranted.

\section{CONCLUSIONS}

In conclusion, we have demonstrated a $26 \%$ prevalence of positive urine TB tests in patients presenting with HIV-associated meningitis. Our results support the expanded use of TB urine diagnostics in patients with meningitis to optimize timely diagnosis and treatment of TB, especially, but not only, in settings such as sub-Saharan Africa where TB infection prevalence is extremely high. Further studies are required to investigate whether disseminated $\mathrm{TB}$ is an independent risk factor for death in cryptococcal meningitis and whether enhanced TB treatment can improve outcomes. We have also shown that urine TB diagnostics are of use in TB meningitis; first as rapid, noninvasive adjunctive tests for suspected TB meningitis and second, possibly, as a prognostic tool in patients with HIV-associated TB meningitis. Further research is required to investigate the lack of concordance between urine TB-LAM and urine Xpert Ultra.

\section{Supplementary Data}

Supplementary materials are available at Open Forum Infectious Diseases online. Consisting of data provided by the authors to benefit the reader, the posted materials are not copyedited and are the sole responsibility of the authors, so questions or comments should be addressed to the corresponding author.

Supplementary Table 1. Demographic, HIV, clinical, and laboratory factors by urine TB diagnostic result.

\section{Acknowledgments}

Financial support. This work was funded by a Wellcome Trust Clinical PhD Fellowship (Grant 210772/Z/18/Z; to F. V. C.), the National Institute of Neurologic Disorders and Stroke (R01NS086312 and K23NS110470; to D. R. B. and D. B. M.), the Fogarty International Center (K01TW010268; to J. R.), and the National Institute of Allergy and Infectious Diseases (T32AI055433 and R01AI145437; to D. R. B. and D. B. M.). F. V. C. is an honorary fellow of the Makerere University-Uganda Virus Research Institute Centre of Excellence for Infection and Immunity Research and Training (MUII-plus). MUII-plus is supported through the DELTAS Africa Initiative (Grant no. 107743). The DELTAS Africa Initiative is an independent funding scheme of the African Academy of Sciences (AAS), Alliance for Accelerating Excellence in Science in Africa (AESA) and supported by the New Partnership for Africa's Development Planning and Coordinating Agency (NEPAD Agency) with funding from the Wellcome Trust (Grant no. 107743) and the UK Government. The MRC/UVRI and LSHTM Uganda Research Unit is jointly funded by the UK Medical Research Council (MRC) and the UK Department for International Development (DFID) under the MRC/DFID Concordat agreement and is also part of the European and Developing Countries Clinical Trials Partnership (EDCTP)-2 programme supported by the European Union.

Potential conflicts of interest. All authors have submitted the ICMJE Form for Disclosure of Potential Conflicts of Interest. Conflicts that the editors consider relevant to the content of the manuscript have been disclosed.

\section{References}

1. Kerkhoff AD, Barr DA, Schutz C, et al. Disseminated tuberculosis among hospitalised HIV patients in South Africa: a common condition that can be rapidly diagnosed using urine-based assays. Sci Rep 2017; 7:10931.

2. Ayaslioglu E, Basar H, Duruyurek N, et al. Disseminated tuberculosis with lymphatic, splenic and scrotal abscesses: a case report. Cases J 2009; 2:6995. 
3. Gupta RK, Lucas SB, Fielding KL, Lawn SD. Prevalence of tuberculosis in postmortem studies of HIV-infected adults and children in resource-limited settings: a systematic review and meta-analysis. AIDS 2015; 29:1987-2002.

4. Jarvis JN, Meintjes G, Williams A, et al. Adult meningitis in a setting of high HIV and TB prevalence: findings from 4961 suspected cases. BMC Infect Dis 2010; 10:67.

5. Hakim JG, Gangaidzo IT, Heyderman RS, et al. Impact of HIV infection on meningitis in Harare, Zimbabwe: a prospective study of 406 predominantly adult patients. AIDS 2000; 14:1401-7.

6. Chen M, Al-Hatmi AM, Chen Y, et al. Cryptococcosis and tuberculosis co-infection in mainland China. Emerg Microbes Infect 2016; 5:e98.

7. Fang W, Zhang L, Liu J, et al. Tuberculosis/cryptococcosis co-infection in China between 1965 and 2016. Emerg Microbes Infect 2017; 6:e73.

8. Ellis J, Cresswell FV, Joshua R, et al. Cryptococcal meningitis and tuberculous meningitis co-infection in HIV-infected Ugandan adults. Open Forum Infect Dis 2018; 5: ofy 193 .

9. Lawn SD, Kerkhoff AD, Burton R, et al. Diagnostic accuracy, incremental yield and prognostic value of determine TB-LAM for routine diagnostic testing for tuberculosis in HIV-infected patients requiring acute hospital admission in South Africa: a prospective cohort. BMC Med 2017; 15:67.

10. Bjerrum S, Kenu E, Lartey M, et al. Diagnostic accuracy of the rapid urine lipoarabinomannan test for pulmonary tuberculosis among HIV-infected adults in Ghana-findings from the DETECT HIV-TB study. BMC Infect Dis 2015; 15:407.

11. Shah M, Hanrahan C, Wang ZY, et al. Lateral flow urine lipoarabinomannan assay for detecting active tuberculosis in HIV-positive adults. Cochrane Database Syst Rev 2016; 2016:CD011420.

12. Gupta-Wright A, Corbett EL, van Oosterhout JJ, et al. Rapid urine-based screening for tuberculosis in HIV-positive patients admitted to hospital in Africa (STAMP): a pragmatic, multicentre, parallel-group, double-blind, randomised controlled trial. Lancet 2018; 392:292-301.

13. Peter JG, Zijenah LS, Chanda D, et al. Effect on mortality of point-of-care, urinebased lipoarabinomannan testing to guide tuberculosis treatment initiation in HIV-positive hospital inpatients: a pragmatic, parallel-group, multicountry, open-label, randomised controlled trial. Lancet 2016; 387:1187-97.

14. World Health Organization. Lateral flow urine lipoarabinomannan assay (LF-LAM) for the diagnosis of active tuberculosis in people living with HIV. Policy update 2019. Geneva: World Health Organization; 2019.

15. Chakravorty S, Simmons AM, Rowneki M, et al. The new Xpert MTB/RIF Ultra: improving detection of Mycobacterium tuberculosis and resistance to rifampin in an assay suitable for point-of-care testing. mBio 2017; 8:e00812-17

16. Bahr NC, Nuwagira E, Evans EE, et al.; ASTRO-CM Trial Team. Diagnostic accuracy of Xpert MTB/RIF Ultra for tuberculous meningitis in HIV-infected adults: a prospective cohort study. Lancet Infect Dis 2018; 18:68-75.

17. Cresswell FV, Tugume L, Bahr NC, et al.; ASTRO-CM team. Xpert MTB/RIF Ultra for the diagnosis of HIV-associated tuberculous meningitis: a prospective validation study. Lancet Infect Dis 2020; 20:308-17.

18. Atherton RR, Cresswell FV, Ellis J, et al. Detection of Mycobacterium tuberculosis in urine by Xpert MTB/RIF Ultra: a useful adjunctive diagnostic tool in HIVassociated tuberculosis. Int J Infect Dis 2018; 75:92-4.

19. Perez-Risco D, Rodriguez-Temporal D, Valledor-Sanchez I, Alcaide F. Evaluation of the Xpert MTB/RIF ultra assay for direct detection of Mycobacterium tuberculosis complex in smear-negative extrapulmonary samples. J Clin Microbiol 2018 56: e00659-18.

20. Wu X, Tan G, Gao R, et al. Assessment of the Xpert MTB/RIF Ultra assay on rapid diagnosis of extrapulmonary tuberculosis. Int J Infect Dis 2019; 81:91-6.

21. Andama A, Jaganath D, Crowder R, et al. Accuracy and incremental yield of urine Xpert MTB/RIF Ultra versus Determine TB-LAM for diagnosis of pulmonary tuberculosis. Diagn Microbiol Infect Dis 2020; 96:114892.

22. Lawn SD, Gupta-Wright A. Detection of lipoarabinomannan (LAM) in urine is indicative of disseminated TB with renal involvement in patients living with HIV and advanced immunodeficiency: evidence and implications. Trans R Soc Trop Med Hyg 2016; 110:180-5.

23. Ellis J, Bangdiwala AS, Cresswell FV, et al. The changing epidemiology of HIVassociated adult meningitis, Uganda 2015-2017. Open Forum Infect Dis 2019; 6:ofz419.

24. Marais S, Thwaites G, Schoeman JF, et al. Tuberculous meningitis: a uniform case definition for use in clinical research. Lancet Infect Dis 2010; 10:803-12.

25. World Health Organization. Global Tuberculosis Report 2019. Geneva: World Health Organization; 2019.

26. Boulware DR, Meya DB, Muzoora C, et al.; COAT Trial Team. Timing of antiretroviral therapy after diagnosis of cryptococcal meningitis. N Engl J Med 2014; 370:2487-98.

27. Songkhla MN, Tantipong H, Tongsai S, Angkasekwinai N. Lateral flow urine lipoarabinomannan assay for diagnosis of active tuberculosis in adults with human immunodeficiency virus infection: a prospective cohort study. Open Forum Infect Dis 2019; 6:ofz132.

28. Wood R, Lawn SD. Challenges facing lipoarabinomannan urine antigen tests for diagnosing HIV-associated tuberculosis. Expert Rev Mol Diagn 2012; 12:549-51. 


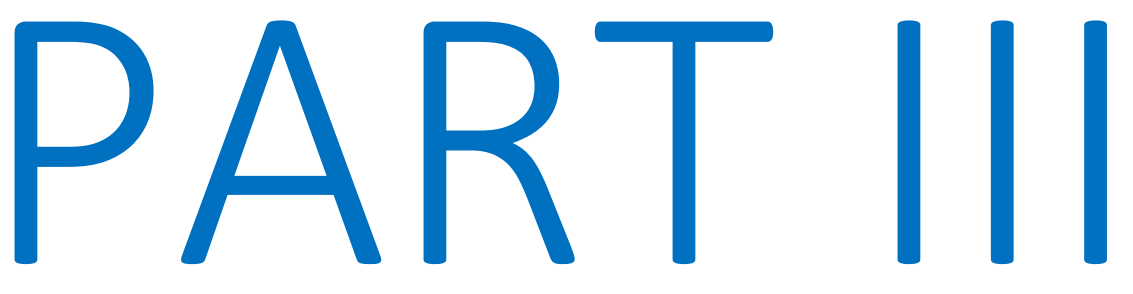

THERAPEUTIC

$$
\text { RESEARCH }
$$





\section{Chapter Seven: Intensified Treatment of Tuberculous Meningitis (review paper)}

\section{Foreword}

As detailed in the introduction (section 1.3) the optimal antibiotic therapy for TBM remains to be determined. TBM treatment recommendations are not evidence-based from PK-PD research in TBM patients, simply an extrapolation of trial data from pulmonary TB in the pre-HIV era. There is a drive to generate data specifically from TBM patients (including children and PLHIV) and investigate the impact of using intensified treatment, including higher doses of first-line TB drugs, additional of adjunctive antibiotics and different routes of administration.

In order to immerse myself in the field I conducted a literature review and led the writing of a review paper, along with expert co-authors from Radboud University, Netherlands and Johns Hopkins University, USA who work in the field of intensified TB treatment trials and PK-PD research. The search strategy can be found in the Appendix 4. This research paper is in fulfilment of PhD objective 5: to review the literature relating to the rationale and best options for intensified antibiotic treatment of TBM and summarise findings in a review paper.

The paper summarises the rationale for intensification of treatment, the properties of antituberculous drugs that might make them useful agents in the treatment of CNS TB and special populations including children, drug-resistant TB and PLHIV. There have been a number of clinical trials on intensified TBM treatment completed, and more underway or planned, which are also summarised in the review paper Table 3. 


\section{RESEARCH PAPER COVER SHEET}

\section{PLEASE NOTE THAT A COVER SHEET MUST BE COMPLETED FOR EACH RESEARCH PAPER INCLUDED IN A THESIS.}

\section{SECTION A - Student Details}

\begin{tabular}{|l|l|}
\hline Student & Dr Fiona Cresswell \\
\hline Principal Supervisor & Prof Alison Elliott \\
\hline Thesis Title & $\begin{array}{l}\text { Improving diagnosis and treatment of HIV-associated } \\
\text { Tuberculous meningitis }\end{array}$ \\
\hline
\end{tabular}

If the Research Paper has previously been published please complete Section B, if not please move to Section C

\section{SECTION B - Paper already published}

\begin{tabular}{|l|l|l|l|}
\hline Where was the work published? & Expert Reviews in Clinical Pharmacology \\
\hline When was the work published? & March 2019 \\
\hline $\begin{array}{l}\text { If the work was published prior to } \\
\text { registration for your research degree, } \\
\text { give a brief rationale for its inclusion }\end{array}$ & \multicolumn{3}{|l|}{} \\
\hline $\begin{array}{l}\text { Have you retained the copyright for the } \\
\text { work?* }\end{array}$ & Yes & $\begin{array}{l}\text { Was the work subject to } \\
\text { academic peer review? }\end{array}$ & Yes \\
\hline
\end{tabular}

*If yes, please attach evidence of retention. If no, or if the work is being included in its published format, please attach evidence of permission from the copyright holder (publisher or other author) to include this work.

\section{SECTION C - Prepared for publication, but not yet published}

\begin{tabular}{|l|l|}
\hline $\begin{array}{l}\text { Where is the work intended to be } \\
\text { published? }\end{array}$ & \\
\hline $\begin{array}{l}\text { Please list the paper's authors in the } \\
\text { intended authorship order: }\end{array}$ & \\
\hline Stage of publication & Choose an item. \\
\hline
\end{tabular}

\section{SECTION D - Multi-authored work}

For multi-authored work, give full details of your role in the research included in the paper and in the preparation of the paper. (Attach a further sheet if necessary)
I handled the literature review process, read and interpretted key papers. As a group we lay out the format for the manuscript and wrote our respective section (I led on the introduction, HIV section, and the discussion). I did the referencing, coordinated comments from co-authors, handled submission and review responses with input 


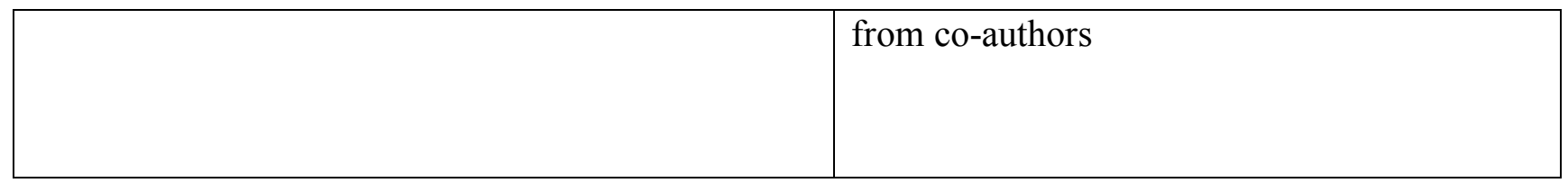

Student Signature:

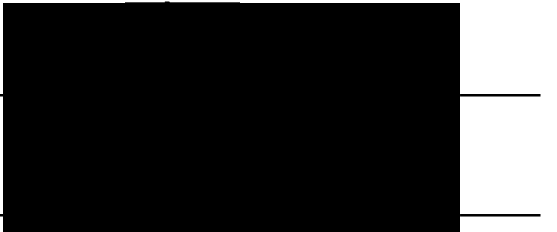

Date:

24th Sept 2020

Supervisor Signature:

Date:

8th October 2020 


\title{
Intensified antibiotic treatment of tuberculosis meningitis
}

\author{
Fiona V. Cresswell ${ }^{\mathrm{a}, \mathrm{b}}$, Lindsey Te Brake ${ }^{c}$, Rachel Atherton ${ }^{\mathrm{b}}$, Rovina Ruslami ${ }^{\mathrm{d}}$, Kelly E. Dooley ${ }^{\mathrm{e}}$, Rob Aarnoutse \\ and Reinout Van $\mathrm{Crevel}^{\mathrm{f}, \mathrm{g}}$
}

\begin{abstract}
${ }^{a}$ Clinical Research Department, London School of Hygiene and Tropical Medicine, London, UK; ${ }^{\circ}$ Research Department, Infectious Diseases Institute, Kampala, Uganda; 'Department of Pharmacy, Radboud Institute of Health Sciences, Radboud Center for Infectious Diseases Radboud university medical center, Nijmegen, The Netherlands; ${ }^{\mathrm{T}}$ BB-HIV Research Centre, Faculty of Medicine, Universitas Padjadjaran, Bandung, Indonesia; ${ }^{D}$ Divisions of Clinical Pharmacology and Infectious Diseases, Department of Medicine, Johns Hopkins University School of Medicine, Baltimore, MD, USA; fDepartment of Internal Medicine and Radboud Center for Infectious Diseases, Radboud university medical center, Nijmegen, the Netherlands; ${ }^{9}$ Centre for Tropical Medicine and Global Health, Nuffield Department of Medicine, University of Oxford, Oxford, UK
\end{abstract}

\begin{abstract}
Introduction: Meningitis is the most severe manifestation of tuberculosis, resulting in death or disability in over $50 \%$ of those affected, with even higher morbidity and mortality among patients with HIV or drug resistance. Antimicrobial treatment of Tuberculous meningitis (TBM) is similar to treatment of pulmonary tuberculosis, although some drugs show poor central nervous system penetration. Therefore, intensification of antibiotic treatment may improve TBM treatment outcomes.

Areas covered: In this review, we address three main areas: available data for old and new anti-tuberculous agents; intensified treatment in specific patient groups like HIV co-infection, drug-resistance, and children; and optimal research strategies.

Expert commentary: There is good evidence from preclinical, clinical, and modeling studies to support the use of high-dose rifampicin in TBM, likely to be at least $30 \mathrm{mg} / \mathrm{kg}$. Higher dose isoniazid could be beneficial, especially in rapid acetylators. The role of other first and second line drugs is unclear, but observational data suggest that linezolid, which has good brain penetration, may be beneficial. We advocate the use of molecular pharmacological approaches, physiologically based pharmacokinetic modeling and pharmacokinetic-pharmacodynamic studies to define optimal regimens to be tested in clinical trials. Exciting data from recent studies hold promise for improved regimens and better clinical outcomes in future.
\end{abstract}

ARTICLE HISTORY

Received 3 August 2018

Accepted 23 November 2018

\section{KEYWORDS}

Tuberculous meningitis; TB meningitis; antituberculous therapy; intensified

\section{Introduction}

\subsection{Brief epidemiology of tuberculous meningitis}

Tuberculosis (TB) is the leading infectious disease killer worldwide. Tuberculous meningitis (TBM), its most dramatic manifestation, makes up 1-2\% of all TB cases in settings where the HIV prevalence is low $[1,2]$. However, in settings where HIV is common, disseminated TB and TBM make up a much greater proportion of TB cases [3]. A meta-analysis of post-mortem studies in low-resource settings showed evidence of active TB in $43 \%$ of deceased HIVinfected individuals, and one study noted meningeal involvement in $28 \%$ of those with disseminated TB $[4,5]$. It is therefore not surprising that $\mathrm{TB}$ is the second leading cause of adult meningitis in many countries with high HIV prevalence $[6,7]$. TBM tends to strike those at the extremes of age. It is particularly common in young children under 5 years of age [8-10], and often the leading cause of childhood bacterial meningitis in TB-endemic areas [11].

\subsection{Pathogenesis and presentation of tuberculous meningitis}

Much remains unclear about the pathogenesis of TBM, but old histopathological studies suggest that pulmonary infection is followed by hematogenous dissemination of bacilli, including the meninges covering the brain, forming so-called 'Rich foci' [12]. Months to years later, disease may reactivate; Rich foci rupture into the subarachnoid space with the dissemination of bacilli into the cerebrospinal fluid (CSF) and development of basal meningitis [13]. In children, the meningitis usually occurs as part of disseminated primary disease rather than a later reactivation, and the same may be true of HIV-infected adults where host-defense mechanisms are diminished [3]. A basal meningitis results in formation of a dense exudate on the brainstem, which can envelop cerebral arteries and cranial nerves and obstruct the flow of CSF. Entrapment of cranial nerves by the exudate can lead to ophthalmoplegia or other cranial nerve palsies, while obstruction of the CSF flow can lead to hydrocephalus. As the most severe consequence, the exudate can lead to vasculitis of blood vessels of the circle of Willis, the vertebrobasilar system, and the perforating branches of the middle cerebral artery, resulting in brain infarctions. To dampen this immunopathology, standard treatment now includes the use of adjuvant corticosteroids $[14,15]$.

TBM presents initially as subacute meningitis, with an insidious onset of low-grade fever, malaise, weight loss, and

CONTACT Reinout van Crevel reinout.vancrevel@radboudumc.nl D Department of Internal Medicine, Radboud University Medical Center, P0 Box 9101, 6500 $\mathrm{HB}$, Nijmegen, The Netherlands

(1) Supplemental data for this article can be accessed here. 
gradually worsening headache, followed by confusion, development of focal neurological symptoms like eye movement disorders or limb paralysis and progressive coma. In small children, early symptoms are non-specific including cough, fever, failure to thrive, irritability or apathy, followed by loss of consciousness, and focal neurological signs [16]. Recognition and diagnosis of TBM remain a huge challenge. In the absence of a widely available, rapid, and reliable diagnostic test for extrapulmonary manifestations of $T B$, clinicians face a management challenge in deciding when anti-tuberculous therapy should be offered.

\subsection{Prognosis of tuberculous meningitis}

It is widely recognized that TBM is the most severe form of TB death and severe neurological sequelae are common. Reported outcomes vary drastically by geographical region, prevalence of HIV co-infection, drug resistance patterns, and time period. We reviewed tuberculous meningitis studies published up to 6 June 2018, to examine the risk of death, and neurological sequelae amongst survivors. Full search strategy and search terms are shown in the supplementary material. After manual screening of abstracts, we included 84 studies; 12 included all ages, 35 included adults only, and 37 included children only. We present data from both retrospective and prospective studies which commenced enrolment within the past 30 years (from 1988 onwards) and which enrolled at least 100 participants $(n=28 ; 4$ all ages, 16 adults, 8 children) plus two additional smaller studies which add to limited evidence within a drug-resistant population in Table 1.

For adults with TBM, risk of death ranged from $7 \%$ to $50 \%$ [35,36] for in-hospital mortality, up to 58\% [37] at five years' follow-up. Amongst survivors, risk of neurological sequelae ranged from $14 \%$ to $45 \%[35,38]$. Amongst studies including only HIV-infected adults, mortality was $41-58 \%[37,39]$. The majority of studies were performed in Asia, with evidence from East and West Africa notably lacking. A study in Uganda [40] examined a TBM population with $96 \%$ HIV prevalence and reported $44 \%$ in-hospital mortality. Seven studies recruited patients with drug-resistant TB; those with isoniazid monoresistance demonstrated a mortality of 31-56\% [41,42] whereas those with rifampicin resistance or multi-drug resistant TB had a mortality of up to $95 \%$ [42].

With regard to pediatric TBM, eight studies recruited exclusively children; those up to October 2012 are more comprehensively reviewed in a meta-analysis from Chiang et al. [43], who reported a risk of death of $19 \%(95 \% \mathrm{Cl} 14.0-26.1)$ and a risk of neurological sequelae among survivors of $54 \%$ (95\% Cl 42.6-64.9) amongst 1636 children studied. Within our included studies, risk of death ranged from $8 \%$ to $29 \%$ $[44,45]$ for in-hospital mortality (excluding a small case series of patients with MDR-TB [46]), and up to 34\% overall [44]. Amongst survivors, risk of severe disability was up to $40 \%$ [47], and all-severity disability up to $88 \%$ [48].

\subsection{Towards better treatment of tuberculous meningitis: outline of this review}

Development of better treatments is a priority in light of the high mortality and neurological disability associated with TBM. Prompt diagnosis and effective antimicrobial treatment are critical to limit bacillary multiplication and associated immunopathology in the central nervous system (CNS). Host-directed therapies targeting immunopathology are an important area of research but outside of the scope of this review [49].

In this review, we focus on intensified antibiotic treatment of TBM. First, we discuss the rationale and options for intensification of antibiotic treatment as well as available data for key anti-tuberculous agents. Secondly, we address the relevance and considerations of intensified treatment in HIV co-infection, drug-resistant TBM, and pediatric populations. Thereafter, we explore ways to accelerate understanding in this field through the application of state-of-the-art and intelligent research tools. We conclude by highlighting knowledge gaps and identifying key areas for future research.

\section{The concept of intensified antibiotic treatment}

\subsection{The challenge of treating tuberculous meningitis}

TBM is more challenging to treat than pulmonary TB (PTB) for a number of reasons. Most importantly, in contrast to antibiotics used to treat PTB, antimicrobials for CNS TB must cross the blood-brain barrier (BBB), and blood-cerebrospinal fluid barrier (BCSFB), and remain in the brain or CSF and be present at the site of infection at sufficient concentrations for a sufficient period of time to kill the TB bacilli (Figure 1) [50-54]. The BBB and the BCSFB separate the extracellular CNS fluid compartment, the interstitial fluid and the CSF from the blood compartment, and transporters, like P-glycoprotein, in endothelial cells actively pump substances out of the brain and into the blood or CSF, thereby protecting the CNS from the free entry of potentially neurotoxic substances $[55,56]$. Despite the presence of these barriers designed to keep foreign materials such as xenobiotics out, resulting in some first-line TB drugs, especially rifampicin and ethambutol, penetrating the BBB and BCSFB poorly, national and international guidelines for antimicrobial treatment for TBM follow the model for PTB, with a 2-month intensive phase followed by a continuation phases of treatment, using the same first-line TB drugs and identical dosing, just prolonged for 9-12 months duration [57-59] The World Health Organisation guidelines suggest replacing ethambutol with streptomycin but this has not been widely adopted and there is little evidence to warrant this [60].

There are at least three other reasons that TBM treatment is difficult. First, the anatomical distribution and quantity of bacilli and their metabolic state (and, thus, likelihood of being killed by anti-tuberculous drugs given their particular mechanisms of action) - and the changes in these factors over time with treatment - are poorly characterized in TBM, making it difficult to develop a new treatment model specific for TBM. Further, the integrity of the BBB and BCSFB related to infection and inflammation, as well as blood supply to the compartments where Mycobacterium tuberculosis resides, are likely to change significantly over the course of treatment as antibiotics and anti-inflammatory medications are given. This may affect the penetration of antibiotics to their site of action. Lastly, it is not clear how mechanisms of bacterial killing (protein synthesis inhibition, disruption of cell wall synthesis, bacterial enzyme inhibition) influence host inflammatory 


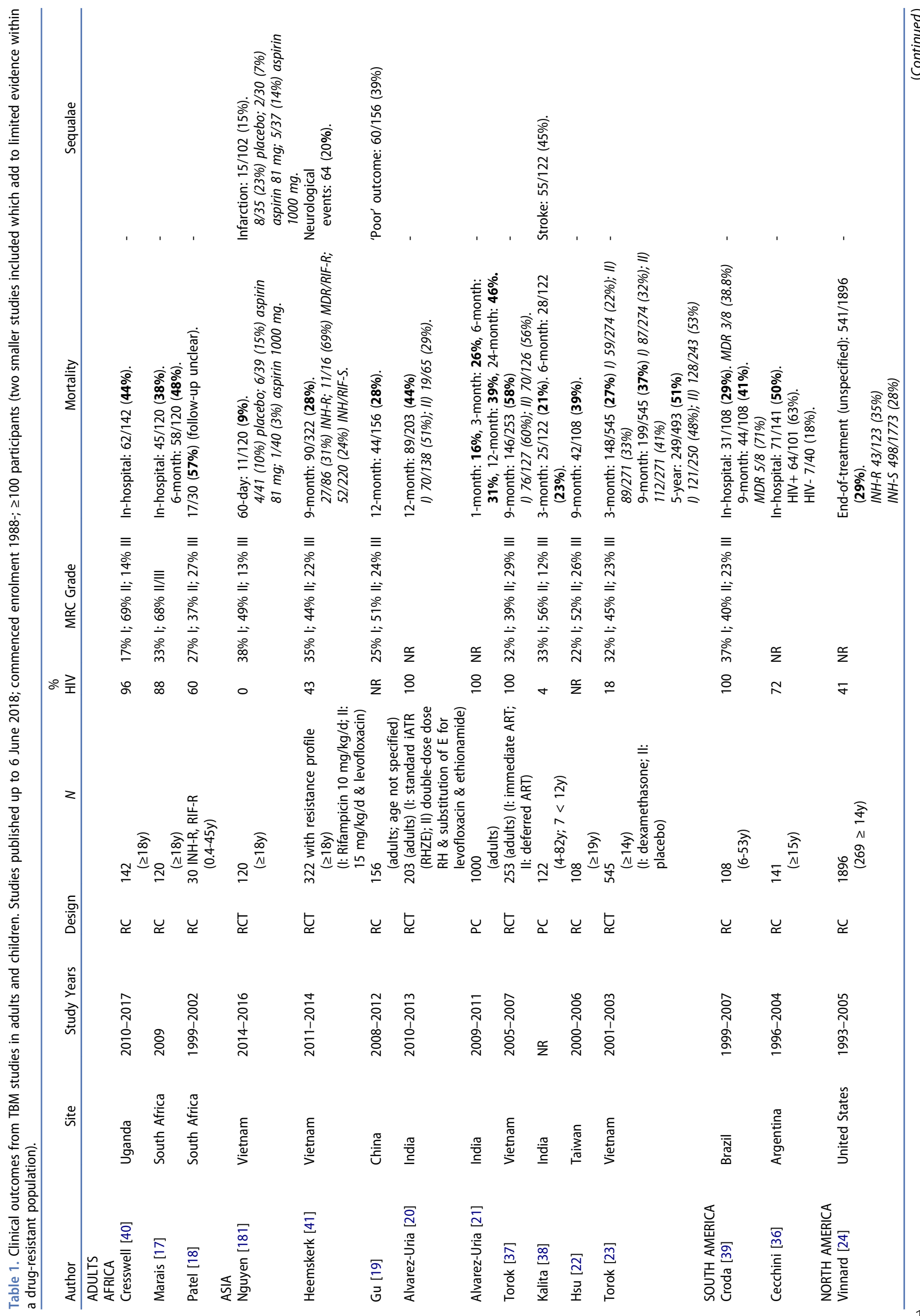




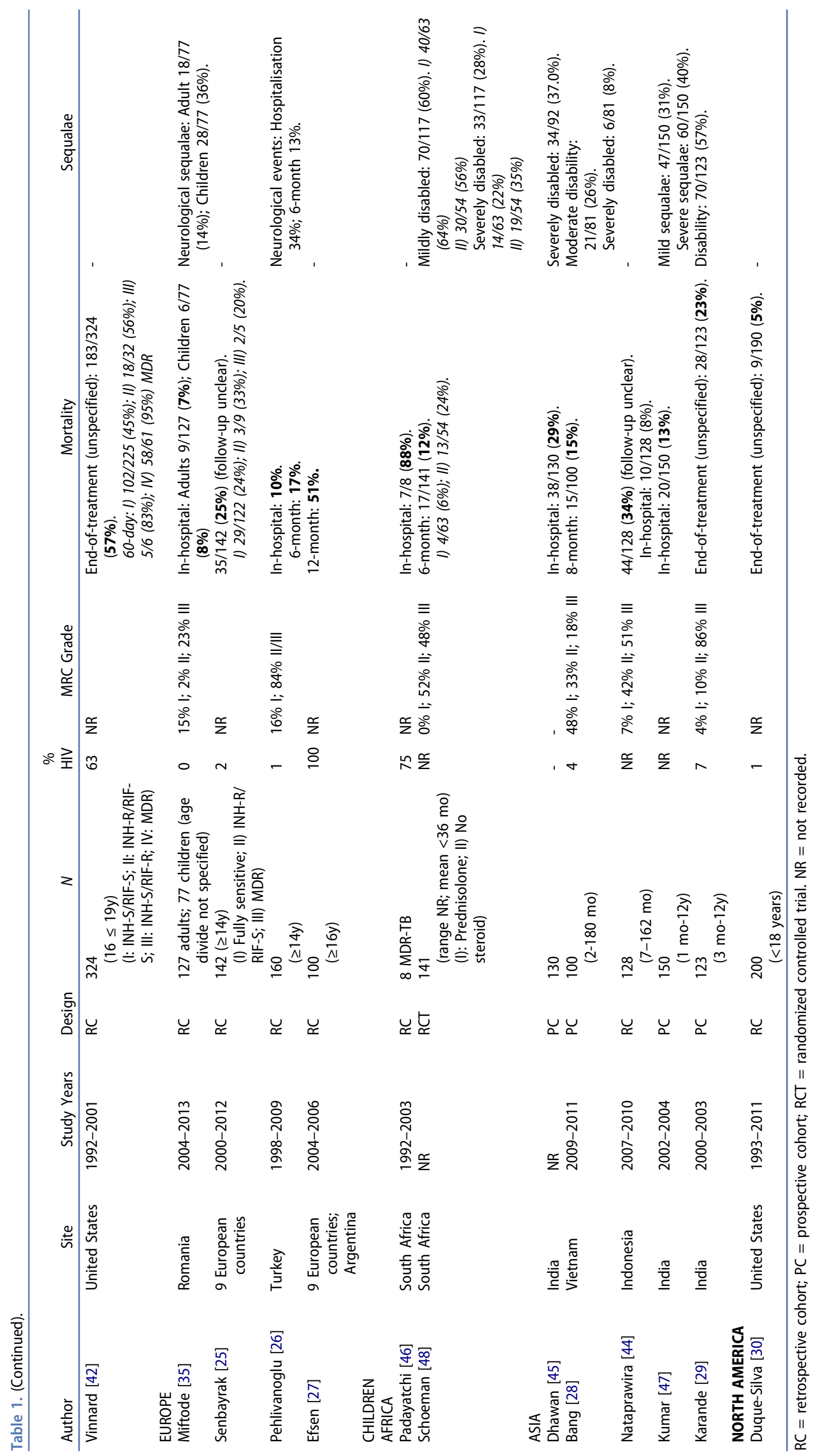




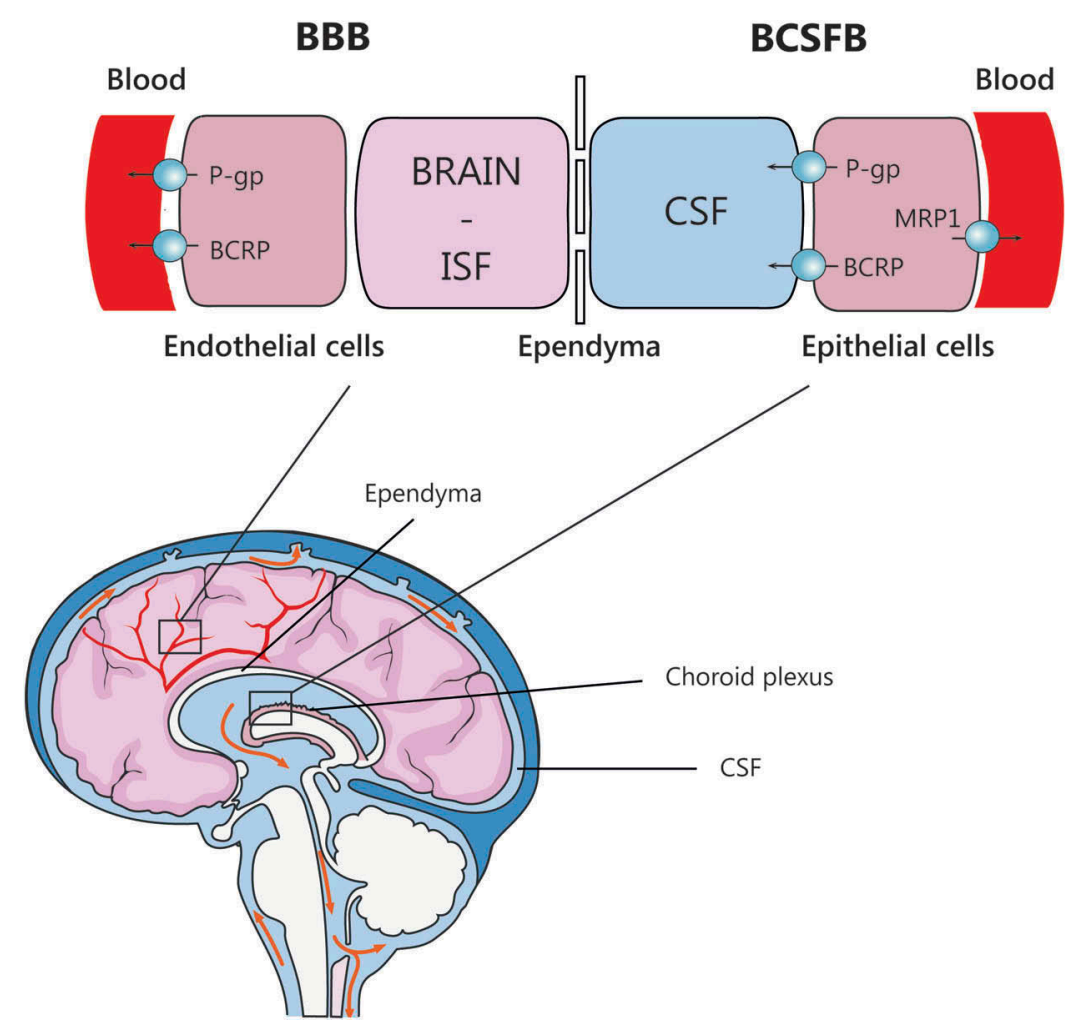

Figure 1. Schematic overview of the relation between the blood-brain barrier (BBB), made up by brain endothelial cells, and the blood-CSF barrier (BCSFB), made up by choroid plexus epithelial cells. The ependymal lining of the cerebral ventricles forms the barrier between brain (and its interstitial fluid, ISF) and CSF. According to this simplified paradigm, there is free exchange of drug between the brain ISF and CSF, and CSF drug concentrations approximate the free drug concentration in plasma that is able to penetrate the BBB, BCSFB, or both. Displayed efflux transporters may actively disturb this equilibrium, by actively transporting drugs into the blood or CSF.

responses and, in turn, vasculitis, strokes, and risk of death. Host, bacterial, and treatment effects are thus intertwined and must be taken into account when investigating optimal treatments for TBM.

\subsection{Strategies to optimize TBM treatment}

In the absence of basic information on the interaction of host, bacteria and treatment effects, there may be different strategies to optimize TBM treatment. One strategy might be prolonging treatment, as is often advocated. However, in one meta-analysis evaluating treatment duration, the risk of relapse was extremely low $(\sim 0.8 \%)$ and virtually identical in patients who got $<6$ months versus $>6$ months of therapy, suggesting that there is no evidence base to support longer treatment as a means to improve response to TBM treatment [61].

A more promising strategy is to increase drug exposures in the CNS. This may be achieved by increasing doses of poorly penetrating drugs, adding, or replacing drugs with anti-tuberculous agents that have better BBB and BCSFB penetration characteristics [62], modification of physicochemical properties of TB drugs, intracerebral injection, olfactory route delivery, tight-junction modulation (e.g. by osmotic opening, liposome or nanoparticlemediated permeation) and, finally, inhibition of efflux mechanisms that pump drugs out of the brain [55,63-65]. Considering the limited availability of some of these interventions in general and particularly in settings where TB is most common, increasing doses of poorly penetrating drugs and the use of agents with improved penetration may form the most obvious way to intensify and improve TBM treatment.

To find the optimal dose of drugs for TBM, whether they penetrate poorly or relatively well across barriers, robust information on the relationship between doses and exposures achieved (pharmacokinetics, PK) and between exposures and efficacy or safety (pharmacodynamics, PD) is indispensable. Ideally, the 'right' dose results in maximum efficacy, with limited adverse effects (Figure 2). Doseresponse or preferably exposure-response evaluations are part of the drug development process. However, historically, for most TB drugs currently in use (Table 2), far less attention has been paid to establishing the relationship between drug concentrations and responses for optimal dose-finding. Furthermore, there has been little interest in exploring PKPD differences among TB disease-type subsets, such as patients suffering from TBM or TBM-HIV coinfection. The paradigm that inadequate exposure results in suboptimal clinical response is nevertheless generally accepted for TB drugs and has been described in multiple studies [66-72]. Nonetheless, such a relationship could not always be identified [73-75], and for most TB drugs dose-response curves such as presented in Figure 2 are lacking, especially with sterilizing effect as a clinical endpoint. The search for the 'right' high-dose of rifampicin is an illustrative example of how a better understanding of TB drug PK and PD could be used to improve treatment regimens. 

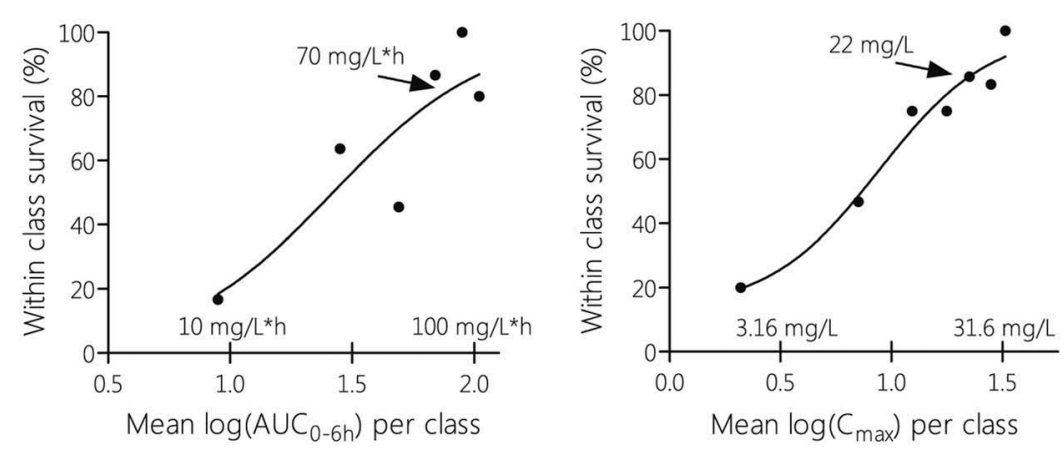

Figure 2. Concentration-response curves displaying the effect of rifampicin plasma $A U C_{0-6 h}$ (left) and $C_{\max }$ (right) exposure on survival, and the corresponding exposure threshold values associated with maximal survival. Te Brake et al. aimed to deduce concentration thresholds predictive of good treatment response in TBM. For this classes of $20 \mathrm{mg} . \mathrm{h} / \mathrm{L}$ for $A U C_{0-6 h}$ and $5 \mathrm{mg} / \mathrm{L}$ for $C_{\max }$ were constructed (and included a minimum of three participants in each class), the percentage survival within each class was calculated. Eight-week survival was used as the response parameter. Concentration-response curves were generated by non-linear regression fitting.

\subsection{Rifampicin}

Rifampicin is an essential component of TBM treatment. Rifampicin-resistant TBM is $69-100 \%$ fatal [41,76-78]. Up to now, no agents have been identified that can replace it for treatment of TBM. However, rifampicin has poor CSF penetration, and at current treatment doses, CSF concentrations of rifampicin barely exceed the minimal inhibitory concentration (MIC) against M. tuberculosis [79]. When interpreting rifampicin CSF concentration data, it is important to keep in mind that rifampicin in highly protein bound and that only the unbound or 'free' rifampicin is biologically active. In the absence of meningeal inflammation, only the 'free' rifampicin crosses the BCSFB. Therefore, the difference of total rifampicin concentrations between plasma and CSF is usually much bigger than the difference between 'free' or active rifampicin concentrations, and the CSF: plasma ratio of total rifampicin will underestimate penetration of unbound (active) rifampicin.

As to the PD of rifampicin, it has been shown that the efficacy of this drug is exposure or concentration dependent, which means that it correlates with exposure to the drug (area under the concentration - time curve, $A \cup C_{0-24 h}$ ) and/or its peak concentration $\left(C_{\max }\right)$ [80]. An increase in rifampicin dose will increase the $A \cup C_{0-24 h}$ and $C_{\text {max }}$ in plasma and at the sites of action in supra-proportional fashion (owing to saturable hepatic clearance), and this is hoped to enhance activity against $M$. tuberculosis.

In search of higher yet safe doses of the cornerstone TB drug rifampicin, several studies have been performed. Studies using an in vitro pharmacodynamics system (IVPDS) or 'hollow fiber model' and studies in mouse models have suggested that higher doses of rifampicin are associated with better results and shorter treatments in PTB [80-82]. Until 2011, 14 clinical trials had evaluated high-dose rifampicin [83]. Before 1985, 13 of these trials were published. These trials evaluated regimens with doses of rifampicin up to $1200 \mathrm{mg}(20 \mathrm{mg} / \mathrm{kg})$ and different dosing intervals (once, twice, thrice, six times per week, and daily). Several trials suggested an advantage in terms of likelihood of culture conversion among patients with PTB receiving at least $900 \mathrm{mg}$ of rifampicin. Hepatotoxicity was rarely observed, and no trend in the occurrence of hepatotoxicity with the rifampicin dose was evident. However, attempts to use high dose rifampicin (900 mg or $15 \mathrm{mg} / \mathrm{kg}$ and more) intermittently rather than daily were met with a high incidence of flu-like syndrome, an allergic reaction which is ascribed to the intermittency of dosing rather than the height of the dose. Therefore, high dose rifampicin should be administered on a daily basis $[83,84]$.

More recently, several trials have evaluated the high dose rifampicin as a strategy for treatment shortening for pulmonary TB. Increasing doses of rifampicin up to $40 \mathrm{mg} / \mathrm{kg}$ daily were associated with a nine-fold increase in total exposure to rifampicin in plasma compared to the standard $10 \mathrm{mg} / \mathrm{kg}$ dose and were safe and tolerable over two weeks $[85,86]$. From the same data, a significant exposure-response relationship could be derived between rifampicin exposure and early bactericidal activity [72]. In another recent clinical trial, a higher daily rifampicin dose of $35 \mathrm{mg} / \mathrm{kg}$ administered over a longer period of 12 weeks together with standard doses of isoniazid, pyrazinamide, and ethambutol decreased the time to culture conversion compared to standard treatment [87]. PK-PD analysis revealed that higher exposures to rifampicin were the intermediary link to the decreased time to culture conversion and showed that this effect of rifampicin did not plateau at the $35 \mathrm{mg} / \mathrm{kg}$ dose [88]. Higher doses of rifampicin up to $20 \mathrm{mg} / \mathrm{kg}$ have also been studied and were safe and tolerable for other diseases such as brucellosis [89], leishmaniasis [90], and other indications [91].

Along with the studies on PTB, high dose rifampicin has also been evaluated as a strategy to reduce mortality in TBM. AlvarezUria administered $900 \mathrm{mg}(\sim 20 \mathrm{mg} / \mathrm{kg})$ of oral rifampicin for a median of 7 days to adults with TBM in India; no safety issues were reported in this setting [92]. In the first in a series, in an open-label, randomised phase II clinical trial in Indonesia, a 33\% higher dose of rifampicin administered intravenously $(600 \mathrm{mg}$ or $13 \mathrm{mg} / \mathrm{kg}$ iv) for 2 weeks resulted in a three-fold higher exposure to rifampicin in plasma and CSF during the first critical days of treatment than $450 \mathrm{mg}$ given orally. In this small study, 6-month mortality was reduced by greater than $50 \%$ [79]. A clear concentration-effect relationship could be identified, with the 'effect' being survival at 8-weeks (Figure 2) and threshold values associated with decreased mortality could be derived through PK-PD modeling, even though data suggested that the exposures that maximized efficacy had not been reached yet [93]. Since 
EXPERT REVIEW OF CLINICAL PHARMACOLOGY $\Rightarrow 7$

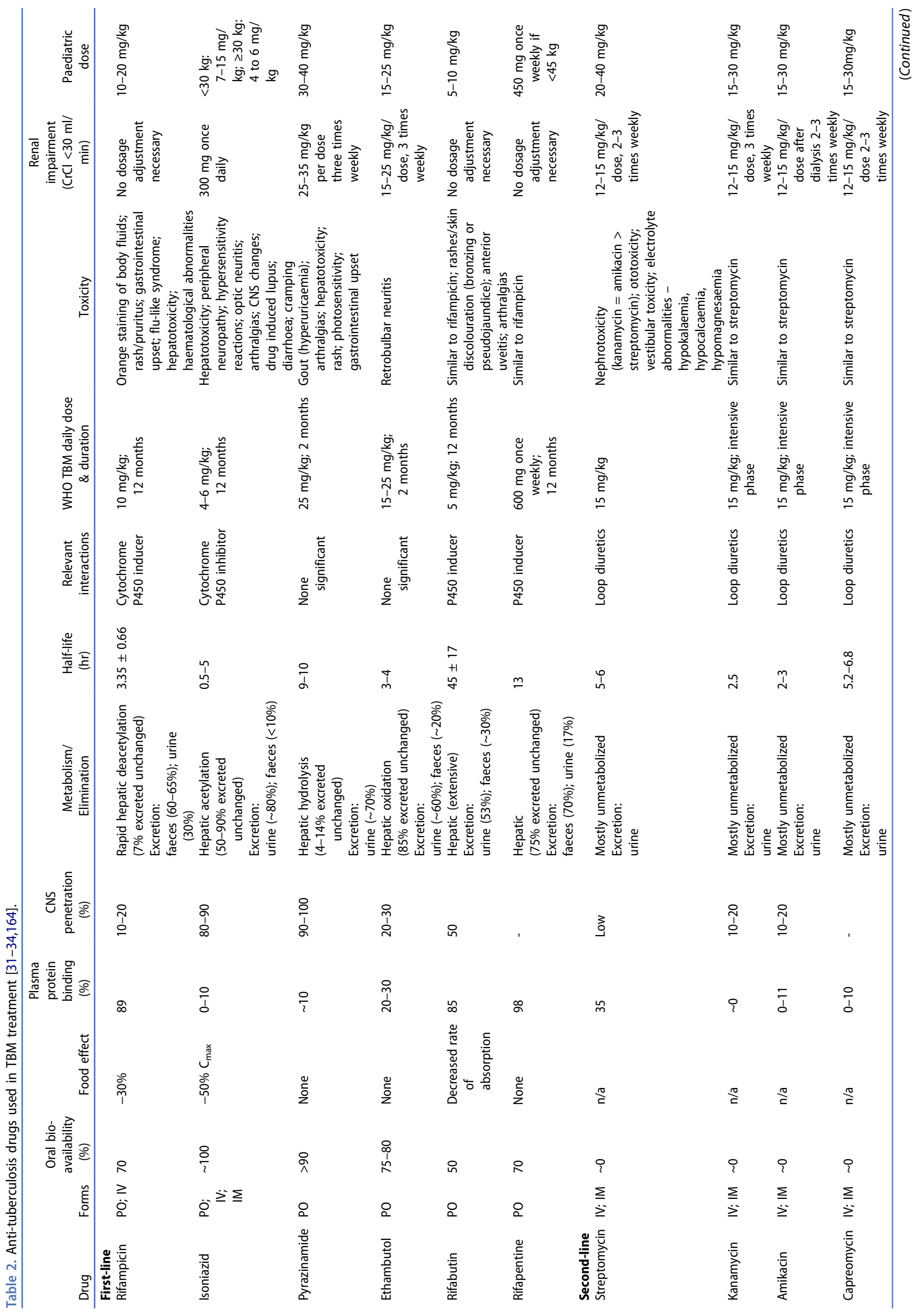




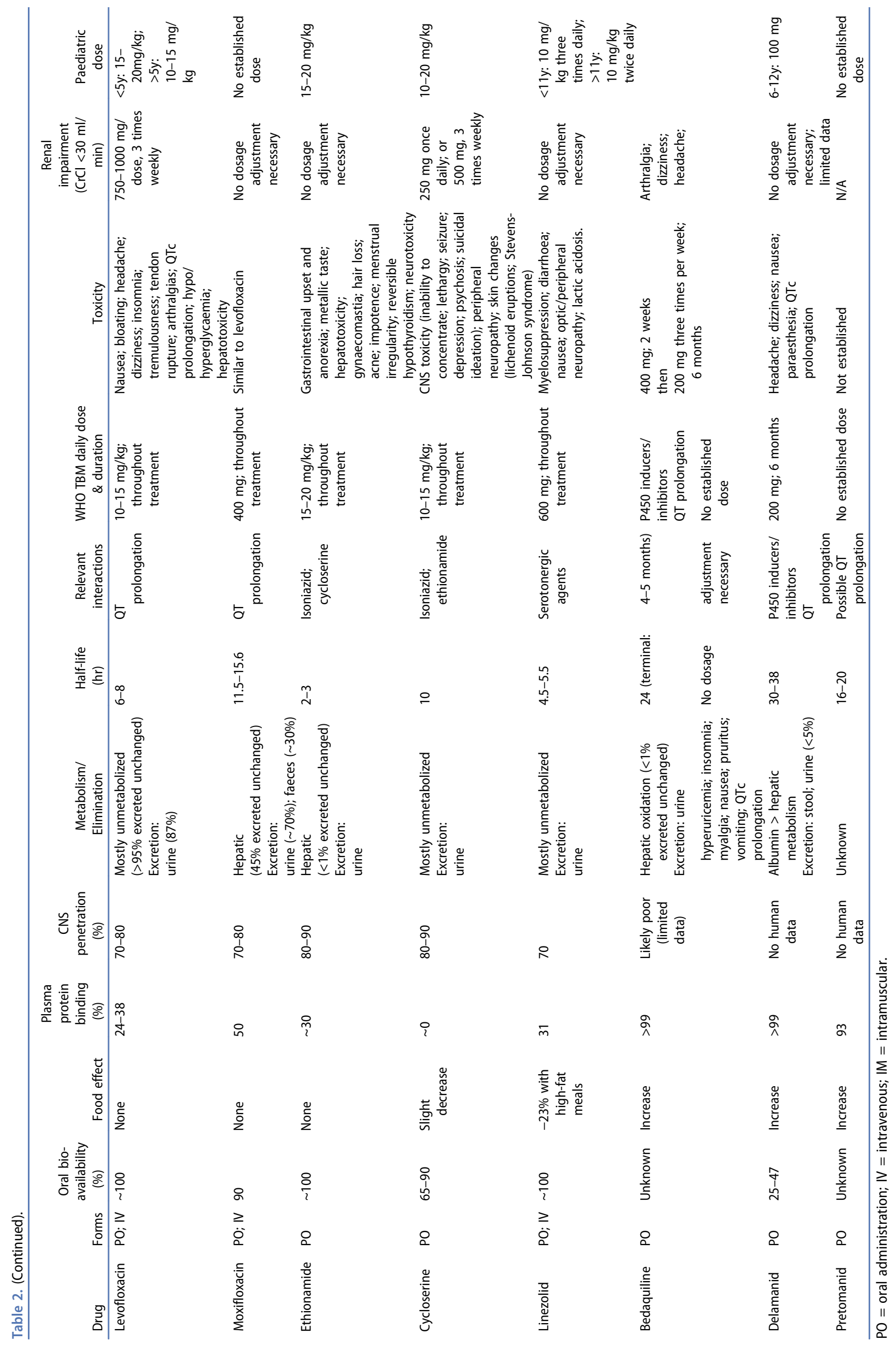


intravenous rifampicin is not widely available and must be administered in a hospital setting, a follow-up pharmacokinetic study evaluated doses of 17 or $20 \mathrm{mg} / \mathrm{kg}$ of oral rifampicin, which resulted in total exposures in plasma approximately similar to the values after $13 \mathrm{mg} / \mathrm{kg}$ iv, but average peak plasma and CSF concentrations were lower with large interindividual variability [94]. In literature, reported rifampicin MICs for susceptible strains ranged from 0.06 to $0.4 \mathrm{mg} / \mathrm{L}[95,96]$. CSF concentrations after 17 or $20 \mathrm{mg} / \mathrm{kg}$ oral rifampicin ranged from 0.125 to $1.06 \mathrm{mg} / \mathrm{L}$ [94] and can, therefore, be considered to have reached MIC, but not by much.

In a third phase II trial, this time double-blinded, randomized, and placebo-controlled, 60 adult TBM patients were assigned to 10,20 , or $30 \mathrm{mg} / \mathrm{kg}$ oral rifampicin combined with standard companion TB drugs for 30 days. The double and triple dose of oral rifampicin led to three and five-fold higher total exposures in plasma in the critical early days of treatment with proportional increases in highest recorded CSF concentrations and without an increase in the incidence of grade $3 / 4$ adverse events. Six-month mortality was $7 / 20$ (35\%), 9/20 (45\%), and 3/20 (15\%) in the 10 , 20 , and $30 \mathrm{mg} / \mathrm{kg}$ groups, respectively $(p=0 \cdot 12)$ [97]. In contrast to the Indonesian studies, a large trial involving 817 TBM patients in Vietnam evaluating to an intensified regimen with 15 (rather than 10) $\mathrm{mg} / \mathrm{kg}$ of rifampicin plus levofloxacin versus standard of care for 8 weeks did not demonstrate a survival benefit for higher doses of rifampicin [98], except for patients with isoniazidmonoresistant TBM [41]. It was postulated that the lack of effect in this study can be explained by the relatively modest dose increase of rifampicin [99].

Thus, the preponderance of the evidence points to a treatment benefit with higher doses of rifampicin, but this benefit is likely only to be seen with significant dose increases. Additionally, rifampicin has been shown in vitro to have a variety of anti-inflammatory effects - inhibiting the production of lipopolysaccharide-induced pro-inflammatory mediators including nitric oxide, cyclooxygenase-2, tumor necrosis factor- $a$, and interleukin-1 $\beta$ and prostaglandin $E_{2}$ [100], as well as reducing microglial activation. Whether such anti-inflammatory benefits are dose-related requires further research.

With regards to other rifamycin antibiotics, rifabutin has been effective in rabbit pneumococcal meningitis, but neither it nor the other licensed rifamycin - rifapentine - have been tested in clinical TBM [101].

\subsection{Other first-line drugs (isoniazid, pyrazinamide, and ethambutol)}

Isoniazid has high early bactericidal activity that kills actively growing bacteria within the first $24 \mathrm{~h}$ and causes substantial decreases in culturable sputum bacilli in PTB over the first 2 weeks of treatment [102]. Isoniazid has excellent CSF penetration [92,93,103-105]. It is a valuable component of TBM treatment, as evidenced by a two-fold higher risk of death in isoniazid-monoresistant compared to drug-susceptible TBM $[42,76]$. Adding a second bactericidal agent (like levofloxacin) does not appear to confer a survival benefit unless the patient is infected with an isoniazid-resistant strain [98]. Isoniazid is metabolized by the genetically polymorphic $\mathrm{N}$-acetyltransferase 2 (NAT2). This enzyme exhibits two main phenotypes of activity: fast and slow. This acetylator status has been linked to both treatment outcome and hepatotoxicity in PTB. In TBM, exposures to isoniazid in plasma and CSF were found to be higher among slow acetylators [106]. Increasing the dose of isoniazid, e.g., to 10-15 mg/kg daily as in treatment for multi-drug resistant TB, may be an option for intensified treatment of TBM, though this remains to be proven.

Pyrazinamide also penetrates well into CSF [107] according to unpublished data from the Ruslami [79] and Yunivita [93,94] Indonesian studies. The drug only displays limited early bactericidal activity in drug-sensitive PTB (first 2-4 days of therapy), which is considered the most critical period in TBM treatment $[108,109]$. Activity thereafter (days 4-14) seems comparable with rifampicin and isoniazid $[108,109]$. In observational studies, pyrazinamide appeared to reduce the risk of death and neurologic sequelae in children with TBM allowing shortening of TBM treatment to 6 months [110]. However, while some pyrazinamide appears to be good, more is not necessarily better, at least in selected patient populations. In one study involving patients with HIV infection (median CD4 count of 41 cells/ $\mathrm{mm}^{3}$ ), high pyrazinamide exposures in CSF were independently associated with increased risk of death and neurotoxicity, even after adjusting for BBB integrity $[37,111]$

The main function of ethambutol is to protect companion drugs against emergence of resistance, but ethambutol has poor CSF penetration, even early in treatment $[104,107,112,113]$. Considering this probably limited contribution, debate has arisen regarding the choice of a fourth drug in treatment of drugsensitive TBM. But efforts to replace ethambutol, or add-on a drug to the first-line regimen have been ineffective so far $[79,114]$ Thus, isoniazid and rifampicin are essential drugs for drug-sensitive TBM, pyrazinamide is helpful, there is not a clear role for ethambutol.

\subsection{Second-line drugs: fluoroquinolones}

Fluoroquinolones have excellent CSF penetration [115,116], and high activity against drug-susceptible bacteria as well as many drug-resistant bacteria. Analysis of fluoroquinolone exposure versus response revealed worse outcomes among TBM patients with lower and higher plasma and CSF exposures than for patients with intermediate exposures (U-shaped exposure-response) [117].

In a phase II trial with factorial design, TBM patients were randomized to a higher intravenous dose of rifampicin and subsequently to a standard dose of moxifloxacin, a double dose of moxifloxacin or no moxifloxacin [79]. Use of moxifloxacin was not associated with a survival benefit in this trial. The higher dose of moxifloxacin was used considering that higher exposures to moxifloxacin may be more effective in TB treatment based on studies in vitro and in mice, and rifampicin decreases the exposure to moxifloxacin by approximately $30 \%$ [118]. Similarly, in a large trial of intensified anti-tuberculous therapy in Vietnam, levofloxacin failed to improve survival unless the disease was caused by bacteria resistant to isoniazid $[41,98]$. A meta-analysis of the use of fluoroquinolones in the management of TBM used data from 1115 patients and concluded that fluoroquinolones cannot be recommended at present, though the evidence was of moderate to low quality 
[119]. In light of two RCTs that have failed to show a benefit of quinolones in combination with current first-line antituberculous agents [98,120], further trials of quinolones are only likely to be warranted in combination with novel agents.

\subsection{Second-line and repurposed drugs: aminoglycosides, cycloserine, ethionamide, clofazimine, and linezolid}

Streptomycin distribution into brain and CSF is limited [107], and while its use in the 1940s decreased mortality from $90 \%$ to $40-80 \%$, this was only with long, complicated, painful treatment with repeated intrathecal injections over months [121], and its contribution to improving outcomes once multidrug regimens were available was small and only seen with intrathecal administration [115,122]. The same constraints apply to amikacin and kanamycin, with limited entry to CSF described in adult and pediatric studies [103,107].

Cycloserine and ethionamide achieve high concentrations in CSF, and the latter has been used successfully as a replacement for ethambutol to enhance TBM regimens in children $[92,123,124]$. Both can cause CNS adverse effects including psychiatric disturbances and there are doselimiting gastrointestinal toxicities for ethionamide. Ethionamide can be changed for the chemically similar prothionamide, and cycloserine for terizidone (containing two molecules of cycloserine) can be used interchangeably in PTB, although there is limited evidence in TBM. Clofazimine does not penetrate CSF well but in a single mouse study showed widespread brain tissue distribution $[125,126]$. Studies in rats suggest that para-aminosalicylic acid can cross the BBB and BCSF barriers [127], in humans the CSF concentrations of PAS are low, even in the presence of meningitis [107].

Linezolid is an approved predominantly bacteriostatic drug, which has shown efficacy against multidrug-resistant (MDR) and extensively drug resistant (XDR) TB [128-130], Two recent reviews support linezolid's efficacy for treating pulmonary MDR or XDR TB, though the optimal dosing that maximizes efficacy while minimizing risk of toxicities (bone marrow suppression and peripheral neuropathy) is not firmly established [128-130]. Janssen and the TB Alliance's Nix-TB trial investigated a shorter, all-oral three-drug regimen for XDR-TB, consisting of bedaquiline, pretomanid and linezolid (600 $\mathrm{mg}$ BID, later changed to $1200 \mathrm{mg}$ QD) for six months $[131,132]$. Dose reduction or interruption due to myelosuppression or neuropathy occurred only after a treatment period of 2-3 months and 4-6 months, respectively, supporting a short-term linezolid intervention in TBM.

Linezolid seems successful in reaching the CSF, with CSF/ serum concentration ratios varying from 0.7 to 1.0 in studies among patients with CNS infections without TB [133-135]. The impressive efficacy of linezolid in the management of critically ill patients with non-TB CNS infections (e.g. penicillinnonsusceptible Streptococcus pneumoniae, vancomycinresistant enterococci, methicillin-resistant Staphylococcus aureus) was reviewed by Ntziora et al. [136].

Only two studies have evaluated the effect of linezolid in patients with TBM, both in China [137]. In a non-randomized study among adults with severe TBM, 16 patients received adjunctive linezolid (600 mg BID, median duration 32 days)
[138]. Compared to the control group, patients showed better improvement in Glasgow Coma Scale, temperature recovery, and normalisation of CSF parameters, and safety was acceptable. No pharmacokinetic data were collected. Secondly, in a small retrospective cohort, children without clinical improvement after two weeks of standard treatment were given linezolid while other children continued on standard treatment only, the addition of linezolid was associated with improved clinical outcome, shortened fever clearance time, and reduced hospital stay. These results suggest that linezolid may be an effective additional drug for treatment of TBM, which is safe when given for a short duration ( $<4$ weeks) [138].

\subsection{New drugs: bedaquiline, delamanid, and pretomanid}

Bedaquiline appears to have poor penetration into CSF, though data are limited, and binding to tubing make drug measurement following lumbar puncture challenging [139]. Delamanid achieves CNS concentrations that are several-fold higher in brain than in plasma in rats [140], but there are no clinical data. Mass spectrometry imaging of rats following intraperitoneal administration of pretomanid demonstrated distribution of pretomanid to certain areas of the brain in uninfected animals [141]; brain or CSF distribution in infected animals or humans has not been assessed.

Thus, the second-line or new drugs with the highest likelihood of providing meaningful benefit include the following: cycloserine or ethionamide (though these drugs carry high-risk of toxicity), linezolid (optimal dosing to be determined), and possibly the nitroimidazoles (e.g. delamanid or pretomanid). It is hoped that new compounds in the TB drug development pipeline (https://www.newtbdrugs.org) will have characteristics associated with high probability of achieving effective concentrations in the brain and CSF, for example, high CNS multiparameter optimization desirability scores [62,142].

\section{Intensified treatment for specific populations}

\subsection{HIV co-infection}

Mortality from TBM is 2 to 3-fold higher in HIV co-infected individuals. Mortality depends on stage of disease at presentation and is modified by HIV infection, with estimates of death in HIV-uninfected adults of $15 \%, 30 \%$, and $50 \%$ and HIVinfected adults of 25\%, 50\%, and $80 \%$, for Medical Research Council stage I, II, or III disease, respectively $[143,144]$. Antiretroviral therapy (ART) naïve patients who present with TBM are at high risk of severe adverse events, most likely due to immune reconstitution inflammatory syndrome (IRIS), if they initiate ART immediately [37], yet delayed ART initiation puts them at risk of opportunistic infections and other complications of AIDS. There are two reasons why intensified antibiotic therapy might be especially relevant for HIVinfected TBM patients.

Firstly, a number of studies have reported that HIV-positive patients achieve lower plasma concentrations of the orally administered first-line anti-tuberculous drugs than their HIVnegative counterparts, particularly those with more advanced 
HIV [145-147]. Possible explanations relate to HIV enteropathy, diarrhea, and wasting (latter leading to under-dosing using current weight-based algorithms) [145,146,148,149]. A recent meta-analysis of rifampicin pharmacokinetic data for 931 individuals concluded that HIV status affects the total exposure to rifampicin in the early days of treatment (AUC $37.2 \mathrm{mg} . \mathrm{h} / \mathrm{L}$ in HIV-positive versus $56.7 \mathrm{mg} . \mathrm{h} / \mathrm{L}$ in HIV-negative, $p=0.003$ ) but not later in therapy [150]. The lower plasma rifampicin exposure in HIV-positive individuals in the initial days of therapy may be particularly relevant in TB meningitis, which carries a high early mortality.

Secondly, compared to those without HIV co-infection, HIVinfected TBM patients generally have less meningeal inflammation, as shown lower CSF white blood cell count or protein [151-154]. When meninges are inflamed the tight junctions between cells of the choroid plexus are disrupted allowing plasma proteins and protein-bound drugs (like rifampicin) to penetrate the CSF more readily [155]. One may, therefore, hypothesize that reduced meningeal inflammation in HIVassociated TBM may result in lower levels of anti-tuberculous drug penetrating the BCSFB, although this has not been studied. However, if this hypothesis is valid, then intensified therapies that penetrate effectively into the CNS, regardless of degree of meningeal inflammation, will be important in HIV-associated TBM.

Theoretically, intensified antibiotic treatment might lower the risk of IRIS in TBM. IRIS is a condition that results from rapid restoration of pathogen-specific immune responses to $M$. tuberculosis resulting in exuberant inflammation [156]. Although such paradoxical reactions have also been described in HIV-uninfected patients, the incidence is much greater (up to $47 \%$ ) in HIV-infected patients on initiating ART $[156,157]$. TBM-associated IRIS presents as a recurrence or deterioration of neurological symptoms and typically occurs within the first three weeks and up to 3 months after ART is initiated, recommenced or switched $[156,158]$. The risk of IRIS in TBM is much higher (RR 9.3, 95\% confidence interval 1.4 to 62.2) in patients with positive baseline CSF culture compared to those with negative CSF cultures [156]. Culture positivity reflects a higher $M$. tuberculosis antigen burden, more rapid eradication of $M$. tuberculosis antigen by intensified treatment may theoretically reduce the risk of subsequent IRIS on ART initiation. It should be emphasized, however, that corticosteroids are currently the only proven therapy for paradoxical reactions, including IRIS, and that the onset of paradoxical reactions does not warrant intensification of antimicrobial therapy [158].

There are two other important considerations in HIV-TB coinfection with regards to intensified treatments. Firstly, there are significant drug-drug interactions between rifamycins and many ART drugs. Rifampicin causes induction of cytochrome P-450 (CYP) enzymes, phase II metabolizing enzymes, and drug transporters, reducing concentrations of many companion drugs $[159,160]$. Little is known about the maximal inductive capacity of rifampicin and whether high dose rifampicin would cause more significant drug-drug interactions, though a few small studies suggest maximal induction already occurs at standard doses of rifampicin [161-163]. The second consideration is the overlapping toxicity profile of many ART and anti-tuberculous agents. For example, linezolid and zidovudine can both cause significant myelosuppression, aminoglycosides and tenofovir can cause renal toxicity, fluoroquinolones and efavirenz can cause hepatoxicity. Whilst such toxicity is relatively easy to monitor and manage in higher income settings, in lower income settings with the scarcity of laboratory tests and personalized drug regimens, drug toxicity can pose a major management dilemma. Current understanding and knowledge gaps regarding TBM-HIV are summarised in Box 1.

\subsection{Drug-resistant TBM}

The treatment of TBM can be complicated by various forms of drug resistance of $M$. tuberculosis. This can be resistance to just one TB drug (monoresistance), multi-drug resistance which refers to resistance to both isoniazid and rifampicin, and extensively-drug resistant TB in which there is also resistance against any fluoroquinolone and at least one injectable TB drug [164].

Isoniazid monoresistance appears to be the most frequent form of drug-resistant TBM. Heemskerk et al. reported isoniazid monoresistance, rifampicin monoresistance and multidrug resistance in $27 \%, 0.3 \%$, and $5 \%$ of 322 TBM patients in Vietnam [41]; and the study by Vinnard et al. showing 10\%, $2 \%$, and $19 \%$ of 324 TBM patients with the same resistance patterns in New York, USA [42]. HIV-infected patients with TBM are at a higher risk of rifampicin-resistant TBM [42,143].

The consequences of resistance to rifampicin and/or isoniazid are enormous, with mortality rates of up to $100 \%$ [42,76-78]. An important reason for this high mortality is the slow detection of drug resistance. The availability of Xpert MTB/RIF and, more recently, Xpert MTB/Rif Ultra tests is relevant in this respect, as these tests enable the detection of $M$. tuberculosis complex and rifampicin resistance within a few hours. Still, the suboptimal sensitivity of these assays in CSF and the lack of susceptibility information on other TB drugs remain a challenge.

If rapid detection of drug-resistant TBM is possible, a drug regimen can be designed based on the resistance pattern. In TBM with monoresistance to isoniazid, available data suggest that use of levofloxacin with a higher dose of rifampicin can improve survival [41]. The best regimen for the treatment of MDR TBM is unknown. Of the first-line agents, pyrazinamide is often added in MDR-TB treatment in general, and this drug shows a high penetration into CSF. It should be realized, however, that MDR-TB isolates are often resistant to pyrazinamide as well and further work is needed to characterize genotypes in varying geographic regions [165]. Furthermore, in line with MDR-TB treatment for PTB, high dose isoniazid (10-15 mg/kg daily) can be considered in case of low-level resistance to isoniazid with mutations in the Inh A promoter region, outside of the katG gene.

The second-line drugs with good penetration into the CSF, are the fluoroquinolones, ethionamide/prothionamide, cycloserine/terizidone, and linezolid, as discussed above. Again, it should be noted that ethionamide/prothionamide and cycloserine/terizidone are associated with CNS disturbances and these may be additive when combined. Use of pyridoxine is recommended to prevent CNS effects. Ethionamide/prothionamide also cause gastrointestinal intolerance. There is some 
Box 1. Implications of HIV coinfection in TBM.

Known

Two to three-fold increased risk of death in HIV co-infection

Diagnosis more challenging: differential diagnosis is broader and CSF picture can be atypical

ART initiation should be delayed til week 8 in TBM.

Increased risk of immune reconstitution inflammatory syndrome (IRIS)

Overlapping drug toxicities with ART e.g. liver injury, peripheral neuropathies, rash

Increased risk of drug-interactions with ART

Increased risk of disseminated TB disease, or HIV-related organ pathology and associated complications

\section{Unknown}

Use of adjunctive corticosteroids is not of proven benefit in HIV-associated TBM

Driver of lower exposure to rifampicin in early days of TB treatment in HIV-infection are not known

Unknown whether levels of other anti-tuberculous drugs are reduced in HIV co-infection

Differential degree of meningeal inflammation in HIVassociated TBM

Theoretical risk of strongyloides super-infection with high-dose corticosteroids

\section{Implications}

- Drivers of increased mortality not well characterized and require further research

- HIV-associated TBM, in particular, may benefit from intensified therapy

- Diagnostic algorithms for TBM have poor sensitivity and specificity in HIV co-infection

- Delayed treatment initiation due to diagnostic uncertainty may be implicated in poor outcomes

- Additional diagnostic tests such as urine TB-LAM may be useful in the diagnostic process for disseminated TBM

- Provide dose-adjusted fluconazole in cryptococcal antigenemia and cotrimoxazole prophylaxis if CD4 $<200$

- Increased pill burden and additional risk of liver toxicity, renal dysfunction, and blood dyscrasias

- IRIS has 30\% associated mortality

- Complicates ART initiation and increases steroid requirements

- Alternative host-directed therapies are required

- Significant drug-related adverse events are 3.8-fold more likely in HIV-TB co-infection than TB mono-infection This includes drug-induced hepatitis which occurs in $10 \%$ of HIV-negative and $20 \%$ of HIV-positive TB patients.

- Individualized therapy to avoid or manage toxicity is challenging in resource-constrained settings (individually formulated drugs are not widely available) so TBM treatment is often interrupted. Interruption of anti-TB drugs in TBM correlates with death

- Availability of safe and personalized treatment is particularly important at HIV-TBM

- PIs: risk of virological failure due to significant reduction in PI exposures with rifampicin co-administration. Rifabutin recommended instead of rifampicin, though not widely available and little data in TBM. In the absence of rifabutin or alternative ART some use double dose lopinavir/ ritonavir, though side effects are considerable.

- INIs: require dose-adjustment with increased pill burden and possible increase in toxicity

- NNRTIs: nevirapine is contraindicated due to marked reduction in exposure with rifampicin co-administration, switch required. Efavirenz levels reduced, though not thought to be clinically significant.

- HIV-positive people frequently have disseminated TB at the time of TBM, including granulomatous hepatitis, which can worsen on initiating antituberculous therapy. Medical course may be more complicated due to multi-system disease at baseline.

Implications

- Corticosteroids can be potentially harmful in people with advanced HIV as they may exacerbate fungal infections and Kaposi's sarcoma. A randomized trial in Asia is currently evaluating the impact of adjunctive corticosteroids in HIV-TBM.

- Undetermined whether HIV-related intestinal immune activation, HIV enteropathy, wasting or alteration in drug transporters are contributory factors in the observed lower rifampicin exposures in HIV, nor whether ART use can modify exposures.

- Further pharmacokinetic studies in HIV-TBM are needed

- Parenteral administration may be preferable in HIV

- Possible reduced clearance of pyrazinamide and isoniazid in HIV coinfected patients with high levels of immune activation

- Reduced meningeal inflammation in HIV likely to result in reduced levels of antimicrobial drug penetration into CSF compartment

- Concurrent antihelminthic treatment may be required to prevent overwhelming strongyloides infection in endemic regions (albendazole or ivermectin). Trial underway in Asia.
Evidence

Thao [183]

Marais [177]

Checkley [2]

Cresswell [151]

Marais [177]

Gupta-Wright

[184]

DHHS Guidelines

[160]

Torok [37]

Marais [177]

Pepper [185]

Heemskerk [98]

Thwaites [14]

Mcllleron [159]

www.hiv-

druginterac

tions.org

Coash [186]

Katrack [154]

Evidence

Thwaites [14]

Prasad [187]

Chabria [188]

Beardsley [189]

Donovan [190]

Stott [150]

Vinnard 2017

[42]

Mcllleron [159]

Vinnard [191]

Mcllleron [159]

Cresswell [151]

Nau [155]

Donovan [190]

$\mathrm{PI}=$ protease inhibitors, INI = integrase inhibitors, NNRTI = Non-nucleoside reverse transcriptase inhibitors 
cross-resistance between ethionamide/prothionamide with isoniazid if the inhA mutation is present.

Based on the above, a combination of TB drugs for MDRTBM could consist of pyrazinamide, a fluoroquinolone (moxifloxacin or levofloxacin), ethionamide/prothionamide, and cycloserine. This does not add up to the recommended five effective agents yet and a combination of ethionamide/ prothionamide and cycloserine may only be tolerated for a short term. Linezolid and high dose isoniazid (in case of lowlevel resistance) are alternatives, and some clinicians may consider aminoglycosides as a fifth drug despite their poor penetration across the BBCSF barrier. There is no clear option for further intensification. Clearly, there is an urgent need for comparative trials on combinations of the available and promising drugs in drug-resistant TBM, though RCTs in drugresistant TBM would face significant logistical challenges due to low patient numbers at any given center.

\subsection{Paediatric TBM}

As noted above, compared to adults, children often have nonspecific presentations. Prodromal symptoms in older children include fever, headache, anorexia, and vomiting. In younger children, presentation is often one of the nonspecific symptoms including failure to thrive, poor appetite, and vomiting. Despite the importance of early initiation of antiTBM therapy, treatment is often delayed, as a diagnosis in the pediatric population is challenging. In addition to the risk of death (which appears to be lower in children than adults) and neurologic sequelae [43], TBM in children occurs in the context of a developing brain. Intellectual impairment is common, and there is a risk of developmental sequelae related to TBM that is unique to children, as the condition affects children during important neurodevelopmental stages [166,167]. Given the high risk of functional and neurodevelopmental impairment following pediatric TBM, any improvement in treatment has the potential not only for reducing the risk of death and functional disability but also for improving quality of life in young children by improving long-term neurocognitive outcomes.

Clinical trials of anti-inflammatory therapy with steroids or thalidomide have been conducted [168-170], but up to now there have been no clinical trials of antimicrobial treatments in pediatric TBM. Likewise, almost all data describing CSF concentrations of TB drugs in TBM are from adults. Best treatment practices, therefore, must be extrapolated largely from adult trials, with doses adjusted for differences in drug disposition in children. Recognizing that standardrecommended pediatric dosing of first-line drugs achieves subtherapeutic concentrations (for targets established for PTB) in a high proportion of children [171-173], it is clear that optimal treatment of TBM or CNS TB will require higher doses of the most life-saving drugs, most notably rifampicin. Based on adult PK targets for rifampicin associated with improvements in mortality and knowledge of the developmental pharmacology of this drug, rifampicin should almost certainly should be dosed at $30 \mathrm{mg} / \mathrm{kg}$ or higher in children [174]. Second-line drugs have not been examined for efficacy expressly in pediatric TBM, though linezolid which was associated with improved clinical outcomes in adults, may have a role in pediatric TBM [137].

While WHO recommends treating paediatric TBM with 2 months of isoniazid, rifampicin, pyrazinamide, and ethambutol followed by 10 months of isoniazid and rifampicin, there is variability in guidelines given the paucity of clinical trials data to inform practice, and in some settings treatment duration is shorter. In South Africa, for example, children are mostly treated for six months using high-dose isoniazid, high-dose rifampicin, standard-dose pyrazinamide, and ethionamide (the latter in place of ethambutol) [124], based on cohort data showing the efficacy of this regimen. While adult trials can lend clues about the best antimicrobial treatment for pediatric TBM, independent trials in children may be especially valuable to look at pediatric-specific outcomes such as neurocognitive and functional outcomes. Phase II and III clinical trials of enhanced antimicrobial treatments for pediatric TBM are enrolling or soon to start [175].

\section{Summary and conclusions}

Meningitis is the most dramatic and devastating manifestation of TB, leaving upwards of $50 \%$ of those affected dead or disabled. While in this review we addressed the possible role of intensified antibiotic treatment, we acknowledge that a number of other factors are also highly relevant. Timely diagnosis and treatment is the most important factor related to patient outcome, and there is a need for earlier presentation to hospital, more sensitive rapid diagnostics, and optimal supportive care [176]. Secondly, more effective or tailored host-directed therapy could help control the immunopathology associated with TBM. Importantly, we must kill the bug to cure the patient, and intensified antibiotic treatment with drugs and doses that reach the site of disease well and slow or arrest infection-related pathology is an intervention that could be applied broadly once compelling evidence of reduced death or disability has been provided by a randomized clinical trial, of which there are number in the pipeline (Table 3). In fact, in some settings, this is already done. As an example, Table 4 contains a treatment regimen for critically ill patients with suspected TBM who are admitted to the intensive care unit in the Netherlands (Table 4). More research is needed to examine the role of higher doses of current drugs or use of alternative drug regimens, but some candidate drugs for use and/or optimization are emerging, as discussed in this review.

A number of priorities for future studies stand out. First, data support the use of a much higher dose of rifampicin for TB in general and for TBM in particular. Toxicity does not seem to be higher compared to standard treatment, noting that the anticipated survival benefit would outweigh a higher potential risk of drug toxicity. Besides high-dose rifampicin, a second approach that needs further study is the use of new drugs, especially linezolid, giving its good penetration and promising clinical effects, although this is based on two observational studies. Delamanid and pretomanid also merit further evaluation. A third priority is research in HIV-infected patients, children, and drug-resistant TBM. HIV-infected patients are at 
Table 3. Summary of clinical studies investigating intensified antimicrobial treatments for TBM.

\begin{tabular}{|c|c|c|c|c|c|c|c|}
\hline \multicolumn{8}{|c|}{ Published } \\
\hline Author & Year & Country & Trial design & $N$ & $\begin{array}{l}\text { Drug regimens tested } \\
\text { (daily doses) }\end{array}$ & $\begin{array}{l}\text { Duration of } \\
\text { intervention }\end{array}$ & $\begin{array}{c}\text { Outcomes } \\
\text { Clinical/Pharmacokinetic }\end{array}$ \\
\hline $\begin{array}{l}\text { Alvarez-Uria } \\
{[92]}\end{array}$ & 2013 & India & $\begin{array}{l}\text { Observational } \\
\text { cohort } \\
\text { (100\% HIV- } \\
\text { positive) }\end{array}$ & $\begin{array}{l}A=138 \\
B=65\end{array}$ & $\begin{array}{l}\text { Prior standard of } \\
\text { care }(\boldsymbol{A}) \text { : } \\
\text { R } 450 \mathrm{mg}, \mathrm{H} 300 \mathrm{mg} \text {, } \\
\text { Z } 1500 \mathrm{mg}, \\
\text { E } 800 \mathrm{mg} \\
\text { compared with } \\
\text { intensified } \\
\text { regimen (B): } \\
\text { LVX } 750 \mathrm{mg}, \mathrm{ETD} \\
750 \mathrm{mg}, \mathrm{Z} 1500 \mathrm{mg}, \\
\text { R } 900 \mathrm{mg}, \mathrm{H} 600 \mathrm{mg}\end{array}$ & $\begin{array}{l}7 \text { days } \\
\text { (IQR 6-9) }\end{array}$ & $\begin{array}{l}\text { Clinical: } \\
21.5 \%(95 \% \mathrm{Cl} 7.3-35.7 \%) \text { absolute } \\
\quad \text { reduction in } 12 \text { month mortality with } \\
\text { regimen B } \\
\text { PK: No PK data }\end{array}$ \\
\hline Ruslami [79] & 2013 & Indonesia & $\begin{array}{l}\mathrm{RCT} \\
\text { Phase II } \\
\text { Factorial } \\
\text { design } \\
\text { (12\% HIV- } \\
\text { positive) }\end{array}$ & 60 & $\begin{array}{l}\text { First randomisation: } \\
\text { R } 450 \mathrm{mg} P O \text { or } \\
600 \mathrm{mg} \text { IV } \\
\text { Second } \\
\text { randomisation: } \\
\text { M } 400 \mathrm{mg} \text { or } 800 \mathrm{mg} \\
\text { or } \mathrm{E} 750 \mathrm{mg} \\
\text { Standard doses of } \\
\text { Z and H used in all } \\
\text { participants }\end{array}$ & 14 days & $\begin{array}{l}\text { Clinical: } \\
\text { Reduction in 6-month mortality in IV } \\
\text { rifampicin recipients (aHR } 0.42 ; 95 \% \mathrm{Cl} \\
0.2-0.9 \text { ). No increased toxicity } \\
\text { PK: } 3 \text {-fold increase in plasma } \mathrm{AUC}_{0-24 \mathrm{~h}} \\
\mathrm{C}_{\max } \text { and } \mathrm{CSF} \mathrm{C}_{\max } \text { with } 600 \mathrm{mg} \mathrm{IV} \\
\text { rifampicin }\end{array}$ \\
\hline Heemskerk [98] & 2016 & Vietnam & $\begin{array}{l}\text { RCT } \\
\text { Phase III } \\
\text { (43\% HIV- } \\
\quad \text { positive) }\end{array}$ & $\begin{array}{l}A=409 \\
B=408\end{array}$ & $\begin{array}{l}\text { Standard of care }(A) \text { : } \\
\text { R } 10 \mathrm{mg} / \mathrm{kg}, \mathrm{H} 5 \mathrm{mg} / \\
\mathrm{kg}, \mathrm{Z} 25 \mathrm{mg} / \mathrm{kg} \text {, } \\
\text { E } 20 \mathrm{mg} / \mathrm{kg} \\
\text { Intensified regimen (B): } \\
\text { R } 15 \mathrm{mg} / \mathrm{kg}, \mathrm{LVX} \\
20 \mathrm{mg} / \mathrm{kg} \\
\text { H } 5 \mathrm{mg} / \mathrm{kg}, \mathrm{Z} 25 \mathrm{mg} / \\
\text { kg, E } 20 \mathrm{mg} / \mathrm{kg}\end{array}$ & 8 weeks & $\begin{array}{l}\text { Clinical: } \\
\text { No reduction in mortality overall (HR } \\
0.94 ; 95 \% \mathrm{Cl} 0.7-1.2, \mathrm{P}=0.66) \text {. } \\
\text { Subgroup analysis of isoniazid- } \\
\text { resistant TBM found that intensified } \\
\text { therapy was significantly associated } \\
\text { with improved survival (HR } 0.34,95 \% \\
\mathrm{Cl} 0.15 \text { to } 0.76, \mathrm{p}=0.01)[41] \\
\text { No increased toxicity. } \\
\text { PK: PK data awaited }\end{array}$ \\
\hline Dian [97] & 2017 & Indonesia & $\begin{array}{l}\text { RCT } \\
\text { Phase II } \\
3 \text { arm, parallel } \\
\text { group (10\% } \\
\text { HIV-positive) }\end{array}$ & $\begin{array}{l}A=20 \\
B=20 \\
C=20\end{array}$ & $\begin{array}{l}\text { A: } \mathrm{R} 450 \mathrm{mg} \text { (standard } \\
\text { dose, } \sim 10 \mathrm{mg} / \mathrm{kg} \text { ) } \\
\text { B: } \mathrm{R} 900 \mathrm{mg} \text { (double } \\
\text { dose, } \sim 20 \mathrm{mg} / \mathrm{kg} \text { ) } \\
\text { C: } \mathrm{R} 1350 \mathrm{mg} \text { (triple } \\
\text { dose, } \sim 30 \mathrm{mg} / \mathrm{kg} \text { ) } \\
\text { Standard dose } \mathrm{H}, \\
\text { Z and E used in all } \\
\text { arms }\end{array}$ & 30 days & $\begin{array}{l}\text { Clinical: Six month mortality: } 7 / 20 \\
\text { (35\%), 9/20 (45\%), } 3 / 20(15 \%) \text { in arms } \\
\text { A, B and C respectively ( } p=0.12) \\
\text { No increase in grade } 3 \text { or } 4 \text { AEs } \\
\text { PK: } 3 \text { and 5-fold higher total plasma } \\
\text { exposures with double and triple dose } \\
\text { rifampicin. Proportional increases in } \\
\text { CSF concentrations. }\end{array}$ \\
\hline Sun [138] & 2014 & China & $\begin{array}{l}\text { Retrospective } \\
\text { cohort } \\
\text { (HIV-negative) }\end{array}$ & $\begin{array}{l}A=17 \\
B=16\end{array}$ & $\begin{array}{l}\text { A: Standard of care } \\
\text { B: Standard of care } \\
\text { plus adjunctive LZD } \\
600 \text { mg twice daily }\end{array}$ & $\begin{array}{l}32 \text { days } \\
\text { (median) }\end{array}$ & $\begin{array}{l}\text { Clinical: Those who received LZD had } \\
\text { more rapid resolution of coma and } \\
\text { normalisation of temperature as well } \\
\text { as higher CSF glucose and lower CSF } \\
\text { white cell count. AEs limited: } 12.5 \% \text { in } \\
\text { LZD group (including } \\
\text { myelosuppression }(n=1 \text { ) and optic } \\
\text { neuropathy }(n=1) \text { ) both resolved on } \\
\text { discontinuing LZD) } \\
\text { PK: no PK done }\end{array}$ \\
\hline Thwaites [117] & 2011 & Vietnam & $\begin{array}{l}\text { RCT } \\
\text { Phase II } \\
4 \text { arm, parallel } \\
\text { group }\end{array}$ & $\begin{array}{l}A=15 \\
B=16 \\
C=15 \\
D=15\end{array}$ & $\begin{array}{l}\text { A: Standard of care } \\
\text { (SOC) } \\
\text { B: SOC plus CIP } \\
\text { C: SOC plus LVX } \\
\text { D: SOC plus GFX }\end{array}$ & 270 days & $\begin{array}{l}\text { Clinical: Worse outcomes amongst } \\
\text { participants with low or high plasma } \\
\text { or CSF exposures, rather than } \\
\text { intermediate exposures (U-shaped } \\
\text { exposure-response) } \\
\text { PK: CSF penetration (ratio CSF AUC } \mathrm{A}_{0-24 \mathrm{~h}} \text { : } \\
\text { plasma AUC } \mathrm{A}_{0-24 \mathrm{~h}} \text { ) greatest for } \\
\text { levofloxacin }(0.74,95 \% \mathrm{Cl} 0.58-1.03) \text {. }\end{array}$ \\
\hline Yunivita [94] & 2016 & Indonesia & $\begin{array}{l}\text { RCT } \\
\text { Phase II, } \\
\text { Open-label, } \\
\text { three-arm, } \\
\text { two-period } \\
\text { (20\% HIV- } \\
\text { positive) }\end{array}$ & $\begin{array}{l}A=11 \\
B=9 \\
C=10\end{array}$ & $\begin{array}{l}\text { A: } \mathrm{R} 750 \mathrm{mg}(\sim 17 \mathrm{mg} / \\
\mathrm{kg}) \\
\text { B: } \mathrm{R} 900 \mathrm{mg}(\sim 20 \mathrm{mg} / \\
\mathrm{kg}) \\
\text { C: R } 600 \mathrm{mg} \text { i.v. }\end{array}$ & 14 days & $\begin{array}{l}\text { Clinical: The study focused on } \\
\text { pharmacokinetics and safety/ } \\
\text { tolerability and was not designed or } \\
\text { powered to study outcome or } \\
\text { relationships between exposures and } \\
\text { outcome. } \\
\text { PK: } 750 \mathrm{mg} \text { and } 900 \mathrm{mg} \text { resulted in } \\
\text { similar total exposures to rifampicin in } \\
\text { plasma as compared with } 600 \mathrm{mg} \text { IV, } \\
\text { but peak plasma concentrations } \\
\text { remain higher with i.v. administration. }\end{array}$ \\
\hline
\end{tabular}


Table 3. (Continued).

\begin{tabular}{|c|c|c|c|c|c|c|c|}
\hline \multicolumn{8}{|c|}{ Upcoming or current TBM clinical trials of intensified therapy } \\
\hline Trial name & Start & Country & $\begin{array}{l}\text { Trial design/ } \\
\text { population }\end{array}$ & Sample size & $\begin{array}{l}\text { Regimens to be } \\
\text { examined }\end{array}$ & $\begin{array}{l}\text { Duration of } \\
\text { intervention }\end{array}$ & Outcome measures \\
\hline $\begin{array}{l}\text { RifT study } \\
\text { ISRCTN42218549 }\end{array}$ & 2018 & Uganda & $\begin{array}{l}\text { Phase II RCT } \\
3 \text { arm, parallel } \\
\text { group } \\
\text { (95-100\% HIV- } \\
\text { positive) }\end{array}$ & 60 & $\begin{array}{l}\text { A: Standard of care } \\
\text { R (R 10 mg/kg) } \\
\text { B: Intravenous } \\
\text { R } 20 \mathrm{mg} / \mathrm{kg} \\
\text { C: Oral R } 35 \mathrm{mg} / \mathrm{kg} \\
\mathrm{H}, \mathrm{Z} \text { and E given at } \\
\text { standard doses in all } \\
\text { arms }\end{array}$ & 8 weeks & $\begin{array}{l}\text { 1. PK parameters in plasma and CSF } \\
\text { ( } C_{\max }, A U C, T>\text { MIC) } \\
\text { 2. Safety } \\
\text { 3. } 8 \text { and } 24 \text {-week mortality } \\
\text { 4. Functional status (Rankin scale) at } 2 \\
\text { and } 24 \text { weeks } \\
\text { 5. Incidence of TBM-IRIS }\end{array}$ \\
\hline $\begin{array}{l}\text { TBM-KIDS } \\
\text { NCT02958709 }\end{array}$ & 2018 & $\begin{array}{l}\text { India } \\
\text { Malawi }\end{array}$ & $\begin{array}{l}\text { Phase II RCT } \\
\text { paediatric }\end{array}$ & 120 & $\begin{array}{l}\text { A: high dose } \\
\mathrm{R} \text { (standard dose } \mathrm{H}, \\
\mathrm{Z}, \mathrm{E} \text { ) } \\
\text { B: high dose } \mathrm{R} \text { and } \\
\text { levofloxacin } \\
\text { (standard dose } \\
\mathrm{H} \text { and } \mathrm{Z} \text { ) } \\
\text { C: standard of care }\end{array}$ & 8 weeks & $\begin{array}{l}\text { 1. PK parameters (plasma, CSF) } \\
\text { 2. Functional outcome (Modified Rankin } \\
\text { Scale) } \\
\text { 3. Safety } \\
\text { 4. Neurocognitve (Mullen Scales of Early } \\
\text { Learning) }\end{array}$ \\
\hline Harvest & 2019 & $\begin{array}{l}\text { Indonesia } \\
\text { South Africa } \\
\text { Uganda }\end{array}$ & $\begin{array}{l}\text { Phase III RCT } \\
2 \text { arm, parallel } \\
\text { design }\end{array}$ & 600 & $\begin{array}{l}\text { A: Standard of care } \\
\text { B: } \mathrm{R} 1500 \mathrm{mg} \text { (Asia) or } \\
1800 \mathrm{mg} \text { (Africa), } \\
\text { equivalent to } \\
\sim 35 \mathrm{mg} / \mathrm{kg} . \mathrm{H}, \\
\mathrm{Z} \text { and E given at } \\
\text { standard doses }\end{array}$ & 8 weeks & $\begin{array}{l}\text { 1. } 6 \text { month survival time } \\
\text { 2. Time to normalisation of } \\
\text { consciousness (GCS 15) } \\
\text { 3. Neurocognitive outcomes } \\
\text { 4. safety and tolerability endpoints } \\
\text { 5. PK-PD endpoints } \\
\text { 6. Cost effectiveness }\end{array}$ \\
\hline ACTG A5384 & 2019 & TBD & $\begin{array}{l}\text { Phase II RCT } \\
2 \text { arm, parallel } \\
\text { design }\end{array}$ & 300 & $\begin{array}{l}\text { A: } 2 \text { months } \mathrm{R}_{35} \mathrm{H}_{10} \\
\mathrm{LZD}_{1200} \mathrm{Z}, 4 \text { months } \\
\mathrm{R}_{35} \mathrm{H}_{10} \\
\text { B: Standard of care }\end{array}$ & 6 months & $\begin{array}{l}\text { 1. } 18 \text { month survival time } \\
\text { 2. Modified Rankin scale at week } 12,24 \text {, } \\
26,48 \text { and } 72 \\
\text { 3. Grade } 3-5 \text { adverse events } \\
\text { 4. Neurocognitive function } \\
\text { 5. Time to GCS }=15 \\
\text { 6. Pharmacokinetic parameters }\end{array}$ \\
\hline
\end{tabular}

$\mathrm{R}=$ rifampicin, $\mathrm{H}=$ isoniazid, $\mathrm{Z}=$ pyrazinamide, $\mathrm{E}=$ ethambutol, ETD = ethionamide, CIP = ciprofloxacin, GFX = gatifloxacin, $\mathrm{LVX}=$ levofloxacin, LZD = linezolid, $M=$ moxifloxacin

much higher risk of dying, with lower average drug exposure and a higher risk of treatment complications like IRIS, drug interactions, and toxicity. Clinical trials in children are a high priority, as there are no published clinical trials in this population to date, and extrapolation of findings in adult patients is difficult in light of different PK-PD and disease characteristics. The considerable incidence of resistant TBM warrants further research in improved identification of drug-resistant pathogens and comparative trials on combinations of available and promising drugs. A fourth priority is the use of innovative research strategies, including molecular pharmacology, Physiologically based pharmacokinetic (PBPK) models, adaptive trial designs, and smart quantitative pharmacology, including PK-PD assessments.

Finally, barriers to implementation of high-quality care for TBM should be examined, prioritized, and addressed. Delays in the presentation to hospital, lumbar puncture and treatment initiation all contribute to poor outcomes. Intensified therapies for TBM holds the potential to make the greatest impact on mortality only if such barriers are addressed in parallel. The global TBM research consortium convenes every 1-2 years and has issued standard case definitions for research [177], standardized methods for enhanced quality and comparability of TBM studies [178], and produced a recent general overview of TBM [49]. We hope this will catalyze high-quality research in the field and translation of research findings to clinical practice.

\section{Expert commentary}

TBM is a highly variable disease, with multiple competing causes of neurologic damage or death. Much remains unknown about immunopathogenesis in different populations and different disease stages, and how best to minimize the immunopathology. Additionally, the optimal antimicrobial chemotherapy regimen remains to be defined, and we have discussed a number of promising options and the immediate clinical trial landscape in this review. However, cases are rare in any one institution, and significant resources are required to care for any adult or child with TBM. Thus, there is much to gain from optimizing design and analysis of clinical trials in TBM. In addition, standardizing of pharmacokinetic approaches across studies will allow for pooling of data or easier comparisons between studies. We believe 
Table 4. Suggested intensified TBM regimen and administration for high resource settings.

\begin{tabular}{|c|c|c|c|}
\hline $\begin{array}{l}\text { Individual } \\
\text { drugs }\end{array}$ & Dose & Formulation & Administration \\
\hline Isoniazid* & $\begin{array}{l}5 \mathrm{mg} / \mathrm{kg} \text { p.o. or i. } \\
\text { v. }\end{array}$ & Tablet $200 \mathrm{mg}$ or injection fluid $100 \mathrm{mg} / \mathrm{ml}, 3 \mathrm{ml}$ & $\begin{array}{l}\text { Orally } \\
\text { i.v. bolus: in } 3-5 \mathrm{~min} \\
\text { i.v. infusion: add the dose to } 100 \mathrm{ml} \mathrm{NaCl} 0.9 \% \text {, administer } \\
\text { within } 0.5 \mathrm{~h}\end{array}$ \\
\hline Rifampicin* & $\begin{array}{l}30-35 \mathrm{mg} / \mathrm{kg} \text { p.o } \\
\text { or } 15 \mathrm{mg} / \mathrm{kg} \text { i.v. }\end{array}$ & $\begin{array}{l}\text { Capsule (150 or } 300 \mathrm{mg} \text { ), tablet }(600 \mathrm{mg} \text { ) or powder for } \\
\text { infusion fluid } 600 \mathrm{mg} \text { (Rifadin }{ }^{\circledR} \text {, Sanofi-Aventis) }\end{array}$ & $\begin{array}{l}\text { Orally } \\
\text { i.v. infusion: dissolve } 600 \mathrm{mg} \text { in } 10 \mathrm{ml} \text { water for injection, add } \\
\text { the correct dose to } 250 \mathrm{ml} \mathrm{NaCl} 0.9 \% \text {, administer in } 1.5 \mathrm{~h} \text {. } \\
\text { Minimal volume is } 100 \mathrm{ml} \text {, administration in } 0.5 \mathrm{~h} \text {. }\end{array}$ \\
\hline Pyrazinamide* & 30 mg/kg p.o. & Tablet $500 \mathrm{mg}$ & Orally \\
\hline Moxifloxacin* & $\underset{* *}{600}$ mg p.o. or i.v. & $\begin{array}{l}\text { Tablet } 400 \mathrm{mg} \text { or infusion fluid, } 1.6 \mathrm{mg} / \mathrm{ml}, 250 \mathrm{ml} \\
\text { (Avelox }{ }^{\oplus}, \text { Bayer) }\end{array}$ & i.v. infusion: infuse in no less than $1 \mathrm{~h}$. No dilution needed. \\
\hline \multicolumn{4}{|c|}{ Optional fifth drug } \\
\hline Linezolid* & $\begin{array}{l}600 \mathrm{mg} \text { bd p.o. or } \\
\text { i.v. }\end{array}$ & Tablet $600 \mathrm{mg}$, or infusion fluid, $2 \mathrm{mg} / \mathrm{mL}, 300 \mathrm{~mL}$ & $\begin{array}{l}\text { Orally, twice daily } \\
\text { i.v. infusion: administer within 30-120 minutes, no dilution } \\
\text { needed. }\end{array}$ \\
\hline Amikacin & $15 \mathrm{mg} / \mathrm{kg}$ i.v. & Injection fluid $250 \mathrm{mg} / \mathrm{ml}, 2 \mathrm{ml}$ & $\begin{array}{l}\text { i.v. infusion: add the dose to } 100-200 \mathrm{ml} \mathrm{NaCl} 0.9 \% \text {, administer in } \\
0.5-1.0 \mathrm{~h} \text {. }\end{array}$ \\
\hline \multicolumn{4}{|l|}{ Other } \\
\hline Dexamethasone & $\begin{array}{c}0.4 \mathrm{mg} / \mathrm{kg} \text { i.v. } \\
\text { (grade } 2 / 3 \text { ), or } \\
0.3 \mathrm{mg} / \mathrm{kg} \text { i.v. } \\
\text { (grade 1) TBM }\end{array}$ & Dexamethasone disodium phosphate, $5 \mathrm{mg} / \mathrm{ml}, 1 \mathrm{ml}$ & $\begin{array}{l}\text { i.v. bolus: in } 2-3 \mathrm{~min} \\
\text { i.v. infusion: add the dose to } 50-500 \mathrm{ml} \mathrm{NaCl} 0.9 \%\end{array}$ \\
\hline \multicolumn{4}{|c|}{$\begin{array}{l}\text { This regimen is used in the intensive care unit at Radboud university medical center, The Netherlands. All doses of drugs can be individualized by measurement of } \\
\text { drug concentrations in plasma or CSF (Therapeutic Drug Monitoring, TDM) } \\
\text { *In patients without risk of malabsorption e.g. ileus medication can be given by mouth or by nasogastric tube in patients unable to take medication by mouth. } \\
\text { ** Higher dose of moxifloxacin is used to counteract the effect of rifampicin, which decreases the exposure to moxifloxacin by approximately } 30 \% \text { [118]. } \\
\text { Of note, Intravenous compatibility of these drugs has not been tested, neither when combined in one infusion fluid nor when co-administered by Y-side. Only } \\
\text { dexamethasone is compatible with amikacin infusion when administered through a Y-side. Therefore, the drugs should be administered separately from each other } \\
\text { and the injection port/infusion system should be flushed with } \mathrm{NaCl} 0.9 \% \text { after each administration. }\end{array}$} \\
\hline
\end{tabular}

there are a variety of innovative strategies that may add to the quality and scientific output of TBM research (Box 2).

Firstly, we can take advantage of preclinical tools to aid us in designing regimens. Molecular pharmacological research using, for example, human liver microsomes, hepatic cell lines, brain capillary endothelial cell lines, and transporter overexpression models can help identify whether TB drugs are substrates, inhibitors and/or inducers of specific metabolic enzymes or drug transporters, including those transporters that control access of molecules to the brain or CSF [179]. It can predict drug-drug interactions mediated by metabolic enzymes or drug transporters, reveal possible synergy between drugs in combinations, and assess tissue penetration/accumulation characteristics [179]. PBPK modeling is another value in silico tool; it combines mechanistically scaled in vitro experimental data about PK and drug distribu-

Box 2. Innovative research strategies and methodology.

(1) Preclinical tools

- Molecular pharmacological research

- Physiologically based pharmacokinetic (PBPK) modeling

(2) Adaptive trial design

(3) Integration of PK and PK-PD assessments

(4) Tools for determination of CNS drug distribution

- PET imaging

- Post-mortem measurement of drug in brain

- Animal models

(5) Validated alternative endpoints

- Functional

- Metabolomic

- Proteomic tion to relevant compartments to simulate in vivo behavior, and can take into account the distribution of demographic and disease characteristics in the target population. In this way, PBPK modeling allows for prediction of drug concentrations in specific tissue compartments, such as the brain or CSF, and helps translate exposure predictions to other doses and populations. Thus, PBPK modeling can improve the efficiency and accuracy of drug, dose, and regimen selection up front.

A second important aspect is the trial design itself. Modern trial designs that are adaptive in nature and allow for testing of multiple regimens, with mid-trial selection of those arms that show enough promise to continue will help avoid late-phase trial failures which are costly in so many ways [87]. In trials for TBM, however, the optimal way to assess regimens mid-study is not clear and is made challenging by relatively low patient numbers and variability in presentation and disease severity.

Thirdly, irrespective of the trial design, integration of PK and PK-PD assessments into study design can enrich the information upon which decisions are made. For example, measurements of drug concentrations in the CSF can be useful in models linking site-of-action PK with outcomes, provided samples are collected properly. Optimal sampling strategies should be employed to choose the best times to collect plasma samples and (generally) the single CSF sample per study participant. Timing of CSF sampling can differ among study participants so that CSF concentrations over a dosing interval can be characterized more fully using population PK modelling. Advanced pharmacometrics (pharmacokineticpharmacodynamic (PK/PD) modelling) is also crucial to maximize both study design and learning from rich and complex data that emerges from interventional trials. Examples include 
the assessment of paediatric doses of high dose rifampicin in TBM based on adult data [174], and the evaluation of PK-PD relationships with high dose rifampicin in PTB.

When interpreting CSF concentration data, it is also important to keep in mind that unbound drug is the active drug, and highly bound drugs may have vastly different concentrations in plasma and CSF but similar free concentrations in the two compartments. Of note, CSF concentrations do not always correlate with concentrations in the brain. While we commonly only have access to CSF to learn about CNS drug distribution, post-mortem measurement of drug in brain can provide critical information about distribution of specific drugs into granulomas and those areas affected by vasculitis and other TB-related brain pathologies. Although animal models may be helpful in this regard, most animal models introduce TB meningitis by intracranial injections, possibly compromising the comparison with human BBB and BCSFB integrity and CNS drug entry.

To simplify TBM trials, having validated alternative endpoints could aid efficiency substantially - including CSF markers, neurocognitive or functional outcomes, microbiologic endpoints. A difference in normalization of routine CSF parameters like glucose or more advanced (e.g. metabolomic [180] or proteomic [181]) markers could be used as surrogate markers of clinical response. Obviously, it is also important to correct the effect of intensified treatment for other factors associated with outcome (age, HIV-status, disease severity, inflammatory phenotype, etc.) to more accurately represent the relationship between the antimicrobial treatment and clinical outcomes [182].

\section{Five-year view}

In the era of 'omics' it is encouraging to see that we are beginning to understand more about the immunopathogenesis of TBM $[157,180,192]$. This may lead to discovery of novel targets for host immunomodulation or antimicrobial effect, as well as identification of repurposed drugs with potential activity in TBM. In five years a number of the enrolling trials of intensified antimicrobial therapy for TBM, listed in Table 3, will have yielded important information on the PK and clinical impact of higher dose rifampicin and linezolid in TBM. We will also have new insights into previously under-studied populations with TBM including children in India and Malawi and HIV co-infected adults in sub-Saharan Africa.

\section{Key issues}

- Timely diagnosis and treatment is the most important factor related to patient outcome, and there is a need for earlier presentation to hospital and more sensitive rapid diagnostics. More effective or tailored host-directed therapy will help control the immunopathology associated with TBM.

- Intensified antibiotic treatment with drugs and doses that reach the site of disease sufficiently to slow or arrest infection-related pathology is an intervention that could be applied quickly and broadly if proven to improve clinical outcomes in randomised trials.
- More research is needed to examine the role of higher doses of current drugs or use of alternative drug regimens. Data support the use of a much higher-dose of rifampicin for TB in general and for TBM in particular. Linezolid, given its good brain penetration and promising clinical effects in two observational studies also warrant further investigation.

- Research in HIV-positive patients, children, and drugresistant TBM is a priority.

- Use of innovative research strategies are essential, including molecular pharmacology, physiologically based pharmacokinetic models, adaptive trial designs, and smart quantitative pharmacology, including pharmacokineticpharmacodynamic assessments.

\section{Funding}

This paper was not funded.

\section{Declaration of interest}

FV Creswell is supported through a Wellcome Trust Clinical PhD Fellowship (210772/Z/18/Z) and is an honorary fellow of the MUll-plus which is supported through the DELTAS Africa Initiative (Grant no. 107743). K Dooley is supported by the Eunice Kennedy Shriver National Institute of Child Health and Human Development (NICHD/NIH: R01HD074944). FV Cresswell, L te Brake, R Ruslami, R Aarnoutse and R van Crevel will receive support from Medical Research Council UK for a phase III RCT "High Dose Oral Rifampicin to Improve Survival from Adult TB Meningitis - (HARVEST) Trial" to be conducted in Uganda, South Africa and Indonesia (MR/S004963/1).The authors have no other relevant affiliations or financial involvement with any organization or entity with a financial interest in or financial conflict with the subject matter or materials discussed in the manuscript apart from those disclosed.

\section{Reviewer disclosures}

Peer reviewers on this manuscript have no relevant financial or other relationships to disclose.

\section{References}

Papers of special note have been highlighted as either of interest $(\bullet)$ or of considerable interest $(\cdot \cdot)$ to readers.

1. Ducomble T, Tolksdorf K, Karagiannis I, et al. The burden of extrapulmonary and meningitis tuberculosis: an investigation of national surveillance data, Germany, 2002 to 2009. Euro Surveill. 2013;18(12). pii: 20436.

2. Checkley AM, Njalale Y, Scarborough M, et al. Sensitivity and specificity of an index for the diagnosis of TB meningitis in patients in an urban teaching hospital in Malawi. Trop Med Int Health. 2008;13 (8):1042-1046.

3. Zumla A, Malon $P$, Henderson J, et al. Impact of HIV infection on tuberculosis. Postgrad Med J. 2000;76(895):259-268.

4. Gupta RK, Lucas SB, Fielding KL, et al. Prevalence of tuberculosis in post-mortem studies of HIV-infected adults and children in resource-limited settings: a systematic review and meta-analysis. Aids. 2015;29(15):1987-2002.

5. Rana FS, Hawken MP, Mwachari C, et al. Autopsy study of HIV-1-positive and HIV-1-negative adult medical patients in Nairobi, Kenya. J Acquir Immune Defic Syndr. 2000;24(1):23-29.

6. Jarvis JN, Meintjes G, Williams A, et al. Adult meningitis in a setting of high HIV and TB prevalence: findings from 4961 suspected cases. BMC Infect Dis. 2010;10:67. 
7. Rajasingham R, Rhein J, Klammer K, et al. Epidemiology of meningitis in an HIV-infected Ugandan cohort. Am J Trop Med Hyg. 2015;92(2):274-279.

8. Starke JR. Tuberculosis of the central nervous system in children. Semin Pediatr Neurol. 1999;6(4):318-331.

9. Principi N, Esposito S. Diagnosis and therapy of tuberculous meningitis in children. Tuberculosis (Edinb). 2012;92(5):377-383.

10. Farinha NJ, Razali KA, Holzel $\mathrm{H}$, et al. Tuberculosis of the central nervous system in children: a 20-year survey. J Infect. 2000;41 (1):61-68.

11. Wolzak NK, Cooke ML, Orth $H$, et al. The changing profile of pediatric meningitis at a referral centre in Cape Town, South Africa. J Trop Pediatr. 2012;58(6):491-495.

12. Ar R. The Pathogenesis of Tuberculosis. C.C. Thomas; 1946.

13. Jain SK, Tobin DM, Tucker EW, et al. Tuberculous meningitis: a roadmap for advancing basic and translational research. Nat Immunol. 2018;19(6):521-525.

14. Thwaites GE, Nguyen DB, Nguyen HD, et al. Dexamethasone for the treatment of tuberculous meningitis in adolescents and adults. N Engl J Med. 2004;351(17):1741-1751.

15. Prasad K, Singh MB, Ryan H. Corticosteroids for managing tuberculous meningitis. In: Cochrane database of systematic reviews. 2016 Apr 28;4:CD002244. doi:10.1002/14651858.CD002244.pub4.

16. Thwaites GE, van Toorn R, Schoeman J. Tuberculous meningitis: more questions, still too few answers. Lancet Neurol. 2013;12 (10):999-1010.

17. Marais S, Pepper DJ, Schutz C, et al. Presentation and outcome of tuberculous meningitis in a high HIV prevalence setting. PLoS One. 2011;6(5):e20077.

18. Patel VB, Padayatchi N, Bhigjee Al, et al. Multidrug-resistant tuberculous meningitis in KwaZulu-Natal, South Africa. Clin Infect Dis. 2004;38(6):851-856.

19. Gu J, Xiao H, Wu F, et al. Prognostic factors of tuberculous meningitis: a single-center study. Int J Clin Exp Med. 2015;8(3):4487-4493.

20. Alvarez-Uria G, Midde M, Pakam R, et al. Initial antituberculous regimen with better drug penetration into cerebrospinal fluid reduces mortality in HIV Infected Patients with tuberculous meningitis: data from an HIV observational cohort study. Tuberc Res Treat. 2013;2013:242604.

21. Alvarez-Uria G, Naik PK, Pakam R, et al. Natural history and factors associated with early and delayed mortality in HIV-infected patients treated of tuberculosis under directly observed treatment short-course strategy: a prospective cohort study in India. Interdiscip Perspect Infect Dis. 2012;2012:502012.

22. Hsu PC, Yang -C-C, Ye -J-J, et al. Prognostic factors of tuberculous meningitis in adults: a 6-year retrospective study at a tertiary hospital in northern Taiwan. J Microbiol Immunol Infect. 2010;43 (2):111-118.

23. Torok ME, Bang ND, Chau TTH, et al. Dexamethasone and long-term outcome of tuberculous meningitis in Vietnamese adults and adolescents. PLoS One. 2011;6(12):e27821.

24. Vinnard C, Winston CA, Wileyto EP, et al. Isoniazid resistance and death in patients with tuberculous meningitis: retrospective cohort study. Bmj. 2010;341:c4451.

25. Senbayrak S, Labeikyte D, Markuckas A, et al. Antituberculosis drug resistance patterns in adults with tuberculous meningitis: results of haydarpasa-iv study. Ann Clin Microbiol Antimicrob. 2015;14:47.

26. Pehlivanoglu F, Yasar KK, Sengoz G. Tuberculous meningitis in adults: a review of 160 cases. Sci World J. 2012;2012:169028.

27. Efsen AM, Panteleev AM, Grint D, et al. TB meningitis in HIV-positive patients in Europe and Argentina: clinical outcome and factors associated with mortality. Biomed Res Int. 2013;2013:373601.

28. Bang ND, Caws M, Truc $T$, et al. Clinical presentations, diagnosis, mortality and prognostic markers of tuberculous meningitis in Vietnamese children: a prospective descriptive study. BMC Infect Dis. 2016;16(1):573.

29. Karande S, Gupta V, Kulkarni M, et al. Prognostic clinical variables in childhood tuberculous meningitis: an experience from Mumbai, India. Neurol India. 2005;53(2): 191-196. discussion 195-6.
30. Duque-Silva A, Robsky K, Flood J, et al. Risk factors for central nervous system tuberculosis. Pediatrics. 2015;136(5):e1276-84.

31. Wishart DS, Feunang YD, Guo AC, et al. DrugBank 5.0: a major update to the drugbank database for 2018. Nucleic Acids Res. 2018;46(D1):D1074-d1082.

32. World Health Organisation. WHO guidelines approved by the guidelines review committee. In: Companion handbook to the WHO guidelines for the programmatic management of drug-resistant tuberculosis. Geneva: World Health OrganizationCopyright (c) 2014.

33. ASHP. AHFS clinical drug information. 2018. American Society of Health-System Pharmacists. [cited 2018 Aug 1]. Available from: http://www.ahfsdruginformation.com/

34. Dart RC. Medical toxicology. Philadelphia: Lippincott, Williams \& Wilkins; 2004.

35. Miftode EG, Dorneanu OS, Leca DA, et al. Tuberculous meningitis in children and adults: a 10-year retrospective comparative analysis. PLoS One. 2015;10(7):e0133477.

36. Cecchini D, Ambrosioni J, Brezzo C, et al. Tuberculous meningitis in HIV-infected and non-infected patients: comparison of cerebrospinal fluid findings. Int J Tuberc Lung Dis. 2009;13(2):269-271.

37. Török ME, Yen NTB, Chau TTH, et al. Timing of initiation of antiretroviral therapy in human immunodeficiency virus (HIV)-associated tuberculous meningitis. Clin Infect Dis. 2011;52(11):1374-1383.

38. Kalita J, Misra UK, Nair PP. Predictors of stroke and its significance in the outcome of tuberculous meningitis. J Stroke Cerebrovasc Dis. 2009;18(4):251-258.

39. Croda MG, Vidal JE, Hernández AV, et al. Tuberculous meningitis in HIV-infected patients in Brazil: clinical and laboratory characteristics and factors associated with mortality. Int J Infect Dis. 2010;14(7):e586-91.

40. Cresswell F, Bangdiwala A, Bahr NC, et al. Can improved diagnostics reduce mortality from tuberculous meningitis? Findings from a 6.5-year cohort in Uganda. Wellcome Open Res. 2018;3:64.

41. Heemskerk AD, Nguyen MTH, Dang HTM, et al. Clinical outcomes of patients with drug-resistant tuberculous meningitis treated with an intensified antituberculosis regimen. Clin Infect Dis. 2017;65(1):20-28.

42. Vinnard C, King L, Munsiff S, et al. Long-term mortality of patients with tuberculous meningitis in New York city: A cohort study. Clin Infect Dis. 2017;64(4):401-407.

43. Chiang SS, Khan FA, Milstein MB, et al. Treatment outcomes of childhood tuberculous meningitis: a systematic review and meta-analysis. Lancet Infect Dis. 2014;14(10):947-957.

44. Nataprawira HM, Ruslianti V, Solek P, et al. Outcome of tuberculous meningitis in children: the first comprehensive retrospective cohort study in Indonesia. Int J Tuberc Lung Dis. 2016;20(7):909-914.

45. Dhawan SR, Gupta A, Singhi $P$, et al. Predictors of neurological outcome of tuberculous meningitis in childhood: a prospective cohort study from a developing country. J Child Neurol. 2016;31 (14):1622-1627.

46. Padayatchi N, Bamber S, Dawood H, et al. Multidrug-resistant tuberculous meningitis in children in Durban, South Africa. Pediatr Infect Dis J. 2006;25(2):147-150.

47. Kumar R, Dwivedi A, Kumar $P$, et al. Tuberculous meningitis in BCG vaccinated and unvaccinated children. I Neurol Neurosurg Psychiatry. 2005;76(11):1550-1554.

48. Schoeman JF, Van Zyl LE, Laubscher JA, et al. Effect of corticosteroids on intracranial pressure, computed tomographic findings, and clinical outcome in young children with tuberculous meningitis. Pediatrics. 1997;99(2):226-231.

49. Wilkinson RJ, Rohlwink U, Misra UK, et al. Tuberculous meningitis. Nat Rev Neurol. 2017;13(10):581-598.

- This recent review paper summarises the current understanding of TBM and highlights research priorities.

50. Ichikawa $H$, Ishikawa $M$, Fukunaga $M$, et al. Quantitative evaluation of blood-cerebrospinal fluid barrier permeability in the rat with experimental meningitis using magnetic resonance imaging. Brain Res. 2010;1321:125-132.

51. Loscher W, Potschka H. Drug resistance in brain diseases and the role of drug efflux transporters. Nat Rev Neurosci. 2005;6 (8):591-602. 
52. Andes DR, Craig WA. Pharmacokinetics and pharmacodynamics of antibiotics in meningitis. Infect Dis Clin North Am. 1999;13 (3):595-618.

53. Sun YX, Minthon L, Wallmark A, et al. Inflammatory markers in matched plasma and cerebrospinal fluid from patients with Alzheimer's disease. Dement Geriatr Cogn Disord. 2003;16(3):136-144.

54. Parrish KE, Sarkaria JN, Elmquist WF. Improving drug delivery to primary and metastatic brain tumors: strategies to overcome the blood-brain barrier. Clin Pharmacol Ther. 2015;97(4):336-346.

55. Begley DJ. Delivery of therapeutic agents to the central nervous system: the problems and the possibilities. Pharmacol Ther. 2004;104(1):29-45.

56. Redzic Z. Molecular biology of the blood-brain and the blood-cerebrospinal fluid barriers: similarities and differences. Fluids Barriers CNS. 2011;8(1):3.

57. America IDSO Official American thoracic society/centers for disease control and prevention/infectious diseases society of America clinical practice guidelines: treatment of drug-susceptible tuberculosis. 2016. [cited 2018 Oct 8]. Available from: https://www.idsociety.org/ practice-guideline/treatment-of-drug-susceptible-tb/

58. Africa, D.o.H.o.S. Standard Treatment Guidelines at Hospital Level for Adults. 2015.

59. Excellence, N.I.o.C. Tuberculosis guideline [NG33]. 2016 [cited 2018 Oct 8]. Available from: https://www.nice.org.uk/guidance/ng33/ chapter/Recommendations\#active-tb

60. WHO. Treatment of tuberculsis guidelines. Fourth ed. Geneva: World Health Organisation; 2010.

61. Jullien S, Ryan H, Modi M, et al. Six months therapy for tuberculous meningitis. Cochrane Database Syst Rev. 2016;9:CD012091.

62. Wager $\Pi$, Hou $X$, Verhoest PR, et al. Moving beyond rules: the development of a central nervous system multiparameter optimization (CNS MPO) approach to enable alignment of druglike properties. ACS Chem Neurosci. 2010;1(6):435-449.

63. Deeken JF, Loscher W. The blood-brain barrier and cancer: transporters, treatment, and Trojan horses. Clin Cancer Res. 2007;13 (6):1663-1674.

64. Mustafa S, Pai RS, Singh G, et al. Nanocarrier-based interventions for the management of MDR/XDR-TB. J Drug Target. 2015;23 (4):287-304.

65. Te Brake LHM, de Knegt GJ, de Steenwinkel JE, et al. The role of efflux pumps in tuberculosis treatment and their promise as a target in drug development: unraveling the black box. Annu Rev Pharmacol Toxicol. 2018;58:271-291.

66. Kimerling $M E$, Phillips $P$, Patterson $P$, et al. Low serum antimycobacterial drug levels in non-HIV-infected tuberculosis patients. Chest. 1998;113(5):1178-1183.

67. Mehta JB, Shantaveerapa H, Byrd RP, et al. Utility of rifampin blood levels in the treatment and follow-up of active pulmonary tuberculosis in patients who were slow to respond to routine directly observed therapy. Chest. 2001;120(5):1520-1524.

68. Weiner M, Burman W, Vernon A, et al. Low isoniazid concentrations and outcome of tuberculosis treatment with once-weekly isoniazid and rifapentine. Am J Respir Crit Care Med. 2003;167(10): 1341-1347.

69. Weiner M, Benator D, Burman W, et al. Association between acquired rifamycin resistance and the pharmacokinetics of rifabutin and isoniazid among patients with HIV and tuberculosis. Clin Infect Dis. 2005;40(10):1481-1491.

70. Chideya S, Winston CA, Peloquin CA, et al. Isoniazid, rifampin, ethambutol, and pyrazinamide pharmacokinetics and treatment outcomes among a predominantly HIV-infected cohort of adults with tuberculosis from Botswana. Clin Infect Dis. 2009;48 (12):1685-1694.

71. Pasipanodya JG, Mcllleron $H$, Burger $A$, et al. Serum drug concentrations predictive of pulmonary tuberculosis outcomes. J Infect Dis. 2013;208(9):1464-1473.

72. Svensson RJ, Svensson EM, Aarnoutse RE, et al. Greater early bactericidal activity at higher rifampicin doses revealed by modeling and clinical trial simulations. J Infect Dis. 2018;218:991-999.
73. Narita $M$, Hisada $M$, Thimmappa B, et al. Tuberculosis recurrence: multivariate analysis of serum levels of tuberculosis drugs, human immunodeficiency virus status, and other risk factors. Clin Infect Dis. 2001;32(3):515-517.

74. Chang $\mathrm{KC}$, Leung $-\mathrm{C}-\mathrm{C}$, Yew $-\mathrm{W}-\mathrm{W}$, et al. Peak plasma rifampicin level in tuberculosis patients with slow culture conversion. Eur J Clin Microbiol Infect Dis. 2008;27(6):467-472.

75. Burhan E, Ruesen $C$, Ruslami $R$, et al. Isoniazid, rifampin, and pyrazinamide plasma concentrations in relation to treatment response in indonesian pulmonary tuberculosis patients. Antimicrob Agents Chemother. 2013;57(8):3614-3619.

76. Tho DQ, Török ME, Yen NTB, et al. Influence of antituberculosis drug resistance and mycobacterium tuberculosis lineage on outcome in HIV-associated tuberculous meningitis. Antimicrob Agents Chemother. 2012;56(6):3074-3079.

77. Seddon JA, Visser DH, Bartens $M$, et al. Impact of drug resistance on clinical outcome in children with tuberculous meningitis. Pediatr Infect Dis J. 2012;31(7):711-716.

78. Thwaites GE, Lan NTN, Dung NH, et al. Effect of antituberculosis drug resistance on response to treatment and outcome in adults with tuberculous meningitis. J Infect Dis. 2005;192(1):79-88.

79. Ruslami R, Ganiem AR, Dian S, et al. Intensified regimen containing rifampicin and moxifloxacin for tuberculous meningitis: an open-label, randomised controlled phase 2 trial. Lancet Infect Dis. 2013;13(1):27-35.

.- This trial found a marked improvement in outcomes with intravenous rifampicin and lends weight to the rationale for intensified therapy for TBM.

80. Jayaram R, Gaonkar S, Kaur P, et al. Pharmacokineticspharmacodynamics of rifampin in an aerosol infection model of tuberculosis. Antimicrob Agents Chemother. 2003;47(7):2118-2124.

81. Rosenthal IM, Tasneen R, Peloquin CA, et al. Dose-ranging comparison of rifampin and rifapentine in two pathologically distinct murine models of tuberculosis. Antimicrob Agents Chemother. 2012;56(8):4331-4340.

82. de Steenwinkel JE, Aarnoutse RE, de Knegt GJ, et al. Optimization of the rifampin dosage to improve the therapeutic efficacy in tuberculosis treatment using a murine model. Am J Respir Crit Care Med. 2013;187(10):1127-1134.

83. Steingart KR, Jotblad S, Robsky $\mathrm{K}$, et al. Higher-dose rifampin for the treatment of pulmonary tuberculosis: a systematic review. Int J Tuberc Lung Dis. 2011;15(3):305-316.

84. Burman WJ, Gallicano K, Peloquin C. Comparative pharmacokinetics and pharmacodynamics of the rifamycin antibacterials. Clin Pharmacokinet. 2001;40(5):327-341.

85. Boeree MJ, Diacon AH, Dawson R, et al. A dose-ranging trial to optimize the dose of rifampin in the treatment of tuberculosis. Am J Respir Crit Care Med. 2015;191(9):1058-1065.

86. Svensson RJ, Aarnoutse RE, Diacon AH, et al. A population pharmacokinetic model incorporating saturable pharmacokinetics and autoinduction for high rifampicin doses. Clin Pharmacol Ther. 2018;103(4):674-683.

87. Boeree MJ, Heinrich N, Aarnoutse R, et al. High-dose rifampicin, moxifloxacin, and SQ109 for treating tuberculosis: a multi-arm, multi-stage randomised controlled trial. Lancet Infect Dis. 2017;17(1):39-49.

88. Svensson EM, Svensson RJ, Te Brake LHM, et al. The potential for treatment shortening with higher rifampicin doses: relating drug exposure to treatment response in patients with pulmonary tuberculosis. Clin Infect Dis. 2018;67(1):34-41.

89. Solera J, Rodríguez-Zapata M, Geijo P, et al. Doxycycline-rifampin versus doxycycline-streptomycin in treatment of human brucellosis due to brucella melitensis. The GECMEI group. grupo de estudio de castilla-la mancha de enfermedades infecciosas. Antimicrob Agents Chemother. 1995;39(9):2061-2067.

90. Kochar DK, Aseri S, Sharma BV, et al. The role of rifampicin in the management of cutaneous leishmaniasis. Qjm. 2000;93(11):733-737.

91. Kissling M, Bergamini N. Rifampicin in free combination with other antimicrobial drugs in non-Tb infections. Clinical data on 650 patients (a review). Chemotherapy. 1981;27(5):368-402. 
92. Alvarez-Uria G, Midde $M$, Pakam R, et al. Initial antituberculous regimen with better drug penetration into cerebrospinal fluid reduces mortality in HIV infected patients with tuberculous meningitis: data from an HIV observational cohort study. Tuberc Res Treat. 2013;2013:242604.

93. Te Brake L, Dian S, Ganiem AR, et al. Pharmacokinetic/pharmacodynamic analysis of an intensified regimen containing rifampicin and moxifloxacin for tuberculous meningitis. Int J Antimicrob Agents. 2015;45(5):496-503.

94. Yunivita V, Dian S, Ganiem AR, et al. Pharmacokinetics and safety/ tolerability of higher oral and intravenous doses of rifampicin in adult tuberculous meningitis patients. Int J Antimicrob Agents. 2016;48(4):415-421.

95. Bemer-Melchior P, Bryskier A, Drugeon HB. Comparison of the in vitro activities of rifapentine and rifampicin against mycobacterium tuberculosis complex. J Antimicrob Chemother. 2000;46 (4):571-576.

96. Rastogi N, Labrousse V, Goh KS. In vitro activities of fourteen antimicrobial agents against drug susceptible and resistant clinical isolates of mycobacterium tuberculosis and comparative intracellular activities against the virulent $\mathrm{H} 37 \mathrm{Rv}$ strain in human macrophages. Curr Microbiol. 1996;33(3):167-175.

97. Dian S, Yunivita. V, Ganiem AR, et al. High dose rifampicin for the treatment of TB meningitis: a dose finding study. 10th International Workshop on Clinical Pharmacology of TB drugs 2017 Oct 16; Atlanta, USA.

- This study of high dose oral rifampicin also noted an improvement in outcomes, and is the second Indonesian study that supports further investigation of high dose rifampicin in a phase III trial

98. Heemskerk AD, Bang ND, Mai NTH, et al. Intensified antituberculosis therapy in adults with tuberculous meningitis. N Engl J Med. 2016;374(2):124-134.

- This is the largest TBM study ever undertaken and was expertly conducted. The intervention reduced mortality in those in isoniazid monoresistant TBM only.

99. van Crevel R, Ruslami R, Aarnoutse R. Therapy for tuberculous meningitis. N Engl J Med. 2016;374(22):2187.

100. Bi W, Zhu L, Wang C, et al. Rifampicin inhibits microglial inflammation and improves neuron survival against inflammation. Brain Res. 2011;1395:12-20.

101. Schmidt H, Zysk G, Reinert RR, et al. Rifabutin for experimental pneumococcal meningitis. Chemotherapy. 1997;43(4):264-271.

102. Isoniazid. Tuberculosis (Edinb). 2008;88(2):112-116.

103. Donald PR, Gent WL, Seifart HI, et al. Cerebrospinal fluid isoniazid concentrations in children with tuberculous meningitis: the influence of dosage and acetylation status. Pediatrics. 1992;89 (2):247-250.

104. Ellard GA, Humphries MJ, Allen BW. Cerebrospinal fluid drug concentrations and the treatment of tuberculous meningitis. Am Rev Respir Dis. 1993;148(3):650-655.

105. Phuapradit P, Supmonchai K, Kaojarern S, et al. The blood/cerebrospinal fluid partitioning of pyrazinamide: a study during the course of treatment of tuberculous meningitis. J Neurol Neurosurg Psychiatry. 1990;53(1):81-82.

106. Yunivita V, R R, Dian S, et al. Pharmacokinetics of isoniazid and the effect of acetylator status in Indonesian tuberculous meningitis patients. in 10th International Workshop on Clinical Pharmacology of TB drugs. 2017 Oct 16; Atlanta, USA.

107. Donald PR. Cerebrospinal fluid concentrations of antituberculosis agents in adults and children. Tuberculosis (Edinb). 2010;90(5):279-292.

.. This review paper gives a comprehensive and expert insight into all the TB drugs and their CNS penetration.

108. Botha FJ, Sirgel FA, Parkin DP, et al. Early bactericidal activity of ethambutol, pyrazinamide and the fixed combination of isoniazid, rifampicin and pyrazinamide (Rifater) in patients with pulmonary tuberculosis. S Afr Med J. 1996;86(2):155-158.

109. Jindani A, Aber VR, Edwards EA, et al. The early bactericidal activity of drugs in patients with pulmonary tuberculosis. Am Rev Respir Dis. 1980;121(6):939-949.
110. Jacobs RF, Sunakorn $P$, Chotpitayasunonah $T$, et al. Intensive short course chemotherapy for tuberculous meningitis. Pediatr Infect Dis J. 1992;11(3):194-198.

111. Torok ME, Aljayyoussi G, Waterhouse $D$, et al. Suboptimal exposure to anti-TB drugs in a TBM/HIV+ population is not related to antiretroviral therapy. Clin Pharmacol Ther. 2018;103(3):449-457.

112. Pilheu JA, Maglio F, Cetrangolo R, et al. Concentrations of ethambutol in the cerebrospinal fluid after oral administration. Tubercle. 1971;52(2):117-122.

113. Gundert-Remy U, Klett M, Weber E. Concentration of ethambutol in cerebrospinal fluid in man as a function of the non-protein-bound drug fraction in serum. Eur J Clin Pharmacol. 1973;6(2):133-136.

114. Heemskerk AD, Bang ND, Thwaites GE. Therapy for tuberculous meningitis. N Engl J Med. 2016;374(22):2188-2189.

115. Donald PR. The chemotherapy of tuberculous meningitis in children and adults. Tuberculosis (Edinb). 2010;90(6):375-392.

116. Alffenaar JW, van Altena R, Bökkerink HJ, et al. Pharmacokinetics of moxifloxacin in cerebrospinal fluid and plasma in patients with tuberculous meningitis. Clin Infect Dis. 2009;49(7):1080-1082.

117. Thwaites GE, Bhavnani SM, Chau TTH, et al. Randomized pharmacokinetic and pharmacodynamic comparison of fluoroquinolones for tuberculous meningitis. Antimicrob Agents Chemother. 2011;55 (7):3244-3253.

118. Nijland HM, Ruslami R, Suroto AJ, et al. Rifampicin reduces plasma concentrations of moxifloxacin in patients with tuberculosis. Clin Infect Dis. 2007;45(8):1001-1007.

119. Rizvi I, Malhotra HS, Garg RK, et al. Fluoroquinolones in the management of tuberculous meningitis: systematic review and meta-analysis. J Infect. 2018;77:261-275.

120. Ruslami R, Ganiem AR, Aarnoutse RE, et al. Rifampicin and moxifloxacin for tuberculous meningitis-authors' reply. Lancet Infect Dis. 2013;13(7):570.

121. Cairns H, Duthie ES. Intrathecal streptomycin in meningitis; clinical trial in tuberculous, coliform, and other infections. Lancet. 1946;2 (6414):153-155.

122. Freiman I, Geefhuysen J. Evaluation of intrathecal therapy with streptomycin and hydrocortisone in tuberculous meningitis. J Pediatr. 1970;76(6):895-901.

123. van Toorn R, Schaaf HS, Laubscher JA, et al. Short intensified treatment in children with drug-susceptible tuberculous meningitis. Pediatr Infect Dis J. 2014;33(3):248-252.

124. Donald PR, Schoeman JF, Van Zyl LE, et al. Intensive short course chemotherapy in the management of tuberculous meningitis. Int J Tuberc Lung Dis. 1998;2(9):704-711.

125. Holdiness MR. Clinical pharmacokinetics of clofazimine. A review. Clin Pharmacokinet. 1989;16(2):74-85.

126. Baijnath S, Naiker S, Shobo A, et al. Evidence for the presence of clofazimine and its distribution in the healthy mouse brain. J Mol Histol. 2015;46(4-5):439-442.

127. Hong L, Jiang W, Pan $\mathrm{H}$, et al. Brain regional pharmacokinetics of p-aminosalicylic acid and its $\mathrm{N}$-acetylated metabolite: effectiveness in chelating brain manganese. Drug Metab Dispos. 2011;39 (10):1904-1909.

128. Agyeman AA, Ofori-Asenso R. Efficacy and safety profile of linezolid in the treatment of multidrug-resistant (MDR) and extensively drug-resistant (XDR) tuberculosis: a systematic review and meta-analysis. Ann Clin Microbiol Antimicrob. 2016;15(1):41.

129. Zhang X, Falagas ME, Vardakas KZ, et al. Systematic review and meta-analysis of the efficacy and safety of therapy with linezolid containing regimens in the treatment of multidrug-resistant and extensively drug-resistant tuberculosis. J Thorac Dis. 2015;7(4):603-615.

130. Alffenaar J-WC, van Altena R, Harmelink IM, et al. Comparison of the pharmacokinetics of two dosage regimens of linezolid in multidrug-resistant and extensively drug-resistant tuberculosis patients. Clin Pharmacokinet. 2010;49(8):559-565.

131. TB alliance. Nix-TB. 2014. [cited 2018 Aug 1]. Available from: https:// www.tballiance.org/portfolio/trial/5089

132. Conradie, F., et al. The Nix-TB Trial Of Pretomanid, Bedaquiline And Linezolid To Treat Xdr-TB. Presented at Conference on Retroviruses 
and Opportunistic Infections 2017; Seattle, Washington, the United States

133. Tsona A, Metallidis S, Foroglou N, et al. Linezolid penetration into cerebrospinal fluid and brain tissue. J Chemother. 2010;22(1):17-19.

134. Luque S, Grau S, Alvarez-Lerma F, et al. Plasma and cerebrospinal fluid concentrations of linezolid in neurosurgical critically ill patients with proven or suspected central nervous system infections. Int J Antimicrob Agents. 2014;44(5):409-415.

135. Beer R, Engelhardt KW, Pfausler B, et al. Pharmacokinetics of intravenous linezolid in cerebrospinal fluid and plasma in neurointensive care patients with staphylococcal ventriculitis associated with external ventricular drains. Antimicrob Agents Chemother. 2007;51 (1):379-382.

136. Ntziora F, Falagas ME. Linezolid for the treatment of patients with central nervous system infection. Ann Pharmacother. 2007;41 (2):296-308.

137. Li H, Lu J, Liu J, et al. Linezolid is associated with improved early outcomes of childhood tuberculous meningitis. Pediatr Infect Dis J. 2016;35(6):607-610.

138. Sun F, Ruan $Q$, Wang J, et al. Linezolid manifests a rapid and dramatic therapeutic effect for patients with life-threatening tuberculous meningitis. Antimicrob Agents Chemother. 2014;58 (10):6297-6301.

139. Akkerman OW, Odish OFF, Bolhuis MS, et al. Pharmacokinetics of bedaquiline in cerebrospinal fluid and serum in multidrug-resistant tuberculous meningitis. Clin Infect Dis. 2016;62(4):523-524.

140. Shibata M, Shimokawa Y, Sasahara K, et al. Absorption, distribution and excretion of the anti-tuberculosis drug delamanid in rats: extensive tissue distribution suggests potential therapeutic value for extrapulmonary tuberculosis. Biopharm Drug Dispos. 2017;38 (4):301-312.

141. Shobo A, Bratkowska D, Baijnath S, et al. Tissue distribution of pretomanid in rat brain via mass spectrometry imaging. Xenobiotica. 2016;46(3):247-252.

142. Drugs, T.W.G.f.N.T. [cited 2018 Aug 1]. Available from: https://www. newtbdrugs.org/

143. Thwaites GE, Duc Bang N, Huy Dung N, et al. The influence of HIV infection on clinical presentation, response to treatment, and outcome in adults with tuberculous meningitis. J Infect Dis. 2005;192 (12):2134-2141.

144. Brancusi F, Farrar J, Heemskerk D. Tuberculous meningitis in adults: a review of a decade of developments focusing on prognostic factors for outcome. Future Microbiol. 2012;7(9):1101-1116.

145. Choudhri SH, Hawken M, Gathua S, et al. Pharmacokinetics of antimycobacterial drugs in patients with tuberculosis, AIDS, and diarrhea. Clin Infect Dis. 1997;25(1):104-111.

146. Gurumurthy P, Ramachandran G, Hemanth Kumar AK, et al. Malabsorption of rifampin and isoniazid in HIV-infected patients with and without tuberculosis. Clin Infect Dis. 2004;38(2):280-283.

147. Daskapan A, Idrus LR, Postma MJ, et al. A systematic review on the effect of HIV infection on the pharmacokinetics of first-line tuberculosis drugs. Clin Pharmacokinet. 2018.

148. Mcllleron $\mathrm{H}$, Rustomjee $\mathrm{R}$, Vahedi $\mathrm{M}$, et al. Reduced antituberculosis drug concentrations in HIV-infected patients who are men or have low weight: implications for international dosing guidelines. Antimicrob Agents Chemother. 2012;56(6):3232-3238.

149. Court R, Chirehwa MT, Wiesner L, et al. Quality assurance of rifampicin-containing fixed-drug combinations in South Africa: dosing implications. Int J Tuberc Lung Dis. 2018;22(5):537-543.

150. Stott KE, Pertinez H, Sturkenboom MGG, et al. Pharmacokinetics of rifampicin in adult TB patients and healthy volunteers: a systematic review and meta-analysis. J Antimicrob Chemother. 2018;2305-2313.

151. Cresswell FV, Bangdiwala AS, Meya DB, et al. Absence of cerebrospinal fluid pleocytosis in tuberculous meningitis is a common occurrence in HIV co-infection and a predictor of poor outcomes. Int J Infect Dis. 2018;68:77-78.

152. Cecchini D, Ambrosioni J, Brezzo $C$, et al. Tuberculous meningitis in HIV-infected patients: drug susceptibility and clinical outcome. Aids. 2007;21(3):373-374.
153. Thwaites GE, Chau TTH, Caws $M$, et al. Isoniazid resistance, mycobacterial genotype and outcome in Vietnamese adults with tuberculous meningitis. Int J Tuberc Lung Dis. 2002;6(10):865-871.

154. Katrak SM, Shembalkar PK, Bijwe SR, et al. The clinical, radiological and pathological profile of tuberculous meningitis in patients with and without human immunodeficiency virus infection. J Neurol Sci. 2000;181(1-2):118-126.

155. Nau $R$, Sorgel $F$, Eiffert $H$. Penetration of drugs through the blood-cerebrospinal fluid/blood-brain barrier for treatment of central nervous system infections. Clin Microbiol Rev. 2010;23 (4):858-883.

156. Marais S, Meintjes G, Pepper DJ, et al. Frequency, severity, and prediction of tuberculous meningitis immune reconstitution inflammatory syndrome. Clin Infect Dis. 2013;56(3):450-460.

157. Marais S, Lai RPJ, Wilkinson KA, et al. inflammasome activation underlying central nervous system deterioration in HIV-associated tuberculosis. J Infect Dis. 2017;215(5):677-686.

158. Meintjes G, Lynen L. Prevention and treatment of the immune reconstitution inflammatory syndrome. Curr Opin HIV AIDS. 2008;3(4):468-476.

159. Mcllleron H, Meintjes G, Burman WJ, et al. Complications of antiretroviral therapy in patients with tuberculosis: drug interactions, toxicity, and immune reconstitution inflammatory syndrome. J Infect Dis. 2007;196(Suppl 1):S63-75.

160. AIDSinfo, Guidelines for the prevention and treatment of opportunistic infections among HIV-exposed and HIV-infected children. 2013. [cited 2018 Aug 1]. Available from: http://aidsinfo.nih.gov/ guidelines/

161. Herman RJ, Nakamura K, Wilkinson GR, et al. Induction of propranolol metabolism by rifampicin. Br J Clin Pharmacol. 1983;16 (5):565-569.

162. Ohnhaus EE, Brockmeyer N, Dylewicz $P$, et al. The effect of antipyrine and rifampin on the metabolism of diazepam. Clin Pharmacol Ther. 1987;42(2):148-156.

163. O'Reilly RA. Interaction of sodium warfarin and rifampin. Studies in man. Ann Intern Med. 1974;81(3):337-340.

164. Davis A, Meintjes G, Wilkinson RJ. Treatment of tuberculous meningitis and its complications in adults. Curr Treat Options Neurol. 2018;20(3):5.

165. Allana S, Shashkina E, Mathema B, et al. pncA gene mutations associated with pyrazinamide resistance in drug-resistant tuberculosis, South Africa and Georgia. Emerg Infect Dis. 2017;23(3):491-495.

166. Wait JW, Schoeman JF. Behaviour profiles after tuberculous meningitis. J Trop Pediatr. 2010;56(3):166-171.

167. Schoeman CJ, Herbst I, Nienkemper DC. The effect of tuberculous meningitis on the cognitive and motor development of children. S Afr Med J. 1997;87(1):70-72.

168. Shah I, Meshram L. High dose versus low dose steroids in children with tuberculous meningitis. J Clin Neurosci. 2014;21(5):761-764.

169. Schoeman JF, Janse van Rensburg A, Laubscher JA, et al. The role of aspirin in childhood tuberculous meningitis. J Child Neurol. 2011;26(8):956-962.

170. Schoeman JF, Springer P, van Rensburg AJ, et al. Adjunctive thalidomide therapy for childhood tuberculous meningitis: results of a randomized study. J Child Neurol. 2004;19(4):250-257.

171. Hiruy $H$, Rogers $Z$, Mbowane $C$, et al. Subtherapeutic concentrations of first-line anti-TB drugs in South African children treated according to current guidelines: the PHATISA study. J Antimicrob Chemother. 2015;70(4):1115-1123.

172. Mukherjee A, Velpandian T, Singla $M$, et al. Pharmacokinetics of isoniazid, rifampicin, pyrazinamide and ethambutol in HIV-infected Indian children. Int J Tuberc Lung Dis. 2016;20(5):666-672.

173. Mlotha R, Waterhouse D, Dzinjalamala F, et al. Pharmacokinetics of anti-TB drugs in Malawian children: reconsidering the role of ethambutol. J Antimicrob Chemother. 2015;70(6):1798-1803.

174. Savic RM, Ruslami R, Hibma JE, et al. Pediatric tuberculous meningitis: model-based approach to determining optimal doses of the anti-tuberculosis drugs rifampin and levofloxacin for children. Clin Pharmacol Ther. 2015;98(6):622-629. 
175. University $\mathrm{JH}$. Optimizing treatment to improve outcomes in children (TBM-KIDS). In: Clinical trials.gov. [cited 2018 Aug 1]. Available from: www.clinicaltrials.gov

176. Figaji AA, Fieggen AG. The neurosurgical and acute care management of tuberculous meningitis: evidence and current practice. Tuberculosis (Edinb). 2010;90(6):393-400.

177. Marais S, Thwaites G, Schoeman JF, et al. Tuberculous meningitis: a uniform case definition for use in clinical research. Lancet Infect Dis. 2010;10(11):803-812.

178. Marais BJ, Heemskerk AD, Marais SS, et al. Standardized methods for enhanced quality and comparability of tuberculous meningitis studies. Clin Infect Dis. 2017;64(4):501-509.

179. Te Brake LH, Russel FGM, van Den Heuvel JJMW, et al. Inhibitory potential of tuberculosis drugs on ATP-binding cassette drug transporters. Tuberculosis (Edinb). 2016;96:150-157.

180. van Laarhoven A, Dian S, Aguirre-Gamboa R, et al. Cerebral tryptophan metabolism and outcome of tuberculous meningitis: an observational cohort study. Lancet Infect Dis. 2018;18(5):526-535.

181. Mai NTH, Dobbs N, Phu NH, et al. A randomised double blind placebo controlled phase 2 trial of adjunctive aspirin for tuberculous meningitis in HIV-uninfected adults. eLife. 2018;7:e33478.

182. van Laarhoven A, Dian S, Ruesen C, et al. Clinical parameters, routine inflammatory markers, and LTA4H genotype as predictors of mortality among 608 patients with tuberculous meningitis in Indonesia. J Infect Dis. 2017;215(7):1029-1039.

183. Thao LTP, Heemskerk AD, Geskus RB, et al. Prognostic models for 9-month mortality in tuberculous meningitis. Clin Infect Dis. 2018;66(4):523-532.

184. Gupta-Wright A, Corbett EL, van Oosterhout JJ, et al. Rapid urine-based screening for tuberculosis in HIV-positive patients admitted to hospital in Africa (STAMP): a pragmatic, multicentre, parallel-group, double-blind, randomised controlled trial. Lancet. 2018;392(10144):292-301.

185. Pepper DJ, Marais S, Maartens G, et al. Neurologic manifestations of paradoxical tuberculosis-associated immune reconstitution inflammatory syndrome: a case series. Clin Infect Dis. 2009;48(11):e96107.

186. Coash M, Forouhar F, Wu CH, et al. Granulomatous liver diseases: a review. J Formos Med Assoc. 2012;111(1):3-13.

187. Prasad $K$, Singh MB, Ryan $H$. Corticosteroids for managing tuberculous meningitis. Cochrane Database Syst Rev. 2016;4: Cd002244.

188. Chabria S, Barakat L, Ogbuagu O. Steroid-exacerbated HIVassociated cutaneous Kaposi's sarcoma immune reconstitution inflammatory syndrome: 'Where a good intention turns bad'. Int J STD AIDS. 2016;27(11):1026-1029.

189. Beardsley J, Wolbers M, Kibengo FM, et al. Adjunctive dexamethasone in HIV-associated cryptococcal meningitis. N Engl J Med. 2016;374(6):542-554.

190. Donovan J, Phu NH, Mai NTH, et al. Adjunctive dexamethasone for the treatment of HIV-infected adults with tuberculous meningitis (ACT HIV): study protocol for a randomised controlled trial [version 1; referees: 1 approved, 1 approved with reservations]. Wellcome Open Res. 2018;3(31).

191. Vinnard C, Ravimohan S, Tamuhla N, et al. Pyrazinamide clearance is impaired among HIV/tuberculosis patients with high levels of systemic immune activation. PLoS One. 2017;12(11): e0187624.

192. Thuong NTT, Heemskerk D, Tram TTB, et al. Leukotriene A4 hydrolase genotype and HIV infection influence intracerebral inflammation and survival from tuberculous meningitis. J Infect Dis. 2017;215(7):1020-1028. 


\section{Chapter Eight: High Dose Intravenous and Oral Rifampicin in the Treatment of Tuberculous Meningitis: A Phase II Open Label Randomised Clinical Trial (research paper)}

\section{Foreword}

Rifampicin is the cornerstone drug in TBM treatment, as evidenced by the fact that meningitis patients with rifampicin-resistant $M$. tuberculosis strains have a near-universal fatal outcome, even with second-line drugs in resource-rich settings. ${ }^{1-3}$ Mortality is much lower in patients with isoniazid monoresistant TBM. ${ }^{4}$

As described in the introduction, there is a growing body of evidence that higher dose rifampicin is safe, improves blood and CSF rifampicin exposures and survival in predominantly HIV-negative Indonesians with TBM. ${ }^{5,6}$ This phase II clinical trial seeks to fill the knowledge gap that exists relating to: 1) the safety of highdose rifampicin in advanced HIV disease and 2) PK parameters from advanced HIV disease, and specifically rifampicin CSF PK data of which there is none from Africa.

This chapter tests the following hypotheses:

- Hypothesis \#3: Intensified treatment of TB meningitis with intravenous rifampicin $(20 \mathrm{mg} / \mathrm{kg}$ ) or high dose oral rifampicin $(35 \mathrm{mg} / \mathrm{kg}$ ) will result in significantly increased blood and CSF exposure during the critical early days of TB treatment as compared to common control (10mg/kg oral rifampicin)

- Hypothesis \#3: High dose rifampicin will be safe in people with advanced HIV disease and may improve functional outcomes and survival at 8- and 24-weeks.

The full clinical trial protocol has been summarised in a protocol paper, which has been published on Wellcome Open Research and is included in Appendix 5. The safety data collected during the 8-week intervention period are presented as a primary endpoint, and the individual details of all the adverse events during the 24-week follow up period are presented in the supplementary material.

This phase II clinical trial provided reassuring data on the safety of high-dose rifampicin in Ugandans with advanced HIV-related immunosuppression. The PK data also revealed how sub-optimal the CSF rifampicin concentrations are with standard TB treatment, with around half of enrolees having undetectable CSF levels. A two-fold increase in rifampicin dose administered intravenously and a 3.5-fold increase in oral dose had a marked impact on both serum and CSF rifampicin exposures, with almost all enrolees having CSF concentrations above the rifampicin MIC (1 mg/L). Given that oral rifampicin $35 \mathrm{mg} / \mathrm{kg}$ achieved good CSF concentrations with no excess toxicity and is a less complex intervention than intravenous rifampicin, it would be the most appropriate option to examine in a phase III clinical trial. 


\section{Bibliography}

1. Tho DQ, Torok ME, Yen NT, et al. Influence of antituberculosis drug resistance and Mycobacterium tuberculosis lineage on outcome in HIV-associated tuberculous meningitis. Antimicrob Agents Chemother 2012; 56(6): 3074-9.

2. Marais S, Pepper DJ, Schutz C, Wilkinson RJ, Meintjes G. Presentation and outcome of tuberculous meningitis in a high HIV prevalence setting. PLoS One 2011; 6(5): e20077.

3. Thwaites GE, Lan NT, Dung NH, et al. Effect of antituberculosis drug resistance on response to treatment and outcome in adults with tuberculous meningitis. J Infect Dis 2005; 192(1): 79-88.

4. Thwaites GE, Chau TT, Caws M, et al. Isoniazid resistance, mycobacterial genotype and outcome in Vietnamese adults with tuberculous meningitis. Int J Tuberc Lung Dis 2002; 6(10): 865-71.

5. Ruslami R, Ganiem AR, Dian S, et al. Intensified regimen containing rifampicin and moxifloxacin for tuberculous meningitis: an open-label, randomised controlled phase 2 trial. Lancet Infect Dis 2013; 13(1): 27-35.

6. Dian S, Yunivita V, Ganiem AR, et al. Double-Blind, Randomized, Placebo-Controlled Phase II DoseFinding Study To Evaluate High-Dose Rifampin for Tuberculous Meningitis. Antimicrob Agents Chemother 2018; 62(12). 


\section{RESEARCH PAPER COVER SHEET}

\section{PLEASE NOTE THAT A COVER SHEET MUST BE COMPLETED FOR EACH RESEARCH PAPER INCLUDED IN A THESIS.}

\section{SECTION A - Student Details}

\begin{tabular}{|l|l|}
\hline Student & Dr Fiona Cresswell \\
\hline Principal Supervisor & Prof Alison Elliott \\
\hline Thesis Title & $\begin{array}{l}\text { Improving diagnosis and treatment of HIV-associated } \\
\text { Tuberculous meningitis }\end{array}$ \\
\hline
\end{tabular}

If the Research Paper has previously been published please complete Section B, if not please move to Section C

\section{SECTION B - Paper already published}

\begin{tabular}{|l|l|l|}
\hline Where was the work published? & \multicolumn{2}{|l|}{} \\
\hline When was the work published? & \multicolumn{2}{|l|}{} \\
\hline $\begin{array}{l}\text { If the work was published prior to } \\
\text { registration for your research degree, } \\
\text { give a brief rationale for its inclusion }\end{array}$ & \multicolumn{3}{|l|}{} \\
\hline $\begin{array}{l}\text { Have you retained the copyright for the } \\
\text { work?* }\end{array}$ & & $\begin{array}{l}\text { Was the work subject to } \\
\text { academic peer review? }\end{array}$ \\
\hline
\end{tabular}

*If yes, please attach evidence of retention. If no, or if the work is being included in its published format, please attach evidence of permission from the copyright holder (publisher or other author) to include this work.

\section{SECTION C - Prepared for publication, but not yet published}

\begin{tabular}{|l|l|}
\hline $\begin{array}{l}\text { Where is the work intended to be } \\
\text { published? }\end{array}$ & $\begin{array}{l}\text { Submitted to Lancet Infectious Diseases on 31st October 2020 } \\
\text { Fiona V Cresswell, David B Meya, Enock Kagimu, Daniel Grint, Lindsey te Brake, John }\end{array}$ \\
\hline $\begin{array}{l}\text { Please list the paper's authors in } \\
\text { the intended authorship order: }\end{array}$ & $\begin{array}{l}\text { Kasibante, Emily Martyn, Morris Rutakingirwa, Carson M Quinn, Micheal Okirwoth, Lillian } \\
\text { Tugume, Kenneth Ssembambulidde, Abdu K Musubire, Ananta S Bangdiwala, Allan Buzibye, } \\
\text { Conrad Muzoora, Elin M Svensson, Rob Aarnoutse, David R Boulware, Alison M Elliott }\end{array}$ \\
\hline Stage of publication & Choose an item. Under review \\
\hline
\end{tabular}

\section{SECTION D - Multi-authored work}

For multi-authored work, give full details of your role in the research included in the paper and in the preparation of the paper. (Attach a further sheet if necessary)
I wrote the full protocol for the clinical trial, handled the regulatory and the day to day operational aspects of the trial. I was involved in coordinating the validation of the rifampicin assay. I did the data cleaning and analysis. I wrote the manuscript, handled comments from co-authors and the submission process 
Student Signature:

Supervisor Signature:
Date: 31 st October 2020

Date: 8th November 2020 


\section{High-dose oral and intravenous rifampicin for the treatment of tuberculous meningitis in predominantly HIV-positive Ugandan adults: a phase II open-label randomised controlled trial}

\section{Authors:}

Fiona V Cresswell MBChB ${ }^{1,2,3}$, David B Meya $\mathrm{PhD}^{2}$, Enock Kagimu MBChB2 , Daniel Grint PhD ${ }^{4}$, Lindsey te Brake $\mathrm{PhD}^{5}$, John Kasibante $\mathrm{MBChB}^{2}$, Emily Martyn $\mathrm{MBBS}^{1}$, Morris Rutakingirwa $\mathrm{MBCh}^{2}$, Carson M Quinn BA ${ }^{6}$, Micheal Okirwoth BSc$^{2}$, Lillian Tugume $\mathrm{MBChB}^{2}$, Kenneth Ssembambulidde MBChB ${ }^{2}$, Abdu K Musubire MD², Ananta S Bangdiwala MS , Allan Buzibye MSc², Conrad Muzoora MD², Elin M Svensson $\mathrm{PhD}^{5,9}$, Rob Aarnoutse PharmD PhD ${ }^{5}$, Alison M Elliott MD*1,3, David R Boulware MD*10

\section{Institutions:}

1. Clinical Research Department, London School of Hygiene and Tropical Medicine, Keppel Street, London, UK

2. Infectious Diseases Institute, Makerere University, Kampala, P.O. Box 22418, Uganda

3. Medical Research Council - Uganda Virus Research Institute - LSHTM Uganda Research Unit, Entebbe, Uganda

4. Tropical Epidemiology Group, London School of Hygiene and Tropical Medicine, Keppel Street, London, UK

5. Department of Pharmacy, Radboud Institute for Health Sciences, Radboud University Medical Centre, Netherlands

6. University of California, San Francisco, California, USA

7. Division of Biostatistics, University of Minnesota, Minneapolis, MN, USA

8. Mbarara University of Science and Technology, Mbarara, Uganda

9. Department of Pharmacy, Uppsala University, Sweden

10. Division of Infectious Diseases and International Medicine, University of Minnesota, Minneapolis, MN, USA

Corresponding author: Dr Fiona Cresswell, Clinical Research Department, London School of Hygiene and Tropical Medicine, Keppel Street, London, WC1E 7HT, UK. Fiona.cresswell@Ishtm.ac.uk

Key words: tuberculous meningitis, TB meningitis, rifampicin, intensified therapy, HIV, TBM

*Joint senior author 


\section{Abstract}

Background: High dose rifampicin may improve outcomes of tuberculous meningitis (TBM). Little safety or pharmacokinetic (PK) data exist on high-dose rifampicin in HIV co-infection, and no cerebrospinal fluid (CSF) PK data exist from Africa. We hypothesized that high-dose rifampicin would increase serum and CSF concentrations without excess toxicity.

Methods: In this phase II open-label trial, Ugandan adults with suspected TBM were randomised to standard-of-care control (PO-10, rifampicin 10mg/kg/day), intravenous rifampicin (IV-20, $20 \mathrm{mg} / \mathrm{kg} /$ day), or high-dose oral rifampicin (PO-35, 35mg/kg/day). We performed serum and CSF PK sampling on day 2 and 14 . The primary outcomes were total exposure $\left(A \cup C_{0-24}\right)$, maximum concentration $\left(C_{\max }\right)$, CSF concentration $\left(\mathrm{C}_{\mathrm{CSF}}\right)$ and grade 3-5 adverse events.

Findings: We enrolled 61 adults, 92\% were HIV-positive, median CD4 count was 50cells/ $\mu \mathrm{L}$ (IQR 46 56). On day 2, geometric mean plasma $A \cup C_{0-24 h r}$ was $42.9 \mathrm{~h} \cdot \mathrm{mg} / \mathrm{L}$ with standard-of-care $10 \mathrm{mg} / \mathrm{kg}$ dosing, 249h.mg/L for IV-20 and 327h.mg/L for PO-35 (P<0.001). In CSF, standard-of-care achieved geometric mean $\mathrm{AUC}_{0-24 \mathrm{hr}} 0.27 \mathrm{mg} / \mathrm{L}$ compared with $1.74 \mathrm{mg} / \mathrm{L}(95 \% \mathrm{Cl} 1.2-2.5)$ for IV-20 and $2.17 \mathrm{mg} / \mathrm{L}(1.6-2.9)$ for PO-35 regimens ( $p<0.001)$. Achieving CSF concentrations above rifampicin minimal inhibitory concentration occurred in 11\% (2/18) of standard-of-care, 93\% (14/15) of IV-20, and 95\% (18/19) of PO-35 participants. Higher serum and CSF levels were sustained at day 14. Adverse events did not differ by dose $(p=0.34)$

Interpretation: High-dose intravenous or oral rifampicin was safe, resulted in 6-8-fold higher exposures than standard of care, and therapeutic CSF levels. Current international guidelines result in sub-therapeutic CSF rifampicin concentration for $89 \%$ of Ugandan TBM patients.

Funding: Wellcome Clinical PhD Fellowship (210772/Z/18/Z) 


\section{Research in Context}

Evidence before this study: We searched PubMed Central for reports on high dose rifampicin in antituberculosis therapies including the following search terms: "high dose" OR "intensified" AND "rifampicin" OR "antitubercul*" AND "tubercul*" OR "mening*" OR "TB", on 2 ${ }^{\text {nd }}$ September 2020.

Rifampicin was first identified as a key antituberculous agent in 1972 and at this time the minimum effective dose $(600 \mathrm{mg}$ ) was used as rifampicin was relatively expensive. Since then, limited advances have been made in the treatment of drug-sensitive TB and there is a need to dose-optimise existing agents. This has promoted rifampicin dose-optimisation studies by the PanACEA consortium who have recently concluded that $40 \mathrm{mg} / \mathrm{kg}$ rifampicin is the maximum tolerable dose in humans. This is particularly relevant for TBM as only about $5 \%$ of plasma rifampicin penetrates into the cerebrospinal fluid (CSF). Recent studies have shown that with the current dosing $(10 \mathrm{mg} / \mathrm{kg})$ rifampicin is often undetectable in the CSF of patients with TBM.

Several key clinical trials have also investigated high-dose rifampicin in TBM in a largely HIV-negative population. An Indonesian phase II trial administered high-dose intravenously (IV) ( $13 \mathrm{mg} / \mathrm{kg}$ IV) or standard dose oral rifampicin $(10 \mathrm{mg} / \mathrm{kg}$ ) with or without moxifloxacin for 2-weeks. Intravenous rifampicin led to a 3-fold increase in plasma and CSF pharmacokinetic (PK) parameters and reduced 6-month mortality (adjusted Hazard Ratio 0.42; 95\% Cl 0.20-0.91; $\mathrm{p}=0.03$ ). Two subsequent Indonesian phase II trials established the safety and PK of oral rifampicin up to a dose of $30 \mathrm{mg} / \mathrm{kg}$. A pharmacokinetic-pharmacodynamic model combining data from 148 participants in three Indonesian trials predicted that increasing the oral rifampicin dose from 10 to $30 \mathrm{mg} / \mathrm{kg}$ would result in an increase in 6-month survival from $\sim 50$ to $~ 70 \%$ and that higher doses would further improve survival.

In contrast, the phase III randomised, double-blind, placebo-controlled trial of 817 adults with TBM in Vietnam investigating oral rifampicin (15 mg/kg) and levofloxacin compared with standard treatment, found improved survival in people with isoniazid monoresistance but no effect overall. A nested pharmacokinetic-pharmacodynamic study of 237 participants within this trial did not find an exposure-response relationship within the range of rifampicin exposure achieved, though the observed exposures in the intensified arm (geometric mean $82.5 \mathrm{~h} \cdot \mathrm{mg} / \mathrm{L}$ ) were considerably lower than the total exposure predicted to improve survival in the Indonesian model (300 h.mg/L). 
The added value of this study: This is the first randomised controlled trial of high-dose rifampicin in TBM in adults in sub-Saharan Africa. We show that a 3.5-fold increase in the oral rifampicin dose results in an 8-fold increase in serum and CSF exposure with no excess toxicity. With standard dosing only $11 \%$ of participants achieve a CSF rifampicin concentration above the minimal inhibitory concentration for rifampicin (1 mg/L), compared to 95\% with rifampicin 35mg/kg and 96\% with $20 \mathrm{mg} / \mathrm{kg}$ IV. Oral administration of rifampicin $35 \mathrm{mg} / \mathrm{kg} /$ day appears to achieve similar exposures as intravenous rifampicin and would be a less complex intervention to study in phase III trials and implement programmatically.

HIV-positive individuals with advanced immunosuppression are at considerably higher risk of adverse drug reactions, 4-fold increased risk of drug induced liver injury, complications of polypharmacy and drug-drug interactions. Prior trials examining high-dose rifampicin have only included a handful of HIV-positive people with CD4 T-cell counts $>200$ cells/ $\mu$. This is the first safety and PK data for considerably higher dose rifampicin in HIV-associated TB and advanced immune suppression (median CD4 T-cell count 50 cells/ $\mu$ ).

Implications of all the available evidence: Our data, taken together with existing data from Indonesia, show a clear increase in rifampicin exposure in the blood and CSF with higher dose rifampicin whilst maintaining an acceptable toxicity profile. Our data provide reassurance that it is safe to use oral rifampicin $35 \mathrm{mg} / \mathrm{kg}$ in HIV-positive adults. The next step is to examine the impact of $35 \mathrm{mg} / \mathrm{kg}$ oral rifampicin on survival and neurological disability in an appropriately powered phase III study in adults with TBM. 


\section{Introduction}

The HIV epidemic has dramatically changed the epidemiology of meningitis in sub-Saharan Africa and M. tuberculosis is now one of the leading causes. ${ }^{1}$ Tuberculous meningitis (TBM) is fatal in 16\% (95\% confidence interval (CI), 10-24\%) of HIV-negative adults and $57 \%(95 \% \mathrm{Cl}, 48-67 \%)$ of people living with HIV (PLHIV). ${ }^{2}$ Long-term disability is seen in around a third of survivors regardless of HIV serostatus. ${ }^{2}$ The treatment of TBM has been extrapolated from trials defining short course pulmonary TB treatment in the 1970s, which used rifampicin dosed at $600 \mathrm{mg}(8-12 \mathrm{mg} / \mathrm{kg})$ daily and did not explore substantially higher doses of rifampicin (Rifampin ${ }^{\circ}$ ) due to its high cost in that era. ${ }^{3}$ Rifampicin is a key drug in TB treatment, it is highly bactericidal and has important sterilising activity, and low plasma exposures have been linked with treatment failure, relapse and evolution of resistance. ${ }^{4}$ There is a growing body of evidence in pulmonary TB suggesting that high-dose rifampicin accelerates the rate of bacillary clearance and the maximum well-tolerated oral dose is 40 $\mathrm{mg} / \mathrm{kg} /$ day. $5,6,7,8,9$ Rifampicin is highly protein-bound so only around $5 \%$ of plasma rifampicin penetrates across the blood-cerebrospinal fluid (CSF) barrier, and at the current $10 \mathrm{mg} / \mathrm{kg}$ dose the majority of TBM patients have undetectable rifampicin in their CSF, the site of disease. ${ }^{10,11}$ Inadequate central nervous system (CNS) drug penetration may be an important contributory factor to early mortality. Improved survival may be achieved by 'intensified therapy': addition of drugs with good CNS penetration, modifying drug doses and/or route of administration to maximise early mycobactericidal activity in the CNS. ${ }^{11}$

In the Vietnamese intensified TBM treatment trial, the intervention arm contained oral rifampicin 15 $\mathrm{mg} / \mathrm{kg} /$ day and levofloxacin $20 \mathrm{mg} / \mathrm{kg} /$ day compared to standard TB treatment (containing rifampicin $\sim 10 \mathrm{mg} / \mathrm{kg} /$ day). ${ }^{12}$ The intensified regimen showed no differential effect on mortality overall, though there was a survival benefit in those with isoniazid-resistant disease. ${ }^{13}$ The recently published pharmacokinetic (PK)-pharmacodynamic (PD) sub-study reported that whilst there was an approximate doubling of plasma and CSF rifampicin exposures in the intensified arm there was no detectable association between rifampicin exposure and survival within the moderate range of exposures observed. ${ }^{14}$ Conversely, data from 148 participants in three phase II Indonesian TBM trials investigating intravenous rifampicin and oral rifampicin dosed at 10, 20 and $30 \mathrm{mg} / \mathrm{kg} / \mathrm{day}$, combined in a model-based meta-analysis found a strong relationship between rifampicin exposure and survival in HIV-negative TBM patients. ${ }^{15,16,17}$ Simulations predicted an increase in 6-month survival 
from approximately $50 \%$ to $70 \%$ upon tripling the oral rifampicin dose to $\sim 30 \mathrm{mg} / \mathrm{kg}$, and that even higher doses would further improve survival. ${ }^{17}$

There is however a stark lack of clinical trial data on the safety and PK of high-dose rifampicin in HIV co-infection. PK data from Asia cannot necessarily be extrapolated to African populations due to differences in HIV serostatus, comorbidities, body size, and pharmacogenomics. PLHIV are at high risk of drug-related toxicity including a 4-fold increased risk of drug-induced liver injury, ${ }^{18}$ complications relating to polypharmacy, and drug-drug interactions. ${ }^{19}$ There is therefore an urgent need to generate data on safety and PK of higher dose rifampicin in African adults with TBM, particularly in PLHIV who experience the greatest burden of disease.

To date, the regimen with the most marked impact on survival is intravenous rifampicin at a of dose once daily $13 \mathrm{mg} / \mathrm{kg} \cdot{ }^{10}$ Intravenous rifampicin is not susceptible to first-pass metabolism, so a higher and less variable drug exposure may be achieved. ${ }^{20}$ However, intravenous rifampicin is not readily available in many TB endemic settings and co-formulation of oral TB drugs is essential to reducing pill burden. Therefore, in this first interventional trial in adults with suspected TBM in sub-Saharan Africa, we sought to test the hypothesis that in a predominantly HIV-positive population high-dose rifampicin, administered orally or intravenously, is safe and increases blood and CSF exposures and attainment of PK targets.

\section{Methods}

\section{Study population and setting}

Participants were recruited from two centres in Uganda: Kiruddu National Referral Hospital, Kampala and Mbarara Regional Referral Hospital, Mbarara; public hospitals which act as referral hospitals for the Central and Western districts respectively. A full description of the trial protocol has been published elsewhere..$^{21}$ Eligible patients were adults ( $\geq 18$ years of age) with suspected TBM, and either microbiological confirmation of meningeal TB (positive CSF AFB smear or CSF Xpert MTB/Rif Ultra) or a low CSF glucose (CSF:plasma ratio $<50 \%$ or CSF glucose $<65 \mathrm{mg} / \mathrm{dl}$ or $<3.6 \mathrm{mmol}$ ) with TBM treatment planned. Patients were ineligible if they had jaundice or known liver cirrhosis; >3 doses of TB treatment within the previous 3 days; discontinued TB treatment in the prior 14 days; known allergy to rifamycins, isoniazid, pyrazinamide, ethambutol; known current/previous rifampicin drug-resistant $M$. tuberculosis infection; concurrent cryptococcal meningitis; known to be currently 
taking any drug that has a clinically relevant interaction with rifampicin (including HIV protease inhibitors or nevirapine); could not attend follow-up visits, pregnant or breastfeeding; known porphyria; known chronic renal failure with creatinine clearance $<10 \mathrm{ml} / \mathrm{min}$; or lack of written informed consent. Participants were subsequently classified in accordance with the TBM uniform case definition. ${ }^{22}$

\section{Randomisation}

Participants were stratified at study entry by site and British Medical Research Council (MRC) disease severity grade (grade I or II/III). Participants were randomly assigned in a 1:1:1 ratio to one of three trial arms. The randomisation list was generated by a computer-generated permutated block randomization algorithm of different sized blocks and sealed envelopes labelled with the randomisation code contained the study arm assignment. Due to the urgent need to begin treatment to reduce mortality, enrolment was not delayed whilst waiting for enrolment blood test results, and participants were withdrawn and replaced a posteriori if their baseline alanine transaminase (ALT) was $>3 x$ upper limit of normal (ULN).

\section{Procedures and Laboratory Investigations}

CSF was tested at the bedside for Cryptococcal antigen (CrAg LFA, IMMY, Norman, USA), glucose (One Touch Select), and lactate (Nova Biomedical; Waltham, USA). In the local microbiology laboratory, CSF underwent Gram's stain, bacteriological culture, cell count and differential, protein estimation, and Xpert MTB/Rif Ultra (Cepheid, Sunnyvale, CA). Blood tests were sent to the Infectious Diseases Institute Core Laboratory in Kampala and the Joint Clinical Research Centre Laboratory in Mbarara, Uganda. Baseline bloods included HIV Ab/Ag test, liver function tests, renal function tests, complete blood count, and $\beta$-HCG for women. Safety bloods including liver function tests were sent on days $3,7,14,28$, and 56, approximately. For pharmacokinetic measurements, we collected blood samples on day two (+/-1) at the following time points: pre-dose and 2-, 4- and 8hours post-dose. In case of intravenous infusion, the first 2-hour sampling point occurred at the end of the infusion. We collected a single CSF sample on day 2, randomized between 2- to 8-hours post dose with collection windows randomized as $2-4,4-6$, or 6-8-hours post dose. On day 14 (+/-2), we collected a single blood and CSF sample between 2 to 8 hours post-dose for PK analysis. Pharmacokinetic samples were transferred immediately to the Kiruddu Hospital Microbiology Laboratory in Kampala or JCRC laboratory in Mbarara protected from light where they were 
centrifuged at $3000 \mathrm{~g}$ for 10 minutes. Following centrifugation, serum or CSF was aliquoted into $1 \mathrm{ml}$ cryovials and immediately cryopreserved at $-80{ }^{\circ} \mathrm{C}$ for later analysis.

\section{Study treatment}

The intervention period in this open label phase II trial was 8 weeks. Participants were randomized to one of three antituberculous therapies: 1) IV-20, high-dose intravenous (IV) rifampicin (20 $\mathrm{mg} / \mathrm{kg} /$ day) administered over 2 hours, alongside oral isoniazid (5 mg/ $\mathrm{kg}$ ), pyrazinamide $(25 \mathrm{mg} / \mathrm{kg}$ ) and ethambutol (20 mg/kg); 2) PO-35, high dose oral rifampicin (35 mg/kg/day), administered as standard fixed dose combination quadruple antituberculous tablets (RHZE) (containing 10mg/kg of rifampicin) along with additional $25 \mathrm{mg} / \mathrm{kg}$ dose given as $300 \mathrm{mg}$ oral rifampicin capsules; 3) standard of care (SOC, control arm) fixed-dose combination RHZE tablets (containing 10 mg/kg/day of rifampicin) according to WHO weight-bands. Detailed dosing tables are provided in the supplementary materials. Where participants were unable to swallow, oral medication was dispersed and given via a nasogastric tube. In the IV-20 arm, after 14 days of IV rifampicin participants were switched to high dose oral rifampicin $(35 \mathrm{mg} / \mathrm{kg})$ for the remaining 6 weeks of the intervention period, as per PO-35 arm. Study drugs were administered under directly observed therapy during hospitalisation. Adjunctive corticosteroids were given routinely as dexamethasone $0.4 \mathrm{mg} / \mathrm{kg} /$ day IV for week $1,0.3 \mathrm{mg} / \mathrm{kg} /$ day IV for week 2 and thereafter as oral prednisolone $80 \mathrm{mg} /$ day and weaned to a stop over the following 6 weeks. Corticosteroids underwent more rapid weaning or discontinuation if clinical indicated. ${ }^{21}$ All participants received a 3-day albendazole course to mitigate Strongyloides hyperinfection risk secondary to high dose steroids, and PLHIV received cotrimoxazole prophylaxis. Antiretroviral therapy (ART) naïve PLHIV, or those who had discontinued ART, initiated ART after completion of the intensive phase of TB treatment (week 8) in accordance with Ugandan guidelines. Current protease inhibitor or nevirapine-based ART was an exclusion criterion due to clinically significant interactions with rifampicin.

\section{Outcome Assessment and Follow-Up}

Participants were reviewed daily during hospitalisation for neurological status and adverse events (AE) ascertainment, before being discharged around day 14 unless their medical condition warranted prolonged hospitalisation. After hospital discharge, participants were reviewed by the study team as outpatients at weeks $4,8,12,18$, and 24 . Where possible a detailed neurocognitive assessment was 
performed at the week 8 and 24 visit. After 24-weeks of follow-up the participants were referred to local TB services to complete a total treatment duration of 9-12 months.

Primary endpoints were 1) pharmacokinetic parameters in serum $\left(A \cup C_{0-24}, C_{\max }\right)$ and CSF concentration $\left(\mathrm{C}_{\mathrm{CSF}}\right) ; 2$ ) composite safety endpoint during the 8-week intervention period (comprising any of the following: a) grade 3-5 AEs including drug-induced liver injury; b) serious AEs (SAEs); or c) discontinuation of rifampicin for $>5$ days for any cause. Secondary endpoints included survival to 8- and 24-weeks, time to normalisation of conscious level (GCS=15), functional status by modified Rankin scale score at 8 and 24-weeks, quantitative neurocognitive performance Z score at 8 and 24 weeks, incidence of TB immune reconstitution inflammatory syndrome.

\section{Pharmacokinetic analysis}

Total rifampicin concentrations were analysed by validated high-performance liquid chromatography with ultraviolet detection (HPLC-UV) in the Infectious Disease Institute Translational Laboratory using an LC-2010C HT system (Shimadzu, Kyoto, Japan). Chromatographic analysis occurred on a PhenylHexyl, $150 \times 3 \mathrm{~mm} \mathrm{ID}, 3 \mu \mathrm{m}$ particle size, analytical column (Thermoscientific, USA). The assay was internally validated and performed well in an external international quality control (QC) programme, accuracy of the QC samples was 94 - 102\% depending on the concentration. The intra-assay and inter-assay coefficients of variation were 2.9 - 3.6\% and $3.4-4.8 \%$ respectively. The calibration curve for rifampicin in plasma covered a range from 0.25 to $15 \mathrm{mg} / \mathrm{L}$. Samples with concentrations above the upper limit of quantitation were diluted with blank plasma before re-analysis. The accuracy and repeatability of this approach was validated. Further details are provided in the supplementary materials.

The PK parameters $C_{\max }$ and area under the time-concentration curve up to 8 hours post dose (AUC $C_{0-}$ 8) were determined using a standard non-compartmental approach with Phoenix WinNonLin (Certara, Princeton, USA) using the log-linear trapezoidal rule. The $\mathrm{AUC}_{0-24}$ was determined using a published population PK model, ${ }^{23}$ modified for IV administration in IV-20 using NONMEM (Icon Development Solutions, Ellicott City, Maryland). $T_{\max }$ was defined as the time to reach maximum concentration. The rifampicin CSF concentration $\left(\mathrm{C}_{\mathrm{CSF}}\right)$ was the measured concentration in the interval of 2 to 8 -hr post dose. Where rifampicin concentrations were below the lower limit of quantification (LLOQ, $0.25 \mathrm{mg} / \mathrm{L}$ ), for the purposes of PK analysis a value of $0.125 \mathrm{mg} / \mathrm{L}$ (half of the LLOQ) was assigned. The difference in log-transformed exposure measures between study arms was 
assessed in a general linear regression model with the pairwise difference of each arm with the control assessed by the Wald t-test with Bonferroni adjustment for multiple testing. The difference in $T_{\max }$ between study arms was tested with Kruskal Wallis test. The between-arm difference in proportion of participants achieving a minimum published $\mathrm{C}_{\max }$ target of $8 \mathrm{mg} / \mathrm{L}^{24}$ an $\mathrm{AUC} \mathrm{C}_{0-24 \mathrm{hr}}$ of $300 \mathrm{~h} \cdot \mathrm{mg} / \mathrm{L}$ (an exposure generating about 65\% of the maximum effect in the Indonesian modelbased PK-PD meta-analysis ${ }^{17}$ ), and a CSF concentration above the rifampicin MIC for the most prevalent M. tuberculosis strain in Uganda (M. tuberculosis Uganda II) was compared with a Chisquared test. ${ }^{25}$

\section{Statistical analysis}

We determined sample size following the assumption that PK parameters are normally distributed on the log scale and derived rifampicin log-transformed $\mathrm{C}_{\max }$ standard deviation from prior research. ${ }^{10}$ The statistical analysis adhered to the published protocol and statistical analysis plan. ${ }^{21}$ We followed CONSORT guidelines, and the primary analyses were conducted by intention to treat (ITT). We compared the number and proportion of individuals who reported any kind of clinical grade 3-5 AE during the study period, and the composite safety endpoint, between arms with a chisquared test. We compared time to experiencing an $\mathrm{AE}$ and time to normalisation of conscious level (GCS=15/15) between treatment arms using a Fine and Gray competing risks proportional hazards regression model and cumulative incidence functions, with death considered a competing risk. Mortality at 8- and 24-weeks post-randomisation was compared between study arms using the risk difference from a generalised linear regression model with binomial distribution and identity link function. Kaplan-Meier curves were used to compare time to death. Participants who were withdrawn or lost to follow-up were censored at the time of their last contact. We compared the mean modified Rankin score at 8- and 24-weeks between trial arms using a linear regression model. In addition, we modelled repeated measures GCS profiles using a linear mixed model with random slope parameter. Data were collected using the DataFax system, and all statistical analyses were conducted using Stata version 13.1 (StataCorp, College Station, Texas, USA).

\section{Ethical considerations and oversight}

Written informed consent was obtained from all participants or their caregiver (where the individual lacked capacity to consent). Those enrolled via surrogate consent repeated the informed consent process at the earliest opportunity after regaining capacity. The trial was approved by the Infectious 
Diseases Institute Scientific Review Committee, the Research Ethics Committees of the London School of Hygiene and Tropical Medicine and Mulago Hospital, the Uganda National Council of Science and Technology, the National Drug Authority and registered (ISRCTN42218549). An independent data safety committee reviewed the accruing safety data. The intravenous and oral rifampicin were donated by Sanofi. Fixed-dose combination antituberculous therapy was produced by Macleod's pharmaceuticals.

\section{Role of the funding source}

The funders had no role in the study design, data collection, analysis, or interpretation of the results, nor writing of the manuscript.

\section{Results}

\section{Study population}

From 14 ${ }^{\text {th }}$ January 2019 through $17^{\text {th }}$ December 2019, 139 adults with suspected TBM were assessed for eligibility of which 61 were enrolled, including 31 (51\%) with microbiologically confirmed TBM, see Figure 1. One participant in the PO-35 arm was withdrawn on day 2 due to baseline ALT >3x ULN. An additional participant was enrolled to replace the withdrawal, and randomised to the SOC care arm giving rise to 21 participants in that arm. One participant withdrew consent for follow-up on day 7. One participant left the hospital against medical advice on day 3 and was lost to follow-up. Participant baseline characteristics are described in Table 1 . The majority of participants (56/61, 92\%) were HIV-positive with a median CD4 count of 50 cells/ $\mu$ I (IQR 46-56), a median HIV viral load of 4815 copies/ml, and 20 (33\%) were on ART with a median duration of 36 days (IQR 16 to 61 days). There were no cases of rifampicin-resistant disease identified by CSF or urine Xpert Ultra. The characteristics at baseline were balanced between the three arms.

\section{Primary outcomes}

Compared to the control, rifampicin administered IV at $20 \mathrm{mg} / \mathrm{kg} /$ day and orally at $35 \mathrm{mg} / \mathrm{kg} / \mathrm{day}$ resulted in greater than proportional increases in geometric mean serum peak and total exposure and CSF concentration. On day 2 in the control arm, serum $\mathrm{C}_{\max }$ was $6.0 \mathrm{mg} / \mathrm{L}(95 \% \mathrm{Cl}, 4.20-8.68)$ and AUC $_{0-24}$ was $42.9 \mathrm{~h} \cdot \mathrm{mg} / \mathrm{L}(95 \% \mathrm{Cl}, 29.2-63.0)$. With IV-20 arm, $\mathrm{C}_{\max }$ increased 6-fold to $36.2 \mathrm{mg} / \mathrm{L}$ (95\% Cl, 31.8-41.2) and $\mathrm{AUC}_{0-24}$ increased 5-fold to $217 \mathrm{~h} \cdot \mathrm{mg} / \mathrm{L}(95 \% \mathrm{Cl}, 202-306 ; \mathrm{p}<0.001$ for each 
comparison with control). In the PO-35 arm, $\mathrm{C}_{\max }$ increased 5 -fold to $29.3 \mathrm{mg} / \mathrm{L}(95 \% \mathrm{Cl}, 23.0-37.5)$ and $\mathrm{AUC}_{0-24}$ increased $~ 8$-fold to $327 \mathrm{~h} \cdot \mathrm{mg} / \mathrm{L}$ (95\%Cl, 248-430; $\mathrm{p}<0.001$ for each). In CSF, with standard-of-care treatment 56\% (10/18) of participants had undetectable rifampicin ( $<0.25 \mathrm{mg} / \mathrm{L})$ and geometric mean concentration was $0.27 \mathrm{mg} / \mathrm{L}(95 \% \mathrm{Cl},<0.25-0.45)$. CSF rifampicin was detectable in all participants in the intensified arms. Geometric mean CSF concentrations were 6fold higher with IV-20 at $1.74 \mathrm{mg} / \mathrm{L}(95 \% \mathrm{Cl}, 1.20-2.53)$ and 8-fold higher with PO-35 at $2.17 \mathrm{mg} / \mathrm{L}$ $(95 \% \mathrm{Cl}, 1.64-2.86 ; \mathrm{p}<0.001$ for each). A CSF concentration at or above $1 \mathrm{mg} / \mathrm{L}$, which is the rifampicin minimal inhibitory concentration for the predominant M. tuberculosis strain in Uganda, ${ }^{25}$ occurred in 11\% (2/18) with standard-of-care, 93\% (14/15) with IV-20, and 95\% (18/19) with PO-35 $(p<0.001)$. PK results are further described in Table 2 and Figure 2.

At day 14 , when rifampicin cytochrome enzyme autoinduction is approximately $90 \%$ established, ${ }^{23}$ geometric mean exposures had decreased in the arms with oral (but not intravenous) rifampicin, but exposures in the intervention arms remained higher than those in the control arm. The median sample collection time post-dose was 2.67 hours (IQR 2.18-3.17) for serum and 2.83 hours (IQR, 2.18-3.17) for CSF on day 14. The geometric mean concentrations increased from $4.58 \mathrm{mg} / \mathrm{L}(95 \% \mathrm{Cl}$, 2.67-7.86 mg/L; $n=13)$ with standard-of-care to $34.2 \mathrm{mg} / \mathrm{L}(95 \% \mathrm{Cl}, 29.2-40.1 ; n=11 ; p<0.001)$ with IV-20 and $15.9 \mathrm{mg} / \mathrm{L}(95 \% \mathrm{Cl}, 6.85-36.9 \mathrm{mg} / \mathrm{L} ; \mathrm{n}=13 ; \mathrm{p}=0.003)$ with PO-35mg/kg. In the CSF at day 14, $11 \%(1 / 9)$ of participants in the standard-of-care arm had detectable rifampicin (>0.25 mg/L), whilst $88 \%$ (7/8) with IV-20 and 89\% (8/9) PO-35mg/kg arm had detectable CSF levels. Geometric mean CSF concentrations were $0.57(0.30-1.11, p=0.001)$ for IV-20 and $0.45(95 \% \mathrm{Cl} 0.21-0.96, p=0.007)$ for PO-35.

During the 8-week intervention period, grade 3 to 5 adverse events occurred in 15 (71\%) of standard-of-care, 10 (50\%) of IV-20, and 11 (55\%) PO-35 participants ( $p=0.342)$. No participants interrupted rifampicin for $>5$ days during the intervention period. The composite safety endpoint did not differ between trial arms ( $p=0.342)$, see Table 3 . There was no difference in time to experiencing an AE when comparing the intervention arms to the control: IV-20 sub-distribution hazard ratio (SHR) $0.734(95 \% \mathrm{Cl}, 0.33-1.65, \mathrm{p}=0.456)$ and $\mathrm{PO}-351.04(95 \% \mathrm{Cl}, 0.53-2.05, \mathrm{p}=0.909)$. The most common grade $\geq 3$ event was elevated ALT, which occurred in $7(11.7 \%)$ of participants overall. This was attributed to drug-induced liver injury (DILI), which occurred in 4 (19\%) participants in the standard-of-care arm, 1 (5\%) with IV-20, and 2 (10\%) with PO-35. ALT elevation was grade 3 in all 
cases, except one episode of grade 4 ALT elevation in the standard-of-care arm. In all instances, liver enzymes improved after interrupting pyrazinamide whilst other TB drugs were continued. There were no episodes of fulminant hepatic failure. Isolated hyperbilirubinaemia, a recognised adverse effect of rifampicin, occurred in three participants (grade 4) during the intervention period, one of which was in the IV-20 arm and the other two were in the control arm.

\section{Secondary outcomes}

Overall, during the 8-week intervention period, 18 (29.5\%) participants died; 5/21, 6/20 and 7/20 in the control, IV-20, and PO-35 arms, respectively. There was no evidence of an association with treatment arm (LR test p-value=0.595). By the end of the 24-week follow-up period, 24 (39.3\%) of participants died; $7 / 21,7 / 20$, and 10/20 in the control, IV-20, and PO-35 arm, respectively. There was no evidence of an association with treatment arm (LR test $p$-value=0.333). Kaplan-Meier survival curves are shown in Figure 3.

Mean modified Rankin scores ( 0 to 6 score on which 0 is asymptomatic and 6 is dead) at week 8 were $2.06,1.84$, and 2.30 ( $p=0.75$ ) and week 24 were $0.86,0.81$, and 1.14 in the standard-of-care, IV-20, and PO-35 arms, respectively ( $p=0.85)$. Time to normalization of Glasgow coma scale did not differ compared to the control: IV-20 sub-distribution hazard ratio (SHR) 1.20 (95\%Cl, 0.58-2.48; $\mathrm{p}=0.630)$ and PO-35 $0.85(95 \% \mathrm{Cl}, 0.41-1.77 ; \mathrm{p}=0.658)$. In the linear mixed model of GCS profiles, GCS increased by $0.13(95 \% \mathrm{Cl}, 0.07-0.18 ; \mathrm{p}=<0.001)$ per day, with no evidence of a difference by arm $(p=0.953)$, see supplementary materials.

\section{Discussion}

In this randomised controlled trial of high-dose rifampicin involving predominantly HIV-positive adults with suspected TBM, both high-dose intravenous and oral rifampicin were safe and resulted in a far greater proportion of participants achieving CSF levels above the rifampicin MIC when compared to standard TB treatment. With the currently WHO-recommended TB therapy only 1 in 10 participants achieved a CSF rifampicin concentration above the MIC. As well as being rapidly bactericidal, rifampicin is the key sterilising drug in the regimen, so achieving adequate levels at the site of disease is essential and attainment of the drug MIC should be considered a bare minimum.

Critical illness is known to alter drug PK, and in the early days of TBM treatment patients may be comatose, have vomiting or require drugs to be given via a nasogastric tube, all of which may further 
jeopardise effective early treatment. HIV infection also appears to alter rifampicin PK in the initial days of therapy. A recent meta-analysis of rifampicin PK data from 70 studies including 3477 participants confirmed that during the initial days of standard TB treatment rifampicin total plasma exposure is indeed reduced in PLHIV (mean $\mathrm{AUC}_{0-24}$ was $37.2 \mathrm{~h} \cdot \mathrm{mg} / \mathrm{L}$ in HIV-positive versus 56.7 $\mathrm{h} \cdot \mathrm{mg} / \mathrm{L}$ in HIV-negative adults, $\mathrm{p}=0.003)$, though this difference diminished at steady-state. ${ }^{24}$ Our participants in the standard-of-care arm had a serum geometric mean $\mathrm{AUC}_{0-24}$ of $42.9 \mathrm{~h} \cdot \mathrm{mg} / \mathrm{L}$, similar to the PLHIV and lower than the HIV-negative group in the meta-analysis. ${ }^{24} \mathrm{It}$ is encouraging to see that even in a population with advanced HIV disease high blood and CSF rifampicin exposures were obtained with oral dosing of rifampicin at $35 \mathrm{mg} / \mathrm{kg} / \mathrm{day}$ in this study.

The increases in rifampicin dosing resulted in greater than proportional increases in serum and CSF exposure: a 2-fold dose-increase administered IV resulted in 6-fold increases in exposure, and a 3.5fold oral dose-increase resulted in a 8-fold increase in exposure. The supra-proportional increase in exposures observed here, and in other studies, is likely to be attributable to 1) saturation of the beta-esterase metabolizing enzymes and/or p-glycoprotein with a reduction in the first-pass effect and thereby increased bioavailability of oral drug; 2 ) saturation of the biliary excretion pathway. ${ }^{23,5}$ Rifampicin induces its own metabolism via the pregnane $\mathrm{X}$ receptor, a phenomenon known as clearance autoinduction, resulting in lower exposure at steady state. Despite this phenomenon, we found that serum and CSF rifampicin concentrations in the interventional arms were still higher than those in the control arm at day 14 when clearance autoinduction is well established. The serum and CSF PK parameters in the intervention arms were comparable, although not formally tested.

In relation to other intensification studies, the day 2 total serum rifampicin exposures achieved in the IV-20 and PO-35 arms were 3- and 4-fold higher respectively than that of the intensified arm of the Vietnamese trial by Heemskerk et al ( $\mathrm{UUC}_{0-24} 82.5 \mathrm{~h} \cdot \mathrm{mg} / \mathrm{L}$ following rifampicin $15 \mathrm{mg} / \mathrm{kg} /$ day). ${ }^{14}$ The total exposure achieved with PO-35 (AUC $0-24327 \mathrm{~h} \cdot \mathrm{mg} / \mathrm{L})$ is comparable to the most recent Indonesian phase II trial, which tested rifampicin $30 \mathrm{mg} / \mathrm{kg} /$ day and reported an $\mathrm{AUC}_{0-24}$ of 294 $\mathrm{h} \cdot \mathrm{mg} / \mathrm{L} .{ }^{15}$ Owing to the difficulty in consistently culturing $M$. tuberculosis, CSF does not lend itself to microbial kill studies. We therefore cannot draw any parallels with the mycobacterial clearance rates in high-dose rifampicin pulmonary TB studies but our data can contribute to a PK-PD model to understand the exposure-response relationship in this Ugandan HIV/TBM population. Recently, PKPD data from the Vietnamese intensification trial has sparked further interest in the importance of 
isoniazid, which has high early bactericidal activity and excellent CSF penetration. ${ }^{14}$ Heemskerk et al found that low isoniazid exposure was predictive of death and linked to a fast metabolizer Nacetyltransferase 2 phenotype. ${ }^{14}$ For an African population this may be less relevant, considering that the proportion of fast acetylators is lower as compared to Asians ethnicities. Apart from this, isoniazid monoresistance (up to $25 \%$ prevalence in some countries) cannot readily be detected at baseline with the Xpert MTB/Rif assay. Therefore, obtaining therapeutic concentrations of other key drugs, especially rifampicin, in the CSF is absolutely critical and can only be achieved by increasing the currently recommended rifampicin dose.

Importantly, despite the substantial increase in rifampicin exposure in the intervention arms, there was no increase in toxicity, in-line with other studies of largely HIV-negative individuals. $5,10,15,26$ The adverse effects of rifampicin are well described and include hypersensitivity reactions featuring fever and rash, thrombocytopaenia, liver toxicity and hyperbilirubinaemia. Recent data from the PanACEA group suggests that rifampicin toxicity is largely idiosyncratic. ${ }^{9}$ Our findings support the notion that drug exposure is not a driver of toxicity in this population. This is reassuring data as PLHIV are at markedly increased risk of drug-related toxicity including cutaneous drug reactions, hypersensitivity, liver toxicity, and complications relating to polypharmacy and immune reconstitution. ${ }^{19,18}$ Due to the critically ill and immunocompromised nature of the trial population, grade 3-5 adverse events were experienced by over half $(59 \%, 36 / 61)$ of participants but largely appeared to be a complication of the underlying disease process, and events were evenly distributed across study arms. Interruption of antituberculous therapy in the treatment of TBM is associated with increased mortality. ${ }^{27}$ We therefore used an algorithm for the management of DILI in which pyrazinamide was interrupted in isolation with regular monitoring of liver function tests, allowing other antituberculous agents to be continued. This was effective in all occurrences of DILI during the intensive phase of treatment. We did not observe any impact of high-dose rifampicin on 8 or 24-week mortality, nor functional outcomes by modified Rankin score. The phase II study was not powered for these clinical endpoints and thus an adequately powered trial is justified having established the safety of high-dose rifampicin in this population.

HIV is central to both the incidence and pathogenesis of TBM. Although HIV status was not an entry criterion for the study, 92\% of those eligible to enrol were PLHIV. Under half were on ART at enrolment, none were virologically suppressed, and median CD4 count was 50 cells/ $\mu$ l. Effective HIV 
treatment can reduce the risk of TB by up to $80 \%$, therefore earlier HIV diagnosis and effective longterm treatment are key upstream interventions to reduce the incidence of TB. ${ }^{28}$ Other important upstream interventions include 1) TB preventative therapy and 2) screening for TB prior to ART initiation. It is noteworthy that a third of our participants were presenting to hospital with TBM in the weeks following ART initiation, suggesting that symptom-based TB screening had failed. Access to urine TB diagnostics (e.g. TB-lipoarabinomannan) in HIV clinic settings is important to reduce unmasking TB immune reconstitution inflammatory syndrome, which can be devastating in the CNS. The roll out of dolutegravir as first-line ART in Uganda began in 2018 and may support improved rates of virological suppression in the future. We dose-adjusted dolutegravir to $50 \mathrm{mg}$ twice daily, which is safe and effective with standard dose rifampicin. ${ }^{29,30}$ A PK study is ongoing in Uganda $\left(\mathrm{SAEFRIF}^{4}\right.$ ) to assess the impact of rifampicin $35 \mathrm{mg} / \mathrm{kg}$ on dolutegravir PK.

We acknowledge that this study has a number of limitations. Due to critical illness and inability to stand, baseline weight was frequently estimated, which may have resulted in imprecision in the weight-based dosing. Ideally, we would have collected blood and CSF samples at more time points, especially in the clearance phase, to allow estimation of elimination rate and half-life. However, we prioritised participant safety and comfort so restricted blood sampling to daylight working hours and performed a single PK lumbar puncture. As with many TBM trials microbiological confirmation was only made in around half of participants. Due to the complexity of performing rifampicin MIC, the MIC was derived from population estimates within the same TB laboratory. ${ }^{25}$

This phase II study provides important evidence that high-dose rifampicin increases CSF and serum exposures in a predominantly HIV-positive population. These data, taken together with data from Indonesian studies, justify a phase III trial to investigate safety in a wider population and the impact of high-dose on survival. We look forward to the outcome of a number of large trials already in the pipeline to examine the clinical impact of high-dose oral rifampicin in isolation (ISRCTN15668391), or with adjunctive linezolid and aspirin (NCT04145258, NCT03927313) in patients with TBM. Whether in future the optimised TBM treatment regimen will include high-dose rifampicin remains to be concluded. 


\section{Conflicts of Interest}

Sanofi donated the intravenous and oral rifampicin. No conflict of interest were declared by any of the investigators.

\section{Acknowledgements / funding}

FVC and this trial were supported by a Wellcome Clinical PhD Fellowship (210772/Z/18/Z). Thank you to the external monitors Ms Mirriam Akello and Ms Sarah Coutinho. Sanofi Pasteur donated the rifampicin to the study. We would like to thank the participants and their caregivers for participating in the study, the Infectious Diseases Institute research office for their support and the meningitis team for their dedication. FVC is an honorary fellow of the Makerere University - Uganda Virus Research Institute Centre of Excellence for Infection and Immunity Research and Training (MUII-plus). MUII-plus is supported through the DELTAS Africa Initiative (Grant no. 107743). The DELTAS Africa Initiative is an independent funding scheme of the African Academy of Sciences (AAS), Alliance for Accelerating Excellence in Science in Africa (AESA) and supported by the New Partnership for Africa's Development Planning and Coordinating Agency (NEPAD Agency) with funding from the Wellcome Trust (Grant no. 107743) and the UK Government. The MRC/UVRI and LSHTM Uganda Research Unit is jointly funded by the UK Medical Research Council (MRC) and the UK Department for International Development (DFID) under the MRC/DFID Concordat agreement and is also part of the EDCTP2 programme supported by the European Union.

\section{Contributors}

FVC, DM, DRB, RA, AME, LtB contributed to study conceptualization and methodology. FVC, DRB, DM, AME contributed to funding acquisition. DRB, DM, AME contributed to supervision. DM, FVC, DRB contributed in administration. FVC, EK, MR, LT, KS, AM, CQ, EM, MO, CM, DM, DRB contributed to investigation. FVC, DG, AB, DB, ES, LtB contributed to statistical analysis. FVC, $A B, M O$ contributed to acquisition of laboratory data. FVC, DRB, RA, AME, LtB, ES contributed to data interpretation. FVC contributed to drafting initial manuscript. FVC, DM, DRB, RA, AME, LtB, LT, KS, EM contributed to manuscript review and editing. All authors approved the final version to be published.

\section{Additional Rift study group members}

Members of the trial steering committee: Professor Guy E Thwaites, Professor Reinout van Crevel, Professor Joseph Jarvis. 
Members of the data safety committee: Professor Robert J Wilkinson, Dr Christine SekaggyaWiltshire, Dr Agnes Kiragga, Dr Amanda Clarke.

Additional contributors to clinical and administrative activities: Jane Frances, Florence Kigundu, Cythia Ahimbisibwe, Carol Karuganda, Alice Namudde, Kiiza Tadeo Kandole, Kathy Huppler Hullsiek, Alisat Sadiq, Mable Kabahubya, Dr Edward Mpoza, Dr Gavin Stead, Dr Samuel Jjunju, Dr Edwin Nuwagira, Dr Nathan Bahr, Dr Joshua Rhein, Darlisha Williams, Rhona Muyise, Eva Laker. 


\section{References}

1. Jarvis JN, Meintjes G, Williams A, Brown Y, Crede T, Harrison TS. Adult meningitis in a setting of high HIV and TB prevalence: findings from 4961 suspected cases. BMC infectious diseases 2010; 10: 67 .

2. Stadelman AM, Ellis J, Samuels THA, et al. Treatment Outcomes in Adult Tuberculous Meningitis: A Systematic Review and Meta-analysis. Open forum infectious diseases 2020; 7(8): ofaa257.

3. van Ingen J, Aarnoutse RE, Donald PR, et al. Why Do We Use 600 mg of Rifampicin in Tuberculosis Treatment? Clinical infectious diseases : an official publication of the Infectious Diseases Society of America 2011; 52(9): e194-9.

4. Nabisere R, Musaazi J, Denti P, et al. Pharmacokinetics, SAfety/tolerability, and EFficacy of high-dose RIFampicin in tuberculosis-HIV co-infected patients on efavirenz- or dolutegravir-based antiretroviral therapy: study protocol for an open-label, phase II clinical trial (SAEFRIF). Trials 2020; 21(1): 181.

5. Boeree MJ, Diacon AH, Dawson R, et al. A dose-ranging trial to optimize the dose of rifampin in the treatment of tuberculosis. American journal of respiratory and critical care medicine 2015; 191(9): 1058-65.

6. Hu Y, Liu A, Ortega-Muro F, Alameda-Martin L, Mitchison D, Coates A. High-dose rifampicin kills persisters, shortens treatment duration, and reduces relapse rate in vitro and in vivo. Frontiers in microbiology 2015; 6: 641.

7. Svensson RJ, Svensson EM, Aarnoutse RE, et al. Greater Early Bactericidal Activity at Higher Rifampicin Doses Revealed by Modeling and Clinical Trial Simulations. The Journal of infectious diseases 2018; 218(6): 991-9.

8. Svensson EM, Svensson RJ, Te Brake LHM, et al. The Potential for Treatment Shortening With Higher Rifampicin Doses: Relating Drug Exposure to Treatment Response in Patients With Pulmonary Tuberculosis. Clinical infectious diseases : an official publication of the Infectious Diseases Society of America 2018; 67(1): 34-41.

9. te Brake L, de Jager V, Narunsky K, et al. Increased Bactericidal Activity But Dose-Limiting Tolerability at 50 mg/kg Rifampicin SSRN 2020.

10. Ruslami R, Ganiem AR, Dian S, et al. Intensified regimen containing rifampicin and moxifloxacin for tuberculous meningitis: an open-label, randomised controlled phase 2 trial. The Lancet Infectious diseases 2013; 13(1): 27-35.

11. Cresswell FV, Te Brake L, Atherton $R$, et al. Intensified antibiotic treatment of tuberculosis meningitis. Expert Rev Clin Pharmacol 2019; 12(3): 267-88.

12. Heemskerk AD, Bang ND, Mai NT, et al. Intensified Antituberculosis Therapy in Adults with Tuberculous Meningitis. The New England journal of medicine 2016; 374(2): 124-34. 
13. Heemskerk AD, Nguyen MTH, Dang HTM, et al. Clinical Outcomes of Patients With DrugResistant Tuberculous Meningitis Treated With an Intensified Antituberculosis Regimen. Clinical infectious diseases : an official publication of the Infectious Diseases Society of America 2017; 65(1): 20-8.

14. Ding J, Thuy Thuong Thuong N, Pham TV, et al. Pharmacokinetics and Pharmacodynamics of Intensive Antituberculosis Treatment of Tuberculous Meningitis. Clinical pharmacology and therapeutics 2020.

15. Dian S, Yunivita V, Ganiem AR, et al. Double-Blind, Randomized, Placebo-Controlled Phase II Dose-Finding Study To Evaluate High-Dose Rifampin for Tuberculous Meningitis. Antimicrob Agents Chemother 2018; 62(12).

16. Yunivita V, Dian S, Ganiem AR, et al. Pharmacokinetics and safety/tolerability of higher oral and intravenous doses of rifampicin in adult tuberculous meningitis patients. International journal of antimicrobial agents 2016; 48(4): 415-21.

17. Svensson EM, Dian S, Te Brake L, et al. Model-based meta-analysis of rifampicin exposure and mortality in Indonesian tuberculosis meningitis trials. Clinical infectious diseases : an official publication of the Infectious Diseases Society of America 2019.

18. Abbara A, Chitty S, Roe JK, et al. Drug-induced liver injury from antituberculous treatment: a retrospective study from a large TB centre in the UK. BMC infectious diseases 2017; 17(1): 231.

19. Lin D, Tucker MJ, Rieder MJ. Increased adverse drug reactions to antimicrobials and anticonvulsants in patients with HIV infection. Ann Pharmacother 2006; 40(9): 1594-601.

20. Loos U, Musch E, Jensen JC, Mikus G, Schwabe HK, Eichelbaum M. Pharmacokinetics of oral and intravenous rifampicin during chronic administration. Klin Wochenschr 1985; 63(23): 1205-11.

21. Cresswell FV, Ssebambulidde K, Grint D, et al. High dose oral and intravenous rifampicin for improved survival from adult tuberculous meningitis: a phase II open-label randomised controlled trial (the RifT study). Wellcome open research 2018; 3: 83.

22. Marais S, Thwaites G, Schoeman JF, et al. Tuberculous meningitis: a uniform case definition for use in clinical research. The Lancet Infectious diseases 2010; 10(11): 803-12.

23. Svensson RJ, Aarnoutse RE, Diacon AH, et al. A Population Pharmacokinetic Model Incorporating Saturable Pharmacokinetics and Autoinduction for High Rifampicin Doses. Clinical pharmacology and therapeutics 2018; 103(4): 674-83.

24. Stott KE, Pertinez H, Sturkenboom MGG, et al. Pharmacokinetics of rifampicin in adult TB patients and healthy volunteers: a systematic review and meta-analysis. The Journal of antimicrobial chemotherapy 2018.

25. Kasule GW, Kateete DP, Joloba ML. Mycobacterium tuberculosis Uganda II is more susceptible to rifampicin and isoniazid compared to Beijing and Delhi/CAS families. BMC infectious diseases 2016; 16: 173. 
26. Seijger C, Hoefsloot W, Bergsma-de Guchteneire I, et al. High-dose rifampicin in tuberculosis: Experiences from a Dutch tuberculosis centre. PloS one 2019; 14(3): e0213718.

27. Thwaites GE, Nguyen DB, Nguyen HD, et al. Dexamethasone for the treatment of tuberculous meningitis in adolescents and adults. The New England journal of medicine 2004; 351(17): 1741-51.

28. Suthar AB, Lawn SD, del Amo J, et al. Antiretroviral therapy for prevention of tuberculosis in adults with HIV: a systematic review and meta-analysis. PLoS Med 2012; 9(7): e1001270.

29. Wang $\mathrm{X}$, Cerrone $\mathrm{M}$, Ferretti $\mathrm{F}$, et al. Pharmacokinetics of dolutegravir $100 \mathrm{mg}$ once daily with rifampicin. International journal of antimicrobial agents 2019; 54(2): 202-6.

30. Dooley KE, Kaplan R, Mwelase N, et al. Dolutegravir-based Antiretroviral Therapy for Patients Coinfected With Tuberculosis and Human Immunodeficiency Virus: A Multicenter, Noncomparative, Open-label, Randomized Trial. Clinical infectious diseases : an official publication of the Infectious Diseases Society of America 2020; 70(4): 549-56. 
Table 1. Baseline characteristics

\begin{tabular}{|c|c|c|c|}
\hline & IV-20 arm & PO-35 arm & Control arm \\
\hline $\mathrm{N}$ randomised & 20 & 20 & 21 \\
\hline Age, median (IQR) years & $33.5(25.5-38.5)$ & $32.5(26.5-38.5)$ & $34.0(27-36)$ \\
\hline Gender, male N (\%) & $13.0(65)$ & $12.0(60)$ & $9.00(42.9)$ \\
\hline Weight, median (IQR), kg & $55(47.5-58.5)$ & $50.5(50-55)$ & $50.0(45-55)$ \\
\hline \multicolumn{4}{|l|}{ HIV details } \\
\hline HIV-positive, N (\%) & $18.0(90)$ & $18(90)$ & $20(95.2)$ \\
\hline HIV-negative, N (\%) & $2(10)$ & $2(10)$ & $1(4.80)$ \\
\hline \multicolumn{4}{|l|}{ Amongst those HIV positive: } \\
\hline CD4 T cell count, median (IQR)cells/ $\mu \mathrm{l}$ & $55(45-59)$ & $50.5(50-55)$ & $50(45-55)$ \\
\hline $\begin{array}{l}\text { HIV viral load, median (IQR) copies/ } \\
\text { ml }\end{array}$ & $\begin{array}{c}7840 \\
(4014-574462)\end{array}$ & $\begin{array}{c}6523 \\
(4815-92346)\end{array}$ & $\begin{array}{c}2333.5 \\
(945-19316)\end{array}$ \\
\hline $\begin{array}{l}\text { Currently receiving ART, N (\%) } \\
\text { Antiretroviral therapy duration, } \\
\text { median (range), weeks }\end{array}$ & $\begin{array}{c}12(60) \\
28(0-62.3)\end{array}$ & $\begin{array}{c}11(55) \\
26(1.6-6.6)\end{array}$ & $\begin{array}{c}4(19.0) \\
61(0.7-195.9)\end{array}$ \\
\hline \multicolumn{4}{|l|}{ MRC TB meningitis grade, $n$ (\%) } \\
\hline । & $3(15)$ & $1(5)$ & $2(9.5)$ \\
\hline ॥ & $13(65)$ & $16(80)$ & $12(57.1)$ \\
\hline III & $4(20)$ & $3(15)$ & $7(33.3)$ \\
\hline \multicolumn{4}{|l|}{ Uniform case definition, $n(\%)$} \\
\hline $\begin{array}{l}\text { Definite (microbiologically } \\
\text { confirmed) }\end{array}$ & $8(40)$ & $12(60)$ & $11(52.4)$ \\
\hline Probable & $4(20)$ & $4(20)$ & $6(28.6)$ \\
\hline Possible & $7(35)$ & $4(20)$ & $1(4.8)$ \\
\hline Not & $1(5)$ & $0(0)$ & $3(14.3)$ \\
\hline \multicolumn{4}{|l|}{ CSF information, median (IQR) } \\
\hline Opening pressure, $\mathrm{mmH} 20$ & $230(155-320)$ & $220(135-300)$ & $135(100-230)$ \\
\hline White cells,cells/ $\mu \mathrm{l}$ ) & $24(4-162.5)$ & $4(4-145)$ & $4(4-122.5)$ \\
\hline Protein, mg/dl & $154.5(105-186)$ & $125(73-188)$ & $92(30-164)$ \\
\hline Glucose, mg/dl & $41(21-68)$ & $44(21-66.7)$ & $38(18-64)$ \\
\hline Lactate, $\mathrm{mmol} / \mathrm{L}$ & $8.5(7.30-9.80)$ & $6.9(2.9-11.1)$ & $8(5.2-10.4)$ \\
\hline
\end{tabular}


Table 2. Rifampicin Pharmacokinetic data by treatment arm

\begin{tabular}{|c|c|c|c|c|}
\hline & IV-20 & PO-35 & Control & $P$ value ${ }^{a}$ \\
\hline \multicolumn{5}{|l|}{$\mathrm{AUC}_{0-8}(\mathrm{~h} \cdot \mathrm{mg} / \mathrm{L})^{\mathrm{b}}$} \\
\hline n observations ${ }^{c}$ & 19 & 19 & 20 & \multirow{4}{*}{$<0.001$} \\
\hline $\begin{array}{r}\text { Geometric mean } \\
(95 \% \mathrm{Cl})\end{array}$ & $\begin{array}{c}163 \\
(142-186)\end{array}$ & $\begin{array}{c}162 \\
(129-203)\end{array}$ & $\begin{array}{c}30.5 \\
(21.7-42.8)\end{array}$ & \\
\hline Ratio to control & 5.33 & 5.31 & - & \\
\hline$P$ value ${ }^{d}$ & $<0.001$ & $<0.001$ & - & \\
\hline \multicolumn{5}{|l|}{$\mathrm{AUC}_{0 \_24}(\mathrm{~h} \cdot \mathrm{mg} / \mathrm{L})^{\mathrm{e}}$} \\
\hline n observations & 19 & 19 & 21 & \multirow{4}{*}{$<0.001$} \\
\hline $\begin{array}{r}\text { Geometric mean } \\
(95 \% \mathrm{Cl})\end{array}$ & $\begin{array}{c}249 \\
(202-306)\end{array}$ & $\begin{array}{c}327 \\
(248-430)\end{array}$ & $\begin{array}{c}42.9 \\
(29.2-63.0)\end{array}$ & \\
\hline Ratio to control & 5.80 & 7.62 & - & \\
\hline$P$ value ${ }^{d}$ & $<0.001$ & $<0.001$ & - & \\
\hline $\mathrm{n}(\%)$ achieving target of $\geq 300 \mathrm{~h} \cdot \mathrm{mg} / \mathrm{L}$ & $7(36.8)$ & $9(47.4)$ & $0(0 \%)$ & $<0.001^{j}$ \\
\hline \multicolumn{5}{|l|}{$\mathrm{C}_{\max }(\mathrm{mg} / \mathrm{l})^{\mathrm{b}}$} \\
\hline \multirow{6}{*}{$\begin{array}{r}\text { Geometric mean } \\
\qquad(95 \% \mathrm{Cl}) \\
\text { Ratio to control } \\
\text { P value }{ }^{d} \\
\text { arget of }>8 \mathrm{mg} / \mathrm{L}\end{array}$} & 19 & 16 & 17 & \multirow{4}{*}{$<0.001$} \\
\hline & $\begin{array}{c}36.2 \\
(31.8-41.2)\end{array}$ & $\begin{array}{c}29.3 \\
(23.0-37.5)\end{array}$ & $\begin{array}{c}6.04 \\
(4.20-8.68)\end{array}$ & \\
\hline & 5.99 & 4.86 & - & \\
\hline & $<0.001$ & $<0.001$ & - & \\
\hline & $20(100)$ & $20(100)$ & $10(47.62)$ & $<0.0001^{f}$ \\
\hline & \multicolumn{4}{|c|}{$T_{\max }$} \\
\hline $\begin{array}{l}\text { Median } \\
\text { (range) }\end{array}$ & $\begin{array}{c}2.35 \\
(1.83-3.85)\end{array}$ & $\begin{array}{c}4.05 \\
(2.17-7.33)\end{array}$ & $\begin{array}{c}2.83 \\
(2.08-8.25)\end{array}$ & $0.002^{g}$ \\
\hline \multicolumn{5}{|l|}{$\mathrm{C}_{\mathrm{CSF}}(\mathrm{mg} / \mathrm{l})$} \\
\hline n observations & 15 & 19 & 18 & \multirow[b]{2}{*}{0.058} \\
\hline $\begin{array}{r}\text { Geometric mean } \\
(95 \% \mathrm{Cl})\end{array}$ & $\begin{array}{c}1.74 \\
(1.20-2.53)\end{array}$ & $\begin{array}{c}2.17 \\
(1.64-2.86)\end{array}$ & $\begin{array}{c}0.27^{h} \\
(0.17-0.45)\end{array}$ & \\
\hline Ratio to control & 6.44 & 8.00 & - & \\
\hline P value $^{d}$ & $<0.001$ & $<0.001$ & & \\
\hline n (\%) with detectable CSF level & $15(100)$ & $19(100)$ & $8(44)$ & $<0.001^{f}$ \\
\hline $\begin{array}{r}\mathrm{n}(\%) \text { with concentration above } \\
\text { rifampicin MIC ( } 1 \mathrm{mg} / \mathrm{L})\end{array}$ & $14(93.3 \%)$ & $18(94.7 \%)$ & $2(11.1 \%)$ & $<0.001^{f}$ \\
\hline $\begin{array}{r}\text { Median (IQR) hours post-dose to CSF } \\
\text { sample }\end{array}$ & $4.70(3.28-5.92)$ & $4.55(3.08-6.20)$ & $\begin{array}{c}4.83(3.78- \\
5.5)\end{array}$ & \\
\hline \multicolumn{5}{|c|}{ 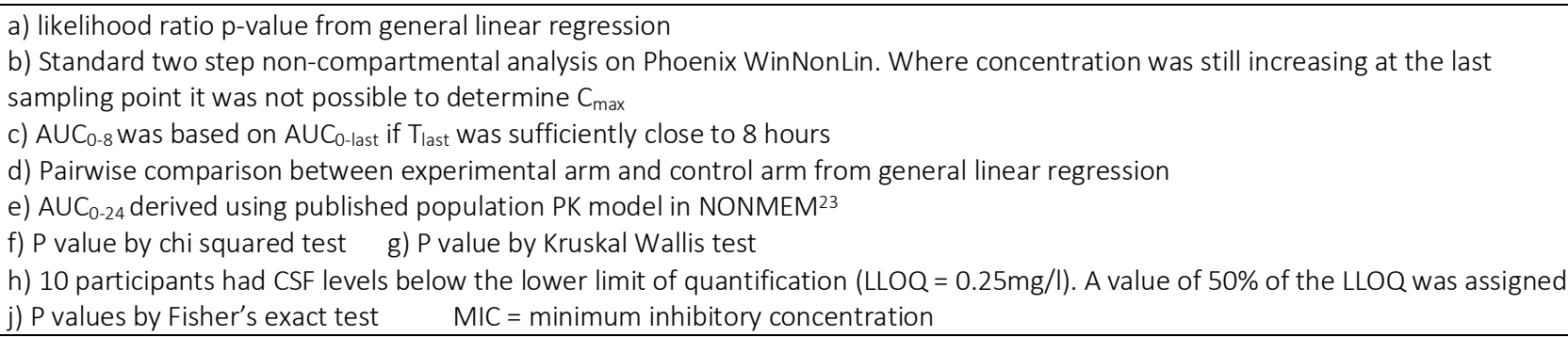 } \\
\hline
\end{tabular}


Table 3. Adverse events by treatment arm during the 8-week interventional period

\begin{tabular}{|c|c|c|c|c|}
\hline & $\begin{array}{l}\text { IV-20 } \\
(n=20)\end{array}$ & $\begin{array}{l}P O-35 \\
(n=20)\end{array}$ & $\begin{array}{l}\text { Control } \\
(n=21)\end{array}$ & $P$ value \\
\hline TOTAL NUMBER OF EVENTS & $\mathrm{N}(\%)$ & $N(\%)$ & $\mathrm{N}(\%)$ & \\
\hline Grade 3 & $6(30)$ & $6(30)$ & $12(57)$ & 0.12 \\
\hline Grade 4 & $3(15)$ & $4(20)$ & $4(19)$ & 0.91 \\
\hline Grade 5 & $5(25)$ & $7(35)$ & $5(24)$ & 0.68 \\
\hline \multicolumn{5}{|l|}{ NEUROLOGICAL EVENT } \\
\hline Cerebrovascular accident & $2(10)$ & $1(5)$ & $1(4.8)$ & \\
\hline Seizures & $1(5)$ & $2(10)$ & $2(9.5)$ & \\
\hline Headache & $0(0)$ & $0(0.0)$ & $1(4.8)$ & \\
\hline Hearing loss & $0(0)$ & $1(5)$ & $0(0)$ & \\
\hline Neuropathy & $0(0)$ & $0(0)$ & $1(4.8)$ & \\
\hline Altered mental status & $2(10)$ & $1(5)$ & $2(9.5)$ & \\
\hline \multicolumn{5}{|l|}{ GENERALISED } \\
\hline Fever & $0(0)$ & $2(10)$ & $1(4.8)$ & \\
\hline Rash (Kaposi's sarcoma) & $0(0)$ & $1(5)$ & $1(4.8)$ & \\
\hline \multicolumn{5}{|l|}{ GASTROINTESTINAL \& HEPATIC } \\
\hline Abdominal pain & $0(0)$ & $1(5.0)$ & $0(0)$ & \\
\hline Dysphagia & $1(5)$ & $0(0)$ & $0(0)$ & \\
\hline Elevated alanine transaminase & $1(5)$ & $2(10)$ & $4(19)$ & \\
\hline Elevated bilirubin & $1(5)$ & $0(0)$ & $2(9.5)$ & \\
\hline \multicolumn{5}{|l|}{ OTHER } \\
\hline Anaemia & $1(5)$ & $1(5)$ & $2(9.5)$ & \\
\hline Hypotension & $1(5)$ & $0(0)$ & $0(0)$ & \\
\hline Thrombosis & $0(0)$ & $0(0)$ & $1(4.8)$ & \\
\hline Elevated creatinine & $1(5)$ & $1(5)$ & $1(4.8)$ & \\
\hline Urinary tract obstruction & $0(0)$ & $1(5)$ & $0(0)$ & \\
\hline Respiratory distress & $1(5)$ & $2(10)$ & $3(14)$ & \\
\hline Low sodium & $1(5)$ & $1(5)$ & $0(0)$ & \\
\hline \multicolumn{5}{|c|}{ AEs in relation to pre-specified secondary endpoint } \\
\hline $\mathrm{N}(\%)$ with a grade $3-5 \mathrm{AE}$ & $10(50)$ & $11(55)$ & $15(71)$ & 0.343 \\
\hline$N(\%)$ with a Serious $A E$ & $8(40)$ & $7(35)$ & $7(33)$ & 0.899 \\
\hline $\begin{array}{l}N(\%) \text { of patient with discontinuation } \\
\text { of rifampicin for }>5 \text { days in week 0-8 }\end{array}$ & 0 & 0 & 0 & \\
\hline Composite of 1 or 2 or 3 & $10(50)$ & $11(55)$ & $15(71.4)$ & 0.343 \\
\hline
\end{tabular}

Values are N (\%). P values by Chi-square test. All Serious AEs were represented within the grade 3-5 AE row and already included in the composite endpoint 
Figure 1. CONSORT diagram

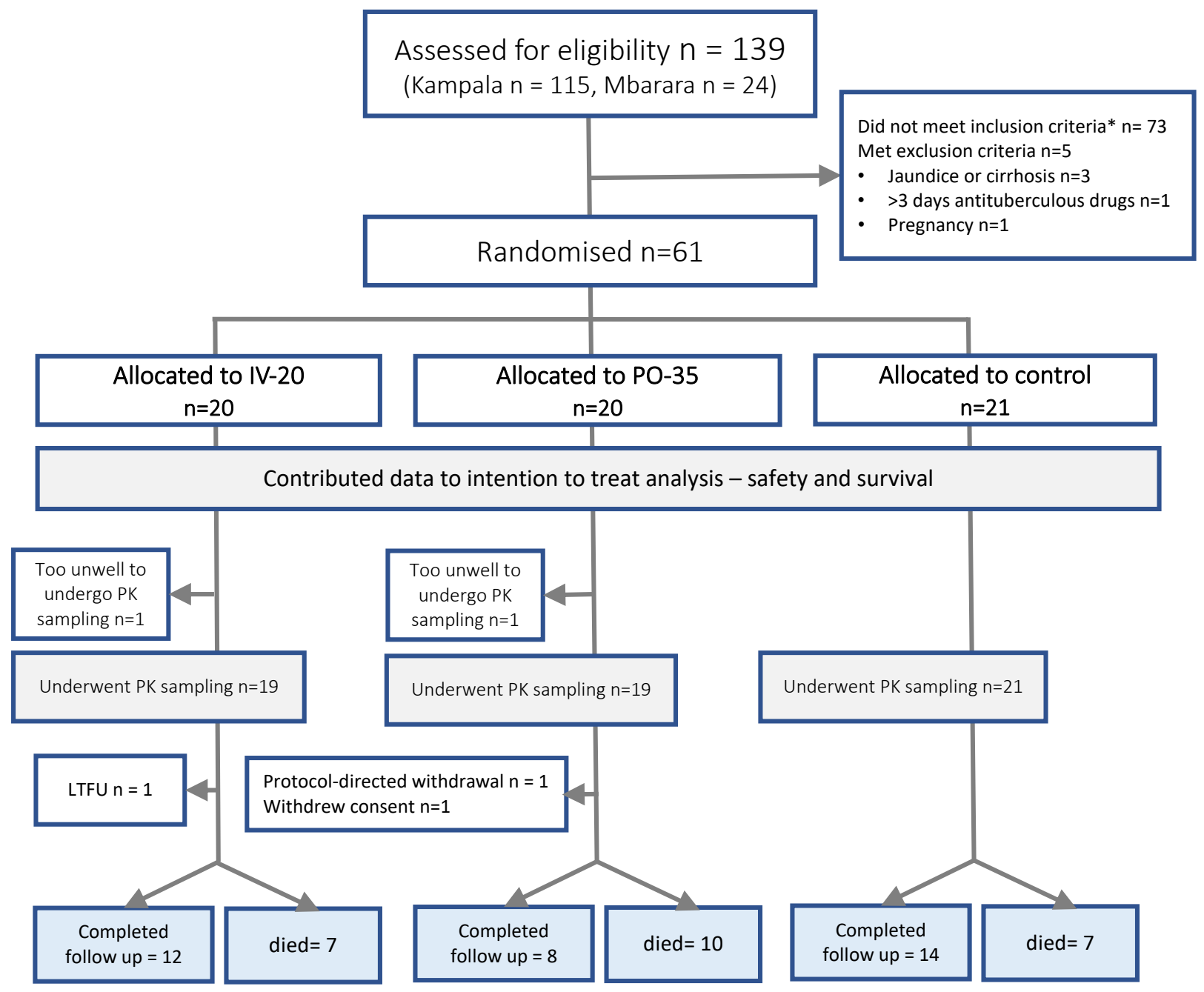

Footnotes:

* Reasons inclusion criteria not met were: research medical officer deemed that the clinical picture was not suggestive of TB meningitis and/or CSF Xpert Ultra was negative and the CSF glucose was not below the inclusion threshold (CSF:plasma ratio $<50 \%$ or CSF glucose $<65 \mathrm{mg} / \mathrm{dl}$ or $<3.6 \mathrm{mmol}$ )

Participants who were LTFU, withdrew consent or withdrawn were censored at their time of last contact

- One LTFU occurred on day 3 when the participant left hospital against medical advice

- One protocol-directed withdrawal was for baseline ALT >3x ULN (withdrawn on day 2, PK visit completed)

- One participant withdrew consent for further follow-up on day 7 after transferring to another facility 
Figure 2 a-c. Pharmacokinetic parameters on day 2

Distribution of rifampicin a) Area under the concentration-time curve from 0 to 24 hours post-dose $\left(A \cup C_{0-24}\right)$, b) maximum concentration in plasma ( $C_{\text {max }}$ ), c) CSF concentration $\left(\mathrm{C}_{\mathrm{CSF}}\right)$, the horizontal red line is the $\mathrm{M}$. tuberculosis Uganda II MIC of $1 \mathrm{mg} / \mathrm{L}$. The $\mathrm{x}$ axis shows the three trial arms: intravenous rifampicin $20 \mathrm{mg} / \mathrm{kg}$, oral rifampicin $35 \mathrm{mg} / \mathrm{kg}$, control rifampicin $10 \mathrm{mg} / \mathrm{kg}$, in combination with standard doses of isoniazid, pyrazinamide, ethambutol and corticosteroids. Red dashed line represents geometric mean concentrations.

a)

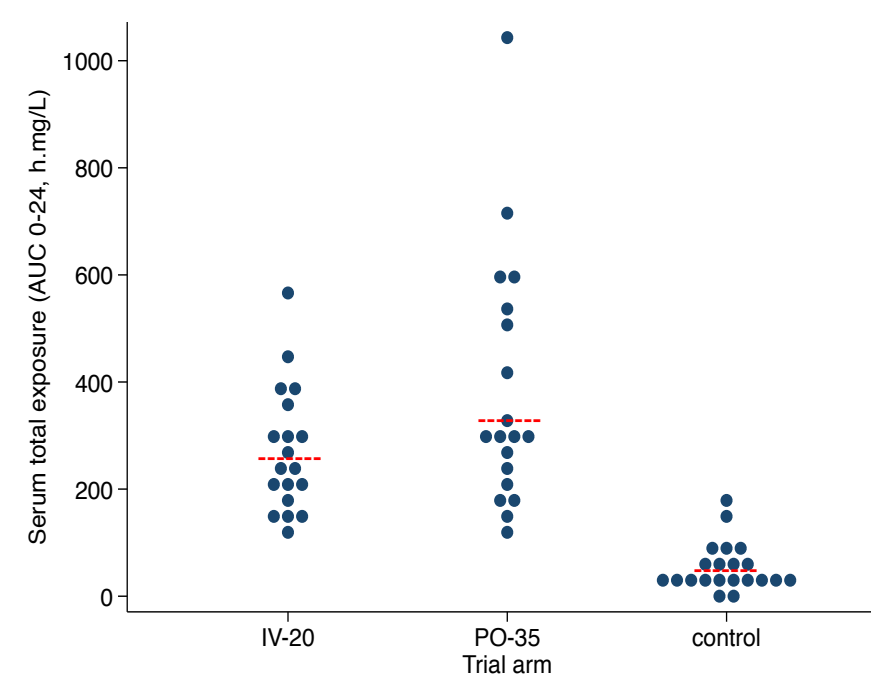

b)

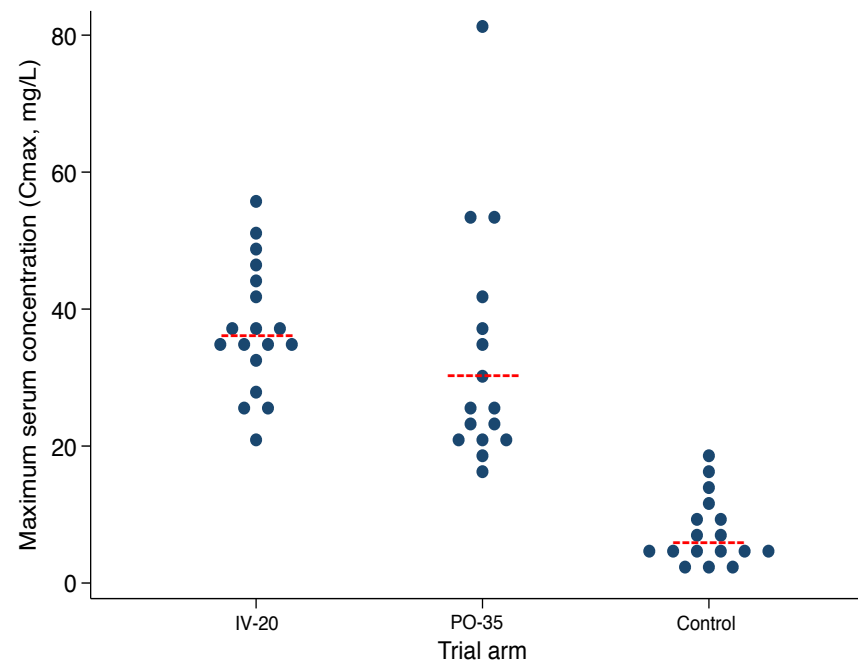

c)

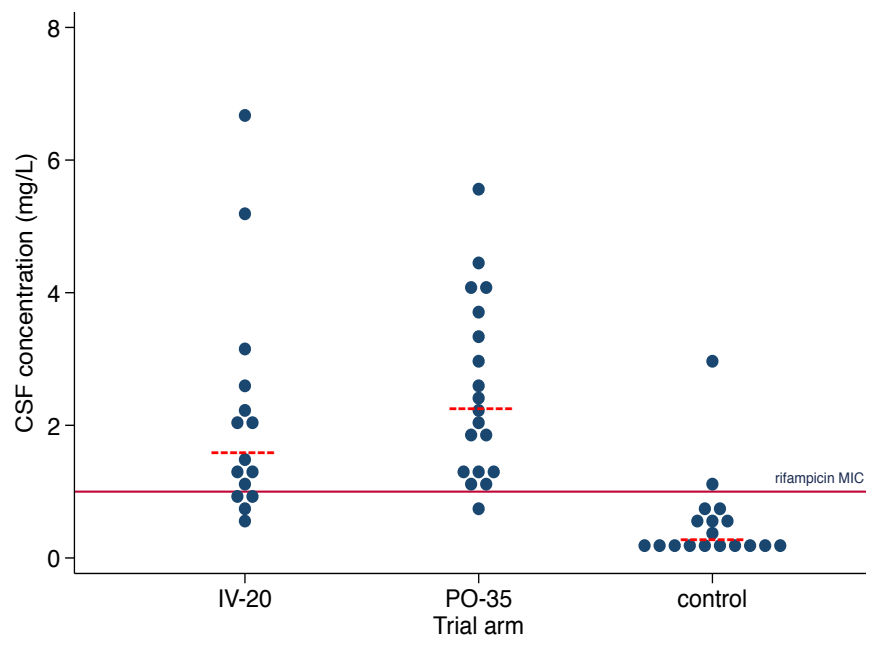


Figure 3. Kaplan-Meier survival

Survival by trial arm. Follow-up time is 24 weeks. Withdrawals $(n=2)$ and loss to follow up $(n=1)$ were censored at their last time of contact. P value by log rank test. The study was not powered for survival, and thus mortality was a secondary endpoint.

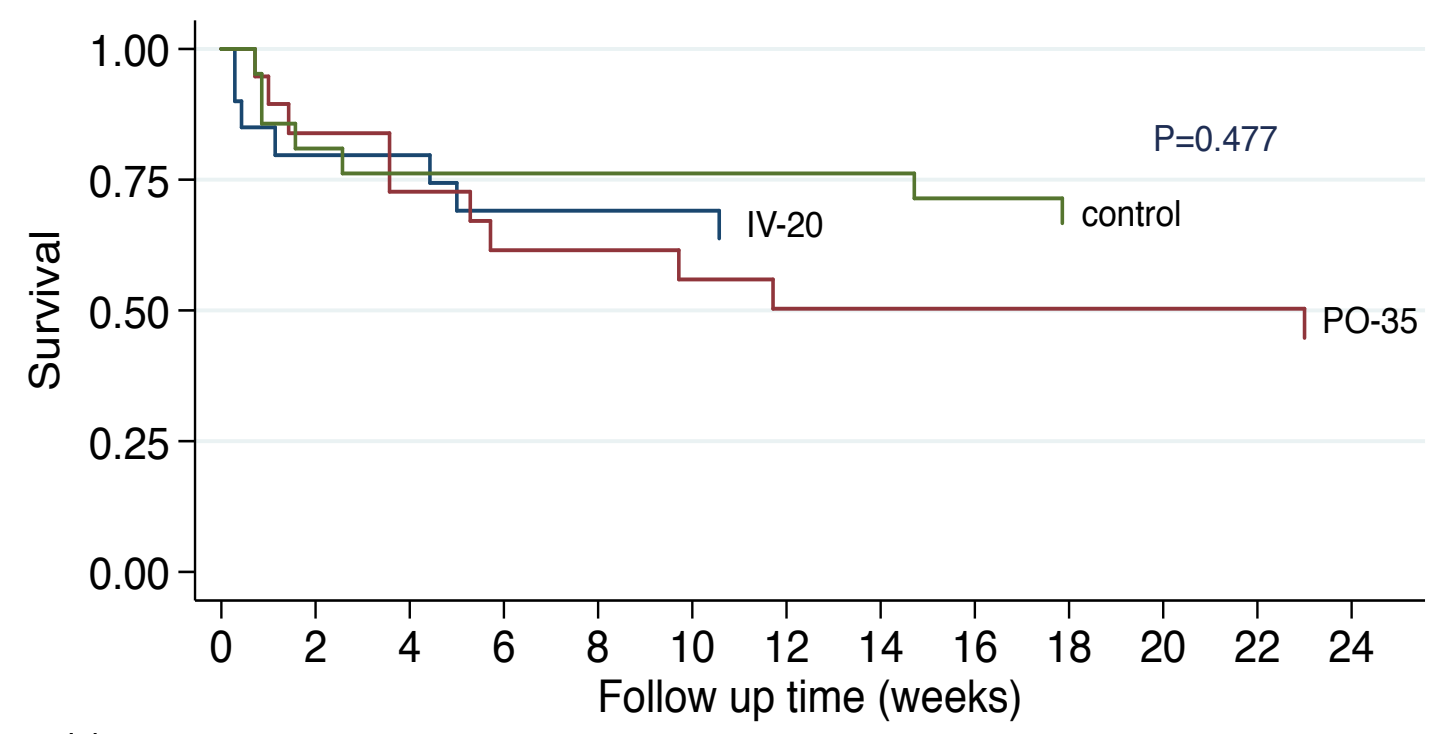

Number at risk

$\begin{array}{rrrrccc}\text { IV-20 } & 20 & 14 & 13 & 12 & 12 & 12 \\ \text { PO-35 } & 20 & 13 & 10 & 9 & 9 & 8 \\ \text { control } & 21 & 16 & 16 & 15 & 14 & 14\end{array}$




\section{Supplementary material}

Figure 1. HIV antiretroviral therapy (ART) status. The blue segments represent participants with recent HIV diagnoses (within 3 months of trial enrolment). The yellow segments represent participants with HIV for greater than 3 months. TLE=tenofovir, lamivudine, efavirenz, TLD=tenofovir, lamivudine, dolutegravir, ATZ/r = atazanavir/ritonavir

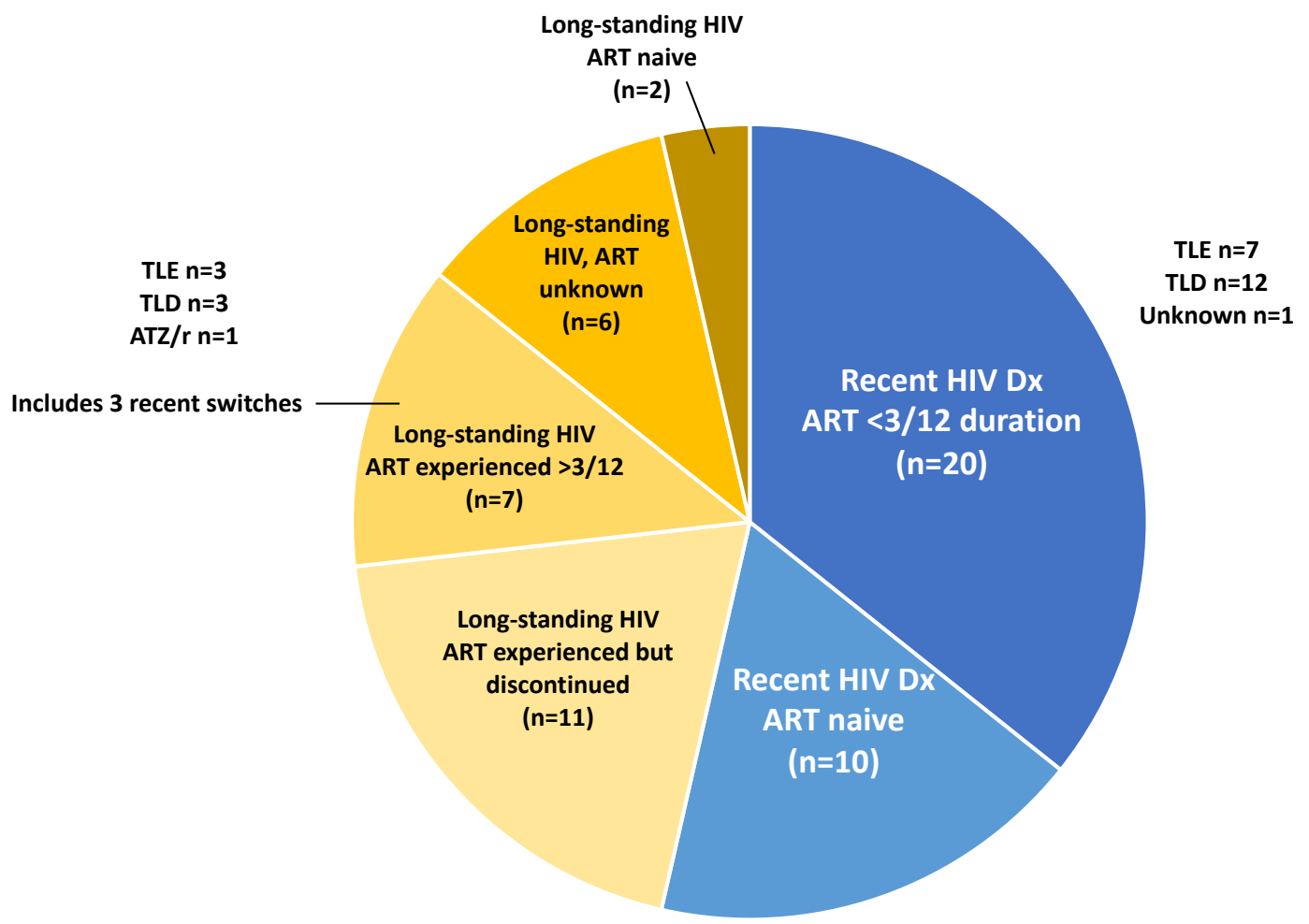


Table 1a-c. Dosing of rifampicin by trial arm

$\mathrm{R}=$ rifampicin $\mathrm{H}=$ isoniazid $\mathrm{Z}=$ pyrazinamide $\mathrm{E}=$ ethambutol

a) Intravenous rifampicin dosing by body weight

\begin{tabular}{|c|c|c|c|c|c|}
\hline Weight (Kg) & $\begin{array}{l}\text { Rifampicin } \\
\text { dose (mg) }\end{array}$ & \multirow{22}{*}{ 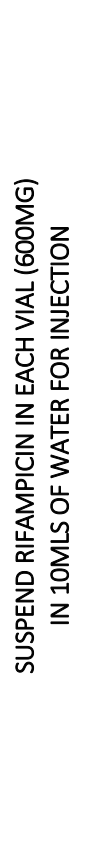 } & $\begin{array}{l}\text { Volume } \\
\text { used }(m L)\end{array}$ & $\begin{array}{l}\text { Number of } \\
\text { vials used }\end{array}$ & \multirow{22}{*}{ 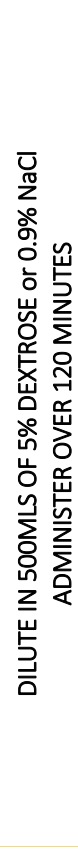 } \\
\hline $28.5-31.49$ & 600 & & 10 & 1.0 & \\
\hline $31.5-34.49$ & 660 & & 11 & 1.1 & \\
\hline $34.5-37.49$ & 720 & & 12 & 1.2 & \\
\hline $37.5-40.49$ & 780 & & 13 & 1.3 & \\
\hline $40.5-43.49$ & 840 & & 14 & 1.4 & \\
\hline $43.5-46.49$ & 900 & & 15 & 1.5 & \\
\hline $46.5-49.49$ & 960 & & 16 & 1.6 & \\
\hline $49.5-52.49$ & 1020 & & 17 & 1.7 & \\
\hline $52.5-55.49$ & 1080 & & 18 & 1.8 & \\
\hline $55.5-58.49$ & 1140 & & 19 & 1.9 & \\
\hline $58.5-61.49$ & 1200 & & 20 & 2.0 & \\
\hline $61.5-64.49$ & 1260 & & 21 & 2.1 & \\
\hline $64.5-67.49$ & 1320 & & 22 & 2.2 & \\
\hline $67.5-70.49$ & 1380 & & 23 & 2.3 & \\
\hline $70.5-73.49$ & 1440 & & 24 & 2.4 & \\
\hline $73.5-76.49$ & 1500 & & 25 & 2.5 & \\
\hline $76.5-79.49$ & 1560 & & 26 & 2.6 & \\
\hline $79.5-82.49$ & 1620 & & 27 & 2.7 & \\
\hline $82.5-85.49$ & 1680 & & 28 & 2.8 & \\
\hline $85.5-88.49$ & 1740 & & 29 & 2.9 & \\
\hline $88.5-91.49$ & 1800 & & 30 & 3.0 & \\
\hline
\end{tabular}

b) Rifampicin dosing in high dose oral rifampicin arm

\begin{tabular}{|l|c|c|c|}
\hline Weight & $\begin{array}{c}\text { Number of RHZE tabs } \\
(\mathbf{1 5 0} / 75 / 400 / 275 \mathbf{~ m g})\end{array}$ & $\begin{array}{c}\text { Additional R 300mg } \\
\text { tablets }\end{array}$ & $\begin{array}{c}\text { Total R dose } \\
(\mathbf{m g})\end{array}$ \\
\hline $30-37 \mathrm{~kg}$ & 2 tabs & 3 tabs & 1200 \\
\hline $38-54 \mathrm{~kg}$ & 3 tabs & 4 tabs & 1650 \\
\hline $55-70 \mathrm{~kg}$ & 4 tabs & 5 tabs & 2100 \\
\hline$\geq 71 \mathrm{~kg}$ & 5 tabs & 6 tabs & 2550 \\
\hline
\end{tabular}

c) Rifampicin dosing in the control arm

\begin{tabular}{|c|c|}
\hline Weight & $\begin{array}{l}\text { Number of RHZE tablets ( } 150 \text { / } \\
75 \text { / } 400 \text { / } 275 \text { mg) }\end{array}$ \\
\hline $30-37 \mathrm{~kg}$ & 2 tabs \\
\hline $38-54 \mathrm{~kg}$ & 3 tabs \\
\hline $55-70 \mathrm{~kg}$ & 4 tabs \\
\hline$\geq 71 \mathrm{~kg}$ & 5 tabs \\
\hline
\end{tabular}




\section{Determination of Rifampicin concentration in plasma and CSF using HPLC-UV}

An in-house high-performance liquid chromatography with ultraviolet detection (HPLC UV) was used to determine the concentration of rifampicin in human plasma. Rifampicin (RIF) was extracted from 1 $\mathrm{ml}$ of plasma after addition of rifapentine (internal standard) using solid phase extraction. HPLC analysis was carried out on a Shimadzu LC-2010C HT system (Kyoto, Japan) equipped with a controller, a quaternary gradient pump, an ultraviolet detector, an autosampler, a column oven, an on-line degasser and was remotely operated by class VP software. The analytical column was a Phenyl-Hexyl, $150 \mathrm{~mm} \times 3 \mathrm{~mm}$ ID, $3 \mu \mathrm{m}$ particle size (Thermoscientific, USA). The mobile phase comprised methanol (eluent1), acetonitrile (eluent2) and a mixture of $10 \mathrm{mM}$ ammonium acetate buffer ( $\mathrm{pH} 5.0$ adjusted with acetic acid)/acetonitrile 98/2 (eluent3) using a gradient elution program. 5\% methanol(eluent1) was pumped constantly over the total run time. The gradient started with $55 \%$ eluent 2 in 27 minutes, then $95 \%$ eluent 2 in 0.5 minutes where it was maintained for 7.5 minutes. The column was conditioned with $5 \%$ eluent 2 for five minutes before the next injection. The injection volume was 10 $\mu \mathrm{L}$. The UV detector was set at $254 \mathrm{~nm}$ over the run time of 40 minutes. The flow rate was $0.45 \mathrm{ml} / \mathrm{min}$ at $40^{\circ} \mathrm{C}$. Unknown concentrations were derived from linear regression analysis of the peak area ratios (analyte/internal standard) vs. concentration curve. The linearity was verified using estimates of correlation coefficient $(r)$. The calibration curve for rifampicin in plasma covered a range from 0.25 to $15 \mathrm{mg} / \mathrm{L}$.

The method was validated according to the published FDA guidelines ${ }^{1}$. The average recovery of RIF from plasma was $100 \%$ while that of the internal standard was $104 \%$. The within batch precision(\%CV) of quality control samples and for dilutions of samples above the upper limit of quantification was 2.88 to $3.57 \%$, and the batch to batch precision was 3.41 to $4.78 \%$. Accuracy was $94-102 \%$. The samples were stable for at least 6hrs on the bench and $48 \mathrm{hrs}$ in the auto sampler. There was no interference with endogenous compound in the 5 plasma samples of volunteers tested. No interference with nevirapine, efavirenz, lopinavir, atazanavir, darunavir, etravirine, rifampicin, isoniazid, pyrazinamide, ethambutol, carbamazepine, phenytoin, saquinavir, vancomycin, ceftriaxone, tenofovir, amikacin, gentamycin, methotrexate, carboplatin and cefotaxime.

Quality control samples spiked in CSF were run in five folds each and analyzed using calibrators spiked in plasma. The deviation from the expected value was between $0.1-15 \%$.

1. US FDA D. Bioanalytical method validation guidance for industry. 2018. 
Table 2. Individualised AE details. This table shows all the AEs in the 24-week follow up period. The safety endpoint was based on AEs during the intervention period (week 0-8), these are represented in aggregate in the trial paper Table 3.

\begin{tabular}{|c|c|c|c|c|c|c|c|c|c|c|c|c|}
\hline $\begin{array}{c}\text { Study } \\
\text { arm }\end{array}$ & ID & Site & $\begin{array}{l}\text { AE start } \\
\text { date }\end{array}$ & Day & Event & Diagnosis & Grade & SAE & $\begin{array}{l}\text { Relationship to } \\
\text { study drug }\end{array}$ & $\begin{array}{c}\text { Relationship to } \\
\text { HIV }\end{array}$ & $\begin{array}{l}\text { Relationship to } \\
\text { TBM }\end{array}$ & Outcome of $A E$ \\
\hline IV-20 & 110038 & Kampala & $16 / 02 / 2019$ & 8 & respiratory distress & aspiration & 3 & no & not related & probably related & probably related & persistent, expect to resolve \\
\hline IV-20 & 110038 & Kampala & $22 / 04 / 2019$ & 73 & seizures & $\begin{array}{l}\text { generalized } \\
\text { seizures }\end{array}$ & 5 & yes & not related & probably related & definitely related & death, unrelated \\
\hline IV-20 & 110038 & Kampala & 25/03/2019 & 45 & seizures & $\begin{array}{l}\text { generalized } \\
\text { seizures }\end{array}$ & 3 & yes & not related & definitely related & definitely related & resolved \\
\hline IV-20 & 110038 & Kampala & $16 / 02 / 2019$ & 8 & seizures & partial seizures & 3 & no & not related & probably related & probably related & persistent, expect to resolve \\
\hline IV-20 & 110065 & Kampala & $13 / 03 / 2019$ & 1 & stroke & TBM & 5 & yes & not related & definitely related & definitely related & death, unrelated \\
\hline IV-20 & 110102 & Kampala & $15 / 04 / 2019$ & 0 & altered mental status & TBM & 5 & yes & not related & probably related & definitely related & death, unrelated \\
\hline IV-20 & 110135 & Kampala & $27 / 05 / 2019$ & 0 & stroke & stroke & 5 & yes & not related & not related & possibly related & severity worsened to grade 5 \\
\hline IV-20 & 110193 & Kampala & $15 / 09 / 2019$ & 39 & dysphagia & candidiasis & 3 & yes & not related & definitely related & not related & resolved \\
\hline IV-20 & 110220 & Kampala & $30 / 08 / 2019$ & 1 & altered mental status & TBM & 5 & yes & not related & definitely related & definitely related & severity worsened to grade 5 \\
\hline IV-20 & 110326 & Kampala & $05 / 12 / 2019$ & 2 & elevated creatinine & acute kidney injury & 3 & no & not related & possibly related & possibly related & resolved \\
\hline IV-20 & 110327 & Kampala & $11 / 12 / 2019$ & 8 & elevated ALT & DILI & 3 & no & $\begin{array}{l}\text { probably } \\
\text { related }\end{array}$ & possibly related & possibly related & resolved \\
\hline IV-20 & 110332 & Kampala & 08/01/2020 & 29 & low sodium & TBM & 4 & yes & not related & not related & probably related & severity worsened to grade 5 \\
\hline IV-20 & 120089 & Mbarara & $11 / 10 / 2019$ & 9 & low haemoglobin & diarrhoea & 4 & no & not related & probably related & not related & resolved \\
\hline IV-20 & 120089 & Mbarara & $30 / 10 / 2019$ & 28 & hypotension & sepsis & 5 & yes & possibly related & possibly related & not related & severity worsened to grade 5 \\
\hline IV-20 & 120089 & Kampala & $31 / 10 / 2019$ & 29 & bilirubin, high & Other & 4 & no & $\begin{array}{c}\text { probably } \\
\text { related }\end{array}$ & possibly related & possibly related & chronic, not expect to resolve \\
\hline PO-35 & 110014 & Kampala & $21 / 01 / 2019$ & 0 & elevated creatinine & acute kidney injury & 4 & no & not related & not related & probably related & resolved \\
\hline PO-35 & 110014 & Kampala & 28/01/2019 & 7 & elevated ALT & DILI & 3 & no & possibly related & possibly related & possibly related & resolved \\
\hline PO-35 & 110071 & Kampala & $25 / 04 / 2019$ & 35 & abdominal pain & Other & 5 & yes & not related & not related & possibly related & severity worsened to grade 5 \\
\hline PO-35 & 110071 & Kampala & 29/03/2019 & 8 & $\begin{array}{l}\text { urinary tract } \\
\text { obstruction }\end{array}$ & Other & 3 & no & not related & not related & not related & persistent, expect to resolve \\
\hline PO-35 & 110124 & Kampala & $24 / 05 / 2019$ & 11 & hearing loss & hearing loss & 4 & no & not related & possibly related & definitely related & chronic, not expect to resolve \\
\hline PO-35 & 110145 & Kampala & $19 / 07 / 2019$ & 37 & fever & TBM & 5 & no & not related & probably related & definitely related & severity worsened to grade 5 \\
\hline PO-35 & 110188 & Kampala & 08/01/2020 & 160 & dysphagia & KS disseminated & 5 & yes & not related & not related & definitely related & severity worsened to grade 5 \\
\hline PO-35 & 110214 & Kampala & 03/09/2019 & 12 & low sodium & TBM & 3 & no & not related & probably related & definitely related & chronic, not expect to resolve \\
\hline PO-35 & 110214 & Kampala & $15 / 09 / 2019$ & 24 & seizures & TBM & 5 & yes & not related & probably related & definitely related & severity worsened to grade 5 \\
\hline PO-35 & 110230 & Kampala & $14 / 09 / 2019$ & 4 & altered mental status & TBM & 5 & yes & not related & definitely related & definitely related & severity worsened to grade 5 \\
\hline PO-35 & 110269 & Kampala & 20/10/2019 & 4 & stroke & stroke & 4 & yes & not related & probably related & definitely related & chronic, not expect to resolve \\
\hline PO-35 & 110269 & Kampala & $24 / 10 / 2019$ & 8 & fever & drug fever & 4 & no & possibly related & possibly related & possibly related & resolved \\
\hline PO-35 & 110269 & Kampala & $11 / 11 / 2019$ & 26 & low haemoglobin & Other & 3 & no & not related & probably related & probably related & persistent, expect to resolve \\
\hline PO-35 & 110291 & Kampala & $07 / 04 / 2020$ & 154 & vomitting & liver cirrhosis & 4 & yes & not related & not related & not related & chronic, not expect to resolve \\
\hline
\end{tabular}




\begin{tabular}{|c|c|c|c|c|c|c|c|c|c|c|c|c|}
\hline PO-35 & 110316 & Kampala & 28/11/2019 & 6 & rash & KS disseminated & 5 & yes & not related & definitely related & definitely related & severity worsened to grade 5 \\
\hline PO-35 & 110333 & Kampala & $16 / 12 / 2019$ & 6 & elevated ALT & DILI & 3 & no & possibly related & possibly related & probably related & resolved \\
\hline PO-35 & 120099 & Mbarara & 10/11/2019 & 6 & respiratory distress & $\begin{array}{l}\text { pneumonia, } \\
\text { bacterial }\end{array}$ & 5 & yes & not related & definitely related & definitely related & death, unrelated \\
\hline PO-35 & 120113 & Mbarara & 24/11/2019 & 3 & seizures & partial seizures & 3 & no & not related & possibly related & probably related & resolved \\
\hline PO-35 & 120113 & Mbarara & 29/11/2019 & 8 & respiratory distress & $\begin{array}{l}\text { pneumocystis } \\
\text { pneumonia }\end{array}$ & 5 & yes & not related & probably related & probably related & severity worsened to grade 5 \\
\hline Control & 110061 & Kampala & 28/03/2019 & 21 & elevated ALT & DILI & 3 & no & possibly related & not related & not related & resolved \\
\hline Control & 110061 & Kampala & 02/05/2019 & 56 & rash & KS disseminated & 3 & no & not related & definitely related & not related & persistent, expect to resolve \\
\hline Control & 110061 & Kampala & 29/05/2019 & 83 & bilirubin, high & DILI & 4 & no & possibly related & possibly related & possibly related & resolved \\
\hline Control & 110087 & Kampala & 06/04/2019 & 4 & stroke & stroke & 5 & no & not related & probably related & definitely related & severity worsened to grade 5 \\
\hline Control & 110109 & Kampala & 28/04/2019 & 3 & altered mental status & TBM & 5 & yes & not related & probably related & definitely related & severity worsened to grade 5 \\
\hline Control & 110144 & Kampala & 28/06/2019 & 16 & seizures & $\begin{array}{l}\text { generalized } \\
\text { seizures }\end{array}$ & 4 & yes & not related & possibly related & probably related & resolved \\
\hline Control & 110144 & Kampala & $24 / 06 / 2019$ & 12 & $\begin{array}{l}\text { neurosensory } \\
\text { alteration }\end{array}$ & TBM & 3 & no & not related & possibly related & not related & persistent, expect to resolve \\
\hline Control & 110144 & Kampala & 01/08/2019 & 50 & respiratory distress & anaemia & 3 & yes & not related & possibly related & probably related & persistent, expect to resolve \\
\hline Control & 110152 & Kampala & 29/06/2019 & 4 & respiratory distress & $\begin{array}{l}\text { pneumonia, } \\
\text { bacterial }\end{array}$ & 3 & no & not related & probably related & probably related & resolved \\
\hline Control & 110159 & Kampala & 05/08/2019 & 34 & headache & $\begin{array}{c}\text { cryptococcal } \\
\text { meningitis }\end{array}$ & 3 & yes & not related & definitely related & not related & resolved \\
\hline Control & 110177 & Kampala & 10/09/2019 & 50 & elevated ALT & DILI & 3 & no & possibly related & possibly related & possibly related & resolved \\
\hline Control & 110195 & Kampala & 06/10/2019 & 55 & fever & sepsis & 3 & yes & not related & possibly related & not related & resolved with sequelae \\
\hline Control & 110195 & Kampala & 07/10/2019 & 60 & bilirubin, high & Other & 4 & no & $\begin{array}{l}\text { probably } \\
\text { related }\end{array}$ & possibly related & possibly related & resolved \\
\hline Control & 110195 & Kampala & $22 / 11 / 2019$ & 102 & respiratory distress & Other & 5 & yes & not related & probably related & probably related & death, unrelated \\
\hline Control & 110228 & Kampala & 11/09/2019 & 2 & elevated creatinine & acute kidney injury & 3 & no & not related & definitely related & definitely related & chronic, not expect to resolve \\
\hline Control & 110228 & Kampala & 09/09/2019 & 0 & altered mental status & TBM & 5 & yes & not related & probably related & definitely related & severity worsened to grade 5 \\
\hline Control & 110250 & Kampala & 15/10/2019 & 13 & elevated ALT & DILI & 4 & no & possibly related & possibly related & probably related & resolved \\
\hline Control & 110251 & Kampala & 27/11/2019 & 57 & bilirubin, high & Other & 4 & no & $\begin{array}{l}\text { probably } \\
\text { related }\end{array}$ & possibly related & possibly related & resolved \\
\hline Control & 110287 & Kampala & 04/12/2019 & 34 & elevated ALT & DILI & 3 & no & possibly related & possibly related & possibly related & resolved \\
\hline Control & 110330 & Kampala & $06 / 04 / 2020$ & 123 & heart failure & heart failure & 5 & yes & not related & not related & not related & severity worsened to grade 5 \\
\hline Control & 110330 & Kampala & $27 / 12 / 2019$ & 22 & fever & Other & 3 & yes & not related & probably related & probably related & resolved \\
\hline Control & 110330 & Kampala & $04 / 02 / 2020$ & 61 & fever & Other & 3 & yes & ed & ated & not related & $\begin{array}{l}\text { persistent, expected to } \\
\text { resolve }\end{array}$ \\
\hline Control & 110344 & Kampala & $21 / 12 / 2019$ & 4 & seizures & TBM & 5 & yes & not related & definitely related & definitely related & severity worsened to grade 5 \\
\hline Control & 120108 & Mbarara & 03/12/2019 & 15 & thrombosis & $\begin{array}{l}\text { deep vein } \\
\text { thrombosis }\end{array}$ & 3 & no & not related & possibly related & possibly related & persistent, expect to resolve \\
\hline Control & 120108 & Mbarara & 22/11/2019 & 4 & respiratory distress & aspiration & 5 & no & not related & not related & possibly related & severity worsened to grade 5 \\
\hline
\end{tabular}


Figure 2. Liver function test trajectories in participants who experienced drug-induced liver injury
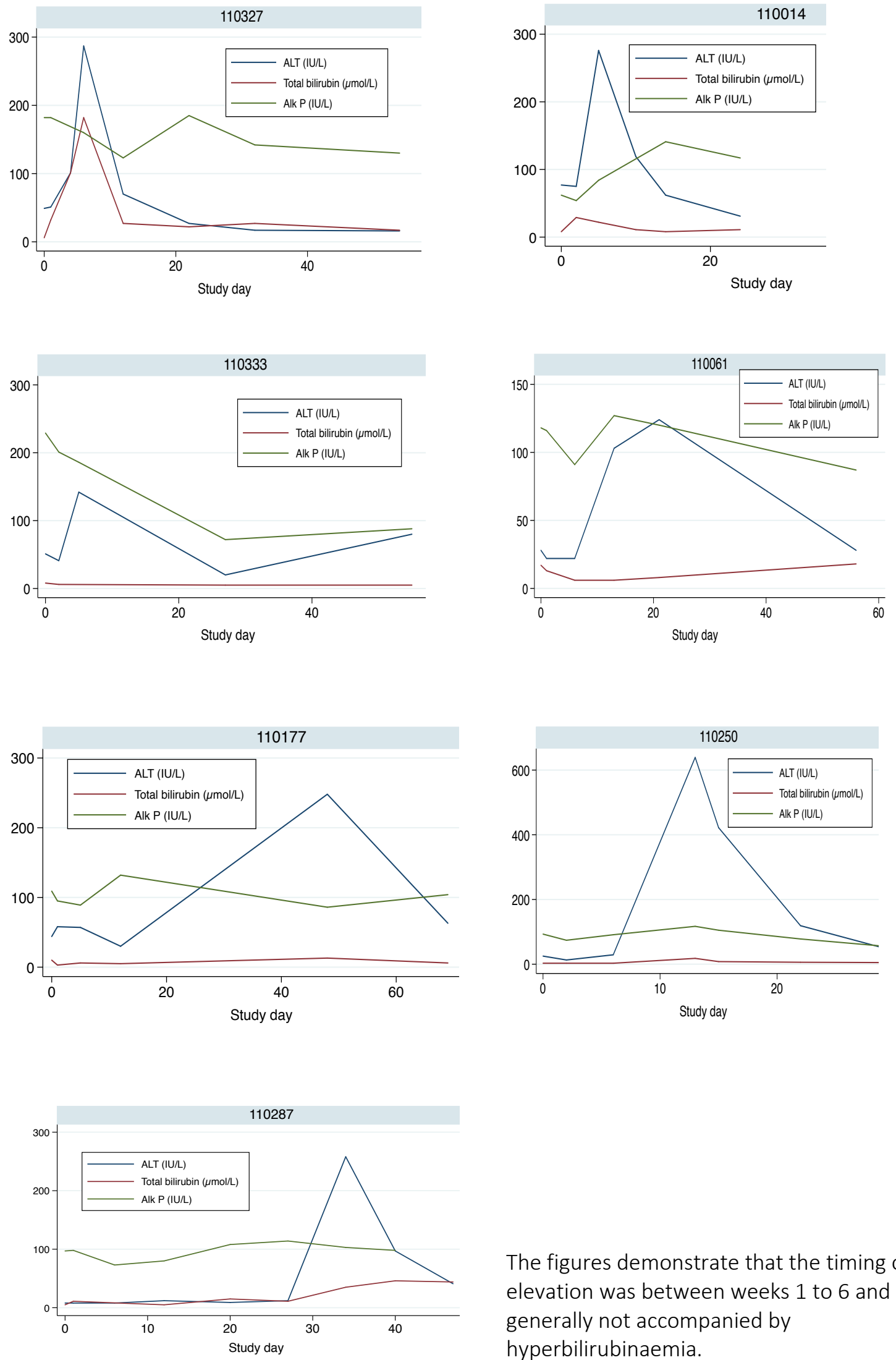

The figures demonstrate that the timing of ALT elevation was between weeks 1 to 6 and was generally not accompanied by hyperbilirubinaemia. 
Figure 3. Adverse event competing risks regressions

Time to experiencing an adverse event (AE) was compared between treatment arms using cumulative incidence functions and a Cox proportional hazards regression model. Death was considered a competing risk. There was no difference in time to experiencing an AE when comparing the intervention arms to the control: IV-20 sub-distribution hazard ratio (SHR) $0.734(95 \% \mathrm{Cl}, 0.33-1.65$, $\mathrm{p}=0.456)$ and $\mathrm{PO}-351.04(95 \% \mathrm{Cl}, 0.53-2.05, \mathrm{p}=0.909)$.

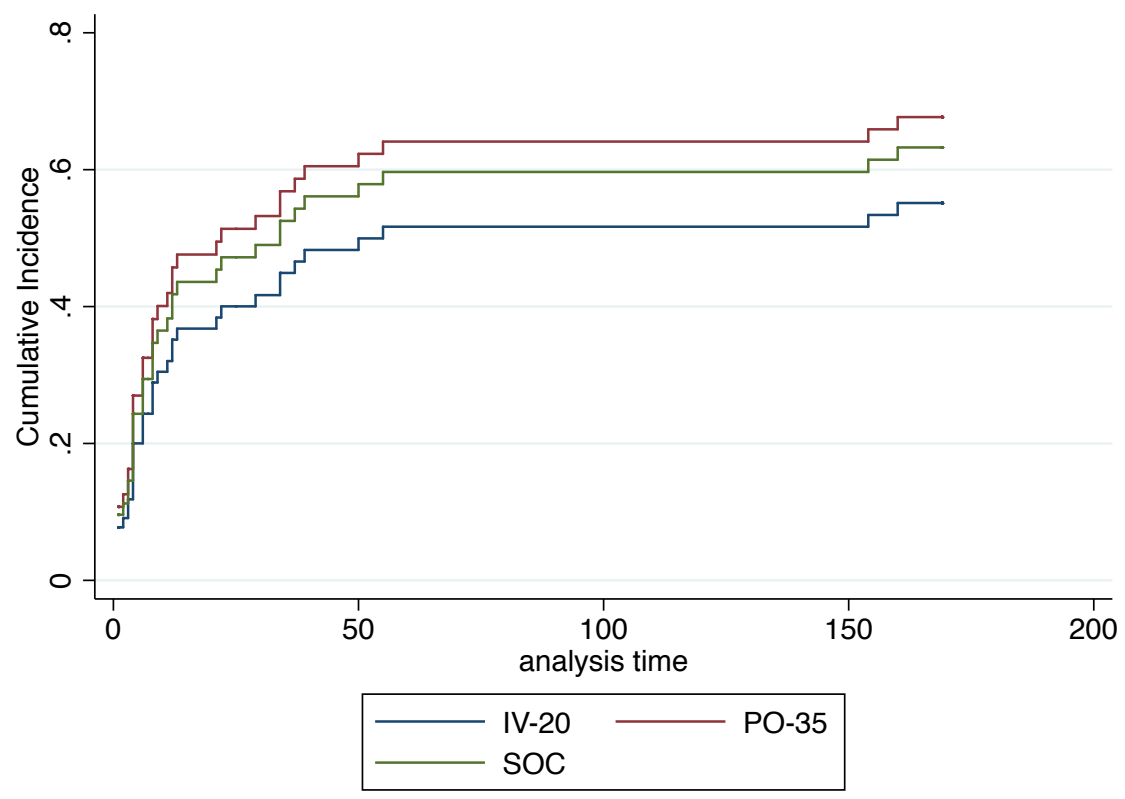

Figure 4. Random effects linear mixed model of Glasgow coma scale score by study day.

In the linear mixed model of GCS profiles, GCS increased by $0.13(95 \% \mathrm{Cl}, 0.07-0.18 ; \mathrm{p}=<0.001)$ per day, with no evidence of a difference by arm $(p=0.953)$.

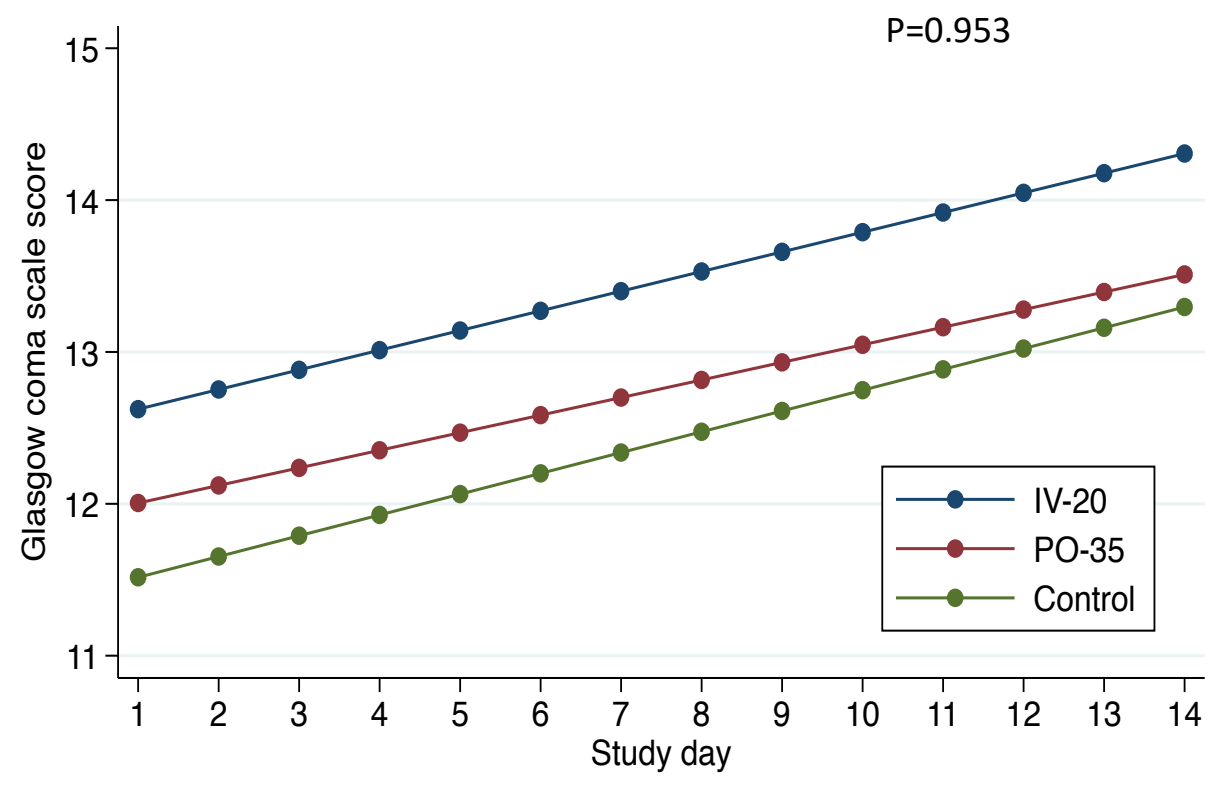




\section{Chapter Nine: A Journey of Hope: giving research participants a voice to share their experiences and improve community engagement around advanced HIV disease in Uganda (research paper)}

\section{Foreword}

Public and community engagement is increasingly recognised as an important part of research activities. Three broad and often overlapping purposes of public engagement are defined as:

1. Transmit - to inspire and inform, change, educate, build capacity and involvement, influence decisions.

2. Collaborate - to collaborate, consider, create or decide something together

3. Receive - to use the views, skills, experience of the public to inspire and inform our own capacity

It is important that public engagement attempts to encompass all three purposes to avoid it becoming a one directional broadcast of a message which may be disconnected with what the community want or need. I believe that our research participants are the greatest asset for community engagement - they have experienced the disease first-hand, they understand the challenges in accessing care that led to them having advanced HIV disease in the first place, they know how to communicate at a level that is accessible and meaningful for the public. As such I endeavoured to include research participants and other community members in all aspects of planning, creation and delivery of the engagement events.

I was successful in securing three small grants to support public engagement activities, the first from LSHTM small grants for public engagement, the second from Makerere University Infection and Immunity Centre of Excellence and the third an LSHTM Continued Development Public Engagement award. Thereafter, I considered the audience with which it was important to engage, not only the community but also with healthcare workers and other stakeholders in the HIV field. The IDI community advisory board were instrumental in thinking about engagement methods and collaboration to maximise the impact of the activities.

As a team we delivered a series of multifaceted events over a 14-month period, which have been described further in an open letter in African Academy of Sciences Open Research. The messages and events have also been summarised in a short video which can be accessed at https://www.youtube.com/watch?v=u0tar-jArPI\&feature=youtu.be 



\section{RESEARCH PAPER COVER SHEET}

\section{PLEASE NOTE THAT A COVER SHEET MUST BE COMPLETED FOR EACH RESEARCH PAPER INCLUDED IN A THESIS.}

\section{SECTION A - Student Details}

\begin{tabular}{|l|l|}
\hline Student & Fiona Cresswell \\
\hline Principal Supervisor & Alison Elliott \\
\hline Thesis Title & $\begin{array}{l}\text { Improving diagnosis and treatment of HIV-associated } \\
\text { Tuberculous meningitis }\end{array}$ \\
\hline
\end{tabular}

If the Research Paper has previously been published please complete Section $B$, if not please move to Section C

\section{SECTION B - Paper already published}

\begin{tabular}{|l|l|l|l|}
\hline Where was the work published? & African Academy of Sciences Open \\
\hline When was the work published? & 27th July 2020 \\
\hline $\begin{array}{l}\text { If the work was published prior to } \\
\text { registration for your research degree, } \\
\text { give a brief rationale for its inclusion }\end{array}$ & \multicolumn{3}{|l|}{} \\
\hline $\begin{array}{l}\text { Have you retained the copyright for the } \\
\text { work?* }\end{array}$ & Yes & $\begin{array}{l}\text { Was the work subject to } \\
\text { academic peer review? }\end{array}$ & Yes \\
\hline
\end{tabular}

*If yes, please attach evidence of retention. If no, or if the work is being included in its published format, please attach evidence of permission from the copyright holder (publisher or other author) to include this work.

\section{SECTION C - Prepared for publication, but not yet published}

\begin{tabular}{|l|l|}
\hline $\begin{array}{l}\text { Where is the work intended to be } \\
\text { published? }\end{array}$ & \\
\hline $\begin{array}{l}\text { Please list the paper's authors in the } \\
\text { intended authorship order: }\end{array}$ & \\
\hline Stage of publication & Choose an item. \\
\hline
\end{tabular}

\section{SECTION D - Multi-authored work}

For multi-authored work, give full details of your role in the research included in the paper and in the preparation of the paper. (Attach a further sheet if necessary)
I coordinated the public engagement work activities and led the writing of the paper, handled the submission and peer-reviewer comments

\section{Student Signature:}

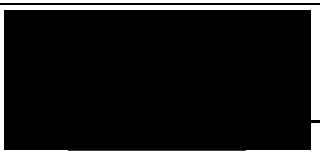

Date: 4th October 2020 
Date: 


\title{
OPEN LETTER
}

\section{REVISED A Journey of Hope: giving research participants a voice}

\section{to share their experiences and improve community}

\section{engagement around advanced HIV disease in Uganda [version}

\section{2; peer review: 1 approved, 1 approved with reservations]}

\author{
Fiona V. Cresswell (D1-3, John Kasibante1, Emily M. Martyn (D1, Lillian Tugume1, \\ Gavin Stead (iD)1, Kenneth Ssembambulidde1, Morris K. Rutakingirwa (iD), \\ Enock Kagimu (D1, Laura Nsangi1 , Carol Namuju (D1, Jane F. Ndyetukira1, \\ Cynthia Ahimbisibwe1, Florence Kugonza1 , Alisat Sadiq ${ }^{1}$, Alice Namudde1, \\ Joanna Dobbin (1D4, Diksha Srishyla5, Carson Quinn (iD), Mable Kabahubya1, \\ Conrad Muzoora(iD7, Stephen Watiti8, David B. Meya1', Alison M. Elliott2,3

\footnotetext{
${ }^{1}$ Research Department, Infectious Diseases Institute, Kampala, 22418, Uganda

${ }^{3}$ MRC UVRI LSHTM Uganda Research Unit, Entebbe, Uganda

${ }^{4}$ Department of Primary Care \& Population Health, University College London, London, UK

6University of California San Francisco Medical Centre, San Francisco, USA

${ }^{7}$ Mbarara University of Science and Technology, Mbarara, Uganda

${ }^{8}$ Mildmay, Entebbe Road, Kampala, Uganda
} \\ ${ }^{2}$ Clinical Research Department, London School of Hygiene and Tropical Medicine, London, WC1E 7HT, UK \\ 5 University of Minnesota, Minneapolis, USA
}

V2 First published: 27 Jul 2020, 3:33

https://doi.org/10.12688/aasopenres.13104.1

Latest published: 29 Oct 2020, 3:33

https://doi.org/10.12688/aasopenres.13104.2

\begin{abstract}
Over the last decade excellent progress has been made globally in HIV management thanks to antiretroviral therapy (ART) rollout and international guidelines now recommending immediate initiation of ART in people living with HIV. Despite this, advanced HIV disease (CD4 less than 200 cells $/ \mathrm{mL}$ ) and opportunistic infections remain a persistent challenge and contribute significantly to HIV-associated mortality, which equates to 23,000 deaths in Uganda in 2018 alone. Our Meningitis Research Team based in Uganda is committed to conducting clinical trials to answer important questions regarding diagnostics and management of HIV-associated opportunistic infections, including tuberculosis and cryptococcal meningitis. However, clinical research is impossible without research participants and results are meaningless unless they are translated into benefits for those affected by the disease. Therefore, we held a series of community engagement events with the aims of 1) giving research participants a voice to share their experiences of clinical research and
\end{abstract}

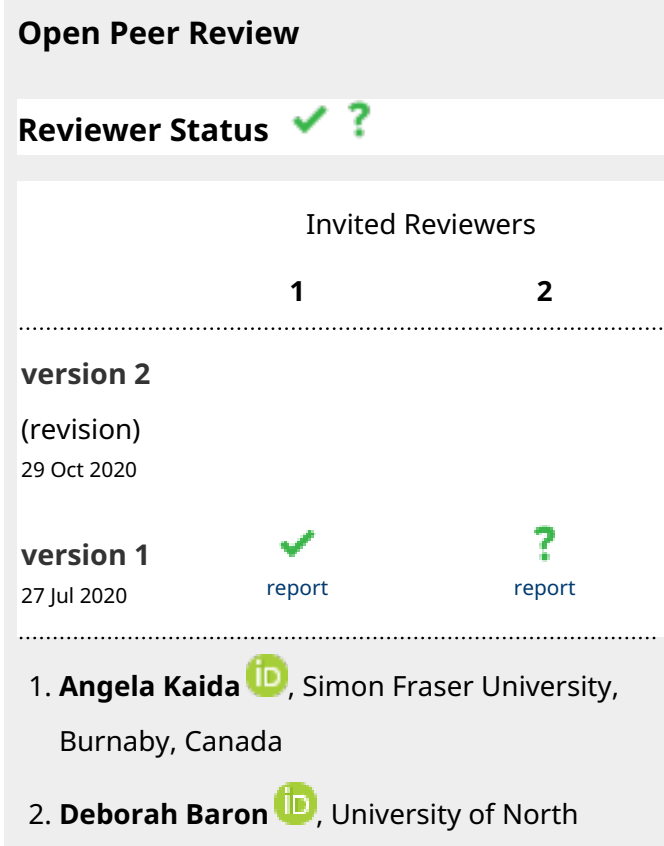


messages of hope around advanced HIV disease with the community, 2) dispelling myths and stigma around HIV, and 3) raising awareness about the complications of advanced HIV disease and local clinical research and recent scientific advances. The purpose of this Open Letter is to describe our community engagement experience in Uganda, where we aimed to give clinical research participants a greater voice to share their experiences. These activities build upon decades of work in HIV community engagement and lays a platform for future research and engagement activities.

\section{Keywords}

Public engagement, advanced HIV diseases, meningitis, clinical research

\section{Carolina, Chapel Hill, USA}

Any reports and responses or comments on the article can be found at the end of the article.

Corresponding author: Fiona V. Cresswell (fionacresswell@doctors.org.uk)

Author roles: Cresswell FV: Conceptualization, Funding Acquisition, Methodology, Project Administration, Resources, Writing - Original Draft Preparation, Writing - Review \& Editing; Kasibante J: Methodology, Project Administration, Writing - Review \& Editing; Martyn EM: Investigation, Methodology, Project Administration, Writing - Original Draft Preparation, Writing - Review \& Editing; Tugume L: Investigation, Methodology, Writing - Review \& Editing; Stead G: Investigation, Methodology, Project Administration; Ssembambulidde K: Investigation, Methodology; Rutakingirwa MK: Investigation, Methodology, Project Administration, Writing - Review \& Editing; Kagimu E: Investigation, Methodology, Project Administration, Writing - Review \& Editing; Nsangi L: Investigation, Methodology, Project Administration; Namuju C: Investigation, Methodology; Ndyetukira JF: Investigation, Methodology, Project Administration;

Ahimbisibwe C: Investigation, Methodology, Project Administration; Kugonza F: Investigation, Methodology; Sadiq A: Investigation, Methodology; Namudde A: Methodology, Project Administration; Dobbin J: Investigation, Methodology, Project Administration, Writing - Review \& Editing; Srishyla D: Investigation, Methodology; Quinn C: Investigation, Methodology, Project Administration, Writing Review \& Editing; Kabahubya M: Investigation, Methodology, Project Administration; Muzoora C: Methodology; Watiti S: Investigation, Methodology, Project Administration, Writing - Review \& Editing; Meya DB: Conceptualization, Funding Acquisition, Investigation, Methodology, Project Administration, Resources, Writing - Review \& Editing; Elliott AM: Funding Acquisition, Investigation, Methodology, Project Administration, Resources, Supervision, Writing - Review \& Editing

Competing interests: No competing interests were disclosed.

Grant information: This work was supported by the African Academy of Sciences (AAS) through a Makerere University/Uganda Virus Research Institute Infection and Immunity Centre of Excellence (MUII-plus) endeavour award. MUII-plus is supported through the Developing Excellence in Leadership, Training and Science in Africa (DELTAS Africa) Initiative [DEL-15-004], which is an independent funding scheme of the AAS's Alliance for Accelerating Excellence in Science in Africa (AESA) and supported by the New Partnership for Africa's Development Planning and Coordinating Agency (NEPAD Agency) with funding from the Wellcome Trust [107743] and the UK Government. FVC is supported by the Wellcome Trust through a Clinical PhD Fellowship [210772/Z/18/Z]. The events were funded through London School of Hygiene and Tropical Medicine (small grant for public engagement and continued development public engagement award) and Makerere University/Uganda Virus Research Institute Infection and Immunity Centre of Excellence endeavour award.

The funders had no role in study design, data collection and analysis, decision to publish, or preparation of the manuscript.

Copyright: @ 2020 Cresswell FV et al. This is an open access article distributed under the terms of the Creative Commons Attribution License, which permits unrestricted use, distribution, and reproduction in any medium, provided the original work is properly cited.

How to cite this article: Cresswell FV, Kasibante J, Martyn EM et al. A Journey of Hope: giving research participants a voice to share their experiences and improve community engagement around advanced HIV disease in Uganda [version 2; peer review: 1 approved, 1 approved with reservations] AAS Open Research 2020, 3:33 https://doi.org/10.12688/aasopenres.13104.2

First published: $27 \mathrm{Jul}$ 2020, 3:33 https://doi.org/10.12688/aasopenres.13104.1 


\section{REVISED Amendments from Version 1}

We have taken on board the reviewers constructive suggestions and modified the manuscript accordingly.

We have elaborated in more detail on who was involved in the 'transmit, receive, collaborate' part of each event.

We have added more about the rich history of HIV community engagement in Uganda

Additional citations have been added as suggested.

Any further responses from the reviewers can be found at the end of the article

\section{Introduction}

Substantial progress has been made in the treatment and prevention of HIV in the last decade thanks largely to the widespread roll out of antiretroviral treatment (ART) and recommendation to treat all people living with HIV (PLHIV) regardless of CD4 cell count. The ambitious UNAIDS "90-90-90" target states that, by 2020 , 90\% of PLHIV should be diagnosed, $90 \%$ of those diagnosed initiated on ART, and $90 \%$ of those on ART should be virally suppressed (HIV viral load $<50$ copies $/ \mathrm{ml}$ ), aiming to reach the Sustainable Development Goal of ending the HIV epidemic by 2030'. The global efforts to achieve these targets are demonstrated by a $55 \%$ decline of AIDS-related deaths between 2004 and $2018^{2}$.

While unquestionable strides in starting people on ART have been made, gains in recent years are decreasing and 23,000 people died from HIV-related illness in 2018 in Uganda alone ${ }^{2}$. This is in part due to the remaining challenge of advanced HIV disease, defined by the World Health Organisation (WHO) as having as CD4 cell count less than 200 cell $/ \mu \mathrm{L}$ or clinical stage III or IV disease ${ }^{3}$. Uganda is making good progress towards the "90-90-90" targets, with $84 \%$ aware of their HIV status, $87 \%$ of those who are HIV positive on treatment and $88 \%$ of those on treatment virally suppressed, however, stigma still acts as a barrier to seeking HIV testing and care ${ }^{4}$. Moreover, those who successfully test may not link to care early enough due to systemic barriers. Data suggest that in sub-Saharan
Africa at least one-third of people starting on ART present with advanced HIV disease, a fact which requires addressing if global targets are to be reached ${ }^{5}$.

Our Meningitis Clinical Research Team based at the Infectious Diseases Institute (IDI) Kampala and Mbarara Regional Referral Hospital Uganda, is dedicated to reducing advanced HIVassociated mortality by improving the diagnosis and treatment of common opportunistic infections, including cryptococcal and tuberculosis meningitis. Together cryptococcal disease and tuberculosis cause over half of HIV-related deaths ${ }^{6,7}$.

Public engagement seeks to overcome the disconnect between scientists and the community, making research more meaningful for the public and scientists alike. It can serve to improve uptake of research studies and can tackle suspicion about the intention of scientists. Three broad and often overlapping purposes (or pillars) of public engagement are: 1) to 'transmit' in order to inspire and inform, change, educate, build capacity and involvement, influence decisions; 2) to 'collaborate' in order to consider, create or decide something together; 3 ) to 'receive' in order to use the views, skills, experience of the public to inspire and inform our own capacity (Figure 1$)^{8}$. The specific roles of the Meningitis Clinical Research Team in 'transmitting', 'receiving' and 'collaborating' varied according to the type of outreach activity.

Globally, there is a strong history of community stakeholder engagement and activism among PLHIV. This is particularly true in Uganda, where community engagement has long been a priority of civil society groups, such as The AIDS Support Organisation (TASO) based on the Greater Involvement of People Living with HIV/AIDS (GIPA) principles ${ }^{9}$. We aimed to build on these efforts and also believe it is critical to ensure research participants have a voice to share their experiences and an opportunity to become advocates of clinical research and the condition being studied; their messages may improve the wellbeing and save the lives of others in their communities. With the support of public engagement funding through London School of Hygiene and Tropical Medicine and Makerere University/Uganda Virus Research Institute Infection and Immunity Centre of Excellence, we designed and

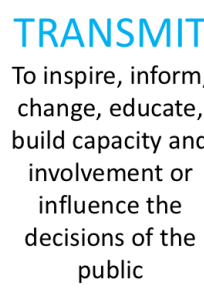

\author{
RECEIVE \\ To use views, skills, \\ experience, \\ knowledge of the \\ public to inspire, \\ inform, change \\ your own capacity \\ or decisions
}

\section{COLLABORATE \\ To collaborate, consider, create or decide something together}

Figure 1. Purposes and pillars of public engagement. 
implemented a series of multifaceted events between December 2018 and January 2020. The overarching aims included giving clinical research participants (who are survivors of advanced HIV disease) a voice in sharing their experiences of clinical research and messages of hope around advanced HIV disease with the community. This included dispelling myths and stigma around HIV, raising awareness about local ongoing clinical research in the field and recent scientific advances. We addressed our aims by engaging a variety of audiences including healthcare workers through knowledge exchange sessions, and thereafter the community and stakeholders in the HIV field through community advisory board meetings, radio, television and two community events. In this letter, using the three pillars of public engagement (transmit, collaborate and receive), we discuss the planning, conduct and outcomes of our public engagement events.

\section{Knowledge exchange with district healthcare workers.} Audience: Health care workers. Purpose: Transmit, receive and collaborate

For PLHIV presenting with symptoms of meningitis or low CD4+ $\mathrm{T}$ cell count, the WHO recommends point-of-care cryptococcal antigen testing (a rapid test for a fungal infection, which causes meningitis in immunocompromised people). However, we noted that most patients were referred on to Kiruddu and Mbarara referral hospitals at a late stage of illness, without prior lumbar puncture (a clinical procedure performed to collect cerebrospinal fluid via a needle placed in the base of the back which is essential for diagnosing meningitis, also known as 'spinal tap') or cryptococcal antigen testing. We visited 21 peripheral health centres in Kampala and 4 centres in Mbarara within the catchment area for Kiruddu and Mbarara hospitals respectively. These were mainly level IV healthcare facilities (HCIV) or district hospitals offering HIV prevention, care and treatment services to HIV-positive clients. In these interactive 'knowledge exchange' sessions the meningitis team had three aims. We transmitted information to the healthcare workers using posters, infographic leaflets and power point presentations detailing the aetiology, pathogenesis, clinical presentation, diagnostic challenges, available treatment options and opportunities that clinical trials present in improving treatment and care of PLHIV. We also 'received' and 'collaborated' with healthcare workers by discussing their experiences and challenges managing advanced HIV disease and potential solutions (e.g. helping with patient referrals and diagnostics such as providing CrAg tests) (Figure 2 and Figure 3).

An average of 40 people attended each session and the audience comprised of healthcare workers including physicians, medical officers, clinical officers, nurses, laboratory personnel, and medical students. The evaluation of these sessions was performed by informal verbal feedback and discussions with staff who attended the teaching sessions. Staff appreciated having up-to-date teaching to ensure they would recognize advanced HIV disease, cryptococcal and tuberculosis meningitis, and felt empowered to refer to the hospital. They also appreciated understanding what resources were available to ensure they could get the best care for their patients. A working relationship was established between the meningitis research team and health workers in the clinics we visited.

\section{Community and stakeholder engagement}

We recognise that challenges in healthcare seeking behaviour in advanced HIV are multifactorial, influenced by policy, healthcare systems, and community cultural, socioeconomic and geographical factors, to name but a few ${ }^{10}$. We therefore undertook a multifaceted approach to maximise community engagement and information dissemination around the Kampala region as follows.

\section{Community Advisory Board Meetings. Audience: Community representative. Purpose: Receive and collaborate}

The IDI Community Advisory Board (CAB; comprised of patient representatives, spiritual leaders, stakeholders and private sector) met with our study team on two occasions to discuss the aims and potential content of our proposed community outreach activities (Figure 4). Our aim within these meetings was to 'receive' opinions and suggestions from the CAB on our proposed outreach activities, and agree appropriate channels of information dissemination and adapting the content and language used to ensure cultural sensitivity Together, we agreed a schedule of events, including a circus event, a television and radio shows, and a 'Journey of Hope' event. The members of the $\mathrm{CAB}$ also activiely 'collaborated' in activities by attending events and a member of the $\mathrm{CAB}$ featured in the TV event.

Circus event in community center. Audience: Adults in the community who may be disengaged from HIV care. Purpose: Transmit

In this event the aim of meningitis research team was to transmit: engaging with hard-to-reach young adults who may be disengaged from HIV care or untested in order to raise awareness about meningitis, the safety of lumbar punctures and ongoing meningitis clinical research. Using a local social circus group, we attracted a large audience of around 250 people using

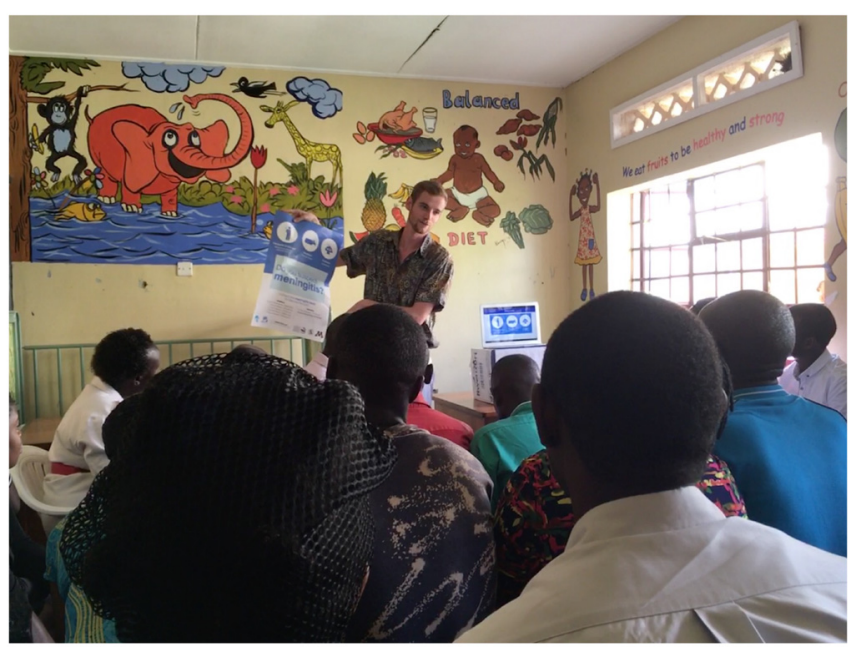

Figure 2. Knowledge exchange session in Western Uganda. 
interactive community performances, music, acrobatics, juggling, fire breathing and a drama sketch of a patient journey through meningitis illness (Figure 5). A trial participant (Mr JS), Dr Meya (Principal Investigator), Dr Stephen Watiti (an HIV advocate) and a Research Medical Officer did a structured question and answer (Q\&A) session with the audience. We conducted formal exit interviews with the audience to ensure that the correct messages had been retained and to receive feedback from attendees. Quotes from exit interviews included "the event corrected wrong thoughts about meningitis, that it's a

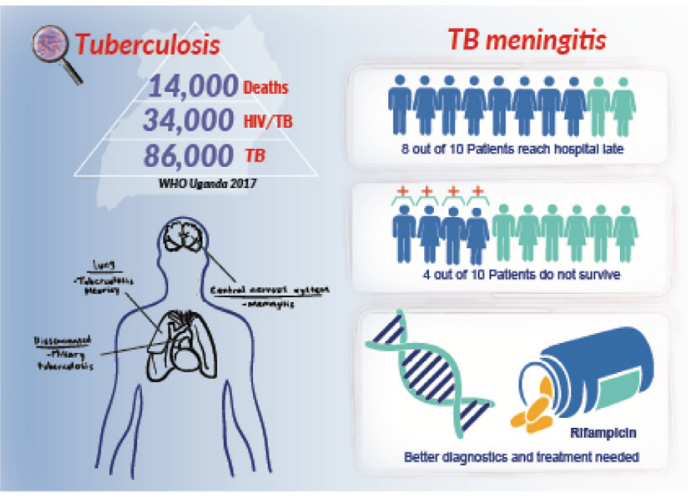

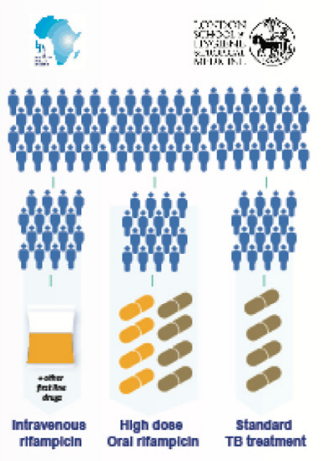
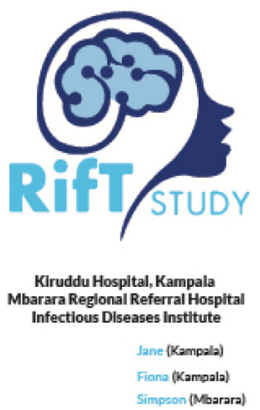

b

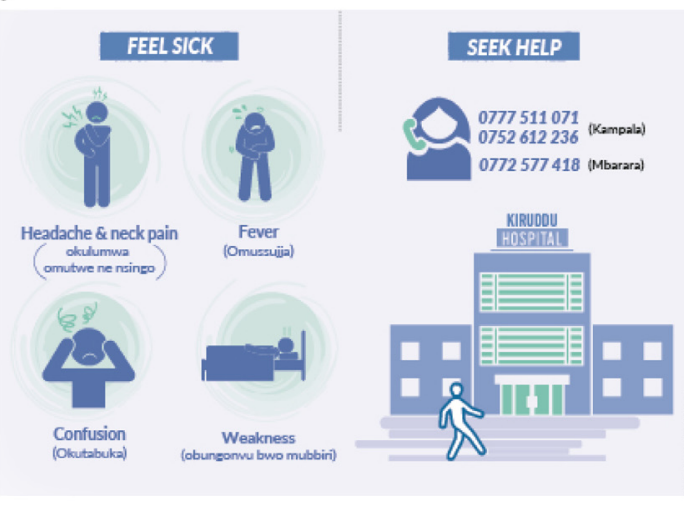

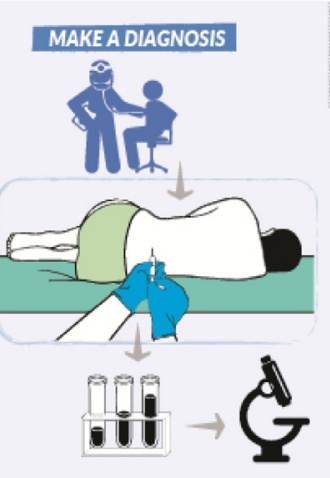

Figure 3. Infographic leaflets distributed to share information about TB meningitis and the RifT clinical trial. The RifT clinical trial studied the safety, tolerability and pharmacokinetics of high dose rifampicin compared to standard of care TBtreatment of adult tuberculous meningitis in Uganda.

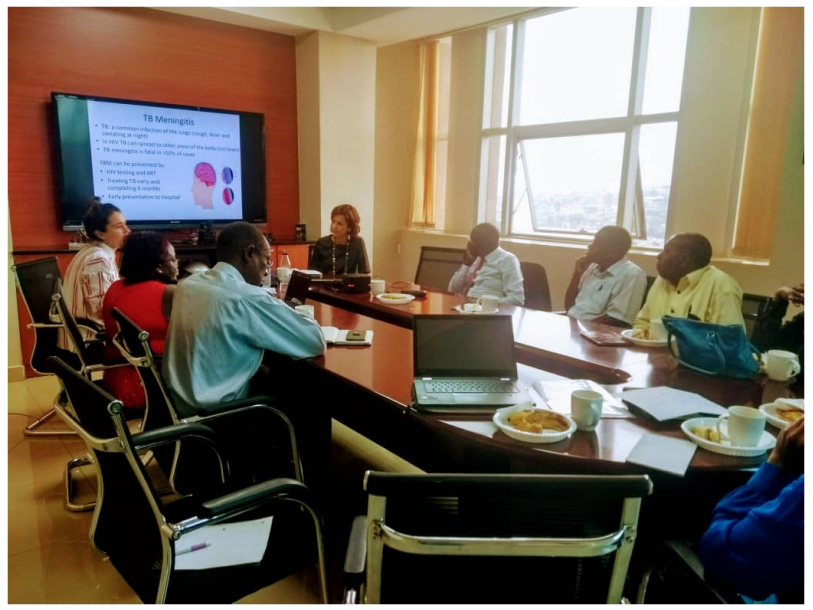

Figure 4. Infectious Diseases Institute Community Advisory Board Meeting.

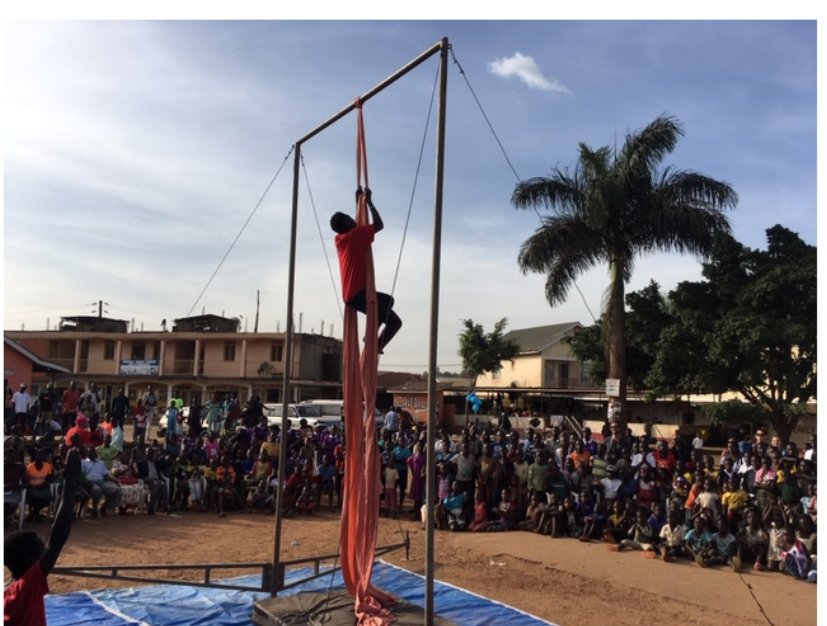

Figure 5. Public audience drawn to the circus event. 
cultural disease or witchcraft", "the event was very good and it helped people or the community to learn many things in this area", "Lumbar punctures are not the cause of death in sick people", "headache, tiredness, neck pain are symptoms of meningitis".

Radio shows on Central Broadcasting Services. Audience: Community members in central districts of Uganda. Purpose: Receive, transmit and collaborate

Radio, described as "Africa's medium of choice in the global age", remains an important medium of communication in Uganda, in part due to its accessibility ${ }^{11}$. We harnessed the popularity of radio in attempting to access hard-to-reach populations and aired a series of events on Central Broadcasting Services (CBS) radio, the largest radio station in Uganda. CBS is home to the most popular Breakfast show in Uganda, and a has a large following across different age groups ${ }^{12}$. The aim of this radio show was to allow the Research Medical Officers to 'transmit' important scientific content to the listeners, and also to 'collaborate' by co-creating the contents of the radio material with former trial participants, who were able to share their experiences - a focal point of the show. (Figure 6). In the week leading into World AIDS Day 2019 pre-recorded material including testimonials from three clinical trial participants who have survived advanced HIV disease were broadcast sharing key messages around symptoms of meningitis, experiences with lumbar punctures and clinical research, their treatment and return to health. On World AIDS day itself, a Research Medical Officer on the team featured on the radio and fielded questions from the presenters and public around advanced HIV disease, consistent with the 'receive' pillar of the public engagement framework.

\section{National Television Show. Audience: Community who} may be disengaged from HIV care or carrying myths or stigma relating to HIV/AIDS. Purpose: Receive, transmit and collaborate

HIV continues to be the most stigmatizing infection in Uganda, in part due to the lack of evidence-based information reaching

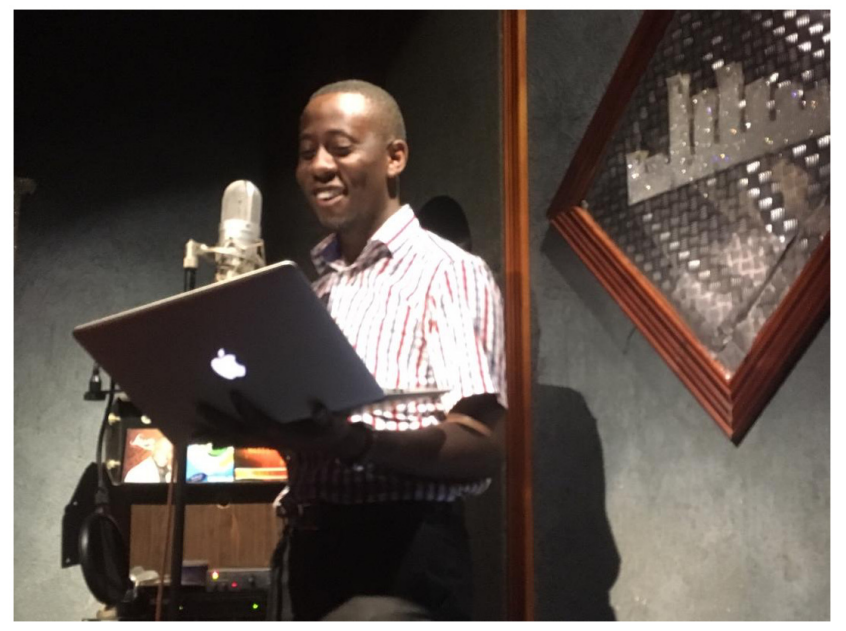

Figure 6. Study physician $\mathrm{Dr}$ Ssembambulidde records material for CBS radio broadcasts. citizens and long-standing myths regarding HIV/AIDS. Few people with HIV can openly talk about their status and this stigma has led to challenges in reaching the UNAIDS 90-90-90 targets, with 70,000 new HIV infections annually in Uganda and 1 in 3 people presenting with advanced HIV disease ${ }^{13}$. The key message of this 1-hour television (TV) show, with a wide national audience was that 'advanced HIV disease is preventable and treatable'. The show was aired on National TV on the World AIDS day 2019 and featured Drs John Kasibante (Research Medical Officer) and Fiona Cresswell (Principal Investigator) and $\mathrm{Mr}$ Tugume, a former research participant (Figure 7). Mr Tugume openly educated people on life as a survivor of advanced HIV disease. He helped demystify key issues such as Undetectable $=$ Untransmissible: early initiation of antiretrovirals to achieve viral suppression and to prevent HIV transmission to loved ones.

During the panel discussion questions arose like "can someone with HIV live with one who doesn't have HIV and she doesn't get the infection?", to which Mr Tugume responded "yes, my wife is HIV-negative, we are raising our son who is also HIV-negative and are soon to have another child". Mr Tugume also helped to reduce anxiety around lumbar punctures when asked "do people die from lumbar punctures?" and replied "No, I got many lumbar punctures when I was being treated for cryptococcal meningitis in Mulago, and here I am talking with you. They didn't kill me but saved my life". This is an example of collaborating with a former research participant to transmit key information to the public. We also received queries and viewpoints from the public.

\section{'A Journey of Hope' - Research participant and stakeholder} event. Audience: Research participants, institutional leaders, stakeholders. Purpose: Transmit and collaborate

'A Journey of Hope' was a celebratory event bringing together clinical trial participants, the IDI Meningitis Research Team, key stakeholders including Centre for Disease Control, U.S. Mission Uganda, Chair of the Mulago Hospital Institutional Review Board, an internationally renowned HIV patient advocate Dr Stephen Watiti, Executive Director of IDI and the research office. The IDI Drama Group performed cultural

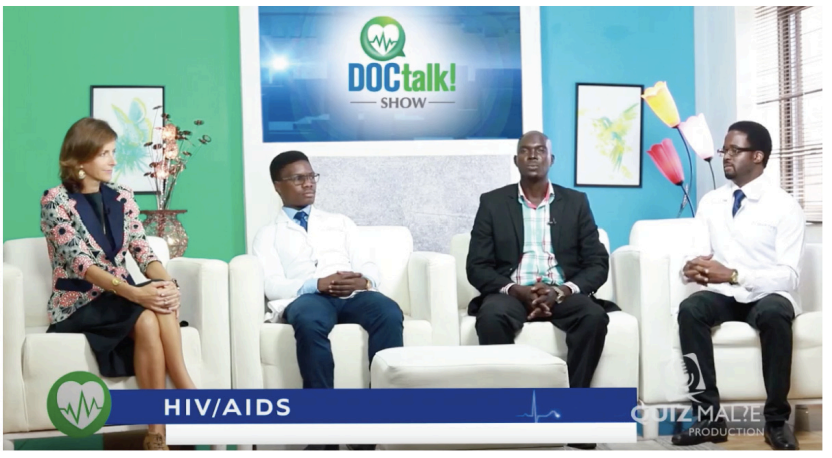

Figure 7. Dr Kasibante, Dr Cresswell and Mr Tugume appear on NTV. 
dances and a dramatization around meningitis (Figure 8) to address stigma and myths surrounding lumbar punctures and meningitis management ('collaborate'). A number of trial participants spoke about their experiences in clinical research. The event was also used as a platform to disseminate scientific results to trial participants and important stakeholders ('transmit'). The 'Journey of Hope' symbolised a very difficult journey for patients and their caretakers, many of whom had at times lost hope and were now celebrating with their families and former doctors, empowered to act as community advocates to improve understanding about advanced HIV. Attendees feasted and cut cake together to celebrate the progress made so far (Figure 9). We hope to repeat this successful event in the future on the completion of further clinical trials.

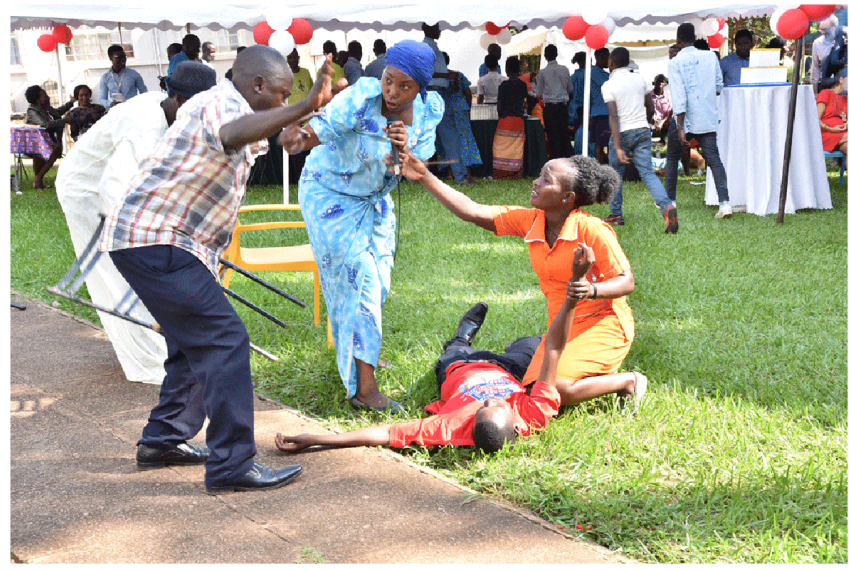

Figure 8. Dramatisation of meningitis illness by IDI drama group.

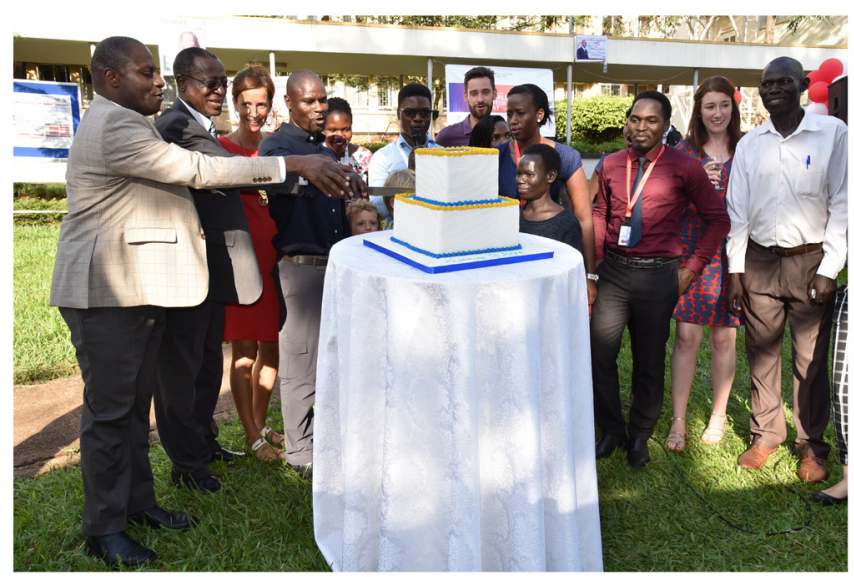

Figure 9. Cake cutting with the research team, study participants and IDI Executive Director.

\section{Discussion}

Whilst it is challenging to measure the exact impact of these activities we know that we engaged with around 500 healthcare workers, 250 members of the public face-to-face, several thousand members of the public through TV and radio, around 80 research participants and a number of key stakeholders in the HIV field. Four former trial participants have come forward as strong advocates for living well with HIV and continue to spread messages of hope in their communities. We learned the importance of harnessing the voices and opinions of the public in planning and conduct of engagement activities and in the planning of future research activities aimed at combating advanced HIV disease. In addition to optimising medical management as stipulated in the WHO advanced HIV care package, we believe addressing late presentation requires a holistic approach, with engagement and education of healthcare workers and the community ${ }^{14,15}$.

While there has been a huge amount of positive work in community engagement in PLHIV in Uganda, sometimes public engagement is can be overlooked in academic research. There can be a focus on volume of publications in peer-reviewed scientific journals leading to a lack of value placed on dissemination to the wider public ${ }^{16}$. However, in an era of digitalisation where 'fake news' surreptitiously invades our media sources, it is more important than ever for researchers to take a stand and accurately disseminate research findings to populations who are most affected. As public-researcher interaction improves, so too will trust in science, which may encourage broader public participation in scientific pursuits such as clinical research. We also feel it is critical to give research participants a voice to share their experiences and become advocates of science in the community.

In this letter we have shared our attempt to close the gap between HIV clinical research and the wider public using research participants as partners in delivering a unique variety of novel engagement activities. Our work lays the foundation for future engagement activities and research into the benefits and best practice around public engagement activities.

\section{Consent}

Written informed consent was obtained from all individuals that are identifiable in the provided figures.

\section{Data availability}

Underlying data

No data are associated with article.

\section{Acknowledgements}

We would like to thank the research participants who contributed to the public engagement work and continue to be advocates in their communities. We would like to thank the IDI community advisory board, drama group and research office for their support in the planning and conduct of the activities. 
1. (UNAIDS) JUNPoHA: 90-90-90 An ambitious target to help end the AIDS epidemic. 2014.

Reference Source

2. UNAIDS: UNAIDS Data 2019. 2019.

Reference Source

3. WHO: Guidelines for managing advanced HIV disease and rapid inititiation of antiretroviral therapy. Geneva, Switzerland: World Health Organization, 2017.

PubMed Abstract

4. Avert: HIV and AIDS in Uganda. Accessed 26th Febraury 2020 Reference Source

5. Benzekri NA, Sambou JF, Ndong S, et al.: Prevalence, predictors, and management of advanced HIV disease among individuals initiating ART in Senegal, West Africa. BMC Infect Dis. 2019; 19(1): 261.

PubMed Abstract | Publisher Full Text | Free Full Text

6. Rajasingham R, Smith RM, Park BJ, et al.: Global burden of disease of HIVassociated cryptococcal meningitis: An updated analysis. Lancet Infect Dis. 2017; 17(8): 873-881.

PubMed Abstract | Publisher Full Text | Free Full Text

7. Letang E, Ellis J, Naidoo K, et al:: Tuberculosis-HIV Co-Infection: Progress and Challenges After Two Decades of Global Antiretroviral Treatment Roll-Out. Arch Bronconeumol. 2020; 56(7): 446-454 PubMed Abstract | Publisher Full Text

8. Science For All: The Public Engagement Triangle. Reference Source
9. https://www.unaids.org/en/resources/documents/2007/20070410 jc1299policybrief-gipa_en.pdf

10. Lahuerta M, Ue F, Hoffman S, et al.: The problem of late ART initiation in Sub-Saharan Africa: a transient aspect of scale-up or a long-term phenomenon? J Health Care Poor Underserved. 2013; 24(1): 359-83. PubMed Abstract | Publisher Full Text | Free Full Text

11. Gunner L, Ligaga D, Moyo D, et al.: Radio in Africa: Publics, cultures, communities. Wits University Press, 2012; 112(446): 160-162. Publisher Full Text

12. GeoPoll: Uganda Media Measurement Report Reference Source

13. https://www.unaids.org/en/regionscountries/countries/uganda

14. Calmy A, Ford N, Meintjes G: The Persistent Challenge of Advanced HIV Disease and AIDS in the Era of Antiretroviral Therapy. Clin Infect Dis. 2018; 66(suppl_2): S103-SS5.

PubMed Abstract | Publisher Full Text | Free Full Text

15. Hakim J, Musiime V, Szubert AJ, et al.: Enhanced Prophylaxis plus Antiretroviral Therapy for Advanced HIV Infection in Africa. N Engl J Med. 2017; 377(3): 233-45.

PubMed Abstract | Publisher Full Text | Free Full Text

16. Pham D: Public engagement is key for the future of science research. NPJ Sci Learn. 2016; 1: 16010.

PubMed Abstract | Publisher Full Text | Free Full Text 


\section{Chapter Ten: Discussion and Conclusion}

\subsection{Summary of findings}

This section summarises the main findings presented in chapters 3 to 8 in relation to the pre-specified PhD hypotheses or objectives.

10.1.1 Objective 1: To assimilate available retrospective data on adults with suspected TBM in Uganda and to describe the TBM population in terms of baseline characteristics, method of diagnosis and clinical outcomes

The retrospective data analysis found that overall just over 1 in 10 (12\%, 195/1672) of the patients presenting with suspected meningitis to Mulago National Referral Hospital and Mbarara Regional Referral Hospital, were treated for TBM in the 2010 - 2017 period, making it the second most common form of meningitis after cryptococcal meningitis, in-line with South African and Zimbabwean reports. $^{1,2}$

TBM was indeed largely seen in PLHIV, particularly those with advanced immunosuppression. In the chapter 4 retrospective cohort, 96\% (187/195) of patients treated for TBM were PLHIV with a median CD4+ T cell count of 78 cells $/ \mu l$, and in the chapter 8 prospective study in 92\% (56/61) of enrolees were PLHIV with a median CD4+ T cell count of 55 cells/ $\mu$. Thanks to the massive scale up of HIV treatment programmes in Uganda, the proportion of TBM patients on ART increased dramatically (from 0\% in the earliest group to 61\% in the latest group) during the 2010 to 2017 retrospective cohort analysis, and in 2019 ART coverage was 48\% (27/56) in Rift trial enrolees. Whilst we lacked data on HIV virological suppression from the chapter 4 retrospective cohort, no one was virologically suppressed in the chapter 8 Rift study population.

HIV treatment characteristics were better described in the Rift study population; among the 56 HIVpositive Rift enrolees, 30 were recent HIV diagnoses, of which $(20,67 \%)$ had initiated ART within the 3 months prior to hospital admission. This suggests that delayed HIV testing and ART initiation has contributed to their advanced HIV disease. Amongst the 26 participants with longer-standing HIV, the majority $(17,65 \%)$ had discontinued their ART or had unknown ART history, suggesting the next biggest contributor to advanced HIV disease was poor engagement with HIV care and treatment. Microbiological confirmation of TBM increased with availability of the Xpert platform, from 3\% (1/33) before Xpert to 41\% (60/147) with routine use of Xpert and culture in the 2013-2017 period, as described in the chapter 4 retrospective cohort. Microbiological confirmation further increased to 
51\% (31/61) with the use of Xpert Ultra in the Rift enrolees in 2019 in chapter 8. This is in keeping with the microbiological-confirmation rate in other TBM trials. ${ }^{3}$ However, this means that around half of TBM cases are still treated empirically in the absence of a microbiological confirmation.

Since the introduction of the Xpert platform in-hospital mortality may have declined. In the chapter 4 retrospective cohort in-hospital, mortality was $57 \%$ in the pre-Xpert group and was $41 \%$ in group where Xpert was used routinely, despite MRC disease severity grade at presentation being stable. In the chapter 5 prospective Xpert Ultra study the in-hospital mortality was 40\% (17/42) in those with definite TBM and in the chapter 8 Rift population, 24-week mortality was 39\% (24/61). Whether the availability of the Xpert platform has directly impacted on clinical outcomes is unclear from the data I have available. Whilst there appears to be a temporal association between the introduction of Xpert and reduced TBM mortality there are a multitude of confounding factors which may have ultimately influenced outcomes, such as improved ART coverage or health system strengthening influencing supportive care received. Despite these marginal improvements in TBM-associated mortality over time, and whilst our reported mortality was a little lower than that in the in the meta-analysis in the Appendix 1 (pooled mortality in PLHIV 57\%, 95\% Cl, 48-67\%), the death-toll from TBM is still unacceptably high.

A key driving factor of poor outcomes is late presentation to hospital. ${ }^{4}$ Only $10 \%$ of the Rift study population presented with MRC grade I disease, compared to about $40 \%$ in Vietnamese studies. ${ }^{5}$ By the time of presentation with MRC grade II/III disease, when mental status is altered +/- focal neurology, there may already be irreversible cerebral infarction, and even the best diagnostics and antimicrobial treatment may be unable to radically change the course of the disease. The difference in health seeking behaviour may therefore be a contributory factor to the difference in survival found between African and Asian settings in the TBM meta-analysis Appendix 1, mortality in African studies ranged from $23-80 \%$ and in Asian studies ranged from 2-67\%. ${ }^{6}$ Ongoing engagement work is needed around health seeking behaviour to encourage earlier presentation to hospital, as well as recognition of meningitis at district health facilities with timely onwards referral.

Whilst acknowledging the limitations that come with using retrospective data, the retrospective cohort in chapter 3 was valuable in understanding the TBM landscape in Uganda. The results from the retrospective data were consistent with data that I collected prospectively in the chapter 5 prospective Xpert Ultra study and in the chapter 8 Rift trial population. 
10.1.2 Hypothesis 1: Xpert MTB/Rif 'Ultra' is significantly more sensitive than Xpert MTB/Rif for the detection of M. tuberculosis in CSF. However, Xpert Ultra's sensitivity will not be perfect and it may fail to diagnose a small number of cases of CNS TB that are evident by metagenomic next generation sequencing, CSF culture or post-mortem examination

In the chapter 5 prospective study of 204 Ugandan adults (96\% HIV-positive) with suspected meningitis, when compared with a reference of probable/definite TBM, test sensitivities were $77 \%$ for Xpert Ultra, 56\% for Xpert, and 61\% for MGIT culture. Xpert Ultra was more sensitive than Xpert when measured against both the uniform case definition of probable/definite ( $77 \%$ versus $56 \%, p=0.001$ ) and the composite microbiological reference standard ( $93 \%$ versus $66 \%, \mathrm{p}=0.006$ ). Xpert Ultra missed 3 cases that were culture positive (including one that was also Xpert positive).

In the limited number of post-mortem examinations performed, one patient had the macroscopic pathological appearance suggestive of TBM (basal meningeal exudate) in a case which had been classified as 'possible' TBM. There were no false negative Ultra results identified amongst the 84 mNGS results available from the Ultra study cohort. However, outside of the Xpert Ultra cohort mNGS has managed to identify M. tuberculosis DNA in two samples negative by Xpert Ultra (Appendix 6b and $5 c)$.

In the chapter 5 Xpert Ultra study I did not include 'possible' TBM in the reference standard as I believed that the 'possible' TBM category is a very non-TB-specific category in advanced HIV immunosuppression, especially in a setting where the diagnostic capacity to exclude other conditions is limited. This was borne out by results from the Biofire meningoencephalitis PCR panel which was used during the Xpert Ultra and study enabled 10 participants (13\%, 10/80) to be re-classified as 'not' TBM prior to the Ultra analysis taking place. In addition, the preliminary results from the CSF mNGS identified viral pathogens in an additional $11 \%$ of 'possible' TBM samples tested (3/27) and the variety of viral pathogens identified in a wider sample set are described in the Appendix 6a.

Data from the chapter 5 Xpert Ultra study also emphasized that TBM can present with a noninflammatory cellular CSF profile in this population. One quarter of patients with confirmed $M$. tuberculosis in CSF had an acellular CSF, so clinicians cannot rely on the classical lymphocyte pleocytosis to make a diagnosis of TBM in PLHIV, and other clinical or CSF parameters may be better predictors to use when making treatment decisions. In the univariate model the presence of CSF white cells (as a binary variable) was associated with definite TBM but the relationship became nonsignificant in the multivariate model. The univariate model showed that that high CSF lactate was the strongest discriminator of confirmed TBM - with every doubling of CSF lactate there was a 6-fold increase in odds of having definite TBM. CSF glucose was also a good discriminator, with every 
doubling in CSF glucose there was a 70\% decrease in the odds of having TBM. It was not possible to examine CSF lactate in the multivariable model due to the limited amount of lactate data but CSF glucose remained a strong predictor of definite TBM. Low GCS (11-14 and $\leq 10$, compared to GCS 15) was strongly associated definite TBM in the univariate model and the trend remained in the multivariable model, albeit with p-values marginally above the 0.05 significance threshold.

Overall, I can conclude that Xpert UItra is significantly more sensitive that Xpert MTB/Rif in the diagnosis of TBM in our study population and only missed a handful of TBM cases that were identified by other means (3 MGIT culture positive, 1 suggestive post-mortem examination, none by mNGS within the chapter 5 study participants).

\subsubsection{Hypothesis 2: Urine diagnostics such as urine TB-LAM and urine Xpert MTB/Rif Ultra will be useful adjunctive tools in diagnosing TB meningitis and will identify patients at the greatest risk of death.}

Access to timely lumbar puncture is not universal in smaller hospitals in Uganda, and many other African settings. Therefore, a non-CSF-based diagnostic approach for TBM would be very valuable. With this in mind, I postulated that a positive urine TB test might help to rapidly differentiate TBM from other forms of meningitis and may have prognostic value. This prompted the chapter 6 prospective observational study of 348 undifferentiated meningitis patients at two referral hospitals. This was an exploratory study and I did not aim to examine sensitivity or specificity of urine LAM or Ultra in this paper.

Urine Alere LAM is only moderately sensitive at detecting TB (40-70\% if CD4+ T cell count $<100$ cells $/ \mu \mathrm{l}$ ), yet despite this there was a staggeringly high prevalence of disseminated TB (detectable by either Alere LAM or urine Ultra) across the cohort of hospitalised meningitis patients: $22 \%$ in cryptococcal meningitis, 50\% in probable and definite TBM and 17\% in other/unknown types of meningitis. The true prevalence of TB is likely to be even higher than that detectable by urine. In the $22 \%(44 / 198)$ of patients in the cryptococcal meningitis group who had a positive urine TB test I believe this represents disseminated TB rather than concurrent TBM and cryptococcal meningitis, which we know is a relatively rare phenomenon. ${ }^{7}$ Whilst there was a difference in prevalence of TB detectable by urine between the four meningitis groups ( $p<0.001)$, a urine LAM or Ultra result could not be used in isolation to make a diagnosis of TBM and should be interpreted in the context of the blood/CSF CrAg results and other CSF parameters (e.g lactate and glucose).

The high burden of urine-detectable disseminated TB in the probable and definite TBM groups is noteworthy when considering pathogenesis and the 3-fold higher mortality from TBM in PLHIV 
compared to HIV-negative TBM. It is evident that HIV-associated TBM is a multisystem disease, with concomitant disease in the kidneys, and therefore probably a number of other organs. Case-fatality in those with a positive urine Ultra was 50\%, $(7 / 14)$ whereas it was $15 \%(3 / 20)$ in those with a negative urine Ultra - a case-fatality similar to that of HIV-negative people with TBM. Patients with definite TBM who had a positive urine Ultra had a 6-fold increased odds of dying (OR 5.67, 95\% Cl, 0.71-12.7, $\underline{p=0.04)}$ compared to those with a negative urine Ultra. There was no detectable association between urine Alere LAM positivity and in-hospital mortality (OR 1.34, 95\% 0.32-5.61, $p=0.69$ ) but this needs to be examined with a larger sample size.

Urine Alere LAM and Ultra results were moderately concordant, possible reasons are postulated in the chapter 6 discussion, and TB-LAM positivity representing non-tuberculous mycobacterial infection must also be considered and further investigated.

\subsubsection{Hypothesis 3: Intensified treatment of TB meningitis with intravenous rifampicin (20 mg/kg) or high dose oral rifampicin $(35 \mathrm{mg} / \mathrm{kg}$ ) will result in significantly increased blood and CSF exposure during the critical early days of TB treatment as compared to common control (10 mg/kg oral rifampicin)}

Through the phase II clinical trial outlined in chapter 8 I demonstrate that with standard of care TB treatment (including R $10 \mathrm{mg} / \mathrm{kg}$ ), the serum geometric mean peak rifampicin concentration $(6.0$ $\mathrm{mg} / \mathrm{L})$ fell below the recommended reference range $(>8 \mathrm{mg} / \mathrm{L})^{8}$ and the serum AUC/MIC $(42.9 \mathrm{~h} \cdot \mathrm{mg} / \mathrm{L})$ fell well below the suggested $271 \mathrm{~h}$ required for a 1 log CFU reduction in murine studies. ${ }^{9}$ In CSF, the rifampicin concentration fell below the lower limit of quantification $(<0.25 \mathrm{mg} / \mathrm{L})$ in around half of participants and only two participants achieved a CSF rifampicin concentration above the MIC of the predominant strain in Uganda. The measured rifampicin exposures with standard TB treatment are sub-optimal, especially considering that rifampicin is the most important drug in TB therapy and meningitis is the deadliest manifestation of TB. A 2-fold rifampicin dose increase administered intravenously $(20 \mathrm{mg} / \mathrm{kg}$ ) yielded a roughly 6-fold increase in serum and CSF exposures. A 3.5-fold oral dose increase $(35 \mathrm{mg} / \mathrm{kg}$ ) yielded a roughly 8 -fold increase in serum and CSF exposures. All the participants on the intervention arms had detectible CSF rifampicin and almost all (93\% and 95\% respectively) had levels above the rifampicin MIC.

10.1.5 Hypothesis 4: High dose rifampicin will be safe in people with advanced HIV disease and may improve functional outcomes and survival at 8- and 24-weeks.

When considering the immunocompromised nature of the study population and severity of the underlying disease, it is not surprising that grade 3-5 adverse events were commonplace in all arms 
(71\% of standard of care, $50 \%$ of IV-20 and $55 \%$ of PO-35, $p=0.343$ ). Overall, the most common grade $>3$ event was elevated ALT, attributed to drug induced liver injury (DILI), and occurred in $7 / 60(11.7 \%)$ of all participants, 4/7 of which were in the control arm. The majority of DILI occurred in the first month of therapy, Figure 10.1. The observed incidence of hepatotoxicity is slightly higher than that reported in the Vietnamese TBM trial (5.5\% overall) and UK TB cohorts $(\sim 7 \%)^{10}$ but less than that in the Indonesian trio of TBM trials (19, 20 and 27\%). ${ }^{11-13}$ The relatively modest incidence of DILI within the study is reassuring considering that HIV is known to be an independent risk factor for DILI (OR $4.40,95 \% \mathrm{Cl}, 1.06-18.3) .{ }^{10}$

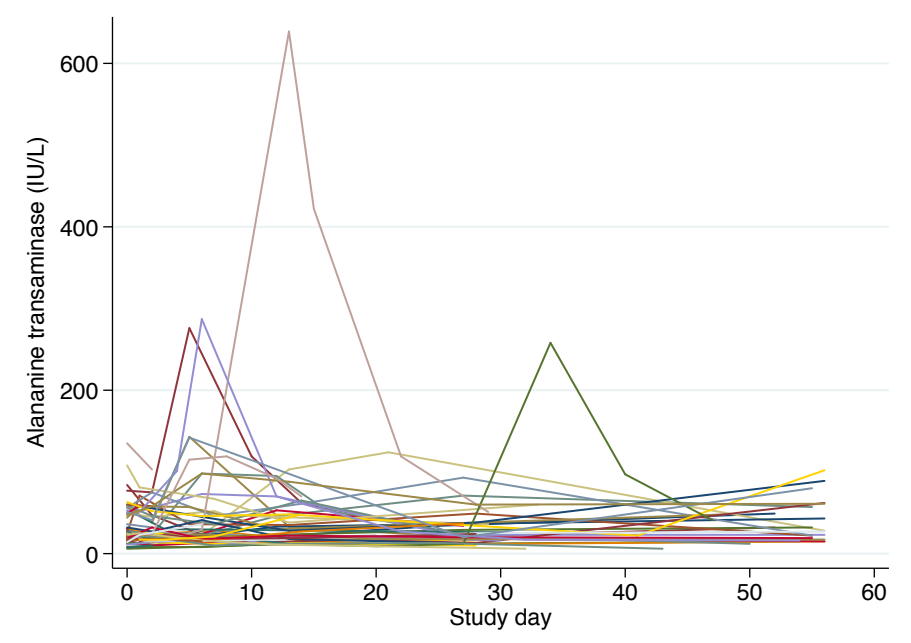

Figure 10.1. Alanine transaminase (IU/L) by study day in the Rift trial

Rifampicin is known to cause hyperbilirubinaemia and an exposure-effect relationship exists.

Unconjugated bilirubin rises after starting rifampicin because of inhibition of bilirubin hepatocellular uptake via OATP and/or glucuronidation by UGTA1A1. ${ }^{14}$ Bilirubin levels then decline to less than pretreatment values upon rifampicin continuation, suggestive of induction of net bilirubin clearance. Clinically detectable jaundice was not a common observation in Rift study enrollees, as the majority of elevations were low grade ( 1 to $3 \times$ upper limit of normal, ULN). There were 3 episodes of isolated hyperbilirubinaemia in the study population and bilirubin elevations ( $\geq$ grade 3 ) also occurred in the context of DILI and were classified as DILI at the time of AE reporting.

There were only two other events that were possibly, probably or definitely related to rifampicin - a persistent fever (possibly related) and hypotension as part of multi-organ failure (possibly related). I did not record subtle grade 1-2 tolerability events as these are challenging to ascertain in a person with altered mental state and a multitude of other disease-related symptoms, and they are of limited clinical relevance.

Seizures occurred in around 8\% (5/61) of the enrolees including 2 fatal seizures and use of certain anticonvulsants (such as phenytoin) added additional complexity to the melee of drug-drug 
interactions with rifampicin and ART. Kaposi's sarcoma was noted at as an AE in two participants after it progressed rapidly and was ultimately fatal several months down the line, though this is unlikely to be related to rifampicin and could have been exacerbated by the corticosteroids recommended as part of TBM treatment (which were promptly discontinued) or may have been the natural course of disease in profoundly immunosuppressed individuals.

In this phase II study I did not detect a difference in modified Rankin score or survival at 8 or 24weeks, although the study was not powered to explore a survival benefit. We performed detailed neurocognitive studies at week 8 and 24 in those who were well enough to engage with the in-depth testing, the results of which I plan to report separately in future.

Owing to the difficulty in diagnosing TBM, only half of the participants included in the study had a microbiologically-confirmed diagnosis and it is plausible that people without TBM were included, which might dilute the clinical effect of the intervention. I can conclude that, within the limited population studied, high-dose rifampicin was safe but no impact on survival or functional status was observed.

\subsection{Limitations}

The specific limitations are discussed in the individual research papers in chapters 3 to 8 . Here I discuss the more general limitations of my work undertaken trying to improve the diagnosis and treatment of TBM in Uganda. I did my best to overcome the multiple obstacles placed by this problematic disease, the three main disease-related challenges are elaborated below.

\section{Challenges posed by the nature of the disease:}

Firstly, although TBM is the second most common aetiology of meningitis in Uganda it is relatively less common than cryptococcal meningitis, and in the absence of a reliable blood or urine antigen test many patients had to be screened and undergo lumbar puncture in ordered to identify cases. As such, recruitment into studies is relatively slow, which limited the size of the studies possible during a PhD across two sites. The phase II clinical trial in chapter 8 was adequately powered from a pharmacokinetic and safety perspective, but we need to further examine safety in a larger study and clinical outcomes in a multicenter phase III trial. A larger sample size would also have been useful for the urine TB diagnostic study in chapter 6 as the confidence intervals were wide and Xpert Ultra and Alere LAM's potential as prognostic markers could have been more accurately examined with a larger study with appropriate adjustment for baseline factors (e.g. CD4+ T cell count) and I have not yet externally validated my findings. I plan to do this within the future research, as described later. 
Secondly, the lack of a gold standard diagnostic test or other specific reference standard for TBM makes both the decision about who to enroll into clinical trials and how to most accurately evaluate diagnostics tests a challenge. The most balanced approach was to present the data stratified by the internationally recognised uniform case definition (definite i.e. microbiologically-confirmed, definite/probable, +/-possible), to explain the range of diagnostic tests undertaken, and state the caveats.

Thirdly, unlike studies in pulmonary TB which use time to culture conversion or serial sputum colony counts, there is currently no validated $M$. tuberculosis quantitative assay or surrogate endpoints for use in TBM studies. Therefore, I was unable to examine the impact of high dose rifampicin on CSF bacillary clearance. Our endpoints were survival and function status by modified Rankin score at 8and 24-weeks, and whilst clinical outcomes are ultimately the most important, they are potentially confounded by other non-TB multi-morbidities in a population with AIDS. Further I have not yet performed a PK-PD analysis of the Rift trial data, though I plan to do this in due course.

Lastly, due to the severity of illness in the patient group collection of clinical samples was seldom straightforward which led to a proportion of patients having incomplete data. For example, in chapter 5, lumbar punctures whereby only a small volume of CSF could be collected led to there being fewer culture and Xpert results than Xpert Ultra. Likewise, collecting urine from patients who were confused and incontinent was challenging and sometimes only enough for the Alere LAM was a collected leading to a mismatch between the number of LAM and Xpert Ultra performed. The pattern of missingness of urine data may not be random; sicker patients are more likely to be incontinent of urine and unable to give a sample, conversely the most unwell patients often have a urethral catheter which then facilitates urine sample collection. Overall, I doubt any major bias has been introduced.

\section{Operational limitations:}

As well as disease-related limitations there were also a number of operational challenges. It would have been ideal to perform a comprehensive work up to better characterize the extent of the TB disease and other co-infections, including sputum Xpert Ultra, TB blood and urine cultures, bacterial blood cultures, chest $x$-ray, abdominal ultrasound and brain imaging on everyone screened and enrolled. The hospital CT scanners were frequently out of service and transporting a neurologically obtunded patient to another center for imaging was deemed too risky in many patients. During the chapter 8 Rift study I prioritized routine CSF microbiology, Ultra and storage for future immunology work above MGIT culture where sample volume was limited. In hindsight a culture and individuallevel rifampicin MIC on every isolate would have more accurately examined the AUC/MIC parameter, at least within the culture-positive patients. 
In Rift trial participants enrolled on DTG-based ART and receiving high-dose rifampicin, I had hoped to perform real-time DTG therapeutic drug monitoring (TDM) to provide reassurance that trough levels were in the therapeutic range. The IDI translational lab DTG assay is on the recently installed liquid chromatography mass spectrometry machine, but unfortunately this was having technical issues that have only recently been overcome, which precluded real-time DTG TDM. DTG was dose-adjusted to $50 \mathrm{mg}$ twice daily shortly after enrolment, but TDM should ideally be performed at week 4 as the rifampicin enzyme induction builds over the first 4 weeks of therapy. A number of stored samples can now be tested, however more definitive results will be provided by the SAEFRIF study underway in Uganda. ${ }^{15}$

I had hoped that we would be able to perform post-mortem examinations in all patients who died in hospital but we only succeeded in performing four post-mortems. We discovered that autopsy studies are highly logistically challenging; post-mortems are not part of normal clinical or cultural practice so the majority of families were understandably reluctant to let their recently deceased loved one be transported to another facility for an alien procedure, especially when the burial normally takes place very quickly. This was compounded by the fact that there was only one pathologist in the country capable of performing the examination, and only during working hours. Minimally invasive autopsy may have been a more successful approach but precludes the macroscopic inspection of the brain.

In this PhD I have focused specifically on the antimicrobial treatment but supportive care of critical illness, host-directed therapies and rehabilitation are also clearly vital considerations in improving treatment and outcomes from TBM.

\subsection{Implications of the findings}

\subsubsection{TBM can be prevented by upstream HIV-related interventions including earlier HIV testing, treatment and TB screening within HIV services.}

The WHO End TB strategy aims to reduce TB deaths by $90 \%$ by 2030 compared with $2015 .{ }^{16}$ Disseminated TB including TBM contributes heavily to the burden of TB deaths ${ }^{17}$ so meeting the End TB target requires the specific drivers of TBM to be addressed. I have illustrated here that the vast majority of TBM patients have advanced HIV, so preventing immunosuppression (as well as preventing HIV transmission in the first place) is likely to be the best approach to reduce the incidence of TBM. The majority of the Rift study population were late HIV diagnoses with an already AIDSdefining CD4+ T cell count. HIV testing interventions may be able to identify PLHIV earlier and link to 
treatment services before immunosuppression is advanced. Diagnosing HIV at the time of hospitalization with an opportunistic illness is a failure of community-based HIV testing services. Equally important is screening for TB in PLHIV. The WHO recommend a TB symptom screen (any of cough, fever, night sweats, or weight loss) ${ }^{18}$ in all PLHIV and urine Alere LAM testing ${ }^{19}$ prior to ART initiation if $C D 4+T$ cell count is $<100$ cells/ $\mu$ or if there are symptoms of TB regardless of CD4+ count. This allows underlying TB to be diagnosed and treated prior to ART initiation, thereby avoiding unmasking TB IRIS which can be fatal, particularly in the CNS. ${ }^{20,21}$ TB screening and treatment prior to ART initiation did not appear to have happened in the 20 Rift trial enrolees who had recently initiated ART, and represents a missed opportunity. In the participants who had been on ART $<35$ days with probable/definite TBM the CSF picture was in keeping with unmasking IRIS [median WBC $140 \mathrm{cell} / \mu$ I (95\% lymphocytes 5\% neutrophils), protein $0.94 \mathrm{~g} / \mathrm{L}$, lactate 9.5 , glucose $1.1 \mathrm{mmol}$ ] as was the CSF transcriptomic picture, which showed upregulation of interferon-gamma signalling, IL-12 signalling and neutrophil degranulation (oral presentation by C Quinn, Union Conference 2020). With the increasing implementation of the WHO HIV test and treat policy ${ }^{22}$ including a push towards same day ART in some settings, the TB screening process must be well embedded within HIV treatment practices to avoid cases of unmasking TBM IRIS.

Even with prompt TB treatment at the time of hospitalization in a relatively well-resourced health service in South Africa, the early mortality from HIV/TB is $22 \%$ within 3 months. ${ }^{23}$ Therefore, community-based interventions which prevent active TB disease arising, or support early diagnosis, are likely to have the greatest impact. TB preventative therapy (TPT) in PLHIV is recommended by WHO and is being rolled out in Uganda, though with limited coverage currently (29\% of household contacts of confirmed TB cases, $46 \%$ of PLHIV newly enrolled into care). ${ }^{24,25}$ This is an effective intervention at a population leve $\left.\right|^{26}$ but is a difficult intervention to implement in an already highly immunosuppressed population unless it is possible to first exclude active TB disease.

The continued lack of access of Alere LAM in both the community HIV clinics and public hospitals is a contributing factor that must be prioritised by the National TB Control Programme. Empowering patients to seek care earlier and recognition of critically ill patients in community settings is also an important piece in the community engagement work around meningitis and advanced HIV disease. Likewise, bigger structural factors that drive the TB/HIV epidemic cannot be ignored.

\subsubsection{Xpert Ultra is the best first test for TBM but should not be the last test}

Xpert Ultra is the best CSF test currently available for TBM in a Ugandan setting, and probably in the rest of sub-Saharan Africa. However, Xpert Ultra cannot be used as a 'rule-out' test for TBM due to its 
imperfect negative predictive value of $93 \%$ ( $95 \% \mathrm{Cl}, 88-96 \%)$. The Xpert Ultra test result must be interpreted with the pre-test probability of TBM in mind, as illustrated in Figure 10.2.

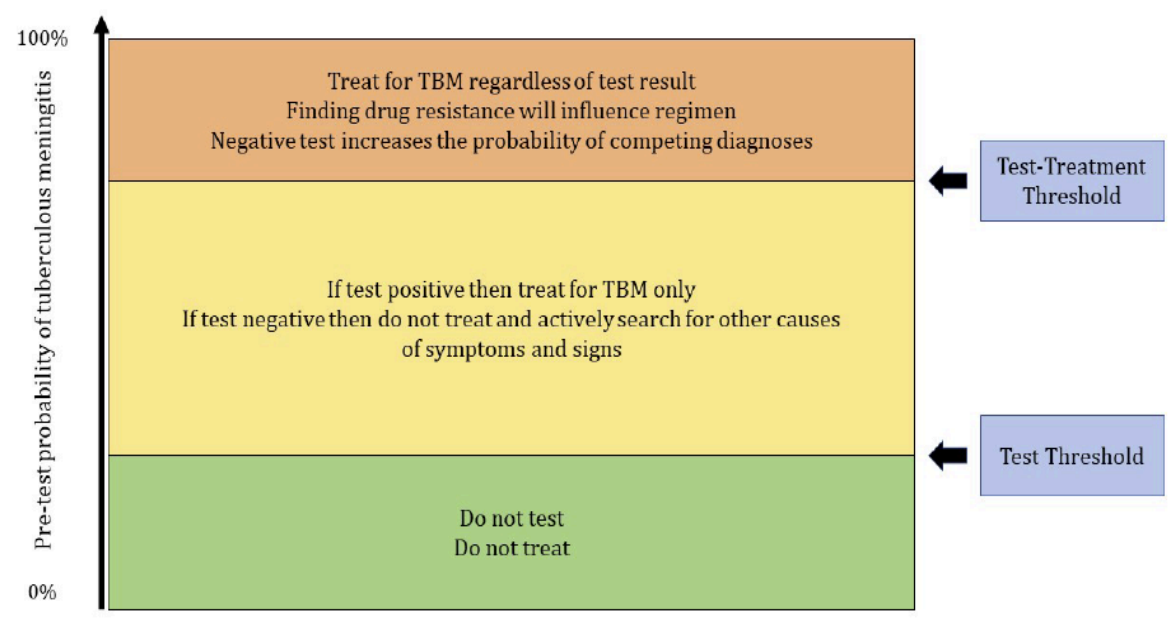

Figure 10.2. Test threshold and testtreatment threshold in relation to pre-test probability of TBM disease. ${ }^{27}$

We may never have a single perfect CSF test for TBM and combining a number of tests including a host and pathogen biomarker might be the most promising approach. I have demonstrated that reliance on a classical CSF picture with lymphocyte pleocytosis is not possible in PLHIV as around 1 in 4 confirmed cases have an acellular CSF. The univariate and multivariate model in the chapter 5 Xpert Ultra paper suggest that low CSF glucose and high CSF lactate are good markers of definite TBM, both of which can be easily, quickly and cheaply be measured at the bedside with a handheld meter. A CSF CrAg is also easily performed at the bedside to exclude cryptococcal meningitis. Urine Alere LAM is helpful (though also commonly positive in other forms of meningitis), and urine Xpert Ultra is supportive of a TBM diagnosis as it is less commonly positive in other forms of meningitis and may be useful as a prognostic indicator, though external validation is needed.

In the same edition of Lancet Infectious Diseases as our chapter 5 paper was the Xpert Ultra study from the Vietnam group, which included 205 adults (15\% PLHIV). ${ }^{28}$ Donovan et al. used a reference standard of definite, probable, or possible TBM, and reported sensitivities of $47 \%$ for Xpert Ultra, $40 \%$ for Xpert, and $48 \%$ for MGIT culture. They concluded that Xpert Ultra was not statistically superior to Xpert based on the reference standard used. ${ }^{28}$ How can we reconcile the different results between the two studies? Differences in CSF volume, CSF processing and study design might have contributed to the difference (in the Vietnam study patients were randomized to either Xpert or Xpert Ultra rather than head-to-head testing). Geographical differences in HIV status, genetics influencing host response, and TB lineage (distinct have different copy number of IS6110 and IS1081) might also have contributed. ${ }^{29}$ The reference standard used in the Vietnamese study is also important; the sensitivity of Xpert Ultra increased to 58\% when 'possible' TBM was dropped from the reference. Remarkably in 
their study roughly one third of cases $(35 \%, 29 / 82)$ in the 'definite' category was positive by ZN smear microscopy in isolation (whilst negative by MGIT culture, Xpert Ultra and Xpert). This unique phenomenon was attributed to their highly experienced resident microscopist and is unlikely to be replicated in any other centers worldwide. Thus, I believe the results from our two Ugandan studies are more generalizable to an African setting and were remarkably consistent - the negative predictive value of Ultra was $94 \%$ in the 2018 study $^{30}$ with cryopreserved samples and $93 \%$ in the chapter 5 prospective study. The negative predictive value is not good enough for Ultra to be used as a rule-out test but is an improvement on Xpert (86\%).

The inherent value of a having a microbiologically-confirmed diagnosis cannot be understated. Firstly, for patients, it may result in increased confidence in the diagnosis and consequently their willingness to comply with long and unpleasant treatment. Secondly, for clinicians, a confirmed diagnosis reduces the risk, time, and cost associated with investigating for other causes of the illness and gives a greater degree of confidence in treating this condition and its associated complications. Thirdly, for policy makers and other stakeholders it is important information for planning of preventative strategies and clinical services. Lastly, for researchers, a microbiologically-confirmed diagnosis can increase the scientific rigour of the study in hand. Everyone stands to gain from a better test being available and the search must continue.

Use of the composite microbiological reference standard does not lend itself to accurately calculating the specificity as the index test is included in the reference standard (i.e. there can be no false positives). When the uniform case definition (excluding the Xpert Ultra result) was used to calculate the specificity of Xpert Ultra it was $96 \%$. The specificities of Xpert and Xpert Ultra are extremely high, ${ }^{31}$ and CSF testing in TBM is not likely to experience the pitfalls that exist with sputum testing in people with prior pulmonary TB who can experience false positive trace calls, as further explained in the chapter 5 paper. So, a positive CSF Xpert Ultra should be interpreted as active TBM disease and treatment initiated immediately.

Finally, the use of CSF test results becomes academic if lumbar puncture needles are not available, which is a common occurrence even at the National Referral Hospital, requiring the family to have available money to buy the needle from an outside pharmacy. Likewise, CrAg LFA tests are often not immediately available to differentiate cryptococcal meningitis from TBM. Health system strengthening to ensure consistent supply of lumbar puncture needles and POC diagnostics will be lifesaving. 


\subsubsection{HIV-associated TBM is a manifestation of disseminated TB disease so supportive care and interventions to optimise the host response are important alongside optimal antimicrobial therapy}

\subsubsection{The prevalence of disseminated TB amongst patients with HIV-associated TBM}

Disseminated TB was highly prevalent in HIV-associated TBM - it was detectable by urine in $50 \%$ of patients with probable or definite TBM. The true proportion with disseminated TB might be more like $70-80 \%$ given the only moderate sensitivity of urine diagnostics. Therefore, TBM may be towards the end of the spectrum of disseminated TB disease, as proposed in Figure 10.3. Recent in-depth clinical phenotyping of hospitalised adults with HIV/TB in Malawi and South Africa has shown that urine LAM/Xpert positivity is associated with a severely ill clinical phenotype ${ }^{32}$ and immune activation, which may contribute to mortality through tissue damage and organ dysfunction. ${ }^{33}$ We therefore cannot consider CNS infection and inflammation in isolation in HIV/TBM.

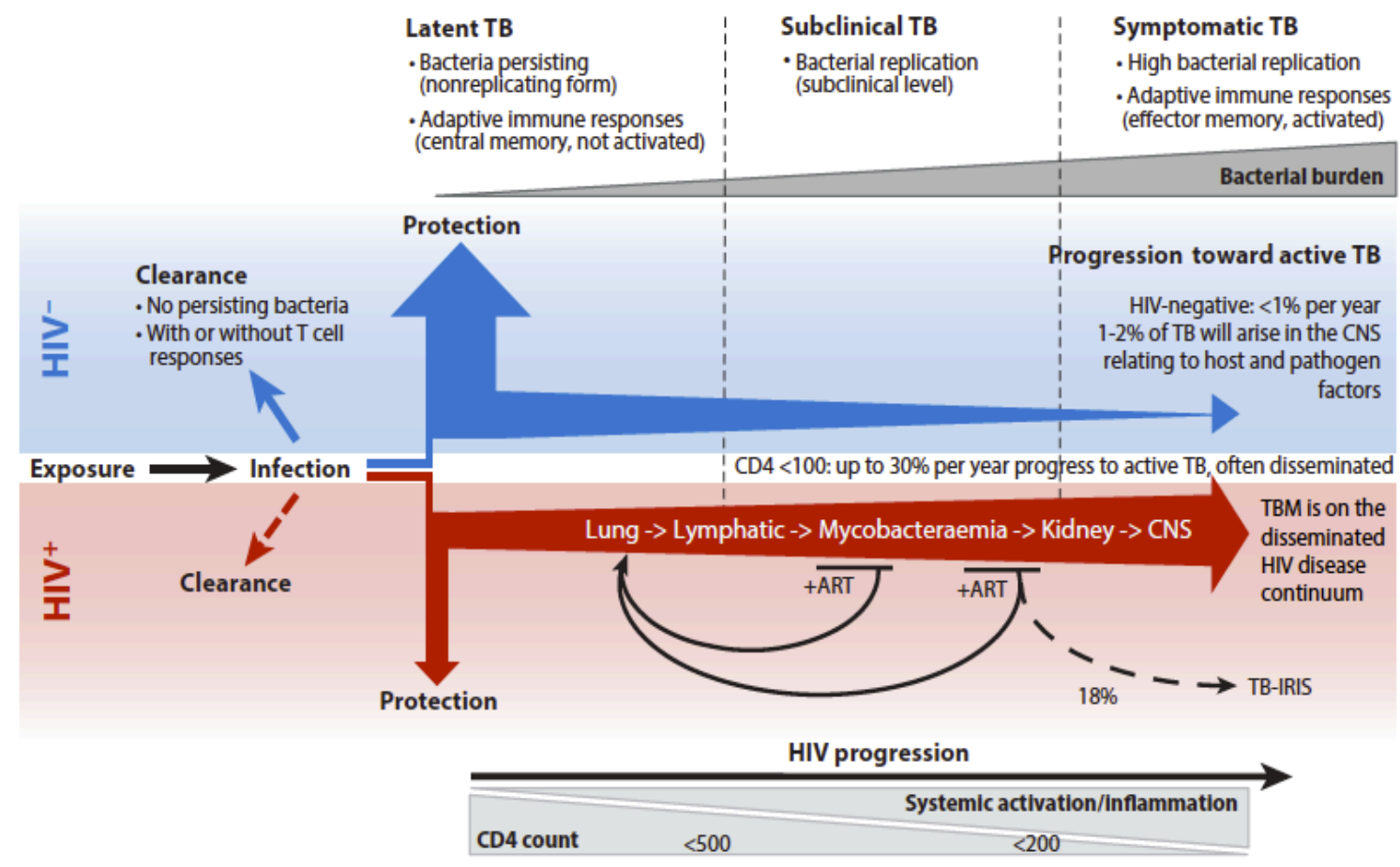

Figure 10.3. Schematic illustrating the natural progression of disease in adults with and without HIV co-infection and the continuum of disseminated HIV disease which has TB meningitis towards the end. TBM = tuberculous meningitis. Shortly after HIV-1 infection and prior to substantial CD4 T cell depletion, the risk of active tuberculosis increases; during the early stages of HIV-1 infection, this risk can rise to approximately $10 \%$ per year; in advanced HIV-1, the risk of active tuberculosis can increase to $30 \%$ per year. While highly beneficial, antiretroviral therapy (ART) does not fully restore the tuberculosisspecific immune response, and ART-treated HIV-infected persons exhibit increased tuberculosis risk despite viral suppression. ART can lead to clinical worsening of patients coinfected with opportunistic pathogens. When immune control 
in the lung and lymphatics fail a mycobacteraemia arises and bacilli are disseminated to multiple organ systems including the spleen, kidneys and CNS (central nervous system). Adapted from Esmail et al. ${ }^{34}$

\subsubsection{Disseminated TB may be a risk factor for death in HIV/TBM}

It is well established that disseminated TB, as evidenced by a positive Alere LAM, is a risk factor for mortality in hospitalised HIV/TB patients. Meta-analysis of 10 studies including 1172 adults with HIV/TB confirmed a 2.5-fold increased mortality risk in patients with positive TB-LAM compared to those with negative urine LAM, even after adjusting for other risk factors for mortality such as CD4+ T cell count. ${ }^{35}$ Positive urine Xpert MTB/Rif is also associated with a 2-fold increased mortality risk, but there is no published data on urine Xpert Ultra and mortality risk.

To the best of my knowledge, the impact of disseminated TB on TBM outcomes has not previously been explored. Case-fatality in definite TBM cases with evidence of disseminated TB disease by a positive urine Xpert Ultra was 50\% (7/14), whereas it was 15\% (3/20) in those with a negative urine Xpert Ultra. The increased mortality in those with HIV/TBM with disseminated TB may relate to multisystem dysfunction and generalised immune dysregulation, rather than specifically CNS-related pathology. There was a similar but less marked trend with urine Alere LAM positivity. This is a small dataset and there is likely to be collinearity with extent of immunosuppression, so this proposition needs to be further explored in a larger study with appropriate adjustment for other baseline variables.

Why might urine Xpert Ultra be a better predictor of mortality than Alere LAM in TBM and what does this tell us? Of the two urine tests, urine Xpert Ultra positivity is perhaps the most representative of renal TB infection which manifests as micro-abscesses in HIV/TB. Whereas urine Alere LAM antigenuria is indicative of glomerular filtration of circulating LAM or LAM fragments from mycobacteraemia or TB disease at a distant site, as well as renal TB itself. ${ }^{36,37}$ In theory, the Xpert assay is designed to detect $M$. tuberculosis DNA associated with bacilli rather than cell free DNA of low molecular weight, as the cartridge has an on-board internal wash step to reduce contamination by cell free DNA pre-amplification. ${ }^{38}$ Therefore, if urine Xpert Ultra positivity truly represents renal TB it may be a marker of the general systemic burden of TB, given that at post-mortem renal TB microabscesses usually occurs when there is also evidence of multi-organ involvement. ${ }^{39-41}$

\subsubsection{Systemic immune dysregulation in disseminated TB}

Disseminated TB is accompanied by a generalised inflammatory response. Professor Meintjes' group have recently done in-depth profiling of the hospitalised adults with HIV/TB in Khayelitsha Hospital, Cape Town, and found an association between disseminated TB and death which was driven by an immune profile dominated by soluble inflammatory mediators associated with the innate immune system and chemotactic signalling (IL1Ra, IL-6 and -8, MIP-1 $\alpha$ and $\beta$, CCL3 and 4, IP-10, CXCL10, 
CCL3). ${ }^{33}$ Similar findings were noted in the STAMP trial participants, those who died had more disseminated TB, higher concentrations of cytokines responsible for innate and pro-inflammatory signaling, elevated markers of inflammation such as C-reactive protein and hepcidin, more marked monocyte activation, and impaired ex vivo functional responses, including cytokine production and phagocyte function, and impaired CD8+ T-cell function. ${ }^{42}$ Higher levels of innate and chemotactic mediators reflect increased activation and recruitment of innate cells into the tissue in response to multi-organ infection. Whilst this is an appropriate response (though ultimately ineffective) it comes with significant immunologic and metabolic costs to the host, including tissue damage and organ dysfunction. ${ }^{33}$ The innate response goes from initially being protective to becoming harmful to the host and ultimately results in death. Interrupting this negative physiological cascade with an appropriate anti-inflammatory drug (as well as or instead of corticosteroids) whilst high-dose rifampicin takes its rapidly bactericidal effect on the infection may be more effective than targeting the bacteria alone.

\subsubsection{The role of co-infections}

The results from the Meintjes' group suggest not only impairment of anti-TB host defenses in patients with disseminated disease and poor outcomes, but potentially also those against bacteria or other coinfections in patients with HIV/TB. Given the general impairment in host defences in HIV/TBM, with the additional insult of high-dose corticosteroids, empirical prophylactic therapy may have a role, particularly antifungal prophylaxis with fluconazole. An ART-naïve Rift study participant who had negative blood and CSF CrAg at baseline went on to develop fulminant cryptococcal meningitis 6 weeks into TBM therapy with adjunctive corticosteroids. The REALITY trial studied an enhanced prophylaxis package consisting of 12 weeks of fluconazole (100mg od) and isoniazid TB preventative therapy, 5 days of azithromycin, and a stat dose of albendazole in addition to the standard of care cotrimoxazole in PLHIV with CD4+ T-cell count $<100$ cells/ $\mu$ at the time of ART initiation, compared to co-trimoxazole alone. ${ }^{43}$ The group who received the enhanced prophylactic package had a $27 \%$ lower relative rate of death at 24 weeks compared to the control group. In the absence of baseline $\mathrm{CrAg}$ testing the enhanced prophylaxis reduced the incidence of cryptococcal disease (9 [1.0\%] vs. 23 [2.6\%], $\mathrm{P}=0.01)$ and candidiasis (10 [1.1\%] vs. $23[2.6 \%], \mathrm{P}=0.02)$ and was well tolerated. ${ }^{43}$ Whilst we are fortunate to have access to CrAg LFA tests in research settings, sadly this is not the case in all routine care settings, so I believe there may be a role for routine fluconazole, dosed of $200 \mathrm{mg}$ daily, in the first 8 weeks of TBM treatment in PLHIV whilst steroids are being used and ART therapy is yet to be initiated/switched.

Whilst most PLHIV routinely receive co-trimoxazole prophylactic therapy, additional antibiotics could also be considered to prevent concomitant bacterial hospital acquired infections, though given the 
widespread antimicrobial resistance this may be counterproductive. In the REALITY trial no reduction in death due to bacterial infections was observed in the group who received azithromycin 500mg daily for 5 days. ${ }^{43}$ The most common blood stream infection in PLHIV is $M$. tuberculosis so this would already be treated by the TBM therapy. ${ }^{44}$ However, with prolonged hospital admissions, intravenous access for dexamethasone and urethral catheterisation patients are also susceptible to other MDR nosocomial infections, which would be interesting to quantify in future studies.

\subsubsection{Adjunctive steroids in HIV/TBM}

The worldwide use of high dose corticosteroids in PLHIV with TBM is questionable given that there is no data confirming a benefit in PLHIV. In the 2004 Thwaites dexamethasone trial there was a trend towards a 9-month survival benefit in the 98 PLHIV within the study but the follow-up period was too short to exclude an increased risk of HIV-related cancers and late fatalities. A prospective study of prednisolone in TB pericarditis with a 2-year follow-up found a 3.3-fold increased risk of HIV-related cancers, particularly Kaposi's sarcoma, with use of corticosteroids in PLHIV. ${ }^{45}$ This is corroborated by a trial of prednisolone in pleural TB which found a significantly higher incidence of Kaposi's sarcoma (4.2 cases/100 person-years compared to 0 cases with placebo). ${ }^{46} \mathrm{~A}$ prospective study is underway to examine the long-term safety of corticosteroids in PLHIV in Asia (NCT03092817) but it is unfortunate that this trial will not include PLHIV in Africa who may experience a quite different array of steroidrelated complications.

In summary, I can infer that HIV-associated TBM is part of the disseminated TB disease spectrum, which is known to co-exist with generalised immune activation, organ dysfunction and susceptibility to co-infections. Corticosteroids may be beneficial in some patients yet harmful in others and a more targeted immunomodulatory therapy (for example aspirin) may be preferable as well as additional prophylactic antifungals until ART-related immune reconstitution has occurred.

\subsubsection{High dose rifampicin should be studied at a larger scale for TBM (and disseminated TB disease) with adequately powered studies}

Based on the observation that the vast majority of TBM patients have CSF levels below the rifampicin MIC in Uganda, it is hard to justify the ongoing use of standard TB therapy for the treatment of TBM. However, changing TB treatment regimens is a complex undertaking from a large-scale programmatic perspective so a very compelling body of evidence must exist for the WHO and national policymakers, to consider the cost-effectiveness and implementation considerations including the pill burden, tolerability and safety in the absence of blood test monitoring in most African settings. 
Therefore, a phase III trial is warranted, and since oral rifampicin $35 \mathrm{mg} / \mathrm{kg}$ achieves therapeutic levels in CSF this is a good regimen to take forward, particularly given that $40 \mathrm{mg} / \mathrm{kg}$ has recently been shown to be the maximum tolerable dose. ${ }^{14}$ Such a study needs to factor into the sample size calculations and analysis plan a priori that a significant proportion of enrolees will not have the disease of interest and non-TB morbidity and mortality is high in PLHIV/AIDS. I would caution against enrolling PLHIV with 'possible' TBM unless a reasonable effort has been made to exclude other pathologies (e.g. CSF viral PCR and brain imaging) or allowing broad eligibility criteria whilst ensuring that the sub-groups of definite/probable TBM are adequately powered for the primary end-point. An adequately powered study will therefore be large and lengthy. Nested within such trials, detailed CSF and blood immune profiling will help better characterize the response to therapy and unique drivers of poor outcomes.

We know that high-dose rifampicin accelerates the time to sputum culture conversion in pulmonary TB, so it may well result in more rapid clearance of disseminated TB disease, albeit a difficult thing to measure microbiologically. I would postulate that a trial of high-dose rifampicin in all forms of disseminated TB (including TBM) as evidenced by positive urine LAM (FujiLAM or Alere) may be valuable, and also quicker to recruit that a TBM-specific study.

\subsection{Future research and engagement}

\subsubsection{Model burden of disease and cost-effectiveness of upstream interventions}

Hospitalized HIV-associated TB patients pose a significant challenge to healthcare services and caregivers; in one report TBM cases account for up to $19 \%$ of hospitalised TB cases, often requiring prolonged hospital stays and rehabilitation periods. ${ }^{47}$ The incidence of TBM in Uganda cannot be ascertained from the data I have available currently, so modelling studies would be useful to understand the burden of TBM nationally, including its contribution to TB deaths, survival with disability, and disability/quality adjusted life years associated with the condition. A modeller at the University of Sheffield (Dr Pete Dodd), investigators from Cape Town, USA, Vietnam and myself are trying to model the global burden of TBM disease by triangulating information from available national level data, TB and TBM cohorts, post-mortem studies and laboratory data.

The cost-effectiveness of upstream interventions could be also modelled. I hypothesize that comprehensive screening for TB in HIV outpatient clinics including consistent access to urine diagnostics (Alere LAM) would be cost-effective in Uganda (as it is in South Africa and Malawi ${ }^{48}$ ) and help to achieve the End TB targets. The availability of urine Alere LAM in hospital settings is also important, but might not be far enough upstream to avert death and disability given how late people 
present to hospital. TPT in outpatient HIV settings has been shown to reduce incident TB and must be more widely implemented. ${ }^{26}$

Policy-makers and other stakeholders in Uganda must prioritise TB screening, access to Alere LAM and TPT in HIV outpatient settings. I plan to ensure that IDI and the many Kampala City Council HIV clinics which it oversees have TB screening and TPT embedded in their standard operating procedures. I will present our findings at an IDI research forum and will invite the National Tuberculosis Control Programme, Center for Disease Control, UNITAID and other stakeholders to advocate for prioritization of TB screening and TPT, alongside community-based HIV testing. In addition, along with the Medical Officers, I will present to the Kiruddu Hospital management, medics and medical students at a grand round to share our findings and advocate for provision of lumbar puncture equipment.

\subsubsection{Continue to improve low and high technology diagnostics for TBM}

According to the WHO's End TB strategy, improved diagnostic tests are required as a means to stop the global tuberculosis epidemic by 2035. Thanks to collaborative efforts from Foundation for Innovative New Diagnostics (FIND), funders and researchers worldwide there are promising non-sputum-based diagnostics in the pipeline, as shown in Figure 10.4, some of which will also have utility in TBM.

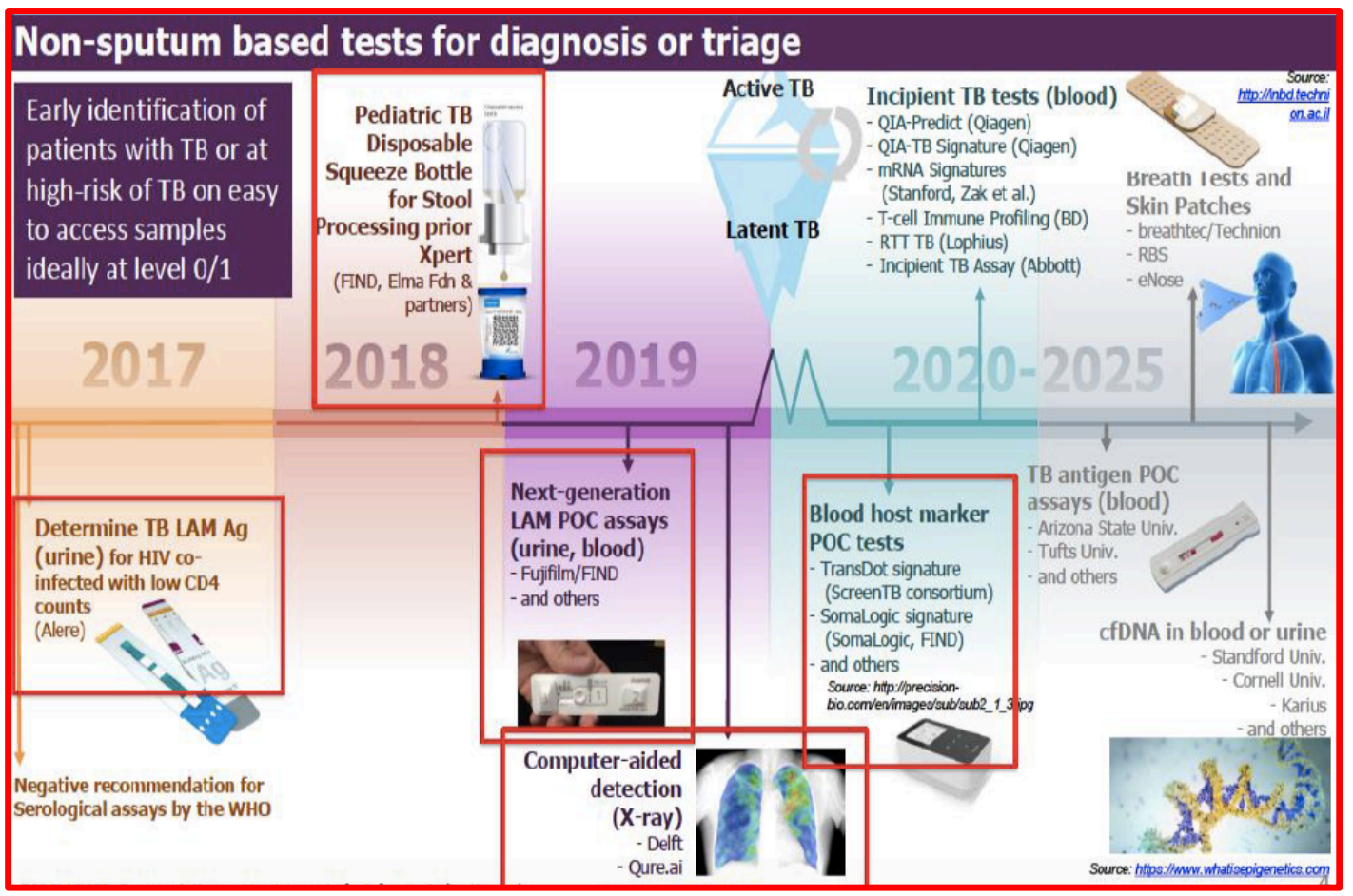

Figure 10.4 Non-sputum-based TB diagnostic pipeline. 
FujiFilm SILVAMP TB-LAM will detect more TB than Alere LAM and its diagnostic performance in TBM needs to be properly evaluated, both on urine and CSF. We have recently conducted a pilot study of FujiLAM on CSF in Uganda on 101 adults with suspected TBM and the results are quite promising: sensitivity of FujiLAM on CSF was 74\% (25/34) for definite TBM and 51\% for definite or probable TBM, compared to 94\% (32/34) and 54\% (32/59) for Xpert Ultra. FujiLAM specificity was surprisingly good 98\% (41/42) for probable/definite TBM (submitted to Clinical Infectious Diseases on $15^{\text {th }}$ October 2020). We are also beginning a collaborative study with FIND examining FujiLAM on urine in suspected TBM patients. FujiLAM has the advantage of being a near patient test with results available in under an hour, so it may have a role in supporting treatment decisions in probable/possible TBM cases, but unlike Xpert Ultra, it does not provide any information on rifampicin resistance so would ideally be used as an adjunctive test. FujiLAM may have a role in reducing the diagnostic lead time in smaller health facilities where the Xpert platform is not rapidly accessible, e.g. facilities where Xpert provided through a hub-and-spoke system, and the turnaround time for results can be several days. Lots of questions remain to be explored regarding the potential of $\underline{\text { NGS }}$ as a diagnostics tool particularly when considering the host immune signature. In some patients a very vigorous host immune response may be so effective at controlling the infection that it renders it impossible to detect the bacilli/DNA with conventional tests, yet the immune signature could provide evidence of M. tuberculosis infection. Using CSF samples collected by the meningitis research team in Uganda beyond the Xpert Ultra study participants, and in collaboration with Dr Wilson and Dr Ramachandran at UCSF, a combined host and pathogen classifier was developed in a training sample set and is now being examined in a larger validation sample set. Preliminary results recently presented at American Academy of Neurology conference (Appendix A6b) found that among 'probable' TBM cases (i.e. negative by Xpert Ultra and $\geq 11$ points) the host transcriptomic classifier predicted that $71 \%$ (5/7) were TBM including one case where $M$. tuberculosis DNA was identified by mNGS. Within 'possible' TBM cases (i.e. negative by Xpert Ultra and $>9$ points), the host transcriptomic signature classified 9/53 (17\%) as TBM, including one mNGS-positive TBM case. mNGS also identified 9 other pathogens (4 viral, 2 bacterial, 2 toxoplasmosis and 1 TBM-viral co-infection) within the 'possible' TBM group. The host and pathogen classifier findings will be submitted for publication by Dr Ramachandran in the near future. The turnaround time, cost and the degree of technical expertise required for mNGS are rate-limiting factors in LMIC settings currently. However, work is underway to create a low-tech host signature assay for the Cepheid Xpert platform.

Researchers at Stanford University have embraced the heterogeneity in host responses between different TB populations and using machine learning techniques on global publicly available TB datasets have identified a combinatory score (TB-score) based on whole blood mRNA levels of only 
three differentially expressed genes (GBP5, DUSP3 and KLF2) which can discriminate between active TB disease and other diseases. ${ }^{49}$ Cepheid have taken this 3-gene signature and developed a prototype host response cartridge (Xpert-MTB-HR-Prototype) that quantifies 3-gene mRNA-levels in whole blood (fingerprick sample). FIND have recently examined the performance of the Xpert-MTB-HRPrototype on bio-banked blood samples from PLHIV/TB: area under the receiver-operatorcharacteristics (ROC) curve was 0.89 (95\% Cl, 0.83-0.94) for active TB against a comprehensive microbiological reference standard. ${ }^{50} \mathrm{~A}$ host response assay for TB would be a huge leap forwards for diagnosis of paucibacillary TB disease and extrapulmonary TB and I am keen to explore this assay in Ugandan TBM suspects. Whether the whole blood and CSF host transcriptional signature differ remains to be determined. Preliminary results of CSF host transcriptional profiling from UCSF suggest that a combination of 4 genes (GBP5, SOD2, FTL, NFKBIA) discriminate TBM from other forms of meningitis but this will continue to be refined on a larger sample set.

A TBM host - pathogen - diagnostic framework remains to be defined; one proposed framework is depicted in Figure 10.5.

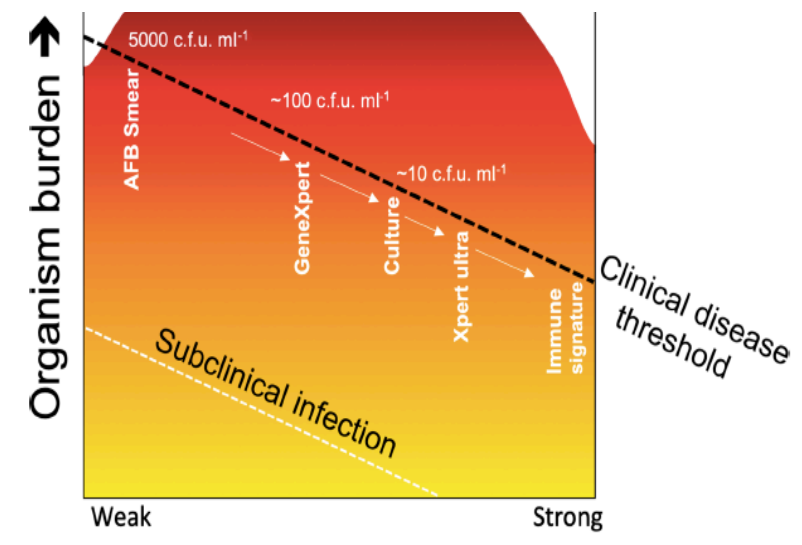

Effective $\mathrm{Th}_{1}$ immune response $\rightarrow$
Figure 10.5. Diagnostic framework for TBM. Along the

horizontal axis is increasing immune response and along the vertical axis is organism burden. As immune response increases organism burden declines, and thus increasingly sensitive tests with lower limits of detection are needed to detect disease. At the far right end the immune response is so strong the bacilli/DNA are undetectable by PCR but an immune signature could be used to diagnose disease. Th1 $=\mathrm{T}$ helper cell 1 (cell-mediated immunity against intracellular bacteria, mediated by IFN- $\gamma$ and IL-2, effector cells macrophages and CD8+ T-cells). ${ }^{51}$

Whilst the technology is exciting and fast-moving we must not neglect the importance of grassroots good clinical decision making. Medical Officers in Uganda and other LMIC settings are often working without senior support having to make highly emotive treatment decisions. It is perhaps not surprising that in the chapter 4 retrospective cohort that there was such a heterogeneity in amount of empirical treatment given between the two Ugandan referral hospital sites.$^{52} \mathrm{~A}$ clinical predication tool built with local data might be able to support clinicians in making these challenging decisions. A smartphone application that uses a data-driven model (that considers presenting clinical features, results of CSF and other diagnostics tests) could serve as a clinical prediction tool to support decisionmaking. I am collaborating with investigators from Witwatersrand University and University of 
Minnesota on an individual patient data meta-analysis and the development of a clinical predication tool, the protocol for which has been published. ${ }^{53}$ My specific role was systematically reviewing abstracts from a comprehensive literature search and identifying RCTs, cohort or cross-sectional studies which met the eligibility criteria. From the studies identified we have gathered data from over 5000 individuals from 7 countries (including Uganda). The data is currently being cleaned and the modelling approach finalised by data scientists on the team.

\subsubsection{Better characterization of the TBM population}

We need to better characterise the patient population from both a microbiological and immunological perspective so the we can understand the different clinical phenotypes, burden of coinfection, co-morbidities and the heterogeneity in the immune response. Extensive microbiological characterisation (including post-mortem examinations) allows for a better estimate of the true TB status and identification of other co-infections and morbidities. It would also be interesting to examine whether these is any relationship between genetic diversity in M. tuberculosis and clinical phenotype, as has recently been described in pulmonary TB. ${ }^{54}$ It is incredible to consider From an immunology perspective, host immunity clearly contributes to TBM pathogenesis, but exactly how still needs to be defined in our Ugandan HIV/TBM population. A fascinating recent report from a study nested in ongoing Vietnamese and Indonesian TBM clinical trials found that active Strongyloides stercoralis infection (by stool microscopy or PCR) modulated CSF inflammatory response (reduced pre-treatment CSF neutrophils, CSF IFN- $\gamma$, and TNF- $\alpha$ ) and was associated with reduced neurological complications by 3 months when compared to patients without active $S$. stercoralis infection (3.8\% [1/26] vs. 30\% [22/110] respectively, $p=0.01)$. Additionally, there was reduced Xpert positivity in the active S. stercoralis group (3.8\% [1/26]) vs. the S. stercoralis uninfected group $(28.2 \%[31 / 110])(p=0.03)^{55} \mathrm{It}$ is incredible to think that a intestinal helminth infection could have an impact on inflammatory response to TB with a knock on effect on diagnostics and disease outcomes. Who knows what impact helminth infections are having on TB disease in Uganda, particularly Schistosoma mansoni infection which is endemic in country. Helminth infection may be an additional contributory factor to the degree of heterogeneity we see in TBM presentation and outcomes and should certainly be explored further.

There may also be heterogeneity between ethnic populations, for example the LTA4H genetic polymorphism in Vietnamese adults which strongly determines steroid responsiveness and survival from TBM, did not have the same impact in Indonesians with TBM. Therefore, we must be cautious about extrapolating findings from one population into another, and multi-country studies are called 
for. Nested within diagnostic or interventional studies in Uganda, we need to characterise cellular populations, cytokines and transcriptomic profiles, and identify responses that correlate with clinical outcomes. This may identify new biomarkers and potential targets for host-directed therapies (HDTs).

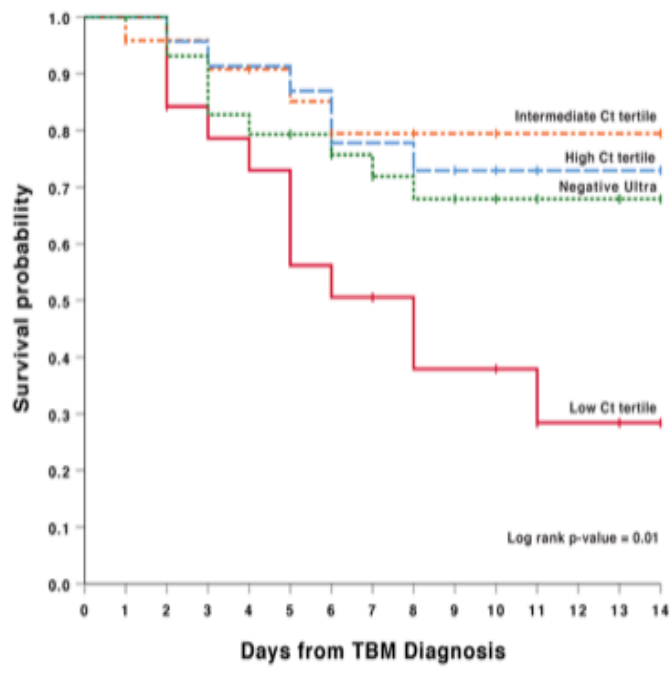

Pathogen-related biomarkers are also important to further explore both in urine (Ultra, LAM, FujiLAM) and CSF (lactate, PCR cycle threshold, FujiLAM). In our Ugandan TBM cohort, recent exploratory analysis of Xpert UItra bacillary load as measured by PCR cycle threshold ( $\mathrm{Ct}$, low $\mathrm{Ct}=$ high bacillary load) suggests that high bacillary load is a predictor of death, Figure 10.6. We need contemporary immune profiling to disentangle these relationships and elucidate a microbiologic and immunologic framework for TBM disease.

Figure 10.6. Kaplan Meier survival estimate by CSF Ultra cycle threshold (Ct) from 101 patients with suspected or confirmed TBM. Where Ultra results were positive the IS1081/IS6110 Ct values were divided into tertiles. The survival of patients with negative Ultra results is shown by the green line. (In press Clinical Infectious Diseases)

The Medical Research Council TBM severity grade is a simple and useful tool for assessing severity but it is not clear whether it informs prognosis in Africans with HIV/TBM. With larger datasets it may be possible to derive and externally validate a prognostic score in HIV/TBM which considers baseline characteristics such as GCS, focal neurology, blood results (CD4+ T cell count, haemoglobin, sodium), CSF results (Xpert Ultra result and Ct, lactate, WCC), urine TB results (LAM or Ultra or both), ART status.

Illuminating the pathogenesis of HIV/TBM is clearly fundamental to improving outcomes. Although immunology studies were outside the scope of this PhD I hope stored samples will be useful in providing some future insights.

\subsubsection{Optimising antimicrobial therapy for TBM}

The momentum around high dose rifampicin is building and a number of clinical trials have recently been funded examining high-dose rifampicin, including three trials in Uganda as outlined below:

1. ALTER - phase II, open-label factorial design RCT. High-dose rifampicin $(35 \mathrm{mg} / \mathrm{kg}$ ) orally for 4 weeks +/- linezolid 1200mg orally for 4 weeks. standard dose H, Z, E. N=60 Site: Masaka Hospital. PI: Dr F. Chow. Sponsor: UCSF, USA. NCT04021121.

https://clinicaltrials.gov/ct2/show/NCT04021121 
2. INTENSE TBM - phase III, open-label placebo-controlled RCT. High-dose rifampicin $35 \mathrm{mg} / \mathrm{kg}$ for 8 weeks, +/- linezolid $1200 \mathrm{mg}$ orally for 4 weeks then $600 \mathrm{mg}$ for 4 weeks, +/- aspirin $100 \mathrm{mg} /$ day. Standard dose H, Z, E. N=768. Site: Mbarara Regional Referral Hospital, Uganda (along with sites in Cote D'Ivoire, Madagascar, South Africa). PI: Sponsor: French National Agency for Research on AIDS and Viral Hepatitis. NCT03927313 https://intensetbm.org/clinical-trial.

3. HARVEST - phase III, two-arm parallel design double-blind placebo-controlled RCT. Standard $\mathrm{RHZE}+$ rifampicin $1800 \mathrm{mg}$ orally 8 weeks versus placebo. $\mathrm{N}=500$. Site: Mulago National Referral Hospital (along with sites in South Africa and Indonesia). PI: Prof Ruslami and Dr Meya. Sponsor: Infectious Diseases Institute, Uganda. ISRCTN15668391. http://www.isrctn.com/ISRCTN15668391

Our group in Uganda are leading the HARVEST trial which is examining an intervention which would be relatively easy to implement if it proves to be efficacious - RHZE as standard combination therapy plus a flat dose of $3 \times 600 \mathrm{mg}$ rifampicin tablets for 8 weeks. Modelling work by collaborators at Radboud University Medical Centre has shown that weight-based dosing of rifampicin yields a small non-clinically relevant decrease in variability in total rifampicin exposure, ${ }^{56}$ so using a flat-dose intervention is a reasonable and less complex approach. Rifampicin is cheap and generically made within Africa which might facilitate implementation.

In the implementation of any novel TB regimen the safety of its use outside of a clinical trial setting (i.e. without routine blood test monitoring) must be considered alongside potential drug interactions with ART or other over-the-counter medicines. Liver toxicity is probably the biggest safety concern in TB cohorts worldwide, it occurred in $11 \%$ of Rift enrolees, and can be fatal if medications are not interrupted. Although, in TBM, interruption of treatment is associated with poor outcomes in itself, so pragmatic guidance needs to be developed about how to manage DILI in a LMIC setting. The approach that I adopted in Rift study (interruption of pyrazinamide in isolation if ALT >3XULN) allowed other TB drugs to be continued, but requires availability of individual formulations of antituberculous agents. This approach, along with tolerating a higher LFT threshold before any drugs are interrupted, will be further explored in a sub-study of the HARVEST trial. Given that high-dose rifampicin can cause a transient hyperbilirubinaemia in the first 1-2 weeks, jaundice may be a misleading early symptom but late onset jaundice may be a worrying feature. Blood biomarkers for DILI risk are being investigated so that high risk patients could be identified at the start of treatment and active liver monitoring planned. ${ }^{57}$

The PanACEA consortium have recently suggested that the most promising programmatic strategy for high dose rifampicin in the treatment of pulmonary TB would be to start TB therapy with a standard rifampicin dose, allowing the body to get used to rifampicin in terms of facilitating clearance autoinduction and bilirubin clearance, before escalating the dose. ${ }^{14}$ After this initial period, a higher 
dose of rifampicin could be introduced in all patients. However, in TBM where there is high early mortality, this approach is unlikely to be successful. Personalized medicine using a low-cost point of care device to estimate rifampicin concentrations in real-time with titration for individual maximum exposures is also an option that needs to be explored. ${ }^{14}$ Outside of the high-dose rifampicin arena, rifapentine, a fellow rifamycin, has recently been shown to be effective in the treatment of pulmonary TB as part of a 4-month treatment regimen with moxifloxacin, isoniazid and pyrazinamide. The CSF pharmacokinetics of rifapentine warrant examination in case it becomes a WHO-recommended regimen. ${ }^{58}$

It is important that diagnostic and immunology studies are nested within RCTs for the reasons outlined in sections above and so that if the interventions don't work we can understand why. Optimal methods and timing of sample collection have recently been outlined in a paper by the TBM consortium. ${ }^{59}$ It is possible that high-dose rifampicin will reduce CSF cell trafficking and immune activation as a result of faster TB killing, conversely more rapid killing might prompt a more florid immune response, we will only know if longitudinal samples are collected for immune profiling.

\subsubsection{Optimising the host status}

In parallel with optimal antimicrobial therapy we must endeavour to optimise the host status, both through supportive care and HDTs to allow high-dose rifampicin time to exert its killing effect. Local supportive care solutions need to be defined and tested as interventions from northern settings tend to generalise poorly to low resource settings; for example, 'early-goal directed therapy for sepsis' and 'goal-directed therapy for suspected acute bacterial meningitis', both paradoxically led to increased mortality in sub-Saharan Africa. ${ }^{60,61}$ Clearly, basic supportive considerations such as nutrition, thromboprophylaxis, co-trimoxazole prophylaxis +/- antifungals are important in this highly immunocompromised group. Anemia has been associated with poor outcomes in HIV/TB so the role of blood transfusion could also be further explored.

Cerebral infarction occurs in around $25 \%{ }^{62}$ of TBM cases and was associated with a twofold increase in mortality in a recent meta-analysis. ${ }^{63}$ TBM is a hypercoagulable inflammatory state, which contributes to the pathogenesis of stroke, and I hypothesise that an adjunctive medication which can reduce the incidence and severity of strokes will be essential to reduce TBM death and disability, of which high-dose aspirin in the most promising. I believe one size does not fit all when it comes to TBM. We need to identify which sub-populations benefit or are harmed by adjunctive corticosteroids and how their ART status intersects with steroid requirements, and likewise who may benefit from 
other HDTs such as aspirin or other immunomodulating drugs to augment or dampen specific immune responses.

In HIV/TBM the timing and choice of ART regimen will also be key to improving outcomes. If the $M$. tuberculosis antigen burden is cleared more rapidly with high-dose rifampicin it may be possible to start ART earlier (rather than waiting til week 8 as is current practice in many settings), which may shorten the period that the individual remains susceptible to opportunistic illnesses. As DTG is rolled out as first-line HIV treatment across Africa, we must confirm that high-dose rifampicin can be used safely with DTG-based ART after dose-adjustment to $50 \mathrm{mg}$ twice daily, both from a PK and safety perspective. Whilst expert opinion is that CYP450 enzyme induction is already maximal with standard dose rifampicin, the were some cautionary findings within the RIFAVIRENZ study (section 1.5.3), so this needs to be confirmed with adequately powered nested PK study (planned within HARVEST trial).

\subsection{Conclusion}

TBM is the second leading cause of adult meningitis in Uganda, is largely seen in people with advanced HIV-related immunosuppression and carries a mortality of approximately $40 \%$. Introduction of the Cepheid Xpert platform in government hospitals has increased rates of microbiological confirmation of TBM to around 50\%, and Xpert Ultra is the best first test for TBM with a sensitivity of 77\% for probable/definite TBM and negative predictive value of 93\%. Disseminated TB, detectable by urine Alere LAM or urine Xpert Ultra, is present in over half of patients with HIV/TBM and urine Ultra positivity may serve as a prognostic biomarker. The disseminated nature of TB disease and associated multi-system dysfunction may be a driver of the excess mortality in HIV-associated TBM.

With currently WHO-recommended TB treatment, CSF rifampicin concentration is subtherapeutic in the majority of TBM patients in Uganda. Compared to standard TB therapy, total serum and CSF exposures are increased by roughly 6 -fold and roughly 8 -fold with intravenous rifampicin $20 \mathrm{mg} / \mathrm{kg}$ and oral rifampicin $35 \mathrm{mg} / \mathrm{kg}$ respectively. Almost all the participants who received high-dose rifampicin had CSF levels above the local M. tuberculosis rifampicin MIC. There was no excess toxicity with high-dose rifampicin but no impact on survival or functional outcomes was observed in this phase II study. An upcoming multi-centre phase III study, including sites in Uganda, Indonesia and South Africa will examine the impact of high-dose rifampicin on survival from TBM. Nested within this trial, through old fashioned pathology studies combined with modern 'omics' approaches, we aim to better understand the different clinical phenotypes and unique drivers of poor outcomes in HIV/TBM including co-infections, immune activation/exhaustion, poly-pharmacy including ART and corticosteroids. 
Whilst we continue to optimise antimicrobial and host-directed therapies for TBM we must also remember that prevention is better than cure. Upstream interventions such as HIV testing, earlier ART initiation and interventions to support ART adherence and retention in care are vital to prevent the apparent main driver of TBM in Uganda - advanced HIV disease. Improved TB screening including access to urine TB diagnostics in outpatient settings and tuberculosis preventative therapy are also important tools in reducing incident TBM and its associated morbidity and mortality in Uganda. 


\section{Bibliography}

1. Jarvis JN, Meintjes G, Williams A, Brown Y, Crede T, Harrison TS. Adult meningitis in a setting of high HIV and TB prevalence: findings from 4961 suspected cases. BMC Infect Dis 2010; 10: 67.

2. Hakim JG, Gangaidzo IT, Heyderman RS, et al. Impact of HIV infection on meningitis in Harare, Zimbabwe: a prospective study of 406 predominantly adult patients. AIDS 2000; 14(10): 1401-7.

3. Heemskerk AD, Bang ND, Thwaites GE. Therapy for Tuberculous Meningitis. N Engl J Med 2016; 374(22): 2188-9.

4. Sheu JJ, Yuan RY, Yang CC. Predictors for outcome and treatment delay in patients with tuberculous meningitis. Am J Med Sci 2009; 338(2): 134-9.

5. Heemskerk AD, Bang ND, Mai NT, et al. Intensified Antituberculosis Therapy in Adults with Tuberculous Meningitis. N Engl J Med 2016; 374(2): 124-34.

6. Stadelman AM, Ellis J, Samuels THA, et al. Treatment Outcomes in Adult Tuberculous Meningitis: A Systematic Review and Meta-analysis. Open Forum Infect Dis 2020; 7(8): ofaa257.

7. Ellis J, Cresswell FV, Joshua R, Kenneth S, Boulware DR. Cryptococcal Meningitis and Tuberculous Meningitis Co-infection in HIV-Infected Ugandan Adults. Open Forum Infect Dis 2018; 5(8): ofy193.

8. Alsultan A, Peloquin CA. Therapeutic drug monitoring in the treatment of tuberculosis: an update. Drugs 2014; 74(8): 839-54.

9. Jayaram R, Gaonkar S, Kaur P, et al. Pharmacokinetics-pharmacodynamics of rifampin in an aerosol infection model of tuberculosis. Antimicrob Agents Chemother 2003; 47(7): 2118-24.

10. Abbara A, Chitty S, Roe JK, et al. Drug-induced liver injury from antituberculous treatment: a retrospective study from a large TB centre in the UK. BMC Infect Dis 2017; 17(1): 231.

11. Ruslami R, Ganiem AR, Dian S, et al. Intensified regimen containing rifampicin and moxifloxacin for tuberculous meningitis: an open-label, randomised controlled phase 2 trial. Lancet Infect Dis 2013; 13(1): 27-35.

12. Yunivita V, Dian S, Ganiem AR, et al. Pharmacokinetics and safety/tolerability of higher oral and intravenous doses of rifampicin in adult tuberculous meningitis patients. Int J Antimicrob Agents 2016; 48(4): 415-21.

13. Dian S YV, Ganiem AR, Achmad TH, Colbers A, te Brake L, van Crevel R, Ruslami R, Aarnoutse R. . High dose rifampicin for the treatment of TB meningitis: a dose finding study. . 10th International Workshop on Pharmacology of TB Drugs,. Atlanta, USA; 2017.

14. te Brake L, de Jager V, Narunsky K, et al. Increased Bactericidal Activity But Dose-Limiting Tolerability at $50 \mathrm{mg} / \mathrm{kg}$ Rifampicin SSRN 2020.

15. Nabisere R, Musaazi J, Denti P, et al. Pharmacokinetics, SAfety/tolerability, and EFficacy of high-dose RIFampicin in tuberculosis-HIV co-infected patients on efavirenz- or dolutegravirbased antiretroviral therapy: study protocol for an open-label, phase II clinical trial (SAEFRIF). Trials 2020; 21(1): 181.

16. World Health Organisation. The End TB Strategy. Geneva, Switzerland: World Health Organisations, 2015. 
17. Ford N, Matteelli A, Shubber Z, et al. TB as a cause of hospitalization and in-hospital mortality among people living with HIV worldwide: a systematic review and meta-analysis. $J$ Int AIDS Soc 2016; 19(1): 20714.

18. Organisation WH. Systamtic Screening for Active Tuberculosis. Geneva, Switzerland. , 2013.

19. World Health Organisation. Lateral flow urine lipoarabinomannan assay (LF-LAM) for the diagnosis of active tuberculosis in people living with HIV. Policy update 2019. Geneva, 2019.

20. Dhasmana DJ, Dheda K, Ravn P, Wilkinson RJ, Meintjes G. Immune reconstitution inflammatory syndrome in HIV-infected patients receiving antiretroviral therapy : pathogenesis, clinical manifestations and management. Drugs 2008; 68(2): 191-208.

21. Hoyo-Ulloa I, Belaunzaran-Zamudio PF, Crabtree-Ramirez B, Galindo-Fraga A, PerezAguinaga ME, Sierra-Madero JG. Impact of the immune reconstitution inflammatory syndrome (IRIS) on mortality and morbidity in HIV-infected patients in Mexico. Int $\mathrm{J}$ Infect Dis 2011; 15(6): e408-14.

22. World Health Organisation. Prevent HIV, test and treat all - WHO support for country impact. Geneva, 2016.

23. Gupta-Wright A, Fielding $\mathrm{K}$, Wilson $\mathrm{D}$, et al. Tuberculosis in hospitalised patients with HIV: clinical characteristics, mortality, and implications from the STAMP trial. Clin Infect Dis 2019.

24. World Health Organisation. WHO consolidated guidelines on tuberculosis: module 1: prevention: tuberculosis preventive treatment. Geneva, 2020.

25. World Health Organisation. Tuberculosis country profiles: Uganda. 2020. https://worldhealthorg.shinyapps.io/tb profiles/? inputs \&lan=\%22EN\%22\&iso2=\%22UG\% $\underline{22}$ (accessed 15th October 2020.

26. Ross J. Isoniazid preventive therapy added to ART to prevent TB disease: an individual participant data meta-analysis. The 51st Union World Conference on Lung Health; 2020; 2020.

27. Boyles TH, Lynen L, Seddon JA, Tuberculous Meningitis International Research C. Decisionmaking in the diagnosis of tuberculous meningitis. Wellcome Open Res 2020; 5: 11.

28. Donovan J, Thu DDA, Phu NH, et al. Xpert MTB/RIF Ultra versus Xpert MTB/RIF for the diagnosis of tuberculous meningitis: a prospective, randomised, diagnostic accuracy study. Lancet Infect Dis 2020.

29. Donovan J, Cresswell FV, Thuong NTT, et al. Xpert MTB/RIF Ultra for the Diagnosis of Tuberculous Meningitis: A Small Step Forward. Clin Infect Dis 2020.

30. Bahr NC, Nuwagira E, Evans EE, et al. Diagnostic accuracy of Xpert MTB/RIF Ultra for tuberculous meningitis in HIV-infected adults: a prospective cohort study. Lancet Infect Dis 2018; 18(1): 68-75.

31. Chakravorty S, Simmons AM, Rowneki M, et al. The New Xpert MTB/RIF Ultra: Improving Detection of Mycobacterium tuberculosis and Resistance to Rifampin in an Assay Suitable for Point-of-Care Testing. MBio 2017; 8(4).

32. Ankur Gupta-Wright, Katherine Fielding, Douglas Wilson, et al. Tuberculosis in Hospitalized Patients With Human Immunodeficiency Virus: Clinical characteristics, Mortality, and Implications From the Rapid Urine-based Screening for Tuberculosis to Reduce AIDS Related Mortality in Hospitalized Patients in Africa. Clinical Infectious Diseases 2020. 
33. Schutz C, Barr D, Andrade BB, et al. Clinical, microbiologic, and immunologic determinants of mortality in hospitalized patients with HIV-associated tuberculosis: A prospective cohort study. PLoS Med 2019; 16(7): e1002840.

34. Esmail H, Riou C, Bruyn ED, et al. The Immune Response to Mycobacterium tuberculosis in HIV-1-Coinfected Persons. Annu Rev Immunol 2018; 36: 603-38.

35. Gupta-Wright A, Peters JA, Flach C, Lawn SD. Detection of lipoarabinomannan (LAM) in urine is an independent predictor of mortality risk in patients receiving treatment for HIVassociated tuberculosis in sub-Saharan Africa: a systematic review and meta-analysis. BMC Med 2016; 14: 53.

36. Kerkhoff AD, Sossen B, Schutz C, et al. Diagnostic sensitivity of SILVAMP TB-LAM (FujiLAM) point-of-care urine assay for extra-pulmonary tuberculosis in people living with HIV. Eur Respir J 2020; 55(2).

37. Lawn SD, Gupta-Wright A. Detection of lipoarabinomannan (LAM) in urine is indicative of disseminated TB with renal involvement in patients living with HIV and advanced immunodeficiency: evidence and implications. Trans R Soc Trop Med Hyg 2016; 110(3): 1805.

38. Blakemore R, Story E, Helb D, et al. Evaluation of the analytical performance of the Xpert MTB/RIF assay. J Clin Microbiol 2010; 48(7): 2495-501.

39. Rana FS, Hawken MP, Mwachari C, et al. Autopsy study of HIV-1-positive and HIV-1-negative adult medical patients in Nairobi, Kenya. J Acquir Immune Defic Syndr 2000; 24(1): 23-9.

40. Kerkhoff AD, Barr DA, Schutz $C$, et al. Disseminated tuberculosis among hospitalised HIV patients in South Africa: a common condition that can be rapidly diagnosed using urinebased assays. Sci Rep 2017; 7(1): 10931.

41. Gupta RK, Lucas SB, Fielding KL, Lawn SD. Prevalence of tuberculosis in post-mortem studies of HIV-infected adults and children in resource-limited settings: a systematic review and meta-analysis. AIDS 2015; 29(15): 1987-2002.

42. Gupta-Wright A. Investigating mortality risk in hospitalised patients in Africa with HIVassociated tuberculosis and positive urine diagnostics: a clinical, epidemiological and immunological study: London School of Hygiene \& Tropical Medicine; 2018.

43. Hakim J, Musiime V, Szubert AJ, et al. Enhanced Prophylaxis plus Antiretroviral Therapy for Advanced HIV Infection in Africa. N Engl J Med 2017; 377(3): 233-45.

44. Crump JA, Ramadhani HO, Morrissey AB, et al. Bacteremic disseminated tuberculosis in subsaharan Africa: a prospective cohort study. Clin Infect Dis 2012; 55(2): 242-50.

45. Mayosi BM, Ntsekhe M, Bosch J, et al. Prednisolone and Mycobacterium indicus pranii in tuberculous pericarditis. N Engl J Med 2014; 371(12): 1121-30.

46. Elliott $A M$, Luzze $H$, Quigley $M A$, et al. A randomized, double-blind, placebo-controlled trial of the use of prednisolone as an adjunct to treatment in HIV-1-associated pleural tuberculosis. J Infect Dis 2004; 190(5): 869-78.

47. Alvarez-Uria G NP, Pakam R, Bachu L, Midde M. . Natural History and Factors Associated with Early and Delayed Mortality in HIV-Infected Patients Treated of Tuberculosis under Directly Observed Treatment Short-Course Strategy: A Prospective Cohort Study in India. . Interdiscip Perspect Infect Dis 2012: 502012.

48. Reddy KP, Gupta-Wright A, Fielding KL, et al. Cost-effectiveness of urine-based tuberculosis screening in hospitalised patients with HIV in Africa: a microsimulation modelling study. Lancet Glob Health 2019; 7(2): e200-e8. 
49. Sweeney TE, Braviak L, Tato CM, Khatri P. Genome-wide expression for diagnosis of pulmonary tuberculosis: a multicohort analysis. Lancet Respir Med 2016; 4(3): 213-24.

50. Erik Södersten, Stefano Ongarello, Anna Mantsoki, et al. Diagnostic accuracy study of a novel blood-based assay for identification of TB in people living with HIV. medRxiv preprint 2020.

51. Bahr NC, Meintjes G, Boulware DR. Inadequate diagnostics: the case to move beyond the bacilli for detection of meningitis due to Mycobacterium tuberculosis. J Med Microbiol 2019; 68(5): 755-60.

52. Cresswell FV, Bangdiwala AS, Bahr NC, et al. Can improved diagnostics reduce mortality from Tuberculous meningitis? Findings from a 6.5-year cohort in Uganda. Wellcome Open Res 2018; 3: 64.

53. Boyles T, Stadelman S, Ellis J, et al. The diagnosis of tuberculous meningitis in adults and adolescents: protocol for a systematic review and individual patient data meta-analysis to inform a multivariable prediction model. Wellcome Open Res 2020; 4.

54. Sousa J, Ca B, Maceiras AR, et al. Mycobacterium tuberculosis associated with severe tuberculosis evades cytosolic surveillance systems and modulates IL-1beta production. Nat Commun 2020; 11(1): 1949.

55. Donovan J TT, Phu NH, et al. . The influence of Strongyloides stercoralis co-infection on the presentation, pathogenesis and outcome of tuberculous meningitis. Journal of Infectious Diseases 2020; In Press. .

56. Susanto BO, Svensson RJ, Svensson EM, Aarnoutse R, Boeree MJ, Simonsson USH. Rifampicin can be given as flat-dosing instead of weight-band dosing. Clin Infect Dis 2019.

57. Rupprechter SAE SD, Oosthuyzen WO, . Cytokeratin-18 has potential as a biomarker of druginduced liver injury in European and African patients on treatment for tuberculosis. medRxiv 2020.

58. Dorman SE. The design and primary efficacy results of Study 31/A5349. 51st Union World Lung Health Conference, 2020; 2020; 2020.

59. Rohlwink UK, Chow FC, Wasserman S, et al. Standardized approaches for clinical sampling and endpoint ascertainment in tuberculous meningitis studies. Wellcome Open Res 2019; 4: 204.

60. Morton B, Stolbrink M, Kagima W, Rylance J, Mortimer K. The Early Recognition and Management of Sepsis in Sub-Saharan African Adults: A Systematic Review and MetaAnalysis. Int J Environ Res Public Health 2018; 15(9).

61. Wall EC, Mukaka M, Denis B, et al. Goal directed therapy for suspected acute bacterial meningitis in adults and adolescents in sub-Saharan Africa. PLoS One 2017; 12(10): e0186687.

62. Wasay M, Khan M, Farooq S, et al. Frequency and Impact of Cerebral Infarctions in Patients With Tuberculous Meningitis. Stroke 2018; 49(10): 2288-93.

63. Wen L, Li M, Xu T, Yu X, Wang L, Li K. Clinical features, outcomes and prognostic factors of tuberculous meningitis in adults worldwide: systematic review and meta-analysis. J Neurol 2019; 266(12): 3009-21. 
APPENDIX 



\title{
Treatment Outcomes in Adult Tuberculous Meningitis: A Systematic Review and Meta-analysis
}

\author{
Anna M. Stadelman, ${ }^{1, a, \odot}$ Jayne Ellis, ${ }^{2, a, \oplus}$ Thomas H. A. Samuels, ${ }^{3}$ Ernest Mutengesa, ${ }^{4}$ Joanna Dobbin, ${ }^{5}$ Kenneth Ssebambulidde, ${ }^{6}$ Morris K. Rutakingirwa, \\ Lillian Tugume, ${ }^{6}$ David R. Boulware, ${ }^{8, \odot}$ Daniel Grint, ${ }^{9}$ and Fiona V. Cresswell ${ }^{5,6,7, \odot}$
}

${ }^{1}$ Division of Epidemiology and Community Health, School of Public Health, University of Minnesota, Minneapolis, Minnesota, USA, ${ }^{2}$ Hospital for Tropical Diseases, University College London Hospitals NHS Foundation Trust, London, UK, ${ }^{3}$ University College London Hospitals NHS Foundation Trust, London, UK, ${ }^{4}$ Hillingdon Hospital, The Hillingdon Hospitals NHS Foundation Trust, Uxbridge, UK, ${ }^{5}$ Clinical Research Department, London School of Hygiene and Tropical Medicine, London, UK, Infectious Diseases Institute, Makerere University, Kampala, Uganda, ${ }^{7}$ MRC-UVRILondon School of Hygiene and Tropical Medicine Uganda Research Unit, Entebbe, Uganda, ${ }^{8}$ Division of Infectious Diseases and International Medicine, Department of Medicine, University of Minnesota, Minneapolis, Minnesota, USA, and ${ }^{9}$ Tropical Epidemiology Group, Department of Infectious Disease Epidemiology, London School of Hygiene and Tropical Medicine, London, UK

Background. There is substantial variation in the reported treatment outcomes for adult tuberculous meningitis (TBM). Data on survival and neurological disability by continent and HIV serostatus are scarce.

Methods. We performed a systematic review and meta-analysis to characterize treatment outcomes for adult TBM. Following a systematic literature search (MEDLINE and EMBASE), studies underwent duplicate screening by independent reviewers in 2 stages to assess eligibility for inclusion. Two independent reviewers extracted data from included studies. We employed a random effects model for all meta-analyses. We evaluated heterogeneity by the $I^{2}$ statistic.

Results. We assessed 2197 records for eligibility; 39 primary research articles met our inclusion criteria, reporting on treatment outcomes for 5752 adults with TBM. The commonest reported outcome measure was 6-month mortality. Pooled 6-month mortality was $24 \%$ and showed significant heterogeneity $\left(I^{2}>95 \% ; P<.01\right)$. Mortality ranged from $2 \%$ to $67 \%$ in Asian studies and from $23 \%$ to $80 \%$ in Sub-Saharan African studies. Mortality was significantly worse in HIV-positive adults at $57 \%$ (95\% CI, 48\%-67\%), compared with 16\% (95\% CI, 10\%-24\%) in HIV-negative adults $(P<.01)$. Physical disability was reported in 32\% (95\% CI, 22\%-43\%) of adult TBM survivors. There was considerable heterogeneity between studies in all meta-analyses, with $I^{2}$ statistics consistently $>50 \%$.

Conclusions. Mortality in adult TBM is high and varies considerably by continent and HIV status. The highest mortality is among HIV-positive adults in Sub-Saharan Africa. Standardized reporting of treatment outcomes will be essential to improve future data quality and increase potential for data sharing, meta-analyses, and facilitating multicenter tuberculosis research to improve outcomes.

Keywords. tuberculous meningitis; mortality; neurological sequelae; systematic review; meta-analysis.

In 2018, 10 million cases of tuberculosis were reported globally [1]; tuberculous meningitis accounts for $1 \%-5 \%$ of these cases [2]. Tuberculous meningitis is the most severe form of tuberculosis and is responsible for a considerable burden of neurological sequelae and mortality; a systematic review of treatment outcomes in 1636 children with tuberculous meningitis estimated a mortality rate of $19.3 \%$ [3]. There is considerable variation in the reported outcomes for adult tuberculous meningitis across available studies; the reasons for this remain unclear. Two recent systematic reviews of adult tuberculous meningitis outcomes reported substantial heterogeneity in mortality and the proportion of deaths among those diagnosed with TBM,

Received 30 March 2020; editorial decision 17 June 2020; accepted 23 June 2020.

aEqual contribution

Correspondence: A. Stadelman, MPH, Infectious Diseases Institute, 2221 University Ave SE, Minneapolis, MN 55455 (stad0110@umn.edu).

Open Forum Infectious Diseases ${ }^{\circledR}$

(C) The Author(s)2020.Published by Oxford UniversityPress on behalf of Infectious Diseases Society of America. This is an Open Access article distributed under the terms of the Creative Commons Attribution License (http://creativecommons.org/licenses/by/4.0/), which permits unrestricted reuse, distribution, and reproduction in any medium, provided the original work is properly cited. DOI: 10.1093/ofid/ofaa257 with pooled estimates of $22.8 \%$ and $24.7 \%$ [4, 5]. However, neither review attempted to explain the variation in treatment outcomes by stratifying studies by HIV status and geographical location. In addition, Wen and colleagues excluded all investigational treatment studies, effectively excluding major treatment randomized controlled trials (RCTs) investigating regimens that have now become the standard of care (eg, adjunctive steroids and delayed antiretroviral therapy [ART] for those with HIV-associated tuberculous meningitis). Furthermore, there is a paucity of data in recent meta-analyses on drug resistance rates, treatment regimens, and steroid use. HIV co-infection has been shown to be a risk factor for death (hazard ratio, 2.5; 95\% CI, 1.9-3.4) in Vietnamese adults with tuberculous meningitis [6], but this remains to be explored systematically in other regions [7-9]. Neurological disability in adult tuberculous meningitis survivors has not been studied in detail in meta-analyses. In 2 recent systematic reviews, the prevalence of disability in adult tuberculous meningitis survivors varied between $29 \%$ and $50 \%[4,5]$. However, neither review provided data on the nature and severity of neurological sequelae in tuberculous meningitis survivors. 
We performed a systematic review and meta-analysis to characterize treatment outcomes, namely all-cause mortality and neurological sequelae, for adult tuberculous meningitis across a range of epidemiological settings. We endeavored to perform a definitive review by including the best quality data available and performing a robust quality assessment of the studies included.

\section{METHODS}

\section{Literature Search Strategy}

This review followed the Preferred Reporting Items for Systematic Reviews and Meta-Analyses (PRISMA) statement for the reporting of systematic reviews and meta-analyses [10]. A systematic electronic search was conducted using MEDLINE and EMBASE with the aim of identifying all studies reporting treatment outcomes in adult tuberculous meningitis from 1988 to the present. This time period corresponds to the WHO recommendation of standard quadruple therapy for the treatment of tuberculosis [1]. Controlled and natural language terms identified key search concepts such as "tuberculosis," "meningitis," "mortality," "complications," and "outcome." The full search strategies are presented in Supplementary Appendix A. Searches were conducted on July 9, 2018.

\section{Study Selection}

A 2-stage sifting process was employed: (1) at title and abstract and (2) at full-text level according to eligibility criteria as detailed below. Sifting was performed in duplicate independently by 2 reviewers, and any unresolved disagreements were resolved by a third independent reviewer. Reference and citation checking were conducted for included articles.

Studies were eligible for inclusion if they (i) included adults (aged $\geq 15$ years) with confirmed or suspected TB meningitis; (ii) utilized diagnostic criteria to systematically evaluate patients for tuberculous meningitis; (iii) reported on at least 1 of the following outcome measures: neurological sequelae, in-hospital mortality, mortality at the end of follow-up; (iv) employed any of the following study designs: consecutive case series, case-control study, cohort study, randomized controlled study, systematic review, or meta-analysis.

The following exclusion criteria were applied: (i) studies with $<10$ participants; (ii) studies limited to specific complications or comorbidities (eg, hydrocephalus, tuberculoma, or surgical intervention); (iii) studies not providing at least a backbone of standard fixed-dose combination antituberculous therapy; (iv) studies not specifying treatment given; (v) studies published before 1988; (vi) studies not written in English; (vii) any systematic review superseded by an updated systematic review; (viii) narrative reviews not adding new data or new analysis of data to existing knowledge.

\section{Data Extraction and Data Synthesis}

Two authors independently extracted data on study characteristics, recruitment populations, and treatment outcomes from eligible studies using a standardized, piloted electronic data capture database (REDCap, Vanderbilt University, Nashville, TN, USA). We captured data on geographical region, number of HIV-positive participants, British Medical Research Council (MRC) tuberculous meningitis grade at presentation, treatment regimens utilized, use of corticosteroids, and outcomes reported at specified time points for each study. Any unresolved disagreements in extraction were resolved by a third independent reviewer.

We used each study's definition of neurological sequelae as reported in the study. For articles that utilized the modified Rankin Scale or the Barthel index, "disability" was defined as "any disability that impeded the patient's ability to carry out tasks they once performed." This is was represented as a score of $>2$ on the Modified Rankin Scale or $<80$ on the Barthel Index.

For systematic reviews, individual study-level data were not extracted or analyzed; only the summary estimates were recorded for comparison, and citation checking was performed to ensure all relevant source manuscripts had been identified.

\section{Data Analysis}

We used the proportion of all-cause deaths and neurological sequelae within each study to define outcomes of tuberculous meningitis for the meta-analyses. As such, all meta-analyses used random effects models and employed the DerSimonian and Laird method on Freeman-Tukey transformed proportions, which is the established approach for this type of analysis [11-13]. We graphically displayed data in forest plots, which display point estimates of tuberculous meningitis outcomes in each study, with $95 \%$ confidence intervals. We generated pooled effect estimates by inverse variance-weighting each individual point estimate, such that the estimates with lower variances contributed more to the pooled estimate [13]. The overall pooled estiamte for mortality was stratified by follow-up outcome reporting time. Interstudy and subgroup heterogeneity were assessed with the $I^{2}$ statistic. All analyses were conducted in Stata, version 15.1 (StataCorp, College Station, TX, USA), with the "metaprop" command [14].

\section{Quality Assessment}

The 39 articles included in the meta-analysis were assessed for study quality using the Downs and Black tool, a 27-item quality assessment checklist [15]. Each study was scored on a 32-point scale for items that examined quality of reporting, external validity, internal validity (bias and confounding), and study power. Study power was estimated according to sample size methodology. Studies were scored as follows; 0 if no sample size calculation was made or reported in the manuscript (given for observational studies); 3 if a power calculation was done but there were insufficient numbers of patients recruited; 5 if the power calculation was done and sufficiently powered. Systematic reviews meeting the inclusion criteria were not 
assessed for risk of bias. As treatment outcomes were of interest in these analyses and not treatment or intervention efficacy, we included all studies regardless of quality assessment score.

\section{Role of Funding Source}

The Fogarty International Center of the National Institutes of Health provided funding fellowship support to the lead author of the study. The funders had no role in the study design, data collection, data analysis, data interpretation, or writing of the report. The corresponding author had full access to all the data in the study and had final responsibility for the decision to submit for publication.

\section{RESULTS}

\section{Search Results, Studies, and Participants Included}

Our searches yielded 2562 reports. After removal of duplicates ( $\mathrm{n}=365), 2197$ studies underwent title and abstract screening, and 264 full texts were reviewed (Figure 1). Thirty-nine studies met our eligibility criteria for inclusion and analysis (Table 1).
These 39 studies were published between 1995 and 2018; 10 (26\%) were case series, 21 (54\%) were cohort studies, and 8 (21\%) were randomized controlled trials. Studies arose from 18 countries, including a range of epidemiological settings; 24 (62\%) were from high-TB burden settings, and 15 (38\%) were from low-TB burden settings. A total of 26 (67\%) studies were conducted in Asia, and 5 (13\%), 5 (13\%), and 2 (5\%) were conducted in Europe, Africa, and the Americas, respectively (Figure 2). Study quality scores ranged from 8 to 32 , with a score of 32 indicating the highest quality. The median quality score for included articles (interquartile range [IQR]) was 18 (15-20). Our meta-analysis includes reported treatment outcomes for 5752 adults with tuberculous meningitis. Participant age ranged from 15 to 88 years. Seven studies included 1078 HIV-positive patients: 302 (28\%) from Africa and 776 (72\%) from Asia. MRC tuberculous meningitis grade was reported in 29 studies, in which $28 \%$ (1354/4761) of participants presented with MRC grade I disease, $48 \%$ (2302/4761) with grade II, and 20\% (967/4761) with grade III. A total of 37 studies ( $n=5623$ participants) reported the classification or uniform case definition of enrolled participants. Of

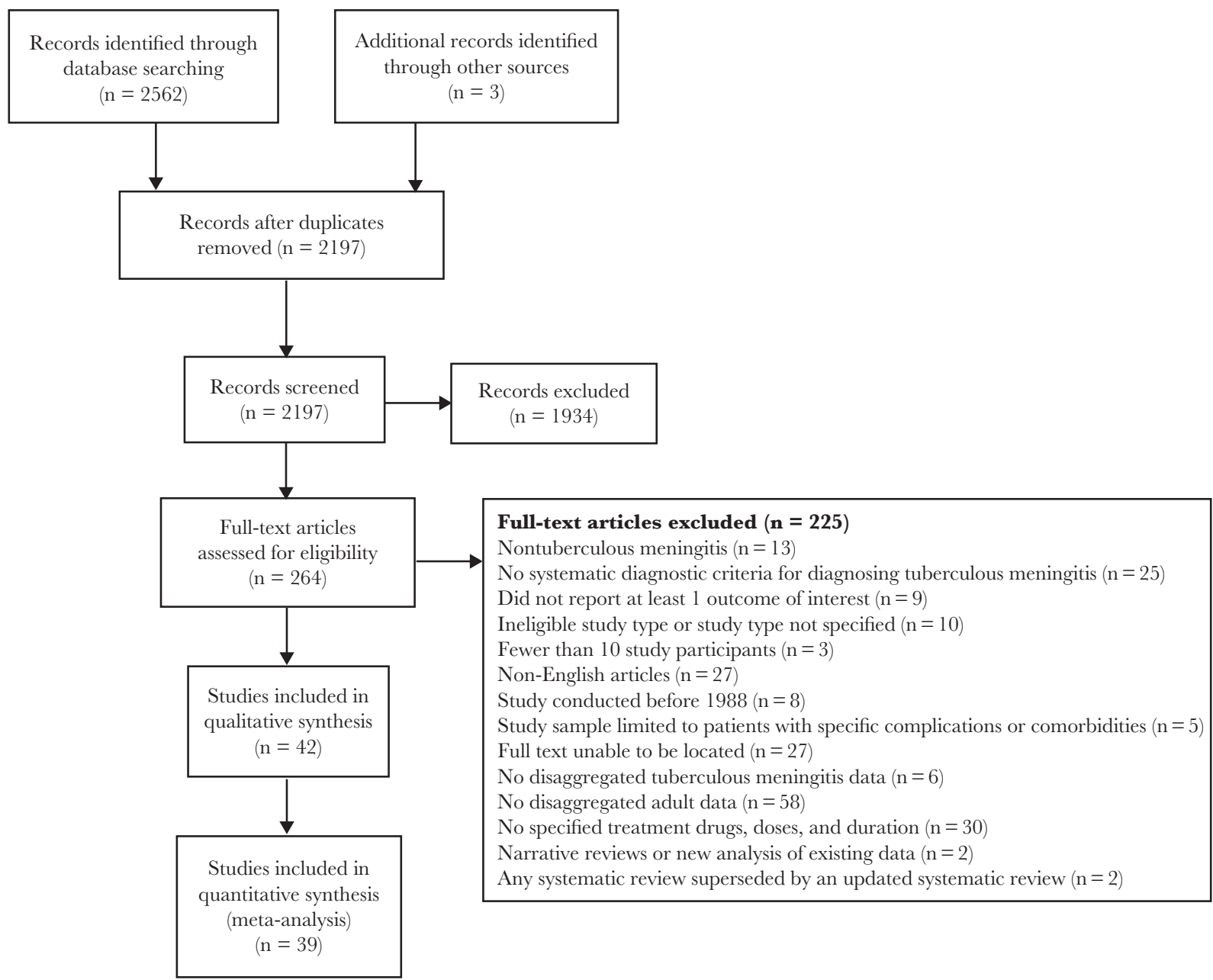

Figure 1. Flow diagram of the study selection process. 


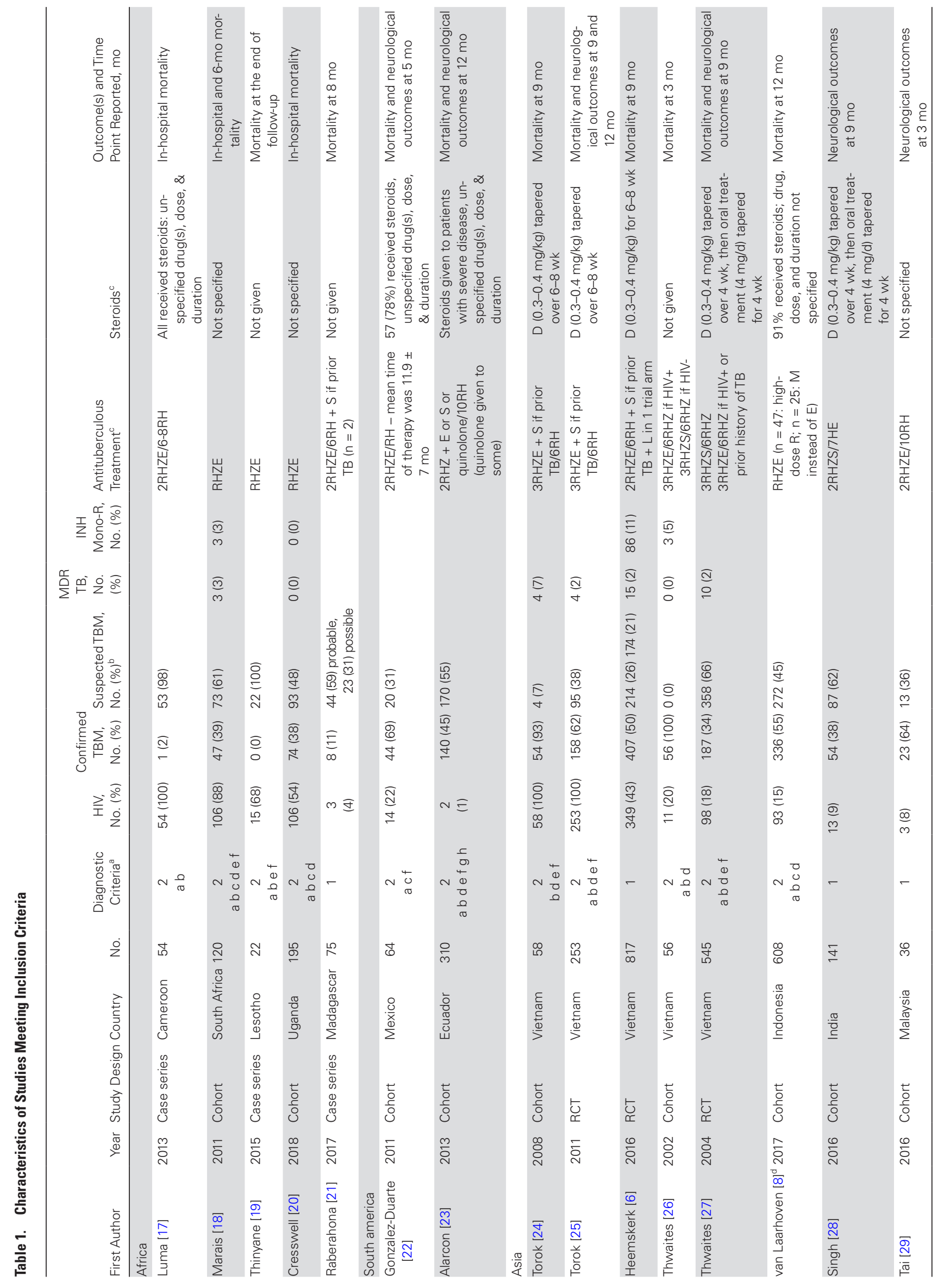

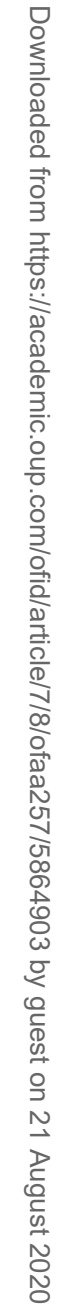




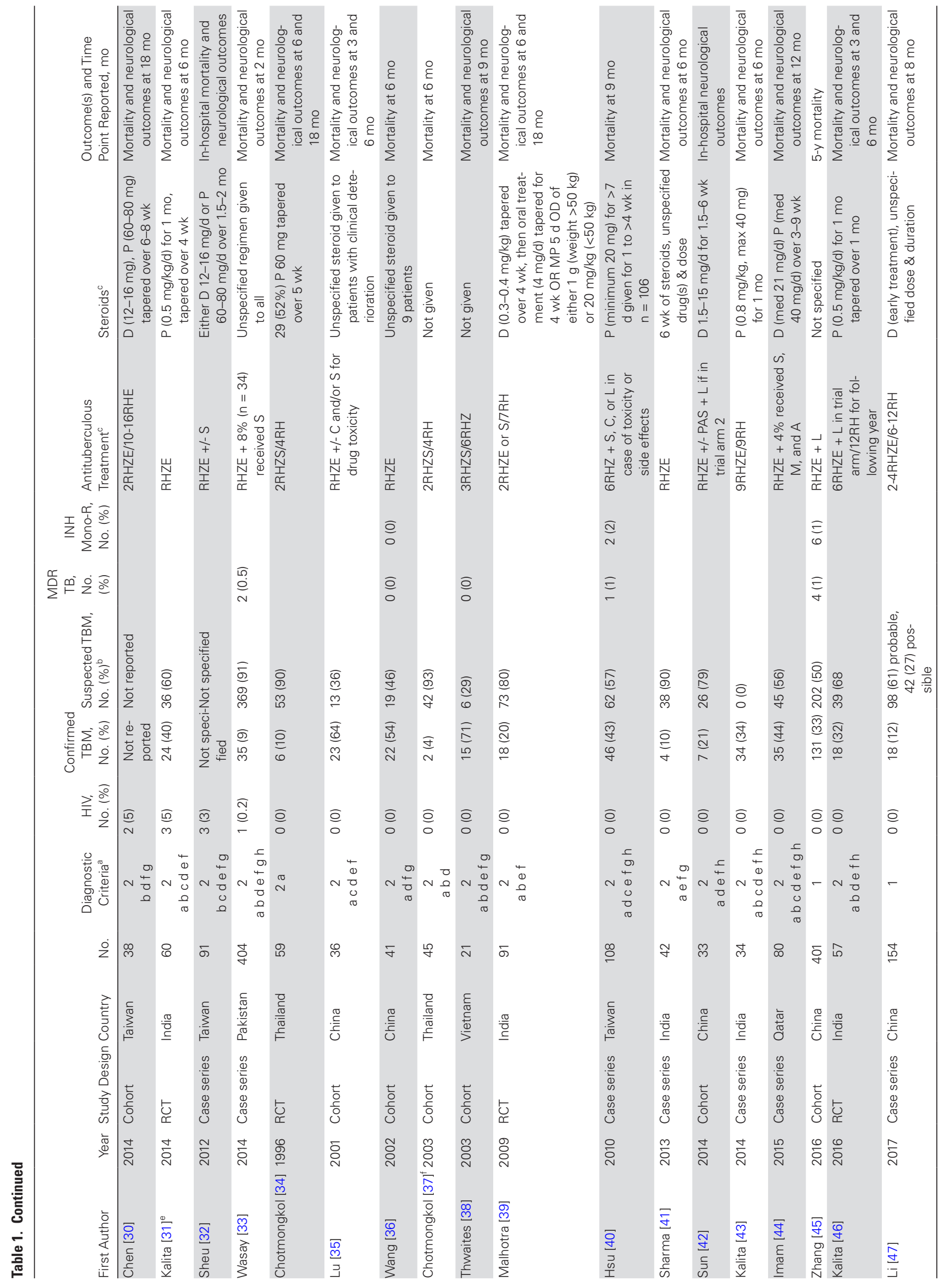




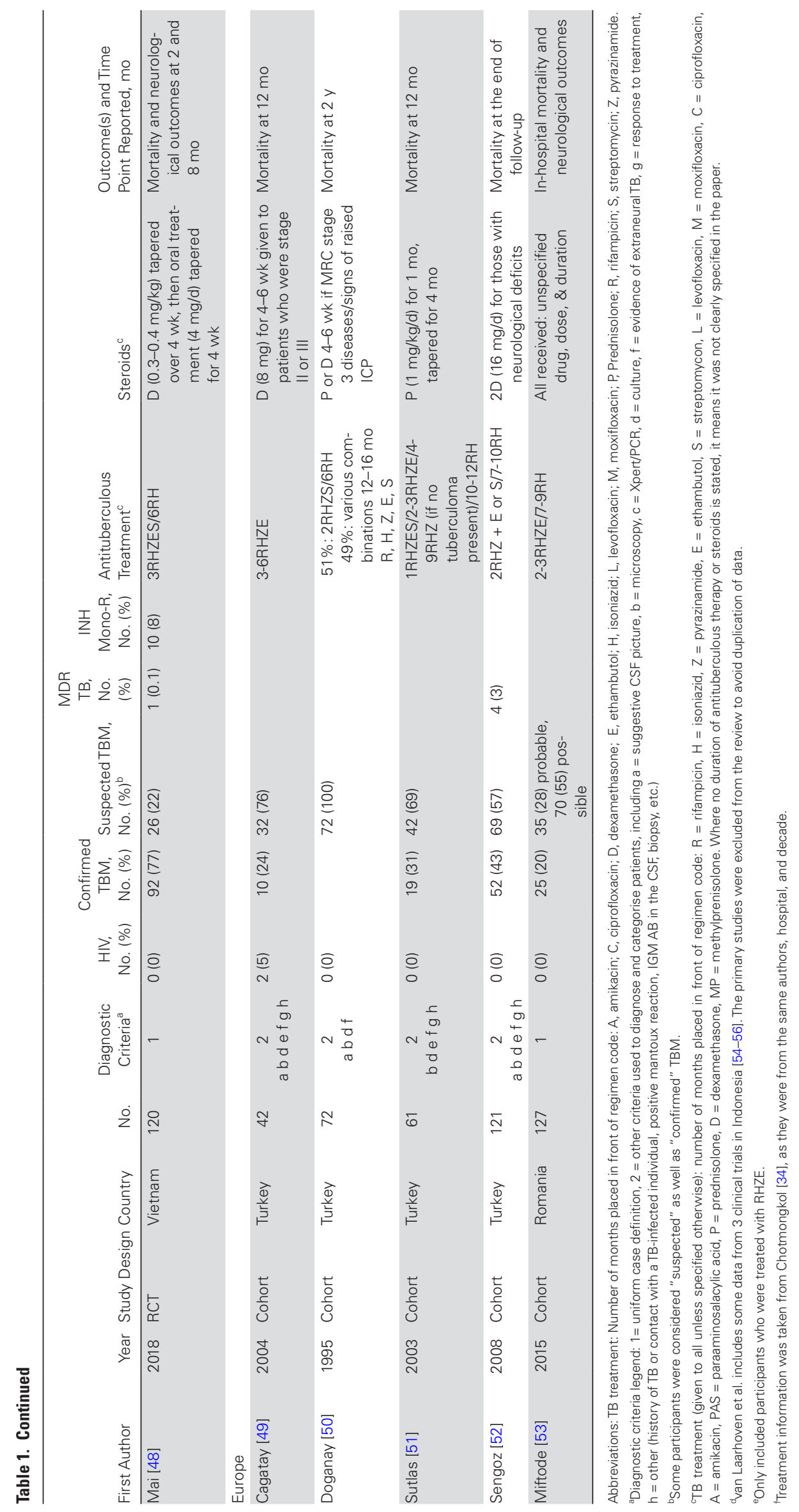

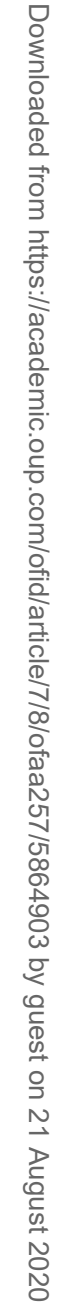




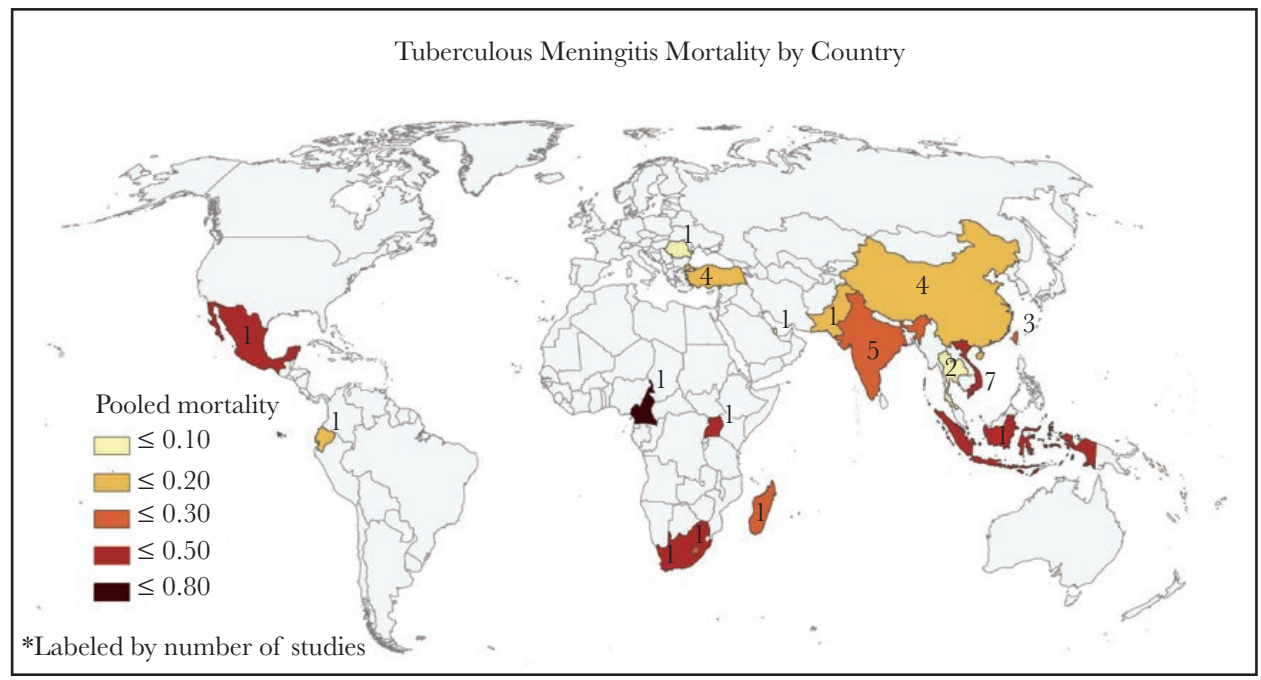

Figure 2. Tuberculous meningitis mortality by country. Pooled mortality for tuberculous meningitis by country. Mortality for countries with only 1 study reflect the reported mortality for that 1 study.

those, $40 \%$ (2243/5623) were microbiologically confirmed tuberculous meningitis and $49 \%(2741 / 5623)$ were suspected tuberculous meningitis, the latter of which included 21\% (1013/5623) with probable tuberculous meningitis and $12 \%$ (663/5623) with possible tuberculous meningitis according to the uniform case definition [16]. Only 12 studies reported on drug resistance rates; 10 studies included patients with multidrug-resistant tuberculosis $(\mathrm{n}=49)$, and 12 studies included patients with mono-drugresistant tuberculosis, of which 10 studies included isoniazid resistance ( $\mathrm{n}=112$ participants), 6 studies included rifampin resistance ( $\mathrm{n}=12$ participants), and 1 study included streptomycin resistance ( $\mathrm{n}=1$ participant).

The most common treatment regimen was standard 4-drug therapy of rifampicin, isoniazid, pyrazinamide, and ethambutol (RHZE) with no additional antituberculous drugs ( $\mathrm{n}=17$ studies). Seven studies used streptomycin in addition to or in replacement for ethambutol (Table 1). The median treatment duration (IQR) was 9 (9-12) months. Corticosteroids were given to all patients in 19 studies and to some participants in 10 studies (Table 1). Treatment outcomes by corticosteroid use were examined in a meta-analysis with included studies, but this was not the aim or design of our meta-analysis, and a significant amount of heterogeneity in mortality between studies was unexplained (Supplementary Appendix B). A Cochrane meta-analysis on corticosteroid use in TBM was published in 2016 [57].

\section{Mortality Assessment and Outcomes}

A wide range of mortality end points were reported: 15\% (6/39) studies reported 1-month mortality, 5\% (2/39) studies reported 2-month mortality, 8\% (3/39) studies reported 3-month mortality, $18 \%(7 / 39)$ studies reported 6-month mortality, $13 \%$
(5/39) studies reported 12-month mortality, and 2\% (1/39) reported 5-year mortality. Other reported outcomes included in-hospital mortality ( $\mathrm{n}=6$ studies) and median time to death ( $\mathrm{n}=4$ studies). In the 6 studies that reported on "in-hospital mortality," only 1 study reported on the length of hospitalization, which ranged from 4 to 10 days until death or discharge. Five studies did not define "in-hospital mortality" in terms of time frame.

To investigate time-specific mortality, articles were grouped by follow-up outcome reporting time point. Articles that reported outcomes $\leq 3$ months were included in the 3 -month reporting category to summarize "early" mortality. Articles that reported outcomes $>3$ months to 6 months were included in the 6-month reporting category. Articles that reported outcomes $>6$ months were included in the 12-month reporting category. Of articles reporting outcomes at 3,6 , and 12 months, pooled mortality was $23 \%$ (95\% CI, $14 \%-35 \%$ ), $23 \%$ (95\% CI, $14 \%-33 \%)$, and $25 \%$ (95\% CI, $17 \%-33 \%)$, respectively (Figure 3). There was significant heterogeneity $\left(I^{2}=95 \% ; P<.01\right)$ for all outcome reporting time points. There was no marked heterogeneity in mortality between outcome reporting time points $(P=.60)$, but it was included in the pooled analysis, resulting in a pooled mortality of $24 \%$ (95\% CI, 19\%-29\%).

\section{Mortality End Points by HIV Status}

Seven studies reported mortality for HIV-positive adults. For HIV-positive adults, pooled mortality was 57\% (95\% CI, 48\%$67 \%$ ), compared with $16 \%$ (95\% CI, 10\%-24\%) in HIV-negative adults (Figure 4). HIV status explained a significant amount of the observed heterogeneity in tuberculous meningitis mortality $(P<.01)$. 


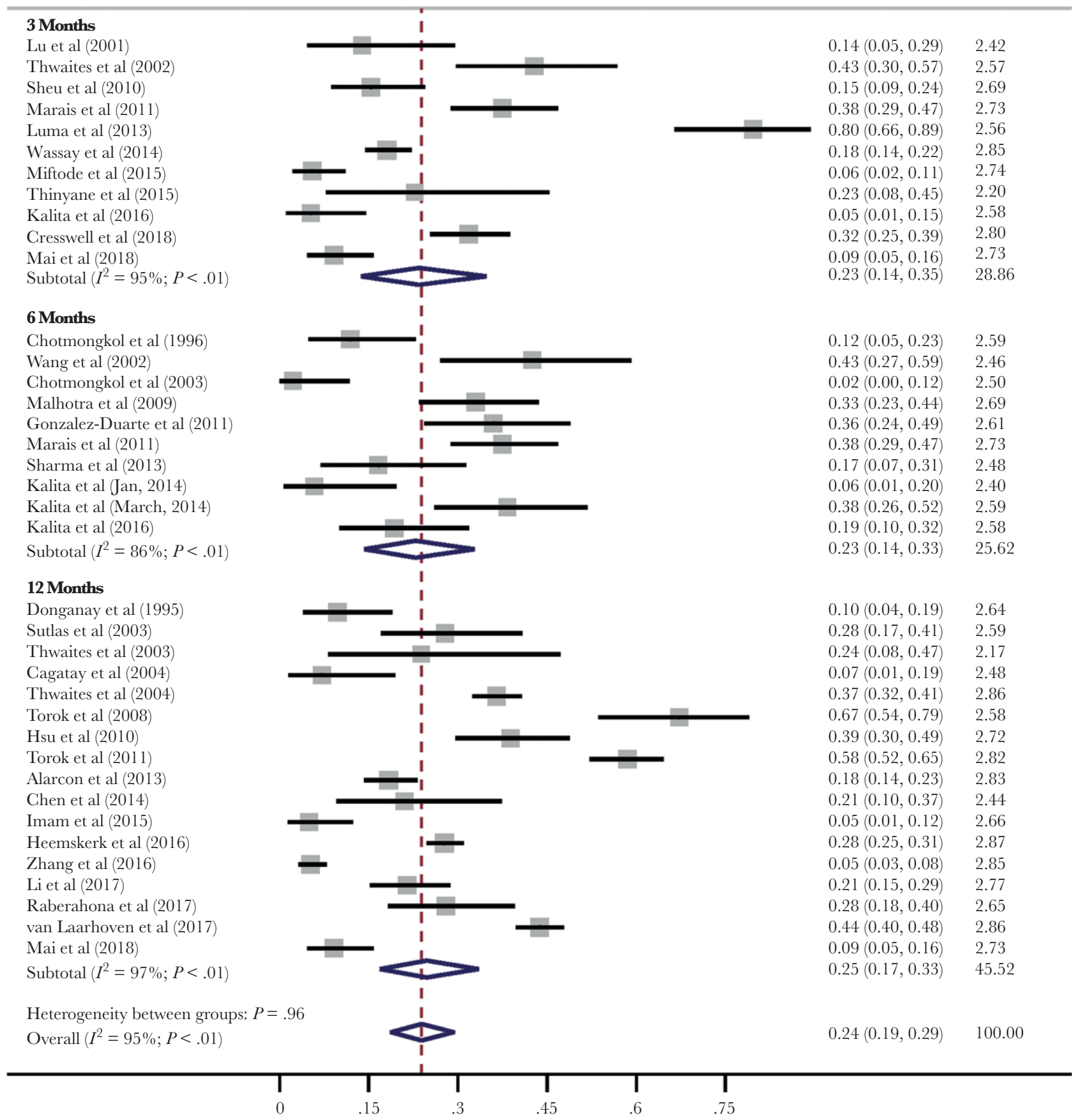

Figure 3. Tuberculous meningitis mortality by outcome reporting time point. Forest plots depicting mortality due to tuberculous meningitis at 3,6 , and 12 months. One study was excluded because the outcome time point was not reported.

\section{Mortality End Points by Geographical Region}

Most studies reporting on tuberculous meningitis mortality were conducted in India and the Asian continent $(\mathrm{n}=27 ; 70 \%)$, where pooled mortality ranged from $2 \%$ to $67 \%$ (Figure 2). The countries reporting the highest tuberculous meningitis mortality were located in Sub-Saharan Africa, where mortality ranged from $23 \%$ to $80 \%$. Continent (Africa vs Asia) explained a significant amount of the observed heterogeneity in tuberculous meningitis mortality $(P=.02)$.

\section{Temporal Variation in Mortality End Points}

To investigate changes in tuberculous meningitis treatment outcomes over time, we conducted a temporal analysis in which individual studies were allocated to 1 of 5 time periods and stratified analyses were conducted. Time periods were subdivided into 5-year windows from 1995 onwards, and pooled mortality was analyzed within each time window. The highest pooled mortality was 31\% (95\% CI, 14\%-51\%) in articles published from 2006 to 2010 , though there was no significant variation 


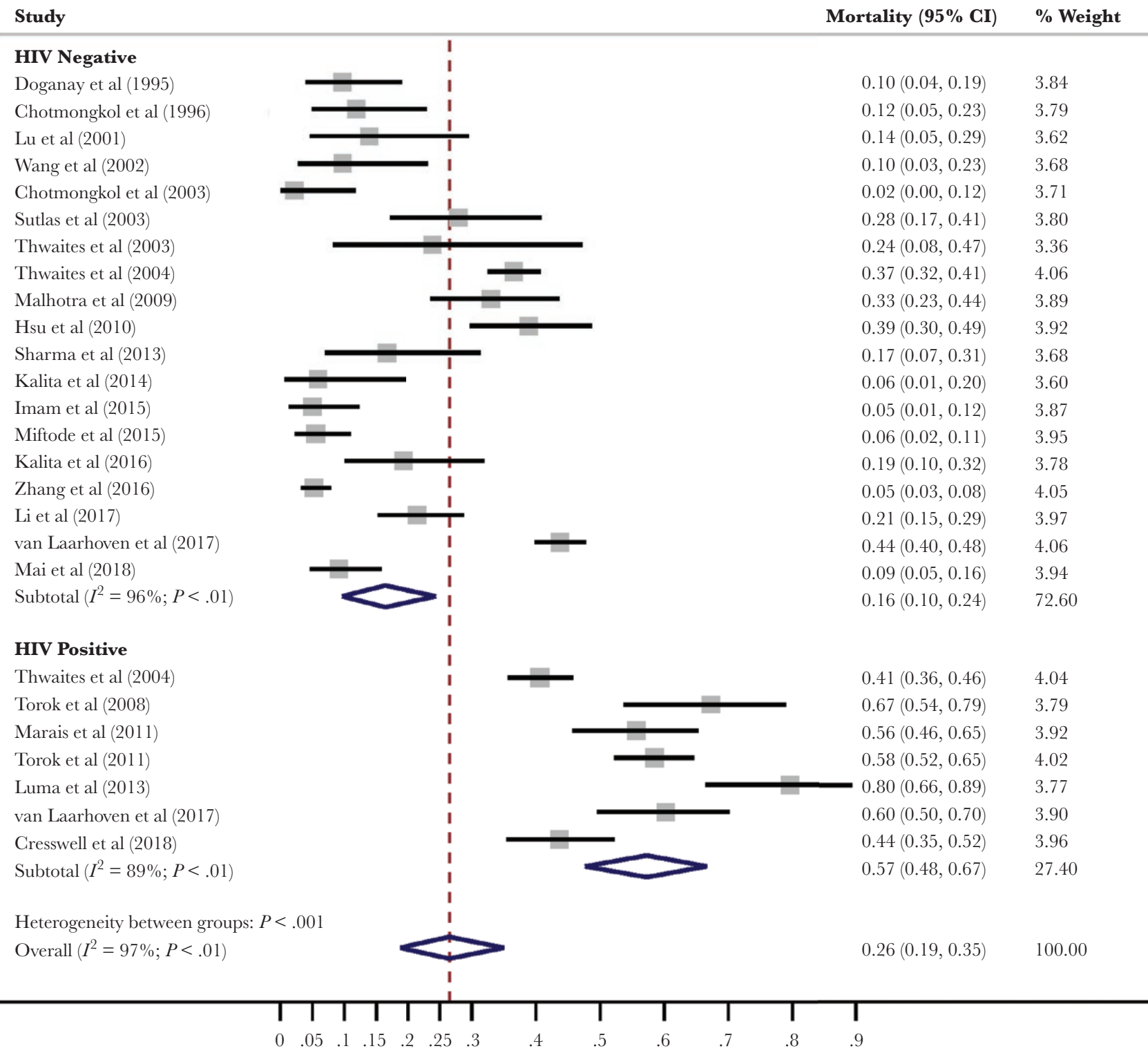

Figure 4. Tuberculous meningitis mortality by HIV status. Forest plot depicting mortality due to tuberculous meningitis stratified by HIV status. HIV status explains a significant amount of the heterogeneity in tuberculous meningitis mortality $(P<.01)$.

by time window (Supplementary Appendix C). In earlier time periods, heterogeneity in survival was greatest, and heterogeneity appears to have declined in more recent time periods.

\section{Neurological Disability}

Functional outcomes among survivors was a prespecified end point in 24 studies; 10 studies reported on functional outcomes using the Modified Rankin Scale score $(n=6)$ or the Barthel Index $(n=5), 10$ studies reported on neurocognitive disability without using a specified scale or measurement tool, and 5 studies reported using "clinical assessments."

The timing and method of neurological assessments varied between studies; the most commonly used outcome assessment was physical disability conducted at the end of follow-up.
In this analysis, participants were considered disabled if there was any indication of functional disability as reported by the Modified Rankin Scale or Barthel Index. Of the studies utilizing the Modified Rankin Scale, the pooled proportion of patients experiencing some level of physical disability was 26\% (95\% CI, $18 \%-35 \%$ ), with considerable heterogeneity (Supplementary Appendix D). Of the studies using the Barthel Index, the proportion of patients experiencing some level of physical disability was $32 \%$ ( $95 \%$ CI, $22 \%-43 \%$ ), with only moderate heterogeneity.

\section{DISCUSSION}

In this rigorous systemic review and meta-analysis, we reviewed treatment outcomes for $>6000$ adults with tuberculous meningitis, and our data clearly demonstrate that the mortality and 
neurological sequelae associated with tuberculous meningitis remain unacceptably high. Although there was significant heterogeneity between studies $\left(I^{2}>95 \%\right)$, the overall risk of death was $23 \%$ at 3 months and $25 \%$ at 12 months. In patients who did survive, neurological sequelae were common, affecting nearly one-third of all patients. Furthermore, our temporal analysis of treatment outcomes indicates that prognosis has improved little over time. Our results are in concordance with 2 recently published systematic reviews, which reported overall morality associated with adult tuberculous meningitis to be $23 \%$ and $25 \%$ and risk of neurological sequelae to be $29 \%$ and $50 \%$, respectively. Our study expands on the current literature through subgroup meta-analyses to evaluate differential treatment outcomes by HIV status and geographical region.

We have demonstrated that patients with HIV-associated tuberculous meningitis have 3-fold higher mortality compared with HIV-negative cohorts; mortality in HIV-negative cohorts ranged between $10 \%$ and $24 \%$, compared with $48 \%-67 \%$ in HIV-positive cohorts $(P<.01)$. Pathogenesis research is urgently needed to investigate the disproportionate mortality associated with HIV co-infection in tuberculous meningitis and to identify potential interventions or preventative measures.

Second, our data demonstrate that despite adoption of standardized treatment regimens for tuberculous meningitis, considerable global disparities in treatment outcomes exist. Pathogenesis work has shown that even within a Vietnamese population, a single genetic polymorphism significantly impacts corticosteroid responsiveness and survival from TBM [58]. The extent of the heterogeneity observed in this meta-analysis raises the possibility that genetic or other latent factors may contribute to outcome, and the current one-size-fits all approach to treatment may be effective in some individuals/populations and less effective in others. Our subgroup meta-analyses indicate that patients in the African continent have a higher mortality compared with all other continents. This may in part be explained by the higher co-prevalence of HIV. However, given the considerable resource limitations including a lack of intensive care facilities typical of many settings in Sub-Saharan Africa, it is likely that the management of commonly encountered complications of tuberculous meningitis including hyponatremia, raised intracranial pressure, hydrocephalus, stroke, and nosocomial infections is suboptimal. Further research is needed to determine the attributable mortality due to a lack of supportive or critical care in Sub-Saharan Africa. Our systematic literature review highlights the historical paucity of clinical studies published from this continent. In order to address the devastatingly poor outcomes from HIV-associated meningitis, particularly for those in Sub-Saharan Africa, we need to design, fund, and deliver more clinical research.

Our meta-analyses of follow-up time-specific mortality at 3 , 6 , and 12 months highlight that $>90 \%$ of tuberculous meningitis deaths occur in the first 3 months. This may justify that 3-month mortality is a reasonable RCT end point, potentially making study trial follow-up shorter and cheaper, thereby accelerating research outputs. However, the considerable heterogeneity found in these analyses, as well as inconsistencies in reporting outcomes, indicates that further evidence is needed to justify a 3-month clinical trial end point. Clinical studies to identify drivers of early mortality in tuberculous meningitis may inform the design of treatment intensification strategies and other adjunctive interventions.

Concerningly, our results demonstrate that minimal improvements in survival have been made over time. There are a number of temporal factors that may have affected outcomes in certain time periods including the height of the HIV epidemic in the 1990-2005 period: ART rollout in the 1995-2010 window, the increasing availability of more rapid diagnostics in the form of the Xpert MTB/Rif assay in the 2010-2020 window facilitating the diagnosis of tuberculous meningitis where it was previously unconfirmed, and, lastly, gradually increasing rates of antituberculous drug resistance worldwide. Reporting bias, which may have varied over time, must also be considered.

Our analysis has several limitations. First, although we only included studies that employed a prespecified diagnostic criterion for tuberculous meningitis, there was considerable variation in the quality of diagnostic criteria used, and diagnostics have changed over time. We chose not to restrict diagnostic criteria to microbiologically confirmed tuberculous meningitis because doing so would have restricted our meta-analysis to $40 \%$ ( $n=2243$ ) of adults, and furthermore we wanted our results to be generalizable to real-world clinical settings where confirmation rates are often only moderate. We do, however, recognize that misclassification of undifferentiated meningitis cases as tuberculous meningitis is common, especially when left to physician discretion, as may have been the case in some of the patients included in our meta-analysis, which would undermine the accuracy of our outcome estimates. Second, in the spirit of generalizability, we chose to include case series, which are primarily descriptive and not wholly representative of the populations they are drawn from. Although this may have posed some unmeasurable bias, we believe that this would not have substantially impacted our results, as mortality and neurological sequelae, our outcomes of interest, would not have measured differently or changed based on study design. Third, the specific antituberculous regimen utilized and drug resistance rates within the cohorts were inconsistently reported in studies; therefore, we were unable to conduct stratified metaanalyses based on drug resistance patterns. The International Tuberculous Meningitis Research Consortium paper on standardized methods for enhanced quality and comparability of tuberculous meningitis studies specifies that it is essential to document the dose, route of administration, and duration of all antituberculosis drugs used in tuberculous meningitis studies [59]. There remain several outstanding questions concerning 
the optimal treatment of tuberculous meningitis, and therefore to facilitate cross-study comparisons and interrogate differences in study outcomes, basic information about the treatment provided is essential.

Finally, there was a considerable lack of standardization of reporting on treatment outcomes. This was particularly marked with respect to reporting of neurological sequelae; first, neurological sequalae were rarely reported (only 10/39 [26\%] studies including any data on neurological sequalae), the tools used were inconsistent ( 9 tools in total), and the time points for assessment were rarely reported. This inconsistent reporting hampered comparison of data across studies. Given the importance of neurological disability in tuberculous meningitis and the importance of developing a standardized evidence base against which to assess new treatments, the International Tuberculous Meningitis Research Consortium recommends that the Modified Rankin Score be used as the first-line tool, which should be recorded at 12 months from antituberculosis treatment initiation in all adults [59]. We support this recommendation, and in addition we would suggest that mortality be routinely reported on at 3,6, and 12 months, if possible, to improve study comparability.

The strengths of this work include its size, with 39 individual studies included from Asia, Africa, Europe, and the Americas, making our estimates broadly generalizable to a range of settings. Our systematic review is larger than 2 previously published systematic reviews of adult tuberculous meningitis [4, 5]. In comparison to Wen et al. [4], we decided to include randomized controlled trials in our systematic review, which enabled us to include the highest quality of trial evidence, and we also reported drug resistance rates within each included study. In comparison to Wang et al. [5], we ascertained variation in treatment outcomes geographically and reported on the nature and severity of reported neurological sequelae. Overall, we assessed a wide range of covariates to investigate the heterogeneity in treatment outcomes observed. To our knowledge, this is the most extensive critical appraisal of tuberculous meningitis outcomes to date.

In conclusion, adult tuberculous meningitis is associated with considerable neurological morbidity and mortality and remains a major challenge in TB-endemic regions. The worst outcomes are observed by those with HIV co-infection in Sub-Saharan Africa, where risk of death is 3-fold higher. Our study was limited by suboptimal reporting on diagnostic criteria utilized, drug resistance rates, and details of treatment regimens used, as well as highly variable outcome reporting. Adoption of standardized reporting systems across tuberculous meningitis studies would not only facilitate cross-study comparisons, but would also improve the quality of research outputs overall and support collaborative research across centers with an aim of improving tuberculous meningitis outcomes globally.

\section{Supplementary Data}

Supplementary materials are available at Open Forum Infectious Diseases online. Consisting of data provided by the authors to benefit the reader, the posted materials are not copyedited and are the sole responsibility of the authors, so questions or comments should be addressed to the corresponding author.

\section{Acknowledgments}

We thank clinical librarian Tom Roper (Brighton and Sussex University Hospitals NHS Trust) for his assistance with developing our search strategy.

Financial support. This work was supported by the Fogarty International Center, National Institutes of Health, USA (R01NS086312, D43TW009345). Authors A.M.S., K.E., M.R., L.T., and D.R.B. are supported by the Fogarty International Center at the National Institutes of Health.

Potential conflicts of interest. The authors declare that they have no competing interests. All authors: no reported conflicts of interest. All authors have submitted the ICMJE Form for Disclosure of Potential Conflicts of Interest. Conflicts that the editors consider relevant to the content of the manuscript have been disclosed.

\section{References}

1. World Health Organization. Global Tuberculosis Report 2019. Geneva: World Health Organization; 2019.

2. Thao LTP, Heemskerk AD, Geskus RB, et al. Prognostic models for 9-month mortality in tuberculous meningitis. Clin Infect Dis 2018; 66:523-32.

3. Chiang SS, Khan FA, Milstein MB, et al. Treatment outcomes of childhood tuberculous meningitis: a systematic review and meta-analysis. Lancet Infect Dis 2014; 14:947-57.

4. Wen L, Li M, Xu T, et al. Clinical features, outcomes and prognostic factors of tuberculous meningitis in adults worldwide: systematic review and meta-analysis. I Neurol 2019; 266:3009-21.

5. Wang MG, Luo L, Zhang Y, et al. Treatment outcomes of tuberculous meningitis in adults: a systematic review and meta-analysis. BMC Pulm Med 2019; 19:200.

6. Heemskerk AD, Bang ND, Mai NT, et al. Intensified antituberculosis therapy in adults with tuberculous meningitis. N Engl J Med 2016; 374:124-34.

7. Woldeamanuel YW, Girma B. A 43-year systematic review and meta-analysis: case-fatality and risk of death among adults with tuberculous meningitis in Africa. J Neurol 2014; 261:851-65.

8. van Laarhoven A, Dian S, Ruesen C, et al. Clinical parameters, routine inflammatory markers, and LTA4H genotype as predictors of mortality among 608 patients with tuberculous meningitis in Indonesia. J Infect Dis 2017; 215:1029-39.

9. Thwaites GE, Nguyen DB, Nguyen HD, et al. Dexamethasone for the treatment of tuberculous meningitis in adolescents and adults. N Engl J Med 2004; 351:1741-51.

10. Moher D, Shamseer L, Clarke M, et al; PRISMA-P Group. Preferred Reporting Items for Systematic Review and Meta-Analysis Protocols (PRISMA-P) 2015 statement. Syst Rev 2015; 4:1.

11. Freeman MF, Tukey JW. Transformations related to the angular and the square root. Ann Math Stat 1950; 21:305.

12. Nyaga VN, Arbyn M, Aerts M. Metaprop: a Stata command to perform metaanalysis of binomial data. Arch Public Health 2014; 72:39.

13. DerSimonian R, Laird N. Meta-analysis in clinical trials. Control Clin Trials $\mathbf{1 9 8 6}$ 7:177-88.

14. Harris RJ, Bradburn MJ, Deeks JJ, et al. Metan: fixed- and random-effects metaanalysis. Stata J 2008; 8:3-28.

15. Downs SH, Black N. The feasibility of creating a checklist for the assessment of the methodological quality both of randomised and non-randomised studies of health care interventions. J Epidemiol Community Health 1998; 52:377-84.

16. Marais S, Thwaites G, Schoeman JF, et al. Tuberculous meningitis: a uniform case definition for use in clinical research. Lancet Infect Dis 2010; 10:803-12.

17. Luma HN, Tchaleu BC, Ngahane BH, et al. Tuberculous meningitis: presentation, diagnosis and outcome in HIV-infected patients at the Douala General Hospital, Cameroon: a cross sectional study. AIDS Res Ther 2013; 10:16.

18. Marais S, Pepper DJ, Schutz C, et al. Presentation and outcome of tuberculous meningitis in a high HIV prevalence setting. PLoS One 2011; 6:e20077.

19. Thinyane KH, Motsemme KM, Cooper VJ. Clinical presentation, aetiology, and outcomes of meningitis in a setting of high HIV and TB prevalence. J Trop Med 2015; 2015:423161.

20. Cresswell FV, Bangdiwala AS, Bahr NC, et al. Can improved diagnostics reduce mortality from tuberculous meningitis? Findings from a 6.5 -year cohort in Uganda. Wellcome Open Res 2018; 3:64. 
Consensus uniform TBM case definition

Marais S, Lancet Infectious Diseases. 2010;10(11):803-12

\begin{tabular}{|c|c|c|c|c|c|}
\hline \multicolumn{3}{|l|}{ A. Uniform case definition } & \multirow[b]{2}{*}{ 2. $\quad$ CSF criteria } & \multirow[b]{2}{*}{ Yes } & \multirow[b]{2}{*}{ Points } \\
\hline 1. Clinical criteria & Yes & Points & & & \\
\hline CNS Symptoms $>5$ days & $\square$ & 4 & Clear appearance & [ & 1 \\
\hline Symptoms: welght loss, night sweats, cough $>2$ wk & $\square$ & 2 & Cells: $10-500 / \mu \mathrm{L}$ & & 1 \\
\hline Contact with an individual with Pulm TB & $\square$ & 2 & Lymphocytes (>50\%) & & 1 \\
\hline Focal neurological deficit (excluding $\mathrm{CN}$ palsies) & $\square$ & 1 & Protein $>1$ g/L or $>100 \mathrm{~m} / \mathrm{dL}$ & $\square$ & 1 \\
\hline Cranial nerve palsy & $\square$ & 1 & CSF:plasma glucose $<50 \%$ or & $\square$ & 1 \\
\hline Altered consciousness & $\square$ & 1 & CSF glucose $<22 \mathrm{mmol}$ or $42 \mathrm{mg} / \mathrm{dl}$ & & \\
\hline \multicolumn{2}{|c|}{ Total category score $(\max =6)$} & & \multicolumn{2}{|c|}{ Total category score $(\max =4)$} & \\
\hline \multicolumn{3}{|l|}{ 3. Cerebral imaging criteria } & \multicolumn{3}{|l|}{ 4. Evidence of tuberculosis elsewhere } \\
\hline Imaging Done $\square$ Not Done $\square$ & Yes & Points & & Yes & Points \\
\hline Hydrocephalus & $\square$ & 1 & CXR suggests focal TB & 10 & 2 \\
\hline Basal meningeal enhancement & $\square$ & 2 & or CXR suggests miliary TB & $\square$ & 4 \\
\hline Tuberculoma & $\square$ & 2 & CT/MRIUSS evidence of TB outside CNS & $\square$ & 2 \\
\hline Infarct & $\square$ & 1 & M.th at another source - sputium, urine LaM & $\square$ & 4 \\
\hline Pre-contrast basal hyperdensity & $\square$ & 2 & Positive non-CSF Xpert & $\square$ & 4 \\
\hline \multicolumn{3}{|c|}{ Total category score $(\max =6)$} & \multicolumn{2}{|c|}{ Total category score $(\max =4)$} & \\
\hline Current TBM category (select one) & & & Total Score & & \\
\hline
\end{tabular}

Definite TB meningitis:

A. AFB seen in CSF; $M$ th cultured from CSF; or a positive CSF Xpert

B. AFB seen in the context of histological changes consistent with TB in brain or spinal cord with suggestive symptoms / signs and CSF changes, or visible meningitis (on autopsy).

Probable TB meningitis (score total 212 with imaging, score total 210 without imaging)

Possible TB meningitis (score total 6-11 with imaging, score total 6-9 without imaging)

Not TBM (score total $\varangle 8$ or another identfied etiology)
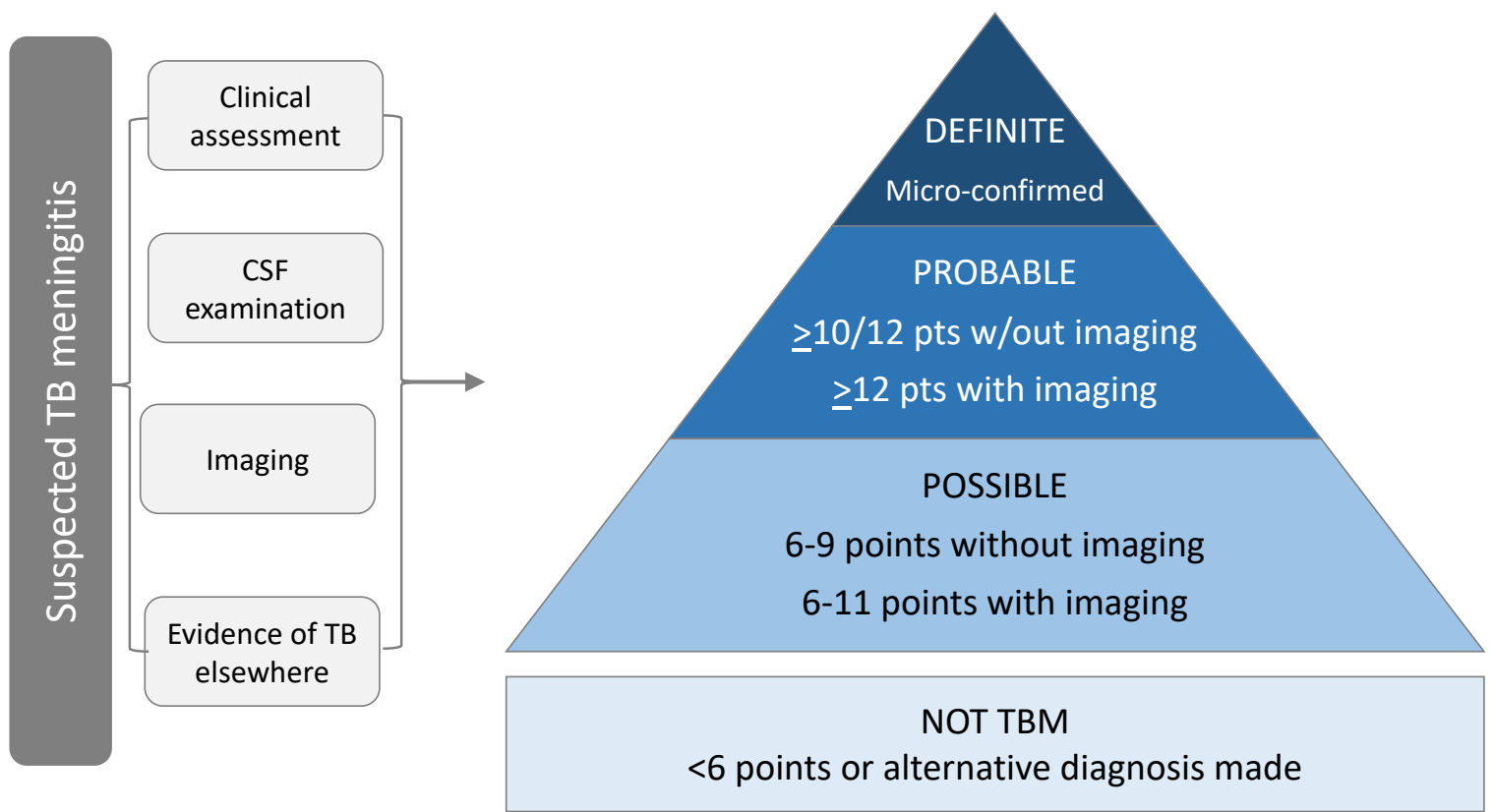
Richard Kwizera (D) 1, Fiona V. Cresswell (D) 1-3, Gerald Mugumya ${ }^{4}$, Micheal Okirwoth (D) 1, Enock Kagimu ${ }^{1}$, Ananta S. Bangdiwala (iD) 5 , Darlisha A. Williams ${ }^{1,5}$, Joshua Rhein (D) 1,5, David R. Boulware (D) 5, David B. Meya ${ }^{1,4,5}$

${ }^{1}$ Infectious Diseases Institute, College of Health Sciences, Makerere University, Kampala, Uganda

${ }^{2}$ Clinical Research Department, London School of Hygiene and Tropical Medicine, London, WC1E 7HT, UK

${ }^{3}$ MRC-UVRI and LSHTM Uganda Research Unit, Entebbe, Uganda

${ }^{4}$ Department of Medicine, School of Medicine, College of Health Sciences, Makerere University, Kampala, Uganda

${ }^{5}$ Division of Infectious Diseases and International Medicine, Department of Medicine, University of Minnesota, Minneapolis, MN, 55455, USA

v2

\author{
First published: 19 Aug 2019, 4:123( \\ https://doi.org/10.12688/wellcomeopenres.15389.1)
}

Latest published: 30 Sep 2019, 4:123(

https://doi.org/10.12688/wellcomeopenres.15389.2)

Abstract

Background: The diagnostic utility of the Mycobacteria tuberculosis lipoarabinomannan (TB-LAM) antigen lateral flow assay on cerebrospinal fluid (CSF) for the diagnosis of tuberculous meningitis (TBM) has not been extensively studied and the few published studies have conflicting results. Methods: Lumbar CSF from 59 HIV-positive patients with suspected TBM was tested with TB-LAM and Xpert MTB/Rif Ultra. The diagnostic performance of CSF TB-LAM was compared to positive CSF Xpert MTB/Rif Ultra (definite TBM) and a composite reference of probable or definite TBM according to the uniform case definition.

Results: Of 59 subjects, 12 (20\%) had definite TBM and five (9\%) had probable TBM. With reference to definite TBM, CSF TB-LAM assay had a diagnostic sensitivity of $33 \%$ and specificity of $96 \%$. When compared to a composite reference of definite or probable TBM, the sensitivity was $24 \%$ and specificity was $95 \%$. There were two false positive tests with TB-LAM (3+ grade). In-hospital mortality in CSF TB-LAM positive patients was $17 \%$ compared to $0 \%$ in those with definite TBM by Xpert MTB/Rif Ultra but negative LAM.

Conclusions: Lumbar CSF TB-LAM has a poor performance in diagnosing TBM. Both urine TB-LAM and Xpert Ultra should be further investigated in the diagnosis of TBM.

\section{Keywords}

Tuberculous meningitis, extra-pulmonary TB, lipoarabinomannan, TB-LAM, Xpert MTB/Rif Ultra, HIV, Diagnostics, cerebrospinal fluid

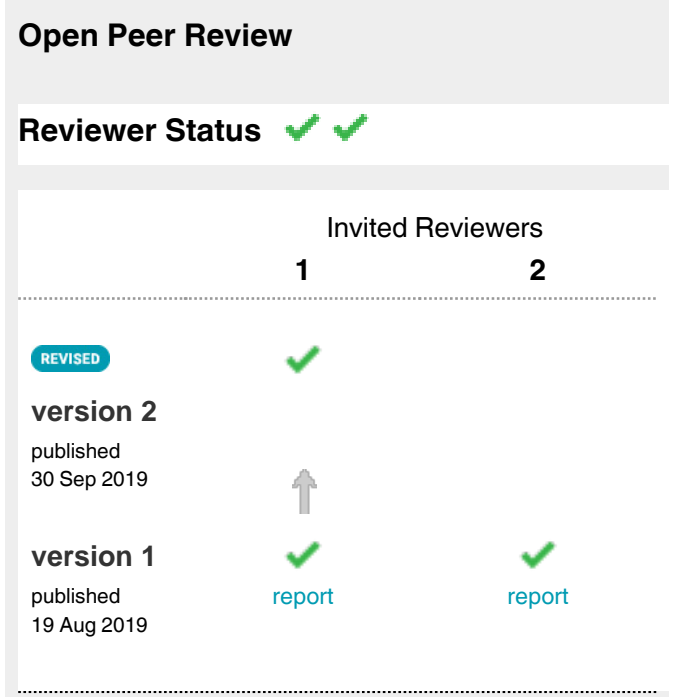

1 Maryline Bonnet (iD), Institute of Research for Development (IRD), Montpellier, France

2 Vinod Patel, University of KwaZulu-Natal (UKZN), Durban, South Africa

Any reports and responses or comments on the article can be found at the end of the article. 
Corresponding author: Richard Kwizera (kwizerarichard@ymail.com)

Author roles: Kwizera R: Conceptualization, Data Curation, Formal Analysis, Funding Acquisition, Investigation, Methodology, Writing - Original Draft Preparation, Writing - Review \& Editing; Cresswell FV: Conceptualization, Formal Analysis, Funding Acquisition, Methodology, Project Administration, Resources, Supervision, Writing - Original Draft Preparation, Writing - Review \& Editing; Mugumya G: Formal Analysis, Investigation, Validation, Writing - Review \& Editing; Okirwoth M: Formal Analysis, Investigation, Methodology, Writing - Review \& Editing; Kagimu E: Data Curation, Investigation, Methodology, Writing - Review \& Editing; Bangdiwala AS: Data Curation, Formal Analysis, Methodology, Validation, Writing - Review \& Editing; Williams DA: Methodology, Project Administration, Resources, Supervision, Writing - Review \& Editing; Rhein J: Project Administration, Resources, Supervision, Validation, Writing - Review \& Editing; Boulware DR: Funding Acquisition, Project Administration, Resources, Supervision, Validation, Writing - Review \& Editing; Meya DB: Funding Acquisition, Project Administration, Resources, Supervision, Validation, Writing - Review \& Editing

Competing interests: No competing interests were disclosed.

Grant information: This work was supported by the Wellcome Trust [107742, 107743 and 210772]. This research was also supported in part by the National Institute of Neurologic Diseases and Stroke (NINDS) and Fogarty International Center [R01NS086312, K01TW010268]. DBM and RK are currently supported through the DELTAS Africa Initiative grant [DEL-15-011] to THRiVE-2, from Wellcome Trust grant [107742] and the UK government. FVC is supported through a Wellcome Clinical PhD Fellowship [210772]. FVC is an honorary fellow of the Makerere University - UVRI Centre of Excellence for Infection and Immunity Research and Training (MUll-plus). MUll-plus is supported through the DELTAS Africa Initiative [107743]. The DELTAS Africa Initiative is an independent funding scheme of the African Academy of Sciences (AAS), Alliance for Accelerating Excellence in Science in Africa (AESA) and supported by the New Partnership for Africa's Development Planning and Coordinating Agency (NEPAD) with funding from the Wellcome Trust [107743] and the UK Government. The MRC/UVRI and LSHTM Uganda Research Unit is jointly funded by the UK Medical Research Council (MRC) and the UK Department for International Development (DFID) under the MRC/DFID Concordat agreement and is also part of the EDCTP2 programme supported by the European Union.

The funders had no role in study design, data collection and analysis, decision to publish, or preparation of the manuscript.

Copyright: $\odot 2019$ Kwizera $R$ et al. This is an open access article distributed under the terms of the Creative Commons Attribution License, which permits unrestricted use, distribution, and reproduction in any medium, provided the original work is properly cited.

How to cite this article: Kwizera R, Cresswell FV, Mugumya G et al. Performance of Lipoarabinomannan Assay using Cerebrospinal fluid for the diagnosis of Tuberculous meningitis among HIV patients [version 2; peer review: 2 approved] Wellcome Open Research 2019, 4 :123 (https://doi.org/10.12688/wellcomeopenres.15389.2)

First published: 19 Aug 2019, 4:123 (https://doi.org/10.12688/wellcomeopenres.15389.1) 


\section{REVISED Amendments from Version 1}

Based on the two reviewers' comments to version 1, major changes were made to the conclusion and Table 1 while minor changes were made to the results section as seen in the tracked changes. We added the suggested reference in the discussion section. Table 1 was modified to present the characteristics of patients with diagnosis of definite, probable, possible and not-TBM. A sentence was added on cerebral imaging. We also added a sentence about the patients who were positive with urine LAM and those who were positive with urine Ultra. We added an explanation about the $17 \%$ of patients had unknown outcome. A limitation was added that TB culture was not used. However, we have found Ultra to be more sensitive than culture $(70 \%$ versus $43 \%$ against definite/probable) for the diagnosis of TBM in our population (Bahr, Lancet ID, 2018). In the conclusion section, we have mentioned the Fuji LAM that is a new LAM POC test that has higher sensitivity in urine than the determine LAM POC and should also be evaluated for diagnosis of TB meningitis both in urine and CSF. Lastly, based on the second reviewer's comments, we would like to say that a cellular CSF is common in this population with advanced HIV disease even in the setting of a confirmed TB or CM infection (Cresswell, Int J Infect Dis 2018). The not-TBM group scored $<6$ points on the uniform case definition $(n=16)$ or had a confirmed alternative diagnosis $(n=10$ with $\mathrm{CM})$.

Any further responses from the reviewers can be found at the end of the article

\section{Introduction}

In many human immunodeficiency virus (HIV) endemic countries, tuberculous meningitis (TBM) is the second most common cause of adult meningitis after cryptococcal meningitis ${ }^{1}$, and accounts for $1-5 \%$ of all tuberculosis (TB) cases ${ }^{2}$. TBM is the most severe form of $\mathrm{TB}$ and causes substantial morbidity and mortality in children and immunocompromised adults ${ }^{3,4}$. HIV infection is known to increase the risk of death in patients with TBM, as does TBM stage at the time of treatment initiation $^{2,5}$. As is the case in cryptococcosis, high-quality nursing care is a critical component in managing TBM patients ${ }^{6}$.

Similarly, diagnosis of TBM is very challenging, especially in resource-limited settings where diagnosis relies on a combination of clinical, radiological and laboratory findings. The World Health Organisation (WHO) recommends Xpert MTB/RIF Ultra for the diagnosis of TBM using cerebrospinal fluid (CSF). Culture has many limitations related to turnaround time and sensitivity, and also requires considerable infrastructure and costs $^{7}$. Therefore, the development of early point of care diagnosis for TBM is a priority. Recent studies have demonstrated that the next generation Xpert MTB/RIF Ultra is the most sensitive diagnostic test in HIV-positive adults ${ }^{7}$. However, Xpert MTB/RIF Ultra is not a bedside test, and thus access to same day results remain a challenge in many settings?

Assays based on the detection of mycobacterial lipoarabinomannan (TB-LAM) antigen in urine have emerged as potential point-of-care tests for extra-pulmonary $\mathrm{TB}^{8}$. There is evidence that urine TB-LAM may help to reduce mortality and predict poor outcomes ${ }^{9,10}$. The WHO recently added the TB-LAM assay onto its essential diagnostic list and recommended TB-LAM in hospitalised HIV positive adults with signs and symptoms of $\mathrm{TB}^{11,12}$. However, there are conflicting results about TB-LAM assay sensitivity for TBM diagnosis ${ }^{13}$ in CSF. With reference to definite TBM, Cox et al. found a $75 \%$ sensitivity using CSF from the fourth ventricle in an autopsy cohort from 91 HIV-infected adults ${ }^{14}$. However, Bahr et al. had no positive TB-LAM tests using lumbar CSF from 67 HIV patients with meningitis ${ }^{13}$. In light of these results, and now that Xpert MTB/RIF Ultra is used instead of Xpert MTB/RIF, we aimed to further explore the utility of CSF TB-LAM test for the diagnosis of TBM among HIV-positive adults presenting with suspected meningitis.

\section{Methods}

Study setting and participants

Between April 2018 and June 2019, we assessed and performed diagnostic lumbar punctures on HIV-positive patients admitted to Mulago National Referral Hospital with suspected meningitis in Kampala, Uganda. Screening for TBM was performed cross-sectionally as part of the High Dose Rifampicin for Tuberculous Meningitis (RIFT) trial (ISRCTN registration number ISRCTN42218549, last updated 24/04/2018) $)^{15}$. Therefore, we did not calculate a sample size for the current study but included all participants that fit the screening criteria for the RIFT trial ${ }^{15}$. All included participants were HIV-infected adults ( $\geq 18$ years old) who provided written informed consent by participant or surrogate, with a suspected diagnosis of TBM (meningitis symptoms, clinical signs of meningism). Demographic information and baseline characteristics for participants were collected through clinical reviews using customized meningitis screening case report forms approved by the relevant ethics committees (Mulago Hospital Research Ethics Committee, Uganda National Council of Science and Technology, and the University of Minnesota). Opening pressures for CSF were measured using a manometer, followed by standard microbiology analysis (CrAg, cell count, protein, glucose, lactate, culture).

\section{Diagnostic tests}

In addition to standard microbiology analysis, CSF was tested with TB-LAM (Alere, Massachusetts, USA), and the test strip interpreted as per manufacturer's instructions. Briefly, the protective foil cover was removed from each test and the strip labelled with the participant's number. Two drops (or $60 \mu \mathrm{L}$ ) of CSF were added to the sample pad. The test was then read after 25 minutes under standard indoor lighting conditions. The reference card was used in interpretation of the results by holding it alongside the patient window. For positive results, purple/gray bars appeared in both the control window and the patient window of the strip. For negative results, one purple/ gray bar appeared in the control window of the strip and no bar appeared in the patient window of the strip. If there was no bar 
in the control window of the strip, the result was considered invalid and the test repeated. The strips were retained and cross checked by a second researcher to corroborate the finding.

CSF was also tested with Xpert MTB/Rif Ultra (Cepheid). Briefly, $2 \mathrm{ml}$ of sample reagent was added to $1 \mathrm{ml}$ of whole CSF and then left to stand at room temperature for 15 minutes. Then, $2 \mathrm{ml}$ of the sample mixture was transferred into the Xpert MTB/Rif Ultra cartridge and loaded into the Xpert machine. The test was run for 90 minutes and results from the assay indicate whether or not Mycobacteria TB (MTB) was detected in the sample. If MTB was detected, the results also stated whether resistance to rifampin was detected.

\section{Test analysis}

Data were analyzed using STATA version 14 (STATA, College Station, Texas). The disease prevalence, sensitivity, specificity, positive predictive values, negative predictive values and test accuracy were estimated at $95 \%$ confidence interval (CI). The diagnostic performance of CSF TB-LAM was compared to positive CSF Xpert MTB/Rif Ultra (definite TBM) and a composite reference of probable or definite TBM according to the uniform case definition ${ }^{16}$. Summaries were made in frequency \& percentages for each baseline characteristic considered as a categorical, and medians (interquartile range) when each characteristic is considered as a continuous variable. For baseline variables with some missing data, we calculated the statistics using the available numbers.

\section{Ethical statement}

Institutional review board approvals for the study and the associated screening process were obtained locally in Uganda (Mulago Hospital Research Ethics Committee, approval number MREC 1260); and from the London School of Hygiene and Tropical Medicine, UK (14388), University of Minnesota (1304M31361) and by the Uganda National Council of Science and Technology (HS136ES). Written informed consent for participation in the study and data publication was obtained from all participants or from their surrogates (e.g. family member or guardian) where the patient had altered mental status and did not have the capacity to provide consent.

\section{Results}

Overall, 59 HIV-positive hospitalized participants with suspected meningitis underwent diagnostic lumbar punctures, of which 20\% (12/59) had definite TBM, 9\% (5/59) had probable TBM, 25\% (15/59) had possible TBM, and 46\% (27/59) had not-TBM ${ }^{17}$. Of those with not-TBM $(\mathrm{n}=27), 10$ had cryptococcosis. Women comprised $50 \%$ of participants with an overall median age for all participants of 33 years (interquartile range [IQR]: 28,40$)$. Only $29 \%$ of the participants were receiving antiretroviral therapy at diagnosis. Among participants reporting a headache $(n=57)$, the median duration of headache was 14 days (IQR: 14, 24). The CSF opening pressures at baseline $(n=45)$ had a median of $200 \mathrm{mmH}_{2} \mathrm{O}$ (IQR: 120, 260). Overall, 55\% $(\mathrm{n}=36)$ had an acellular CSF, whilst those with a CSF lymphocytic pleocytosis had a median CSF white blood cell of 160 cells/ $\mu \mathrm{L}$ (IQR: 135, 268) (Table 1). Only about $10 \%$ of the participants had cerebral imaging done as the CT scanner was dysfunctional for part of the study period. About twenty five percent of the patients had a positive TB-LAM while $20 \%$ had a positive urine MTB/Rif Ultra.

With respect to the reference standard of definite TBM (positive CSF Xpert TB/Rif Ultra), the CSF TB-LAM assay had a sensitivity of $33 \%$ (4/12), specificity of $96 \%$ (45/47), positive predictive value (PPV) of 67\% (4/6), and negative predictive value (NPV) of $85 \%$ (45/53). When compared to a composite reference of definite/probable TBM, the TB-LAM assay had a sensitivity of $24 \%$ (4/17), specificity of $95 \%$ (40/42), PPV of 67\% (4/6), NPV of 76\% (40/53) (Table 2). There were two false positive tests with TB-LAM (3+ grade), without any CSF pleocytosis, normal protein, normal glucose, negative cryptococcal antigen, and normal CSF opening pressure. One patient was discharged alive without TB therapy. The second patient had a headache for 60 days at presentation, but they were lost to follow up (i.e. self-discharged) without an etiologic diagnosis. In-hospital mortality in CSF TB-LAM positive patients was $17 \%(1 / 6)$ compared to $0 \%(0 / 8)$ in those with definite TBM by Xpert MTB/Rif Ultra but negative LAM. About $17 \%$ of patients had unknown outcome. This was because the study population included patients screened for a clinical trial but only a minority were subsequently enrolled into the trial. We endeavoured to follow screen failures through to hospital discharge but this was not possible in all cases.

\section{Conclusion}

In conclusion, a rapid CSF point of care test for TBM is needed; however, this study demonstrated a poor diagnostic performance of the existing Alere TB-LAM on CSF among HIV-associated tuberculous meningitis. Our results corroborate the findings of a recent Zambian study which demonstrated 22\% sensitivity for CSF LAM against a reference standard of TB culture ${ }^{18}$. While the relatively modest sample size is a limitation, a larger sample size is unlikely to fundamentally alter the findings of sensitivity. One explanation could be that TB-LAM is likely not be found in sufficient quantities in lumbar CSF. TB culture was not used, which is also a limitation of the accuracy analysis. However, Xpert Ultra has a sensitivity that is greater than culture in our setting?. The novel Fujifilm SILVAMP TB-LAM (FujiLAM) assay has been shown to have higher sensitivity in urine than the Alere TB-LAM and 
Table 1. Characteristics of the study population.

\begin{tabular}{|c|c|c|c|c|c|c|c|c|c|c|}
\hline \multirow[t]{2}{*}{ Baseline characteristics } & \multicolumn{2}{|c|}{ Overall $(\mathrm{N}=59)$} & \multicolumn{2}{|c|}{ Definite TBM $(n=12)$} & \multicolumn{2}{|c|}{ Probable TBM $(n=5)$} & \multicolumn{2}{|c|}{ Possible TBM $(n=15)$} & \multicolumn{2}{|c|}{ Not-TBM $(n=27)$} \\
\hline & $\mathbf{N}^{*}$ & Statistic & $\mathbf{N}^{*}$ & Statistic & $\mathbf{N}^{*}$ & Statistic & $\mathbf{N}^{*}$ & Statistic & $\mathbf{N}^{*}$ & Statistic \\
\hline Women, n (\%) & 58 & $29(50)$ & 12 & $6(50)$ & 5 & $3(60)$ & 15 & $4(26.7)$ & 26 & $16(61.5)$ \\
\hline Age in years, median (IQR) & 58 & $33(28-40)$ & 12 & $29(28-33)$ & 5 & $26(24-34)$ & 15 & $35(32-43)$ & 26 & $34(26-46)$ \\
\hline On ART, n (\%) & 47 & $29(62)$ & 11 & $7(63.6)$ & 2 & $2(100)$ & 11 & $3(27.3)$ & 23 & $17(73.9)$ \\
\hline Headache, n (\%) & 57 & $46(81)$ & 12 & $10(83.3)$ & 5 & $4(80)$ & 14 & $12(85.7)$ & 26 & $20(76.9)$ \\
\hline $\begin{array}{l}\text { Duration of headache, } \\
\text { median (IQR) days }\end{array}$ & 45 & $14(14-24)$ & 10 & $17.5(14-30)$ & 4 & $14(10.5-17.5)$ & 12 & $17.5(14-31.5)$ & 19 & $14(7-30)$ \\
\hline $\begin{array}{l}\text { Glasgow Coma Scale score, } \\
\text { mean (SD) }\end{array}$ & 55 & $13(2.6)$ & 12 & $12.5(2.9)$ & 5 & $11.8(2.4)$ & 14 & $12.7(2.9)$ & 24 & $14.3(2.1)$ \\
\hline CSF CrAg positive, n (\%) & 58 & $10(17)$ & 12 & $0(0)$ & 5 & $0(0)$ & 15 & $0(0)$ & 26 & $10(38.4)$ \\
\hline $\begin{array}{l}\text { CSF Opening Pressure, } \\
\text { median (IQR) mmH2o }\end{array}$ & 45 & $200(120-260)$ & 7 & $180(70-240)$ & 3 & $260(95-400)$ & 13 & $190(120-270)$ & 22 & $215(120-260)$ \\
\hline Acellular CSF, n (\%) & 55 & $36(55)$ & 11 & $3(27.3)$ & 5 & $1(20)$ & 14 & $11(78.6)$ & 25 & $21(84)$ \\
\hline $\begin{array}{l}\text { CSF WBC in those with CSF } \\
\text { WBC pleocytosis, median } \\
\text { (IQR) cells/ } / \mu \mathrm{L}\end{array}$ & 55 & $160(135-268)$ & 8 & $280(162.5-575)$ & 4 & $173(130-237.5)$ & 3 & $80(35-160)$ & 4 & $145(87.5-210)$ \\
\hline $\begin{array}{l}\text { CSF protein, median (IQR) } \\
\text { mg/dL }\end{array}$ & 52 & $57(28-141)$ & 11 & $184(107-316)$ & 5 & $158(147-215)$ & 13 & $44(35-72)$ & 23 & $31(22-61)$ \\
\hline $\begin{array}{l}\text { CSF glucose, median (IQR) } \\
\text { mg/dL }\end{array}$ & 32 & $65(34-82)$ & 7 & $44(19.8-61)$ & 3 & $90(68-108)$ & 8 & $86(56.3-104)$ & 14 & $61(31-80)$ \\
\hline $\begin{array}{l}\text { CSF lactate, median (IQR) } \\
\mathrm{mmol} / \mathrm{L}\end{array}$ & 36 & $3.9(2.2-9)$ & 8 & $9.7(8.2-11.2)$ & 4 & $9.2(6.3-11.1)$ & 8 & $3.4(2.3-8.1)$ & 16 & $2.4(1.9-3.8)$ \\
\hline $\begin{array}{l}\text { Duration of hospitalization, } \\
\text { median (IQR) days }\end{array}$ & 46 & $7(4-14)$ & 9 & $11(9-14)$ & 4 & $14.5(10-16.5)$ & 9 & $4(4-14)$ & 24 & $5(2-12.5)$ \\
\hline $\begin{array}{l}\text { Status at discharge } \\
\text { Alive, n (\%) } \\
\text { Dead, n (\%) } \\
\text { Unknown, n (\%) }\end{array}$ & 59 & $\begin{array}{l}40(68) \\
9(15) \\
10(17)\end{array}$ & 12 & $\begin{array}{l}8(66.7) \\
3(25) \\
1(8.3)\end{array}$ & 5 & $\begin{array}{l}2(40) \\
1(20) \\
2(40)\end{array}$ & 15 & $\begin{array}{l}8(53.3) \\
2(13.3) \\
5(33.3)\end{array}$ & 27 & $\begin{array}{l}22(81.5) \\
3(11.1) \\
2(7.4)\end{array}$ \\
\hline
\end{tabular}

Data presented are percentages (\%), medians and interquartile ranges (IQR). $N=$ number of participants with data for each parameter. * Participants with data available. ART $=$ antiretroviral therapy, $\mathrm{CSF}=$ cerebrospinal fluid, $\mathrm{WBC}=$ white blood cells.

Table 2. Summary of diagnostic performance of cerebrospinal fluid mycobacterial lipoarabinomannan assay for tuberculous meningitis.

\begin{tabular}{|l|c|c|c|c|c|c|}
\hline Reference standard & $\begin{array}{c}\text { Disease } \\
\text { prevalence }\end{array}$ & Sensitivity & Specificity & PPV & NPV & Test Accuracy \\
\hline $\begin{array}{l}\text { Definite/probable } \\
\text { TBM }\end{array}$ & $28.8 \%(17 / 59)$ & $23.5 \%(4 / 17)$ & $95.2 \%(40 / 42)$ & $66.7 \%(4 / 6)$ & $75.5 \%(40 / 53)$ & $74.6 \%(44 / 59)$ \\
\hline $\mathbf{9 5 \%}$ Cl & 17.8 to $42.1 \%$ & 6.8 to $49.9 \%$ & 83.8 to $99.4 \%$ & 28.7 to $90.8 \%$ & 70.1 to $80.2 \%$ & 61.6 to $85 \%$ \\
\hline Definite TBM & $20.3 \%(12 / 59)$ & $33.3 \%(4 / 12)$ & $95.7 \%(45 / 47)$ & $66.7 \%(4 / 6)$ & $84.9 \%(45 / 53)$ & $83.1 \%(49 / 59)$ \\
\hline $\mathbf{9 5 \%}$ Cl & 10.9 to 32.8\% & 9.9 to $65.1 \%$ & 85.5 to $99.5 \%$ & 29.3 to 90.6\% & 78.9 to $89.4 \%$ & 71 to 91.6\% \\
\hline
\end{tabular}

Data presented are the percentage, numerator/denominator, and 95\% confidence intervals $(\mathrm{CI})$. Test Accuracy $=$ overall probability that a patient will be correctly classified. PPV $=$ Positive predictive value, NPV = negative predictive value, TBM = tuberculous meningitis.

warrants evaluation for diagnosis of $\mathrm{TB}$ meningitis both in urine and $\mathrm{CSF}^{19}$.

\section{Data availability}

Underlying data

Figshare: CSFLAM_data set revised.xlsx. https://doi.org/10.6084/ m9.figshare.9415853.v1 $1^{17}$
Data are available under the terms of the Creative Commons Zero "No rights reserved" data waiver (CC0 1.0 Public domain dedication).

Acknowledgements

We thank institutional support from the IDI research Appendix 3 


\section{Intensified antibiotic treatment of tuberculous meningitis}

ID of request: 13495

Date of request: 4 th June, 2018

Date of completion: 6th July, 2018

If you would like to request any articles or any further help, please contact: Tom Roper at tom.roper@bsuh.nhs.uk

Please acknowledge this work in any resulting paper or presentation as: Evidence search: Intensified antibiotic treatment of tuberculous meningitis. Tom Roper. (6th July, 2018).

BRIGHTON, UK: Brighton and Sussex Library and Knowledge Service.

\section{Sources searched}

EMBASE (87)

MEDLINE (155)

Date range used (5 years, 10 years): No restrictions

Limits used (gender, article/study type, etc.): None

Search terms and notes (full search strategy for database searches below):

MEDLINE and EMBASE were searched with text word and controlled vocabulary terms. Duplicates were removed, but no relevance screen undertaken. References supplied by the search requester were used to make a test set, against which iterations of the strategy could be tested.

A search of Cochrane Central located four papers, all of which were also retrieved by the MEDLINE and EMBASE searches.

Some commentary on the search strategy may be helpful. Taking MEDLINE as the examples, lines 1-3 locate the concept of tuberculous meningitis, while lines 5-7 locate antibiotic treatment. I then combined those concepts with AND and then the string intensif*, This is the most simple form of the search and returns 11 results in MEDLINE, and exactly the same number in EMBASE.

I then added further lines to expand the concept of intensified treatment. Line 23 looks for increased doses, while lines 24 and 25 look for combinations of drugs. Lines 26 to 40 retrieve the specific drugs you mentioned, and lines 41 and 42 find other anti-tubercular treatments that might be used. The final lines bring all these together.

For more information about the resources please go to: https://www.bsuh.nhs.uk/library/.

\section{Contents}

\section{B. Search History}

Source Criteria

Results

1. Medline "TUBERCULOSIS, MENINGEAL"/

6850

2. Medline ((TB OR tubercul*) ADJ2 mening*).ti,ab

6694

3. Medline ((TB OR tubercul*) ADJ2 (brain OR cerebral OR neurological)).ti,ab

929

4. Medline (1 OR 2 OR 3)

5. Medline exp ANTI-BACTERIAL AGENTS / 
6. Medline ((anti-bacterial OR anti-mycobacterial OR antibacterial OR antimycobacterial OR bacteriocidal) ADJ1 (agent ${ }^{\star}$ OR compound*)).ti,ab

9703

7. Medline (antibiotic ${ }^{\star}$ OR anti-biotic* OR anti-microbial OR antimicrobial ${ }^{\star}$ ).ti,ab

391914

8. Medline (5 OR 6 OR 7)

849977

9. Medline (4 AND 8)

2673

10. Medline (intensif $\left.{ }^{\star}\right) . t i, a b$

27057

11. Medline (9 AND 10)

11

12. EMBASE "TUBERCULOUS MENINGITIS"/

6409

13. EMBASE ((TB OR tubercul*) ADJ2 mening $\left.{ }^{\star}\right) . t i, a b$

5995

14. EMBASE ((TB OR tubercul*) ADJ2 (brain OR cerebral OR neurological)).ti,ab

831

15. EMBASE (12 OR 13 OR 14)

8843

16. EMBASE exp "ANTIBIOTIC AGENT"/

1222782

17. EMBASE ((anti-bacterial OR anti-mycobacterial OR antibacterial OR

11832

18. EMBASE (antibiotic* OR anti-biotic* OR anti-microbial OR antimicrobial ${ }^{\star}$ ).ti,ab

516096

19. EMBASE (16 OR 17 OR 18)

20. EMBASE (15 AND 19)

1422112

1892

21. EMBASE (intensif*).ti,ab

48787

22. EMBASE (20 AND 21)

11

((increas* OR higher OR greater OR larger OR non-standard OR

23. Medline nonstandard) ADJ2 (dose OR course OR treatment OR therapy OR regimen)).ti,ab

24. Medline "DRUG THERAPY, COMBINATION"/

198218

156462

25. Medline ((combination OR additional) ADJ2 (dose OR course OR treatment OR therapy OR regimen)).ti,ab

98861

26. Medline ISONIAZID/

27. Medline (isoniazid).ti,ab

14320

28. Medline ETHAMBUTOL/

3764

29. Medline

(ethambutol).ti,ab

4769

30. Medline PYRAZINAMIDE/

3075

31. Medline

(pyrazinamide).ti,ab

3471

32. Medline LINEZOLID/

2608

33. Medline (linezolid).ti,ab

4950

34. Medline (moxifloxacin).ti,ab

4006

35. Medline LEVOFLOXACIN/

2920

36. Medline (levofloxacin).ti,ab

6618

37. Medline

STREPTOMYCIN/

21618

38. Medline

(streptomycin).ti,ab

19951

39. Medline

ETHIONAMIDE/

1273

40. Medline

(ethionamide).ti,ab

895

41. Medline

exp "ADMINISTRATION, INTRAVENOUS"/

136688

42. Medline (intravenous ADJ (adminstration OR infusion OR dose OR course OR treatment OR therapy OR regimen)).ti,ab

33103

43. Medline (23 OR 24 OR 25)

423600

44. Medline (41 OR 42)

158915 
45. Medline

(26 OR 27 OR 28 OR 29 OR 30 OR 31 OR 32 OR 33 OR 34 OR 35 OR 36 OR 37 OR 38 OR 39 OR 40)

66653

46. Medline exp "ANTIBIOTICS, ANTITUBERCULAR"/

41976

47. Medline

((antitubercular OR tuberculostatic) ADJ (treatment OR therapy OR regimen)).ti,ab

1152

48. Medline (46 OR 47)

43043

49. Medline (45 OR 48)

102036

50. Medline (43 OR 44) AND 49

10355

51. Medline (9 AND 50)

158

((increas* OR higher OR greater OR larger OR non-standard OR

52. EMBASE nonstandard) ADJ2 (dose OR course OR treatment OR therapy OR 183035 regimen)).ti,ab

53. EMBASE *"DRUG COMBINATION"/

2250

54. EMBASE ((combination OR additional) ADJ2 (dose OR course OR treatment OR

121867

55. EMBASE ISONIAZID/

54009

56. EMBASE (isoniazid).ti,ab

17629

57. EMBASE ETHAMBUTOL/

27872

58. EMBASE (ethambutol).ti,ab

6840

59. EMBASE PYRAZINAMIDE/

22124

60. EMBASE (pyrazinamide).ti,ab

4700

61. EMBASE LINEZOLID/

17476

62. EMBASE (linezolid).ti,ab

7197

63. EMBASE (moxifloxacin).ti,ab

5733

64. EMBASE LEVOFLOXACIN/

31026

65. EMBASE (levofloxacin).ti,ab

10460

66. EMBASE STREPTOMYCIN/

51063

67. EMBASE (streptomycin).ti,ab

18204

68. EMBASE ETHIONAMIDE/

5294

69. EMBASE (ethionamide).ti,ab

1009

70. EMBASE "INTRAVENOUS DRUG ADMINISTRATION"/

370088

71. EMBASE (intravenous ADJ (adminstration OR infusion OR dose OR course OR

35033

72. EMBASE (52 OR 53 OR 54)

301692

73. EMBASE (70 OR 71)

389820

74. EMBASE (55 OR 56 OR 57 OR 58 OR 59 OR 60 OR 61 OR 62 OR 63 OR 64 OR 65

150357

75. EMBASE exp "TUBERCULOSTATIC AGENT"/

150733

76. EMBASE $\begin{aligned} & \text { ((antitubercular OR tuberculostatic) ADJ (treatment OR therapy OR } \\ & \text { regimenti,ab }\end{aligned}$

1504

77. EMBASE (75 OR 76)

151192

78. EMBASE (74 OR 77)

225605

79. EMBASE (72 OR 73) AND 77

7168

80. EMBASE 20 AND 79

89 


\section{2 approved]}

Fiona V. Cresswell (D) 1,2, Kenneth Ssebambulidde (D)2, Daniel Grint (D)3, Lindsey te Brake ${ }^{4}$, Abdul Musabire ${ }^{2}$, Rachel R. Atherton (iD) 2 , Lillian Tugume ${ }^{2}$, Conrad Muzoora ${ }^{5}$, Robert Lukande ${ }^{6}$, Mohammed Lamorde ${ }^{2}$, Rob Aarnoutse ${ }^{4}$, David Meya ${ }^{2,7}$, David R. Boulware (D) 2,7* Alison M. Elliott (iD) 1,8*

${ }^{1}$ Clinical Research Department, London School of Hygiene and Tropical Medicine, London, WC1E 7HT, UK

${ }^{2}$ Clinical Research, Infectious Diseases Institute, Kampala, Uganda

${ }^{3}$ Tropical Epidemiology Group, London School of Hygiene and Tropical Medicine, London, WC1E 7HT, UK

${ }^{4}$ Department of Pharmacy, Radboud University Medical Centre, Nijmegan, Netherlands

${ }^{5}$ Mbarara University of Science and Technology, Mbarara, Uganda

${ }^{6}$ Department of Pathology, College of Health Sciences, Makerere University, Kampala, Uganda

${ }^{7}$ Division of Infectious Diseases, University of Minnesota, Minneapolis, USA

${ }^{8} \mathrm{MRC}$ - UVRI - LSHTM Uganda Research Unit, Entebbe, Uganda

*Equal contributors

V1

First published: $10 \mathrm{Jul}$ 2018, 3:83 (doi: 10.12688/wellcomeopenres.14691.1) Latest published: 10 Jul 2018, 3:83 (doi: 10.12688/wellcomeopenres.14691.1)

\section{Abstract}

Background: Tuberculous meningitis (TBM) has 44\% (95\%Cl 35-52\%) in-hospital mortality with standard therapy in Uganda. Rifampicin, the cornerstone of TB therapy, has $70 \%$ oral bioavailability and $\sim 10-20 \%$ cerebrospinal fluid (CSF) penetration. With current WHO-recommended TB treatment containing $8-12 \mathrm{mg} / \mathrm{kg}$ rifampicin, CSF rifampicin exposures frequently fall below the minimal inhibitory concentration for $M$. tuberculosis. Two Indonesian phase II studies, the first investigating intravenous rifampicin $600 \mathrm{mg}$ and the second oral rifampicin $\sim 30 \mathrm{mg} / \mathrm{kg}$, found the interventions were safe and resulted in significantly increased CSF rifampicin exposures and a reduction in 6-month mortality in the investigational arms. Whether such improvements can be replicated in an HIV-positive population remains to be determined.

Protocol: We will perform a phase II, open-label randomised controlled trial, comparing higher-dose oral and intravenous rifampicin with current standard of care in a predominantly HIV-positive population. Participants will be allocated to one of three parallel arms (I:I:I): (i) intravenous rifampicin $20 \mathrm{mg} / \mathrm{kg}$ for 2 -weeks followed by oral rifampicin $35 \mathrm{mg} / \mathrm{kg}$ for 6 -weeks; (ii) oral rifampicin $35 \mathrm{mg} / \mathrm{kg}$ for 8-weeks; (iii) standard of care, oral rifampicin $10 \mathrm{mg} / \mathrm{kg} /$ day for 8-weeks.

Primary endpoints will be: (i) pharmacokinetic parameters in plasma and CSF; (ii) safety. We will also examine the effect of higher-dose rifampicin on survival

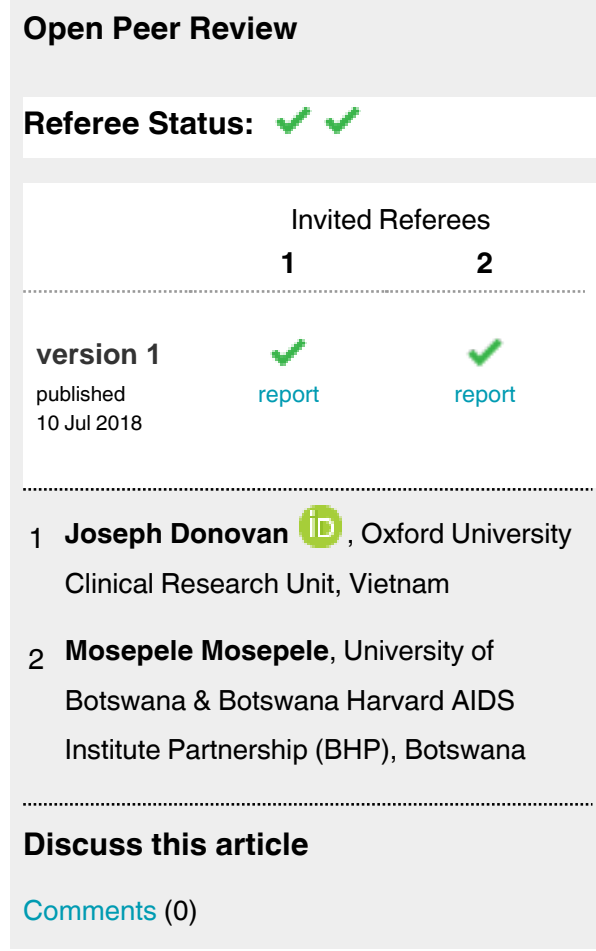


time, neurological outcomes and incidence of immune reconstitution inflammatory syndrome. We will enrol 60 adults with suspected TBM, from two hospitals in Uganda, with follow-up to 6 months post-enrolment.

Discussion: HIV co-infection affects the bioavailability of rifampicin in the initial days of therapy, risk of drug toxicity and drug interactions, and ultimately mortality from TBM. Our study aims to demonstrate, in a predominantly HIV-positive population, the safety and pharmacokinetic superiority of one or both investigational arms compared to current standard of care. The most favourable dose may ultimately be taken forward into an adequately powered phase III trial.

Trial registration: ISRCTN42218549 (24 ${ }^{\text {th }}$ April 2018)

Keywords

TBM, Tuberculous Meningitis, TB, rifampicin, Ultra, HIV

Corresponding author: Fiona V. Cresswell (fiona.cresswell@Ishtm.ac.uk)

Author roles: Cresswell FV: Conceptualization, Funding Acquisition, Methodology, Project Administration, Resources, Writing - Original Draft Preparation, Writing - Review \& Editing; Ssebambulidde K: Investigation, Methodology, Project Administration, Writing - Original Draft Preparation; Grint D: Methodology, Supervision, Writing - Review \& Editing; te Brake L: Conceptualization, Investigation, Methodology, Writing Review \& Editing; Musabire A: Investigation, Methodology, Project Administration, Writing - Review \& Editing; Atherton RR: Project Administration, Writing - Original Draft Preparation; Tugume L: Investigation, Methodology, Project Administration, Writing - Review \& Editing; Muzoora C: Investigation, Methodology, Project Administration, Resources; Lukande R: Investigation, Methodology, Project Administration; Lamorde M: Conceptualization, Methodology; Aarnoutse R: Conceptualization, Methodology; Meya D: Conceptualization, Investigation, Methodology, Project Administration, Resources, Supervision, Writing - Review \& Editing; Boulware DR: Conceptualization, Funding Acquisition, Investigation, Methodology, Project Administration, Resources, Supervision, Writing - Review \& Editing; Elliott AM: Conceptualization, Funding Acquisition, Project Administration, Resources, Supervision, Writing - Original Draft Preparation

Competing interests: Sanofi are donating IV and oral Rifidin for the purposes of the trial. Sanofi were not responsible for protocol design and will be independent from the analysis and reporting of the trial.

Grant information: The trial is funded through a Wellcome Trust Clinical PhD Fellowship [210772], sponsored by London School of Hygiene and Tropical Medicine, UK and hosted by the Infectious Diseases Institute, Uganda. The funders have had no role in the trial design, and will not be involved in the collection, analysis, and interpretation of data; in the writing of the report; or in the decision to submit the paper for publication. We acknowledge the support offered by the individual sites and staff at Mulago and Mbarara Hospitals; the Trial Steering Committee \& Data Safety Committee for monitoring the trial.

The funders had no role in study design, data collection and analysis, decision to publish, or preparation of the manuscript.

Copyright: @ 2018 Cresswell FV et al. This is an open access article distributed under the terms of the Creative Commons Attribution Licence, which permits unrestricted use, distribution, and reproduction in any medium, provided the original work is properly cited.

How to cite this article: Cresswell FV, Ssebambulidde K, Grint D et al. High dose oral and intravenous rifampicin for improved survival from adult tuberculous meningitis: a phase II open-label randomised controlled trial (the RifT study) [version 1; referees: 2 approved] Wellcome Open Research 2018, 3:83 (doi: 10.12688/wellcomeopenres.14691.1)

First published: 10 Jul 2018, 3:83 (doi: 10.12688/wellcomeopenres.14691.1) 


\section{Introduction}

\section{Background}

Worldwide, 10.4 million people are estimated to have fallen ill with Tuberculosis (TB) in 2016, resulting in 1.3 million deaths, and an additional 374,000 TB deaths in people living with $\mathrm{HIV}^{1}$. The African region carries the greatest burden relative to its population; 281 cases for every 100,000 people, more than double the global average of 133 per 100,000. Additionally, of the 1.2 million HIV-infected people who developed TB worldwide, $74 \%$ were in the African World Health Organisation (WHO) region ${ }^{2}$.

The true incidence of TB meningitis is not known due to lack of accurate diagnostics and scarce epidemiological data in many TB endemic regions. Central nervous system (CNS) TB accounts for $\sim 1 \%$ of notified $\mathrm{TB}$ cases in Germany and the USA, but this proportion is likely to be significantly higher in HIV endemic regions where TB frequently manifests as disseminated disease ${ }^{3,4}$. As such, in the wake of the HIV epidemic; TB meningitis (TBM) is one of the leading causes of meningitis in both adults and children in Africa ${ }^{5,6}$. It is estimated there is likely to be well over 100,000 cases annually worldwide.

\section{Prognosis of TBM}

$\mathrm{TBM}$, the most devastating form of $\mathrm{TB}$, is associated with a mortality of $7-28 \%$ of HIV-uninfected ${ }^{7}$, and $29-63 \%$ of HIVinfected patients ${ }^{8,9}$. The driver of this 2 to 3 -fold increased risk of death in HIV co-infection has not yet been elucidated. Neuro-disability is seen in up to $50 \%$ of survivors, regardless of their HIV serostatus. TBM cases account for up to $19 \%$ of all hospitalized HIV-associated TB cases and pose a significant challenge to healthcare services and care-givers, often requiring prolonged hospital stays and long rehabilitation periods ${ }^{10}$. Delays in seeking medical care, diagnosis, and initiation of treatment are contributing factors to the high morbidity and mortality, particularly in resource-limited settings.

\section{Clinical TBM management}

Early treatment with anti-tuberculous therapy and adjunctive corticosteroids is the most effective way of reducing death and disability from TBM. However, currently morbidity and mortality remains unacceptably high ${ }^{5}$. Whilst the $4^{\text {th }}$ edition of the WHO treatment guidelines suggest substituting ethambutol with streptomycin during the intensive phase of treatment this has not been widely adopted for a number of reasons, and treatment for TBM does not differ from that of pulmonary $\mathrm{TB}$, with rifampicin $(\mathrm{R})$, isoniazid $(\mathrm{H})$, pyrazinamide $(\mathrm{Z})$ and ethambutol (E) given for two months (intensive phase) followed by rifampicin and isoniazid (continuation phase), except that continuation phase is prolonged to complete 9-12 months ${ }^{2,11}$.

Due to variable TB drug penetration across the blood-brain barrier and blood-cerebrospinal fluid (CSF) barrier, optimal treatment regimens for pulmonary $\mathrm{TB}$ may not be the most effective options for TBM. Inadequate CNS drug penetration may be an important contributory factor to the high early mortality in $\mathrm{TBM}^{12}$. Improved early treatment outcomes may be achieved by altering drug selection, drug doses, and routes of administration to ensure adequate drug delivery to the site of disease and maximal early mycobactericidal activity in the CNS.

\section{Rifampicin in TBM}

Rifampicin is the cornerstone drug in the treatment of TBM, as evidenced by the fact that those infected with rifampicinresistant Mycobacterium tuberculosis (M.tb) strains have a near-universal fatal outcome, even with treatment with secondline drugs in resource-rich settings ${ }^{5,13,14}$. Mortality is not nearly so high in patients with isoniazid mono-resistant $\mathrm{TBM}^{6}$.

In addition to male sex and low body weight, which are associated with more rapid drug clearance ${ }^{15}$, malabsorption may contribute to reduced systemic drug bioavailability in HIV-infected persons ${ }^{16-21}$. A recent comprehensive meta-analysis of rifampicin pharmacokinetics confirmed that during the initial days of TB treatment rifampicin total plasma exposure to rifampicin is reduced in HIV co-infected adults (AUC $37.2 \mathrm{mg} . \mathrm{h} / \mathrm{L}$ in HIV-positive versus $56.7 \mathrm{mg} . \mathrm{h} / \mathrm{L}$ in HIV-negative, $\mathrm{p}=0.003$ ). Interestingly, this association did not persist in steady-state ( $>7$ days on therapy, once saturation of first-pass metabolism and the establishment of metabolic autoinduction is well established $)^{22}$. Lower plasma levels of antituberculous agents are predictive of poorer outcomes in pulmonary $\mathrm{TB}^{23}$, thus the lower plasma levels of rifampicin seen in HIV-positive individuals in the initial days of TBM therapy may be particularly relevant in TB meningitis, which carries a high early mortality. Specific factors that potentially play a role in reduced bioavailability include HIV-related enteric infections ${ }^{17,18}$, HIV-associated enteropathy and chronic intestinal immune activation affecting regulation of drug transporters. Patients with TBM often present while critically ill, are vomiting and frequently receive drugs through a nasogastric tube. Such factors may further associate with reduced TB drug concentrations, which could explain the lower plasma rifampicin concentrations observed in patients with TBM compared to those with pulmonary TB taking the same dose from the same setting ${ }^{24,25}$.

Rifampicin is largely protein bound and thus has limited CSF penetration, with reported CSF concentrations often reaching $10-20 \%$ of those in plasma ${ }^{25,26}$. Suggested target concentrations of rifampicin are $>8 \mathrm{mcg} / \mathrm{ml}$ at the site of disease, but CSF rifampicin concentrations seldom exceeded the minimum inhibitory concentration (MIC; $0.25 \mathrm{mcg} / \mathrm{ml}$ ) against $M$. tuberculosis at the standard oral adult dose $(8-12 \mathrm{mg} / \mathrm{kg})^{12}$. Currently, little is known about rifampicin concentrations in brain matter, spinal cord or meninges and how plasma levels or inflammation affect tissue concentrations.

The evidence for the currently recommended dose of rifampicin $(10 \mathrm{mg} / \mathrm{kg} / \mathrm{day})$ in TBM treatment is scant, and this dose falls on the steep part of the dose-response curve for sterilising effect ${ }^{27}$. Recent studies by the Pan-African Consortium for the Evaluation of Antituberculous Antibiotics in pulmonary TB have shown that rifampicin dosed at $35 \mathrm{mg} / \mathrm{kg}$ is associated with improved early bactericidal activity and 2-fold faster rate of sputum conversion (Hazard Ratio 1.99, 95\% CI:1.21-3.29) as compared to standard therapy. Furthermore, this dose was safe 
and well tolerated, with a $14 \%$ grade $3-5$ adverse event incidence as compared with $10 \%$ with standard 4-drug TB therapy with rifampicin dosed at $10 \mathrm{mg} / \mathrm{kg}^{28}$.

Ruslami et al. investigated the safety and pharmacokinetic (PK) profiles of higher than normal dose intravenous (IV) rifampicin as well as oral moxifloxacin in adults with TBM. Although not powered to detect a mortality benefit, improved survival was observed in patients receiving $600 \mathrm{mg}$ IV rifampicin $(\sim 13 \mathrm{mg} / \mathrm{kg} /$ day $)$ compared to the oral standard dose $(\sim 10 \mathrm{mg} / \mathrm{kg} /$ day $)$. Patients who received IV rifampicin had a more rapid resolution of coma (4 versus 5 days), reduced mortality at 8 -weeks $(24 \%$ versus $55 \%)$ and 6-months (34\% versus $65 \%$ ); (adjusted Hazard Ratio $=0.42$; 95\% CI 0.20-0.91, p=0.03) compared with those who received oral standard dose rifampicin. The change in rifampicin dose and mode of delivery was associated with a 3-fold increase in plasma area under the timeconcentration curve (AUC) and maximum concentration $\left(\mathrm{C}_{\max }\right)$ and a 3-fold increase in $\mathrm{CSF} \mathrm{C}_{\text {max }}$ from 0.21 to $0.60 \mathrm{mcg} / \mathrm{mL}$.

A second phase II study in Indonesia evaluating increased oral doses of $30 \mathrm{mg} / \mathrm{kg}$ or $20 \mathrm{mg} / \mathrm{kg}$ versus standard of care $(10 \mathrm{mg} / \mathrm{kg})$ also reported no increase in toxicity in the high dose arms and

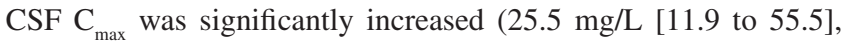
$18.1 \mathrm{mg} / \mathrm{L}$ [2 to 43.6 ], $7.2 \mathrm{mg} / \mathrm{L}$ [2.2 to 14.1 ] respectively, $<0.001$ ). In those with microbiologically confirmed TBM there was a trend towards lower 6-month mortality in the $30 \mathrm{mg} / \mathrm{kg}$ arm compared to standard of care (7\% versus $36 \%$ (HR 0.16 [0.02 to 1.34$], \mathrm{p}=0.09)^{29}$.

In contrast, a large Vietnamese trial of an intensified TBM treatment containing oral rifampicin $15 \mathrm{mg} / \mathrm{kg} /$ day versus standard of care showed no differential effect on mortality $(27.7 \%$ versus $27.9 \%$, hazard ratio $0.94 ; 95 \% \mathrm{CI} 0.73$ to $1.22 ; \mathrm{p}=0.66)^{30}$

The conflicting evidence from prior trials evaluating the benefits of higher than standard doses of rifampicin for TBM creates a situation of equipoise that can only be answered with an adequately powered phase III trial examining a rifampicin dose with proven pharmacokinetic-pharmacodynamic superiority to the standard of care. This phase II study aims to demonstrate pharmacokinetic superiority of one or both of the investigational arms so that the most favourable dose may ultimately be taken forward into a phase III study. Additionally, it is important to generate PK and safety data from HIV-positive African adults, as HIV co-infection can have a significant impact on TBM outcomes, pharmacokinetics and drug toxicity.

\section{Protocol}

This is version $1.2 .1,19^{\text {th }}$ February 2018.

\section{Hypotheses}

Our primary hypothesis is that intravenous rifampicin $(20 \mathrm{mg} / \mathrm{kg})$ and high dose oral rifampicin $(35 \mathrm{mg} / \mathrm{kg})$ will result in significantly increased plasma and CSF exposure during the critical early days of TBM treatment as compared to standard control (10mg/kg oral rifampicin).
Our secondary hypotheses are that high dose rifampicin will lead to improved early mycobacterial clearance from the CNS, reduced inflammatory response, and thereby will result in more rapid resolution of coma, improved long-term functional status, reduced TBM immune reconstitution inflammatory syndrome (IRIS) incidence and lower mortality.

\section{Main study objectives}

Primary objective. Our primary objective is to determine whether higher-dose rifampicin, delivered either orally at $35 \mathrm{mg} / \mathrm{kg} /$ day or intravenously at $20 \mathrm{mg} / \mathrm{kg} / \mathrm{day}$ for 2 -weeks (followed by orally at $35 \mathrm{mg} / \mathrm{kg} /$ day for 6-weeks) is safe and provides exposure profiles that are favourable compared to the $10 \mathrm{mg} / \mathrm{kg}$ standard dose oral rifampicin.

Secondary objectives. Our secondary objectives are to observe whether greater rifampicin exposure in CSF is associated with any clinical benefit including more rapid resolution of coma, improved long-term functional status, reduced TBM-IRIS incidence and lower mortality.

\section{Ancillary studies}

Two ancillary studies will be conducted within the RifT trial, the second of which will only take place at Mulago Hospital.

Evaluation of the diagnostic accuracy of Xpert MTB/Rif Ultra (Ultra) in TBM. Our hypothesis is that CSF Ultra is significantly more sensitive than CSF Xpert MTB/Rif or culture in the diagnosis of TBM.

Descriptive study of brain tissue rifampicin concentrations on autopsy specimens. Our hypothesis is that brain tissue concentrations will correlate with plasma and CSF concentration and will be significantly higher in the investigational arms.

\section{Design and setting}

RifT is a three arm, parallel group, phase II open label randomised controlled trial (see Figure 1), evaluating three rifampicin regimens over an 8-week intervention period, as follows:

A. Intravenous $20 \mathrm{mg} / \mathrm{kg} /$ day rifampicin for 2-weeks (followed by oral rifampicin $35 \mathrm{mg} / \mathrm{kg} /$ day for 6-weeks)

B. Oral $35 \mathrm{mg} / \mathrm{kg} /$ day rifampicin for 8 -weeks

C. Standard of care oral rifampicin $(\sim 10 \mathrm{mg} / \mathrm{kg} / \mathrm{day})$ for 8-weeks

All participants will receive a standard backbone of oral TB treatment (consisting of isoniazid $\sim 5 \mathrm{mg} / \mathrm{kg}$ /day, pyrazinamide $\sim 25 \mathrm{mg} / \mathrm{kg} / \mathrm{day}$, ethambutol $\sim 20 \mathrm{mg} / \mathrm{kg} /$ day) and daily IV dexamethasone $(0.4 \mathrm{mg} / \mathrm{kg} / \mathrm{day})$ for 1 week then weaned over a 6-8 week period, as per WHO guidelines.

The trial will be set in two hospitals in Uganda: Mulago National Referral Hospital and Mbarara Regional Referral Hospital. The study population will be adults ( $\geq 18$ years of age) with 


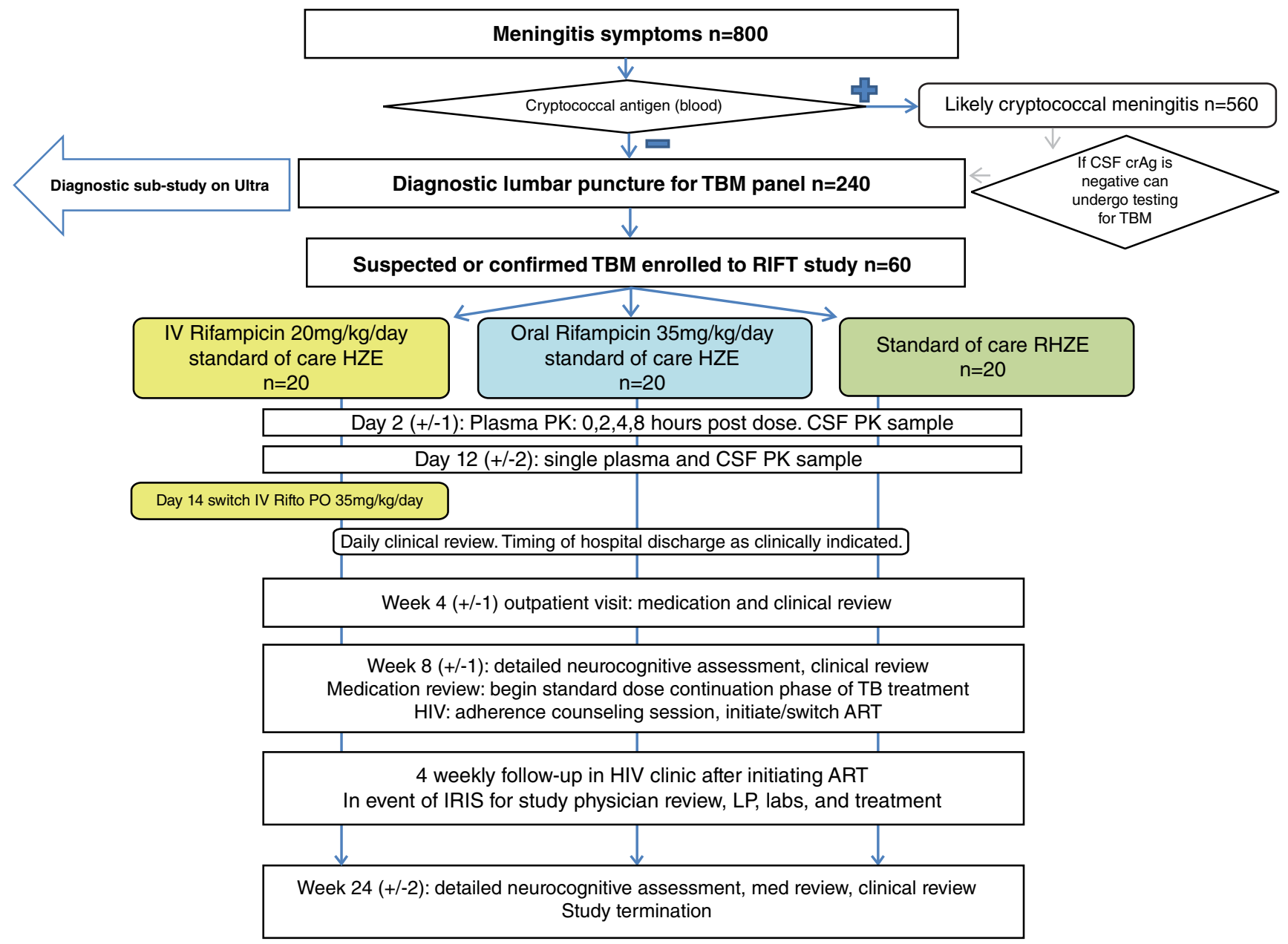

Figure 1. Study flow chart.

a diagnosis of TBM and treatment for TBM planned by the attending physician.

\section{Endpoints}

\section{Primary end points}

1. Pharmacokinetic parameters

Plasma and CSF rifampicin area under the curve $\left(\mathrm{AUC}_{0-24 \mathrm{~h}}\right)$, and maximum concentration $\left(\mathrm{C}_{\max }\right)$

\section{Adverse events}

We will use a composite safety endpoint for the 8-week intervention period that includes clinical grade 3-5 adverse events (AEs) (as classified by Division of AIDS (DAIDS) Toxicity Scale) or all serious adverse events (SAEs), drug-induced liver injury (DILI), grade 3-5 defined by alanine transaminase (ALT) $>5 \mathrm{x}$ upper limit of normal (ULN), or discontinuation of rifampicin for $>5$ consecutive days in the first 8 weeks for any cause.

\section{Secondary endpoints}

1. Mortality

Mortality at 8- and 24-weeks post randomisation will be compared between study arms.
2. Time to normalization of conscious level

We will determine days from randomisation until observation of a GCS of 15 which is achieved for $\geq 2$ consecutive days.

\section{Functional status}

The modified Rankin scale at 8 and 24 weeks will be used in determining functional outcomes.

\section{TB immune reconstitution inflammatory syndrome}

TB-IRIS will be diagnosed according to published case definitions ${ }^{31}$. TB-IRIS will be determined independently by 2 trial clinicians blinded to treatment allocation.

\section{Inclusion and exclusion criteria}

Inclusion criteria. Participants to be included in the study are consecutive patients $\geq 18$ years with clinically suspected TBM based on meningitis symptoms, clinical signs of meningism and anti-tuberculous chemotherapy planned by the attending physician. In addition, they must have either a bedside CSF glucose to plasma ratio $<50 \%$, or absolute CSF glucose $<40 \mathrm{mg} / \mathrm{dl}$ or $2.2 \mathrm{mmol} / \mathrm{l}$ or a positive CSF acid fast bacilli (AFB) smear or positive Xpert MTB/Rif or Ultra. Written informed consent must be given by either by the participant or by surrogate if the participant has altered mental state. 
Exclusion criteria. Patients with jaundice or known liver cirrhosis will not be eligible to enrol. Additionally, those who have received more than 3 doses of TB treatment within the previous 3 days, or have discontinued TB treatment in the prior 14 days, or those with known allergy to rifamycins, isoniazid, pyrazinamide, ethambutol or study drug excipients are not eligible. Those with known current/previous rifampicin drug-resistant $M$. tuberculosis infection or those with concurrent cryptococcal meningitis or those known to be currently taking any drug that has a clinically relevant interaction with rifampicin or other first-line TB drugs, including ritonavir, atazanavir or darunavir will not be eligible. Individuals who cannot or are unlikely to attend regular clinic visits or are pregnant or breastfeeding or where we lack consent from participant or family members are not eligible. Those with known porphyria or known chronic renal failure with $\mathrm{eGFR}<10 \mathrm{ml} / \mathrm{min}$ are not eligible.

\section{Randomisation and treatment allocation}

Adults who have given consent for, and undergone, a diagnostic screening lumbar puncture and are eligible for enrolment will then be approached for enrolment consent after which they will be randomised. Randomisation will occur at study entry and must occur prior to the 4th dose of TB treatment. Participants will be randomised by a computer generated permutated block randomization algorithm of different sized blocks with a 1:1:1 randomization ratio into the three arms described above.

Randomisation will be stratified by:

1. Clinical site

2. British Medical Research Council (BMRC) TBM disease grade I or II/III at time of consent

All randomised participants will initiate their allocated study TB treatment within 24 hours of randomisation, and preferably the same day. As TBM is a medical emergency, initiation of standard of care TB treatment will not be delayed whilst waiting for randomisation.

Treatment discontinuation. Due to the urgent need to begin TBM therapy to reduce mortality, enrolment will not be delayed whilst waiting for enrolment blood test results and participants will be replaced a posteriori if baseline ALT $>3 x$ ULN. As infection with a rifampicin resistant TB strain is an exclusion criterion, all patients in whom rifampicin resistant $\mathrm{TB}$ is identified after randomisation may have their treatment altered as per physician judgement. Once randomised, analysis is by modified intent-to-treat (ITT).

\section{Interventions}

Diagnostic lumbar puncture. At the time of screening, after informed consent has been received, a diagnostic lumbar puncture will be performed with a $\sim 10 \mathrm{ml} \mathrm{CSF}$ sample collected. The sample with then be tested with an enhanced TB diagnostic panel including Xpert MTB/Rif, Ultra, MGIT culture, Biofire meningoencephalitis multiplex polymerase chain reaction and next generation sequencing.

\section{Antituberculous therapy}

\section{Intravenous rifampicin arm (R20IV)}

For the first 14 days study drug will be administered as intravenous rifampicin $20 \mathrm{mg} / \mathrm{kg}$ once daily. Study medications will be dispensed during hospitalization to ward staff who will administer the drugs to the participants under directly observed therapy. Rifampicin $600 \mathrm{mg}$ will be reconstituted with $10 \mathrm{ml}$ of sterile water for a dilution of $60 \mathrm{mg}$ per $\mathrm{ml}$ of reconstituted solution. The weight-specific volume of rifampicin solution will be drawn up (Table 1) then further diluted in $500 \mathrm{ml} \mathrm{5 \%}$ Dextrose solution. The solution will be administered as a continuous infusion via a peripheral venous catheter over 2 hours. Thereafter the line will be flushed with $5 \mathrm{ml} 0.9 \%$ normal saline after which the cannula will be capped. Other antituberculous drugs (isoniazid, pyrazinamide and ethambutol) will be given orally by weight (Table 2) as intact tablets unless they are unable to swallow voluntarily, e.g. in participants with a depressed level of consciousness, in which case tablets will be crushed and given via nasogastric tube.

After 14 days of intravenous rifampicin the participant will be switched to high dose oral rifampicin $(35 \mathrm{mg} / \mathrm{kg}$ ) for the remaining 6 weeks of the intervention period administered as per the participants in the high dose oral rifampicin arm.

High dose oral rifampicin arm (R35PO) Oral rifampicin at $25 \mathrm{mg} / \mathrm{kg}$ for 8 weeks, in addition to standard fixed dose combination quadruple antituberculous tablets containing $\sim 10 \mathrm{mg} / \mathrm{kg}$ of rifampicin, will be administered for the first 8 weeks. Fixed-dose combination tablets according to weight bands as presented (Table 3) will be dispensed with additional 300mg oral rifampicin tablets to make the rifampicin dose up to $\sim 35 \mathrm{mg} / \mathrm{kg} /$ day. All study drugs will be taken orally during this period (either as intact tablets or via nasogastric tube). Study drugs will be administered under directly observed therapy by nursing staff whilst the participant remains in hospital. After discharge study drugs will be dispensed to participants at the week 4 visit. Fixed dose combination antituberculous therapy will be prescribed through routine care pathways initially via the hospital and subsequently via linked TB clinics according to National Guidelines. Participants will be weighed at each visit and TB drug dose will be adjusted as necessary. At the end of the intervention period (week 8) the participant begins standard of care continuation phase TB treatment (Table 4).

Control arm, standard of care arm (R10PO) The control arm will receive standard of care TB treatment: Oral rifampicin $\sim 10 \mathrm{mg} / \mathrm{kg}$, isoniazid $\sim 5 \mathrm{mg} / \mathrm{kg}$, pyrazinamide $\sim 25 \mathrm{mg} / \mathrm{kg}$, ethambutol $\sim 20 \mathrm{mg} / \mathrm{kg}$ in fixed-dose combination tablets according to weight bands for 8 weeks (Table 4). Participants will receive intact tablets or via nasogastric tube. Fixed dose combination antituberculous therapy will be prescribed through routine care pathways initially via the hospital and subsequently via at TB clinics according to National Guidelines.

Pharmacokinetic sampling. Intensive plasma PK sampling will take place on day $2(+/-1)$ at hours $0,2,4$ and 8 post dose 
Table 1. Intravenous administration of rifampicin by weight for day 0-14 in the intravenous rifampicin arm (arm R20IV).

\begin{tabular}{|c|c|c|c|c|c|}
\hline Weight (Kg) & $\begin{array}{l}\text { Rifampicin dose } \\
(\mathrm{mg})\end{array}$ & \multirow{22}{*}{ 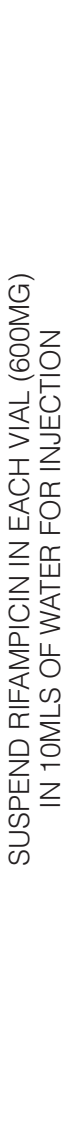 } & $\begin{array}{l}\text { Volume } \\
\text { used }(m L)\end{array}$ & $\begin{array}{l}\text { Number of } \\
\text { vials used }\end{array}$ & \multirow{22}{*}{ 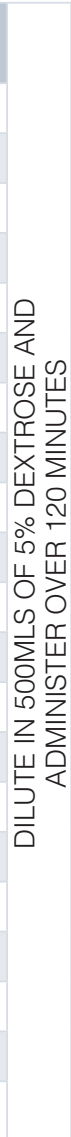 } \\
\hline $28.5-31.49$ & 600 & & 10 & 1.0 & \\
\hline $31.5-34.49$ & 660 & & 11 & 1.1 & \\
\hline $34.5-37.49$ & 720 & & 12 & 1.2 & \\
\hline $37.5-40.49$ & 780 & & 13 & 1.3 & \\
\hline $40.5-43.49$ & 840 & & 14 & 1.4 & \\
\hline $43.5-46.49$ & 900 & & 15 & 1.5 & \\
\hline $46.5-49.49$ & 960 & & 16 & 1.6 & \\
\hline $49.5-52.49$ & 1020 & & 17 & 1.7 & \\
\hline $52.5-55.49$ & 1080 & & 18 & 1.8 & \\
\hline $55.5-58.49$ & 1140 & & 19 & 1.9 & \\
\hline $58.5-61.49$ & 1200 & & 20 & 2.0 & \\
\hline $61.5-64.49$ & 1260 & & 21 & 2.1 & \\
\hline $64.5-67.49$ & 1320 & & 22 & 2.2 & \\
\hline $67.5-70.49$ & 1380 & & 23 & 2.3 & \\
\hline $70.5-73.49$ & 1440 & & 24 & 2.4 & \\
\hline $73.5-76.49$ & 1500 & & 25 & 2.5 & \\
\hline $76.5-79.49$ & 1560 & & 26 & 2.6 & \\
\hline $79.5-82.49$ & 1620 & & 27 & 2.7 & \\
\hline $82.5-85.49$ & 1680 & & 28 & 2.8 & \\
\hline $85.5-88.49$ & 1740 & & 29 & 2.9 & \\
\hline $88.5-91.49$ & 1800 & & 30 & 3.0 & \\
\hline
\end{tabular}

\begin{abstract}
Table 2. Daily number of tablets of isoniazin, pyrazinamide and ethambutol by weight during the first 14 days for the intravenous rifampicin study arm (arm R20IV).
\end{abstract}

\begin{tabular}{|c|c|c|c|}
\hline $\begin{array}{c}\text { Baseline } \\
\text { weight } \mathbf{( k g})\end{array}$ & $\begin{array}{c}\text { ISONIAZID } \\
\mathbf{1 0 0} \mathbf{~ m g}^{*}\end{array}$ & $\begin{array}{c}\text { PYRAZANAMIDE } \\
\mathbf{5 0 0} \mathbf{~ m g}^{*}\end{array}$ & $\begin{array}{c}\text { ETHAMBUTOL } \\
\mathbf{4 0 0} \mathbf{~ m g}\end{array}$ \\
\hline $\mathbf{3 0 - 3 3}$ & 1.5 & 1.5 & 1 \\
\hline 34 & 1.5 & 1.5 & 2 \\
\hline $35-44$ & 2 & 2 & 2 \\
\hline $45-54$ & 2.5 & 2.5 & 2 \\
\hline 55 & 3 & 3 & 2 \\
\hline $56-64$ & 3 & 3 & 3 \\
\hline 65 & 3 & 3.5 & 3 \\
\hline$\geq 66$ & 3.5 & 3.5 & 3 \\
\hline
\end{tabular}

as well as a single CSF sample. Where possible a single plasma and CSF sample will be collected on day 14 (+/- 2 days).

Blood test monitoring. Routine blood monitoring will take place on days $1,3,7$ and 14 , week 4 and week 8 to monitor for any rifampicin-related toxicity (Table 5 and Table 6).
Neurocognitive assessment. Where possible a detailed neurocognitive assessment will take place on week 8 and week 24 .

Antiretroviral therapy. HIV-positive participants who are ART naïve or who have defaulted ART will initiate ART after completion of the intensive phase of TB treatment (at week 8) 
Table 3. Daily administration of oral rifampicin during the first 8 weeks in the high dose oral rifampicin arm (R35PO) and week 2-8 in arm R20IV.

\begin{tabular}{|l|c|c|c|}
\hline Weight & $\begin{array}{c}\text { Number of RHZE tabs } \\
(\mathbf{1 5 0 / 7 5 / 4 0 0 / 2 7 5 ~} \mathbf{~ m g})\end{array}$ & $\begin{array}{c}\text { Additional rifampicin } \\
\text { 300 } \mathbf{m g} \text { tablets }\end{array}$ & $\begin{array}{c}\text { Total rifampicin } \\
\text { dose (mg) }\end{array}$ \\
\hline $30-37 \mathrm{~kg}$ & 2 tabs & 3 tabs & 1200 \\
\hline $38-54 \mathrm{~kg}$ & 3 tabs & 4 tabs & 1650 \\
\hline $55-70 \mathrm{~kg}$ & 4 tabs & 5 tabs & 2100 \\
\hline$\geq 71 \mathrm{~kg}$ & 5 tabs & 6 tabs & 2550 \\
\hline
\end{tabular}

Table 4. Daily number of fixed dose tablet in the control arm (arm R10PO) during week $0-8$ and for all arms during the continuation phase of treatment.

\begin{tabular}{|c|c|c|c|}
\hline \multirow[t]{2}{*}{ Weight } & \multirow{2}{*}{$\begin{array}{l}\text { Intensive Phase (week 0-8) } \\
\text { RHZE fixed dose combination daily } \\
\left(150 / 75 / 400 / 275 \mathrm{mg}^{3}\right.\end{array}$} & \multicolumn{2}{|c|}{$\begin{array}{l}\text { Continuation Phase } \\
\text { (month 3-12) }\end{array}$} \\
\hline & & $\mathrm{RH}(150 / 75)^{3}$ & RH $(300 / 150)^{3}$ \\
\hline $30-37 \mathrm{~kg}$ & 2 tabs & 2 tabs & \\
\hline $38-54 \mathrm{~kg}$ & 3 tabs & 3 tabs & \\
\hline $55-70 \mathrm{~kg}$ & 4 tabs & & 2 tabs \\
\hline$\geq 71 \mathrm{~kg}$ & 5 tabs & & 2 tabs \\
\hline
\end{tabular}

in accordance with WHO and Ugandan guidelines. An efavirenz -based regimen or dose-adjusted (twice daily) dolutegravirbased regimen would be used in accordance with Ugandan HIV treatment guidelines (currently under revision) ${ }^{32}$. Participants who were enrolled on a failing efavirenz-based regimen will be switched at week 8 to a dose-adjusted dolutegravir-based regimen. Current protease inhibitor or nevirapine-based ART is an exclusion criteria so we do not anticipate having participants on these agents. All participants will be registered at the Infectious Diseases Institute HIV clinic or the Mbarara Hospital HIV clinic such that their HIV care lies within the national HIV service framework.

\section{Adverse events and safety reporting}

The principles of ICH GCP require that both investigators and sponsors follow specific procedures when notifying adverse events or reactions in clinical trials. The definitions of the EU Directive 2001/20/EC Article 2 based on the principles of ICH GCP apply to this trial protocol. All adverse events will be assessed for seriousness, causality and expectedness. Causality in relation to study drug (rifampicin) is assessed as unrelated, unlikely, possible, probable or definite based on temporal relationship and clinical judgement. If the event is serious and unrelated or unlikely to be related it is classified as an SAE. If the event is possibly, probably or definitely related it is classified as a Serious Adverse Reaction (SAR). Expectedness of the adverse reaction is assessed using the summary of product characteristics (SPC) at the time of the event. An unexpected adverse reaction is one not previously reported in the $\mathrm{SPC}$, or one that is more frequent or more severe than previously reported. If a SAR is assessed as being unexpected it becomes a suspected unexpected serious adverse reaction (SUSAR). Intensity will be graded using the DAIDS toxicity scale.

The Trial Management Group at the Infectious Diseases Institute (IDI) must be notified of all grade 3-5 AEs, SAEs and SARs within 24 hours, thereafter they are responsible for reporting SAEs, SUSARs to the Sponsor, regulatory authorities and ethics committees in accordance with local regulatory and institutional guidelines.

Interim analyses. Interim analysis of safety will occur after 24 participants ( 8 per arm) have completed the week 8 visit. The committee can modify the frequency of interim analysis and early termination could occur if the data safety committee decides there is an unacceptable level of toxicity in any of the investigational arms.

\section{Data collection}

Baseline and subsequent assessment. Participants will be followed as in-patients for the first 14 days and then approximately 4 weekly as outpatients until week 24, see Table 5 and Table 6. If a trial participant dies during the in-patient period additional consent will be sought from family members for a post-mortem examination to explore cause of death and levels of rifampicin in brain tissue. Recruitment will continue for 12-18 months with an additional 6 months follow-up period. The trial will be considered closed when the last participant has completed 24 weeks in the study, all SAEs resolved and all follow-up and laboratory reports have been received. 
Table 5. In-patient schedule of events.

\begin{tabular}{|c|c|c|c|c|c|c|c|c|}
\hline Study visit & Screening & Enrolment D1 & Day 2 & Day 3 & Day 7 & Day 10 & Day 14 & $\begin{array}{c}\text { Further } \\
\text { Hospitalization }\end{array}$ \\
\hline Visit window (days) & & & \pm 1 & \pm 1 & \pm 1 & \pm 1 & \pm 2 & \\
\hline Screening Consent & $x$ & & & & & & & \\
\hline Assess eligibility criteria & & $x$ & & & & & & \\
\hline Informed enrolment consent & & $x$ & & & & & & \\
\hline \multicolumn{9}{|c|}{ Clinical history and examination } \\
\hline Past medical history & $(X)$ & & & & & & & \\
\hline Medication review & $(X)$ & $x$ & $x$ & $x$ & $x$ & $x$ & $x$ & $x$ \\
\hline Document HIV status & $(X)$ & & & & & & & \\
\hline Current symptoms & $(X)$ & $x$ & $x$ & $x$ & $x$ & $x$ & $x$ & $x$ \\
\hline Examination & $(X)$ & $x$ & $x$ & $x$ & $x$ & $x$ & $x$ & $x$ \\
\hline GCS score & $(X)$ & $x$ & $x$ & $x$ & $x$ & $x$ & $x$ & $x$ \\
\hline BMRC disease grade & & $x$ & $x$ & $x$ & $x$ & $x$ & $x$ & $x$ \\
\hline Adverse events assessment ${ }^{b}$ & & $x$ & $x$ & $x$ & $x$ & $x$ & $x$ & $x$ \\
\hline \multicolumn{9}{|c|}{ Investigations } \\
\hline HIV-test (if not known positive) & $(X)$ & & & & & & & \\
\hline Cryptococcal antigen & $(X)$ & & & & & & & \\
\hline Sodium/Potassium & $x$ & $x$ & & & $x$ & & $x$ & $x$ \\
\hline Glucose (bedside) & $(X)$ & & & & & & & \\
\hline Creatinine $^{h}$ & $x$ & $x$ & & & $x$ & & $x$ & $x$ \\
\hline Hepatic panela & $x$ & $x$ & & $x$ & $x$ & & $x$ & $x$ \\
\hline Blood Count (including differential) & $x$ & $x$ & & & $x$ & & & $x$ \\
\hline CD4 count if HIV-positive & $x$ & $x$ & & & & & & \\
\hline Pregnancy test & Women & & & & & & & \\
\hline CSF sample (aim >10ml, as per SOP ${ }^{\circ}$ ) & $(X)$ & & $x^{d}$ & \multicolumn{3}{|c|}{$\begin{array}{l}\text { as clinically indicated } \\
\text { (storage of remaining } \\
\text { specimen) }\end{array}$} & $\begin{array}{c}X \\
\text { (Paired } \\
\text { plasma) }\end{array}$ & $\begin{array}{c}\text { X } \\
\text { (as clinically } \\
\text { indicated) }\end{array}$ \\
\hline Plasma PK sampling 0, 2, 4, 8 hrs & & & $x$ & & & & & \\
\hline Sparse plasma PK (one sample) & & & & & & & $x$ & \\
\hline Chest radiograph & $(+/-)$ & & & & & & & \\
\hline CT head & $+/-$ & $+/-$ & & & & & & \\
\hline Abdominal Ultrasound Scan & & $x$ & & & & & & \\
\hline Urine sample ${ }^{g}$ & $(X)$ & & & & & & & \\
\hline Blood/DNA/RNA storage & & with consent & & & & & & with consent \\
\hline Approx. volume blood (mL) & 0 & 20 & 20 & 3 & 7 & 0 & 7 & 7 \\
\hline
\end{tabular}

()$=$ part of routine medical care

${ }^{a}$ Hepatic panel $=$ alanine aminotransferase (ALT), alkaline phosphatase (ALP) and bilirubin. Hepatitis BsAg, Hepatitis C Ab and INR will be added if baseline ALT is elevated. In the event of DILI hepatic panel will be performed more regularly.

${ }^{\mathrm{b}}$ Adverse events will be recorded according to DAIDS toxicity scale

${ }^{c}$ SOP $=$ standard operating procedure detailing exact processing and testing algorithm of CSF

${ }^{d}$ CSF PK will be timing randomised to early (2-4hrs) or medium (4-6hrs) or late interval (6-8hrs) post dose.

Contrast enhanced CT head is indicated if there is focal neurology at baseline or during enrolment period

Urine sample may be collected for testing with LAM (lipoarobinmannan) or urine Xpert MTB/Rif Ultra as part of TB work-up

Additional renal monitoring will be undertaken in those with abnormal baseline creatinine

Baseline bloods must occur at either screening or enrolment visit. It is likely these visits will be on the same day. If baseline bloods were done at screening and enrolment occurs $>48$ hours later baseline blood tests will be repeated. 
Table 6. Out-patient schedule of events.

\begin{tabular}{|c|c|c|c|c|c|c|}
\hline Study visit & Week 4 & Week 8 & Week 12 & Week 18 & Week 24 & Sick Visit \\
\hline Visit window (weeks) & \pm 1 & \pm 1 & \pm 1 & \pm 2 & \pm 2 & As needed \\
\hline Dispensing of study drug & & $x$ & \multicolumn{4}{|c|}{$\begin{array}{l}\text { standard fixed dose therapy til } 12 \text { months as } \\
\text { per local guidelines }\end{array}$} \\
\hline Interim history & $x$ & $x$ & $x$ & \multirow{15}{*}{ 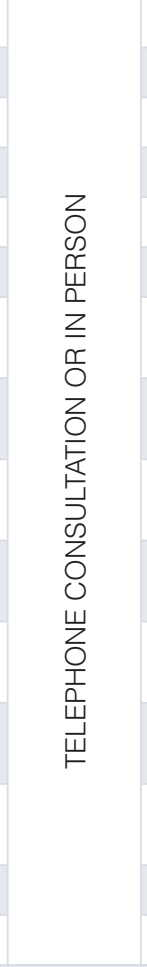 } & $x$ & $x$ \\
\hline Adverse events assessment & $x$ & $x$ & $x$ & & $x$ & $x$ \\
\hline Medication review & $x$ & $x$ & $x$ & & $x$ & $x$ \\
\hline Examination & $x$ & $x$ & $x$ & & $x$ & $x$ \\
\hline Modified Rankin score & & $x$ & & & $x$ & \\
\hline Detailed neurocognitive follow-up & & $x$ & & & $x$ & \\
\hline $\begin{array}{l}\text { Center for Epidemiological Studies } \\
\text { of Depression Scale (CES-D) }\end{array}$ & & $x$ & & & $x$ & \\
\hline Sodium, potassium & & & & & & $\begin{array}{l}X \text { (if clinically } \\
\text { indicated) }\end{array}$ \\
\hline Creatinine & & & & & & $\begin{array}{l}X \text { (if clinically } \\
\text { indicated) }\end{array}$ \\
\hline Hepatic Panel ${ }^{a}$ & $x$ & $x$ & & & & $\begin{array}{l}X \text { (if clinically } \\
\text { indicated })\end{array}$ \\
\hline FBC, differential ${ }^{b}$ & & & & & & $\begin{array}{l}X \text { (if clinically } \\
\text { indicated) }\end{array}$ \\
\hline Storage bloods ${ }^{c}$ & & $x$ & & & & $\begin{array}{l}\text { X (IRIS event } \\
\text { only) }\end{array}$ \\
\hline CSF with storage & & & & & & $\begin{array}{l}X \text { (if clinically } \\
\text { indicated) }\end{array}$ \\
\hline ART counselling ${ }^{d}$ & $x$ & $x$ & & & & \\
\hline Commence/switch ART ${ }^{d}$ & & $x$ & & & & \\
\hline Total volume of blood (ml) & & 20 & & & & 20 \\
\hline
\end{tabular}

aHepatic panel $=$ ALT, ALP, bilirubin +/- INR

${ }^{\mathrm{b}} \mathrm{FBC}$; full/complete blood count

'Blood will be collected and stored for research if storage consent has been received, refer to site SOP.

dHIV-infected patients not receiving effective ART, ART counselling to be performed by qualified counsellors as per standard clinic procedures. Physician discretion allowed for timing of ART initiation.

Data handling and data management. Source documents are made up of detailed case report forms (CRFs), laboratory results, radiology results and other relevant documents. Data entry will occur via the DataFax system, whereby the paper-based case record forms are scanned, emailed to a server, and data entered by intelligent character recognition. After an initial automated error-checking, secondary review for accuracy is then performed by the DataFax team at the Infectious Diseases Institute, Uganda. The DataFax system allows for automated data queries to alert for any missing data on an ongoing basis. Second, this also allows for permanent archiving and potential remote review by oversight bodies. Study forms will be harmonized between all study sites enabling multi-site data management. The investigator will retain study essential source documents for 20-years after the completion of the study, as per Ugandan guidelines. Digital images of the source documents will be retained for an indefinite period.
Quality control and assurance. Site monitoring is conducted to ensure that the human subject protection, study procedures, laboratory, study intervention administration, and data collection processes are of high quality and meet the sponsor, ICH E6 and regulatory guidelines. The study may be subject to audit by the London School of Hygiene \& Tropical Medicine under their remit as Sponsor, as well as other regulatory bodies to ensure adherence to Good Clinical Practice.

\section{Statistical considerations}

Sample size. Approximately 800 adults presenting with symptoms of meningitis will be assessed, of which it is anticipated two-thirds will have cryptococcal meningitis. The circa 240 patients with negative CSF cryptococcal antigen (crAg) will undergo a comprehensive TBM diagnostic panel (Xpert MTB/Rif, Xpert Ultra, mycobacterial growth indicator tube (MGIT) culture, and/or next generation sequencing) from which we anticipate 
60 people with suspected or confirmed TBM will be recruited into the randomised trial.

The sample size is determined following the assumption that PK parameters are normally distributed on the log scale. Estimated rifampicin log-transformed $\mathrm{C}_{\max }$ standard deviation is taken from Ruslami et al. ${ }^{1}$ The global study significance level (alpha) will be $10 \%$, which is appropriate for phase II studies. Using the Bonferonni correction for multiple testing, each individual comparison will be made with alpha of $5 \%$. Group sample sizes of $n=15$ achieve $90 \%$ power to reject the null hypothesis of equal means when the log-transformed population mean difference is $0.54 \mathrm{mg} / \mathrm{L}$ with standard deviation of $\pm 0.36 \mathrm{mg} / \mathrm{L}$ in the control group and $\pm 0.48 \mathrm{mg} / \mathrm{L}$ ( $33 \%$ higher) in the experimental group, with a significance level of 0.05 using a two-sided, two sample unequal variance t-test. If the standard deviation differs from our estimate, the overall detectable effect size at $90 \%$ power will be 1.5 times the standard deviation, and 1.27 times the standard deviation at $80 \%$ power. We will not formally compare equivalence of the two investigational arms as this would require a larger sample size. Comparison of the two investigational arms will be primarily descriptive.

This phase II study would be underpowered to detect a survival benefit unless the effect size is large. A one-sided log rank test with 20 subjects per arm provides $80 \%$ power at 0.05 significance to detect a hazard ratio of 0.285 when $40 \%$ of controls survive 8 -weeks. This assumes no more than 1 person drops out or is lost to follow-up per arm. The prior lost to follow up rate has been $<1 \%$ in recent Ugandan meningitis trials.

Statistical methods. After data cleaning, analysis will proceed according to the pre-specified analysis plan using Stata version 13. The trial will be reported in accordance with CONSORT guidelines and primary analyses will be conducted by the participant's originally assigned group (ITT). Analyses will be conducted on individuals from both study sites together and all analyses will be adjusted for site, BMRC grade and any baseline variables associated with missingness of the analysis outcome.

\section{Primary analysis}

\section{Pharmacokinetic analysis}

The following pharmacokinetic parameters will be calculated using standard two-stage approach involving calculation of pharmacokinetic parameters in those patients in whom a full PK curve was recorded:

1. $\mathrm{C}_{\max }$ - the peak concentration of the drug after administration

2. $\mathrm{T}_{\max }$ - the time to reach $\mathrm{C}_{\max }$

3. $\mathrm{AUC}_{0-24}$ - area under the time concentration curve from hour 0 to hour 24

4. Time $>$ MIC is the duration of time where the drug concentration level is greater than the minimal inhibitory concentration for the $M$. tuberculosis isolate (either the individual or the median MIC of the study population)

\section{Safety analysis}

The number and proportion of individuals who reported any kind of clinical grade 3-5 AE during the study period will be presented by intervention arm. Data for safety will be analysed in accordance with the ITT principle. The main analysis will be a composite safety endpoint over the 8-week intervention period:

- AEs, clinical Grade 3-5 as classified by DAIDS Toxicity Scale

- All SAEs

- DILI, grade 3-5 (ALT >3x ULN with symptoms or $5 \mathrm{x}$ ULN without symptoms)

- Discontinuation of rifampicin for $>5$ days in the first 8 weeks for any cause

Time to experiencing an adverse event will be compared between treatment arms using cumulative incidence functions and a Cox proportional hazards regression model adjusted for site and BMRC TBM grade. Death will be considered a competing risk. The individual subgroups of AEs that make up the composite endpoint will be summarised. A secondary analysis of rifampicin-related toxicity (probable or definitely related, as assigned on the adverse events case record form) will be presented by intervention arm.

\section{Clinical outcomes}

Survival at 8 and 24 weeks will be calculated using the risk difference, from a generalised linear regression model adjusted for site and BMRC TBM grade. Kaplan-Meier curves will also be used to compare time to death between treatment arms, with censoring and loss to follow-up handled as previously described. Cox proportion hazards regression will be used to assess the effects of rifampicin dose, plasma/CSF rifampicin concentration, HIV, MRC severity grade and baseline GCS on survival.

When calculating time to normalization of GCS continuousvalued secondary endpoints will be compared with general linear models or Wilcoxon rank-sum tests as appropriate. Death can be considered a competing risk. $\chi^{2}$ test will be used for comparison of proportions of patients with TBM-IRIS by intervention arm.

Secondary analysis. We will explore the relationship between rifampicin exposures and clinical outcomes using a population pharmacokinetic-pharmacodynamic model. We will consider $\mathrm{C}_{\max }$ and $\mathrm{AUC}_{0-24 \mathrm{~h}}$ as the exposure variables. Survival, proportion with normalisation of conscious level and functional status (as determined by modified Rankin score) are the response variables.

Planned subgroup analyses. Specified high-risk subgroups are of interest because of potential different responses to intravenous rifampicin from both an efficacy and safety perspective, and the clinical utility in future physician decision-making. With standard therapy, worse clinical outcome is associated 
with altered mental status, high organism burden. Subgroups of interest include:

1. TBM Diagnostic category (Definite, Probable, Possible) ${ }^{33}$

2. British Medical Research Council (BMRC) TBM disease severity $^{7}$

3. CSF inflammation (CSF white blood cells) by tertiles

4. CD4 at study entry

5. ART status at study entry

Analysis of ancillary studies. The results of ancillary studies will be reported separately from the main trial.

Findings of the diagnostic sub-study will be reported in line with the Standards for the Reporting of Diagnostic Accuracy studies (STARD) guidelines. Sensitivity and specificity will be calculated against a composite reference standard (any positive CSF test for $M$. tuberculosis) and against the published uniform case definition of 'probable' or 'definite' $\mathrm{TBM}^{33}$. A latent class analysis will also be performed.

The studies on brain tissue concentration at autopsy will be primarily descriptive.

\section{Ethical considerations}

Confidentiality. Participants will be identified only by means of a coded identification number specific to each participant. All participant-related information (including CRFs, laboratory results, radiology reports etc.) will be kept strictly confidential. All records will be kept in a secure, locked location and only research staff will have access to the records. With consent, information relevant for the future HIV care will be shared with relevant HIV clinic for continuity of care and patient safety.

All computerized databases will identify participants by numeric codes only, and will be password-protected. Upon request, participant records will be made available to the study sponsor, monitors, applicable regulatory entities, including the Uganda National Council of Science and Technology, Uganda National Drug Authority, Mulago Institutional Review Board, or London School of Hygiene and Tropical Medicine.

Consent. An estimated $>80 \%$ of the study population will have altered mental status at initial hospital presentation. Those presenting with meningitis and altered mental status are at the greatest risk of death, and enhanced TBM therapy may have the greatest benefit for them. This hypothesis deserves testing via inclusion into the clinical trial. Secondly, as most persons with TBM present with altered mental status, in order to make results generalizable to all TBM patients, we wish to offer enrolment to all persons. Subjects unable to give informed consent due to altered mental status may have surrogate consent provided by proxy from their caregiver/next of kin.

For subjects enrolled by surrogate consent, if and when the participant regains the physical and mental capacity to give consent, information will be provided to them and written informed consent will be sought for continuation in the trial. If a patient or representative declines to give consent for continuation at this stage, his/her wishes will be respected.

A person who speaks and understands the language of the informed consent document, but does not read and write, can be enrolled in a study by "making their mark" or via a thumbprint on the informed consent document. In this event, an impartial, literate third party must witness the entire consent process and sign the informed consent document. The witness's name, signature, and relationship must be recorded on the informed consent document. A member of the study team is not an impartial third party.

Supplementary File 1 contains the participant information sheet and consent form.

Sample use and storage. Additional consent will be sought for the long-term storage of samples (including blood, spinal fluid, DNA/RNA) for use in future research. In this case, samples will be stored for the purposes of future research, unless the subject asks for them to be destroyed. Alternatively, subjects may give limited consent to the collection and testing of samples for the purposes of this research only, after which the samples will be destroyed.

Withdrawals. Subjects may withdraw from the study at any time by withdrawing subject consent, and will be eligible to continue to receive TB treatment from a primary $\mathrm{TB}$ clinic of their choice. Subjects enrolled in the study but choosing to leave the hospital early against medical advice will continue to participate in the study if they wish. Additional phone calls by study personnel will encourage the subject to seek follow up TB care and to re-join the trial per the on-going schedule of events. Assessment of vital status will continue via telephone calls at a minimum, unless consent is completely withdrawn. Participants will be asked if they would like their accrued data to be destroyed.

Ethical approval. The investigators have obtained approval from the Research Ethics Committees of the London School of Hygiene and Tropical Medicine (14388), as well as the Mulago Hospital Institutional Review Board (MHREC1260), the Uganda National Council of Science and Technology, the Ugandan National Drug Authority and Mbarara Hospital.

Any further amendments will be submitted and approved by each ethics committee.

\section{Trial committees}

The trial sponsor is London School of Hygiene and Tropical Medicine (LSHTM; Keppel Street, London, WC1E 7HT, UK; Tel: 0207 6368636). The trial management group (TMG) will oversee day to day management of the trial and is formed of the principal investigators (PI), site PI in Mbarara, a neurologist and a statistical advisor. The TMG will meet weekly. The trial steering committee (TSC) has members of the TMG and independent members (Professor Guy Thwaites (Chair), Professor Alison Elliott, Professor Reinout van Crevel, Dr Joe Jarvis, Dr Frank 
Mugabe (Head of National TB and Leprosy Programme), a patient representative). The TSC provides supervision for the trial and advice through the independent Chair. The data safety committee (DSC) (Professor Robert Wilkinson (Chair), Dr William Worodria, Dr Mindy Clarke, Dr Agnes Kiragga (independent statistician), Dr Christine Sekaggya-Wiltshire) will advise the TSC regarding continuation, modification or premature closure of the trial. The DMC is independent from the Sponsor.

\section{Publication policy}

We will share results though presentations at scientific conferences and in peer-reviewed open-access journals. De-identified individual patient data will be stored on LSHTM secure data repository (LSHTM Data Compass) for patients who have given consent to data sharing at the time of enrolment.

\section{Discussion}

Tuberculous meningitis is highly fatal despite current WHO recommended therapy, particularly in HIV-positive people, who are 2-3 times more likely to die than their HIV-negative counterparts. Recent evidence from Indonesian phase II studies suggests that significantly higher dose IV or oral rifampicin enhances CNS penetration and may reduce mortality. However, there is currently no available pharmacokinetic data on highdose rifampicin for TBM in an HIV-positive African population. Furthermore, intravenous rifampicin is not widely available so finding a bioequivalent oral dose, that reaches target levels in the $\mathrm{CNS}$, is a priority.

In this phase II study we anticipate the sample size will not be sufficient to detect small differences in mortality between the treatment arms. However, the pharmacokinetic and pharmacodynamic data will be used to define optimal rifampicin dose, and route of administration in an HIV-positive population, and this will be taken forward into a larger phase III randomised controlled trial, if safe and tolerable.

\section{Data availability}

No data are associated with this article.

\section{Competing interests}

Sanofi are donating IV and oral Rifidin for the purposes of the trial. Sanofi were not responsible for protocol design and will be independent from the analysis and reporting of the trial.

\section{Grant information}

The trial is funded through a Wellcome Trust Clinical PhD Fellowship [210772], sponsored by London School of Hygiene and Tropical Medicine, UK and hosted by the Infectious Diseases Institute, Uganda.

The funders have had no role in the trial design, and will not be involved in the collection, analysis, and interpretation of data; in the writing of the report; or in the decision to submit the paper for publication.

We acknowledge the support offered by the individual sites and staff at Mulago and Mbarara Hospitals; the Trial Steering Committee \& Data Safety Committee for monitoring the trial.

\section{Acknowledgements}

FVC is an honorary fellow of the Makerere University - Uganda Virus Research Institute Centre of Excellence for Infection and Immunity Research and Training (MUII-plus). MUII-plus is supported through the DELTAS Africa Initiative (Grant no. 107743). The DELTAS Africa Initiative is an independent funding scheme of the African Academy of Sciences (AAS), Alliance for Accelerating Excellence in Science in Africa (AESA), and supported by the New Partnership for Africa's Development Planning and Coordinating Agency (NEPAD Agency) with funding from the Wellcome Trust (Grant no. 107743) and the UK Government. Dr Brigitte Demers (Sanofi Pasteur) has been instrumental is facilitating the donation of Rifidin. We acknowledge the institutional support of Dr Andrew Kambugu and the research office and the Infectious Diseases Institute as well as the clinical trials office at the London School of Hygiene and Tropical Medicine.

\section{Supplementary material}

Supplementary File 1: Participant information sheet and consent form.

Click here to access the data.

1. Organisation WH: Global Tuberculosis Report 2017. Geneva: World Health Organisation, 2017

Reference Source

2. Organisation WH: Global Tuberculosis Report 2016. Geneva, Switzerland: World Health Organisation, 2016.

Reference Source
3. Ducomble T, Tolksdorf K, Karagiannis I, et al:: The burden of extrapulmonary and meningitis tuberculosis: an investigation of national surveillance data, Germany, 2002 to 2009. Euro Surveill. 2013; 18(12): pii: 20436. PubMed Abstract | Publisher Full Text

4. Control CfD: Reported Tuberculosis in the United States, 2016. 2017. Reference Source 


\section{Appendix 6a}

Viral Causes of Meningitis Detected by Metagenomic Next-generation Sequencing in a Ugandan Tuberculous Meningitis Cohort

Presented at American Academy of Neurology 2020

Neurology. 2020;94(15)

Authors: Carson Quinn, Prashanth Ramachandran, Morris Rutakingirwa, Ananta Bangdiwala, Enock Kagimu, Kiiza Tadeo Kandole, Fiona Cresswell, David Meya, David Boulware, Michael Wilson

Objective: To identify viral infections by metagenomic next-generation sequencing (mNGS) in Ugandan HIV-infected patients with presumed tuberculous meningitis (TBM).

Background: Most Ugandan patients with HIV infection and meningitis whose CSF tests negative for cryptococcal antigen are treated as presumed TBM. Viral meningitis masquerading as TBM needs to be considered.

Design/Methods: CSF was obtained from 71 patients at Kiruddu National Referral Hospital in Kampala, Uganda, who were classified according to the Uniform Case Definition as definite $(n=16)$, probable $(n=7)$, or possible TBM $(n=48)$. After total RNA and DNA were extracted, and unbiased cDNA and DNA libraries were sequenced, viruses were identified using a custom bioinformatics pipeline.

Results: Viruses other than HIV-1 were identified in 37 patients, of which 6 were likely pathogenic (measles virus ( $n=2)$, Wesselsbron virus $(n=1)$, rubella virus $(n=1)$, herpes simplex virus (HSV)-1 $(n=1)$, and HSV-2 $(n=1)$ ). All $(n=6)$ were in patients classified as possible TBM (definite and probable TBM, $\mathrm{n}=0$ ). Only the HSV-2 infected patient received contemporaneous multiplex PCR testing given lack of clinical improvement on anti-tubercular therapy. The patient with HSV-1 presented with headache, fever, and focal neurologic deficits, followed by pneumonia and death. One patient with measles virus was co-infected with Cryptococcus neoformans and did not improve with antifungal treatment. The other measles virus-infected patient had seizures progressing to coma and death. The patient infected with rubella virus had cutaneous Kaposi Sarcoma and a milder presentation of meningism after initiating antiretrovirals. Wesselsbron virus, an endemic flavivirus, was found in a patient with meningism, confusion, and hepatomegaly, who died from respiratory failure despite anti-tubercular therapy.

Conclusions: The Uniform Case Definition of possible TBM, while sensitive, is not specific. mNGS of CSF identified alternate candidate viral etiologies in $12.5 \%(n=6)$ of possible TBM cases. Two infections (HSV-1, HSV-2) were treatable, and three (two measles virus and one rubella virus) were vaccine preventable. 
Appendix 6b

Improving Tuberculous Meningitis Diagnosis - A Combined Host and Pathogen Classifier

\section{Presented at American Academy of Neurology 2020.}

\section{Neurology. 2020;94(15).}

Authors: Prashanth Ramachandran, Akshaya Ramesh, Fiona Cresswell, Carson Quinn, Morris Rutakingirwa, Ananta Bangdiwala, Enock Kagimu, David Meya, Emily Crawford, David Boulware, Michael Wilson.

Objective: To assess the diagnostic performance of metagenomic next-generation sequencing (mNGS) combined with host differential gene expression for tuberculous meningitis (TBM) versus other causes of meningitis.

Background: GeneXpert RIF/MTB Ultra is 70\% sensitive for definite/probable TBM. Definite TBM is microbiologically proven. Probable TBM has negative microbiology but scores $\geq 10$ points on the case definition scale. 90-98\% of mNGS sequencing data reflect host gene expression. Host transcriptomic signatures may differ by pathogen and thus, enhance diagnostic utility. We assessed CSF mNGS and transcriptomics for identifying TBM and other pathogens misclassified as TBM.

Cohort: 157 HIV-infected Ugandan adults with sub-acute meningitis: Definite $(n=15)$, probable $(n=7)$, and possible ( $n=53$ ) TBM; non-TB meningitis ( $n=82$ ).

Method: Unbiased RNA and DNA libraries were sequenced. We performed metagenomic analysis through a custom bioinformatics pipeline. Transcriptomic diagnostic classifier was developed using 22 samples (11 TB + 11 other), which, along with cases with co-infections, were not included in the final analysis.

Results: mNGS was $80 \%$ concordant (12/15) against definite TBM with 3 additional cases of TBM detected (1 probable and 2 possible). Host transcriptomics displayed 83\% (5/6) sensitivity (inclusive of mNGS-positive TBM), 78\% (62/79) specificity, and 98\% negative predictive value. Among probable TBM cases the host transcriptomic classifier predicted 71\% (5/7) were TBM including the 1 mNGSpositive TBM case. Within possible TBM, mNGS identified 9 other pathogens ( 4 viral, 2 bacterial, 2 toxoplasmosis and 1 TBM-viral co-infection). The host transcriptomic signature classified 9/53 possible TBM cases as TBM, including the 1 mNGS-positive TBM case.

Conclusions: mNGS and host transcriptomics combined for $77 \%(17 / 22)$ sensitivity against definite/probable TBM. mNGS alone detected TB or alternate pathogens in 19\% (10/53) of possible TBM cases. Further optimization of the classifier is required to increase accuracy. The combined assay will be assessed on a validation cohort with results presented at the meeting. 


\section{Appendix 6c \\ Illuminating Tuberculous Meningitis with Metagenomics and FLASH Enrichment}

\section{Presented at American Academy of Neurology}

Authors: Prashanth Ramachandran, Fiona Cresswell, Charles Langelier, Saharai Caldera, Amy Lyden, Aaron McGeever, Josh Batson, Rene Sit, Norma Neff, Katherine Travisano, Lillian Khan, Joe DeRisi, David Boulware, Emily Crawford, Michael Wilson

Objective: To enhance detection of Mycobacterium tuberculosis meningitis (TBM) and anti-microbial resistance (AMR) mutations in HIV-infected Ugandan adults with meningitis, using metagenomic next generation sequencing (mNGS) of CSF coupled with CRISPR-Cas9 enrichment technology.

Background: TBM diagnosis is difficult. mNGS permits broad detection of CNS pathogens in a single assay. Further, targeted enrichment of low abundance genes by up to $10^{5}$-fold is possible with Finding Low Abundance Sequences by Hybridization (FLASH), which is a novel CRISPR-Cas9 technology. Combining these technologies could improve detection of TB and associated AMR mutations.

Design/Methods: Cohort: 15 patients with subacute meningitis: 6 definite TBM, 4 Cryptococcus (1 meningitis, 3 symptomatic antigenemia), 1 potential Cryptococcus-immune reconstitution inflammatory syndrome (IRIS), and 4 unknown

Method: DNA was extracted from CSF, and unbiased cDNA libraries were sequenced on an Illumina NextSeq. In parallel, FLASH enrichment was performed by incubating phosphatase blocked-DNA fragments with recombinant Cas9 and 314 computationally designed guide RNAs targeting 46 TBAMR genes, allowing adapter ligation of the exposed ends and preferential amplification.

Results: Unbiased mNGS: Low abundance (0.03-6.41 rpm) TB reads were identified in $100 \%$ of the definite TB samples ( $n=6$ ), and Cryptococcus neoformans was detected in $100 \%$ of cryptococcal cases $(n=4)$. Among the 4 unknowns, Cryptococcus $(n=1)$ and Toxoplasma gondii $(n=1)$ were detected. The first FLASH experiment demonstrated up to a 100-fold increase in TB read abundance in definite TBM cases and detection of an additional case of TBM that had initially been mis-classified as Cryptococcus paradoxical IRIS.

Conclusions: We report the development of a novel TBM and AMR CSF diagnostic protocol combining the agnostic ability of mNGS with FLASH, a CRISPR-Cas9 tool that enriches pathogen sequences of interest. These promising preliminary data suggest the possibility of developing an "all-in-one" assay to detect TB, any associated AMR genes and any additional or alternate infections. 